


$$
z-10
$$




A CATALOGUE

OF THE

\section{COLLECTION OF BIRDS}

FORMED BY THE LATE

HUGH EDWIN STRICKLAND, M.A. 
晒ondon: C. J. CLAY, M.A. \& SON, CAMBRIDGE UNIVERSTTY PRESS WAREHOUSE, 17, PAternoster Row.

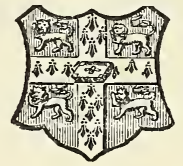

(Cambridge: DEIGHTON, BELL, AND CO. ILeipsin: F. A. BROCKHAUS. 
on that group, taken in conjunction with the special articles upon the various families and genera scattered through the work, furnishes the best guide at present extant to the classification of those interesting though lowly organisms. Upon Bacterium and the Schizomycetes we find a considerable quantity of new information brought in, as also upon the parasitic insects and Acarina and the Infusoria, derived from the recent publications of Mégnin and Andrew Murray upon the former groups, and from the valuable manual of the Infusoria of Mr. Saville Kent. We are sorry to note, however, that while fully availing himiself of the last-mentioned important work, the editor has entirely passed over the most magnificent work that has appeared of recent years upon any group of Protozoa, namely Prof. Leidy's 'Monograph of the Freshwater Rhizopoda of North America.' This is the more to be regretted as, since its publication, a manual founded upon it has been published in America, and there can be no doubt that many of the genera proposed by Prof. Leidy will be frequently referred to in the literature of the microscope.

We had noted several other points in which it seems to us that there is room for improvement; but fault-finding is an unsatisfactory business, and all the deficiencies that we could indicate would but very slightly derogate from the general excellence of the book. Its chief value consists in the immense mass of varied information upon all subjects of interest to microscopists, collected in its pages in a most convenient form for reference ; and from the mode of treatment adopted it is, as we have before pointed out, well fitted to serve as a guide in the investigation of many departments of natural history quite outside the domain of microscopic work. On this ground we can recommend it to all students of natural history, and especially to those located in country places at a distance from libraries. To such workers it will prove invaluable as a general book of reference.

The plates with which the volume is illustrated are for the most part the same as in the last edition; but five new ones have been added, bringing the whole number up to fifty-three, a large proportion of them coloured. The immense number of figures contained in these plates, with the numerous woodcuts scattered through the text, render this one of the best-illustrated volumes with which we are acquainted.

\section{A Catalogue of the Collection of Birds formed by the late Hugh} Edwin Strickland, M.A., F.R.S., \&c. By Osbert Salvin, M.A., F.R.S., Strickland Curator in the University of Cambridge. Cambridge University Press, 1882.

THE title of the present volume fully explains its contents, and renders much further explanation unnecessary. It is a descriptive catalogue of the extensive collection of birds formed by the late Mr. Strickland and bequeathed by his widow to the Cambridge Ann. \& Mag. N. Hist. Ser. 5. Vol. xi. 
University. Attached to the 'Catalogue' is a supplement or list showing the sources whence the specimens (about 6000 in number) were obtained. Such a work will greatly enhance the value of this collection, to which it forms an almost exhaustive guide; and as such it will doubtless be warmly welcomed by most working ornithologists. Appended to each species is the reference to its original description and to works containing its geographical distribution; but we think that the value and interest of the "Catalogue" would have been considerably increased if the latter item had been briefly sketched out in a similar manner to that in the British-Museum Catalogues of Birds. The general arrangement adopted, subject to certain neccssary modifications and additions, is that elaborated by Messrs. Sclater and Salvin in their 'Nomenclator Avium Neotropicalium,' which has for its basis the system of Huxley.

In the earlier portions of the work (the only ones at present we have had the opportunity of carefully examining) we notice that Mr. Salvin does not admit the distinctness of T'urdus magellanicus from Turdus fallitandicus. The latter bird is, we believe, an island form confined to the Falklands, whilst the former is found in various parts of South America. Again, we fail to see why the genus Merula should be disregarded when the genera Oreocincla, Geocichla, Petrocincla, and Zoothera are recognized. The name Oreocincla Heinii of Cabanis surely has the precedence over that of O. iodura of Gould, although the former naturalist erroneously gave "Japan" as the locality for his species. We also notice that Mr. Salvin (following Messrs. Blanford and Dresser in their celebrated 'Monograph of the Chats ') makes the Saxicola leucomela of Pallas synonymous with the Saxicola lugens of Lichtenstein, although these two birds are quite distinct. Again, upon what grounds is Cetti's Warbler included in the subfamily Ruticillinæ? MI. Salvin also makes this bird synonymous with the Bradypterus platurus (? platyurus) of Swainson. The type of this species (from S. Africa) is in the Cambridge Museum, and was identified as "nothing but Cetti's Warbler " by Mr. Dresser in his 'Birds of Europe,' a conclusion shown to be totally erroneous by Mr. Seebohm in 'The Ibis' for 1878, p. 380. Swainson's generic name will stand for this South-African species; but his specific name must give place (if the law of priority is enforced) to that bestowed by Vieillot; and it will consequently stand as Bradypterus brachypterus (Vieill.). Moreover Cetti's Warbler has no claim whatever to be included in the genus Bradypterus, nor has it the slightest claim to such a generic title. The type of this genus (B. brachypterus) has twelve tail-feathers, whereas the group of Warblers amongst which Cetti's Warbler is included (Cettia) is distinguished by having only ten tail-feathers.

We must also strongly protest against the changing of many wellknown names-names familiar to us from our childhood-of such birds as the Garden-Warbler, the Whitethroat, the Dartford Warbler, the Reed-Warblers, and the Chiffchaff, and substituting: for them unknown synonyms raked up from a just and well-merited oblivion, or transferring the name of one species to another until it ceases to have any definite meaning.

But apart from these faults and inaccuracies it is impossible to overestimate the value of such a Catalogue, dry enough, it is true, to a non-scientific reader, but to the ornithologist working with the birds in this magnificent collection truly a "friend in need." 


\section{A CATALOGUE}

OF THE

\section{COLLECTION OF BIRDS}

FORMED BY THE LATE

\section{HUGH EDWIN STRICKLAND, M.A.}

FELLOW OF THE ROYAL, LINNEAN, GEOLOGICAL AND ROYAL GEOGRAPHICAL SOCIETIES, \&C. \&C.,

DEPCTY READER OF GEOLOGY IN THE UNIVERSITY OF OXFORD.

OSBERT SALVIN, M.A., F.R.S., \&C. STRICKLAND CURATOR IN THE UNIVERSITY OF CAMBRIDGE.

CAMBRIDGE : AT THE UNIVERSITY PRESS. 


\section{Cambriege:}

PRINTED BY C. J. CLAY, M.A. \& SON, AT THE UNIVERSITY PRESS. 


\section{PREFACE.}

THE collection of Bird skins, here catalogued, was formed by the late Hugh Edwin Strickland, M.A., F.R.S., of Oriel College, Oxford, and Deputy Reader of Geology in that University, who at the age of forty-two years was killed by a railway train whilst in the pursuit of his scientific labours on the 14th of September, 1853. Begun by him when yet a boy, for the label of one of the specimens (No. 3051 $a$ ) bears date August, 1822, the formation of this collection continued to be a great object of interest with him throughout his life; but most of the specimens obtained by him prior to 1833 were mounted and placed in cases in the hall at Apperley Court, near Tewkesbury, his father's seat, where they still are.

In the year last mentioned, however, his brother Algernon Strickland, a midshipman in the Royal Navy, visiting the Cape of Good Hope, Mauritius, and Southern India, obtained about 90 specimens now in the collection; and in the winter of 1835-36, Strickland himself made a voyage to Greece and Asia Minor, where he collected upwards of 100 others.

In 1838 STRICKLAND largely increased his collection by purchasing about 1200 specimens from his cousin Nathaniel Constantine Strickland. Another cousin, Arthur Strickland, purchased at the same time some 500 additional specimens, which also became the property of $\mathrm{H}$. E. STRICKLand in 1850 . The specimens thus acquired form no inconsiderable part of the whole collection. They had been originally obtained, as I am informed, partly from captains of merchantmen, and partly from dealers. 
The most important part of the present collection, however, was got together between 1838 and 1853; and the names of most of the best known ornithologists and collectors of that period appear on the labels as contributors to it, among whom Andersson, Baird, Blyth, Boys, Brandt, Gosse, Hodgson, Jerdon, Petherick, may be specially mentioned.

The whole number of skins is 6006. About one-third of which, including those obtained by STRICKLaNd himself, and by his brother Algernon, may be assigned to the collectors themselves. The rest, including the specimens bought from Nathaniel Constantine Strickland in 1838 and 1850, were generally obtained from dealers in England and Scotland.

Though the classification of Birds was a subject that, as his writings shew, constantly occupied STRICKLAND's attention, it is clear that up to the time of his sudden death his opinions on few if any points were matured. It is true that amongst his papers he left in MS. a comparatively complete catalogue of birds, extracted from the literature of ornithology, in which the various synonyms, and range of each species, as far as was then known, are elaborately set down. Each species is treated of on a separate slip of paper, and these slips are arranged in a certain order; but it is obvious on examination that the arrangement was dictated merely by convenience and cannot be taken to indicate any definite opinions.

This arrangement might have been used as STRICKLAND's system of classification, but a moment's reflection shews the impropriety of publishing such a system as the one finally adopted by its author; for by doing so it is evident that STRICKLAND would be supposed to have held views on many questions concerning which he never publicly expressed himself. I therefore had to seek another classification, and I naturally inclined to follow that used in the Nomenclator Avium Neotropicalium, published in 1873 by $\mathrm{Mr}$ Sclater and myself, which has as its basis the system of Huxley. Into this classification, which treats of South American birds only, I have 
inserted the species of the rest of the world, as represented in the collection, wherever I thought they could be most conveniently placed.

The references given in the catalogue under each species are; (1) to the original description, (2) to some work where the species is treated of either as regards its geographical distribution or its classification. In almost every case where STRICKLAND has mentioned a species, even incidentally, in his writings, I have given the reference; and also the synonyms, if any, under which the species was named in the collection. Where, however, a species appears to me to have been wrongly determined, I have not thought it expedient to quote the wrong name unless the statement had been published.

As this Catalogue has been a longer time in passing through the press than I at first anticipated (it contains about 12000 references), several recent important works could not be quoted in the earlier portion.

In preparing the Catalogue, and in re-arranging the collection in conformity therewith, I must gratefully acknowledge the patient help which I have constantly received from Mrs H. E. Strickland, without whose minute and accurate knowledge of almost every individual specimen, my attempts to trace their history would have resulted only in failure. To Mrs STRICKLAND I am also indebted for the following list, shewing the sources whence the collection was derived. To this I have added the approximate number of specimens to be referred to each.

It remains to be said that the collection was presented to the University of Cambridge by Mrs Strickland in 1867 under conditions which will be found in the Ordinationes Academice Cantabrigiensis (ed. 1877, p. 208); and that the chief events of Strickland's life are told in his Memoirs by his father-in-law, the late Sir William Jardine, published in 1858, to which is added a selection from his principal scientific papers. 



\section{ORIGIN OF THE SPECIMENS IN THE COLLECTION.}

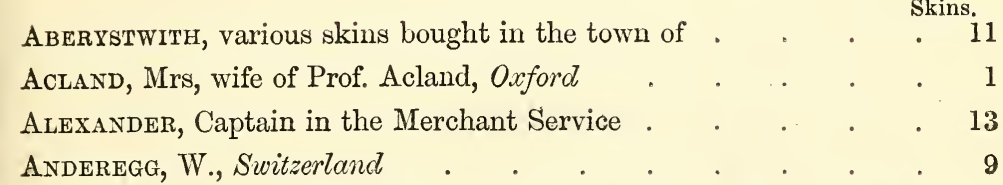

Andersson, C. J., a Swedish traveller in South Western Africa between the years 1849 and 1867. One of his collections of birds from Damara-land was described by Strickland and Sclater in the 'Contributions to Ornithology' for 1852, p. 141 et seq. A volume on the whole of his collections of birds was subsequently compiled by Mr J. H. Gurney, 'Notes on the Birds of Damara-land,' 1872, where a memoir of Andersson will be found _ . . . $\quad$. 99 Argent, Dealer, 32, Bishopsgate Street Without, London · . 262 Ashmolean Museum, Oxford . . . . . • . . 1 Askew, Captain in the Merchant Service . . . . . 339 Audubon, J. J., Author of 'The Birds of North America' and other works .

Baird, S. F., Professor, formerly of Dickinson College, Carlisle, Pennsylvania, now Secretary to the Smithsonian Institution, Washington. . . . . . . . . . . 66 Ball, Miss . . . . . . . . . . . 1 Barnes, H. M. B., Wareham, Dorsetshire . . . . . . 18 Bartlett, A. D., Superintendent of the Zoological Gardens, London 2 BeLr, G., Dealer, Whitehaven . . . . . . . . 3 Bell-Whitehouse . . . . . . . . . . 2 Birmingham, various skins bought in the town of . . . . 54 
Burth, E., formerly Curator of the Museum of the Asiatic Society of

Bengal. Author of various papers on Indian birds, \&c. A correspondent of Strickland's for several years . . . . 353

Boissoneau, Dealer, Paris . $\quad$. . . . . . $\quad .10$

Bors, W. J. E., Captain. An energetic collector of birds in Northern

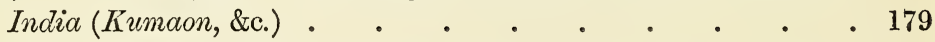

Brandt, J. F., Curator of the Museum of St Petersburg. Author

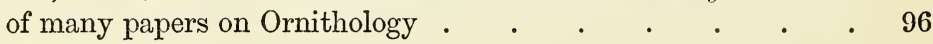

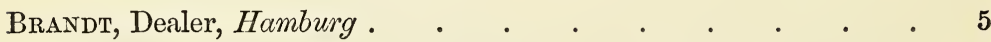

Bristol, a skin bought in the town of . . . . . . 1

Brown, E., Burton-on-Trent, Curator of the Museum . . $\quad$. 35

Brown, Rev. J. . . . . . . . . . . 1

Brown, T., Capt., Manchester, Curator of the Museum of the Natural

History Society . . . . . . . . . . 38

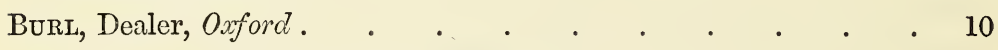

CARFrae, Dealer, George Street, Edinburgh . . . . . 21

Cartwright, Miss F., Aunt to H. E. Strickland . . . . 1

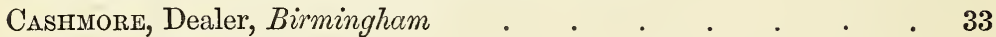

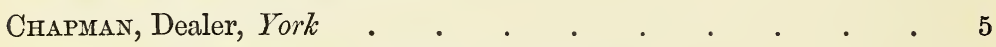

Constancia, José Senor, Antigua, Guatemala. . 212

Coulon, L., Curator of the Museum at Neuchatel a $\quad$. $\quad$. $\quad$ - 6

Cuming, H., Gower Street, London. Traveller in the Philippine

Islands and elsewhere . . . . . . . . . 45

DARwin, C., Naturalist in H.M.S. "Beagle." Author of the "Origin of

Species,' \&c. One skin from the Galapagos Islands . . . . 1

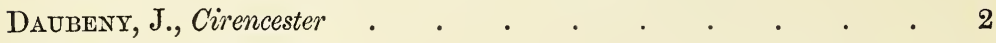

Dewgard, Dealer, Oxford ․ . . . . . . . . $\quad$. 73

Deyrolle, Dealer, Paris . . . . . . . . . . . 1

Dillwyn, L. L., Swansea . . . . . . . . . . . 2

Dresden, various skins bought in the town of . . . . . 11

Drummond, H. M., now Lt.-Col. H. M. Drummond-Hay, Seggieden,

Perthshive . . . . . . . . . . . 2

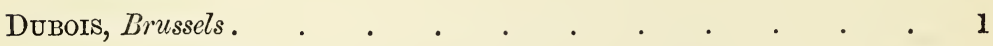

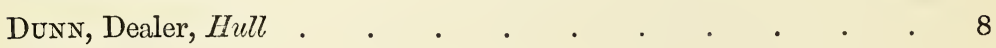

Dutton . . . . . . . . . . . . . 7

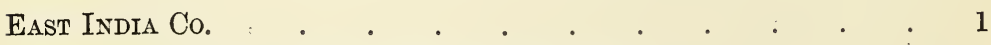

EDINBURGh, various skins bought in the town of . . . . 17 
Elaterts

Erton, T. C., Eyton Hall, Wellington, Salop. Author of 'Osteologia Avium' and other works

Favier, Collector of birds at Tangier, $N$. Africa (See 'Irby Birds of Gibraltar') .

Fenton, Dealer, George Street, Edinburgh . $\quad$. $\quad$. $\quad . \quad$ 8

Fenwick • • • • . . . . . • • • 8

Fergusson, Dr, Sierra Leone, West Africa . . . . . . 1

Forbes, E., Professor, Edinburgh . . . . . . . . 11

Fraser, H., Dealer, Dingrvall . . . . . . . . 1

Fraser, L., formerly Curator to the Museum of the Zoological Society of London. Traveller in North and West Africa and subsequently in Ecuador, South America. Author of 'Zoologia typica,' \&c.

GaLeotri, traveller in Mexico, where he made large botanical collections

GARDner, Dealer, London .

Goatrey, Chipping Norton, Oxfordshire . . . . . . . 1

Gordon, Dr C.

Gosse, P. H. Author of 'The Birds of Jamaica,' \&c.

Gould, J. Author of 'The Birds of Australia' and many other works .

Graham, Dealer, Iork

Graves, T., H.M.S. 'Beacon' .... . . . . 1

Green, F., Dealer, Leeds .

Hartladb, Dr. G. Hamburg. Author of many papers on OrnithoHARVEY, Lincoln

Havelu, Dealer, 77, Oxford Street, London

Hay, Lord A., afterwards Lord Walden and Marquess of Tweeddale.

Author of many papers on Ornithology . . . . . . 8

Heath, Rev. T., Missionary in the Samoan Islands . . . . 3

Hodgson, B. H., formerly resident at Nepal. Author of many papers

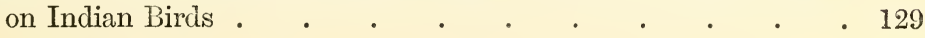

Holden . . . . . . . . . . . . 1

Houme, F., Fellow of Corpus Christi College, Oxford . 6

Honsfield, Dr T., Curator of the East India Co. Museum and Author of many papers on Ornithology . . . . . . 5 HyerT 
IsAaC, Dealer, Liverpool

Number of Skins.

IsaACson, Zoological Gardens, Liverpool . . . . . . . 12

Jameson, Prof., Quito, Ecuador

JARDINE, Sir W., Bart., Jardine Hall, Lockerby N.B., Father-in-law to

H. E. Strickland. Author of various Ornithological works .

JERDON, T. C., Surgeon in the Madras Army. Author of 'The Birds of India' and many papers on Indian Ornithology . . . 288

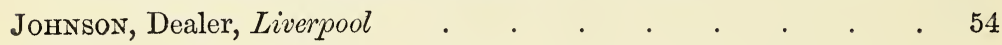

KeLLETr, Capt., H.M.S. 'Herald' . . . . . . . . 4

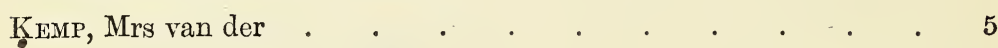

KinberG, J. G., Lund, Sweden . . . . . . $\quad$. 50

KIRK, J., a collector of birds in the Island of Tobago . . . 4

Kintland, W., Curator of the Ashmolean Museum, Oxford . $\quad 58$

$\mathrm{KOCH}$. . . . . . . . . . 3

Leadbeater, Dealer, 19, Brewer Street, Golden Square, London $\quad$. 15

LEEDS, various skins bought in the town of . $\quad$. $\quad . \quad 3$

Lrverpool, various skins bought in the town of . . . . 25

Lloyd, G., Stank Hill, Warwickshire . . . . . . . . 34

Lockwood, Rev. J. . . . . . . . . . . 3

Longchamps, E., De Selys, Belgium. Author of various papers on

Ornithology . . . . . . . . . . 6

Lowe, R. T, for many years resident in Madeira . . . . 1

MACDONALD . . . . . . . . . . . . . 72

MACMURdo . . . . . . . . . . . . 1

Manchester, Nat. Hist. Soc. . . . . . . . . 18

Mans, T. G., a traveller in Mexico, where he made a collection of birds. . . . . . . . . . . . . 33

Mansfield, Dealer, Birmingham . . . . . . . 38

Mather, Dealer, Theatre Square, Liverpool . . . . . 43

MorrIs, Rev. F. O., Author of various works on Ornithology . $\quad 1$

Murray, J., Capt. R.N., Dumfriesshire . . . . . 3

NASH, Capt. . . . . . . . . . . . 41

Nichols, Dealer . . . . . . . . . . . 1

Osborn, Dealer . . . . . . . . . . 4

Osman, Dealer, Oxford . . . . . . . . . . 21 
Passerini, C., Assistant Prof. of Zoology in the Museum of Nat.

Hist. Parma

Pearson, Capt., a traveller and collector in Ceylon . . . . . 11

Penrose, Rev. J., Cousin to H. E. Strickland . . . . . . 1

Perrot, Mrs, Worcestershire . . . . . . . . I

Petherick, J., H.M. consul at Kordofan. For an account of a col-

lection of birds formed by him, see Strickland, 'P. Z. S.' 1850,

p. 214 . . . . . . . . . . . . 110

Plymouth, a specimen bought in . . . . . . . 1

Pratimgton, Dr. Two Sumatra birds . . . . . . 2

RhAM, De . . . . . . . . . . . . . . 1

Riccioli . . . . . . . . . . . . . . 1

Robins, Dealer, Worcester . . . . . . . . 3

Robinson, T., Dealer, Worcester . . . . . . . . $\quad 21$

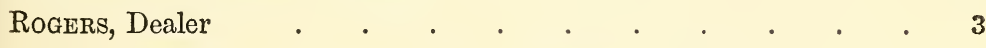

Schomburgk, R., traveller in British Guiana . . . . . 5

Sclater, P. L., Secretary to the Zoological Society of London. Author of many works on Ornithology . . . . . . 17

Seaman, Dealer, Liverpool . . . . . . . . . 12

Sмiтh, Sir A., traveller in South Africa . . . . . . 18

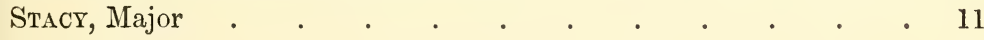

Staunton . . . . . . . . . . . . . 1

Stievens, S., Natural History Agent and Auctioneer, King Street, Covent Garden, London . . . . . . . . . 302

Strange . . . . . . . . . . . . . 1

Strickland, Algernon, brother of H. E. Strickland, a Midshipman in the Royal Navy. Died in the West Indies in 1835 . .

Strickland, Arthur, son of Sir W. Strickland, Bart., of Boynton, Yorkshire, and cousin of H. E. Strickland . . . . . . 434

Strickland, Hugh Edwin (see antea) . . . . . . . 301

Strickland, Nathaniel Constantine, brother of Arthur Strickland and cousin of H. E. Strickland . . . . . . . . $\quad$. 839

Stuchiury, Dealer, 47, Theobald Road, London . . . . 6

TAYLOR, Col. J. . . . . . . . . . . 25

Telfatr, Mrs, Mauritius . . . . . . . . . . . 3 
Thomas, Dealer, London Road, Liverpool

TOWNSEND .

TUCKer, Dealer, 13, Quadrant, London . . . . . . 12

Verreaux, Maison Verreaux, Paris a . . . . . $\quad$. 8

WALLACE, A. R., traveller on the Amazon and in the East. Author of 'The Amazon and Rio Negro,' 'The Malay Archipelago,' \&c. 14

WARE

WARWICK, Museum, skins obtained from . . . . . . 5

Weaver, Curator of the Museum at Birmingham . . . . 5

Whitehaven, G. B. M. . . . . . . . . $\quad$. 1

Williams, Dealer, City Road, London . • . . . . 28

Wilson, E., Tenby, South Wales . . . . . . . . . 4

WISE . . . . . . . . . . . I 1

Worcester, Nat. Hist. Soc. $\quad . \quad$. $\quad . \quad$. $\quad . \quad$. 1

No origin recorded $\quad . \quad$. $\quad . \quad . \quad . \quad . \quad . \quad . \quad . \quad .141$ 


\section{ADDENDA AND CORRIGENDA.}

Page 26, line 29, for Arkaan read Arakan

, 31, , 12, before Kartoum add h

,, 38, ,, 23, for A. read Arthur

,, 65, head of page, for Purider read PARIDze

,, 77, line 22, for Matthew read Mather

, 79, , 26, for Luzononsis read Luzonensis

, 79, , 27, for luzononsis read luzonensis

, 83, ,, 12, after - insert $e$

,, 108, ,, 1, del. a Malacsa (Johnson) 1837.-

, 125, ,, 35, for $c$ Damara-land read $d$ Damara-land

,, 146, , 30, for Matthew read Mather

, 147, ,, 13, for Matthew read Mather

,, 168, „, 1, 3, 10, 17, for Anthotreptes read Anthothreptes

,, 173, „, 7, for Temm. read Temminck, Man. d’Orn. i. p. Ixxxvi. (1820)

, 173, , , 20, for $d$ read $a$

, 175, , 9, for 1852 read 1844

,, 201, ,, 6, del. -b

,; 201, ,, 12, del. -b

, 202, , 12, for 1840 read 1850

, 203, ,, 5, for Liverpool read Edinburgh

, 225, , 5, 6, 7, 8, for Hapalospiza read Haplcspiza

, 226, „, 8, 9, 11, 14 for Porphyriospiza read Porphyrospiza

, 235, ,, 14, after 1833 insert -

,, 288, ,, 33, for Garurlus read Garrulus

,, 309, ,, 22, after S. America insert (Thomas)

", 310, ," 24, before Pelz. insert Pitangus parvus,

"326, , 4, for Lipaugu read Lipaugus

,, 352, , , 26, for Mather read Thomas

, 369, , 16, for 1824 read 1834

, 375, ,2 28, for second $b$ read $c$

, 379, ,, 19, for Ocellatus read ocellatus

, 491, ,, 7, for $e$ read $a$

, 502, ,, 28, for Arthur read $A$.

,, 506, after line 15 insert

$2472 \%$ Harpagus diodon.

Falco diodon, Temm. P1. Col. 198.

Harpagus diodon, Strickl. Orn. Syn. p. 102; Sharpe, Cat. B.

i. p. $361 a$ S. America (Arthur Strickland). 
Page 576, line 14, del. (Arthur Strickland) 1850. -b.

, 588, , 23, del. -b.

, 621, after line 19 insert

3031*. Larus glaucodes.

Larus glaucodes, Meyen, Act. Ac. Leop. xvi. Suppl. p. 115, pl. 24 : Saund. P. Z. S. 1878, p. 203.

$a,-b$ Chili (Capt. Brown) 1842.

Page 634, line 30, for 1826 read 1828

,, 634, , 32, del. -b. 


\title{
CLASS.-AVPS.
}

\author{
SUB-CLASS.-AVES CARINATA.
}

\section{ORDER.-PASSERES.}

SUB-ORDER.-OSCINES.

SECTION.-OSCINES DENTIROSTRES.

\section{FAMILY.-TURDIDEE.}

\section{Catharus.}

Catharus, Bonaparte, Consp. Av. i. p. 278. (1850).

\section{Catharus melpomene.}

Turdus melpomene, Cab. Mus. Hein. i. p. 5.

Catharus melpomene, Scl. Cat. Am. B. p. 1. a Guatemala (J. Constancia) 1848.

This species was for some time supposed to be identical with C. aurantiirostris, Hartl. (Rev. Zool. 1850, p. 158, and Contr. Orn. 1851, pl. 72), from Venezuela; but the rufous margins of the primaries distinguish it from the more southern bird.

\section{TURDUS.}

Turdus, Linnæus, Syst. Nat. i. p. 291 (1766) (partim).

\section{Turdus viscivorus.}

Turdus viscivorus, Linn, l. s. c.: Newton, ed. Yarr. Brit. B. i. p. 258 .

S. C. 
Turdus hodgsoni, Lafr.: Jerd. B. Ind. i. p. 531.

$a,-b$ Worcestershire (H. E. Strickland) 1833. -c N. India (W. J. E. Boys) 1848. -d (T. C. Eyton) 1850.

Stated by Strickland to be found near Smyrna during winter (P.Z.S. 1836, p. 98). Though Jerdon's latest views (Ibis 1872, p. 138) respecting $T$. hodgsoni were that it was not separable from the European bird, $c$ and $d$ both have the large bill and other peculiarities characteristic of the Himalayan race.

\section{Turdus pilaris.}

Turdus pilaris, Linn. Syst. Nat. i. p. 291 : Strickl. P. Z. S. 1836, p. 98 : Newton, ed. Yarr. Brit. B. i. p. 272.

a Surrey (H. E. Strickland) 1827. - b Worcestershire (H. E. Strickland) Feb. 10, 1844.

Found by Strickland at Smyrna during the winter of 1835-36.

\section{Turdus atrigularis.}

Turdus atrigularis, Temm. Man. d'Orn. i. p. 169 (1820): Newton, ed. Yarr. Brit. B. i. p. 276.

Planesticus atrogularis, Jerd. B. Ind. i. p. 529.

a Siberia (J. F. Brandt) 1842. -b (Gardner) 1844. -c, -d (Bt. at Stevens's) 1845. —e, -f Nepal (B. H. Hodgson 198, 199) 1845.

Specimen $a$ is that described and figured by Prof. Newton $l$. $c$.

\section{Turdus musicus.}

Turdus musicus, Linn. Syst. Nat. i. p. 292: Newton, ed. Yarr. Brit. B. i. p. 264.

$a$ Worcestershire (H. E. Strickland) 1832.

Observed by Strickland at Smyrna during the winter of $1835-36$. (P. Z. S. 1836, p. 98).

\section{Turdus iliacus.}

Turdus iliacus, Linn. Syst. Nat. i. p. 292: Newton, ed. Yarr. Brit. B. i. p. 268.

a Worcestershire (HI. E. Strickland) 1830. - b Worcestershire (H. E. Strickland) 1833. - c (N. C. Strickland) 1838.

Observed by Striclzland at Smyrna during the winter of $1835-36$. (P. Z. S. 1836, p. 98). 


\section{Turdus mustelinus.}

Turdus mustelinus, Gm. Syst. Nat. i. p. 817 : Baird, Brew. \& Ridgw. N. Am. B. i. p. 7.

a ô Pennsylvania, 30 April, 1840 (Kinberg) 1840. - b ํ Carlisle, Pennsylvania, 30 April, 1846 (S. F. Baird) 1847.

\section{Turdus swainsoni.}

Turdus swainsoni, Cab. in Tsch. Faun. Per. p. 187: Baird, Brew. \& Ridgw. N. Am. B. i. p. 14.

Turdus minimus, Lafr. Rev. Zool. 1848, p. 5.

a N. America (N. C. Strickland) 1838. - b Bogota (Bt. at Stevens's) 1845. —c o N. W. America (Wosnessenski, 1840) (J. F. Brandt) 1846. - d f Carlisle, Pennsylvania, 12 May, 1846 (S. F. Baird) 1847 .

\section{Turdus pallasi.}

Turdus pallasi, Cab. Wiegm. Arch. 1847, i. p. 205 : Baird, Brew. \& Ridgw. N. Am. B. i. p. 18.

$a \mathrm{~N}$. America (Askew). - $b$ q Carlisle, Pennsylvania, 26 Ap. 1843 (S. F. Baird) 1847.

\section{Turdus fuscescens.}

Turdus fuscescens, Steph. Shaw's Zool. x. p. 182: Baird, Brew. \& Ridgw. N. Am. B. i. p. 9.

a N. America (N. C. Strickland) 1838. - b ô Carlisle, Pennsylvania, May 1845 (S. F. Baird) 1847.

\section{Turdus jamaicensis.}

Turdus jamaicensis, Gm. Syst. Nat. i. p. 809: Gosse, B. Jamaica p. 142 ; \& Ill. pl. 24 : Scl. Cat. Am. B. p. 3.

a Jamaica (P. H. Gosse) 1848.

\section{Turdus albicollis.}

Turdus albicollis, Vieill. N. Dict. d'Hist. N. xx. p. 226 : Scl. \& Salv. Ex. Orn. p. 141, pl. 71.

a Brazil (N. C. Strickland) 1838.

\section{Turdus leucomelas.}

Turdus leucomelas Vieill. N. Dict. d'Hist. N. xx. 238: Scl. \& Salv. Ex. Orn. p. 143, pl. 72. 
a (Thomas) 1842. - b (Bt. at Stevens's) 1844. - c Brazil? (Argent) 1852. —d (Bt. at L'pool) 1853.

$c$ is apparently a Bolivian skin.

\section{Turdus unicolor.}

Turdus unicolor, Tickell, J. A. S. B. ii. p. 577 (1833).

T. dissimilis, Blyth, J. A. S. B. xvi. pp. 144, 474.

Geocichla unicolor, Jerd. B. Ind. i. p. 519.

a (Cashmore) 1839. - b Madras (T. C. Jerdon) 1846. c Kumaon (W. J. E. Boys) 1847.

\section{Turdus pallidus.}

Turdus pallidus, Gm. Syst. Nat. i. p. 815: Wald. Ibis, 1871 , p. 167.

Turdus rufulus, Drap. Dict. Class x. p. 443 (1826).

Turdus modestus, Eyt. P. Z. S. 1839, p. 103.

a Malacca (Thomas) 1840. - b Malacca (P. L. Sclater) 1844.

In his Notes on certain species of birds from Malacca (P.Z.S. 1846, p. 99) Strickland gives a short description of a specimen of this species under Eyton's title.

\section{Turdus migratorius.}

Turdus migratorius, Linn. Syst. Nat. i. p. 292: Baird, Brewer \& Ridgw. N. Am. B. i. p. 25.

$a$ N. America (Askew) 1834. -b N. America (Thomas) 1839. — Mexico (T. Mann) 1844.

\section{Turdus falklandicus.}

Turdus falklandii, Quoy \& Gaim. Voy. de l'Uranie, p. 104: Scl. Cat. Am. B. p. 4.

Turdus magellanicus, King, P. Z. S. 1830, p. 14.

a (N. C. Strickland) 1838.

Found in Chili, Patagonia and the Falkland Islands.

\section{Turdus grayi.}

Turdus grayi, Bp. P. Z. S. 1837, p. 118: Scl. Cat. Am. B. p. 4.

a Guatemala ( $J$. Constancia) 1848. 
19. Turdus fumigatus.

Turdus fumigatus, Licht. Verz. Doubl. p. 38: Scl. Cat. Am. B. p. 4 .

$a$ (Gardner) 1845. -b Brazil ? (Argent) 1852.

$b$ is probably a Bolivian skin.

\section{Turdus rufiventris.}

Turdus rufiventris, Vieill. N. Dict. d'Hist. N. xx. p. 226 : Scl. Cat. Am. B. p. 5.

a Brazil (Bt. at Aberystwith) 1833.

\section{Turdus olivaceus.}

Turdus olivaceus, Linn. Syst. Nat. i. p. 292: Layard, B. S. Afr. p. 128.

$a($ Askew) 1839. - 6 S. Africa (Dr A. Smith) 1843.

\section{Tardus rufitorques.}

Turdus rufitorques, Hartl. Rev. Zool. 1844, p. 214: Du Bus, Esq. Orn. tt. 19, 20 : Scl. Cat. Am. B. p. 6.

$a$ Guatemala (J. Constancia) 1845. -b Guatemala (J. Constancia) 1848.

\section{Turdus merula.}

Turdus merula, Linn. Syst. Nat. i. p. 295: Newton, ed. Yarr. Brit. B. i. p. 280.

$a,-b,-c$ Worcestershire (H. E. Strickland) June, 1834. -d Gloucestershire (H. E. Strickland) Jan., 1850.

Observed by Strickland near Sinyrna (P. Z. S. 1836, p. 97).

\section{Turdus serranus.}

Turdus serranus, Tsch. Wiegm. Arch. x. pt. i. p. 280, \& Faun. Per. p. 186: Scl. \& Salv. P. Z. S. 1870, p. 783.

Merula atrosericea, Lafr. Rev. Zool. 1848, p. 3.

a Bogota (J. Gould) 1844.

\section{Turdus simillimus.}

Turdus simillimus, Jerd. Madr. Journ. x. p. 253 (1839).

Merula simillima, Jerd. B. Ind. i. p. 524.

a, -b Madras ("T. simillimus" T. C. Jerdon) 1845. 


\section{Turdus nigripileus.}

Turdus nigripileus, Lafr. Rev. Zool. 1840, p. 65.

Merula nigropileus, Blyth, J. A. S. B. xvi. p. 148 : Jerd. B. Ind. i. p. 523.

$a$ (Gardner) 1846.

Found throughout Southern India (Jerdon).

\section{Turdus boulboul.}

Lanius boulboul, Lath. Ind. Orn. p. 89.

Merula boulboul, Blyth, J. A. S. B. xvi. p. 147 : Jerd. B. Ind. i. p. 525 .

Turdus pőcilopterus, Vig. P. Z. S. 1831, p. 54: Gould, Cent. B. Him. pl. 14.

a Nepal (B. H. Hodgson 184) 1845. -b, -c Kumaon (W. J. E. Boys) 1847. -d N. India (Williams) 1848. -e N. India (W.J. E. Boys) 1848.

\section{Turdus poliocephalus.}

Ash-headed Thrush, Lath. Syn. Suppl. ii. p. 373.

Turdus poliocephalus, Lath. Ind. Orn. Suppl. p. xliv. No. 25.

Merula nestor, Gould, P. Z. S. 1835, p. 186: Jard. \& Selb. Ill. Orn. iv. pl. 37.

a Australia (Bt. at Stevens's) 1846.

The true habitat of this species is Norfolk Island. Mr Gould (Ann. \& Mag. N. H. xi. p. 336) identifies his T. nestor with T. fuliginosus, Lath. (Ind. Orn. Suppl. p. xlii. No. 14), after seeing the Earl of Derby's unpublished drawings of Australian Birds. But as Latham's description is not satisfactory the bird had better bear the name $T$. poliocephalus applied by the same author to the Norfolk Island Thrush.

\section{Turdus collaris.}

Turdus collaris, Soret, Rev. Zool. 1840, p. 2.

Turdus albicollis, Royle, Ill. Him. Bot. pl. 8. f. 3. (nec Vieill.). Merula albocincta, Blyth, J. A. S. B. xvi. p. 148 (1847): Jerd. B. Ind. i. p. 526.

a (T. C. Eyton), 1850.

Found throughout the Himalayas as high as $10,000 \mathrm{ft}$. 


\section{Turdus castaneus.}

Merula castanea, Gould, P. Z. S. 1835, p. 185: Gray \& Mitch. Gen. B. pl. 56 : Jerd. B. Ind. i. p. 526.

a India (W. J. E. Boys) 1847. - b (T. C. Eyton) 1850.

\section{Turdus torquatus.}

Turdus torquatus, Linn. Syst. Nat. i. p. 296 : Newton, ed. Yarr. Brit. B. i. p. 287.

a Britain (Askew) 1838.

\section{Turdus gigas.}

Turdus gigas, Fraser, P. Z. S. 1840, p. 59: Scl. \& Salv. Ex. Orn. p. 139, pl. 70.

a Bogota (Bt. at Stevens's) 1846.

Found in the Andes from Columbia to Bolivia.

\section{Turdus fuscater.}

Turdus fuscater, Lafr. \& D’Orb. Syn. Av. i. p. 16 : Scl. Cat. Am. B. p. 5.

a Chili? (N. C. Strickland) 1838.

Originally described from Bolivian specimens. The species is also found in the Argentine Republic, but not in Western Chili.

\section{Turdus flavipes.}

Turdus flavipes, Vieill. N. Dict. d'Hist. N. xx. p. 277 : Scl. Cat. Am. B. p. 6.

Turdus carbonarius, Licht. Verz. Doubl. p. 37.

a, -b Brazil (G. Lloyd) 1838. — Bahia (Dutton) 1846.

\section{OREOCINCLA.}

Oreocincla, Gould, P. Z. S. 1837, p. 145.

\section{Oreocincla lunulata.}

Turdus lunulatus, Lath. Ind. Orn. Suppl. p. xlii.

Oreocincla lunulata, Gould, B. Austr. iv. pl. 7.

a (N.C. Strickland) 1838.

Found in New South Wales and Van Diemen's Land.

The Javan Thrush of this form was considered by Strickland to be perhaps the same as the Australian bird (Ann. \& Mag. N. H. 
xi. p. 336). The Javan bird is however distinct and is O. horsfieldi, Bp. $=$ (O. malayana, Sundev. T. varius Vig. \& Horsf. et Strickl. ut supra, nec Pallas).

\section{Oreocincla dauma.}

Turdus dauma, Lath. Ind. Orn. p. 362.

Oreocincla dauma, Jerd. B. Ind. i. p. 533.

Oreocincla whitei, Hodgs. Zool. Misc. p. 83. 1846.

a Nepal (B. H. Hodgson 194) 1845. -b India (E. Blyth)

\section{Oreocincla iodura.}

Oreocincla iodurus, Gould, Ann. \& Mag. N. H. ser. 4. ix. p. 401.

Turdus iodurus, Newton, ed. Yarr. Brit. B. i. p. 256.

a (N. C. Strickland) 1838.

\section{Oreocincla mollissima.}

Turdus mollissimus, Blyth, J. A. S. B. xi. p. 188.

Oreocincla mollissima, Jerd. B. Ind. i. p. 533.

a (Bt. at Edinburgh) 1852.

Originally described from specimens from Darjeeling. The species has also been obtained throughout the Himalayas and in Western China.

\section{GEOCICHLA}

Geocichla "Kuhl," Gould, P. Z. S. 1836, p. 7.

\section{Geocichla citrina.}

Turdus citrinus, Lath. Ind. Orn. p. 350.

Geocichla citrina, "Kuhl."__Jerd. B. Ind. i. p. 517.

Petrocincla cyanota, Hodgs. Gray's Zool. Misc. p. 83.

Petrocossyphus citrinus, G. R. Gray, Cat. Hodgs. Coll. (1846) p. 79 1846.

a, - b Nepal (B. H. Hodgson 584) 1845. —c India (E. Blyth)

In 1844 (Änn. \& Mag. N. H. xiii. p. 35) Strickland pointed out that the Thrush from India at that time called G. rubecula should properly be called G. citrina, the true $G$. rubecula from Java being

1 This generic name has been attributed to Kuhl, but I have been unable to find where that author employed it. Agassiz gives two references to Kuhl's works both of which (Nomenel. Zool. Aves, p. 32, and Add. p. 9) are wrong. 
probably distinct. This view is doubtless correct, but the only bird then in Strickland's collection called by him G. citrina appears to me to be referable to $G$. cyanonota, Jard.

\section{Geocichla cyanonota.}

Turdus cyanotus, Jard. and Selb. Ill. Orn. t. 46.

Geocichla cyanotus, Jerd. B. Ind. i. p. 517.

a India (N. C. Strickland) 1838. - b Madras (T. C. Jerdon) 1845 .

$a$ seems to have been referred by Strickland in 1844 (Ann. \& Mag. N. H. xiii. p. 35) to G. citrina.

\section{Geocichla rubecula.}

Geocichla rubecula, Gould, P. Z. S. 1836, p. 7.

a (Bt. at Birmingham) 1849.

Agrees with Gould's descriptions of the Javan Geocichla.

\section{Petrocincla ${ }^{1}$.}

Petrocincla, Vigors, Zool. Journ. ii. p. 396 (1825).

\section{Petrocincla saxatilis.}

Turdus saxatilis, Linn. Syst. Nat. i. p. 294.

Petrocincla saxatilis, Vig. l. s. c.

a ô Tuscany (C. Passerini), 1835.

\section{Petrocincla solitaria.}

Turdus solitarius, P. I. S. Müller, Syst. Nat. Suppl. p. 142.

Monticola solitarius, Walden, Trans. Zool. Soc. ix. p. 192. a Philippine Is. (J. Gould), 1846.

\section{Petrocincla cyanus.}

Turdus cyanus, Linn. Syst. Nat. i. p. 296.

Petrocossyphus cyaneus, Jerd. B. Ind. i. p. 511 : Dresser, B. Eur. appendix A.

Petrocincla pandoo, Sykes, P. Z. S. 1832, p. 87.

Petrocincla affinis, Blyth, J. A. S. B. xii. p. 177.

1 In 1841 (Ann. \& Mag. N. H. vii. p. 26) Strickland adrocated the use of Boie's generic name Monticola (Isis, 1822, p. 552) for the Rock-Thrushes, and again in Contrib. Orn. 1852, p. 147. But the latter name having been introduced without any definition of characters ought not to supersede Vigors's welldefined generic appellation (Rules of Nomenclature, clause 12). 
a Rome (H. E. Strickland) Dec. 1836. -b Himalaya (N.C. Strickland) 1838. - c India (Havell) 1839. —d (Stevens) 1843. e Madras (T. C. Jerdon) 1845. - f India ("P. affinis," E. Blyth) 1846. $-g$ N. India (W. J. E. Boys) 1848. - h (T. C. Eyton) 1850. - $i$ Tangiers (F. Favier) 1851.

\section{Petrocincla erythrogastra.}

Turdus erythrogaster, Vig. P. Z. S. 1831, p. 171: Gould, Cent. B. Him. t. 13.

Orocetes erythrogastra, Jerd. B. Ind, i. p. 514.

$a$ (Carfrae) 1840. -b (Stevens) 1844. -c, -d Nepal (B. H. Hodgson 351) 1845. -e (W. J. E. Boys) 1847. $-f$ N. India (W.J.E. Boys) 1848. - g Himalaya (T. C. Eyton) 1850.

\section{Petrocincla rupestris.}

Turdus rupestris, Vieill. N. Dict. d'Hist. Nat. xx. p. 281.

Petrocincla rupestris, Layard, B. S. Afr. p. 129.

Turdus rupicola, Licht. Verz. Doubl. p. 38. 1838.

a S. Africa (N. C. Strickland) 1838. - b (N. C. Strickland)

\section{4\%. Petrocincla explorator.}

Turdus explorator, Vieill. N. Dict. d'Hist. N. xx. p. 260.

Petrocincla explorator, Layard, B. S. Afr. p. 130.

Turdus perspicax, Shaw, Nat. Misc. xxii. pl. 961. $a$ S. Africa (N. C. Strickland) 1838.

\section{Petrocincla cinclorhyncha.}

Petrocincla cinclorhyncha, Vig. P. Z. S. 1831, p. 172: Gould, Cent. B. Him. t. 19.

Orocetes cinclorhynchus, Gray, Jerd. B. Ind. i. p. 514.

a India (Gardner) 1844. —b Kumaon (W. J. E. Boys) 1847.

\section{Petrocincla brevipes.}

Petrocincla brevipes, Waterh. Alex. Exp. ii. p. 263.

Monticola brevipes, Strickl. \& Scl. Contr. Orn. 185̃2, p. 147.

\section{a, -b Damaraland (C. J. Anderson) 1852.}

The specimens described in Strickland's and Sclater's paper l. s. c.. 


\section{MiMOcICHLA.}

Mimocichla, Baird, Rev. Am. B. p. 35, (1864) ex Sclater P. Z. S. 1859, p. 336 (as a subgenus).

\section{Mimocichla aurantia.}

Turdus aurantius, Gm. Syst. Nat. i. p. 832: Scl. Cat. Am. B. p. 6.

Semimerula aurantia, Baird, Rev. Am. B. p. 34.

Merula leucogenys, Gosse, B. Jamaica, p. 136, \& Ill. pl. 23. Mimocichla aurantia, Scl. \& Salv. Nomencl. Av. Neotr. p. 2. a Jamaica (N. C. Strickland) 1838. -b Jamaica (P. H. Gosse) 1848.

\section{Melanotis.}

Melanotis, Bonaparte, Consp. Av. i. p. 276, (1850).

\section{Melanotis hypoleucus.}

Melanotis hypoleucus, Hartl. Rev. Zool. 1851, p. 460: Scl. Cat. Am. B. p. 7.

$a$ Guatemala (J. Constancia) 1845.

\section{GaLEoscoptes.}

Galeoscoptes, Cabanis, Mus. Hein. i. p. 82 (1850).

\section{Galeoscoptes carolinensis.}

Muscicapa carolinensis, Linn. Syst. Nat. i. p. 328.

Galeoscoptes carolinensis, Cab. Mus. Hein. i. p. 82 : Baird, Brew. \& Ridgw. N. Am. B. i. p. 52. 1834.

$a$ N. America (Askew) 1834. - b N. America (Mansfield)

\section{HARPORHYNCHUS.}

Harporhynchus, Cabanis, Wiegm. Arch. 1848, i. p. 98.

\section{Harporhynchus rufus.}

Turdus rufus, Linn. Syst. Nat. i. p. 393.

Harporhynchus rufus, Cab. l. c. s.: Baird, Brew. \& Ridgw. N. Am. B. i. p. 39.

$a$ N. America (N. C. Strickland) 1838. 


\section{ZOOTHERA.}

Zoothera, Vigors, P. Z. S. 1831, p. 172.

\section{Zoothera monticola.}

Zoothera monticola, Vig. l. s. c.: Gould, Cent. B. Him. t. 22 : Jerd. B. Ind. i. p. 509.

a Darjeeling (E. Blyth) 1850.

\section{Mimus.}

Mimus, Boie, Isis, 1826, p. 972.

55. Mimus polyglottus.

Turdus polyglottus, Linn. Syst. Nat. i. p. 293.

Mimus polyglotitus, Bp. Consp. i. p. 276 : Scl. Cat. Am. B. p. 8 : Baird, Brew. \& Ridgw. N. Am. B. i. p. 49.

a (Askew) 1834.

Found in North America as far south as Mexico.

\section{Mimus lividus.}

Turdus lividus, Licht. Verz. Doubl. p. 39.

Mimus lividus, Max. Beitr. iii. p. 653: Scl. Cat. Am. B. p. 9.

$a$ (Arthur Strickland) 1840. -b (Dewgard) 1851.

A Brazilian species.

\section{5\%. Mimus gilvus.}

Turdus gilvus, Vieill. Ois. d'Am. Sept. ii. p. 15, pl. 68 bis.

Mimus gilvus, Gray, Gen. B. : Scl. Cat. Am. B. p. 9.

a Tobago ( $M r$ Kirk) 1844. -b Tobago (Sir W. Jardine) 1844. - c, -d Trinidad (Argent) 1851.

\section{Mimus calandria.}

Orpheus calandria, Lafr. \& D’Orb. Syn. Av. i. p. 17.

Mimus calandria, Gray, Gen. B.: Scl. P. Z. S. 1859, p. 343 : Cat. Am. B. p. 10.

$a$ (N. C. Strickland), 1838.

Found in Bolivia and the Argentine Republic.

59. Mimus thenca.

Turdus thenca, Mol. Saggio Stor. Nat. Chili, p. 250 (1782). 
Mimus thenca, Darwin, Zool. Voy. Beagle, iii. p. 61: Scl. Cat. Am. B. p. 10.

a Chili (E. Brown) 1842.

\section{Irimus longicaudatus.}

Mimus longicaudatus, Tsch. Archiv f. Naturg. 1844, pt. i. p. 280, \& Faun. Per. p. 190, t. xv. f. 2.

$a$ (N. C. Strickland) 1838.

A species of Western Peru.

MytopHoneus.

Myophonus Temminck, Pl. Col. 170 (26 July, 1823).

\section{Myiophoneus temmincki.}

Myiophonus temmincki, Vig. P. Z. S. $1830-31$, p. 171: Gould, Cent. B. Him. pl. 21. Jerd. B. Ind. i. p. 500.

a Himalayas (Carfrae) 1840. $-b,-c$ Nepal (B. H. Hodgson 224) 1845. -d (W. J. E. Boys) 1847.

\section{MIyiophoneus horsfieldi.}

Myiophonus horsfieldi, Vig. P. Z. S. 1830-31, p. 35: Gould, Cent. B. Him. pl. 20 : Jerd. B. Ind. i. p. 499.

a Madras (T. C. Jerdon) 1845.

63. Myiophoneus flavirostris.

Turdus flavirostris, Horsf. Linn. Trans. xiii. p. 149.

Myiophonus flavirostris, Vig. Memoir of Sir S. Rafles, p. 660.

a Java (Arthur Strickland) 1840.

\section{CHatops.}

Chcetops, Swainson, Faun. Bor. Am. p. 486 (1831).

64. Chrtops pycnopygius.

Sphenceacus pycnopygius, Scl. Contr. Orn. 1852, p. 148, pl. 102.

Chcetops pycnopygius, Sharpe, Cat. Afr. B. No. 226 : Gurney, in And. B. Damara-l. p. 117.

a Damara-land (C. J. Andersson) 1852.

The type of the species described in Strickland and Sclater's Paper in the "Contributions" $l$. $c$. 
COSSYPHA.

Cossypha, Vigors, Zool. Journ. ii. p. 396 (1825).

65. Cossypha caffira.

Motacilla caffra, Linn. Mant. p. 527.

Bessonornis caffra, Layard, B. S. Afr. p. 132.

$a,-b$ Cape of Good Hope (A. Strickland) 1832.

\section{Cossypha albicapilla.}

Turdus albicapillus, Vieill. N. Dict. d'Hist. N. xx. p. 254.

Cossypha leucoceps, Sw. Classif. B. ii. p. 232. 1838.

a Africa (Johnson) 1837. - b W. Africa (N. C. Strickland)

\section{Cossypha verticalis.}

Hartl. Beitr. z. Orn. W. Afr. p. 23 : Syst. Orn. W. Afr. p. 77. a W. Africa (Cashmore) 1839.

\section{Cossypha bicolor.}

Muscicapa bicolor, Sparrm. Mus. Carls. ii. t. 46.

Cossypha bicolor, Finsch \& Hartl. Vög. Ost-Afr. p. 281.

Turdus reclamator, Vieill. N. Dict. d'Hist. N. xx. p. 280.

Cossypha vociferans, Sw. Zool. Ill. iii. t. 180.

Bessonornis vociferans, Layard, B. S. Afr. p. 130. 1852 .

a Cape of Good Hope (Askew) 1837. - b (Bt. at Edinburgh)

\section{Cossypha natalensis.}

Bessonornis natalensis, Smith, Zool. S. Afr. t. 60 : Layard, B. S. Afr. p. 131.

$a$ Port Natal? (Williams) 1846.

\section{ro. Cossypha semirufa.}

Petrocincla semirufa, Rüpp. Neue Wirbelth. p. 81.

Cossypha semirufa, Guér. Rev. Zool. 1843, p. 322. a Abyssinia (E. Verreaux) 1850.

\section{Cossypha signata.}

Cossypha signata, Sundevall, Öfvers. K. Vet. Ak. Förh. 1850, p. 101.
a (N. C. Strickland) 1838. 


\section{Cittocincla.}

Kittacincla, Gould, P. Z. S. 1836, p. 7.

\section{Cittocincla macrura.}

Long-tailed Thrush, Lath. Syn. ii. pt. i. p. 72. undè

Turdus macrourus, Gm. Syst. Nat. i. p. 820.

Kittacincla macrura, Gould, l. s. c.

$a$ India (N. C. Strickland) 1838. -b Malacca (Mather) 1840 .

\section{Copsychus.}

Copsychus, Wagl. Syst. Av. (1827) Genus Gracula, Fol. 20, p. 2.

\section{Copsychus saularis.}

Gracula saularis, Linn. Syst. Nat. i. p. 165.

Copsychus saularis, Jerd. B. Ind. ii. p. 114.

$a$ India (Askew) 1834. - b Malacca (N. C. Strickland) 1838. -c (N. C. Strickland) 1838. - d India (W. Kirtland) 1838. - e India (Stevens) 1843. - $f$ India (E. Blyth) 1851. -g (Major Stacy).

Strickland (Ann. \& Mag. N. H. vi. p. 422) refers Gryllivora brevirostris, Sw. to Turdus amoenus, Horsf. and (Ann. \& Mag. N. H. xviii. p. 108) in a note to his translation of Sundevall's article on the Birds of Calcutta draws attention to the Malaccan Copsychus having three instead of four lateral rectrices white. Mr Sharpe (Ibis, 1875, p. 37) doubts the value of this distinction, and unites the Malaccan with the Indian bird. See also Lord Tweeddale (Ibis, 1871, p. 175).

In 1842 (P. Z. S. p. 168) Strickland referred the Chinese Copsychus to C. longirostris, Sw. 


\section{FAMILY.TIMELIID AE. \\ SUB-FAMILY.-CRATEROPODIN E.}

Crateropus.

Crateropus, Swainson, Faun. Bor. Am. ii. p. 487 (1831).

\section{Crateropus bicolor.}

Crateropus bicolor, Jard. Edinb. Journ. Nat. \& Geogr. Sc. iii. p. 97: Waterhouse, Alex. Exp. ii. p. 263: Layard, B. S. Afr. p. 133: Strickl. \& Scl. Contr. Orn. 1852, p. 145: Gurney, in And. B. Damara-l., p. 121.

$a$ S. Africa (Capt. Alexander) 1838. -b Damara-land (C. J. Andersson) 1852.

In their paper on Birds of Damara-land Strickland and Sclater give a description of the young of this species.

\section{Crateropus platycercus.}

Crateropus platycercus, Sw. B. W. Afr. i. p. 271, \& Classif. B. ii. p. 234 .

$a$ (Mansfield) 1839.

A West-African species, the specimen agreeing with Swainson's type.

\section{Malacocercus.}

Malacocercus, Swainson, Zool. Ill. ser. 2, pl. 127 (183233).

\section{Malacocercus terricolor.}

Pastor terricolor, Hodgs. J. A. S. B. v. p. 771. (descr. nulla). Malacocercus terricolor, Jerd. B. Ind. ii. p. 59.

M. bengalensis, Blyth (ex Briss.) Cat. Birds As. Soc. p. 140 (1849).

a Nepal (B. H. Hodgson 371) 1845. -b India (E. Blyth " $M$. terricolor (Hodgs.) Turdus canorus, Linn.") 1846. -c Malwa (W. J. E. Boys) 1847.

Strickland's dissent from Blyth's identification of this species with Turdus canorus, Linn. is given by Blyth in a footnote to a paper in the J. A. S. B. xvi. p. 453. 
77. Malacocercus griseus.

Turdus griseus, Gm. Syst. Nat. i. p. 824.

Malacocercus griseus, Jerd. B. Ind. ii. p. 60.

$a \hat{\delta},-b$ o Madras (A. Strickland) Sep. 1833. - c India (N. C. Strickland) 1838.

The name M. striatus, considered by Strickland (Ann. \& Mag. N. H. viii. p. 372) to be a synonym of $M$. griseus, belongs to the Ceylonese species, which is distinct from the Indian bird (cf. Blyth, Ibis, 1867, p. 300).

\section{Malacocercus somervillii.}

Timalia somervillii, Sykes, P. Z. S. 1832, p. 88.

Malacocercus somervillii, Jerd. B. Ind. ii. p. 63.

a India (Stevens) 1843. - b Madras ("M. somervillii (orientalis)" T. C. Jerdon) 1845.

\section{Malacocercus malcolmi.}

Timalia malcolmi, Sykes, P. Z. S. 1832. p. 88.

Malacocercus malcolmi, Jerd. B. Ind. ii. p. 64.

$a$ India (Gardner) 1844. $-b$ S. India (T. C. Jerdon " $M$. malcolmi") 1850.

\section{Malacocercus earlii.}

Malacocercus earlii, Blyth, J. A. S. B. xiii. p. 369, (1844) : et xliii. extr. No. p. 118 : Jerd. B. Ind. ii. p. 68.

a Arakan (E. Blyth) 1847.

81. Malacocercus chatarrhœa.

Cossyphus caudatus, Dumeril, fide Blyth.

Chatarrhœa caudata, Jerd. B. Ind. ii. p. 67.

Timalia chatarcea, Frankl. P. Z. S. 1830-1, p. 118.

a India "(M. caudatus (Dum.), Timalia chatarrhœa, Frankl., Megalurus isabelinus Sw." E. Blyth) 1846. -b $\hat{o}$ India (W. J. E. Boys) 1847. —c S. India (T. C. Jerdon) 1850.

The separation of this bird from the more slender-billed forms of Malacocercus such as M. earlii seems hardly justifiable. But should it prove distinct the right place for it seems to be in the genus Argya, Less. (Traité d'Orn. p. 402, (1831), an older name than Malacocercus, Swains., and one the larger genus should bear, were all these closely allied forms united together.

S. C. 
82. Malacocercus subrufus.

Layardia subrufa, Jerd. B. Ind. ii. p. 66.

$a$ (Bt. at Stevens's) 1843.

Agrees with an Indian specimen in the Swainson collection and with Jerdon's description.

\section{Megalurus.}

Megalurus, Horsfield, Trans. Linn. Soc. xiii. p. 158, (1820).

83. Megalurus palustris.

Megalurus palustris, Horf. l. s. c.: Jerd. B. Ind. ii. p. 70 : Wald. Trans. Z. S. ix. p. 189. 1846.

$a$ India? (Gardner) 1844. -b Philippine Islands (J. Gould)

SPHEN

Sphenceacus, Strickland, Ann. \& Mag. N. H. vi. p. 421, (1841).

\section{Sphenæacus africanus.}

Motacilla africana, Gm. Syst. Nat. i. p. 958.

Sphenceacus africanus, Strickland, l. s. c.

Drymoica africana, Layard, B. S. Afr. p. 95.

a Cape of Good Hope (N. C. Strickland) 1838.

\section{Sphenæacus punctatus.}

Synallaxis punctata, Quoy \& Gaim. Voy l'Astrolabe, i. p. 255. t. 18. f. 2.

Sphencacus punctatus, Buller, B. N. Zeal. p. 128. pl. 13. a New Zealand (Askew) 1840.

\section{Chetornis.}

Chcetornis, G. R. Gray, Gen. B. i. p. 167. pl. 48. f. 9. (1848).

86. Chætornis striata.

Megalurus striatus, Jerd. Madr. Journ. xiii. a. p. 169.

Choetornis striata, G. R. Gray, l. s. c.: Jerd. B. Ind. ii. p. 72. a Madras (T. C. Jerdon) 1845. -b India (E. Blyth) 1846. 


\section{SPHENURA.}

Sphcenura, Lichtenstein, Verz. Doubl. p. 40, (1823): Strickl. Ann. \& Mag. N. H. xiii. p. 35.

\section{Sphenura brachyptera.}

Turdus brachypterus, Lath. Ind. Orn. Suppl. p. xliii.

Sphenura brachyptera, Licht. l. s. c.

Dasyornis australis, Vig. \& Horsf. Trans. L. S. xv. p. 231 : Gould, B. Austr. iii. pl. 32.

$a$ New South Wales (McDonald) 1838.

\section{PsOPHODES.}

Psophodes, Vigors \& Horsfield, Trans. Linn. Soc. xv. p. 328, $(1825)^{1}$.

\section{Psophodes crepitans.}

Muscicapa crepitans, Lath. Ind. Orn. Suppl. p. li.

Psophodes crepitans, Vig. \& Horsf. l. s. c.: Gould, B. Austr. iii. pl. 15.

a Australia (N. C. Strickland) 1838. - b New South Wales (N. C. Strickland) 1838.

\section{HyPERGERUS.}

Hypergerus, Reichenbach, Av. Syst. pl. liv. (1850)².

\section{Hypergerus atriceps.}

Moho atriceps, Less. Traité d'Orn. p. 646.

Crateropus oriolides, Sw. B. W. Afr. p. 280. pl. 31

$a$ W. Africa (N. C. Strickland) 1838. - b W. Africa (Gardner) 1845 .

These specimens agree with Swainson's type of his $C$. oriolides marked as coming from Senegal.

I The affinity of this form to the Crateropodince has been pointed out by Strickland (Ann. \& Mag. N. H. vi. p. 420).

2 No types whatever are indicated under the generic names introduced in this work of Reichenbach's. But the names of the types were, I believe, communicated by letter to G. R. Gray, and published by him in his "Catalogue of the Genera and Subgenera of Birds" (1855). 


\section{SUB-FAMILY.-GARRULACIN Æ.}

Garrulax.

Garrulax, Lesson, Traité d'Orn. 647 (1831).

90. Garrulax leucolophus.

Corvus leucolophus, Hardw. Trans. Linn. Soc. xi. p. 208. t. 15.

Cinclosoma leucolophum, Gould, Cent. B. Him. t. 18.

Garrulax leucolophus, Gray. Gen. B. app. p. 5 : Cat. Hodgs. Coll. (1846), p. 82 : Jerd. B. Ind. ii. p. 35.

a Himalaya (N. C. Strickland) 1838. -b Nepal (B. $H$. Hodgson 205) 1845.

Strickland (Ann. \& Mag. N. H. viii. p. 372) placed G. belangeri Less. (Bél. Voy. Ois. t. 4) as a synonym of G. leucolophus, correcting Gray (Gen. B. p. 37), who placed it under G. perspicillatus (Gm.). G. belangeri, however, is now considered to be a distinct species (cf. Blyth. B. Burma J. A. S. B. xliii. extr. No. p. 107).

\section{Garrulax perspicillatus.}

Turdus perspicillatus, Gm. Syst. Nat. i. p. 830.

Garrulax perspicillatus, Gray, Gen. B. p. 27 : Swinhoe, P.Z. S. 1871 , p. 371.

$a$ E. Indies (Arthur Strickland) 1840.

A common species in China.

\section{Garrulax pectoralis.}

Ianthocincla pectoralis, Gould, P. Z. S. 1835. p. 186.

Garrulax pectoralis, Gray, Gen. B.i. p. 225: Jerd. B. Ind. ii. p. 39.

$a$ India (E. Blyth) 1846. - b Darjeeling (E. Blyth) 1850.

\section{Garrulax moniliger.}

Cinclosoma moniliger, Hodgs. As. Res. xix. p. 147.

Garrulax moniliger, Blyth, J. A. S. B. xii. p. 949 : Jerd. B. Ind. ii. p. 40.

$a$ India (E. Blyth) 1846. 
94. Garrulax albigularis.

Ianthocincla albogularis, Gould, P. Z. S. 1835, p. 187.

Garrulax albogularis, Gray, Gen. B. i. p. 225: Cat. Hodgs. Coll. (1846), p. 82 : Jerd. B. Ind. ii. p. 38.

a Himalaya (Havell) 1839. - b Nepal (B. H. Hodgson 204) 1845 .

\section{Garrulax ruficollis.}

Ianthrocinclà ruficollis, Jard. \& Selb. Ill. Orn. ser. 2. t. 21 : Less. Bélanger Voy. t. 5.

Garrulax ruficollis, Blyth, J. A. S. B. xii. p. 950 : Jerd. B. Ind. ii. p. 38.

a India ("G. ruficollis" E. Blyth) 1846.

96. Garrulax cœruleatus.

Cinclosoma corruleatus, Hodgs. As. Res. xix. p. 147.

Garrulax coruleatus, Gray, Cat. Hodgs. Coll. (1846), p. 82: Jerd. B. Ind. ii. p. 36.

$a$ Himalaya (Williams) 1847. —b Darjeeling ("G.corulatus" E. Blyth) 1850 .

\section{TROCHALOPTERUM.}

Trochalopteron, Hodgson, J. A. S. B. xii. p. 952 (1843): Zool. Misc. p. 83 (1844).

\section{Trochalopterum erythrocephalum.}

Cinclosoma erythrocephalum, Vig. P. Z. S. 1831, p. 171: Gould, Cent. B. Him. t. 17.

Trochaloptera erythrocephalum, Jerd. B. Ind. ii. p. 43.

Garrulax? erythrocephalus, Gray, Cat. Hodgs. Coll. (1846), p. 83.

a Nepal (B. H. Hodgson 820) 1845. -b Kumaon (W. J. E. Boys) 1847.

\section{Trochalopterum lineatum.}

Cinclosoma lineatum, Vig. P. Z. S. 1831, p. 56.

Trochalopteron lineatum, Jerd. B. Ind. ii. p. 50.

a Nepal (B. H. Hodgson 317) 1845. - b Kumaon (W. J. E. Boys) 1847.

Hodgson's specimen was sent to Strickland as T. setifer, having 
been thus named doubtless by mistake. See Jerdon's note on this point (B. Ind. ii. p. 51, foot-note).

\section{Trochalopterum cachinnans.}

Crateropus cachinnans, Jerd. Madras Journ. x. p. 256, t. 7. Trochalopteron cachinnans, Jerd. B. Ind. ii. p. 48.

a Madras (T. C. Jerdon) 1845. -b India (T. C. Eyton) 1850.

100. Trochalopterum phœniceum.

Ianthocincla phonicea, Gould, Icon. Av. t. 3.

Trochalopteron phoniceum, Jerd. B. Ind. ii. p. 48.

a Darjeeling ("G. phoeniceus" E. Blyth) 1850.

\section{Trochalopterum rufogulare.}

Ianthocincla rufogularis, Gould, P. Z. S. 1835, p. 48.

Trochalopteron rufogulare, G. R. Gray, Cat. Hodgs. Coll. (1846) p. 84: Jerd. B. Ind. ii. p. 47.

a Nepal (B. H. Hodgson 187) 1845. -b Himalaya (Stevens) 1850.

\section{Trochalopterum variegatum.}

Cinclosoma variegatum, Vig. P. Z. S. 1830-1, p. 56: Gould, Cent. B. Him. t. 16.

Trochalopteron variegatum, Jerd. B. Ind. ii. p. 45.

a Simla (T. C. Eyton) 1850.

\section{Gampsorhynchus.}

Gampsorhynchus, Blyth, J. A. S. B. xiii. p. 370 (1844).

103. Gampsorhynchus rufulus.

Gampsorhynchus rufulus, Blyth, l. s. c. Jerd. B. Ind. ii. p. 14.

a Arakan (E. Blyth) 1847.

Blyth's label to this specimen has been lost.

Actinodura.

Actinodura, Gould, P. Z. S. 1836, p. 17.

104. Actinodura egertoni.

Actinodura egertoni, Gould, l. s. c. p. 18: Jerd. B. Ind. ii. p. 52.

a Darjeeling ("A. egertoni" E. Blyth) 1850. 
105. Actinodura nipalensis.

Sibia nipalensis, Hodgs. As. Res. xix. p. 145.

Actinodura nipalensis, G. R. Gray, Cat. Hodgs. Coll. (1846), p. 84: Jerd. B. Ind. ii. p. 53.

a Nepal (B. H. Hodgson 257) 1845.

\section{Sibia.}

Sibia, Hodgson, J. A. S. B. viii. p. 37 (1840).

106. Sibia picaoides.

Sibia picaoides, Hodgs. J. A. S. B. viii. p. 38 : G. R. Gray, Cat. Hodgs. Coll. (1846) p. 88 : Jerd. B. Ind. ii. p. 55.

a Nepal (B. H. Hodgson 246) 1845.

\section{0\%. Sibia capistrata.}

Cinclosoma capistratum, Vig. P. Z. S. 1830-1, p. 56.

Sibia capistrata, Jerd. B. Ind. ii. p. 54.

Sibia nigriceps, Hodgs. J. A. S. B. viii. p. 38 (1839).

a Himalaya (N. C. Strickland) 1838. - b Nepal (B. H. Hodgson 258) 1845. —c Gogra Hills (W. J. E. Boys) 1847.

\section{Cinclosoma.}

Cinclosoma, Vigors \& Horsfield, Trans. Linn. Soc. xv. p. 219 (1825).

\section{Cinclosoma punctatum.}

Turdus punctatus, Lath. Ind. Orn. Suppl. p. xliv.

Cinclosoma punctatum, Vig. Gould, B. Austr. iv. t. 4.

a Australia? (Askew), 1834.-b Australia (Askew), 1834.c Australia (N. C. Strickland) 1838.

\section{Grammatoptila.}

Grammatoptila, Reichenbach, Av' Syst. t. 1xxxv. (1850).

\section{Grammatoptila striata.}

Garrulus striatus, Vig. P. Z. S. 1831, p. 7 : Gould, Cent. B. Him. t. 37.

Grammatoptila striata, Jerd. B. Ind. ii. p. 11.

a India ("Keropia striata" E. Blyth) 1846.-Kumaon (W. J. E. Boys) 1847. 


\section{Turnagra.}

Turnagra, Lesson, Compl. Buff. ii. p. 216 (1837).

\section{Turnagra crassirostris.}

Turdus crassirostris, Gm. Syst. Nat. i. p. 815.

Turnagra crassirostris, Gray, Buller, B. N. Zeal. p. 139.

$a$ New Zealand (Fenton) 1850.

G. R. Gray (Gen. B. i. p. 227 and Hand-list. i. p. 284) places 'I' $a$ nagra capensis, Sparrm. as a synonym of this species, a view from which Strickland dissented (Ann. \& Mag. N. H. vii. p. 27), considering Sparrman's name applicable to the bird subsequently called Lanius corvinus by Shaw.

\section{SUB-FAMILY.-TIMELIIN $Æ$.}

\section{TIMELIA.}

Timalia, Horsfield, Trans. Linn. Soc. xiii. p. 150 (1820).

\section{Timelia nigricollis.}

Timalia nigricollis, Temm. Pl. Col. 594. f. 2.

$a$ Malacca (Mather) 1840.

112. Timelia leucotis.

Timalia leucotis, Strickl. Contr. Orn. 1848 p. 63, pl. 10.

a Malacca (Gardner) 1847.

The type of the species as described $l$. c.

\section{Timelia erythroptera.}

Timalia erythroptera, Blyth, J. A. S. B. xi. p. 794.

Brachypteryx acutirostris, Eyton, Ann. \& Mag. N. H. xvi. p. 228.

a Malacca (N. C. Strickland) 1838.

According to Strickland (P. Z. S. 1846, p. 103) a true Timelia.

\section{Malacopterum.}

Malacopteron, Eyton, P. Z. S. 1839, p. 102.

\section{Malacopterum magnum.}

Malacopteron magnum, Eyton, l.s.c.

a Malacca (Mather) 1840.

Compared by Strickland with Eyton's type. 
115. Malacopterum cantori.

Alcippe cantori, Moore, P. Z. S. 1854, p. 277.

a Malacca (Askew) 1839.

Agrees with a specimen of Wallace's in Lord Tweeddale's collection which has been compared and identified with Moore's type.

116. Malacopterum magnirostre.

Alcippe magnirostris, Moore, P. Z. S. 1854, p. 277.

$a$ Malacca (Argent) 1850.

Agrees with specimens thus named in Lord Tweeddale's collec. tion and with Moore's description.

Mixornis.

Mixornis, Hodgson, J. A. S. B. xiii. p. 380 note (1844): Zool. Misc. p. 83 (1844).

117. Mixornis gularis.

Timalia gularis, Horsf. Zool. Res. Jav. pl.-f. 2.

a Malacca (Gardner) 1844.

Dumetia.

Dumetia, Blyth, Cat. Birds As. Soc. p. 140 (1849).

118. Dumetia albogularis.

Malacocercus? albogularis, Blyth, J. A. S. B. xvi. p. 453.

Pellorneum albogularis, Blyth, J. A. S. B. xxi. p. 357.

Dumetia albogularis, Jerd. B. Ind. ii. p. 26.

$a,-b$ S. India (T. C. Jerdon, "Timelia hyperythra") 1850.

Macronus.

Macronus, Jardine \& Selby, Ill. Orn. iii. p. 150 (183-).

119. Macronus ptilosus.

Macronus ptilosus, Jard. \& Selb. l. s. c.

a Malacca (Mather) 1840.

Pellornecm.

Pellorneum, Swainson, Faun. Bor. Am. ii. p. 487 (1831). 
120. Pellorneum ruficeps.

Pellorneum ruficeps, Sw. l. s. c.: Jerd. B. Ind. ii. p. 27.

a India (Gardner) 1844. -b (Gardner) 1847. —c Himalaya (Stevens) 1850.

\section{TURDINUS.}

Turdinus, Blyth, J. A. S. B. xiii. p. 382 (1844).

121. Turdinus macrodactylus.

Malacopteron macrodactylum, Strickl. Ann. \& Mag. N. H. xiii. p. 417.

Turdinus macrodactylus, Blyth, l. s. c.

Brachypteryx albogularis, Hartl. Rev. Zool. 1844, p. 401 : Strickl. P. Z. S. 1846, p. 103.

a Malacca (Mather) 1840.

This specimen is the type of the species.

\section{Drymocataphus.}

Drymocataphus, Blyth, J. A. S. B. xviii. pt. 2, p. 815 (1850).

\section{Drymocataphus nigrocapitatus.}

Brachypteryx nigrocapitata, Eyt. P. Z. S. 1839, p. 103.

Goldana capistratoides, Strickl. Contr. Orn. 1849. p. 128, pl. 23.

a Malacca (Mather) 1840.

In a MS. note to his paper (ut s. c.) Strickland refers this species to Blyth's genus Drymocataphus.

\section{TRICHOSTOMA.}

Trichastoma, Blyth, J. A. S. B. xi. p. 795 (1842).

123. Trichostoma abboti.

Malacocincla abboti, Blyth, J. A. S. B. xiv. p. 601 (1845).

a Arkaan (E. Blyth) 1847. -b (Bt. at Birmingham) 1849.

With reference to the figures (Contr. Orn. 1849, pl. 22) of Trichostoma umbratile and T. celebense Strickland has written in his copy, "The black streak through the eye of both figures is a fancy of the colourer. It does not exist in the birds. H. E. S." 


\section{AlCIPPE.}

Alcippe, Blyth, J. A. S. B. xiii. p. 384 (1844).

\section{Alcippe atriceps.}

"Brachypteryx atriceps, Jerd." Blyth, l. s. c. Alcippe atriceps, Jerd. B. Ind. ii. p. 19. a (Gardner) 1840.

Forests of Southern India.

125. Alcippe magnirostris.

Alcippe magnirostris, Wald. J. A. S. B. xliii. extra No. p. 115 (nec Moore).

A. phayrei, Blyth, op. cit. xiv. p. 601.?

$a$ (Askew) 1837.

Lord Tweeddale, who has kindly examined for me this and other specimens of this obscure family, says "that it is identical with his type specimen of $A$. magnirostris, but that it may be $A$. phayrii, Blyth." This name Blyth seems latterly to have considered a synonym of $A$. nipalensis, supposing his title to have been founded upon what was probably the young of that species. A. nipalensis, however, is a much smaller bird, and differs in the coloration of the head.

\section{Alcippe nipalensis.}

Siva nipalensis, Hodgs. Indian Review, 1838, p. 89.

Alcippe nipalensis, Jerd. B. Ind. ji. p. 18.

a Nepal (B. H. Hodgson 357) 1845. -b India (E. Blyth) 1846.

\section{StACHYRIS.}

Stachyris, Hodgson, J. A. S. B. xiii. p. 378 (1844).

\section{2\%. Stachyris pyrrhops.}

S. pyrrhops, Hodgson. l. s. c. p. 379 : Jerd. B. Ind. ii. p. 21. 1850 .

a Nepal (B. H. Hodgson 410) 1845. -b Himalaya (Stevens)

\section{Stachyris ruficeps.}

Stachyris ruficeps, Blyth, J. A. S. B. xvi. p. 452: Jerd. B. Ind. ii. p. 27.

a N. India (Williams) 1848. 


\section{Pyctorhis.}

Pyctorhis, Hodgson, Zool. Misc. p. 83 (1844).

129. Pyctorhis sinensis.

Parus sinensis, Gm. Syst. Nat. i. p. 1012.

Timalia horsfieldi, Jard. \& Selb. Ill. Orn. iii. pl. 119.

Timalia hypoleuca, Frankl. P. Z. S. 1830-1, p. 118.

Pyctorhis sinensis, Jerd. B. Ind. ii. p. 15.

a India (Havell) 1839. -b Madras (T. C. Jerdon) 1850.

\section{Pomatorhinus.}

Pomatorhinus, Horsfield, Trans. Linn. Soc. xiii. p. 164 (1820). 130. Pomatorhinus borneensis.

Pomatorhinus borneensis, Cab. Mus. Hein. p. 84: Salvad. An. Mus. Civ. Genov. v. p. 210.

Pomatorhinus montanus, S. Müll. (nec Horsf.)

a Malacca (Argent).

131. Pomatorhinus ruficollis.

Pomatorhinus ruficollis, Hodgs. As. Res. xix. p. 182: Zool. Misc. p. 83: Jerd. B. Ind. ii. p. 29.

a Nepal (B. H. Hodgson 239) 1845. -bq Kumaon (W. J.E. Boys) 1847.

\section{Pomatorhinus erythrogenys.}

Pomatorhimus erythrogenys, Vig. P. Z. S. 1830-1, p. 173 : Gould, Cent. B. Him. pl. 55 : Hodgs. Zool. Misc. p. 83 : Jerd. B. Ind. ii. p. 31.

$a$ Nepal (B. H. Hodgson 237) 1845.

\section{Pomatorhinus leucogaster.}

Pomatorhinus leucogaster, Gould, P. Z. S. 1837, p. 137 : Jerd. B. Ind. ii. p. 30.

a India (Stevens) 1844.

\section{Pomatorhinus schisticeps.}

Pomatorhinus schisticeps, Hodgs. As. Res. xix. p. 180 : Jerd. B. Ind. ii. p. 29: Blyth, J. A. S. B. xliii. extr. No. p. 113. a Arakan (E. Blyth) 1847. 


\section{Pomatorhinus horsfieldi.}

Pomatorhinus horsfieldi, Sykes, P. Z. S. 1832, p. 89 : Jerd. B. Ind. ii. p. 31.

$a$ India (Gardner) 1844. - b S. India ("P.horsfieldi" T. C. Jerdon) 1850.

136. Pomatorhinns temporalis.

Pomatorhinus temporalis, Vig. \& Horsf. Trans. L. S. xv. p. 330 : Gould, B. Austr. iv. pl. 20.

Pomatorhinus trivirgatus, Temm. Pl. Col. 20.

a Australia (N.C. Strickland) 1838.

\section{FAMILY.-CINCLID $Æ$.}

\section{CINCLUS.}

Cinclus, Bechstein, Naturg. Deutschl. iii. p. 808 (1802).

\section{3\%. Cinclus aquaticus.}

Cinclus aquaticus, Bechst. l. s. c.: Newton ed. Yarr. Brit. B. i. p. 241: Salvin, Ibis, 1867, p. 113.

a Rosshire (Hyett) 1844. - b N. Wales (H. E. Strickland) Jan. 1851.

138. Cinclus leucogaster.

Cinclus leucogaster, Brandt, Tchihatcheff's Voy. Altai, p. 417: Bp. Consp. p. 252 : Salvin, Ibis, 1867, pp. 117, 382.

$a$ W. Siberia (Brandt) 1844.

Doubtless a typical specimen.

139. Cinclus asiaticus.

Cinclus asiaticus, Sw. Faun. Bor. Am. ii. p. 174: Salv. Ibis, 1867, p. 120.

Hydrobata asiatica, Jerd. B. Ind. i. p. 506.

Cinclus pallasi, Gould, Cent. B. Him. t. 24.

a Kumaon (W. J. E. Boys) 1847. -b N. India (W. J. E. Boys) 1848. 


\section{Cinclus mexicanus.}

Cinclus mexicanus, Sw. Phil. Mag. 1827, p. 368 : Salv. Ibis, 1867, p. 120.

Cinclus unicolor, Bp. Comp. List. p. 19.

a Mexico (T. Mann) 1844.

\section{FAMILY.-HENICURIDÆ.}

\section{Henicurus.}

Enicurus, Temm. Pl. Col. 160. Livr. 27 (July 1823).

\section{Henicurus schistaceus.}

Enicurus schistacens, Hodgs. As. Res. xix. p. 189 : Jerd. B. Ind. ii. p. 214.

Henicurus schistaceus, Elwes, Ibis, 1872, p. 253.

a (T. C. Eyton) 1850.

142. Henicurus immaculatus.

Enicurus immaculatus, Hodgs. As. Res. xix. p. 190: Jerd. B. Ind. ii. p. 213.

Henicurus immaculatus, Elwes, Ibis, 1872, p. 254: Blyth, J. A. S. B. xliii. extr. No. p. 97. a Arakan (E. Blyth) 1847 .

\section{Henicurus scouleri.}

Enicurus scouleri, Vig. P. Z. S. 1830-1, p. 174: Gould, Cent. B. Him. pl. 28: Jerd. B. Ind. ii. p. 214.

Henicurus scouleri, Elwes, Ibis, 1872, p. 255.

a $f$ Kumaon (W. J. E. Boys) 1847.

144. Henicurus maculatus.

Enicurus maculatus, Vig. P. Z. S. 1830-1, p. 9 : Gould, Cent. B. Him. pl. 27 : Jerd. B. Ind. ii. p. 212.

Henicurus maculatus, Elwes, Ibis, 1872, p. 260.

a Himalaya (N.C. Strickland) 1838. -b Kumaon (W.J. E. Boys) 1847. 


\section{FAMILY.-SYLVIID A. \\ SUB-FAMILY.-SAXICOLIN E.}

SAXICOLA.

Saxicola, Bechstein, Orn. Taschenb. p. 216 (1802).

\section{Saxicola cnanthe.}

Motacilla enanthe, Linn. Syst. Nat. i. p. 352.

Saxicola enanthe, Strickl. P. Z. S. 1836 , p. 98 ; 1850. p. 216 : Blanf. \& Dresser, P. Z. S. 1874, p. 218: Newton, ed. Yarr. Brit. B. i. p. 347.

$a,-b$ Aberdovey (H. E. Strickland) Aug. 1833. —c (Askew) 1834. —d Smyrna (H.E. Strickland) April, 1836. — e, - $f$ Britain? (N. C. Strickland) 1838. —g Senegal (Isaacson) 1840. - Kartoum Oct. 1848 (J. Petherick) 1848.

\section{Saxicola albicollis.}

Motacilla stapazina, Linn. Syst. Nat. i. p. 331 (partim). Enanthe albicollis, Vieill. N. Dict. d'Hist. N. xxi. p. 424.

Saxicola aurita, Temm. Man. d'Orn. i. p. 341 : Strickl. P. Z. S. 1836, p. 98.

a f Morea (H. E. Strickland) May 1836. - b o Smyrna (H. E. Strickland) April, 1836.

\section{Saxicola stapazina.}

Saxicola stapazina, Linn. Syst. Nat. i. p. 331 (partim). Enanthe stapazina, Vieill. N. Dict. d'Hist. N. xxi. p. 428. Saxicola stapazina, Temm. Man. d'Orn. i. p. 239.

$a$ S. Europe (Bt. at Dresden) 1845.

In 1843 Strickland, in his notes to Col. Drummond-Hay's Catalogue of Birds found in Corfu \&c. (Ann. \& Mag. N. H. xii. pp. 416, 424) refers to the supposed identity of S. stapazina and $S$. albicollis (aurita), and remarks that the former having been found breeding in Corfu, the distinctness of the latter would be proved on its being found to breed in Crete. In a notice of Malherbe's 'Faune Ornithologique de la Sicile' (Ann. \& Mag. N. H. xiv. p. 432) attention is drawn to his assertion of their distinctness. Though Strickland as late as 1849 (Contr. Orn. p. 60) still seemed undecided on the 
point, the question cannot now be considered an open one, as the fact of the distinctness of the two species is quite settled.

\section{Saxicola deserti.}

Saxicola deserti, Temm. Pl. Col. 359, f. 2 : Strickl. P. Z. S. 1850, p. 216 : Blanf. \& Dresser, P. Z. S. 1874, p. 224.

Saxicola atrogularis, Blyth, J. A. S. B. xvi. p. 130.

a ô Kartoum, Oct. 1848 (J. Petherick) 1848. -b N. India (Stevens) 1850.

\section{Saxicola leucomela.}

Motacilla leucomela, Pall. N. Comm. Petr. xiv. p. 584. t. 22. f. 3.

Saxicola leucomela, Temm. Man. d’Orn. i. p. 243 : Blanf. \& Dresser, P. Z. S. 1874, p. 225.

Saxicola lugens, Licht. Verz. Doubl. p. 33.

$a$ (Askew) 1837.

Asia and N. E. Africa.

150. Saxicola morio.

Saxicola morio, Ehr. Symb. Phys. fol. aa: Blanf. \& Dresser, P. Z. S. 1874, p. 225.

Saxicola leucomela, Jerd. B. Ind. ii. p. 131 (nec Pallas). a $\hat{\delta}$ Siberia (J. F. Brandt) 1846.

\section{Saxicola picata.}

Saxicola picata, Blyth, J. A. S. B. xvi. p. 131 : Blanf. \& Dresser, P. Z. S. 1874, p. 227.

$a,-b$. N. India (W. J. E. Boys) 1847.

\section{Saxicola leucura.}

Turdus leucurus, Gm. Syst. Nat. i. p. 820.

Saxicola leucura, Blanf. \& Dresser, P. Z. S. 1874, p. 228.

a Dalmatia (L. Coulon) 1836.

\section{Saxicola opistholeuca.}

Saxicola opistholeuca, Strickland, Contr. Orn. 1849, p. 60. t. 13 : Blanf. \& Dresser, P. Z. S. 1874, p. 229.

a N. India (W. J. E. Boys) 1848.

Probably the type of the species, at one time in Mr Sclater's collection. 
154. Saxicola isabellina.

Saxicola isabellina, Cretzschm. Rüpp. Atl. p. 52. t. 34 b: Blanf. \& Dresser, P. Z. S. 1874, p. 228.

Saxicola onanthe, Jerd. B. Ind. ii. p. 132.

a Benares (W. J. E. Boys) 1847. -b N. India (W. J. E. Boys), 1847. —c q Kartoum, Nov. 1848 (J. Petherick) 1848.

$a$ and $b$ were both considered by Strickland to belong to $S$. exnanthe; $c$ is referred to in Strickland's paper on the Birds procured in Kordofan by Petherick (P. Z. S. 1850, p. 216).

\section{Saxicola leucomelæna.}

Saxicola leucomelcena, Burchell, Travels in S. Afr. i. p. 335: Isis, 1823, p. 153: Strickl. \& Scl. Contr. Orn. 1852, p. 146.

a Damara-land (C. J. Andersson) 1852.

\section{Saxicola pollux.}

Saxicola pollux, Hartl. P. Z. S. 1865, p. 747 : Blanf. \& Dresser, P. Z. S. 1874, p. 235.

a $q \mathrm{~S}$. Africa (Dr A. Smith).

\section{5\%. Saxicola familiaris.}

Saxicola familiaris, Steph. in Shaw's Zool. xiii. Suppl. p. 241 : Gurney, in And. B. Damara-1. p. 103.

a Cape of Good Hope (A. Strickland) 1832.

\section{Saxicola galtoni.}

Erythropygia galtoni, Strickland, Contr. Orn. 1852, p. 147.

Saxicola galtoni, Blauf. \& Dresser, P. Z. S. 1874, p. 237.

a Damara-land (C. J. Andersson) 1852.

The type of the species as described by Strickland (l. s. c.) This bird has been identified by many writers with $S$. spercita (Lath.), by others with S.familiaris, Steph.; but see Blanford's and Dresser's remarks on this point (l. s. c.). It is distinct from though closely allied to the preceding species, which I believe to be S. familiaris, Steph.

\section{Saxicola sinuata.}

Luscinia sinuata, Sundev. K. Sv. Vet. Akad. Hand. ii. p. 44. (1857).

Saxicola sinuata, Layard, B. S. Afr. p. 106 ; Blanf. \& Dresser, P. Z. S. 1874 , p. 238. 
a §ิ Cape of Good Hope (A. Strickland) 1833.

Considered by Strickland to be either $S$. sperata, Lath. or $S$. familiaris Steph.

\section{Saxicola bifasciata.}

Saxicola bifasciata, Temm. Pl. Col. 472. f. 2: Blanf. \& Dresser, P. Z. S. 1874, p. 239.

Sylvia hottentota, Gm. Syst. Nat. i. p. 965.

a S. Africa (N. C. Strickland) 1838. - b Damara-land (C. J. Andersson) 1852.

In their paper on Birds from Damara-land (Contr. Orn. 1852, p. 146), Strickland and Selater identify this bird with Gmelin's S. hottentota, but Blanford and Dresser state that they found it quite impossible to determine the 'Grand Motteux du Cap de B. Espérance,' of Buffon upon which Gmelin bestowed his name.

\section{Saxicola infuscata.}

Saxicola infuscata, Smith, Zool. S. Afr. t. 28 : Gurney, in And. B. Damara-1. p. 107.

Saxicola sp. Strickl. \& Scl. Contr. Orn. 1852, p. 146. No. 29. a Damara-land (C. J. Andersson) 1852.

\section{MYRMECOCICHLA.}

Myrmecocichla, Cabanis, Mus. Hein. i. p. 8 (1850).

\section{Myrmecocichla formicivora.}

Saxicola formicivora, Vieill. N. Dict. d'Hist. Nat. xxi. p. 421.

Myrmicocichla formicivora, Layard, B. S. Afr. p. 110.

$a$ S. Africa (Askew) 1834.

\section{Myrmecocichla æthiops.}

Myrmecocichla cethiops, Cab. Mus. Hein. i. p. 8 (ex Licht. MS.).

a S. Africa (Thomas) 1842.

This specimen agrees best with examples in the British Museum bearing this name. 
Pratincola.

Pratincola, Koch, Syst. d. Baier. Zool. i. p. 190 (1816).

164. Pratincola rubicola.

Motacilla rubicola, Linn. Syst. Nat. i. p. 332.

Pratincola rubicola, Koch, l.s. c.

Saxicola rubicola, Strickl. P. Z. S. 1836, p. 98: Newton, ed. Yarr. Brit. B. i. p. 339.

$a \hat{\delta},-b \hat{\delta}$ juv. $-c$ f Worcestershire (H. E. Strickland) May, 1834. - $d \hat{\delta}$ Worcestershire (H. E. Strickland) March, 1834. -e f Smyrna (H. E. Strickland) 20 Nov. 1835.

\section{Pratincola indica.}

Pratincola indica, Blyth, J. A. S. B. xvi. p. 120 : Jerd. B. Ind. ii. p. 124.

Saxicola saturatior, Hodgs. Zool. Misc. p. 83.

a Nepal (B. H. Hodgson 300) 1845. - b q Madras ("Saxicola rubicola fem." T. C. Jerdon) 1845. —c Benares (W. J. E. Boys) 1847. — - d juv. India ("Pratincola indica" E. Blyth) 1850.

\section{Pratincola pastor.}

Pratincola pastor, Strickl. Ann. \& Mag. N. H. xiii. p. 410 (1844): Layard, B. S. Afr. p. 111.

$a$ \& Cape of Good Hope (A. Strickland) 29 April, 1832. -b $\hat{\delta}$ Cape of Good Hope (A. Strickland) 18 April, 1832.

The types of the species as described by Strickland $l . s . c$.

\section{6\%. Pratincola caprata.}

Motacilla caprata, Linn. Syst. Nat. i. 335.

Saxicola caprata, G. R. Gray, Cat. Hodgs. Coll. (1846) p. 71.

Pratincola caprata, Jerd. B. Ind. ii. 123: Wald. Trans. Z. S. ix. p. 193.

a (Askew) 1834. - b Luzon (Cuming) 14 June, 1839. c Madras ("Saxicola caprata" T. C. Jerdon) 1845. —d Nepal (B. H. Hodgson 420) 1845. - e Benares (W. J. E. Boys) 1847. -f (Gardner) 1847.

\section{Pratincola ferrea.}

Rubicola ferrea, Hodgs. Zool. Misc. p. 83 (descr. nulla).

Saxicola ferrea, G. R. Gray, Cat. Hodgs. Coll. (1846) p. 71 (descr. nulla). 
Pratincola ferrea, Hodgs. Blyth, J. A. S. B. xvi. p. 129 : Jerd. B. Ind. ii. p. 127.

a Himalaya ( $N$. C. Strickland) 1838. -b Nepal (B. H. Hodgson 416) 1845. -c Kumaon (W. J. E. Boys) 1847. -d India (W. J. E. Boys) 1847.

\section{Pratincola rubetra.}

Motacilla rubetra, Linn. Syst. Nat. i. p. 332.

Saxicola rubetra, Newton, ed. Yarr. Brit. B. i. p. 9.

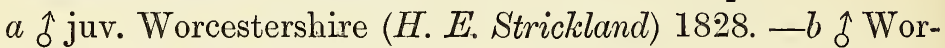
cestershire (H. E. Strickland) 28 April, 1832. - $c$ o Worcestershire (H. E. Strickland) May, 1834.

\section{THAMNOBIA.}

Thamnobia, Swainson, Faun. Bor. Am. ii. p. 489 (1831).

\section{Thamnobia fulicata.}

Motacilla fulicata, Linn. Syst. Nat. i. p. 336.

Thamnobia fulicata, Jerd. B. Ind. ii. p. 121.

a Philippine Islands? (N. C. Strickland) 1838. - b India (N. C. Strickland) 1838.

If the Philippine origin of specimen $a$ were free from doubt. we should have the first authentic proof of the presence of the species in those islands since Brisson's statement that he received his type from there through M. Aubrey (see Walden, Trans., Z. S. ix. p. 1933).

\section{Thamnobia cinnamomeiventris.}

Turdus cinnamomeiventris, Lafr. Mag. Zool. 1836, tt. 55, 56. Saxicola albiscapulata, Layard, B. S. Afr. p. 106. (nec Rüpp.) 1838.

$a$ S. Africa (Askew) 1834. - b S. Africa (N. C. Strickland)

\section{Thamnobia cambaiensis.}

Sylvia cambaiensis, Lath. Ind. Orn. p. 554.

Thamnobia cambaiensis, Jerd. B. Ind. ii. p. 122.

$a$ N. India (N. C. Strickland) 1838.

\section{PeNTHOL}

Pentholcea, Cabanis, Mus. Hein. i. p. 40 (1850).

173. Pentholæa frontalis.

Saxicola frontalis, Swains, Birds W. Afr. ii. p. 46. 
Pentholcea frontalis, Cab. l. s. c. a Africa (Arthur Strickland) 1840.

\section{Petreca.}

Petroica, Swainson, Zool. Ill. ser. 2. i. pl. 36 (1832-33).

\section{Petrœca multicolor.}

Muscicapa multicolor, Vig. \& Horsf. Trans. Linn. Soc. xv. p. 243.

Petroica multicolor, Swains. Classif. B. ii. p. 240 : Gould, B. Austr. iii. pl. 3.

$a$ New South Wales (N. C. Strickland) 1838. - b (Dewgard) 1851.

\section{Petroca rosea.}

Petroica rosea, Gould, P. Z. S. 1839, p. 142.

Erythrodryas rosea, Gould, B. Austr. iii. pl. 2.

a New South Wales (N. C. Strickland) 1838.

\section{Petrœca phœnicea.}

Petroica phonicea, Gould, P. Z. S. 1836, p. 105 : B. Austr. iii. pl. 6.

$a,-b$ New South Wales (N. C. Strickland) 1838.

\section{7\%. Petrœca goodenovi.}

Muscicapa goodenovi, Vig. \& Horsf. Trans. Linn. Soc. xv. p. $24 \check{.}$.

Petroica goodenovi, Gould, B. Austr. iii. pl. 5.

a New South Wales (N. C. Strickland) 1838.

\section{Petrœca cucullata.}

Muscicapa cucullata, Lath. Ind. Orn. Suppl. p. li.

Petroeca cucullata, Strickl. Ann. \& Mag. N. H. xi. p. 337.

a Australia (Stevens), 1842.

Strickland (l.s. c.) identified $P$. bicolor, Swains., a name long borne by this bird, with Latham's description drawn up from one of the Earl of Derby's 'New South Wales Drawings'.

179. Petrœca macrocephala.

Parus macrocephalus, Gm. Syst. Nat. i. p. 1013. 
Petroeca macrocephala, Gray, Zool. Ereb. \& Terr. Birds, p. 6. Myiomoira macrocephala, Buller B. N. Zeal. p. 126. a New Zealand (Fenton) 1850.

Sialia.

Sialia, Swainson, Zool. Journ. iii. p. 173 (1827).

\section{-180. Sialia wilsoni.}

Motacilla sialis, Linn. Syst. Nat. i. p. 336.

Sialia wilsoni, Swains. l.s.c.

Sialia sialis, Baird, Brew. \& Ridgw. N. Am. Birds, i. p. 62.

$a$ N. America (Askew) 1833. —b Bermuda (Stevens) 1843. - c (Arthur Strickland) 1840. -d N. America (Gardner) 1845. -e Guatemala (J. Constancia) 1845.

181. Sialia mexicana.

Sialia mexicana, Swains. Faun. Bor. Am. ii. p. 202 : Baird, Brew. \& Ridgw. N. Am. Birds i. p. 65.

a Mexico (T. Mann) 1844.

\section{ORIGMA.}

Origma, Gould, P. Z. S. 1837, p. 148.

182. Origma rubricata.

Sylvia rubricata, Lath. Ind. Orn. Suppl. p. lv.

Origma rubricata, Gould, B. Austr. iii. t. 69.

a (A. Strickland) 1850.

An Australian species.

\section{SUB-FAMILY.-RUTICILLIN AE.}

\section{Ruticilla.}

Ruticilla, C. L. Brehm, Isis. 1828, p. 1280¹.

\section{Ruticilla phœnicurus.}

Motacilla phœnicurus, Linn. Syst. Nat. i. p. 335.

1 In stating (Ann. \& Mag. N. H. vi. p. 422) that Phønicura, Sw. (1831) should be employed instead of Ruticilla "Ray" Strickland overlooked Brehm's use of the latter name. 
Ruticilla phonicurus, Newton, ed. Yarr. Brit. B. i. p. 329.

$a \hat{\delta},-b+$ Worcestershire (H. E. Strickland) May, 1837. -c Gloucestershire (H. E. Strickland) June, 1850.

184. Ruticilla titys.

Sylvia tithys, Scop., Ann. i. Hist. Nat. i. p. 157.

Phcenicura tithys, Jard. \& Selb., Strickl. P. Z. S. 1836. p. 98.

Ruticilla titys, Newton, ed. Yarr. Brit. B. i. p. 333.

$a$ f, -b ô Smyrna (H. E. Strickland) Dec. 1835.

Found by Strickland throughout the winter on the bare rocky hills near Smyrna.

\section{Ruticilla rufiventris.}

Enanthe rufiventris, Vieill. N. Dict. d'Hist. N. xxi. p. 431.

Ruticilla rufiventris, Jerd. B. Ind. ii. p. 137.

Phonicura atrata, Jard. \& Selb. Ill. Orn. pl. 86. f. 3.

$a$ India (Askew) 1837. - $b$ o Madras ("Phonicura atrata" T. C. Jerdon) 1845. —c, —d India ("Ruticilla atrata" E. Blyth) 1846.

186. Ruticilla cæruleocephala.

Phonicura coeruleocephala, Vig. P.Z. S. 1830-1, p. 35 : Gould, Cent. B. Him. t. 25, f. 2.

Ruticilla cceruleocephala, Jerd. B. Ind. ii. p. 141.

a N. India (W. J. E. Boys) 1848. - b Himalaya (Stevens) 1850 .

\section{8\%. Ruticilla frontalis.}

Phonicura frontalis, Vig. P. Z. S. 1830-1. p. 172: Gould, Cent. B. Him. t. 26. f. 1.

Ruticilla frontalis, Jerd. B. Ind. ii. p. 141.

Phoenicura tricolor, Hodgs. Zool. Misc. p. 83.

a Himalaya (N. C. Strickland) 1838. -b Nepal (B. H. Hodgson 483) 1845. —c Bugaisir (W. J. E. Boys) 1847.

\section{Ruticilla fuliginosa.}

Phoenicura fuliginosa, Vig. P. Z. S. 1830-1, p. 35.

Ruticilla fuliginosa, Jerd. B. Ind. ii. p. 142.

Phoenicura rubricauda, Hodgs. Zool. Misc. p. 68. 
$a,-b \mathrm{Nepal}$ (B. H. Hodgson 298, 418) 1845. -c, -d Kumaon (W. E. J. Boys) 1847. -e N. India (W. J. E. Boys) 1848.

\section{Chimarrhornis.}

Chaimarrornis, Hodgson, Zool. Miscell. p. 82 (1844).

189. Chimarrhornis leucocephala.

Phonicura leucocephala, Vig. P. Z. S. 1830-1, p. 35 : Gould, Cent. B. Him. pl. 26, f. 2.

Chainarrornis leucocephala, Hodgs. l. s. c.: Jerd. B. Ind. ii. p. 143.

a Nepal (B. H. Hodgson 297) 1845. -b Kumaon (W. J. E. Boys) 1847. —c Himalaya (Stevens) 1849.

LARVIVORA.

Larvivora, Hodgson, J. A. S. B. vi. p. 102 (1837.)

190. Larvivora cyanea.

Larvivora cyanea, Hodgs. l. s. c. : Jerd. B. Ind. ii. p. 145. a Madras (T. C. Jerdon) 1845.

\section{CyaneCula.}

Cyanecula, C. L. Brehm, Isis, 1828, p. 1280.

191. Cyanecula suecica.

Motacilla suecica, Linn. Syst. Nat. i. p. 336.

Cyanecula suecica, Dresser, B. Eur.

Phonicura suecica, Strickl. P. Z. S. 1836, p. 98.

Ruticilla suecica, Newton, ed. Yarr. Brit. B. i. p. 321.

a W. Siberia (J. F. Brandt) 1844. - b Lapland (E. De Selys Longchamps) 1845. —c Madras (T. C. Jerdon) 1845. —d Britain (W. Kirtland) 1846. - e Siberia (J. F. Brandt) 1846. $-f,-g$ India (E. Blyth) 1846. - $h$ India (W. J. E. Boys) 1847. -i Marwar (W. J. E. Boys) 1847.

Strickland believed that he saw this bird near Smyrna in April.

192. Cyanecula wolfi.

Sylvia wolfi, C. L. Brehm, Beitr. z. Vögelk. ii. p. 173: Dresser, B. Eur. (partim).

a $\hat{o}$ Spain (Boissoneau) 1839. 


\section{DAUlias.}

Daulias, Boie, Isis, 1831, p. $542^{1}$.

\section{Daulias luscinia,}

Motacilla luscinia, Linn. Syst. Nat. i. p. 328.

Daulias luscinia, Newton, ed. Yarr. Brit. B. p. 312.

Philomela luscinia, Strickl. P. Z. S. 1836, p. 98.

a $\hat{\delta}$ Worcestershire (H. E. Strickland) 1833.

Heard by Strickland on April 5th near Hushak in Asia Minor.

IANTHIA.

Ianthia, Blyth, J. A. S. B. xvi. p. 133 (1847): Nemura, Hodgs. P. Z. S. 1845, p. 27 (nec Latreille).

\section{Ianthia rufilata.}

Nemura rufilata, Hodgs. l. s. c.

Ianthia rufilata, Blyth, l. s. c.: Jard. Contr. Orn. 1850, p. 28. pl. 44 .

Ianthia cyanura, Jerd. B. Ind. ii. p. 146, (nec Pall. $c f$. Blyth, Ibis, 1867, p. 16).

a Kumaon (W. J. E. Boys) 1847. -b N. India (W. J. E. Boys) 1848.

\section{Calliope.}

Calliope, Gould, Birds of Eur. t. 114. (1836).

\section{Calliope camtschatkensis.}

Turdus camtschatkensis, Gm. Syst. Nat. i. p. 817.

Calliope lathami, Gould, l. s. c.

Calliope camtschatkensis, Strickland, Ann. \& Mag. N. H. vi. p. 422 : Jerd. B. Ind. ii. p. 150.

$a,-b$ India (E. Blyth) 1846.

\section{Erithacus.}

Erithacus, Cuvier, Leç. d'Anat. Comp. i. Tab. 2. (1800).

\section{Erithacus rubecula.}

\section{Motacilla rubecula, Linn. Syst. Nat. i. p. 337.}

1 See Strickland's remarks respecting the generic title for the Nightingale, Ann. \& Mag. N. H. vi. p. 422. 
Erithacus rubecula, Newton, ed. Yarr. Brit. B. i. p. 305 : Dresser, B. Eur.

a Oxfordshire (N. C. Strickland) 10 July 1833. - b f Worcestershire (H. E. Strickland) Feb. 1837.

\section{Bradypterus.}

Bradypterus, Swainson, Classif. Birds, ii. p. 241 (1837).

19\%. Bradypterus sericeus.

Sylvia sericea, Natt. Temm. Man. d'Orn. i. p. 197.

Bradypterus sericeus, Dresser, B. Eur.

Bradypterus platurus, Sw. l. s. c.

Cettia altisonans, Gould, B. Eur. ii. pl. 114.

a Italy (Boissoneau) 1839.

\section{AEDON。}

Aedon, Boie, Isis, 1826, p. $972^{1}$.

\section{Aedon galactodes.}

Sylvia galactotes, Temm. Man. d'Orn. i. p. 182 (1820). Aedon galactodes, Newton, ed. Yarr. Brit. B. i. p. 355. $a \hat{\delta},-b q \operatorname{Morea}(H$. E. Strickland) May 1836.

\section{Aedon paena.}

Erythropygia paena, Smith, Rep. Exp. Afr. p. 46 : Zool. S. Afr. t. 30 .

AEdon pœna, Layard, B. S. Afr. p. 99.

$a$ S. Africa (Capt. Alexander) 1838.

200. Aedon leucophrys.

Sylvia leucophrys, Vieill. N. Dict. d'Hist. N. xi. p. 191. AEdon leucophrys, Layard, B. S. Afr. p. 99.

Turdus pipiens, Steph. Shaw's Zool. xiii. Suppl. p. 202.

$a$ S. Africa (Bought at Birmingham) 1843.

\section{Aedon coryphæus.}

Sylvia coryphceus, Vieill. N. Dict. d'Hist. N. xi. p. 177.

Bradypterus coryphous, Layard, B. S. Afr. p. 100.

a ई̊ Cape of Good Hope (A. Strickland) 1833.

1 See Strickland's note as to the position of this genus (Ann. \& Mag. N. H. vi. p. 422). 


\section{SUB-FAMILY.—SYLVIIN Æ.}

\section{SYLVIA.}

Sylvia, Scopoli, Annus i. Hist. Nat. p. 154 (1769).

202. Sylvia atricapilla.

Motacilla atricapilla, Linn. Syst. Nat. i. p. 332.

Sylvia atricapilla, Newton, ed. Yarr. Brit. B. i. p. 418.

a f juv. $-b$ \& Worcestershire (H. E. Strickland) 1834.

\section{Sylvia orphea.}

Sylvia orphea, Temm. Man. d'Orn. p. 107 (1815): Newton, ed. Yarr. Brit. B. i. p. 423 (et auctt. plurr.)

Motacilla hortensis, Gm. Syst. Nat. i. p. 955, ex D'Aubent. Pl. Enl. 579. f. 1. ?

Curruca hortensis, Strickland, Ann. \& Mag. N. H. viii. p. 372. 1837.

$a$ \&, - b f Zante (H. E. Strickland) May 1836. -c (Askew)

$c$ seems to belong to the large Indian form $S$. jerdoni.

\section{Sylvia salicaria.}

Motacilla salicaria, Linn. Syst. Nat. i. p. 330.

Sylvia salicaria, Newton, ed. Yarr. Brit. B. i. p. 414.

Sylvia hortensis, var. B. Lath. Ind. Orn. p. 507 (et auctt.plurr. nec Motacilla hortensis, Gm.).

a f Zante (H. E. Strickland) 19 May 1836. -b Worcestershire (Robinson) 1840.

205. Sylvia subalpina.

Sylvia subalpina, Bonelli, Temm. Man. d'Orn. i. p. 214 (1820): Dresser, B. Eur.

Sylvia passerina, Temm. Man. d'Orn. i. p. 213 (nec Gm.).

a Hymettus (Bought at Dresden) 1845. $-b$ Tangiers ( $F$. Favier) 1848.

\section{Sylvia conspicillata.}

Sylvia conspicillata, La Marm. Mem. Ac. Tor, 1819: Dresser, B. Eur.

$a q,-b$ ก S. Europe (N. C. Strickland) 1838. 


\section{Sylvia curruca.}

Motacilla curruca, Linn. Syst. Nat. i. p. 329.

Sylvia curruca, Newton, ed. Yarr. Brit. B. i. p. 410 : Jerd. B. Ind. ii. p. 209.

a Madras (T. C. Jerdon) 1845. - b ई Worcestershire (H. E. Strickland) 1833. -c $\hat{\circ}$ Worcestershire (H. E. Strickland) 6 May, 1837. —d (Askew) 1837.

\section{Sylvia affinis.}

Curruca affinis, Blyth, J. A. S. B. xiv. p. 564.

Sylvia affinis, Jerd. B. India, ii. p. 209.

a Madras ("C. affinis, Blyth" T. C. Jerdon) 1846.

\section{Sylvia rufa.}

Motacilla rufa, Bodd. Tab. P1. Enl. p. 35.

Sylvia rufa, Newton, ed. Yarr. Brit. B. i. p. 406.

Sylvia cinerea, Lath. et auctt. plurr.

Curruca cinerea, Strickl. P. Z. S. 1836, p. 98.

$a$ Tuscany (C. Passerini) Aug. 1833. - b Worcestershire (H. E. Strickland) 1834. —c f Worcestershire (H. E. Strickland) 12 May, 1837.

Seen by Strickland near Smyrna.

\section{Sylvia nisoria.}

Sylvia nisoria, Bechst. Naturg. Deutschl. iii. p. 547 : Dresser, B. Eur.

a S. Europe (P. L. Sclater).

\section{Sylvia melanocephala.}

Motacilla melanocephala, Gm. Syst. Nat. i. p. 970.

Curruca melanocephala, Strickl. P. Z. S. 1836, p. 98.

$a$ ㅇ 20 Nov. - $b$ î 10 Dec. Smyrna (H. E. Strickland) 1835. c $\hat{\delta} 18$ May, -d $q 21$ May Zante (H. E. Strickland) 1836.

Strickland says that this bird remains throughout the year at Smyrna.

\section{MelizophiLus.}

Melizophilus, Leach, Syst. Cat. Mamm. \& Birds in Brit. Mus. p. 25 (1816). 
212. Melizophilus undatus.

Pittechou de Provence, D'Aubent. Pl. Enl. 655, f. i. undè.

Motacilla undata, Bodd. Tab. Pl. Enl. p. 40.

Melizophilus undatus, Newton, ed. Yarr. Brit. B. p. 398.

a Tuscany (C. Passerini) 1836.

\section{SUB-FAMILY.-PHYLLOSCOPIN AE.}

\section{Phylloscopus ${ }^{1}$.}

Phylloscopus, Boie, Isis, 1826, p. 970.

\section{Phylloscopus viridanus.}

Phylloscopus viridanus, Blyth, J. A. S. B. xiii. p. 967 : Seebohm, Ibis, 1877, p. 73.

$a,-b$ India (E. Blyth) 1846.

Sent to Strickland by Blyth, as $Y$. viridanus, three years after he had named the species.

\section{Phylloscopus lugubris.}

Phylloscopus lugubris, Blyth, J. A. S. B. xii. p. 968 : Seebohm, Ibis, 1877 , p. 78.

a India (E. Blyth) 1846.

Sent by Blyth as a specimen of his P. lugubris.

\section{Phylloscopus occipitalis.}

"Phyllopneuste occipitalis, Jerd." apud Blyth.

Phylloscopus occipitalis, Blyth, J. A. S. B. xiv. p. 593 : Seebohm, Ibis, 1877, p. 80.

a ô India (Stevens) 1844.

\section{Phylloscopus viridipennis.}

Phylloscopus viridipennis, Blyth, J. A. S. B. xxiv. p. 275 : Seebohm, Ibis, 1877, p. 82.

a India (E. Blyth) 1846.

1 For the names of the species of this intricate genus I am indebted to $\mathrm{Mr} \mathrm{H}$. Seebohm, who examined the skins in the Strickland collection at the time he was compiling his exhaustive paper published in the Ibis $(1877$, p. 66 et seqq.). 


\section{1\%. Phylloscopus indicus.}

Sylvia indica, Jerd. Madr. Journ. xi. p. 6.

Phylloscopus indicus, Jerd. B. Ind. ii. p. 194 : Seebohm, Ibis, 1877, p. 87.

$a$ N. India (W. J. E. Boys) 1848.

\section{Phylloscopus sibilatrix.}

Motacilla sibilatrix, Bechst. Naturf. xxvii. p. 47.

Phylloscopus sibilatrix, Newton, ed. Yarr. Brit. B. i. p. 427 : Seebohm: Jbis, 1877, p. 89.

a \& Boynton (Arthur Strickland) 1 May, 1830.

$b \hat{\delta}$ Worcestershire (H. E. Strickland) 1833.

In his Commentary on Gray's Genera of Birds Strickland pointed out that Bechstein's name $P$. sibilatrix antedated Montagu's title Sylvia sylvicola applied to the same species (Ann. \& Mag. N. H. vi. p. 422). He followed Bonaparte in using the name Phyllopneuste, a term never formally introduced generically, and which is now properly merged in Phylloscopus (cf. Newton, op. cit. p. 442, note).

\section{Phylloscopus trochilus.}

Motacilla trochilus, Linn. Syst. Nat. i. p. 338.

Phylloscopus trochilus, Boie, Seebohm, Ibis, 1877, p. 90.

a Trollhätten (N. C. Strickland) 25 May, 1831. -b § Wor-

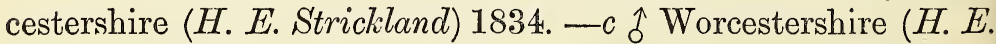
Strickland) 12 May, 1837. —d Kartoum (J. Petherick) 1848.

The Kartoum specimen $(d)$ was correctly identified with this species by Strickland (P. Z. S. 1850, p. 216).

\section{Phylloscopus bonellii.}

Sylvia bonellii, Vieill. N. Dict. d'Hist. N. xxviii. p. 91.

Phylloscopus bonellii, Seebohm, Ibis, 1877, p. 94.

Sylvia nattereri, Temm. Man. d'Orn. i. p. 227.

$a$ Savoy (P. L. Sclater) $18 \check{0} 0$.

\section{Phylloscopus collybita.}

Sylvia collybita, Vieill. N. Dict. d'Hist. N. xi. p. 235.

Phylloscopus collybita, Newton, ed. Yarr. Brit. B. i. p. 437 : Seebohm, Ibis, 1877, p. 95.

Phyllopneuste rufa, auctt. plurr.

$a$ Lombardy (Bt. in Parma) 1834.-b Smyrna (H. E. Strick- 
land) 20 Nov. 1835. -c ô Smyrna (H. E. Strickland) 22 Nov. 1835. - - Europe (N. C. Strickland) 1838.

$c$ is the type of Strickland's Sylvia brevirostris (P.Z.S. 1836, p. 98). This Mr Seebohm pronounces without hesitation to be identical with $P$. collybita (l.s. c.).

\section{Phylloscopus tristis.}

Phylloscopus tristis, Blyth, J. A. S. B. xii. p. 966 : Seebohm, Ibis, 1877 , p. 97. 1847.

a India ("P. tristis" E. Blyth) 1846. -b India (E. Blyth)

\section{Phylloscopus affinis.}

Motacilla affinis, Tickell, J. A. S. B. ii. p. 576.

Phylloscopus affinis, Blyth, Seebohm, Ibis, 1877, p. 100.

a India (E. Blyth) 1846. -b Madras (T. C. Jerdon) 1846. $-c,-d$ Arakan (E. Blyth) 1847.

$a$ is one of the birds Blyth (J. A. S. B. xvi. p. 442) speaks of as having long been regarded by him as the young of $P$. lugubris, under which name he sent it to Strickland. The species is omitted from Blyth's Birds of Burma (J. A. S. B. xliii. extr. No.).

\section{Phylloscopus superciliosus.}

Motacilla superciliosa, Gm. Syst. Nat. i. p. 975.

Phylloscopus superciliosus, Newton, ed. Yarr. Brit. B. i. p. 443 : Seebohm, Ibis, 1877, p. 102.

Phylloscopus modestus, Blyth, J. A. S. B. xii. p. 963.

$a,-b$ India ("P. modestus" E. Blyth) 1846.

\section{Culicipeta.}

Culicipeta, Blyth, J. A. S. B. xii. p. 968 (1843).

\section{Culicipeta burkii.}

Sylvia burkii, Burton, P. Z. S. 1835, p. 153.

Culicipeta burkii, Jerd. B. Ind. ii. p. 199.

$a$ India (E. Blyth) 1846. 


\section{REgUlus.}

Regulus, Cuvier, Leç. d'Anat. Comp. i. tab. 2 (1800).

\section{Regulus cristatus.}

Motacilla regulus, Linn. Syst. Nat. i. p. 338.

Regulus cristatus, Newton, ed. Yarr. Brit. B. i. p. 449.

$a \hat{\delta},-b \hat{\delta}$ Worcestershire (H. E. Strickland) Feb. 1834. -c young.

\section{2\%. Regulus satrapa.}

Regulus satrapa, Licht. Verz. Doubl. p. 35: Baird, Brew. \& Ridgw. N. Am. B. i. p. 73.

$a,-b$ N. America (N. C. Striclcland) 1838.

\section{Regulus calendula.}

Motacilla calendula, Linn. Syst. Nat. i. p. 337.

Regulus calendula, Baird, Brew. \& Ridgw. N. Am. B. i. p. 75. $a$ N. America (Askew) 1834. -b Mexico (Galeotti) 1845.

\section{Regulus ignicapillus.}

Sylvia ignicapilla, Brehm, Temm. Man. d'Orn. i. p. 232.

Regulus ignicapillus, Strickl. P. Z. S. 1836, p. 98: Newton, ed. Yarr. Brit. B. i. p. 456.

a ô Smyrna (H. E. Strickland) \& Dec. 1835.

According to Strickland this bird frequents the olive-groves near Smyrna.

\section{SERICORNIS.}

Sericornis, Gould, P. Z. S. 1837, p. 133.

230. Sericornis humilis.

Sericornis humilis, Gould, l. s. c.: B. Austr. iii. pl. 47. a Australia (Gardner) 1845.

\section{Sericornis osculans.}

Sericornis osculans, Gould, P. Z. S. 1847, p. 2: B. Austr. iii. pl. 48.

a S. Australia (Argent) 1851. 
Acan'rhiza.

Acanthiza, Vigors \& Horsfield, Trans. Linn. Soc. xv. p. 224 $(182.5)$.

\section{Acanthiza nana.}

Acanthiza nana, Vig. \& Horsf. l. s. c. p. 226 : Gould, B. Austr. iii. pl. 60 .

a New South Wales (N. C. Strickland) 1838. - b New South Wales (Argent) 1851.

233. Acanthiza diemenensis.

Acanthiza diemenensis, Gould, P. Z. S. 1837, p. 146 : B. Austr. iii. pl. 54.

a New South Wales (N. C. Strickland) 1838. - b Van Diemen's Land (Argent) 185 1.

\section{Acanthiza lineata.}

Acanthiza lineata, Gould, P. Z. S. 1837, p. 146; B. Austr. iii. pl. 61 .

$a$ S. Australia (Argent) 1851.

\section{Acanthiza reguloides.}

Acanthiza reguloides, Vig. \& Horsf. 'Trans. L. S. xv. p. 226 : Gould, B. Austr. iii. pl. 62.

$a$ New South Wales (N. C. Strickland) 1838. -b (Argent) 1851 .

\section{Acanthiza chrysorrhœa.}

Saxicola chrysorrhea, Quoy \& Gaim. Zool. Voy. Astrol. i. p. 198, Ois. pl. 10 f. 2.

Acanthiza chrysorrhcea, Gould, B. Austr. iii. pl. 63. a Australia (Dewgard) 1851.

\section{SUB-FAMILX.-ACROCEPHALIN 2 .}

\section{HyPOLAIS.}

Hippolais (err.), C. L. Brehm, Isis, 1828, p. 1283.

\section{Hypolais pallida.}

Curruca pallida, Hempr. \& Ebrenb. Symb. Phys. fol. 67. Hypolais pallida, Dresser \& Blanf. Ibis, 1874, p. 339. a f̊ Zante (H. E. Strickland) 18 May, 1836. 


\section{Hypolais icterina.}

Sylvia icterina, Vieill. N. Dict. d'Hist. N. xi. p. 194.

Hypolais icterina, Newton, ed. Yarr. Brit. B. i. p. 360.

a ô Zante (H. E. Strickland) 18 May, 1836.

\section{Hypolais caligata.}

Sylvia caligata, Licht. in Eversm. Reise n. Buchara, p. 128. Hypolais caligata, Dresser, B. Eur.

Phyllopneuste rama (Sykes), Jerd. B. Ind. ii. p. 189 (partim).

a Madras ("Sylvia rama," T. C. Jerdon) 1846. -b $\hat{\delta}$ N. India (W. J. E. Boys) 184.8. -c (A. Strickland) 1850.

\section{Hypolais rama.}

Sylvia rama, Sykes, P. Z. S. 1832, p. 89.

a Madras (A. Strickland) 1833. -b Madras (T. C. Jerdon) 1846.

\section{Hypolais olivetorum.}

Salicaria olivetorum, Strickl. in Gould's Birds Eur. ii. t. 107. Hypolais olivetorum, Dresser, B. Eur.

a $\hat{\delta}$ Zante (H. E. Strickland) 21 May, 1836.

The type of the species as described by Strickland.

\section{Arundinax.}

Arundinax, Blyth, J. A. S. B. xiv. p. 595 (1845).

\section{Arundinax aedon.}

Muscicapa cedon, Pall. Reise, iii. p. 695.

Arundinax olivaceus, Blyth, l.s.c.: Jerd. B. Ind. ii. p. 157.

$a$ India (Askew) 1840. -b India ("Arundinax olivaceus, Bl.," E. Blyth) 1846.

\section{ACROCEPHALUS.}

Acrocephalus, Naum. Naturg. Vög. Deutschl. Nachtr. p. 201 (1819).

\section{Acrocephalus arundinaceus.}

Turdus arundinaceus, Linn. Syst. Nat. i. p. 296.

Acrocephalus arundinaceus, Strickland, Ann. \& Mag. N. H. vi. p. 421 : Newton, ed. Yarr. Brit. B. i. p. 364.

a France (Kinberg). 


\section{Acrocephalus stentoreus.}

Curruca stentorea, Hempr. \& Ehrenb. Symb. Phys. fol. bb. Acrocephalus stentoreus, S. S. Allen, Ibis, 1864, p. 97, pl. 1. Acrocephalus brunnescens, Jerd. B. Ind. ii. p. 154.

a Madras ("Sylvia turdoides," T. C. Jerdon) 1846. -b f Ferozepore (W. J. E. Boys) 1847.

\section{Acrocephalus streperus.}

Sylvia strepera, Vieill. Faun. Fran. p. 219, t. 99, f. 1.

Acrocephalus streperus, Newton, ed. Yarr. Brit. B. i. p. 369.

Acrocephalus salicarius, Strickl. Ann. \& Mag. N.H. vi. p. 421 (nec Linn.).

a $f$ Weston, Oxfordshire (N. C. Strickland) 17 Aug. 1833. -b ई̂ Worcestershire (H. E. Strickland) 6 May, 1837.

\section{Acrocephalus palustris.}

Sylvia palustris, Bechst. Naturg. Deutschl. iii. p. 639, t. 26. Acrocephalus palustris, Dresser, B. Eur. a Belgium (E. De Selys Longchamps) 1845.

\section{4\%. Acrocephalus dumetorum.}

Sylvia montana, Sykes, P. Z. S. 1832, p. 89 (nec Horsf.).

Acrocephalus dumetorum, Blyth, J. A. S. B. xviii. p. 815 : Dresser, B. Eur.

a (N. C. Strickland) 1838. -b Madras (T. C. Jerdon) 1845. - c Madras ("Sylvia montana"? T. C. Jerdon) 1846. —d India (W. J. E. Boys) 1848.

\section{Acrocephalus agricola.}

Sylvia agricola, Jerd. Madr. Journ. xiii. pt. 2, p. 131.

Calamoherpe agricola, Blyth, J. A. S. B. xiv. p. 59.. Acrocephalus agricolus, Jerd. B. Ind. ii. p. 156.

a India ("Calamoherpe agricola, Jerd.," E. Blyth) 1846. -b Madras ("Sylvia agricola, Jerd.," T. C. Jerdon) 1846. —c India ("Calamoherpe montana," E. Blyth) 1846.

\section{Acrocephalus schœnobænus.}

Motacilla schœenobanus, Linn. Syst. Nat. i. p. 329.

Acrocephalus schoenobcenus, Newton, ed. Yarr. Brit. B. i. p. 376. 
Sylvia phragmitis, Bechst. (et auctt. pl.) Strickl. P.Z. S. 1836, p. 98.

a §ิ Oxfordshire (N. C. Strickland) 10 May 1834. - b Worcestershire (Robinson) 1840. —c France (Kinberg).

Seen at Smyrna by Strickland in December.

\section{Acrocephalus nævius.}

Fauvette tachetée, D'Aubent. Pl. Enl. 581, f. 3, undè

Motacilla ncevia, Bodd. Tabl. Pl. Enl. p. 35.

Sylvia locustella, Lath. et auctt. plurr.

Acrocephalus ncevius, Newton, ed. Yarr. Brit. B. i. p. 384.

Locustella rayi, Gould, B. Eur.

a $\hat{\delta}$ Worcestershire (H. E. Strickland) 5 May, 1837.

In his commentary on Gray's Genera of Birds (Ann. \& Mag. N. H. vi. p. 421, and viii. p. 371), Strickland advocated the use of Gould's name Locustella rayi for this bird as being the oldest known to him. Gray's proposal to adopt $L$. avicula from Ray he shews to be inadmissible.

\section{Acrocephalus certhiola.}

Motacilla certhiola, Pall. Zoogr. Rosso-As. i. p. 509.

Locustella rubescens, Blyth, J. A. S. B. xiv. p. 582 : Jerd. B. Ind. ii. p. $\mathbf{1 6 0 .}$

a India (E. Blyth) 1851. - b Bengal ("Locustella rubescens," E. Blyth) 1851.

\section{SUB-FAMILY.-DRYMCCIN $A$.}

\section{ORTHOTONUS.}

Orthotomus, Horsfield, Trans. Linn. Soc. xiii. p. 165 (1820).

\section{Orthotomus longicauda.}

Motacilla longicauda, Gm. Sys. Nat. i. p. 954.

Orthotomus longicauda, Strickl. Ann. \& Mag. N. H. xiii. p. 35 : Jerd. B. Ind. ii. p. 165.

a India? (Arthur Strickland) 1840. - b, —c India (" O. lon- 
gicauda," E. Blyth) 1846. - d Bengal ("O. longicauda, O. lingoo, Sykes," E. Blyth) 1851.

The synonymy of this species as far as then known is given by Strickland in his notes on Blyth's list of birds from the vicinity of Calcutta $l$. s. $c$.

\section{Orthotomus atrigularis.}

Orthotomus atrigularis, Temm. Pl. Col. Livr. 101: Salvad. Ann. Mus. Genov. v. p. 249 : Sharpe, Ibis, 1877, pp. 16, 113.

a (Bt. at Birmingham) 1849.

A Bornean and Malaccan species.

\section{Orthotomus ruficeps.}

Edela ruficeps, Less. Traité d'Orn. p. 309.

Orthotomus ruficeps, Salvad. Ann. Mus. Civ. Genov. v. p. 248.

a Malacca (Gardner) 1850.

Originally obtained by Labillardière, not in New Holland, as stated by Lesson, but most probably at Batavia, where D'Entrecasteaux's expedition touched. Strickland (Ann. \& Mag. N. H. viii. p. 371), considered Lesson's bird to be the same as 0 . edela, Temm. and Motacilla sepium, Rafl. (nec Horsf.).

\section{Cisticola.}

Cisticola, Kaup, Skizz. Nat. Syst. d. Eur. Thierw. p. 119 (1829).

\section{Cisticola cursitans.}

Sylvia cisticola, Temm. Man. d'Orn. i. p. 228 (1820).

Prinia cursitans, Frankl. P. Z. S. 1830-1. p. 118.

Cisticola cursitans, Dresser, B. Eur.

Cisticola schœnicola, Bp. Jerd. B. Ind. ii. p. 174: Blyth, J. A. S. B. xliii. extr. No. p. 119.

$a$ (Askew) 1837. - b Constantinople (Boissoneau) 1839. c Madras (T. C. Jerdon) 1845. - d Nepal ("Cisticola cursitans," E. Blyth) 1846. -e Arakan (E. Blyth) 1847. 


\section{Cisticola exilis.}

Malurus exilis, Vig. \& Horsf. Trans. L. S. xv. p. 223.

Cisticola exilis, Gould, B. Austr. iii. pl. 42.

$a$ S. Australia (Argent) 1852.

\section{DRYMGCA.}

Drymoica, Swainson, Zool. Journ. iii. p. 168 (1827).

257. Drymoca textrix.

Sylvia textrix, Vieill. N. Dict. d'Hist. N. xi. p. 208.

Drymoica textrix, Layard, B. S. Afr. p. 85.

a Cape of Good Hope (A. Strickland) 1833.

\section{Drymœca maculosa.}

Petite Fauvette tachetée du Cap, D'Aub. Pl. Enl. 752, f. 2. undè

Motacilla maculosa, Bodd. Tabl. Pl. Enl. p. 47.

Motacilla macrura, Gm. Syst. Nat. i. p. 953. 1840.

a $\hat{o}$ Cape of Good Hope (A. Strickland) 1832. -b (Askew)

\section{Drymoca subruficapilla.}

Drymoica subruficapilla, Smith, Zool. S. Afr. pl. 76, f. 2 : Layard, B. S. Afr. p. 91.

a Cape of Good Hope (A. Strickland) 1832.

\section{Drymœca chiniana.}

Drymoica chiniana, Smith, Zool. S. Afr. pl. 79 : Gurney, in And. B. Damara-l. p. 86.

Drymoea levaillanti, Strickl. \& Scl. Contr. Orn. 1852, p. 147.

Cisticola campestris, Gould, P. Z. S. 1845, p. 20.

Cisticola magna, Gould, B. Austr. iii. pl. 41.

$a \mathrm{~S}$. Africa (N. C. Strickland) 1838. - b Damara-land (C. J. Andersson) 1851.

$a$ is the specimen figured in Gould's 'Birds of Australia.' 
Strickland seems to have doubted its Australian origin, as I find a note to that effect in his copy of Gould's work. Gould's names have usually been associated with $D$. levaillanti, but on comparing this specimen $\mathrm{Mr}$ Sharpe and I agreed that it corresponds best with D. chiniana.

\section{Drymœea lais.}

Drymoica lais, Hartl. \& Finsch, Vög. O. Afr. p. 237.

$a$ (N. C. Strickland) 1838.

A South African species.

262. Drymœca smithi.

Drymoica smithi, Bp. Consp. Av. i. p. 283.

Drymoica ruficapilla, Smith, Zool. S. Afr. Pl. 73, f. 1.

$a$ S. Africa (Stevens) 1845 .

\section{Drymœeca lugubris.}

Cisticola lugubris, Rüpp. Neue Wirbelth. p. 111.

a Abyssinia (E. Verreaux) 1850.

\section{Drymœca flavicans.}

Sylvia flavicans, Vieill. N. Dict. d'Hist. N. ii. p. 175.

Drymoeca flavicans, Strickl. \& Scl. Contr. Orn. 1852, p. 148: Gurney, in And. B. Damara-l. p. 84.

a, -b Damara-land (C. J. Andersson) 1852.

265. Drymœca inornata.

Prinia inornata, Sykes, P. Z. S. 1832, p. 89.

Drymoipus inornatus, Jerd. B. Ind. ii. p. 178.

Prinia fusca, Hodgs. P. Z. S. 1845, p. 29.

a Madras ("Prinia inornata," T. C. Jerdon) 1845. - b India ("Prinia inornata," E. Blyth) 1846. — A Arakan (E. Blyth) 1847. -d Bengal (E. Blyth) 1851.

\section{Drymœca sylvatica.}

Prinia sylvatica, Jerd. Madr. Journ. xi. p. 4.

Drymoipus sylvaticus, Jerd. B. Ind. ii. p. 181.

a, -b Madras ("Prinia sylvatica," T. C. Jerdon) 1845. 


\section{Drymøca jerdoni?}

Prinia sp.? Blyth, J. A. S. B. xi. p. 883.

Prinia inornata, Blyth, J. A. S. B. xiii. p. 376.

Drymoica jerdoni, Blyth, J. A. S. B. xvi. p. 459.

a Madras ("Prinia jerdoni, Blyth ?" T. C. Jerdon) 1846. -b S. India (T. C. Jerdon) 1847. —c S. India ("P. jerdoni, Blyth ?" T. C. Jerdon) 1850.

These specimens, sent by Jerdon as doubtfully referable to $D$. jerdoni, Blyth, were considered by Strickland certainly to belong to that species. They agree fairly with Blyth's description, being similar to D. sylvatica, but decidedly smaller. Jerdon (B. Ind. ii. p. 180) refers this bird with doubt to what he calls $D$. longicaudata, Tick. But this name cannot be thus used, for Tickell in employing it was bestowing no new title but merely misapplying Gmelin's name for Orthotomus longicauda.

\section{Drymcea crinigera.}

Suya criniger, Hodgs. As. Res. xix. p. 183 : Jerd. B. Ind. ii. p. 183.

Drymoica criniger, Blyth, Cat. B. As. Soc. p. 142.

a Nepal (B. H. Hodgson, 415) 1845. - b India ("Suya criniger," E. Blyth) 1846.

\section{Printa.}

Prinia, Horsfield, Trans. Linn. Soc. xiii. p. 165 (1820).

\section{Prinia socialis.}

Prinia socialis, Sykes, P. Z. S. 1832, p. 89 : Jerd. B. Ind. ii. p. 170.

a India (Gardner) 1844. - b Madras ("P. socialis," T. C. Jerdon) 1846.

\section{2\%0. Prinia hodgsoni.}

Prinia gracilis, Jerd. Madr. Journ. ii. p. 3 (nec Rüpp.).

Prinia hodgsoni, Blyth, J. A. S. B. xiii. p. 376 : Jerd. B. Ind. ii. p. 173.

a Madras ("P. gracilis," T. C. Jerdon) 1845. 


\section{Prinia flaviventris.}

Orthotomus flaviventris, Deless. Rev. Zool. 1840, p. 101.

Prinia flaviventris, Jerd. B. Ind. ii. p. 252.

a India ("Prinia flaviventris," E. Blyth) 1846.

272. Prinia stewarti.

Prinia stewarti, Blyth, J. A. S. B. xvi. p. 455 : Jerd. B. Ind. ii. p. 171.

a (Argent) 1851.

A species found in Central and North-Western India.

\section{Prinia buchanani.}

Prinia buchannani, Blyth, J. A. S. B. xiii. p. 376.

Franklinia buchannani, Jerd. B. Ind. ii. p. 186.

Prinia rufifrons, Jerd. Madr. Journ. xi. p. 4.

1846.

$a,-b$ Madras ("Prinia rufifrons, Jerd." T. C. Jerdon)

\section{Malurus.}

Malurus, Vieillot, Anal. p. 44. (1816).

\section{Malurus cyaneus.}

Motacilla cyanea, Gm. Syst. Nat. i. p. 991.

Malurus cyaneus, Vieill. Gould, B. Austr. iii. pl. 18.

$a,-b$ New South Wales (N. C. Strickland) 1838.

\section{Malurus sp.?}

$a$ New South Wales (N. C. Strickland) 1838.

A skin of a female bird perhaps belonging to $M$. longicaudatus, Gould (B. Austr. iii. pl. 48).

\section{Malurus elegans.}

Malurus elegans, Gould, B. Austr. iii. pl. 22.

a Australia (N. C. Strickland) 1838. - b West Australia (Dewgard) 1849.

$a$ is a skin of a female bird perhaps belonging to this species. 


\section{2\%\%. Malurus lamberti.}

Malurus lamberti, Vig. \& Horsf. Trans. L. S. xv. p. 221 : Gould, B. Austr. iii. pl. 24.

$a$ S. Australia (Argent) 1852.

\section{Malurus leucopterus.}

Malurus leucopterus, Quoy \& Gaim. Zool. Voy. l'Uran. Zool. p. 108. pl. 23, f. 2: Gould, B. Austr. iii. pl. 25.

$a$ S. Australia (Argent) 1852.

\section{Malurus melanocephalus.}

Scarlet-backed Warbler, Lewin, Birds of N. Holland, pl. xiv. Malurus melanocephalus, Vig. \& Horsf. Trans. L. S. xv. p. 222: Gould, B. Austr. iii. pl. 26.

$a$ New South Wales (N.C. Strickland) 1838. -b (Argent) 1852.

STIPITURUS.

Stipiturus, Lesson, Traité d'Orn. p. 414 (1831). 280. Stipiturus malacurus.

Muscicapa malachura, Lath. Ind. Orn. Suppl. p. lii. Stipiturus malachurus, Less. Gould, B. Austr. iii. pl. 31. $a,-b$ New South Wales (N. C. Strickland) 1838.

\section{HAPALIS.}

Apalis, Swainson, Zool. Ill. 2nd Ser. iii. pl. 119 (1832-33).

\section{Hapalis thoracica.}

Le Plastron noir, Le Vaill. Ois. d'Afr. pl. 123.

Motacilla thoracica, Shaw, Nat. Misc. xxii. pl. 969.

Apalis thoracica, Sw. l.s. c. : Layard, B. S. Afr. p. 93.

$a$ S. Africa (Sir W. Jardine) 1842.

\section{DRYODROMAS.}

Dryodromas, Finsch \& Hartlaub, Vögel O. Afr. p. 239 (1870). 
282. Dryodromas flavida.

Drymoca flavida, Strickl. \& Scl. Contr. Orn. 1852, p. 148.

Dryodromas flavida, Gurney, in And. B. Damara-l. p. 96.

a Damara-land (C. J. Andersson) 1852.

\section{SylvietTa.}

Sylvietta, Lafresnaye, Rev. Zool. 1839, p. 258.

\section{Sylvietta rufescens.}

Dicceum rufescens, Vieill. N. Dict. d'Hist. N. ix. p. 407: Layard, B. S. Afr. p. 84.

Sylvietta rufescens, Gray, Gurney, in And. B. Damara-1. p. 77.

Sylvietta brachyura, Lafr. l. s. c., Strickl. \& Scl. Contr. Orn. 1852, p. 148.

$a$ S. Africa (Capt. Alexander), 1838. - $b$ Damara-land (C. J. Andersson) 1852.

\section{HyLACOLA.}

Hylacola, Gould, P. Z. S. 1842, p. 135.

\section{Hylacola pyrrhopygia.}

Acanthiza pyrrhopygia, Vig. \& Horsf. Trans. L. S. xv. p. 227.

Hylacola pyrrhopygia, Gould, l.s. c.: B. Austr. iii. pl. 39.

$a$ New South Wales $\left(M^{\circ}\right.$ Donald) 1838. $-b$ Australia $(N . C$. Strickland) 1838.

\section{Chthonicola.}

Chthonicola, Gould, P. Z. S. 1847, p. 35.

\section{Chthonicola sagittata.}

Sylvia sagittata, Lath. Ind. Orn. Suppl. p. liv.

Anthus minimus, Vig. \& Horsf. Trans. L. S. xv. p. 230.

Chthonicola minima, Gould, B. Austr. iii. p. 72.

Chthonicola sagittata, Gould, Handb. B. Austr. i. p. 390.

a Australia (N. C. Strickland) 1838. - b Australia (Stevens) 1842. 


\section{SUB-FAMILY.-ACCENTORIN AE.}

\section{ACCENTOR.}

Accentor, Bechstein, Orn. Taschenb. i. p. 191 (1802).

\section{Accentor collaris.}

Sturnus collaris, Scop. An. i. Hist. Nat. p. 131.

Accentor collaris, Dresser, B. Eur.: Newton, ed. Yarr. Brit. B. i. p. 296.

Motacilla alpina, Gm. Syst. Nat. i. p. 957.

a Switzerland (L. Coulon) 1836.

\section{8\%. Accentor atrigularis.}

Accentor atrogularis, Brandt, Bull. Ac. St Petersb. 1843, p. 40.

$a$ W. Siberia (J. F. Brandt) 1844.

Doubtless a typical specimen.

\section{Accentor modularis.}

Motacilla modularis, Linn. Syst. Nat. i. p. 329.

Accentor modularis, Strickland, P. Z. S. 1836, p. 98 : Newton, ed. Yarr. Brit. B. i. p. 301.

a $\hat{o}$ Worcestershire (H. E. Strickland) 1833. - b ^ Smyrna (H. E. Strickland) 10 Dec. 1835.

Stated by Strickland to be rare at Smyrna.

\section{EPHTHIANURA.}

Ephthianura, Gould, P. Z. S. 1837, p. 148.

\section{Ephthianura albifrons.}

Acanthiza albifrons, Jard. \& Selb. Ill. Orn. t. 56.

Ephthianura albifrons, Gould, B. Austr. iii. p. 64.

a New South Wales (ḾDonald) 1838. - b Australia (Gardner) 1845.

290. Ephthianura aurifrons.

Ephthianura aurifrons, Gould, P. Z. S. 1837, p. 148: B. Austr. iii. t. 65.

$a$ New South Wales (Argent) 1851. 
SUB-FAMILY.-POLIOPTILIN Æ.

\section{Polioptila.}

Polioptila, Sclater, P. Z. S. 1855, p. 11.

\section{+ 291. Polioptila cærulea.}

Motacilla carulea, Linn. Syst. Nat. i. p. 337.

Polioptila ccerulea, Scl. Baird, Brew. \& Ridgw. N. Am. B. i. p. 78 .

a Mexico (T. Mann) 1844. - b Guatemala (J. Constancia) 1848.

\section{Polioptila leucogastra.}

Polioptila leucogastra, Max, Beitr. iii. p. 710: Sclater, Cat. Am. B. p. 12.

Culicivora atricapilla, Sw. Zool. Ill. 2nd Ser. pl. 57.

a Brazil (Arthur Strickland) 1840. -b (Mather) 1840.

\section{Polioptila buffoni.}

Figuier à tête noire de Cayenne, Buff. Pl. Enl. 704, f. 1.

Polioptila buffoni, Scl. P. Z. S. 1861, p. 128.

a (N. C. Strickland) 1838.

Found in the northern portions of S. America,-Guiana, Columbia, \&c.

\section{SUB-FAMILY.-MYIADECTIN ДE。}

\section{MriadeCtes.}

Myiadestes, Swainson, Nat. Libr. Ornith. x. Flycatchers, p. 132 (1838).

\section{+ 294. Myiadectes obscurus.}

Myiadestes obscurus, Lafr. Rev. Zool. 1839, p. 99 : Scl. \& Salv. Ex. Orn. p. 49, pl. 35.

$a$ Guatemala (J. Constancia) 1848.

\section{Myiadectes solitarius.}

Myiadestes solitarius, Baird, Rev. Am. B. p. 421.

Myiadestes genibarbis, Gosse, B. Jam. p. 198 (nec Swains.).

Myiadestes armillatus, Scl. Cat. Am. B. p. 47 (nec Vieill.).

a Jamaica (P. H. Gosse) 1848. 


\section{FAMILY.-PANURIDE.}

\section{Panurus.}

Panurus, Koch, Syst. d. Baierisch. Zool. p. 216 (1816).

\section{Panurus biarmicus.}

Parus biarmicus, Linn. Syst. Nat. i. p. 342.

Panurus biarmicus, Koch, l. s. c.: Newton, ed. Yarr. Brit. B. i. p. 511.

$a$ Britain (Askew) 1838. - $b$ q Britain (W. Kirtland) 1843.

\section{FAMILY.-PARIDAE.}

\section{Parus.}

Parus, Linnæus, Syst. Nat. i. p. 341 (1766) (partim).

29\%. Parus major.

Parus major, Linn. l. s. c.: Strickl. P. Z. S. 1836, p. 99 : Newton, ed. Yarr. Brit. B. i. p. 479.

a f Oxfordshire (N. C. Strickland) 14 Sept. 1833. - b of Smyrna (H. E. Strickland) 13 Nov. 1835. - c ô Worcestershire (H. E. Strickland) June 1838.

\section{Parus monticola.}

Parus monticolus, Vig. P. Z. S. 1830-, p. 22 : Gould. B. Him. pl. 29, f. 2 : Hodgs. Zool. Misc. p. 83 : Jerd. B. Ind. ii. p. 277.

$a$ Himalaya (Askew) 1837. - b Nepal (B. H. Hodgson, 398) 1845.

\section{Parus lugubris.}

Parus lugubris, Natt. Temm. Man. d'Orn. i. p. 294: Strickl. P. Z. S. 1836 , p. 99 : Dresser, B. Eur.

a ô Smyrna (H. E. Strickland) 12 Nov. 1835. - b Dalmatia (E. De Selys Longchamps) 1845.

300. Parus cinctus.

Parus cinctus, Bodd. Tabl. Pl. Enl. p. 44, ex D'Aub. Pl. Enl. 708, f. 3.

Parus sibiricus, Gm. Syst. Nat. i. p. 1013.

$a \mathrm{~N}$. Europe (J. G. Kinberg) 1843. 
301. Parus ater.

Parus ater, Linn. Syst. Nat. i. p. 341: Newton, ed. Yarr. Brit. B. i. p. 489.

$a$ f. $-b$ f Worcestershire (H. E. Strickland) Jan. 1834. -c (Askew) 1837.

302. Parus palustris.

Parus palustris, Linn. Syst. Nat. i. p. 341: Newton, ed. Yarr. Brit. B. i. p. 495.

a ơ Oxfordshire (N. C. Striclitand) 25 Sept. 1833. -b Worcestershire (T. Robins) Jan. 1838.

\section{Parus atricapillus.}

Parus atricapillus, Linn. Syst. Nat. i. p. 341 : Baird, Brew. \& Ridgw. N. Am. B. i. p. 96.

$a$ (Arthur Strickland) 1840. - b Fort William, Lake Superior, Dec. 1824 (Arthur Strickland) 1840.

\section{Parus meridionalis.}

Parus meridionalis, Scl. P. Z. S. 1856, p. 293 : Cat. Am. B. p. 14.

a Mexico (T. Mann) 1844.

\section{Parus rufescens.}

Parus rufescens, Towns. J. Ac. Nat. Sc. Phil. vii. p. 190: Baird, Brew. \& Ridgw. N. Am. B. i. p. 104.

$a$ N. W. America (Wosnessenski) 1846.

306. Parus cinereus.

Parus cinereus, Vieill. N. Dict. d'Hist. N. xx. p. 316 : Jerd. B. Ind. ii. p. 278.

Parus atriceps, Horsf. Trans. L. S. xiii. p. 160.

a (Askew) 1837. - b Malacca (Havell) 1839. -c Ceylon (Stevens) 1843. - d f Kumaon (W. J. E. Boys) 1847. -e S. India (T. C. Jerdon) 1850 .

\section{Parus niger.}

Parus niger, Vieill. N. Dict. d'Hist. N. xx. p. 32.5 .

Parus leucopterus, Sw. Layard, B. S. Afr. p. 113.

a Africa (Askew) 1834. - b Cape of Good Hope (N. C. Strickland) 1838. 


\section{Parus leucopterus.}

Parus leucopterus, Sw. B. W. Afr. ii. p. 42.

a (Stevens) 1844.

A West African species.

309. Parus afer.

Parus afer, Gm. Syst. Nat. i. p. 1010.

Parus cinerascens, Vieill. N. Dict. d'Hist. N. xx. p. 316 : Strickl. \& Scl. Contr. Orn. 1852 p. 149 : Layard, B. S. Afr. p. 113.

$a$ (N. C. Strickland) 1838. - b Damara-land (C.J.Andersson) 1852.

\section{Parus cæruleus.}

Parus cceruleus, Linn. Syst. Nat. i. p. 341 : Strickl. P. Z. S. 1836, p. 99 : Newton, ed. Yarr. Brit. B. i. p. 483.

$a \hat{\delta}$ Worcestershire (H. E. Strickland) 1833. - b Smyrna (H. E. Strickland) 24 Nov. 1835.

\section{Parus teneriffæ.}

Parus teneriffce, Less. Traité d'Orn. p. 456: Godman, Ibis, 1872, p. 172.

Parus cceruleanus, Malh. Rev. Zool. 1842, p. 46,

a ô Tangiers (F. Favier) 1851.

\section{Parus cyanus.}

Parus cyanus, Pall. Nov. Comm. Petrop. xiv. pl. 13, f. 1: Dresser, B. Eur.

$a$ Siberia (J. F. Brandt) 1841.

\section{LOPHOPHANES.}

Lophophanes, Kaup, Skizz. Nat. Syst. d. Eur. Thierw. p. 92 (1829).

\section{Lophophanes cristatus.}

Parus cristatus, Linn. Syst. Nat. i. p. 340 : Newton, ed. Yarr. Brit. B. i. p. 499.

Lophophanes cristatus, Kp. l. s. c.

a $\hat{\delta}$ Trollhätten, Sweden (N. C. Strickland) 26 May, 1831. 


\section{Lophophanes bicolor.}

Parus bicolor, Linn. Syst. Nat. i. p. 340.

Lophophanes bicolor, Baird, Brew. \& Ridgw. B. i. p. 87.

$a \mathrm{~N}$. America 1834.

\section{Lophophanes melanolophus.}

Parus melanolophus, Vig. P.Z. S. 1830-1, p. 23: Gould, Cent. B. Him. pl. 30, f. 2 .

Lophophanes melanolophus, Jerd. B. Ind. ii. p. 273.

a Himalaya (N. C. Strickland) 1838. - b ô Kumaon ( W. J. E. Boys) 1847. -c ô India (W. J. E. Boys) 1848.

\section{Lophophanes xanthogenys.}

Parus xanthogenys, Vig. P. Z. S. 1830- , p. 23: Gould, Cent. B. Him. pl. 29, f. 1: Hodgs. Zool. Misc. p. 83.

Machlolophus xanthogenys, Jerd. B. Ind. ii. p. 279.

a Nepal (B. H. Hodgson 397) 1845. -b ô Kumaon (W.J. E. Boys) 1847.

\section{1\%. Lophophanes spilonotus.}

Parus spilonotus, Blyth, Cat. B. Mus. As. Soc. p. 103: Contr. Orn. 1852 , p. 49 , pl. 87, f. 2.

Machlolophus spilinotus, Jerd. B. Ind. ii. p. 281.

a (T. C. Eyton) 1849.

Both Blyth (Catal.) and Jerdon $\left(l_{0} s . c.\right)$ give a reference to the 18th vol. of the Journ. As. Soc. Beng. for the first description of this species, but I can find there no trace of such a description. The species seems to have been first formally noticed in Blyth's paper on the Indian Pari in the Contr. Orn. for 1852.

\section{Melanochlora.}

Melanochlora, Lesson, Rev. Zool. 1839, p. 42.

\section{Melanochlora flavocristata.}

Parus flavocristatus, Lafr. Mag. Zool. 1837, cl. ii. pl. 80.

Parus sultaneus, Hodgs. Ind. Rev.1838, p. 31 ; Zool. Misc. p. 83.

Melanochlora sultanea, Jerd. B. Ind.ii. p. 282.

a Malacca (Johnson) 1837, -b Malacca (N. C. Strickland) 1838. - c Nepal (B. H. Hodgson 345) 1845.

Strickland considered Malaccan and Himalayan specimens to be specifically identical. (P. Z. S. 1846, p. 100.)

S. C. 
ACREDULA.

Acredula, Koch, Syst. d. Baier. Zool. p. 199 (1816).

319. Acredula caudata.

Parus caudatus, Linn. Syst. Nat. i, p. 342.

A credula caudata, Koch, l. s. c.: Newton, ed. Yarr. Brit. B. i. p. 504 .

$a$ Oxfordshire (N. C. Strickland) 23 Sept. 1833. - b Worcestershire (H. E. Strickland) Jan. 1837.

\section{EGITHALiscus.}

AEgithaliscus, Cabanis, Mus. Hein. i. p. 90 (1850).

320. Ægithaliscus erythrocephalus.

Parus erythrocephalus, Vig. P. Z. S. 1830-1, p. 23: Gould, Cent. B. Him. pl. 30, f. 1: Hodgs. Zool. Misc. p. 83.

Egithaliscus erythrocephalus, Jerd. B. Ind. ii. p. 270.

a $\mathrm{Nepal}$ (B. H. Hodgson 389) 1845. -b ô Kumaon (W.J.E. Boys) 1847.

\section{Psaltriparus.}

Psaltriparus, Bonaparte, Compt. Rend. xxxi. p. 478 (1850).

\section{Psaltriparus melanotis.}

Parus melanotis, Hartl., Rev. Zool. 1844, p. 216.

Psaltriparus melanotis, Scl. Cat Am. B. p. 13: Baird, Brew. \& Ridgw. N. Am. B. i. p. 108.

a Guatemala (J. Constancia) 1845.

\section{AGITHALUS.}

Agithalus, Boie, Isis, 1822 , p. 556.

\section{2. \#igithalus pendulinus.}

Parus pendulinus, Linn. Syst. Nat. i. p. 342.

AEgithalus pendulinus, Boie: Dresser, B. Eur.

a Italy (Boissoneau) 1839.

\section{Egithalus capensis.}

Parus capensis, Gm. Syst. Nat. i. p. 1011.

Egithalus capensis, Sw. Classif. B. ii. p. 246.

Paroides capensis, Layard, B. S. Afr. p. 114.

$a$ S. Africa (Stevens) 1842. 
Falcunculus.

Falcunculus, Vieill. Anal. p. 40 (1816).

324. Falcunculus frontalis.

Lanius frontalis, Lath. Ind. Orn. Suppl. p. xviii.

Falcunculus frontalis, Vieill. Gould, B. Austr. ii. pl. 79.

$a$ New South Wales (N.C. Strickland) 1838. $-b$ New South Wales (Argent) 1852.

\section{OREGCA.}

Oreoïca, Gould, P. Z. S. 1837, p. 151.

\section{Oreœca gutturalis.}

Crested Thrush, Lewin, Birds N. Holl. pl. ix.

Falcunculus gutturalis, Vig. \& Horsf. Trans. L. S. xv. p. 212.

Oreoïca gutturalis, Gould, l. s. c.; B. Aust. ii. pl. 81.

Oreica cristata, Gray, Ann. \& Mag. N. H. xi. p. 190.

$a,-b$ New South Wales (Isaac) 1842. — c (T. C. Eyton) 1849.

According to Strickland a true Parus (P. Z. S. 1846, p. 100).

Vigors and Horstield's specific name must certainly be used for this bird. Lewin gave no Latin title, and even if he had done so his work was published eleven years after the memoir in the Linn. Trans.

\section{PARISOMA.}

Parisoma, Swains. Faun. Bor. Am. i. p. 490 (1831).

\section{Parisoma subcæruleum.}

Silvia subccerulea, Vieill. N. Dict. d'Hist. N. xi. p. 188.

Parisoma subccruleum, Strickl. \& Scl. Contr. Orn. 1852, p. 149: Gurney in And. B. Damara-1. p. 77.

Parisoma rufiventer, Layard, B. S. Afr. p. 115.

$a$ Damara-land (C.J. Andersson) 1852. - b S. Africa (Dr A. Smith).

Placed by Strickland and Sclater with the Sylviida, but by Gurney in the Paridoe.

\section{MоноUа.}

Mohoua, Lesson, Compl. Buff. ix. Ois. p. 139 (1837).

\section{2\%. Mohoua ochrocephala.}

Muscicapa ochrocephala, Gm. Syst. Nat. i. p. 944. 
Orthonyx ochrocephala, Buller, B. New Zeal. p. 103, pl. 12. $a$ New Zealand (Fenton) 1850.

\section{FAMILY.-SITTIDA.}

SitTA.

Sitta, Linnæus, Syst. Nat. i. p. 177 (1766).

328. Sitta europæa.

Sitta europcea, Linn. Syst. Nat. i. p. 177: Dresser B. Eur. Sitta asiatica, Gould, B. Eur. pl. 236.

$a$ W. Siberia (J. F. Brandt) 1844.

\section{Sitta syriaca.}

Sitta syriaca, Ehrenb. Temm. Man. d'Orn. i. p. 286 (1820): Strickl. P. Z. S. 1836, p. 100.

Sitta rupestris, Cantraine, Temm. Man. d'Orn. i. p. 287 (1820).

a f Smyrna (H. E. Strickland) 30 Nov. 1835. —b ồ Smyrna (H. E. Strickland) 2 Dec. 1835.

Found by Strickland frequenting rocky hills near Smyrna,

\section{Sitta cæsia.}

Sitta ccesia, Wolf, Tasch. deutsch. Vög. i. p. 128: Dresser, B. Eur.: Newton. ed. Yarr. Brit. B. i. p. 473.

Sitta europcea, Strickl. P. Z. S. 1836, p. 100.

$a$ f Smyrna (H. E. Strickland) 10 Dec. 1835. -b Switzerland (H. E. Strickland) Aug. 1836. —c Worcestershire (Robinson) 1840.

Found by Strickland near Smyrna frequenting groves of ancient olive-trees.

\section{Sitta carolinensis.}

Sitta carolinensis, Lath. Ind. Orn. p. 262: Baird, Brew. \& Ridgw. N. Am. B. i. p. 114.

$a$ N. America (Askew)1833. -b (N. C. Strickland) 1838.

\section{Sitta canadensis.}

Sitta canadensis, Linn. Syst. Nat. i. p. 177: Baird, Brew. \& Ridgw. N. Am. B. i. p. 118.

a N. America (Arthur Strickland) 1840. 
333. Sitta pusilla.

Sitta pusilla, Lath. Ind. Orn. p. 263: Baird, Brew. \& Ridgw. N. Am. B. i. p. 122. $a \hat{\delta},-b \hat{o}$ United States (J. F. Baird) 1847.

334. Sitta himalayensis.

Sitta himalayensis, Jard. \& Selb. Ill. Orn. pl. 144: Jerd. B. Ind. i. p. 385.

Sitta nipalensis, Hodgs. J. A. S. B. v. p. 779.

a Nepal (B. H. Hodgson 401) 1845. - $b$ q Gogra Hills. (W.J. E. Boys), 1847.

\section{Sitta castaneiventris.}

Sitta castaneiventris, Frankl. P. Z. S. 1830-1, p. 121: Jard. \& Selb. Ill. Orn. pl. 145: Jerd. B. Ind. i. p. 386.

a (E. Brown) 1850.

A species inhabiting Central and Southern India (Jerdon).

336. Sitta cinnamomeiventris.

Sitta cinnamomeiventris, Blyth, J. A. S. B. xl. p. 459 : Jerd. B. Ind. i. p. 387.

a. India (Gardner) 1844. - b Nepal (B. H. Hodgson 401) 1845. $-c \neq$ N. India (W. J. E. Boys) 1848.

\section{Dendrophila.}

Dendrophila, Swainson, Classif. B. ii. p. 318 (1837).

33\%. Dendrophila corallina.

Sitta corallina, Hodgs. J. A. S. B. v. p. 779.

Sitta frontalis, Horsf. Jerd. B. Ind. i. p. 388.

a Malacca (N. C. Strickland) 1838. -b India (E. Blyth) 1846. -c ô India (W.J.E. Boys) 1848.

\section{Tichodroma ${ }^{1}$.}

Tichodroma, Illiger, Prodr. p. 210 (1811).

\section{Tichodroma muraria.}

Certhia muraria, Linn. Syst. Nat. i. p. 185.

1 Referred by Strickland to the Sittince the tail not being stiffened. (Ann. \& Mag. N. H. vi. p. 420.) 
Tichodroma muraria, Ill.: Dresser, B. Eur. a Switzerland (W. Anderegg) 1836.

\section{SITTELla.}

Sittella, Swainson, Classif. B. ii. p. 317 (1837).

\section{Sittella chrysoptera.}

Sitta chrysoptera, Lath. Ind. Orn. Suppl. p. xxxii.

Sittella chrysoptera, Sw.: Gould, B. Austr. iv. pl. 101.

$a$ New South Wales (N. C. Strickland) 1838.

\section{Sittella leucoptera.}

Sittella leucoptera, Gould, P. Z. S. 1839, p. 144: B. Austr. iv. pl. 103.

a Australia (T. C. Eyton) 1846.

\section{Climacteris ${ }^{1}$.}

Climacteris, Temminck, Man. d'Orn. i. p. lxxxv. (1820).

\section{Climacteris leucophæa.}

Certhia leucophcea, Lath. Ind. Orn. Suppl. p. xxxvi.

Climacteris leucophoea, Strickl. Ann. \& Mag. N. H. xi. p. 336. Climacteris picumnus, Temm.: Gould, B. Austr. iv. pl. 98. a Australia (Arthur Strickland) 1850. -b.

\section{Climacteris scandens.}

Climacteris scandens, Temm. Pl. Col. 281, f. 2: Gould, B. Austr. iv. pl. 93.

$a$ New South Wales (N. C. Strickland) 1838.

\section{Climacteris erythrops.}

Climacteris erythrops, Gould, P. Z. S. 1840, p. 148; B. Austr. iv. pl. 95 .

$$
a \text { New South Wales ( } M^{c} \text { Donald) } 1838 .
$$

\section{Climacteris rufa.}

Climacteris rufa, Gould, P. Z. S. 1840 p. 149; B. Austr. iv. pl. 94.

a $f$ Perth, W. Australia, 30 Mar. 1839 (Arthur Strickland) 1850.

1 Referred to the Sittina by Strickland. (Ann. \& Mag. N. H. vi. p. 420.) 


\section{FAMILY._CERTHIIDAE.}

Certhia.

Certhia, Linnæus, Syst. Nat. i. p. 184 (1766) (partim)

345. Certhia familiaris.

Certhia familiaris, Linn. Syst. Nat. i. p. 184: Dresser, B. Eur.

a $\hat{\delta}$ Worcestershire (H. E. Strickland) Jan. 1834. - b ô Worcestershire (H. E. Strickland) Feb. 1834.

\section{Certhia himalayana.}

Certhia himalayana, Vig. P. Z. S. 1830-1, p. 174: Jerd. B. Ind. i. p. 380. 1847.

a India (Gardner) 1844. - $b$ ô Kumaon (W. J. E. Boys)

347. Certhia americana.

Certhia americana, Bp. Consp. Av. i. p. 225.

Certhia familiaris var. americana, Baird, Brew. \& Ridgw. N. Am. B. p. 125.

a f Carlisle, Pennsylvania 11 Ap. 1845 (S. F. Baird) 1847.

\section{FAMILY.-LIOTRICHIDE.}

\section{LIOTHRIX.}

Leiothrix, Swainson, Faun. Bor. Am. ii. p. 490 (1831).

348. Liothrix luteus.

Mésange de Nanquin, Sonn. Voy. aux Indes \&c. i. p. 205, pl. xiv. f. 2 unde

Sylvia lutea, Scop. Del. Faun. Fl. Insubr. ii. p. 96.

Leiothrix luteus, Jerd. B. Ind. ii. p. 250.

Tanagra sinensis, Gm. Syst. Nat. i. p. 897.

$a$ (Dewgard) 1846. - b India (Stevens) 1847.

\section{Liothrix argentauris.}

Mesia argentauris, Hodgs. Ind. Rev. ii. p. 88; Zool. Misc. p. 83.

Liothrix argentauris, Blyth, Cat. Calc. Mus. p. 99 : Jerd. B. Ind. ii. p. $2 \check{1} 1$.

a Nepal (B. H. Hodgson 308) 1845. 


\section{Liothrix vinipectus.}

Siva vinipectus, Hodgs. Ind. Rev. ii. p. 89 ; Zool. Misc. p. 83.

Proparus vinipectus, Jerd. B. Ind. ii. 257.

Liothrix vinipectus, Blyth, Cat. Calc. Mus. p. 100.

a Nepal (B. H. Hodgson 479) $184 \check{.}$

\section{Minla.}

Minla, Hodgson, India Rev. ii. p. 32 (1838).

\section{Minla ignitincta.}

Minla ignotincta, Hodgs. l. s. c.: Jerd. B. Ind. ii. p. 254.

a Darjeeling (E. Blyth) 1850.

\section{Minla castaneiceps.}

Minla castaneiceps, Hodgs. Ind. Rev. ii. p. 33 ; Zool. Misc. p. 83: Jerd. B. Ind. ii. p. 255.

a Nepal (B. H. Hodgson 477) 1845.

Siva.

Siva, Hodgson, India Rev. ii. p. 88 (1838).

\section{Siva strigula.}

Siva strigula, Hodgs. Ind. Rev. ii. p. 89 ; Zool. Misc. p. 83: Jerd. B. Ind. ii. p. 252.

a Nepal (B. H. Hodgson 314) 1845. -b India (W. J. E. Boys) 1847. —c Himalaya (Williams).

\section{Siva cyanuroptera.}

Siva cyanuroptera, Hodgs. Ind. Rev. ii. p. 88 : Jerd. B. Ind. ii. p. 2503.

$a$ India (E. Blyth) 1846.

\section{IxULUS.}

Ixulus, Hodgson, J. A. S. B. xiv. p. 562 (1845).

\section{Ixulus flavicollis.}

Yuhina flavicollis, Hodgs. As. Res. xix. p. 167: G. R. Gray, Cat. Hodgs. Coll. (1846) p. 74.

Ixulus flavicollis, Hodgs. J. A. S. B, xiv. p. 562. 
a Nepal (B. H. Hodgson 312) 1845. —b Darjeeling ("Ixulus ruficollis" E. Blyth) 1850.

\section{YUHINA.}

Yuhina, Hodgson, As. Res. xix. p. 165 (1836).

\section{Yuhina gularis.}

Yuhina gularis, Hodgs. l. s. c. p. 166, pl. 9, f. 3: G. R. Gray, Cat. Hodgs. Coll. (1846) p. 74: Jerd. B. Ind. ii. p. 261.

a Nepal (B. H. Hodgson 309) 1845.

\section{5\%. Yuhina occipitalis.}

Yuhina occipitalis, Hodgs. As. Res. xix. p. 167 : G. R. Gray, Cat. Hodgs. Coll. (1846) p. 74: Jerd. B. Ind. ii. p. 261.

a Nepal (B. H. Hodgson 310) 1845.

\section{HERPORNIS.}

Erpornis, Hodgs. J. A. S. B. xiii. p. 379 (1844).

\section{Herpornis xantholeuca.}

Erpornis xantholeuca, Hodgs. l. s. c. Jerd. B. Ind. ii. p. 264.

Herpornis xantholeuca, Blyth, J. A. S. B. xliii. extr. No. p. 110.

$a \operatorname{Arakan}($ E. Blyth) 1847.

\section{Pterythrius ${ }^{1}$ 。}

Pteruthius, Swainson, Faun. Bor. Am. ii. p. 491 (1831). 359. Pterythrius erythropterus.

Lanius erythropterus, Vig. P. Z. S. 1830-1, p. 22 : Gould, Cent. B. Him. pl. 11.

Pteruthius erythropterus, Sw. Classif. B. ii. p. 249 : Jerd. B. Ind. ii. p. 245.

a f India (Stevens) 1844. -b India (W. J. E. Boys) 1848. —c Darjeeling (E. Blyth) 1850.

I The etymology of this name is given by Strickland (Ann, \& Mag. N. H. vii. p. 29). 
Allotrius.

Allotrius, Temminck, Pl. Col. Livr. 99 (1836).

360. Allotrius œnobarbus.

Allotrius cenobarbus, Temm. Pl. Col. 589, f. 2 : Jerd. B. Ind. ii. p. 246.

Pteruthrius xanthochlorus, Hodgs. J. A. S. B. xvi. p. 448. a I Dhee, Kumaon (W. J. E. Boys) 1847.

\section{Cutia.}

Cutia, Hodgson, J. A. S. B. v. p. 772 (1836).

\section{Cutia nipalensis.}

Cinclosoma nipalense, Hodgs. As. Res. xix. p. 145.

Cutia nipalensis, Hodgs. l. s. c: Jerd. B. Ind. ii. p. 247. a India ("Cutia nipalensis, Hodgs." E. Blyth) 1846.

\section{LIOPTILUS.}

Lioptilus, Cab. Mus. Hein. i. p. 88 (1850).

\section{Lioptilus nigricapillus.}

Le Merle à Calotte noir, Le Vaill. Ois. d'Afr. iii. p. 48, pl. 108. Turdus nigricapillus, Vieill. N. Dict. d'Hist. N. xx. p. 256. Lioptilus nigricapillus, Cab. l. s. c.

$a$ S. Africa (Brandt) 1845.

\section{COCHOA.}

Cochoa, Hodgson, J. A. S. B. v. p. 358 (1836).

\section{Cochoa purpurea.}

Cochoa purpurea, Hodgs. l. s. c. : Jerd. B. Ind. ii. p. 243.

a India ("Cochoa purpurea, Hodgs." E. Blyth) 1846.

The position of this genus is by no means established. Blyth (Cat. Calc. Mus. p. 194), without assigning it to any Family, places it between the Ampelidae and Euryloemidoe, at the same time suggesting its alliance with Pterythrius. Jerdon places it in the Liotrichince. By other writers it has been classed with the Ampelidae (Gray), Campephagidae (Bp.\&Wallace), Dicruridee(Bp.), and Sturnidce (Sundevall). In placing the genus here I follow Jerdon. Strickland's specimen is but a fragment. 


\section{FAMILY-TROGLODYTID AE.}

\section{DONACOBIUS.}

Donacobius, Swainson, Zool. Ill. 2nd Ser. pl. 72 (1831).

Cichla, Wagl. Syst. Av. fol. 20, p. 2 (1827) (partim).

\section{Donacobius atricapillus.}

Turdus atricapillus, Linn. Syst. Nat. i. p. 295.

Donacobius vociferus, Sw. l. s. c.

Donacobius atricapillus, Bp. Consp. i. p. 277: Scl. Cat. Am.

B. p. 16.

Cichla atricapilla, Strickl. Ann. \& Mag. N. H. vii. p. 27. a (N. C. Strickland) 1837.

A common widely-ranging South America species.

\section{Campylorhynchus ${ }^{1}$.}

Campylorhynchus, Spix, Av. Bras. i. p. 77 (1824).

\section{Campylorhynchus capistratus.}

Picolaptes capistratus, Less. Rev. Zool. 1842, p. 174.

Campylorhynchus capistratus, Scl. Cat. Am. B. p. 17.

$a$ Guatemala (J. Constancia) 1845.

\section{Campylorhynchus jocosus.}

Campylorhynchus jocosus, Scl. P. Z. S. 1859, p. 371.

a $\hat{\jmath}$ Cinco Señores, Mexico Sep. 1844 (Galeotti) 1845.

An immature bird.

\section{6\%. Campylorhynchus zonatus.}

Picolaptes zonatus, Less. Cent. Zool. pl. 70.

Campylorhynchus zonatus, Scl. Cat. Am. B. p. 17.

$a$ Mexico (Galeotti) 1845. - b Guatemala (J.Constancia) 1845. - c Guatemala (J. Constancia) 1848.

368. Campylorhynchus nuchalis.

Campylorhynchus nuchalis, Cab. Archiv f. Naturg. 1847, i. p. 206 : Scl. Cat. Am. B. p. 17.

\section{$a$ Trinidad (Argent) 1853.}

1 According to Strickland rightly placed in the Troglodytida. (Ann. \& Mag. N. H. vi. p. 423.) 


\section{Campylorhynchus variegatus.}

Turdus variegatus, Gm. Syst. Nat. i. p. 817.

Campylorhynchus variegatus, Gray, Scl. Cat. Am. B. p. 16.

Campylorhynchus scolopaceus, Spix, Av. Bras. i. p. 17.

$a$ S. America (Thomas) 1840.

\section{3\%0. Campylorhynchus unicolor.}

Campylorhynchus unicolor, Lafr. Rev. Zool. 1846, p. 93: Scl. Cat. Am. B. p. 16.

$a$ Bolivia (Argent) 1852.

\section{Cinnicerthia.}

Cinnicerthia, Less. Descr. d. Mamm. et Ois. p. 286.

\section{Cinnicerthia unirufa.}

Limnornis unirufa, Lafr. Rev. Zool. 1840, p. 105.

Cinnicerthia unirufa, Scl. P. Z. S. 1855, p. 143: Cat. Am. B. p. 18.

Cinnicerthia unicolor, Less. l. s. c.

$a$ Bogota (J. Gould) 1844.

Catherpes.

Catherpes, Baird, Birds N. Am. p. 356 (1858).

\section{+ 372. Catherpes mexicanus.}

Thryothorus mexicanus, Sw. Zool. Ill. ser. 2, i. pl. 11.

Catherpes mexicanus, Baird, l. s. c.: Scl. Cat. Am. B. p. 18. a (Arthur Strickland) 1840.

A common species in the highlands of Mexico.

\section{Cyphorhinus.}

- Cyphorhinus, Cabanis in Tsch. F. P. Av. p. 183 (1846).

\section{Cyphorhinus musicus.}

L'Arada, Buff. Pl. Enl. 706. f. 2. undè

Formicarius musicus, Bodd. Tabl. Pl. Enl. p. 44.

Cyphorhinus musicus, Gray: Scl. Cat. Am. B. p. 19.

$a$ (Bt. at Liverpool) 1853.

A species peculiar to Guiana. 


\section{THRYOPHILUS.}

Thryophilus, Baird, Rev. Am. B. i. p. 127 (1864).

\section{Thryophilus rufalbus.}

Thryophilus rufalbus, Lafr. Rev. Zool. 1845, p. 337: Scl. Cat. Am. B. p. 20.

Thryophilus rufalbus, Baird, l. s. c.

a Guatemala (J. Constancia) 184ら.

\section{Thryophilus leucotis.}

Thryothorus leucotis, Lafr. Rev. Zool. 1845, p. 338.

Thryophilus leucotis, Scl. \& Salv. Nomencl. Av. Nentr. p. 6. $a$ (W. Kirtland) 1843.

A species found throughout the northern portions of South America to the Isthmus of Panama.

\section{Thryophilus longirostris.}

Thryothorus longirostris, Vieill. N. Dict. d'Hist. N. xxxiv. p. 56: Scl. Cat. Am. B. p. 20.

a Brazil (N. C. Strickland) 1838.

\section{3\%\%. Thryophilus coraya.}

Le Coraya, Buff. Pl. Enl. 701. f. 1, undè

Turdus coraya, Gm. Syst. Nat. i. p. 825.

Thryothorus coraya, Scl. Cat. Am. B. p. 21.

$a$ (Matthew) 1840. - b Brazil (Argent) 1853.

\section{Thryothorus.}

Thriothorus, Vieillot, Anal. p. 45 (1816).

\section{Thryothorus ludovicianus.}

Sylvia ludoviciana, Lath. Ind. Orn. ii. p. 548.

Thryothorus ludovicianus, Licht. Verz. Doubl. p. 35: Strickl. Ann. \& Mag. N. H. viii. p. 371: Baird, Brew. \& Ridgw. N. Am. B. i. p. 142 .

a Reed's Creek, Minnesota Dec. 1839 (S. F. Baird), 1847.

\section{Thryothorus rutilus.}

Thryothorus rutilus, Vieill. N. Dict. d'Hist. N. xxxiv. p. 56: Scl. Cat. Am. B. p. 21.

a Brazil (Arthur Strickland) 1840. -b Tobago (Kirk) 1844. -c Trinidad (Argent) 1852. 
TROGLODYTES.

Troglodytes, Vieillot, Ois. Am. Sept. ii. p. 52 (1807).

\section{Troglodytes parvulus.}

Motacilla troglodytes, Linn. Syst. Nat. i. p. 337.

Troglodytes parvulus, Koch, Syst. d. Baierisch. Zool. p. 161: Newton, ed. Yarr. Brit. B. i. p. 460.

Troglodytes europceus, Strickl. P. Z. S. 1836, p. 98.

a Weston, Oxfordshire, 20 Aug. 1833 (N. C. Strickland), 1833. -b Smyrna 4 Dec. 1835 (H. E. Strickland), 1835.

\section{Troglodytes nipalensis.}

Troglodytes nipalensis, Hodgs.: Blyth, J. A. S. B. xiv. p. 589: Jerd. B. Ind. i. p. 491.

Troglodytes subhimalayensis, Hodgs. Zool. Misc. p. 82.

a Nepal (B. H. Hodgson) 1845.

\section{Troglodytes hyemalis.}

Troglodytes hyemalis, Vieill. N. Dict. d'Hist. N. xxxiv. p. 514: Troglodytes parvulus var. hyemalis, Baird, Brew. \& Ridgw. N. Am. B. i. p. 155.

$a \mathrm{~N}$. America (J. G. Kinberg) 1845.

\section{Troglodytes ædon.}

Troglodytes aedon, Vieill. Ois. Am. Sept. ii. p. 52, pl. 107 : Baird, Brew. \& Ridgw. N. Am. B. i. p. 149.

a Philadelphia, Pennsylvania (S. F. Baird) 1847.

\section{Troglodytes furvus.}

Motacilla furva, Gm. Syst. Nat. i. p. 994 .

Troglodytes furvus, Vieill. Gal. Ois. pl. 167: Scl. Cat. Am. B. p. 23 : Scl. \& Salv. Nomencl. Av. Neotr. p. 7.

Troglodytes hypaëdon, Scl. P. Z. S. 1861, p. 128.

$a($ Askew) 1833. - b, —c (N. C. Strickland) 1838. - d Guatemala (J. Constancia) 1851.

\section{CistothorUs.}

Cistothorus, Cab. Mus. Hein. i. p. 77 (1850). 385. Cistothorus stellaris.

Troglodytes stellaris, "Licht." Naum. Vög.Deutschl. iii. p. 724. 
Cistothorus stellaris, Cab. l. s. c.: Baird, Brew. \& Ridgw. N. Am. B. i. p. 159.

$a$ Massachusetts (J. G. Kinberg) 1845.

\section{Cistothorus palustris.}

Certhia palustris, Wils. Am. Orn. ii. p. 58, pl. 12, f. 4.

Cistothorus palustris, Baird, B. N. Am. p. 364.

$a$ N. America (J. G. Kinberg) 1845. -b (Dewgard) 1849.

\section{FAMILY.-MOTACILLID $\mathbb{E}$.}

\section{Motacilla.}

Motacilla, Linnæus, Syst. Nat. i. p. 328 (1766) (partim).

\section{8\%. Motacilla alba.}

Motacilla alba, Linn. Syst. Nat. i. p. 331: Newton, ed. Yarr. Brit. B. i. p. 548.

Motacilla dukhunensis, Sykes, P. Z. S. 1832, p. 91.

$a$ N. Europe (Kinberg) 1843. - b Nepal (B.H.Hodgson 135). -c ô Jucknie 13 Nov. 1841 (W. J. E. Boys). —d, -e India (E. Blyth) 1846. $-f$ \& N. India (W. J. E. Boys) 1848.

\section{Motacilla lugubris.}

Motacilla lugubris, Temm. Man. d'Orn. i. p. 253 (1820): Newton, ed. Yarr. Brit. B. i. p. 538.

Motacilla yarrelli, Gould, P. Z. S. 1837, p. 73.

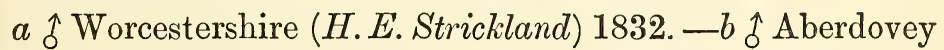
(H. E. Strickland) Aug. 1833. —c o Britain (Askew) 1837. —d q Worcestershire (H. E. Strickland) 25 Mar. 1840. -e Tangiers (F. Favier) 1841.

\section{Motacilla luzononsis.}

Motacilla luzononsis, Scop. Del. Faun. et Fl. Insubr. ii. p. 95: Wald. Trans. Z. S. ix. p. 198: Jerd. B. Ind. ii. p. 218.

a India ("Motacilla luzoniensis," E. Blyth) 1846. -b India (E. Blyth). 
390. Motacilla maderaspatensis.

Motacilla maderaspatensis, Gm. Syst. Nat. i. p. 961.

Motacilla maderaspatana, Briss. Jerd. B. Ind. ii. p. 21 .

Motacilla variegata, Steph. Shaw's Zool. xiii. Suppl. p. 234.

Motacilla picata, Frankl. P. Z. S. 1830-1, p. 119.

$a$ (Askew) 1833. - b Marras (Stuchbury) 1836. -c (Askew) 1840. —d S. India ("Motacilla maderaspatana," T. C. Jerdon) 1850.

\section{Motacilla longicauda?}

Motacilla longicauda, Rüpp, Neue Wirb. p. 84, t. 29, f. 2.

Motacilla capensis, Linn. Strickl. P. Z. S. 1850, p. 217.

a f Kartoum Oct. 1848 (J. Petherick) 1848.

A young bird probably of this species.

\section{Motacilla capensis.}

Motacilla capensis, Linn. Syst. Nat. i. p. 383: Layard, B. S. Afr. p. 118.

a Cape of Good Hope (A. Strickland) 1832.

\section{Motacilla flaviventris.}

Motacilla flaviventris "Verreaux," Hartl. Orn. Beitr. z. Faun. Madag. p. 39.

$a$ (E. De Selys Longchamps) 1845 .

A species found exclusively in Madagascar.

\section{Motacilla melanope.}

Motacilla melanope, Pall. Reise, iii. p. 696: Dresser, B. Eur. Motacilla sulphurea, Bechst. Nat. Deutschl. iii. p. 459 (1807): Newton, ed. Yarr. Brit. B. i. p. 552.

$a$ Lago Maggiore (H. E. Strickland) July 1836. - b Madras ("Motacilla boarula," T. C. Jerdon) 1845.

395. Motacilla flava.

Motacilla flava, Linn. Syst. Nat. i. p. 331: Newton, ed. Yarr. Brit. B. i. p. 558.

Motacilla viridis, Gm. Syst. Nat. i. p. 962.

Budytes viridis, Jerd. B. Ind. ii. p. 222.

Motacilla cinereicapilla, Savi, Orn. Tosc. iii. p. 216.

Motacilla neglecta, Gould, P. Z. S. 1832, p. 129. 
$a,-b$ Norway (N. C. Strickland) 18 May 1832. -c, -d Lago Maggiore (H. E. Strickland) Aug. 1836. -e E. Indies (N. C. Strickland) 1838. $-f$ India (Havell) 1839. -g, - $h$ S. India ("Budytes viridis" T. C. Jerdon) 1844. - i Madras ("Budytes viridis" T. C. Jerdon) 1845. - j, -k, - l India ("Budytes viridis" E. Blyth) 1846. $-m$ Benares (W. J. E. Boys) 1847. - n Malwa (W. J. E. Boys) 1847. - o f (W. J. E. Boys) 1848. - $p$ Sierra Leone (Dr Gordon) 1848.

\section{Motacilla rayi.}

Motacilla flava, Ray, Gould, P. Z. S. 1832, p. 129: B. Eur. pl. 145.

Budytes rayi, Bp. Comp. List, p. 18.

$a,-b \hat{\delta},-c$ o Worcestershire (H. E. Strickland) May 1838. - d Gloucestershire (H. E. Strickland) 4 Aug. 1840.

\section{9\%. ITotacilla melanocephala.}

Motacilla melanocephala, Licht. Verz. Doubl. p. 36.

Budytes melanocephala, Bp. Comp. List, p. 19: Strickl. P. Z. S. 1850 , p. 217.

a Dalmatia (L. Coulon) 1836. - b ई Buchara March 1842 (J. F. Brandt) 1846. —c o Kartoum Oct. 1848 (J. Petherick) 1848.

\section{Motacilla citreoloides.}

Budytes citreoloides, Hodgs. Zool. Misc. p. 83 (descr. mulla).

Motacilla citreoloides, Dresser, B. Eur. (sub M. citreola).

$a,-b$ Nepal (B. H. Hodgson 732) 1845. -c $\hat{\delta}$ Benares (W. J. E. Boys) 1847. —d N. India (W. J. E. Boys) 1847.

\section{Motacilla citreola.}

Motacilla citreola, Pall. Reise, iii. p. 696.

Budytes citreola, Jerd. B. Ind. ii. p. 225.

a ô India (W. J. E. Boys) 1848 .

400. Motacilla indica.

Motacilla indica, Gm. Syst. Nat. i. p. 962.

Nemoricola indica, Jerd. B. Ind. ii. p. 226.

a Malacca (N. C. Strickland) 1838.

S. C. 
ANTHUS.

Anthus, Bechstein, Nat. Deutschl. iii. p. 704 (1807).

\section{Anthus obscurus.}

Alauda obscura, Lath. Ind. Orn. p. 494. (? nec Gmelin).

Anthus obscurus, Newton, ed. Yarr. Brit. B. i. p. 586.

Alauda petrosa, Mont. Trans. L. S. iv. p. 41.

a Aberdovey (H. E. Striclcland) Aug. 1833. -b (N.C. Strickland) 1838.

Gmelin's name Alauda obscura (Syst. Nat. i. p. 801), based upon a description of Cetti's (Stor. Nat. di Ucc. Sard. p. 150), has been placed by Gray with doubt as a synonym of Anthus campestris. Should it be applicable (as seems not impossible) to any other Anthus than the present, Montagu's name will have to be used for the Rock-Pipit. Plate 138 of Gould's Birds of Europe, as pointed out by Strickland (Ann. \& Mag. N. H. viii. p. 372), represents this species, but the propriety of adding Anthus rupestris, Nilss. to its synonyms may be questioned ( $c f$. Newton $l$. s. c.).

\section{Anthus spipoletta.}

Alauda spinoletta, Linn. Syst. Nat. i. p. 288.

Anthus spipoletta, Newton, ed. Yarr. Brit. B. i. p. 581.

Anthus aquaticus, Bechst. Nat. Deutschl. iii. p. 745: Strickl. P. Z. S. 1836 , p. 99.

a Smyrna (H. E. Strickland) 10 Dec. 1835. - b Pyrenees (E. De Selys Longchamps) 1845. —c N. India (W. J. E. Boys) 1848.

\section{Anthus cervinus.}

Motacilla cervina, Pall. Zoogr. Rosso-As. i. p. 511.

Anthus cervinus, Dresser, B. Eur.

Anthus sp. ? Strickl. P. Z. S. 1850, p. 217.

a f Kartoum Oct. 1848 (J. Petherick), 1848.

\section{Anthus rosaceus.}

Anthus rosaceus, Hodgs. Zool. Misc. p. 83 (descr. nulla): Dresser, B. Eur. (sub A. cervinus).

a Nepal (B. H. Hodgson 736) 1845.

Distinguishable from $A$. cervinus by its pale yellow axillaries. 
405. Anthus pratensis.

Alauda pratensis, Linn. Syst. Nat. i. p. 287.

Anthus pratensis, Strickl. P. Z. S. 1836, p. 99: Newton, ed. Yarr. Brit. B. i. p. 575.

a Worcestershire (H. E. Strickland) 1830. - b f Smyrua (H. E. Strickland) 20 Nov. 1835.

\section{Anthus striolatus.}

Anthus striolatus, Blyth, J. A. S. B. xvi. p. 435.

Corydalla striolata, Jerd. B. Ind. ii. p. 233.

$a$ (N. C. Strickland) 1838. - b (Gardner) 1845. -c Madras (T. C. Jerdon) 1845. - d S. India ("Anthus malayanus" T. C. Jerdon) 1850. —S. India ("Anthus striolatus, Bl." T. C. Jerdon) 1850.

I believe all these specimens are referable to the species called A. striolatus by Blyth, being unable to detect any tangible difference between the specimens sent by Jerdon as $A$. striolatus and $A$. malayanus.

\section{0\%. Anthus rufulus.}

Anthus rufuhus, Vieill. N. Dict. d'Hist. N. xxvi. p. 494: Gal. des Ois. i. t. 161.

Corydalla rufula, Jerd. B. Ind. ii. p. 232.

Cichlops ubiquitarius, Hodgs. Zool. Misc. p. 83.

a Nepal (B. H. Hodgson 4.32) 1845. -b India ("Anthus agilis" E. Blyth) 1846.

I follow Jerdon in placing these specimens under this name. Whether those I place under $A$. striolatus are really different is quite doubtful. Mr Brooks (Ibis, 1874 p. 460) maintains positively that there are two species distinguishable by their song, but the characters he gives (a usually longer hind claw and larger bill) by which alone skins can be differentiated do not help one much. Lord Tweeddale (Ibis, 1874, p. 141) doubts the propriety of separating A. striolatus from A. rufulus.

\section{Anthus trivialis.}

Alauda trivialis, Linn. Syst. Nat. i. p. 288.

Anthus trivialis, Newton, ed. Yarr. Brit. B. i. p. 569.

Anthus arboreus, Bechst. Nat. Deutschl. iii. p. 706 . 
Pipastes arboreus, Jerd. B. Ind. ii. p. 229.

Anthus maculatus, Hodgs. Zool. Misc. p. 83.

$a \hat{\delta},-b q$ Trollhätten, Sweden (N. C. Strickland) 24 May, 1831. -c $\hat{\delta}$ Worcestershire (H. E. Strickland) 1834. -d $\hat{\delta}$ Worcestershire (H. E. Strickland) April, 1837. -e Madras (T. C. Jerdon) 1845. - $f$ Nepal (B. H. Hodgson 435) 1845. - g (Stevens). - $h$ India (Major Stacy).

\section{Anthus australis.}

Anthus australis, Vig. \& Horsf. Trans. L. S. xv. p. 229: Gould, B. Austr. iii. pl. 73.

Agrodroma bistriata, Sw. An. in Menag. p. 316.

a Australia (N. C. Strickland) 1838.

\section{Anthus sp.?}

$a$ (A. Strickland) 1850.

A very young bird marked on the label by J. Verreaux as the young of $A$. hasselti, Temm., but upon what grounds I am unable to determine. According to Lord Tweeddale (Trans. Z. S. ix. p. 198 note), this name appears only to exist in Gray's Hand-list (i. p. 252, No. 3655).

\section{Anthus lugubris.}

Corydalla lugubris, Wald. Trans. Z. S. ix. p. 198.

$a$ South of the island of Manilla (H. Cuming) 14 June, 1839.

\section{Anthus rufus.}

Petite Alouette de Buenos Ayres, D'Aub. Pl. Enl. 738, f. i. undè Alauda rufa, Gm. Syst. Nat. i. p. 798.

$a$ (Mansfield) 1834. -b (N. C. Strickland) 1838. -c (Bt. at Birmingham) 1849. —d q Mexiana Dec. 1848 (A. R. Wallace), 1849. -e Brazil (Argent) 1851.

Boddaert (Tab. p. 46) gives to the subject of Pl. Enl. 738 fig. 1 the name Alauda nigra, but it is obvious from Buffon's names which he quotes that he meant his title for the second figure of the same plate. Alauda nigra, Bodd. = Centrites nigra, Cab. \& Hein., and belongs to the Tyrannidae. 


\section{Anthus correndera.}

Correndera, Azara, Apunt. para los Pax. de Parag. ii. p. 2, No. 145 undè

Anthus correndera, Vieill. N. Dict. d'Hist. N. xxvi. p. 491 : Scl. Cat. Am. B. p. 24.

a Chili (Capt. Brown) 1842.

\section{Anthus campestris.}

Alauda campestris, Linn. Syst. Nat. i. p. 288.

Anthus campestris, Newton, Ed. Yarr. Brit. B. i. p. 592.

Agrodroma campestris, Strickl. Ann. \& Mag. N. H. viii.

p. 374: Jerd. B. Ind. ii. p. 234.

Anthus rufescens, Temm. Man. d'Orn. i. p. 267.

a Morea (H. E. Striclcland) May 1836. -b N. India (W. J. E. Boys) 1847.

\section{Anthus richardi.}

Anthus richardi, Vieill. N. Dict. d'Hist. N. xxvi. p. 491: Newton, ed. Yarr. Brit. B. i. p. 598.

Corydalla richardi, Jerd. B. Ind. ii. p. 231.

a Italy ( $E$. De Selys Longchamps) 1845. - b Madras (T. C. Jerdon) 1845. —c (Gardner) 1845. —d India (E. Blyth) 1846.

\section{Anthus pyrrhonotus.}

Alauda pyrrhonotha, Vieill. N. Dict. d'Hist. N. i. p. 361.

Anthus pyrrhonotus, Gurney, Ibis, 1871, p. 156.

$a$ \& Cape of Good Hope (A. Strickland) 15 April, 1832. - b (Askew) 1837. -c (N. C. Strickland) 1838.

\section{Macronyx.}

Macronyx, Swainson, Zool. Journ. iii. p. 344 (1827).

\section{1\%. Macronyx capensis.}

Alauda capensis, Linn. Syst. Nat. i. p. 288.

Macronyx capensis, Sw. Classif. B. ii. p. 293.

$a \hat{\delta},-b q$ Cape of Good Hope (A. Strickland) 7 Mar. 1833. 


\section{Macronyx croceus.}

Alauda crocea, Vieill. N. Dict. d'Hist. N. i. p. 365.

Macronyx flavigaster, Sw. B. W. Afr. i. p. 215.

a Senegal (Isaacson) 1840.

\section{Heterura.}

Heterura, Hodgson, P. Z. S. 1845, p. 33.

\section{Heterura sylvana.}

Heterura sylvana, Hodgs. l. s. c.: Strickl. Contr. Orn. 1848, p. 26, pl. 6 : Jerd. B. Ind. ii. p. 239.

a f Kumaon (W. J. E. Boys) 1847.

The specimen figured in "The Contributions to Ornithology."

\section{Cinclorhamphus ${ }^{1}$.}

Cinclorhamphus, Gould, P. Z. S. 1837 p. 150.

\section{Cinclorhamphus cruralis.}

Megalurus cruralis, Vig. \& Horsf. Trans. L. S. xv. p. 228.

Cinclorhamphus cruralis, Gould, l. s. c.: B. Austr. iii. pl. 74. a Australia (N. C. Strickland) 1838.

\section{Cinclorhamphus rufescens.}

Anthus rufescens, Vig. \& Horsf. Trans. L. S. xv. p. 230.

Cinclorhamphus rufescens, Gould, B.Austr. iii. pl. 76. $a$ New South Wales (McDonald) 1838.

1 The absence of the first primary in C.cruratis and the length of the second point to the Motacillidee as the position of this genus. C. rufescens, however, has a small first primary, the second being long, as in C. cruralis. The formation of the wing is quite different from that of Megalurus, Malacocercus, \&c., with which genera Strickland associated Cinclorhamphus (Contr. Orn. 1848, p. 26). Notwithstanding the presence in some species of a small first primary I consider this genus should be placed in the Motacillidoe. 


\section{FAMILY.-MNIOTILTIDA.}

\section{SiURUs.}

Seiurus, Swainson, Zool. Journ. iii. p. 171 (1827).

\section{Siurus auricapillus.}

Motacilla auricapilla, Linn. Syst. Nat. i. p. 334.

Seiurus auricapillus, Sw. l. s. c.: Baird, Brew. \& Ridgw. N. Am. B. i. p. 280.

Siurus auricapillus, Scl. \& Salv. Ibis, 1859, p. 9.

$a$ N. America (N. C. Strickland) 1838. - b Guatemala ( $J$. Constancia) 1851.

\section{Siurus noveboracensis.}

Motacilla noveboracensis, Gm. Syst. Nat. i. p. 958.

Seiurus noveboracensis (Gm.) : Aud. B. N. Am. iii. pl. 199 : Baird, Brew. \& Ridgw. N. Am. B. i. p. 283.

a Louisiana (J. J. Audubon) 18- . -b (N. C. Strickland) 1838. - c Carlisle, Pennsylvania 14 May, 1846 (S. F. Baird) 1847. — $d$ Tobago (Sir W. Jardine) 1848.

\section{Siurus ludovicianus.}

Turdus ludovicianus, Aud. Orn. Biogr. i. p. 99.

Seiurus ludovicianus, Bp., Baird, Brew. \& Ridgw. N. Am. B. i. p. 287.

Siurus ludovicianus, Salv. \& Scl. Ibis, 1860, p. 273.

$a$ Guatemala (J. Constancia) 1848.

\section{Mniotilta.}

Mniotilta, Vieillot, Anal. p. $4 \check{5}$ (1816).

\section{Mniotilta varia.}

Motacilla varia, Linn. Syst. Nat. i. p. 333.

Mniotilta varia (Linn.), Scl. \& Salv. Ibis, 1859, p. 10 : Baird, Brew. \& Ridgw. N. Am. B. i. p. 180.

a N. America (N. C. Strickland) 1838. -b Guatemala (J. Constancia) 1848. $\rightarrow c$ Guatemala (J. Constancia) 1851. 


\section{PARULA.}

Parula, Bonaparte, Comp. List, p. 20 (1838).

\section{Parula americana.}

Parus americanus, Linn. Syst. Nat. i. p. 341.

Parula americana, Gosse, B. Jam. p. 154: Scl. \& Salv. Ibis, 1859, p. 10 : Baird, Brew. \& Ridgw. N. Am. B. i. p. 208.

a Carlisle, Pennsylvania 5 May, 1847 (S. F. Baird), 1847. -b Jamaica (P. H. Gosse) 1848. —c Guatemala (J. Constancia) 1848.

\section{2\%. Parula pitiayumi.}

Sylvia pitiayumi, Vieill. N. Dict. d'Hist. N. xi. p. 276.

Parula pitiayumi, Scl. Cat. Am. B. p. 26.

Sylvia brasiliana, Licht. Verz. Doubl. p. 35.

a Brazil (Askew) 1837. -b Brazil ? (N. C. Strickland) 1838.

\section{Parula superciliosa.}

Conirostrum superciliosum, Hartl. Rev. Zool. 1844, p. 215.

Parula superciliosa, Scl. \& Salv. Ibis, 1859, p. 10.

Parula mexicana, Licht., Bp. Consp. i. p. 310.

$a$ Guatemala (J. Constancia) 1848.

\section{HeLminthophaga.}

Helminthophaga, Cabanis, Mus: Hein. i. p. 20 (1850).

\section{Helminthophaga pinus.}

Certhia pinus, Linn. Syst. Nat. i. p. 187.

Helminthophaga pinus, Baird, Brew. \& Ridgw. N. Am. B. i. p. 105 .

Sylvia solitaria, Wils. Am. Orn. ii. pl. 15, f. 4. a ô Philadelphia (E. Wilson) 1848.

\section{Helminthophaga chrysoptera.}

Motacilla chrysoptera, Linn. Syst. Nat. i. p. 333.

Helminthophaga chrysoptera, Baird, Brew. \& Ridgw. N. Am. B. i. p. 192.

a (Bought at Liverpool) 1853.

A species of the Eastern states of America, migrating in winter to the Antilles, Central America, and Columbia. 


\section{Helminthophaga ruficapilla.}

Sylvia ruficapilla, Wils. Am. Orn. iii. p. 120, pl. 27, f. 3.

Helminthophaga ruficapilla, Baird, Brew. \& Ridgw. N. Am. B. i. p. 196.

$a$ (Dewgard) 1846. - b o Carlisle, Pennsylvania 8 May, 1845̃, (S. F. Baird) 1847. —c Guatemala (J. Constancia) 1848. -d Guatemala (J. Constancia) 1851.

\section{HeLminthotherus.}

Helmintherus, Rafinesque, Journ. de Phys. Ixxxviii. p. 417 (1819).

\section{Helminthotherus vermivorus.}

Motacilla vermivora, Gm. Syst. Nat. i. p. 951.

Helmintherus vermivorus, Baird, Brew. \& Ridgw. N. Am. B. i. p. 187.

Vermivora fulvicapilla, Sw. Classif. B. ii. p. 245.

$a,-b$ N. America (N. C. Strickland) 1838.

\section{Dendraeca.}

Dendroica, G. R. Gray, Gen. B. App. p. 8 (1842).

433. Dendrœca virens.

Motacilla virens, Gm. Syst. Nat. i. p. 985.

Dendroica virens, Scl. \& Salv. Ibis, 1859, p. 11: Baird, Brew. \& Ridgw. i. p. 261.

$a$ N. America (Askew) 1839. $-b,-c$ Guatemala ( $J$. Constancia) 1845. - $d$, -e Guatemala ( $J$. Constancia) 1848.

\section{Dendrœca townsendi.}

Sylvia townsendi, Towns. Journ. Ac. N. Sc. Phil. viii. p. 191. Dendroica townsendi, Scl. \& Salv. Ibis, 1859, p. 11: Baird, Brew. \& Ridgw. N. Am. B. i. p. 265.

$a$ Guatemala (J. Constancia) 1848.

435. Dendrœca occidentalis. 190.

Sylvia occidentalis, Towns. Journ. Ac. N. Sc. Phil. vii. p. 
Dendroica occidentalis, Baird, Brew. \& Ridgw. N. A.m. B. i. p. 266 .

Dendrœea niveiventris, Salv. P. Z. S. 1863, p. 187, pl. 24, f. 2. a Guatemala (J. Constancia) 1845.

436. Dendrœca nigrescens.

Sylvia nigrescens, Towns Journ. Ac. N. Sc. Phil. vii. p. 191.

Dendroica nigrescens, Scl. P. Z. S. 1858, p. 298: Baird, Brew. \& Ridgw. N. Am. B. i. p. 258.

$a$ San Pedro, Mexico, Oct. 1844 (Galeotti) 1845.

\section{3\%. Dendrœca cærulescens.}

Motacilla ccerulescens, Gm. Syst. Nat. i. p. 960.

Dendroica ccerulescens, Baird, Brew. \& Ridgw. N. Am. B. i. p. 254 .

Motacilla canadensis, Linn. Syst. Nat. i. p. 336 (nec p. 334).

$a$ N. America (Askew) 1840. - b (Argent) 1852.

\section{Dendrœca coronata.}

Motacilla coronata, Linn. Syst. Nat. i. p. 333.

Dendroeca coronata, Baird, Brew. \& Ridgw. N. Am. B. i. p. 227.

$a$ N. America (Aslew) 1834. -b N. America (N. C. Strickland) 1838.

\section{Dendrœca blackburniæ.}

Motacilla blackburnice, Gm. Syst. Nat. i. p. 977.

Rhimamphus blackburnice, Scl. P. Z. S. 1855, p. 143.

Dendroica blackburnia, Baird, Brew. \& Ridgw. N. Am. B. i. p. 237.

a N. America, 1848. -b, -c Bogota (Bt. at Stevens's) 1845.

\section{Dendrœca castanea.}

Sylvia castanea, Wils. Am. Orn. ii. p. 97, pl. 14, f. 4.

Dendroica castanea, Baird, Brew. \& Ridgw. N. Am. B. i. p. 251.

$a$ N. America (N. C. Strickland) 1838.

\section{Dendrœca pennsylvanica.}

Motacilla pennsylvanica, Linn. Syst. Nat. i. p. 333. 
Dendroica pennsylvanica, Baird, Brew. \& Ridgw. N. Am. B. i. p. 245 . 1846.

a N. America, 1838. -b (Gardner) 1845. -c (Dewgard)

\section{Dendrœca striata.}

Muscicapa striata, Forst. Phil. Trans. 1xii. p. 428.

Dendroica striata, Baird, Brew.\& Ridgw. N. Am. B. i. p. 248.

a N. America (N. C. Strickland) 1838. -b (Arthur Strickland) 1840. — (J. G. Kinberg) 1845.

\section{Dendrœca æstiva.}

Motacilla cestiva, Gm. Syst. Nat. i. p. 996.

Dendroica restiva, Scl. \& Salv. Ibis, 1859, p. 11 : Baird, Brew. \& Ridgw. N. Am. B. i. p. 222.

$a \mathrm{~N}$. America (Askew) 1833. -b (W. Kirtland) 1843. $-c$ N. America (J. G. Kinberg) 1844. —d Mexico (Galeotti) 1845. - e Guatemala ( $J$. Constancia) 1845. $-f$ N. W. America (Wosnessenski, J. F. Brandt) 1846. -g Trinidad (Argent) 1852.

\section{Dendrœca pinus.}

Sylvia pinus, Wils. Am. Orn. iii. p. 25, pl. 19, f. 4.

Dendroica pinus, Baird, Brew. \& Ridgw. N. Am. B. i. p. 268. $a \mathrm{~N}$. America (Cashmore) 1839.

\section{Dendrœca olivacea.}

Sylvia olivacea, Giraud, B. Texas, p. 14, pl. 7, f. 2.

Rhimamphus olivaceus, Scl. P. Z. S. 1856, p. 291.

Dendroca olivacea, Baird, Brew. \& Ridgw. N. Am. B. i. p. 258.

$a,-b$ Mexico (T. Mann) 1844.

\section{Dendrœca maculosa.}

Motacilla maculosa, Gm. Syst. Nat. i. p. 984.

Dendroica maculosa, Baird, Brew. \& Ridgw. N. Am. B. i. p. 232.

$a$ N. America (N. C. Strickland) 1838. -b (E. Brown) 1850. 


\section{Dendrœca palmarum.}

Motacilla palmarum, Gm. Syst. Nat. i. p. 951.

Dendroica palmarum, Baird, Brew. \& Ridgw. N. Am. B. i. p. 273.

$a$ Carlisle, Pennsylvania, 3 May, 1847 (S. F. Baird), 1847.

\section{Dendrœca dominica.}

Motacilla dominica, Linn. Syst. Nat. i. p. 334.

Dendroica dominica, Baird, Brew. \& Ridgw. N. Am. B. i. p. 240.

Dendroca superciliosa, Gm., Scl. \& Salv. Ibis, 1860, p. 274.

$a$ Guatemala ( $J$. Constancia) 1845. - 6 Savannah, Carolina (S. F. Baird) 1847.

\section{Dendrœca decora.}

Dendroica gracice var. decora, Baird, Brew. \& Ridgw. N. Am. B. i. p. 244.

$a$ Guatemala (J. Constancia) 1848.

\section{Dendreca discolor.}

Sylvia discolor, Vieill. Ois. Am. Sept. ii. p. 37, pl. 98.

Sylvicola discolor, Gosse, B. Jam. p. 159.

Dendroica discolor, Baird, Brew. \& Ridgw. N. Am. B. i. p. 276.

a Jamaica (P. H. Gosse) 1848.

\section{Geothlypis.}

Geothlypis, Cabanis, Archiv f. Naturg. 1847, p. 316.

\section{Geothlypis trichas.}

Turdus trichas, Linn. Syst. Nat. i. p. 293.

Geothlypis trichas, Cab., Baird, Brew. \& Ridgw. N. Am. B. i. p. 297.

Trichas personatus, Sw. Classif. B. ii. p. 247.

$a$ N. America (Askew) 1833. - b (Askew) 1837. - c, - d (Arthur Strickland) 1840. 
452. Geothlypis æquinoctialis.

Motacilla cequinoctialis, Gm. Syst. Nat. i. p. 972.

Geothlypis cequinoctialis, Scl. Cat. Am. B. p. 27.

$a$ (Askew) 1834. — b Trinidad (Argent) 1852.

\section{Geothlypis philadelphia.}

Sylvia philadelphia, Wils. Am. Orn. ii. p. 101, pl. 14, f. 6.

Geothlypis philadelphia, Baird, Brew. \& Ridgw. N. Am. B. i. p. 301.

a Carlisle, Pennsylvania 18 May, 1841 (S. F. Baird), 1847.

\section{Geothlypis macgillivrayi.}

Sylvia macgillivrayi, Aud. Orn. Biogr. V. p. 75.

Geothlypis macgillivrayi, Scl. \& Salv. Ibis, 1859, p. 10 : Baird, Brew. \& Ridgw. N. Am. B. i. p. 303.

a Guatemala (J. Constancia) 1845. - b, -c Guatemala ( $J$. Constancia) 1848.

\section{Myiodioctes.}

Myiodioctes, Audubon, Synopsis Am. B. p. 48 (1839).

\section{Myiodioctes mitratus.}

Motacilla mitrata, Gm. Syst. Nat. i. p. 977.

Myiodioctes mitratus, Baird, Brew. \& Ridgw. N. Am. B. i. p. 314 .

a N. America (Arthur Strickland) 1840.

456. Myiodioctes canadensis.

Muscicapa canadensis, Linn. Syst. Nat. i. p. 327.

Myiodioctes canadensis, Scl. P. Z. S. 1855, p. 143 : Scl. \& Salv. Ibis, 1859, p. 11 : Baird, Brew. \& Ridgw. N. Am. B. i. p. 320.

a N. America (N. C. Strickland) 1838. - b Bogota (Bt. at Stevens's) 1845. - c, -d Guatemala (J.Constancia) 1848.

\section{Myiodioctes pusillus.}

Motacilla pusilla, Wils. Am. Orn. iii. p. 103. pl. 26, f. 4.

Myiodioctes pusillus, Scl. \& Salv. Ibis, 1859, p. 11 : Baird, Brew. \& Ridgw, N. Am. B. i. p. 317. 
a Guatemala ( $J$. Constancia) 1845. - $b$ N. W. America (Wosnessenski, J. F. Brandt) 1846. - c f Carlisle, Pennsylvania 19 May, 1847 (S. F. Baird) 1847.

\section{Basileuterus.}

Basileuterus, Cabanis, Schomb. Guian. iii. p. 666 (1848).

\section{Basileuterus vermivorus.}

Sylvia vermivora, Vieill. N. Dict. d'Hist. N. xi. p. 278.

Basileuterus vermivorus, Scl. P. Z. S. 1865, p. 283.

a (Cashmore) 1839. - b Brazil ? (Argent) 1852.

\section{Basileuterus hypoleucus.}

Basileuterus hypoleucus, Bp. Consp. i. p. 313.

a (N. C. Strickland) 1838.

A Brazilian species.

460. Basileuterus bivittatus.

Muscicapa bivittata, D’Orb. \& Lafr. Syn. Av. i. p. 50: D'Orb. Voy. Ois. p. 324.

Basileuterus bivittatus, Scl. P. Z. S. 1865, p. 285.

a Bogota (Bt. at Stevens's) 1845.

\section{Basileuterus delattrii.}

Basileuterus delattrii, Bp. C. R. xxxviii. p. 383: Scl. \& Salv. Ibis, 1860, p. 274 : Scl. P. Z. S. 1865, p. 284.

$a,-b$ Guatemala ( $J$. Constancia) 1845.

462. Basileuterus cinereicollis.

Basileuterus cinereicollis, Scl. P. Z. S. 1864, p. 166; 1865, p. 285 , pl. 9 , f. 2.

a Bogota (Bt. at Stevens's) 1845. -b (Gardner) 1841.

463. Basileuterus stragulatus.

Muscicapa stragulata, Licht. Vèrz. Doubl. p. 55.

Basileuterus stragulatus, Scl. P. Z. S. 1865, p. 285.

a Brazil (N. C. Strickland) 1838. _b Brazil (Dewgard) 1849. 
464. Basileuterus leucoblepharus.

Sylvia leucoblephara, Vieill. N. Dict. d'Hist. N. xi. p. 206.

Basileuterus leucoblepharus, Scl. P. Z. S. 1865, p. 285.

a Brazil (N. C. Strickland) 1838.

465. Basileuterus nigricristatus.

Trichas nigrocristatus, Lafr. Rev. Zool. 1840, p. 230.

Basileuterus nigrocristatus, Scl. Cat. Am. B. p. 36.

a Bogota? (Gardner) 1844.

466. Basileuterus flaveolus.

Myiothlypis flaveolus, Baird, Rev. Am. B. i. p. 252.

Basileuterus flaveolus, Scl. \& Salv. Nomencl. Av. Neotr. p. 10.

a (Bt. at Birmingham) 1849.

467. Basileuterus lutoiviridis.

Myiothlypis luteoviridis, Bp. Consp. i. p. 311.

Basileuterus luteoviridis, Scl. \& Salv. Nomencl. p. 10.

$a$ Bogota (J. Gould) 1844. -b,

\section{SETOPHAGA.}

Setophaga, Swainson, Zool. Journ. iii. p. 360 (1827).

\section{Setophaga ruticilla.}

Motacilla ruticilla, Linn. Syst. Nat. i. p. 336.

Setophaga ruticilla, Baird, Brew. \& Ridgw. N. Am. B. i. p. 322.

$a$ N. America (Arthur Strickland) 1834. - b N. America (Burl) 1834. - c (Bt. at Stevens's) 1845. —d Trinidad (Argent) 1851.

469. Setophaga picta.

Setophaga picta, Sw. Zool. Ill. Ser. 2, i. pl. 3 : Scl. \& Salv. Ibis, 1859, p. 12.

a Guatemala (J. Constancia) 1845. - 6 Guatemala (J. Constancia) 1848. 


\section{Setophaga miniata.}

Muscicapa miniata, Sw. Phil. Mag. 1827, p. 368.

Setophaga miniata, Scl. P. Z. S. 1856, p. 292.

Setophaga flammea, Kaup. P. Z. S. 1851, p. 49 : Scl. \& Salv. Ibis, 1859, p. 12.

$a,-b$ Mexico (T. Mann) 1844. $-c,-d$ Guatemala (J. Constancia) 1845.

\section{Setophaga verticalis.}

Setophaga verticalis, D’Orb. \& Lafr. Syn. Av. p. 50 : D'Orb. Voy. Ois. p. 330, pl. 35, f. 1 : Scl. Cat. Am. B. p. 37.

a Bogota (Bt. at Stevens's) 1845.

\section{Setophaga brunneiceps.}

Setophaga brunneiceps, D’Orb. \& Lafr. Syn. Av. p. 50 : D'Orb. Voy. Ois. p. 329, pl. 34, f. 3 : Scl. Cat. Am. B. p. 37.

a Bolivia (Argent) 1852.

\section{Setophaga ornata.}

Setophaga ornata, Boiss. Rev. Zool. 1840, p. 70 : Scl. Cat. Am. B. p. 37.

a Bogota (Bt. at Stevens's) 1845. - b Bogota (Dewgard) 1849. —c (Argent) 1851.

\section{Ergaticus.}

Ergaticus, Baird, Rev. Am. B. p. 264 (1865) (as a sub-genus). 474. Ergaticus ruber.

Setophaga rubra, Sw. Phil. Mag. 1827, p. 368.

Cardellina rubra, Baird, $l_{\text {. s. c. }}$

Ergaticus ruber, Scl. \& Salv. Nomencl. Av. Neotr. p. 11. $a,-b$ Mexico (T. Mann) 1844. 


\section{FAMILY.PYCNONOTIDE.}

\section{SUB-FAMILY.-PYCNONOTINAE.}

\section{Hypsipetes.}

Hypsipetes, Vigors, P. Z. S. 1830-1, p. 43.

\section{Hypsipetes psaroides.}

Hypsipetes psaroides, Vig. l. s. c.: Gould, Cent. B. Him. pl. x. : Jerd. B. Ind. ii. p. 71.

$a,-b$ Nepal (B. H. Hodgson 242) 1845.

\section{Hyposipetes malaccensis.}
Hypsipetes malaccensis, Blyth, J. A. S. B. xix. p. 574.
a Malacca (Fenwick) 1844. -b Java (Brandt) 1845.

\section{7\%. Hypsipetes philippensis.}

\section{Turdus philippensis, Gm. Syst. Nat. i. p. 814 (1788).}

Galgulus philippensis, Kittlitz, Kupf. p. 8, t. 12, f. 2 (1832).

Hypsipetes philippensis, Strickl. Ann. \& Mag. N. H. xiii. p. 413 (1844): Wald. Trans. Z. S. ix. p. 191.

a Manilla, Philippine Islands (H. Cuming) 1839. - -b (Gardner) 1844.

Lord Tweeddale (l. s. c.) has noticed the fact that the same name has been given to this bird by three different authors independently of one another. A marginal note in Strickland's copy of the 'Annals' shews that he recognized the identity of his own and Kittlitz's bird. Specimen $a$ is the type of Strickland's description.

\section{Hypsipetes maclellandi.}

Hypsipetes maclellandi, Horsf. P. Z. S. 1837, p. 159 : Jerd B. Ind. ii. p. 79.

Hypsipetes viridis, Hodgs. Zool. Misc. p. 83.

a Himalaya (N. C. Strickland) 1838. -b India (W. J. E. Boys) 1847.

S. C. 
479. Hypsipetes olivacea.

Hypsipetes ganeesa? Jard. \& Selb. Ill. Orn. iii. pl. 148 (nec Sykes).

Hypsipetes olivacea, Jard. \& Selb. Ill. Orn. Ser. 2 under pl. 1 : Newton, Orn. Misc. ii. p. 51.

a (P. L. Sclater) 1853.

A species peculiar to the island of Mauritius.

Hevixus.

Hemixos, Hodgson, J. A. S. B. xiv. p. 572 (1845).

480. Hemixus fiavala.

Hemixos flavala, Hodgs. l. s. c.: Jerd. B. Ind. ii. p. 80 : Blyth J. A. S. B. xliii. Extr. No. p. 133.

$a$ India (Stevens) 1844. -b, -c Kumaon (W. J. E. Boys) 1847. - d Arakan (E. Blyth) 1847.

$d$ agrees with the Indian specimens and apparently differs from H. hildebrandi, Hume. Blyth's label has been lost.

\section{ALCURUS.}

Alcurus, Hodgson, J. A. S. B. xii. p. 955 (1843).

481. Alcurus striatus.

Trichophorus striatus, Blyth, J. A. S. B. xi. p. 184.

Alcurus striatus, Blyth, J. A. S. B. xii. p. 955 : Jerd. B. Ind. ii. p. 81.

a India ("Alcurus striatus" E. Blyth) 1846.

\section{Criniger.}

Criniger, Temm. Man. d'Orn. i. p. 60 (1820).

482. Criniger barbatus.

Trichophorus barbatas, Temm. Pl. Col. 88.

a Africa (Mansfield) 1838.

483. Criniger ictericus.

Criniger? ictericus, Strickland, Ann. \& Mag. N. H. xiii. p. $411(1844)$. 
Criniger ictericus, Jerd. B. Ind. ii. p. 82.

a (Askew) 1839.

The type of the species as described by Strickland. The bird is found in the forests of Malabar and in Ceylon (Jerdon).

484. Criniger flaveolus. .

Trichophorus flaveolus, Gould, P. Z. S. 1836, p. 6.

Criniger flaveolus, Jerd. B. Ind. ii. p. 83.

Trichophorus xanthogaster, Hodgs. Zool. Misc. p. 83.

a Darjeeling (E. Blyth) 1850.

485. Criniger phæocephalus.

Ixos (Trichixos) phaocephalus, Hartl. Rev. Zool. 1844, p. 401.

Criniger gularis, Strickl. P. Z. S. 1846, p. 101 (nec Horsf.).

a Malacca (Stevens) 1844. -b Malacca (Argent) 1851.

TRACHYCOMUS.

Trachycomus, Cabanis, Mus. Hein. i. p. 109 (1850). 486. Trachycomus ochrocephalus.

Turdus ochrocephalus, Gm. Syst. Nat. i. p. 821.

Criniger ochrocephalus, Blyth, J. A. S. B. xiv. p. 570.

Trachycomus ochrocephalus, Cab. l. s. c.

a Malacca (J.W. Lockwood) 1837.

\section{Phyllostrephus.}

Phyllastrephus, Swainson, Faun. Bor. Am. ii. p. 486 (1831). 48\%. Phyllostrephus caponsis.

Phyllastrephus capensis, Sw. Classif. B. ii. p. 229 : Layard, B. S. Afr. p. 141.

$a$ S. Africa (Weaver) 1843.

\section{ANDROpadus.}

Andropadus, Swainson, Faun. Bor. Am. ii. p. 485 (1831) (as a subgenus).

488. Andropadus importunus.

Turdus importunus, Vieill. N. Dict. d'Hist. N. xx. p. 266. 
Criniger importunus, Layard, B. S. Afr. p.137. Andropadus vociferus, Sw. Classif. B. ii. p. 228. $a$ (Osman) 1846.

A South African species.

\section{Andropadus latirostris.}

Andropadus latirostris, Strickl. P.Z. S. 1844, p. 100 : Fraser, Zool. Typ. pl. 35.

$a$ (Mansfield) 1832.

Originally described from specimens obtained by Fraser at Fernando Po. As Strickland possessed this specimen at the time he characterized the species, and as its label is inscribed "A. latirostris," the specimen may fairly be considered a typical one.

\section{Pycnonotus.}

Pycnonotus, "Kuhl," Boie, Isis, 1826, p. 973."

490. Pycnonotus capensis.

Turdus capensis, Linn. Syst. Nat. i. p. 295.

Pycnonotus capensis, Layard, B. S. Afr. p. 138: Newton, Brit. B. i. p. 247.

a Cape of Good Hope (A. Strickland) 1832.

491. Pycnonotus tricolor.

Ixos tricolor, Hartl. Ibis, 1862, p. 341.

Pycnonotus tricolor, Sharpe, P. Z. S. 1871, p. 131, pl. vii. f. 2.

Pycnonotus capensis (Le Vaill.), Strickl. \& Scl. Contr. Orn. 1852, p. 145.

$a$ (Stevens) 1843. - b Damara-land (C. J. Andersson) 1852.

According to Mr Sharpe the Damara-land bird agrees with that from Angola described as above by Dr Hartlaub.

\section{Pycnonotus barbatus.}

Turdus barbatus, Desf. Mem. Ac. Sc. 1787, p. 500, pl. 13.

Ixos obscurus, Temm. Man. d'Orn. iv. p. 608 (1840).

Pycnonotus barbatus, Dresser. B. Eur.

$a$ (Thomas) 1840.

Found in North and West Africa.

1 Cf. Newton, ed. Yarr. Brit. B. i. p. 247, note. 
493. Pycnonotus arsinoe.

Turdus arsinoe, Licht. Verz. Doubl. p. 39.

Pycnonotus arsinoe, Gray, Gen. B. i. p. 237.

Pycnonotus barbatus, Strickl. P. Z. S. 1850, p. 217 : Contr. Orn. 1851, p. 132, (nec Desf.).

a Kordofan ( $J$. Petherick) 1848.

In his paper on Kordofan birds, and again in his notes on birds from the Gaboon river W. Africa, Strickland treated this species as identical with $P$. barbatus. Their differences seem now to be generally recognized.

494. Pycnonotus bengalensis.

Pycnonotus bengalensis, Blyth, J. A. S. B. xix. p. 566.

Ixos pygceus, Hodgs. Zool. Misc. p. 84 (descr. nulla).

Pycnonotus pygoeus, Jerd. B. Ind. ii. p. 93.

Pycnonotus cafer, Strickl. Ann. \& Mag. N. H. xiii. p. 37 (doubtfully of Linnæus).

a Africa ? (N. C. Strickland) 1838. - b Nepal (B. H. Hodgson 207) 1845 .

\section{Pycnonotus hæmorrhous.}

Red-vented Flycatcher, Brown. Ill. pl. 31, f. 2, undè

Muscicapa homorrhousa, Gm. Syst. Nat. i. p. 941.

Pycnonotus hcemorrhousa, Strickl. Ann. \& Mag. N. H. xiii. p. 37.

Pycnonotus homorrhous. Jerd. B. Ind. ii. p. 94.

Homatornis pusillus, Blyth, J. A. S. B. x. p. 841.

$a$ (Askew). $-b,-c$ Madras (A. Strickland) 1833. - d Madras (Stevens) 1843.

This specific name was based on the Ceylonese and not the Chinese bird, as Blyth, following Cassin, erroneously asserts. (Ibis, 1867, p. 8). ( $C f$. Walden, Ibis, 1866, p. 318 note.)

\section{Pycnonotus jocosus.}

Lanius jocosus, Linn. Syst. Nat. i. p. 138.

Otocompsa jocosa (L.), Jerd. B. Ind. ii. p. 92.

Lanius emeria, Linn. Syst. Nat. i. p. 137.

Musçicapa emeria, Linn. Syst. Nat. i. p. 326. 
Ixus emeria, Wald. J. A. S. B. xliii. extr. No. p. 135. a E. Indies (P. L. Sclater).

The Chinese bird to which Linnæus gave the name $L$. jocosus does not, according to Swinhoe (P. Z. S. 1863, p. 277), differ from the Bengal Bulbul, upon which Linnæus bestowed the name emeria twice over ( $c f$. Wald. l. s. c.). No question of the priority of these names arising, jocosus having hitherto been almost universally used, ought to be retained.

\section{9\%. Pycnonotus fuscicaudatus.}

Otocompsa fuscicaudata, Gould, P. Z. S. 1865, p. 664: Blyth, Ibis, 1867, p. 8.

$a$ E. Indies (N. C. Strickland) 1838.

Found in Southern India, and distinguishable from $P$. bengalensis and $P$. hocmorrhous by the absence of white at the extremity of the tail.

\section{Pycnonotus goiavier.}

Muscicapa goiavier, Scop. Del. Fl. et Faun. Ins. ii. p. 96.

Ixus goiavier, Walden, Trans. Z. S. ix. p. 190.

Muscicapa psidii, Gm. Syst. Nat. i. p. 941.

a Philippine Islands (H. Cuming) 1840.

\section{Pycnonotus leucogenys.}

Brachypus leucogenys, Gray \& Hardw. Ill. Ind. Zool. ii. pl. 35. f. 3.

Ixos leucogenys, Hodgs. Zool. Misc. p. 84.

Otocompsa leucogenys, Jerd. B. Ind. ii. p. 90.

a Nepal (B. H. Hodgson 208) 1845. - b Kumaon (W. J. E. Boys) 1847.

\section{Pycnonotus leucotis.}

Ixos leucotis, Gould, P. Z. S. 1836, p. 6.

Otocompsa leucotis, Jerd. B. Ind. ii. p. 91.

a (W. J. E. Boys) 1847. —b N. India (W. J. E. Boys) 1848.

\section{Pycnonotus crocorrhous.}

Yellow-vented Fly-catcher, Brown, Ill. Zool. pl. 31.f. 1. undè Pycnonotus crocorrhous, Strickl. Ann. \& Mag. N. H. xiii. p. 412.

a Malacca (N. C. Strickland) 1838.

The type of the species as described by Strickland, l. s. c. 
Ixus.

Ixos, Temm. Pl. Col. Livr. 64 (1825).

502. Ixus luteolus.

Hcematornis luteolus, Less. Rev. Zool. 1840, p. 354.

Ixos luteolus, Jerd. B. Ind. ii. p. 84.

Pycnonotus flavirictus, Strickl. Ann. \& Mag. N. H. xiii. p. 413 (1844).

a Arakan (E. Blyth) 1847. - b S. India ("Pycnonotus flavirictus" T. C. Jerdon) 1850 .

Neither of these specimens is Strickland's type of his $P$. flavirictus, which he states that he bought of a dealer as coming from Madras. This was in 1844 or at an anterior date. The species is not included in Blyth's Catalogue of the Birds of Burma (J. A. S. B. xliii, extr. No.).

503. Ixus flavescens.

Pycnonotus flavescens. Blyth, J. A. S. B. xiv. p. 568.

Ixus flavescens, Blyth, J. A. S. B. xliii. extr. No. p. 134. a Arakan (E. Blyth) 1847.

\section{Ixus xantholæmus.}

Pycnonotus xantholaimus, Jerd. J. A. S. B. xiv. p. 568.

Ixos xantholcemus, Jerd. B. Ind. ii. p. 85.

a Madras ("Brachypus xantholaimus" T. C. Jerdon) 1845.

Doubtless one of Jerdon's typical specimens.

\section{Ixus finlaysoni.}

Pycnonotus finlaysoni, Strickl. Ann. \& Mag. N. H. xiii. p. 411 (1844).

Ixus finlaysoni, Blyth, J. A. S. B. xiv. p. 568 : xliii. extr. No. p. 134.

$a$ (Askew) 1840. -b Arakan ("Pycnonotus finlaysoni" $E$. Blyth) 1845 .

$a$ appears to be Strickland's type.

\section{Ixus simplex.}

Pycnonotus simplex, Less. Rev. Zool. 1839, p. 167.

Pycnonotus plumosus, Blyth, J. A. S. B. xiv. p. 567.

a Malacca (Gardner) 1844. 
IOLE.

Iole, Blyth, J. A. S. B. xiii. p. 386 (1844).

50\%. Iole olivacea.

Iole olivacea, Blyth $l$. s. c.

a Malacca (Fenwick) 1844. - b Malacca (Stevens) 1844.

508. Iole viridescens.

Iole virescens, Blyth, J. A. S. B. xiv. p. 573 (nec Ixus virescens, Temm.).

Iole viridescens, Blyth, Ibis, 1867, p. 7 ; J. A. S. B. xliii. extr. No. p. 133.

a India ("Iole virescens" E. Blyth) 1845. -b Malacca (Argent) 1850.

\section{Microtarsus.}

Microtarsus, Eyton, P. Z. S. 1839, p. 102.

509. Microtarsus melanoleucus.

Microtarsus melanoleucus, Eyton, l. s. c.

a Malacca (Stevens) 1843. - b, - c Malacca (W. Kirtland) 1843.

\section{RuBigula.}

Rubigula, Blyth, J. A. S. B. xiv. p. 576 (1845).

510. Rubigula dispar.

Turdus dispar, Horsf. Trans. L. S. xiii. p. 150 : Temm. Pl. Col. 137.

Rubigula dispar, Blyth, l. s. c.

a (Fenton) 1850.

Found in Java, Sumatra, \&c.

511. Rubigula melanictera.

Yellow-breasted Fly-catcher, Brown, Ill. p. 80. pl. 32. f. 1. undè Muscicapa melanictera. Gm. Syst. Nat. i. p. 941.

Rubigula melanictera, Walden, Ibis, 1866, p. 316. $a$ Ceylon (E. Blyth) 1850.

512. Rubigula flaviventris.

Vanga flaviventris, Tickell. J. A. S. B. ii. p. 573. 
Rubigula flaviventris, Jerd. B. Ind. ii. p. 88.

Alcurus melanocephalus, Hodgs. Zool. Misc. p. 83.

a Nepal (B. H. Hodgson 245-"Pycnonotus melanocephalus (Gray), Brachypus plumifera, Gould " E. Blyth) 1845.

\section{BRAChYPodius.}

Brachypodius, Blyth, J. A. S. B. xiv. p. 576 (1845).

\section{Brachypodius melanocephalus.}

Lanius melanocephalus, Gm. Syst. Nat. i. p. 309.

Brachypodius melanocephalus, Blyth, l. s. c.

Pycnonotus melanocephalus, Strickl. P. Z. S. 1846, p. 101.

a Malacca (W. Kirtland) 1843. - b Batavia (Argent).

\section{IXODIA.}

Ixodia, Blyth, J. A. S. B. xiv. p. 577 (1845).

\section{Ixodia cyaniventris.}

Pycnonotus? cyaniventris, Blyth, J. A. S. B. xi. p. 792.

Ixodia cyaniventris, Blyth, l. s. c.

a Malacca (Thomas) 1842.

Noticed by Strickland in his paper on Malacca birds (P. Z. S. 1846, p. 101) as the smallest species of Pycnonotus with which he was acquainted.

\section{SUB-FAMILY.-PHYLLORNITHIN $\nexists$.}

\section{Phyllornis. ${ }^{1}$}

Phyllornis, "Boie”, Temm. Pl. Col. Livr. 81 (1829).

515. Phyllornis jerdoni.

Phyllornis jerdoni, Blyth, J. A. S. B. xii. p. 392 : Jerd. B Ind. ii. p. 97.

Chloropsis cochinsinensis, Jerd. Madr. Journ. x. p. 247.

I Placed by Strickland (Ann. \& Mag. N. H. xiv. p. 116)in the "Tenuirostres" (Meliphagida), but by Jerdon and Blyth with the Bulbuls (Pycnonotida). A reference to the 'Isis' (without date or page) is usually given as the work in which this reputed genus of Boie's was founded. I can find no earlier mention of it than $1829(l . s . c$.$) , and it becomes a question whether Chloropsis, (Jardine and$ Selby, is not an older title. 
$a, \hat{\delta}-b q \mathrm{~S}$. India ("Chloropsis cochinsinensis of my Catal." T. C. Jerdon) 1844. -c o India? (Bt. at Stevens's) 1844. -d India (E. Blyth).

\section{Phyllornis chlorocephalus.}

Phyllornis chlorocephalus, Wald. Ann. \& Mag. N. H. ser. 4. vii. p. 241 : Blyth, J. A. S. B. xliii. extr. No. p. 137.

a India (E. Blyth) 1846. - b Arakan ("Phyllornis cochinsinensis (near)" E. Blyth) 1846.

Blyth's label to specimen $a$ has been lost. The skin is that of a male, and probably was obtained by Blyth at the same time as $b$ (a female) in Arakan.

51\%. Phyllornis icterocephalus.

Phyllornis icterocephala, Lesson, Rev. Zool. 1840, p. 164: Tweeddale, Ibis, 1877, p. 305.

Phyllornis malabaricus, Temm. Pl. Col. 512. f. 2. (nec Gm.). Phyllornis moluccensis, Gray, Strickl. P. Z. S. 1846, p. 100.

a Malacca (Johnson) 1837. - b Malacca (Mather) 1840.

\section{Phyllornis aurifrons.}

Phyllornis aurifrons, Temm. Pl. Col. 484. f. 1: Strickl. P. Z. S. 1846. p. 100 : Jerd. B. Ind. ii. p. 99.

Chloropsis aurifrons. Hodgs. Zool. Misc. p. 82.

a (Bt. at Stevens's) 1844. - b Nepal (B. H. Hodgson 253) 1845 .

\section{Phyllornis hardwickii.}

Chloropsis hardwickii, Jard. \& Selb. Ill. Orn. ii. Addenda, p. 1.

Phyllornis hardwickii, Jerd. B. Ind. ii. p. 100 : Gould, B. Asia, pt. xiii.

Chloropsis cyanopterus, Hodgs. Zool. Misc. p. 82.

$a,-b,-c$ Nepal (B. H. Hodgson 250, 251) 1845. —d India ("Phyllornis hardwickii, fem." E. Blyth) 1846.

\section{Phyllornis malabaricus.}

Turdus malabaricus, Gm. Syst. Nat. i. p. 837.

Phyllornis malabaricus, Strickl. P. Z. S. 1846, p. 160 : Gould, B. Asia, pt. xiii.: Jerd. B. Ind. ii. p. 28.

a Madras ("Chloropsis malabaricus" T. C. Jerdon) 1845. 


\section{Phyllornis viridis.}

Turdus viridis, Horsf. Trans. L. S. xiii. p. 148.

Phyllornis viridis, Tweeddale, Ibis, 1877, p. 305.

Phyllornis sonnerati, Jard. \& Selb. Ill. Orn. pl. 100.

a Malacca (Capt. Nash) 1837.

\section{EGTTHINA.}

AEgithina, Vieillot, Anal. p. $44(1816)^{1}$.

522. Itgithina tiphia。

Motacilla tiphia, Linn. Syst. Nat. i. p. 331

Iora typhia, Jerd. B. Ind. ii. p. 103.

Sylvia leucoptera, Vieill. Ois. Am. Sept. ii. pl. 84.

a Malacca (Johnson) 1837. - b India " Iora typhic" E. Blyth) 1846. - c India ("Iora zeylonica" E. Blyth) 1846. -d India (W. J. E. Boys) 1847. -e India (W. J. E. Boys) 1848. -f; -g India (E. Blyth) 1851. - $h$ f Java.

Of specimens $f$ and $g$ Blyth writes "Iora. Although I never saw the zeylonica plumage here at any season, yet indications in this state are sometimes seen of it." Strickland (Ann \& Mag. N. H. vi. p. 422) considered 2 . scapularis (Horsf. Trans. L. S. xiii. p. 152) to be the same as $\mathcal{E}$. tiphic (L.), but subsequently (Ann. \& Mag. N. H. xiii. p. 205) he states that Dr Horsfield informed him that blackheaded specimens of this bird never occurred in Java. In 1842 (Ann. \& Mag. N. H. viii. p. 372 ) he united $R$. scapularis and $L$. zeylonica to $\mathbb{E}$. typhia, a view which may still prove correct.

\section{Ifgithina zeyIonica.}

Ceylon Black-cap, Brown, Ill. Zool. pl. 15 f. 2, undè Motacilla zeylonica, Gm. Syst. Nat. i. p. 964. Iura zeylonica, Jerd. B. Ind. ii. p. 101.

1 This generic name was based by Vieillot on his Sylvia leucoptera, which, judging from the figure, is identical with Motacilla tiphia, Linn., the locality N. America being erroneous. Accordingly Egithina, Vieill. must supplant Iora, Horsf., a view taken by Gray and Lord Tweeddale. In a note to Blyth's 'Further Observations on the Ornithology of Calcutta' (Ann. \& Mag. N. H. xiv. p. 42) Strickland says that he is disposed to place Iora (= Egithina) with the Orioline, and gives his reasons for this arrangement. 
a Malacca (Johnson) 1837. - b Ceylon (Bt. at Stevens's) 1843. —c, - d Madras ("Iora zeylonica" T. C. Jerdon) 1845, - e Java.

$a$ is a female with a single wing-bar. $e$ is a male in the full "zeylonica" plumage, and only differs from the Ceylonese bird in having two white bars on each wing. The correctness of the locality assigned to the later specimen is open to question.

\section{4. झ̈githina viridissima.}

Iora viridissima, Bp. Consp. i. p. 397.

Egithina viridissima, Tweeddale, Ibis, 1877 p. 304, pl. 5. a Malacca (N. C. Strickland) 1838.

\section{SUBFAMILY.-IRENIN $巴$.}

\section{IRENA.}

Irena, Horsfield, Trans. Linn. Soc. xiii. p. 153 (1820). 525. Irena puella.

Coracias puella, Lath. Ind. Orn. i. p. 171.

Irena puella, Jerd. B. Ind. ii. p. 105 : Blyth, J. A. S. B. xliii. extr. No. p. 138 : Sharpe. Cat. B. Brit. Mus. iii. p. 268.

$a$ India (E. Blyth) 1846. -b Arakan ("Irena puella" E. Blyth) 1846. — S. India ("Irena puella" T. C. Jerdon) 1850 .

\section{Irena cyanea.}

Muscicapa cyanea, P. J. Begbie, Ann. \& Mag. N. H. xvii. p. 407.

Irena puella, Strickl. Ann. \& Mag. N. H. xvii. p. 407, note (nec Lath.).

Irena malayensis, Horsf. \& Moore, Cat. B. i. p. 274.

a Malacca (N. C. Strickland) 1838. - b Malacca (W. Kirtland) 1843. 
FAMILY.-ORIOLIDAE.

\section{ORIOLUS.}

Oriolus, Linnæus, Syst. Nat. i. p. 160 (partim). 52\%. Oriolus galbula.

Oriolus galbula, Linn. l. s. c.: Strickl. P. Z. S. 1836, p. 98 ; 1850, p. 217 : Newton, ed. Yarr. Brit. B. i. p. 233: Sharpe, Cat. B. Brit. Mus. iii. p. 191.

a France (Bt. in Paris) 1830. -b §o Smyrna (H. E. Strickland) April 1834. -c (Askew) 1837. -d Kartoum Oct. 1848, (J. Petherick) 1848.

\section{Oriolus kundoo.}

Oriolus kundoo, Sykes, P. Z. S. 1832, p. 87 : Jerd. B. Ind. ii. p. 107 : Sharpe, Cat. B. Brit. Mus. iii. p. 194.

Oriolus aureus, Jerd. Madr. Journ. x. p. 261.

$a$ (Askew) 1833. - b Himalaya (N.C. Strickland) 1838. -c Madras (T. C. Jerdon) 1845. -d Rohilcund (W. J. E. Boys) 1847.

In his notes on Blyth's list of birds from the vicinity of Calcutta (Ann. \& Mag. N. H. xiii. p. 37) Strickland pointed out that Jerdon's name $O$. aureus must give place to Sykes's older title.

529. Oriolus auratus.

Oriolus auratus, Vieill. N. Dict. d'Hist. N. xviii. p. 194; Gal. des Ois. i. t. 83 : Sharpe, Cat. B. Brit. Mus. iii. p. 195.

$a$ Senegal (N. C. Strickland) 1838. -b Senegal (Isaacson) 1840 .

\section{Oriolus maculatus.}

Oriolus maculatus, Vieill. N. Dict. d'Hist. N. xviii. p. 194: Sharpe, Cat. B. Brit. Mus. iii. p. 199.

a Batavia, Java (Argent) 1851.

\section{Oriolus acrorhynchus.}

Oriolus acrorhynchus, Vig. P. Z. S. 1830-1, p. 97.

Broderipus acrorhynchus, Bp., Wald. Trans. Z. S. ix. p. 185. 
Oriolus chinensis, Sharpe, Cat. B. Brit. Mus. iii. p. 203.

$a$ South of the island of Manilla (H. Cuming) June 1839. -b Philippine Islands (J. Gould) 1846.

532. Oriolus chinensis.

Oriolus chinensis, Linn. Syst. Nat. i. p. 160: Strickl. P. Z. S. 1842, pp. 166, 168: Swinhoe, P. Z. S. 1863, p. 282.

a Malacca (Mather) 1840.

533. Oriolus sp?

$a$ (Askew) 1837.

A young bird which I have not been able to determine satisfactorily.

\section{Oriolus xanthonotus.}

Oriolus xanthonotus, Horsf. Trans. L. S. xiii. p. 152; Zool. Res. pl. 12 : Sharpe, Cat. B. Brit. Mus. iii. p. 213.

a Malacca (Askew). -b Malacca (Thomas) 1840.

\section{Oriolus melanocephalus.}

Oriolus melanocephalus, Linn. Syst. Nat. i. p. 160: Strickl. Ann. \& Mag. N. H. xiii. p. 37 : Jerd. B. Ind. ii. p. 110 : Sharpe, Cat. B. Brit. Mus. iii. p. 215.

a Madras ("Oriolus melanocephalus" T. C. Jerdon) 1845. 6 Mhow (W.J. E. Boys) 1847.

\section{Oriolus ceylonensis.}

Oriolus ceylonensis, Bp. Consp. i. p. 347: Jerd. B. Ind. ii. p. 111 : Holdsw. P. Z. S. 1872, p. 453.

$a,-b$ Ceylon (Capt. Pearson) 1840. $-c$ S. India (Argent).

53\%. Oriolus larvatus.

Oriolus larvatus, Licht. Verz. Doubl. p. 20 : Sharpe, Cat. B. Brit. Mus. iii. p. 217.

Oriolus capensis, Sw. Class. B. ii. p. 237.

$a,-b$ Cape of Good Hope (N. C. Strickland) 1838.

\section{Oriolus trailli.}

Pastor traillii, Vig. P. Z. S. 1830-1, p. 175 : Gould, Cent. B. Him. pl. 35. 
Oriolus traillii, Gray, Cat. Hodgs. Coll. (1846) p. 87: Jerd. B. Ind. ii. p. 112 : Sharpe, Cat. B. Brit. Mus. ii. p. 222.

a Nepal (B. H. Hodgson 261) 1845.

539. Oriolus viridis.

Gracula viridis, Lath. Ind. Orn. Suppl. p. xxviii.

Oriolus viridis, Gould, B. Austr. iv. pl. 13 : Sharpe, Cat. B. Brit. Mus. iii. p. 212.

Mimeta viridis, Vig. \& Horsf.'Trans. L. S. xv. p. 326.

a New Holland (N. C. Strickland) 1838. - b Australia (Askew) 1841. - c Australia (Arthur Strickland) 1850.

\section{SPHECOTHERES.}

Sphecotheres, Vieillot, Anal. p. 42 (1816).

\section{Sphecotheres maxillaris.}

Turdus maxillaris, Lath. Ind. Orn. Suppl. p. xliii.

Sphecotheres maxillaris, Gray, Sharpe, Cat. B. Brit. Mus. iii. p. 224.

"Sphecotheres australis,"Sw. (ubi ?) Gould, B. Austr. iv. pl. 15. Sphecotera viridis, Vieill. Anal. p. 68.

a Australia (Weaver) 1843. - b Australia (Bt. at Birmingham) 1843.

\section{FAMILY.-VIREONID $\mathbb{E}$.}

\section{VIREOSXLVIA。}

Vireosylvia, Bonaparte, Comp. List, p. 26 (1838).

541. Vireosylvia olivacea.

Muscicapa olivacea, Linn. Syst. Nat. i. p. 327.

Vireosylvia olivacea, Bp., Baird, Brew. \& Ridgw. N. Am. B. i. p. 363.

Lanius agilis, Licht. Verz. Doubl. p. 49.

Vireosylvia agilis, Scl. Am. B. p. 43. 
a Brazil (N. C. Strickland) 1834. - b, -c (N. C. Strickland) 1838. - $d$ Brazil (N. C. Strickland) 1838. - e N. America (J. G. Kinberg) 1845. - $f$ ई Carlisle, Pennsylvania 4 May, 1846 (S. F. Baird) 1847. - g Trinidad (Argent) 1851.

\section{Vireosylvia flaviviridis.}

Vireosylvia flavoviridis, Cassin, Pr. Ac. Phil. v. p. 152 ; vi. pl. 2: Scl. \& Salv. Ibis, 1859, p. 12: Scl. Cat. Am. B. p. 44. a Guatemala (J. Constancia) 1851.

\section{Vireosylvia gilva.}

Muscicapa gilva, Vieill. Ois. Am. Sept. i. p. 65. pl. 34.

Vireosylvia gilva, Scl. P. Z. S. 1856, p. 298: Baird, Brew. \& Ridgw. N. Am. B. i. p. 368.

$a \hat{\delta}$ S. Pedro, Mexico, Dec. 1844 (Galeotti) 1845. - $b \hat{\delta}$ Carlisle, Pennsylvania 1 May 1847 (S. F. Baird) 1847.

\section{Vireosylvia josephæ.}

Vireo josepho, Scl. P. Z. S. 1859, p. 137, pl. 154.

Vireosylvia josepha, Scl. \& Salv. Nomencl. Av. Neotr. p. 12. a Bogota (Bt. at Stevens's) 1845.

\section{Vireosylvia flavifrons.}

Vireo flavifrons, Vieill. Ois. Am. Sept. i. p. 85. pl. 54.

Vireosylvia flavifrons, Scl. \& Salv. Nomencl. Av. Neotr. p. 12.

Lanivireo flarifrons, Baird, Brew. \& Ridgw. N. Am. B. i. p. 379. 18.38.

a (Stuchbury) 1836. - b N. America (N. C. Strickland)

\section{Vireosylvia solitaria.}

Muscicapa solitaria, Wils. Am. Orn. ii. p. 143. pl. 17, f. 6. Lanivireo solitarius, Baird, Brew. \& Ridgw. i. p. 373.

Vireosylvia solitaria, Scl. \& Salv. Nomencl. Av. Neotr. p. 12. $a$ N. America (Brandt) 1845. -b Mexico (Galeotti) 1845. -c f Carlisle, Pennsylvania 6 May, 1845 (S. F. Baird) 1847. 


\section{Vireo.}

Vireo, Vieillot, Ois. Am. Sept. i. p. 83 (1807).

\section{Vireo noveboracensis.}

Muscicapa noveboracensis, Gm. Syst. Nat. i. p. 947.

Vireo noveboracensis, Bp., Baird, Brew. \& Ridgw. N. Am. B. i. p. 385 .

a New York (Bt. at Dresden) 1845.

\section{Vireo belli.}

Vireo belli, Aud. B. Am. vii. p. 333, pl. 485: Baird, Brew. \& Ridgw. N. Am. B. i. p. 389.

a Mexico (Galeotti) 1845.

\section{Hylophilus.}

Hylophilus, Temm. Pl. Col. Livr. 29 (1823).

\section{Hylophilus pœcilotis.}

Hylophilus poecilotis. Temm. Pl. Col. 173. f. 2: Scl. Cat. Am. B. p. 44 .

a Brazil (N. C. Strickland) 1838.

550. Hylophilus amaurocephalus.

Sylvia poecilotis Max. Beitr. iii. p. 715 (nec Temm.).

Sylvia amaurocephala, Nordm. Erm. Reise um d. Erde. Vög. p. 14.

$a$ (Bt. at Liverpool) 1853.

Agrees with Bahia specimens of this species.

551. Hylophilus semibrunneus.

Hylophilus semibrunneus, Lafr. Rev. Zool. 1845, p. 341. a Bogota (Bt. at Stevens's) 184.5.

552. Hylophilus muscicapinus.

Hylophilus muscicapinus, Scl. \& Salv. Nomencl. Av. Neotr. p. 156.

$a$ (Williams) 1846.

This is evidently a Cayenne skin, the country where alone the species has as yet been found.

S. C. 


\section{Hylophilus hypoxanthus.}

Hylophilus hypoxanthus, Pelz. Orn. Bras. pp. 71, 136. a (Argent) 1851.

Originally described from specimens obtained by Natterer on the Rio Içanna and Rio Vaupé.

\section{Hylophilus thoracicus.}

Hylophilus thoracicus, Temm. Pl. Col. 173. f. 1: Scl. Cat. Am. B. p. 44.

a Brazil (N. C. Strickland) 1838.

\section{CYCLORHIS.}

Cyclarhis, Swainson, Zool. Journ. i. p. 294 (1824).

Cyclortis, Id. op. cit. iii. p. 162 (1827).

\section{Cyclorhis flavipectus.}

Cyclorhis flavipectus, Scl. P. Z. S. 1858, p. 448; Cat. Am. B. p. 45.

a. (Bt. at Stevens's) 1846.

A species found in the north-western portions of South America and in Central America as far north as Costa Rica.

556. Cyclorhis flaviventris.

Cyclorhis flaviventris, Lafr. Rev. Zool. 1842, p. 133 : Scl. \& Salv. Ibis, 1859, p. 13: Scl. Cat. Am. B. p. 45.

$a$ (Gardner) 1845. -b Guatemala (J. Constancia) 1848.

55\%. Cyclorhis ochrocephala.

Cyclorhis ochrocephala, Tsch. Arch. f. Naturg. 1845, i. p. 362: Scl. Cat. Am. B. p. 45.

a Brazil (Bt. at Aberystwith) 1833.

558. Cyclorhis albiventris.

Cyclorhis albiventris, Scl. \& Salv. Nomencl. Av. Neotrop. pp. 13, 156.

a Brazil (Johnson) 1837.

Agrees with Bahia specimens. 
559. Cyclorhis nigrirostris.

Cyclorhis nigrirostris, Lafr. Rev. Zool. 1842, p. 133: Scl. Cat. Am. B. p. 46.

a Bogota (Gardner) 1845 .

\section{FAMILY.-DICRURIDAE.}

Dicrurus.

Dicrurus, Vieillot, Anal. i. p. 41 (1816).

560. Dicrurus balicassius.

Corvus balicassius, Linn. Syst. Nat. i. p. 157.

Dicrurus balicassius, Wald. Trans. Z. S. ix. p. 180. pl. 31, f. 1 : Sharpe, Cat. B. Brit. Mus. iii. p. 230.

Edolius viridescens, Gould, P. Z. S. 1836, p. 6.

a Philippine Islands 13 May 1840 (H. Cuming) 1840.

561. Dicrurus annectens.

Buchanga annectens, Hodgs. Ind. Rev. i. p. 326.

Dicrurus annectens, Sharpe, Cat. B. Brit. Mus. iii. p. 231.

Dicrurus balicassius, Jerd. B. Ind. i. p. 430 (nec Linn.).

a Malacca (Capt. Nash) 1837. - Malacca (Gardner) 1845.

Chibia.

Chibia, Hodgson, Ind. Rev. i. p. 324 (1837).

562. Chibia hottentotta.

Corvus hottentottus, Linn. Syst. Nat. i. p. 155.

Chibia hottentotta, Strickl. Ann. \& Mag. N. H. xiii. p. 36 : Jerd. B. Ind. i. p. 439.

a India (Havell) 1839. - b Nepal (B. H. Hodgson 564) 1845.

Chaptia.

Chaptia, Hodgson, Ind. Rev. i. p 326 (1837).

563. Chaptia ænea.

Dicrurus ceneus, Vieill. N. Dict. d'Hist. ix. p. 586.

Chaptia cenea, Jerd. B. Ind. i. p. 433 : Sharpe, Cat. B. Brit. Mus. iii. p. 243.

$a$ (Gardner) 1846.

A species found throughout India and in Assam and Burma. 
564. Chaptia malayensis.

Chaptia malayensis, Blyth, J. A. S. B. xv. p. 294 : Sharpe, Cat. B. Brit. Mus. iii. p. 244.

a Malacca (Capt. Nash) 1837.

\section{Buchanga.}

Buchanga, Hodgson, Ind. Rev. i. p. 326 (1837).

\section{Buchanga atra.}

Muscicapa atra, Herm. Obs. Zool. p. 208.

Buchanga atra, Sharpe, Cat. B. Brit. Mus. iii. p. 246.

Dicrurus macrocercus, Vieill. N. Dict. d'Hist. N. ix. p. 588: Jerd. B. Ind. i. p. 427.

Buchanga albirictus, Hodgs. Ind. Rev. i. p. 326.

$a,-b$ (N. C. Strickland) 1838. - c India (Bt. at Stevens's) 1843. - $d$ S. India ("Dicrurus balacassius of my Catal., Sykes \&c." T. C. Jerdon) 1844. - e Nepal (B. H. Hodgson 551) 1845. -f, -g Madras (T. C. Jerdon) 1845. - h (Argent) 1852.

$b, c, d, e$, all have a few white feathers at the gape upon which character Hodgson founded his $B$. albirictus.

\section{Buchanga longicaudata.}

Dicrurus longicaudatus, A. Hay, Madr. Journ. xiii. pt. 2, p. 121 : Jerd. B. Ind. i. p. 430.

Buchanga longicaudata, Wald.: Sharpe, Cat. B. Brit. Mus. iii. p. 249.

$a$ Malacca (Askew) 1834. - b Madras ("Dicrurus longicaudatus" T. C. Jerdon) 1845. — c India ("Dicrurus pyrrhops, Hodgs." E. Blyth). $-d$ N. India (W. J. E. Boys) 1848.

\section{Buchanga leucogenys.}

Buchanga leucogenys, Wald. Ann. \& Mag. N. H. (4) v. p. 219 : Sharpe, Cat. B. Brit. Mus. iii. p. 251.

$a$ Malacca (Williams) 1848. 
568. Buchanga cærulescens.

Lanius carulescens, Linn. Syst. Nat. i. p. 134.

Dicrurus ccerulescens, Jerd. B. Ind. i. p. 432.

Buchanga carulescens, Sharpe, Cat. B. Brit. Mus. iii. p. 252.

$a$ S. India ("Dicrurus corrulescens" T. C. Jerdon) 1844. -b f Jucknie 16 Nov. 1847 (W. J. E. Boys) 1847. — India ("Dicrurus ccerulescens" E. Blyth).

569. Buchanga assimilis.

Corvus adsimilis, Bechst. Lath. Allg. Ueb. d. Vög. ii. p. 362 (1794); Kurze Ueb. p. 117 (1811).

Dicrurus adsimilis, Wald. Trans. Z. S. ix. p. 181.

Buchanga assimilis, Sharpe, Cat. B. Brit. Mus. iii. p. 247 (partim).

Dicrurus musicus, Vieill. et auctt. plurr.

$a$ (Askew) 1834. - b (Bt. at Stevens's) 1843. -c Africa (Brandt) 1845. —d S. Africa (Gardner) 1846. -e S. Africa (Dewgard) 1849.

570. Buchanga divaricata.

Muscicapa divaricata, Licht. Verz. Doubl. p. 52.

Dicrurus divaricatus, Strickl. P. Z. S. 1850, p. 217 : Strickl. $\&$ Scl. Contr. Orn. 1852, p. 144.

$a$ Kordofan 30 April, 1848 (J. Petherick) 1848. - $b$ Damaraland (C.J. Andersson) 1852.

\section{BHRINGA.}

Bhringa, Hodgson, Ind. Rev. i. p. 325 (1837).

\section{Bhringa remifer.}

Edolius remifer, Temm. Pl. Col. pl. 178.

Bhringa remifer, Jerd. B. Ind. i. p. 434.

Bhringa tectirostris, Hodgs. l. s. c.

Dicrurus remifer, Strickl. Ann. \& Mag. N. H. xiii. p. 36.

a Nepal (B. H. Hodgson 554) 1845.

\section{Dissemurus.}

Dissemurus, Gloger, Handb. p. 347 (1842). 


\section{Dissemurus grandis ?'.}

Edolius grandis, Gould, P. Z. S. 1836, p. 5.

Dicrurus grandis, Strickl. Ann. \& Mag. N. H. xiii. p. 36.

Edolius paradiseus (L.), Jerd. B. Ind. i. p. 435.

a Eastern Ghats ("Edolius dentirostris, Hay" T. C. Jerdon) 1845. - - India ("Edolius bengalensis" E. Blyth) 1846.

These specimens have a well-developed crest, but it is not so large in either as in some examples of $D$. grandis I have seen, nor is the bill so long. They probably belong to the bird described as D. orissce, (A. Hay, Madr. Journ. x. p. 121) should that race meet with ultimate recognition as distinct from $D$. grandis.

\section{Dissemurus malabaricus.}

Muscicapa malabarica, Scop. Del. Faun. et Fl. Ins. ii. p. 96. Edolius malabaricus, Jerd. B. Ind. i. p. 437.

Dicrurus malabaricus, Strickl. Ann. \& Mag. N. H. xiii. p. 36. a S. India ("Edolius malabaricus" T. C. Jerdon) 1850.

\section{Dissemurus formosus.}

Edolius malabaricus, (Lath.) Horsf. Trans. L. S. xiii. p. 145 (nec Lath.).

Dissemurus formosus, Cab. Mus. Hein. i. p. 111 : Tweedd. Ibis, 1878, p. 81.

a Malabar (N. C. Strickland) 1838.

This specimen agrees closely with one of Dr Horsfield's examples in the University Museum. It bears a ticket inscribed "Lanius malabaricus, Lath. Malabar Shrike. Irides red." The crest is less developed than in Jerdon's specimen of D. malabaricus. The locality "Malabar" marked upon the ticket is probably conjecture.

\section{Dissemurus platyurus.}

Dicrurus platurus. Vieill. N. Dict. d'Hist. N. ix. p. 558.

Dissemurus platurus, Tweedd. Ibis, 1877, p. 313.

Edolius rangoonensis, Gould, P. Z. S. 1836, p. 5 : Jard. \& Selb. Ill. Orn. n. s. pl. 38.

1 The materials available in the Strickland Collection are not sufficient for me to add anything towards the elucidation of the question whether there be one or more species of this form. At least a dozen names have been proposed for various races, all of which $\mathrm{Mr}$ Sharpe places as synonymous with $D$. paradiseus (L.). 
a Malacca (Capt. Nash) 1837. - b Malacca (Bt. at Birmingham) 1843. - C India ("Edolius rangoonensis, Gould" E. Blyth) 1846.

None of these specimens are in good condition, but so far as I can see belong to the species or race to which the above name seems applicable.

\section{FAMILY.-CAMPEPHAGIDE.}

\section{Graucalus.}

Graucalus, Cuvier, Règn. Anim. i. p. 341. (1817).

\section{Graucalus melanops.}

Corvus melanops, Lath. Ind. Orn. Suppl. p. xxiv.

Graucalus melanops, Gould, B. Austr. ii. pl. 55: Strickl. Ann. \& Mag. N. H. viii. p. 338 : Hartl. J. f. Orn. 1864, p. 437.

a Australia (Askew) 1833. - $b$ New South Wales ( $N . C$ 。 Strickland) 1838.

\section{5\%\%. Gravcalus macæi.}

Grancalus macei, Less. Traité d'Orn. p. 349: Hartl. J. f。 Orn. 1864, p. 439 : Jerd. B. Ind. i. p. 417.

a Africa (!) (Askew) 1833. - b India (Lord A. Hay) 1845. -c, -d Madras ("Graucalus macei Less" T. C. Jerdon) 1845. -e India ("Graucalus papuensis male" E. Blyth) 1846. - $f$ India ("Graucalus papuensis fem." E. Blyth) 1846. -g (Bt. at Birmingham) 1849 .

\section{Graucalus striatus.}

Choucas de la Nouvelle Guinée, D'Aub. Pl. Enl. 629 undè

Corvus striatus, Bodd. Tabl. Pl. Enl. p. 38.

Graucalus striatus, Wald. Trans. Z. S. ix. p. 175.

$a$ Calaguan, Philippine Islands 30 May 1837 (H. Cuming) 1837. -b Philippine Islands 13 May 1840 (H. Cuming) 1840. -c Malacca (Thomas) 1843. $\_d$ Philippine Islands (J. Gould) 1846.

The Malaccan skin $(c)$ is that of a female and differs in no way from the Philippine Island specimens of the same sex. 


\section{Graucalus javensis.}

Ceblepyris javensis, Horsf. Trans. L. S. xiii. p. 145.

Graucalus javensis, Hartl. J. f. Orn. 1864, p. 441.

a Malacca (Arthur Strickland) 1840.

The Malaccan origin of this specimen is doubtful.

\section{Grancalus sp.?}

$a$ (Arthur Strickland) 1840.

Strickland identified this specimen with Lanius robustus, (Lath. Ind. Orn. Suppl. p. xviii.) and it agrees fairly with Latham's description, except as regards dimensions, (the specimen being eleven instead of twenty-one inches long), and as regards the black band on the tail said to exist in $L$. robustus, but which this skin does not possess. Gould (Ann. \& Mag. N. H. xi. p. 235) considers L. robustus, Lath. to be one of the numerous forms of Graucalus mentalis, and he may be right. The present bird seems to me to be more nearly allied to $G$. atriceps, Müll. \& Schl, from which, however, it differs in having the tips of the lateral rectrices white.

\section{Graucalus mentalis.}

Graucalus mentalis, Vig. \& Horsf. Trans. L. S. xv. p. 217 : Gould, B. Austr. ii. pl. 56: Hartl. J. f. Orn. 1864, p. 439.

$a$ (Gardner) 1844.

An Australian species.

\section{Graucalus lineatus.}

Ceblepyris lineatus, Sw. Zool. Journ. i. p. 466.

Graucalus swainsoni, Gould, B. Austr. ii. pl. 58: Hartl. J. f. Orn. 1864, p. 445 .

a Australia (Stevens) 1843.

\section{Graucalus cæsius.}

Ceblepyris coesia, Licht. Verz. Doubl. p. 51.

Campephaga ccesia, Hartl. J. f. Orn. 1865, p. 157.

Campephaga levaillanti, Temm.: Layard, B. S. Afr. p. 153.

$a$ Cape of Good Hope (N. C. Strickland) 1838. - b S. Africa (Van der Kemp) 1843.

584. Graucalus pectoralis.

Graucalus pectoralis, Jard. \& Selb. Ill. Orn. ii. pl. 57. 
Ceblepyris pectoralis, Sw. B. W. Afr. i. p. 249.

Campephaga pectoralis, Hartl. J. f. Orn. 1865, p. 158. $a$ (W. Kirtland) 1838.

A species found in West Africa and in Abyssinia.

\section{VoLVOCIVORA.}

Volvocivora, Hodgson, Ind. Rev. i. p. 328 (1837).

\section{Volvocivora melanoschistus.}

Volvocivora melaschistos, Hodgs. l. s. c.: Jerd. B. Ind. i. p. 415.

Volvocivora lugubris (Sundev.) : Hartl. J. f. Orn. 1865, p. 161.

$a,-b$ India (Havell) 1839. — $\quad$ (Askew) 1840. —d India (Gardner) -e Nepal (B. H. Hodgson 517) 1845. - $f$ India ("Campephaga fimbriata, melaschistos Hodgs." E. Blyth) 1846. - g India (E. Blyth).

Strickland (Ann. \& Mag. N, H. xviii. p. 109), considered this species to be in all probability identical with Ceblepyris fimbriata, Temm.

\section{Volvocivora sykesi.}

Lalage sykesi, Strickl. Ann. \& Mag. N. H. xiii. p. 36 ; xiv. p. 44.

Volvocivora sykesi, Hartl. J. f. Orn. 1865, p. 163; Jerd. B. Ind. i. p. 414.

$a,-b$ (Arthur Strickland) 1840. - $c$ S. India ("Ceblepyris fimbriatus male, Lalage sykesi, Strickl." T. C. Jerdon) 1844. -d India ("Campephaga sykesi," E. Blyth) 1846.

\section{LALAGE。}

Lalage, Boie, Isis, 1826, p. 972.

\section{Lalage terat.}

Merle des Indes orientales, D'Aub. Pl. Enl. 273. f. 2 undè

Turdus terat, Bodd. Tabl. Pl. Enl. p. 17.

Lalage terat, Salvad. Ann. Mus. Genov. v. p. 145.

Turdus orientalis, Gm. Syst. Nat. i. p. 821 ex D'Aub.

Lalage orientalis, Hartl. J. f. Orn. 1865, p. 166. 1848.

a Malacca (N. C. Strickland) 1838. -b Malacca (Williams) 
Cassin (Pr. Ac. Phil. 1864, p. 251) refers this species to Turdus dominicus (P. L. S. Müller, Natursyst. Suppl. p. 145) and calls the bird Lalage dominica. But Müller, in giving a description perhaps applicable to the Lalage, bestowed upon it a name latinized from the French title of a very different species, figured on the same plate as the Lalage.

\section{Lalage tricolor.}

Ceblepyris tricolor, Sw. Zool. Journ. i. p. 467.

Ceblepyris humeralis, Gould, P. Z. S. 1837, p. 143.

Campephaga humeralis, Gould, B. Austr. ii. pl. 63.

Lalage humeralis, Hartl. J. f. Orn. 1865, p. 164.

a New South Wales (N. C. Strickland) 1838 .

\section{SYMMORPHUS.}

Symmorphus, Gould, P. Z. S. 1837, p. 145.

589. Symmorphus leucopygius.

Symmorphus leucopygius, Gould, l. s. c.: Hartl. J. f. Orn. 1865, p. 169.

a Norfolk Island (Stevens) 1846.

\section{Campephaga.}

Campephaga, Vieillot, Anal. p. 39, (1816).

590. Campephaga phoonicea.

Ampelis phoenicea, Lath. Ind. Orn. p. 367.

Campephaga phonicea, Sw. Orn. W. Afr. i. p. 252, pls. $27,28$.

Lanicterus phoniceus, Hartl. J. f. Orn. 1865, p. 172.

$a$ W. Africa (Askew). - b, $-c$ W. Africa (N. C. Strickland) 1838. - $d$ Senegal (N. C. Strickland) 1838. -e W. Africa (G. Hartlaub) 1845 .

\section{Pericrocotus.}

Pericrocotus, Boie, Isis, 1826, p. 972.

\section{Pericrocotus brevirostris.}

Muscipeta brevirostris, Vig. P. Z. S. 1830-1, p. 43.

Phonicornis brevirostris, Gould, Cent. B. Him. pl. 8.

Pericrocotus brevirostris, Jerd. B. Ind. i. p. 421. 
a Nepal (B. H. Hodgson 292) 1845. - b Madras ("Phœenicornis brevirostris" T. C. Jerdon) 1845.

\section{Pericrocotus flammeus.}

Muscicapa flammea, Forst. Zool. Ind. pl. xv.

Pericrocotus flammeus, Jerd. B. Ind. i. p. 420.

$a$ (Askew) 1837. - b India (Bt. at Stevens's) 1844.

Strickland (Ann. \& Mag. N. H. xv. p. 275) pointed out that Phoenicornis Alammeus of Jerdon's 'Illustrations' (pl. ii.) is certainly the same as Muscicapa flammea of Temminck, and hence its range extends to Java and Sumatra. He further states that it is not the "Oranor" of Levaillant (Ois. Afr. pl. 155) as supposed by Jerdon, that bird being identical with Pericrocotus peregrinus.

\section{Pericrocotus speciosus.}

Turdus speciosus, Lath. Ind. Orn. p. 363.

Pericrocotus speciosus, Strickl. Ann. \& Mag. N. H. xiii. p. 36 : Jerd. B. Ind. i. p. 419.

Phoenicornis princeps, Gould, Cent. B. Him. pl. 7.

a (T. C. Eyton). -b, -c Nepal (B. H. Hodgson 291) 1845. - $d$, -e India ("Pericrocotus speciosus" E. Blyth) 1846. -f Kumaon (W. J. E. Boys) 1847.

In the 'Annals' (l. s. c.) Strickland pointed out the identity of $P$. princeps, Gould and Turdus speciosus, Latham.

\section{Pericrocotus peregrinus.}

Parus peregrinus, Linn. Syst. Nat. i. p. 342.

Pericrocotus peregrinus, Jerd. B. Ind. i, p. 423.

a Himalaya (N. C. Strickland) 1838. -b India ("Pericro" cotus peregrinus" E. Blyth). -c (W. J. E. Boys) 1847. -d, -e S. India "Phonicornis peregrinus" T. C. Jerdon), 1850.

Identified by Strickland with the "Oranor" of Levaillant (Ann. \& Mag. N. H. xv. p. 275).

\section{Pericrocotus roseus.}

Muscicapa rosea, Vieill. N. Dict. d'Hist. N. xxi. p. 486. Pericrocotus roseus, Jerd. B. Ind. i. p. 422.

a India (Bt. at Stevens's) 1844. - b Madras (" Phonicornis 
roseus" T. C. Jerdon) 1845. $-c,-d$ India ("Pericrocotus roseus" E. Blyth) 1846.

The bird, called with doubt "Phoenicura miniata, Temm." by Sundevall in his paper on the Birds of Calcutta, is stated by Strickland to be referable to this species (Ann. \& Mag. N. H. xviii. p. 251).

\section{Pericrocotus erythropygius.}

Muscicapa erythropygia, Jerd. Madr. Journ. xi. p. 17.

Pericrocotus erythropygius, Jard. Contr. Orn. 1848, p. 23. pl. 4 : Jerd. B. Ind. i. p. 424.

a Chunar (W. J. E. Boys) 1847.

\section{Pericrocotus cinereus.}

Pericrocotus cinereus, Lafr. Rev. Zool. 1845, p. 94: Wald. Trans. Z. S. ix. p. 179.

Pericrocotus modestus, Strickl. P. Z. S. 1846, p. 102.

a M.alacca (Havell) 1839.

The type of $P$. modestus, Strickl. A note on the label shews that Strickland recognized its identity with the bird previously described by Lafresnaye.

\section{FAMILY.-LANIIDE。}

\section{SUB-FAMILY.-LANIIN $A$.}

\section{LANIUS.}

Lanius, Linn. Syst. Nat. i. p. 134 (1766) (partim). 598. Lanius excubitor.

Lanius excubitor, Linn. Syst. Nat. i. p. 135 : Newton, ed. Yarr. Brit. B. i. p. 199.

$a,-b$ N. Europe (J. G. Kinberg) 1843.

\section{Lanius ludovicianus.}

Lanius ludovicianus, Linn. Syst. Nat. i. p. 134.

Collurio ludovicianus, Baird, Brew. \& Ridgw. N. Am. B. i. p. 418 .

$a$ N. America (J. G. Kinberg) 184.5. $-b$ United States (S. F. Baird) 1847. 
600. Lanius excubitorides.

Lanius excubitorides, Sw. F. Bor. Am. ii. p. 115, pl. 34.

Collurio ludovicianus, var. excubitorides, Baird, Brew. \& Ridgw. N. Am. B. i. p. 421.

a Columbia (Galleoti) 1845.

601. Lanius algeriensis.

Lanius algeriensis, Less. Rev. Zool. 1839, p. 134: Dresser, B. Eur.

$a$ (Arthur Strickland) 1840. -b Morocco (J. G. Kinberg) 1844 .

602. Lanius lahtora.

Collurio lahtora, Sykes, P. Z. S. 1832, p. 86.

Lanius lahtora, Jerd. B. Ind. i. p. 400 : Sharpe \& Dresser, P. Z. S. 1870 , p. 595 : B. Eur.

Lanius algeriensis, Strickl. P. Z. S. 1850, p. 217 (nec Lesson).

a Himalaya (N. C. Strickland) 1838. -b India (Askew) 1839. — c Kordofan (J. Petherick) 1848.

The Kordofan specimen differs from Indian examples in having the forehead white instead of black, and were this character constant it would be justifiable to keep the North-African bird distinct under the name $L$. dealbatus, De Fil. Abyssinian examples, (L. fallax Finsch. \& Hartl.), however, are intermediate, and it would seem as if one form passed gradually into the other.

603. Lanius borealis.

Lanius borealis, Vieill. Ois. Am. Sept. i. p. 80. pl. 50 : Baird, Brew. \& Ridgw. N. Am. B. i. p. 415.

$a$ N. America (Askew) 1837. - b N. America (N. C. Strickland) 1838. —c Massachusetts (J. G. Kimberg) 1845.

\section{Lanius minor.}

Lanius minor, Gm. Syst. Nat. i. p. 308: Strickl. Ann. \& Mag. N. H. xii. p. 414; P. Z. S. 1830, p. 97: Strickl. \& Scl. Contr. Orn. 1852, p. 144: Newton, ed. Yarr. Brit. B. i. p. 205 : Dresser, B. Eur.

$a$ Siberia (T. C. Eyton) 1832. -b Smyrna (H. E. Strickland) 1836. —c Zante (H. E. Strickland) 28 May 1836. - c Damaraland (C. J. Andersson) 1852. 
605. Lanius sp.?

$a$ (N. C. Strickland) 1838.

An immature bird perhaps the young of $L$. minor.

606. Lanius collaris.

Lanius collaris, Linn. Syst. Nat. i. p. 135: Strickl. \& Scl. Contr. Orn. 1852, p. 144: Layard, B. S. Afr. p. 157.

$a,-b$ Cape of Good Hope (A Strickland) 1833. - $(N . C$. Strickland) 1838. -d (Argent) 1851.

\section{0\%. Lanius subcoronatus.}

Lanius subcoronatus, Smith, Ill. Zool. S. Afr. pl. 68: Layard, B. S. Afr. p. 157.

a Damara-land (C. J. Andersson) 1852.

608. Lanius nubicus.

Lanius nubicus, Licht, Verz. Doubl. p. 47 : Strickl. P. Z. S. 1850, p. 217.

a Himalaya? (N. C. Strickland) 1838. -b (Stevens) 1845. $c,-d$ Kordofan (J. Petherick) 1848.

609. Lanius erythronotus.

Collurio erythronotus, Vig. P. Z. S. 1830-1, p. 22: Gould, Cent. B. Him. pl. 12. f. 2.

Lanius erythronotus, Jerd. B. Ind. i. p. 402.

$a$ Madras (A. Strickland) 1833. -b (N. C. Strickland) 1838. -c India (Stevens) 1844. —d Gorruckpore (W. J. E. Boys) 1847.

610. Lanius tephronotus.

Collurio tephronotus, Vig. P. Z. S. 1830-1, p. 43.

Lanius tephronotus, Jerd. B. Ind. i. p. 403.

a India " Lanius tephronotus" E. Blyth) 1846. - b India (E. Blyth) 1846.

\section{Lanius nigriceps.}

Collurio nigriceps, Frankl. P. Z. S. 1830-1, p. 117.

Lanius nigriceps, Jerd. B. Ind. i. p. 494.

Lanius bicolor, Hodgs. Ind. Rev. i. p. 446.

a Madras ("L. nigriceps," T. C. Jerdon). -b Nepal (B. H. Hodgson 229) 1845. - India ("Lanius nigriceps, Frankl.; 
nasutus, Scop.; antiguanus, Gm.; tricolor, Hodgs." E. Blyth) 1846.

Two forms of this bird occur in India to which the names of $L$. nigriceps Frankl. and L. bicolor, Hodgs. have been applied : $a$ belongs to the former, $b$ and $c$ to the latter race. It is also not improbable that Scopoli's name $L$. nasutus will have to be used for one or both of these birds, on which point see Lord Tweeddale's remarks, Trans. Z. S. ix. p. 169.

\section{Lanius vittatus.}

Lanius vittatus, Valenc. Dict. Sc. Nat. xl. p. 227.

Collurio hardwickii, Vig. P. Z. S. 1830-1, p. 42: Gould, Cent. B. Him. pl. 12.

Lanius hardwickii, Jerd. B. Ind. i. p. 405.

a Himalaya (N.C.Strickland) 1838. - b India (E. Blyth) 1846. -c Rohilkund (W. J. E. Boys) 1847.

\section{Lanius collurio.}

Lanius collurio, Linn. Syst. Nat. i. p. 136: Strick. P. Z. S. 1836, p. 97 ; 1850, p. 217 : Newton, ed. Yarr. Brit. B. i. p. 209.

$a$ Worcestershire (H. E. Strickland) 1832. -b, - c, Aberdovey (H. E. Strickland) Aug. 1833. - d Smyrna (H. E. Strickland) April 1836. - e (N. C. Strickland) 1838. $-f$ Kordofan (J. Petherick) 1848.

\section{Lanius cristatus.}

Lanius cristatus, Linn. Syst. Nat. i. p. 134: Strickl. Ann. \& Mag. N. H. xiv. p. 44: Jerd. B. Ind. i. p. 406: Wald. Ibis, 1867, 212.

Lanius melanotis, "Cuv." Puch. Arch. d. Mus. vii. p. 324: Strickl. Ann. \& Mag. N. H. xiii. pp. 36, 205.

Lanius ferrugiceps, Hodgs. Ind. Rev. i. p. 446.

$a,-b$ (N. C. Strickland) 1838. -c Nepal (B. H. Hodgson 431) 1845. — d Madras (T. C. Jerdon) 1845. - e India ("Lanius melanotis" E. Blyth) 1846. - $f$ India (E. Blyth) 1846. -g (E. Brown) 1850.

In 1846 Strickland (P. Z. S. p. 102) retracted his opinion expressed in 1844 (Ann. \& Mag. N. H. xiv. p. 44) as to the distinctness of this bird from $L$. lucionensis and its allies and placed all under the 
latter name. He several times insisted upon the rejection of the name cristatus on account of its inapplicability to this species.

615. Lanius lucionensis.

Lanius lucionensis, Linn. Syst. Nat. i. p. 135: Strickl. Ann. \& Mag. N. H. xiv. p. 44; P. Z. S. 1842, p. 167: Wald. Ibis, 1867, p. 215; Trans. Z. S. ix. p. 171.

$a,-b$ Calaguan, Philippine Islands (H. Cuming) 30 May 1837. $-c,-d$ Philippine Islands ( $H$. Cuming).

616. Lanius superciliosus.

Lanius superciliosus, Lath. Ind. Orn. Suppl. p. xx: Strickl. Ann. \& Mag. N. H. xiv. p. 44: Wald. Ibis, 1867, p. 218.

a Malacca (W. Kirtland) 1843.

617. Lanius magnirostris.

Lanius magnirostris, Less. Bélang. Voy, p. 251: Wald. Ibis, 1867, p. 220.

$a($ E. Brown) 1850. -b (Carfrae) 1850.

Found in the Malay peninsula and in Sumatra. At one time (Ann. \& Mag. N. H. xiii. p. 205), Strickland considered this species to be probably the same as $L$. superciliosus; subsequently (op. cit. xviii. p. 168) to be a variety of $L$. phonicurus, Pallas.

\section{Lanius arenarius.}

Lanius arenarius, Blyth, J. A. S. B. xv. p. 304: Strickl. P. Z. S. 1850, p. 217: Jerd. B. Ind. i. p. 407: Wald. Ibis, 1867, p. 223.

$a$ N. India (W. J. E. Boys) 1847.

619. Lanius anderssoni.

Enneoctonus anderssoni, Strickl. Contr. Orn. 1852, p. 145.

Lanius anderssoni, Wald. Ibis, 1867, p. 224.

$a$ Damara-land (C. J. Andersson) 1852.

The type of the species.

\section{Lanius isabellinus.}

Lanius isabellinus, Hempr. \& Ehr. Sym. Phys. Av. i. fol. $e$ : Strickl. P. Z. S. 1850, p. 217: Wald. Ibis, 1867, p. 224.

a Kordofan (J. Petherick) 1848. 


\section{Lanius auriculatus.}

Lanius auriculatus, P. L. S. Müller, Natursyst. Suppl. p. 71: Newton, ed. Yarr. Brit. i. p. 215.

Lanius rufus, Briss.: Strickl. P. Z. S. 1836, p. 97.

Lanius rutilus, Lath. et auctt. plurr.

a, -b Smyrna (H. E. Strickland) April 1836.

\section{Lanius rutilans.}

Lanius rutilans, Temm. Man. d'Orn. iii. p. 601. a Senegal (Isaacson) 1840.

\section{NilaUs.}

Nilaus, Swainson, Zool. Journ. iii. p. 162 (1827).

\section{Nilaus brubu.}

Lanius brubu, Lath. Ind. Orn. Suppl. p. xx.

Nilaus capensis, Sw. l. s. c. (ex Shaw).

Nilaus brubu, Strickl. Ann. \& Mag. N. H. viii. p. 30: Strickl. \& Scl. Contr. Orn. 1852, p. 145.

$a(N . C$. Strickland) 1838.

Found in West and South Africa.

\section{Corvinella.}

Corvinella, Lesson, Traité d'Orn. p. 372 (1831) (as a subgenus).

\section{Corvinella capensis.}

Tanagra capensis, Sparrman, Mus. Carls. pl. 54.

Corvinella capensis, Strickl. Ann. \& Mag. N. H. vii. pp. 27,30 .

Lanius corvinus, Shaw, Gen. Zool. vii. p. 337: Layard, B. S. Afr. p. 157.

Lanius cissoides, Vieill. : Sw. B. W. Afr. i. p. 233.

$a$ Senegal (N. C. Strickland) 1838. -b (Dewgard) 1851.

S. C. 
EURyCEPHALUS.

Eurocephalus, A. Smith, Rep. Expl. C. Afr. p. 52 (1836).

\section{Eurycephalus anguitimens.}

Eurocephalus anguitimens, Smith, l. s. c.:- Strickl. \& Scl. Contr. Orn. 1852, p. 145: Gurney in And. B. Damara-l. p. 140.

a Damara-land (C. J. Andersson) 1852.

\section{Telephonus.}

Telophorus, Swainson, F. Bor. Am. ii. p. 481 (1831).

\section{Telephonus senegalus.}

Lanius senegalus, Linn. Syst. Nat. i. p. 137.

Telephonus senegalus, Strickl. Ann. \& Mag. N. H. vii. p. 30 : Strickl. \& Scl. Contr. Orn. 1852, p. 145.

Lanius erythropterus, Shaw, Gen. Zool. viii. p. 307.

Telephonus erythropterus, Sw. B. S. Afr. i. p. 235.

a Senegal (N. C. Strickland) 1838.

\section{2\%. Telephonus longirostris.}

Telephonus longirostris, Sw. An. in Menag. p. 283: Layard, B. S. Afr. p. 161. 1842.

$a$ (N. C. Strickland) 1838. -b S. Africa (Sir W. Jardine)

\section{Telephonus remigialis.}

Telephonus remigialis, Finsch \& Hartl. Orn. Ost. Afr. p. 340. Linn.).

Telephonus senegalus, Strickl. P. Z. S. 1850 p. 217 (nec

a Kordofan (J. Petherick) 1848.

\section{Telephonus gutturalis.}

Merle à Collier, du Cap de Bonne-Espérance. D'Aub. Pl. Enl. 272 undè.

Turdus gutturalis, P. Z. S. Müller, Natursyst. Suppl. p. 144.

Lanius bacbakiri, Shaw, Gen. Zool. vii. p. 319.

Telephonus bacbakiri, Layard, B. S. Afr. p. 161.

$a,-b$ Cape of Good Hope (A. Strickland) 1832. $-c$ Cape of Good Hope (N. C. Strickland) 1838. 


\section{LANIARIUS.}

Laniarius, Vieill. Anal. p. 41 (1816).

\section{Laniarius barbarus.}

Lanius barbarus, Linn. Syst. Nat. i. p. 137.

Malaconotus barbarus, Sw. B. W. Afr. i. p. 243, pl. 24.

a Africa (N. C. Strickland) 1833.

631. Laniarius atrococcineus.

Lanius atrococcineus, Burch. Zool. Journ. i. p. 461, pl. 18.

Laniarius atrococcineus, Strickl. \& Scl. Contr. Orn. 1852, p. 145: Gurney, in And. B. Damara-l. p. 144.

$a,-b$ Damara-land (C. J. Andersson) 1852.

\section{Laniarius quadricolor.}

Laniarius quadricolor, Cassin, Pr. Ac. Phil. 1851, p. 245: Layard, B. S. Afr. p. 165.

a Port Natal (T. C. Eyton) 1850.

633. Laniarius icterus.

-Lanius icterus, Cuv. Régn. An. i. p. 352 (1829).

Laniarius icterus, Gray, Gen. B.

Malaconotus olivaceus (Vieill.), Sw. B. W. Afr. i. p. 237.

$a$ W. Africa (N. C. Strickland) 1838. -b (T. C. Eyton) 1845.

634. Laniarius sulfureopectus.

Lanius sulfureopectus, Less. Traité d'Orn. p. 373.

Malaconotus similis, Smith, Ill. Zool. S. Afr. pl. 46.

a Senegal (N. C. Strickland) 1838.

635. Laniarius rubiginosus.

Malaconotus rubiginosus, Sundev. Öfvers. K. Vet. Ac. Förh. 1850, p. 106.

Laniarius rubiginosus, Layard, B. S. Afr. p. 164.

a Cape of Good Hope (N. C. Strickland) 1838. - b S. Africa (Sir W. Jardine) 1842.

\section{Dryoscopus.}

Dryoscopus, Boie, Isis, 1826, p. 973.

636. Dryoscopus cubla.

Lanius cubla, Lath. Ind. Orn. Suppl. p. xx.

Dryoscopus cubla, Boie, l. s. c. 
Laniarius cubla, Layard, B. S. Afr. p. 163. 1850 .

$a$ S. Africa (Stevens) 1843. - b Damara-land (C.J. Andersson)

\section{3\%. Dryoscopus gambensis.}

Lanius gambensis, Licht. Verz. Doubl. p. 48.

Dryoscopus gambensis. Hartl. Syst. Orn. W. Afr. p. 110.

$a$ W. Africa (Johnson) 1837.

\section{Dryoscopus ferrugineus.}

Lanius ferrugineus, Gm. Syst. Nat. i. p. 306.

Dryoscopus ferrugineus, Strickl. Ann. \& Mag. N. H. vii. p. 29.

Laniarius ferrugineus, Layard, B. S. Afr. p. 164.

$a$ (Askew) 1834. -b (N. C. Strickland) 1.838.

\section{TEPHRODORNIS.}

Tephrodornis, Swainson, F. Bor. Am. ii. p. 482 (1831).

639. Tephrodornis pondicerianus.

Muscicapa pondiceriana, Gm. Syst. Nat. i. p. 939.

Tephrodornis pondiceriana, Jerd. B. Ind. i. p. 410.

a Madras (Stuchbury) 1836. —b (N. C. Strickland) 1838. c India ("Tephrodornis muscicapoides; Keroula indica, Gray; T. superciliosus, Sw." T. C. Jerdon) 1844. —d Malwa (W. J. E. Boys) 1847.

\section{Tephrodornis pelvica.}

Tenthaca pelvica, Hodgs. Ind. Rev. i. p. 447.

Tephrodornis pelvica, Jerd. B. Ind, i. p. 409.

a India ("Tephrodornis pelvica" T. C. Jerdon) 1846. -b Arakan (E. Blyth) 1847.

\section{Tephrodornis grisola.}

Tephrodornis grisola, Blyth, J. A. S. B. xii. p. 180*: Jerd. B. Ind. i. p. 411.

a Malacca (Mansfield) 1839.

642. Tephrodornis gularis.

Lanius gularis, Raffl. Trans. L. S. xiii. p. 304. 
Tephrodornis gularis, Blyth, J. A. S. B. xv. p. 304.

a Malacca (W. Kirtland) 1843. -b Malacca (Argent) 1850.

Originally described from Sumatran specimens with which Malaccan probably agree, but specimens from the two localities have not so far as I am aware been compared.

\section{Prionops.}

Prionops, Vieillot, Anal. p. 41 (1816).

643. Prionops plumatus.

Lanius plumatus, Shaw, Gen. Zool. vii. p. 292.

Prionops plumatus, Sw. B. S. Afr. i. p. 246 : Sharpe, Cat. B. Brit. Mus. iii. p. 320.

a Africa (Bt. at Dresden) 184ら. - b Africa (Johnson).

\section{MELANORNIS.}

Melcenornis, G. R. Gray, List, Gen. B. p. 36 (1840) vice Melasoma, Sw. (nec Latr.)

\section{Melænornis edolioides.}

Melasoma edolioides, Sw. B. W. Afr. i. p. 257, pl. 29.

Melcenornis edolioides, Gray: Sharpe, Cat. B. Brit. Mus. iii. 315.

$a$ (N. C. Strickland) 1838.

Agrees with Swainson's type from Senegal.

BRADYORNIS.

Bradyornis, Smith, Ill. Zool. S. Afr. pl. 113 (1847).

\section{Bradyornis mariquensis.}

Bradyornis mariquensis, Smith, l. s. c.: Sharpe, Cat. B. Brit. Mus. iii. p. 308.

Saxicola mariquensis, Layard, B. S. Afr. p. 109.

$a$ S. Africa (Capt. Alexander) 1838.

\section{Sigelus.}

Sigelus, Cabanis, Mus. Hein. i. p. 68 (1850).

\section{Sigelus silens.}

Lanius silens, Shaw, Gen. Zool. vii. p. 330. 
Sigelus silens, Cab. l. s. c.

Laniarius silens, Layard, B. S. Afr. p. 162.

Bradyornis silens, Sharpe, Cat. B. Brit. Mus. iii. p. 308. 1843.

a S. Africa (Sir W. Jardine) 1842. —b S. Africa (Stevens)

\section{COLLYRIOCINCLA.}

Colluricincla, Vigors \& Horsfield, 'Trans. Linn. Soc. xv. p. 214 (1825).

\section{4\%. Collyriocincla harmonica.}

Turdus harmonicus, Lath. Ind. Orn. Suppl. p. xli.

Colluricincla harmonica, Strickl. Ann. \& Mag. N. H. xi. p. 336 : Gould, B. Austr. ii. pl. 70.

Collyriocincla harmonica, Sharpe, Cat. B. Brit. Mus. iii. p. 290.

a Madras (!) (Stuchbury) 1836. - b Australia (Mansfield) 1839. —c (Dewgard) 1851.

\section{Collyriocincla rufiventris.}

Colluricincla rufiventris, Gould, P. Z. S. 1840, p. 164; B. Austr. ii. pl. 75.

Collyriocincla rufiventris, Sharpe, Cat. B. Brit. Mus. iii. p. 292.

a Near Perth, W. Australia 27 March 1839 (T. C. Eyton) 1846.

649. Collyriocincla rufogaster.

Colluricincla rufogaster, Gould, P. Z. S. 1845, p. 80.

Pinarolestes rufigaster. Sharpe, Cat. B. Brit. Mus. iii. p. 296. a (Dewgard) 1851.

Originally described from $\mathrm{New}$ South Wales specimens.

650. Collyriocincla rectirostris.

Colluricincla rectirostris, Jard. \& Selb. Ill. Orn. ser. 2 pl. 31. Collyriocincla rectirostris, Sharpe, Cat. B. Brit. Mus.iii. p. 291. Colluricincla selbyi, Gould, B. Austr. ii. pl. 77.

a Australia (N. C. Strickland) 1838. - b Australia (Askew) 1841 . 
SUBFAM. PACHYCEPHALINA.

\section{Pachycephala.}

Pachycephala, Swainson, Zool. Journ. ii. p. 174 (1827).

651. Pachycephala rufiventris.

Sylvia rufiventris, Lath. Ind. Orn. Suppl. p. liv.

Pachycephala rufiventris, Strickl. Ann. \& Mag. N. H.xi. p.336.

Pachycephala pectoralis, Vig. \& Horsf.: Gould, B. Austr. ii. pl. 67 (nec Lath.).

$a$ N. S. Wales (Askew). -b N. S. Wales (N. C. Strickland). 652. Pachycephala melanura.

Pachycephala melanura, Gould, P. Z. S. 1842, p. 134; B. Austr. ii. pl. 66.

a Australia (Askew) 1840. -b (Argent).

\section{Pachycephala glaucura.}

Pachycephala glaucura, Gould, P. Z. S. 1845, p. 12 ; B. Austr. ii. p. 65 .

$a$ (Stevens) 1845.

Found in Tasmania.

\section{Pachycephala xanthoprocta.}

Pachycephala xanthoprocta, Gould, P. Z. S. 1837, p. 149.

a Norfolk Island (Stevens).

\section{EOPSALTRIA.}

Eopsaltria, Swainson, Classif. B. ii. p. 240 (1839) (?)

655. Eopsaltria australis.

Muscicapa australis, Lath. Ind. Orn. Suppl. p. 1. Eopsaltria australis, Gould, B. Austr. iii. pl. 11. a Australia, (N. C. Strickland) 1858.

\section{FAMILY.-MUSCICAPIDA.}

\section{MoNARCHA.}

Monarcha, Vigors \& Horsfield, Trans. Linn. Soc. xv. p. 254 (1826).

656. Monarcha melanopsis.

Muscicapa melanopsis, Vieill. N. Dict. d'Hist. N. xxi. p. 450. 
Muscipeta carinata, Sw. Zool. Ill. pl. 147.

Monarcha carinata, Vig. \& Horsf. l. s. c.: Gould, B. Austr. ii. pl. 95, et auctt.

a Australia (N. C. Strickland) 1838.

In his MS. "Ornithological Synonyms" Strickland employs Vieillot's name for this species as being prior to that of Swainson. In this view I think he is undoubtedly correct.

\section{5\%. Monarcha dimidiata.}

Monarches dimidiatus, Hartl. \& Finsch, P.Z. S. 1871, p. 28. $a,-b$ Samoan Islands (J. Gould) 1850.

Determined by Dr Finsch by comparison with the types in the Bremen Museum. The specimens from which the original descriptions were made came from the island of Rarotonga and not from the Samoan islands.

\section{Muscicapa.}

Muscicapa, Linnæus, Syst. Nat. i. p. 324 (1766) (partim).

\section{Muscicapa grisola.}

Muscicapa grisola, Linn. Syst. Nat. i. p. 328 : Newton, ed. Yarr. Brit. B. i. p. 220.

a Weston, Oxfordshire (N. C. Strickland) 28 May 1834. $b$ Corfu (H. E. Strickland) Sept. 1835.

659. Muscicapa atricapilla.

Muscicapa atricapilla, Linn. Syst. Nat. i. p. 326: Newton, ed. Yarr. Brit. B. i. p. 229.

a Madras (Stuchbury) 1836. - b (N. C. Strickland) 1838. c Reighton, Yorkshire (H. E. Strickland) May 1840.

The locality assigned to $a$ is questionable.

\section{Muscicapa fuscula.}

Muscicapa fuscula, Sundev. Öfvers. K. Vet. Ac. Förh. 1850, p. 105: Layard, B. S. Afr. p. 148.

$a$ (Askew) 1837. - b S. Africa (Dr A. Smith).

661. Muscicapa latirostris.

Muscicapa latirostris, Raffles, Trans. L. S. xiii. p. 312. 
Alseonax latirostris, Jerd. B. Ind. i. p. 459. 1845 .

a Madras ("Muscicapa poonensis, Sykes" T. C. Jerdon)

\section{Erythrosterna.}

Erythrosterna, Bonaparte, Comp. List., p. 25 (1838).

\section{Erythrosterna parva.}

Muscicapa parva, Bechst. Naturgesch. Deutschl. iv. p. 505 : Newton, ed. Yarr. Brit. i. p. 224.

Erythrosterna parva, Bp. l. s. c.: Jerd. Ibis, 1872, p. 128.

a Nepal (B. H. Hodgson) 1845.

Strickland wrote on the label of this specimen "No doubt = parva of Europe as figured by Gould."

\section{Erythrosterna leucura.}

Muscicapa leucura, Gm. Syst. Nat. i. p. 939.

Erythrosterna leucura. Bp. Consp. i. p. 418: Jerd. B. Ind. i. p. 481 .

a Madras ("Muscicapa leucura" T. C. Jerdon) 1845. 6 India ("Siphya? leucura, fem." E. Blyth) 1846.

Strickland (Ann. \& Mag. N. H. xiii. p. 35) corrected Blyth's statement (J. A. S. B. xi. p. 791) that the Indian species of this form is the M. leucura Latham but not of Gmelin, the fact being that Gmelin gave a Latin name to Latham's bird, which the latter states came from the Cape of Good Hope. Strickland further suggested that as the description agrees fairly well with the Indian bird, the South African locality assigned to it was probably erroneous.

\section{Erythrosterna melanoleuca.}

Muscicapa maculata, Tick. J. A. S. B. ii. p. 574, fide Jerd. B. Ind. i. p. 483.

Muscicapula melanoleuca, Blyth, J. A. S. B. xii. p. 940. a Arakan (E. Blyth) 1847. -b India (W. J. E. Boys) 1848.

I do not see how Tickell's name can possibly be used for this species. Its author gave no description of his own of the bird, supposing he had before him the "Pied Flycatcher" of Linnæus as represented by Bewick. 


\section{Muscicapula.}

Muscicapula, Blyth, J. A. S. B. xii. p. 939 (1843).

\section{Muscicapula superciliaris.}

Muscicapa superciliaris, Jerd. Madr. Journ. xi. p. 16.

Muscicapula superciliaris, Blyth, l. s. c.: Jerd. B. Ind. i. p. 470. $a$ Sucktusghar (W.J. E. Boys) 1847.

\section{XANTHOPYGIA.}

Xanthopygia, Blyth, J. A. S. B. xvi. p. 123 (1847).

\section{Xanthopygia narcissina.}

Muscicapa narcissina, Temm. Pl. Col. 577. f. 1.

Xanthopygia chrysophrys, Blyth, l. s. c. p. 124.

a (E. Blyth) 1846.

A specimen in bad condition. It appears to have been sent to Strickland shortly before Blyth described it as above. A label bears the following note in Blyth's MS. : "What is this? I suspect it to be Australian and have a nearly allied species from Malacca." The true habitat of the species is China and Japan.

\section{Hemipus.}

Hemipus, Hodgson, P. Z. S. 1845, p. 32.

667. Hemipus picatus.

Muscicapa picata, Sykes, P. Z. S. 1832, p 85.

Hemipus piccecolor, Hodgs. l. s. c.

Hemipus picatus, Jerd. B. Ind. i. p. 412.

a Madras ("Muscicapa picata," T. C. Jerdon) 1845. 6 India ("Lanaria picata (Sykes) Bl." E. Blyth) 1846.

668. Hemipus obscurus.

Muscicapa obscura, Horsf. Trans. L. S. xiii. p. 146.

Hemipus obscurus, Sharpe, Cat. B. Brit. Mus. iii. p. 305. a Malacca (N. C. Strickland) 1838.

\section{HrLtota.}

Hyliota, Swainson, Classif. B. ii. p. 260 (1837).

669. Hyliota flavigaster.

Hyliota flavigaster, Sw. B. W. Afr. ii. p. 47 ; Flyc. p. 228. pl. 28. 
$a$ W. Africa (Asleew) 1841.

A male of this species of which a female (one of Swainson's types) is in the University Museum. In the "Contributions to Ornithology" (1851, pp. 132, 163) Strickland refers to Muscicapa violacea of Verreaux MS. as a second species of this genus.

\section{MICRACA.}

Microeca, Gould, P. Z. S. 1840, p. 172.

\section{Micrœca fascinans.}

Loxia fascinans, Lath. Ind. Orn. Suppl. p. xlvi. Micrceca fascinans, Gould, Ann. \& Mag. N. H. xi. p. 337. Microeca macroptera, Gould, B. Austr. ii. pl. 93. $a$ (Argent) 1851. -b (Dewgard) 1851.

An Australian species.

\section{HEMICHELIDON。}

Hemichelidon, Hodgson, P. Z. S. 1845, p. 32.

\section{Hemichelidon sibirica.}

Muscicapa sibirica, Gm. Syst. Nat. i. p. 936.

Hemichelidon sibiricus, Swinh. P. Z. S. 1863, p. 288.

Hemichelidon fuliginosus, Hodgs. l. s. c.: Jerd. B. Ind. i. p. 458.

$a$ India (W. J. E. Boys) 1847.

\section{Nilitava.}

Niltava, Hodgson, India Rev. i. p. 650 (1837).

\section{Niltava sundara.}

Niltava sundara, Hodgs. l. s. c. : Jerd. B. Ind. i. p. 473.

a Nepal (B. H. Hodgson 142) 1845. - b Kumaon (W. J. E. Boys) 1847. —c Himalaya (Bt. at Stevens's) 1850.

\section{Niltava grandis.}

Chaitaris grandis, Blyth, J. A. S. B. xi. p. 189.

Niltava grandis, Jerd. B. Ind. i. p. 476. a India ("Niltava grandis" E. Blyth) 1846. 


\section{Cyornis.}

Cyornis, Blyth, J. A. S. B. xii. p. 940 (1842).

\section{Cyornis rubeculoides.}

Phœnicura rubeculoides, Vig. P. Z. S. 1830-1, p. 3э: Gould, Cent. B. Him. pl. 25, f. 1.

Cyornis rubeculoides, Blyth, l. s. c. : Jerd. B. Ind. i. p. 466.

Niltava brevipes, Hodgs. Ind. Rev. i. p. 651.

$a$ (Mansfield) 1839. —b Nepal (B. H. Hodgson 137) 1845. -c, -d India ("Cyornis rubeculoides" E. Blyth) 1846.

675. Cyornis jerdoni.

Cyornis banyumas, Jerd. B. Ind. i. p. 466 (nec Horsf.).

Cyornis jerdoni, G. R. Gray, Hand-l. i. p. 325: Jerd. Ibis, 1872 , p. 125.

a Madras (T. C. Jerdon) 1845.

\section{6\%6. Cyornis ruficauda.}

Muscicapa ruficauda, Sw. Flyc. p. 251.

Saxicola erythropygia, Sykes, P. Z. S. 1832, p. 92.

$a,-b$ India (Bt. at Stevens's) 1844.

$a$ bears the following note in Strickland's handwriting: "Same as Saxicola erythropygia of Sykes at India House = Musc. ruficauda, Sw." Sykes's name is usually supposed to have been applied to the female of Pratincola caprata.

\section{Stoparola.}

Stoparola, Blyth, J. A. S. B. xvi. p. 125 (1847).

6\%7. Stoparola melanops.

Muscicapa melanops, Vig. P. Z. S. 1830-1, p. 171 : Gould, Cent. B. Him. pl. 6.

Stoparola melanops, Blyth, l. s. c.

Eumyias melanops, Cab. Jerd. B. Ind. i. p. 463.

a Nepal (B. H. Hodgson 288) 1845. - b India ("Stoparola melanops, Musc. lazulina, Sw." E. Blyth) 1846. -c Benares (W. J.E. Boys) 1846.

\section{Siphia.}

Siphia, Hodgson, India Rev. i. p. 651 (1837).

678. Siphia strophiata.

Siphia strophiata, Hodgs. l. s. c. : Jerd. B. Ind. i. p. 479. 
a India (Bt. at Stevens's) 1844. - b Nepal (B. H. Hodgson 424) 1845 .

\section{Sisura.}

Seisura, Vigors \& Horsfield, Trans. Linn. Soc. xv. p. 249 (1826).

\section{Sisura inquieta.}

Turdus inquietus, Lath. Ind. Orn. Suppl. xl.

Seisura inquieta, Gray, Ann. \& Mag. N. H. xi. p. 191: Gould, B. Austr. ii. pl. 87.

a New South Wales (McDonald) 1838.

\section{MYiagRA.}

Myiagra, Vigors \& Horsfield, Trans. Linn. Soc. xv. p. 250 (1826).

\section{Myiagra plumbea.}

Myiagra plumbea, Vig. \& Horsf. l. s. c. p. 254: Gould, B. Austr. ii. pl. 89.

Todus rubecula, Lath. Ind. Orn. Suppl. xxxii. ?

$a$ (N. C. Strickland) 1838. - $b$ New South Wales (McDonald) 1838.

From his MS. notes Strickland appears to have considered this bird to be the Todus rubecula of Latham, but as some doubt must always hang about the application of this name, Vigors \& Horsfield's unquestionable title had best be adhered to.

\section{Myiagra nitida.}

Myiagra nitida, Gould, P. Z. S. 1837, p. 142 ; B. Austr. ii. pl. 91 .

$$
a,-b \text { Australia (N. C. Strickland) } 1838 .
$$

\section{Hуротнуміs.}

Hypothymis, Boie, Isis, 1826, p. 973.

\section{Hypothymis azurea.}

Gobe-mouche bleu, des Philippines, D’Aub. Pl. Enl. 666, f. 1 undè

Muscicapa azurea, Bodd. Tabl. P1. Enl. p. 41. 
Hypothymis azurea, Wald. Trans. Z. S. ix. p. 182.

Myiagra azurea, Jerd. B. Ind. i. p. 450.

a Malacca (N. C. Strickland) 1838. -b Malacca (Cashmore) 1839. —c ("Muscicapa ccerulea" T. C. Jerdon) 1850. —d India (Bt. at Stevens's).

\section{Elminia.}

Elminia, Bonaparte, Compt. Rend. xxxviii. pp. 388, 652 (1854).

\section{Elminia longicauda.}

Myiagra longicauda, Sw. Flyc. p. 210, pl. 25.

Elminia longicauda, Bp. l. s. c.

$a$ (N. C. Strickland) 1838.

Agrees with West African examples of this species and with Swainson's type.

\section{Platystira.}

Platysteira, Jardine \& Selby, Ill. Orn. i. pl. 9 (1826).

684. Platystira cyanea.

Muscicapa cyanea, Müll. Natursyst. Suppl. p. 170.

Platystira cyanea, Gray: Sharpe, Ibis, 1873, p. 158.

Muscicapa melanoptera, Gm. Syst. Nat. i. p. 939.

$a,-b$ West Africa (Johnson) 1847.

685. Platystira capensis.

Muscicapa capensis, Linn. Syst. Nat. i. p. 327.

Platystira capensis, Gray, Gen. B. i. p. 257.

Batis capensis, Sharpe, Ibis, 1873, p. 161.

$a$ S. Africa (Dewgard) 1849.

\section{Platystira senegalensis.}

Muscicapa senegalensis, Linn. Syst. Nat. i. p. 327.

Platystira senegalensis, Hartl. Orn. W. Afr. p. 93.

Batis senegalensis, Sharpe, Ibis, 1873, p. 163.

a (E. Brown) 1850.

A West African species. 
68\%. Platystira pririt.

Muscicapa pririt, Vieill. N. Dict. d'Hist. N. xxi. p. 486.

Platystira pririt, Gray: Strickl. \& Scl. Contr. Orn. 1852, p. 144.

Batis pririt, Sharpe, Ibis, 1873, p. 168.

a W. Africa (N. C. Strickland) 1838. -b S. Africa (Bt. at Stevens's) 1842. — Damara-land (C. J. Andersson) 1852.

\section{LANIOTURDUS.}

Lanioturdus, Waterhouse, Alex. Exp. App. p. 264 (1838).

\section{Lanioturdus torquatus.}

Lanioturdus torquatus, Waterh. l. s. c.: Sharpe, Ibis, 1873, p. 170.

Platystira albicauda, Strickl. Contr. Orn. 1852, p. 144.

a Damara-land (C. J. Andersson) 1852.

The type of $P$. albicauda, Strickl.

\section{Stenostira.}

Stenostira, Bonaparte, Consp. Av. i. p. 316 (1850).

689. Stenostira scita.

Muscicapa scita, Vieill. N. Dict. d'Hist. N. xxi. p. 74.

Stenostira scita, Bp. l. s. c.

Platystira longipes, Sw., Layard, B. S. Afr. p. 143.

a S. Africa ( $\operatorname{Dr}$ A. Smith).

\section{RHIPIDURA ${ }^{1}$.}

Rhipidura, Vigors \& Horsfield, Trans. Linn. Soc. xv. p. 246 (1826).

\section{Rhipidura flabellifera.}

Motacilla flabellifera, Gm. Syst. Nat. i. p. 943.

Rhipidura flabellifera, Gray, Gen. B. p. 32 (1840): Buller, B. New Zeal. p. 143.

$a$ New Zealand (Askew) 1840.

Strickland (Ann. \& Mag. viii. p. 373) draws attention to the fact that the $R$. flabellifera of Vigors \& Horsfield is not the same as $M$. flabellifera, $\mathrm{Gm}$. but must be called $R$. albiscapa, Gould.

1 Strickland in his review of Jerdon's 'Illustrations of Indian Ornithology' (Ann. \& Mag. N. H. xv. p. 275) says that it is impossible to separate by welldefined generic characters Swainson's genus Leucocerca from Rhipidura. 


\section{Rhipidura albiscapa.}

Rhipidura flabellifera, Vig. \& Horsf. Trans. L. S. xv. p. 246. (nec Gm.).

Rhipidura albiscapa, Gould, P. Z. S. 1840, p. 113 ; B. Austr. ii. pl. 83.

a Australia (Askew) 1840.

692. Rhipidura rufifrons.

Muscicapa rufifrons, Lath. Ind. Orn. Suppl. p. l.

Rhipidura rufifrons, Vig. \& Horsf. Trans. L. S. xv. p. 248 : Gould, B. Austr. ii. pl. 84.

a Australia (N. C. Strickland) 1838.

\section{Rhipidura albofrontata.}

Rhipidura albofrontata, Franklin, P. Z. S. 1830-1, p. 116.

Leucocerca albofrontata, Jerd. B. Ind. i. p. 452.

a India (N. C. Strickland) 1838. -b (Gardner) 1844. -c S. India ("Leucocerca albofrontata" T. C. Jerdon) 1850.

\section{Rhipidura pectoralis.}

Leucocerca pectoralis, Jerd. Ill. Ind. Zool. sub pl. 2 ; B. Ind. i. p. 453 .

Rhipidura pectoralis, Blyth, J. A. S. B. xii. p. 935.

a Madras ("Leucocerca pectoralis" T. C. Jerdon) 1845.

\section{Rhipidura fuscoventris.}

Rhipidura fuscoventris, Franklin, P. Z. S. 1830-1, p. 117.

Leucocerca fuscoventris, Jerd. B. Ind. i. p. 451. 1846.

$a,-b$ Madras (T. C. Jerdon) 1845. -c India (E. Blyth)

Strickland (Ann. \& Mag. N. H. xviii. p. 175 note) correctly supposed the $R$. sannio of Sundevall to be the same as Franklin's R. fuscoventris, but he is wrong in adding to the synonomy of the species the name $L$. pectoralis, Jerdon. $a$ bears on a label in Blyth's handwriting "Rhipidura fuscoventris," Broad-tailed Flycatcher "of Latham."

\section{Rhipidura nigritorquis.}

Rhipidura nigritorquis, Vig. P. Z. S. 1830-1, p. 97.

Leucocerca nigritorquis, Wald. Trans. Z. S. ix. p. 182. 
a Luzon, Philippine Islands (H. Cuming) 14 June 1839.

Determined by Strickland, with doubt, to be the Muscicapa philippensis, Gm. (Syst. Nat. i. p. 943) but Gmelin's description is far from complete.

\section{CRyptolopha.}

Cryptolopha, Swainson, Flyc. p. 198 (1838).

\section{9\%. Cryptolopha cinereocapilla.}

Muscicapa cinereocapilla, Vieill. N. Dict. d'Hist. N. xxi. p. 489.

Cryptolopha cinereocapilla, Blyth, J. A. S. B. xv. p. 290 : Jerd. B. Ind. i. p. 455.

Cryptolopha poiocephala, Sw. Flyc. p. 200, pl. 23.

a Himalaya (Havell) 1839. $-b, \multimap$ Rohilcund (W. J. E. Boys) 1847.

Strickland (Ann. \& Mag. N. H. vii. p. 29) points out that Swainson's $C$. poiocephala should be called $C$. ceylonensis, Sw. but both names are later than Vieillot's. He also shews (Ann. \& Mag. N. H. xviii. p. 174) that the bird described by Sundevall as Muscicapa nitida, Lath. is the same as the present species.

\section{TERPSIPHONE.}

Terpsiphone, Gloger, Froriep's Notizen, xvi. p. 278 (1827) vice Muscipeta, Cuv. (1817) (nec Koch 1816) ${ }^{1}$.

\section{Terpsiphone paradisi.}

Muscicapa paradisi, Linn. Syst. Nat. i. p. 324.

Tchitrea paradisi, Jerd. B. Ind. i. p. $44 \tilde{\partial}$.

$a$ (N. C. Strickland) 1838. $-b,-c,-d,-e$ Ceylon (Capt. Pearson) 1840. $-f$ (Bt. at Stevens's) 1843. -g India (E. Blyth) 1851.

\section{Terpsiphone affinis.}

Tchitrea affinis, "A. Hay," Blyth, J. A. S. B. xv. p. 292 : Jerd. B. Ind. i. p. 448.

1 Though Gloger's name was proposed merely as a substitute for Cuvier's, it may still be used for this genus, as the antecedent use of MIuscipeta by Koch precludes the adoption of that title. Tchitrea, Lesson, was introduced four years later than Terpsiphone, Gloger.

S. C. 
a Nepal (B. H. Hodgson 283) 1845. - b S. India ("Muscipeta paradisi" T. C. Jerdon) 1850.

The locality "S. India" assigned to $b$ is open to doubt. The specimen agrees accurately with Jerdon's description of this species.

\section{Terpsiphone melanogaster.}

Muscipeta melanogaster, Sw. B. W. Afr. ii. p. 55.

a W. Africa (Askew) 1837. - b W. Africa (Sir W. Jardine) 1842. -c.

These specimens agree with examples in the Swainson collection, probably the types of the species.

\section{Terpsiphone rufiventer.}

Muscipeta rufiventer, Sw. B. W. Afr. ii. p. 53, pl. 4.

$a$ (N. C. Strickland) 1838. - b W. Africa (W. Kirtland) 1843. — $\mathrm{C}$. Africa (Gardner) 1846.

\section{Terpsiphone cristata.}

Muscicapa viridis (!) Müll. Natursyst. Suppl. p. 171.

Muscicapa cristata, Gm. Syst. Nat. i. p. 398.

Tchitrea cristata, Layard, B. S. Afr. p. 145.

Muscipeta perspicillata, Sw. B. W. Afr. ii. p. 60.

a Cape of Good Hope (N. C. Strickland) 1838. - b S. Africa (N. C. Strickland) 1838. - C S. Africa (Dewgard) 1849. -d (Argent) 1852.

Jules Verreaux, apparently recognizing specimen $a$ as having been skinned by himself, wrote across the label in 1870 "tué par J. Verreaux au Knysa, 1827." Müller's name for this species is utterly inadmissible.

\section{Terpsiphone incii.}

Tchitrea incei, "Gould," Horsf. \& Moore, Cat. B. E. I. Co. Mus. p. 392.

a Malacca (Johnson) 1837. -b Malacca (Matthew) 1840. -c Malacca (Williams) 1847. —d (Williams).

c seems certainly to belong to this species, but I am not so sure as regards $a, b, \& d$. The latter are all female or immature birds, and my materials are insufficient to determine them satisfactorily. 


\section{Philentoma.}

Philentoma, Eyton, Ann. \& Mag. N. H. xvi. p. 229 (1845).

\section{Philentoma velata.}

Drymophila velata, Temm. Pl. Col. 334.

Philentoma velatum, Horsf. \& Moore, Cat. B. E. I. Co. Mus. p. 392 : Wald. Ibis, 1872, p. 373.

a Malacca (Bt. at Stevens's) 1843. -b Malacca (Bt. at Stevens's) 1844. —c Borneo (Leadbeater) 1849. -d Malacca (Chapman) 1849.

\section{Philentoma pyrrhoptera.}

Muscicapa pyrrhoptera, Temm. Pl. Col. 596. f. 2.

Myiagra pyrrhoptera, Strickl. P. Z. S. 1846, p. 101.

a Malacca (Matthew) 1840. - b Malacca (Gardner) 1846.

Strickland (l. s. c.) considered that this bird occupies an intermediate position between Myiagra and Muscipeta (= Terpsiphone), but that it was hardly sufficiently distinct from the former to form a generic division.

\section{FAMILY.-AMPELIDE.}

AMPELIS.

Ampelis, Linnæus, Syst. Nat. i. p. 297 (1766) (partim).

\section{Ampelis cedrorum.}

Ampelis garrulus $\beta$, Linn. l. s. c.

Bombycilla cedrorum, Vieill. Ois. Am. Sept. i. p. 88, pl. 57.

Ampelis cedrorum, Scl. \& Salv. Ibis, 1859, p. 13 : Scl. Cat. Am. B. p. 46 : Baird, Brew. \& Ridgw. N. Am. B. i. p. 401.

$a$ N. America (Askew) 1837. -b N. America (N. C. Strickland) 1838. - c Canada (Barnes) 1848. - d, -e Guatemala (J. Constancia) 1851.

\section{Ptilogonys.}

Ptiliogonatus, Swainson, Zool. Journ. iii. p. 164 (1827).

\section{0\%. Ptilogonys cinereus.}

Ptiliogonys cinereus, Sw. Phil. Mag. 1827, p. 363 ; Zool. Ill. ser. 2, pls. $62,120$. 
Ptilogonys cinereus, Scl. \& Salv. Ibis, 1859, p. 13 : Scl. Cat. Am. B. p. 47.

$a$ (Arthur Strickland) 1840. -b Mexico (T. Mann) 1844. -c Guatemala (J. Constancia) 1845. - $d$ Guatemala (J. Constancia) 1851.

\author{
SECTION.-OSCINES LATIROSTRES.
}

\title{
FAMILY.-HIRUNDINID $\mathbb{E}$.
}

\section{HiRundo.}

Hirundo, Linnæus, Syst. Nat. i. p. 343 (1766) (partim).

\section{Hirundo rustica.}

Hirundo rustica, Linn. l. s. c.: Strickl. P. Z. S. 1836, p. 99 : Strickl. \& Scl. Contr. Orn. 1852, p. 144: Jerd. B. Ind. i. p. 157 (partim).

a Weston, Oxfordshire, Aug. 1833 (N. C. Strickland) 1833. $-b,-c$ Off Ascension, March (A. Strickland) 1833. - $d$ Worcestershire, June (H. E. Strickland) 1838. -e India ("Hirundo jewan, Sykes" E. Blyth) 1846.-f, -g India (E. Blyth) 1846. - $h$ Jucknie (W. J. E. Boys) 1847. — i Damara-land (C. J. Andersson) 1852.

Observed by Strickland with other Hirundines near Smyrna.

\section{Hirundo erythrogaster.}

Hirondelle à ventre roux de Cayenne, D'Aub. Pl. Enl. 724, f. 1, undè,

Hirundo erythrogaster, Bodd. Tabl. Pl. Enl. p. 45.

Hirundo horreorum, Barton, Fragm. N. Hist. Penns. p. 17, et auctt. plurr.

$a$ (N. C. Strickland) 1838. - b ô Carlisle, Pennsylvania, 11 May 1846 (S. F. Baird) 1847. —c, —d Guatemala ( $J$. Constancia) 1848. -e Brazil ? (Argent) 1852.

Specimen $e$ is probably from Bolivia. 
710. Hirundo gutturalis.

Hirundo gutturalis, Scop. Del. Faun. et Fl. Ins. p. 96 : Jerd. B. Ind. i. p. 157 (partim): Wald. Trans. Z. S. ix. p. 184.

a Java (Askew) 1837. - b Calaguan, Philippine Islands, 30 May (H. Cuming) 1837. - c Malacca (N. C. Strickland) 1838. -d Luzon, -e Panay, Philippine Islands (H. Cuming) 1840. -f Madras ("Hirundo jewan" T. C. Jerdon) 1845. - g Madras (T. C. Jerdon) 1845. - $h$ Malacca (Osman) 1846. - $i$ Calcutta ("Hirundo rustica" E. Blyth) 1851.

Neither $f$ nor $i$ labelled respectively $H$. jewan and $H$. rustica by Jerdan and Blyth have a dark pectoral band. I therefore refer them to H. gutturculis.

\section{Hirundo daurica.}

Hirundo daurica, Linn. Mant. p. 528: Jerd. B. Ind. i. p. 160.

$a$ India (Bt. at Stevens's) 1843. -b India (W. J. E. Boys) 1847.

\section{Hirundo filifera.}

Hirundo filifera, Steph. Shaw's Zool. xiii. pt. 2, p. 78 : Jerd. B. Ind. i. p. 159.

$a$ Madras (T. C. Jerdon) 1845. $-b,-c$ N. India (W. J. $E$. Boys) 1848.

\section{Hirundo nigricans.}

Hirundo nigricans, Vieill. N. Dict. d'Hist. N. xiv. p. 523. Collocalia arborea, Gould, B. Austr. ii. pl. 14.

Hylochelidon nigricans, Gould, Handb. B. Austr. i. p. 111.

a Australia (N. C. Strickland) 1838. -b Australia (T. C. Eyton) 1849.

In a MS. note in Gould's "Birds of Australia" Strickland remarks "No doubt $=H$. nigricans, Vieill. though he overlooked the fulvous rump."

\section{Hirundo cucullata.}

Hirondelle à tête rousse du Cap de Bonne-Espérance, D'Aub. Pl. Enl. 723, f. 2, undè, 
Hirundo cucullata, Bodd. Tabl. Pl. Enl. p. 45 : Sharpe, P. Z. S. 1870 , p. 318.

$a$ S. Africa (N. C. Strickland) 1838.

\section{Hirundo albigularis.}

Hirundo albigularis, Strickl. Contr. Orn. 1849, p. 17, pl. 15: Sharpe, P. Z. S. 1870, p. 308.

a S. Africa (N. C. Strickland) 1838. - b S. Africa (Dewgard) 1849 .

The types of the species.

\section{Hirundo bicolor.}

Hirundo bicolor, Vieill. Ois. Am. Sept. i. p: 61, pl. 31 : Baird, Rev. Am. B. p. 297: Baird, Brew. \& Ridgw. N. Am. B. i. p. 344.

a (Gardner) 1845. - b Carlisle, Pennsylvania, 16 March, 1840 (S. F. Baird) 1847.

\section{1\%1\%. Hirundo albiventris.}

Hirondelle à ventre blanc, de Cayenne, D'Aub. Pl. Enl. 546, f. 2, undè,

Hirundo albiventris, Bodd. Tabl. P1. Enl. p. 32.

Hirundo leucoptera, Gm. Syst. Nat. i. p. 1022 : Cab. Schomb. Guian. iii. p. 672.

a Guiana (R. Schomburgk) 183-. —b (Seaman) 1840. -c Brazil (Argent) 1851.

\section{Hirundo meyeni.}

Hirundo meyeni, Cab. Mus. Hein. i. p. 48 : Baird, Rev. Am. B. p. 302 .

Hirundo leucopyga, Meyen. Nov. Act. Ac. L. C. 1834, Suppl. p. 73, pl. 10 (nec Licht).

a Chili (Capt. Brown) 1842.

\section{Hirundo leucorrhoa.}

Hirundo leucorrhoa, Vieill. N. Dict. d'Hist. N. xiv. p. 519": Baird, Rev. Am. B. p. 301.

Hirundo frontalis, Gould, Zool. Voy. Beagle, iii. p. 40. a S. America (N. C. Strickland) 1838. 


\section{Hirundo euchrysea.}

Hirundo euchrysea, Gosse, B. Jam. p. 68; Ill. pl. 12 : Baird, Rev. Am. B. p. 30 t.

a Jamaica (Chapman) 1849.

\section{LAGENOPLASTES.}

Lagenoplastes, Gould, Handb. B. Austr. ii. p. 112 (1865).

\section{Lagenoplastes ariel.}

Collocalia ariel, Gould, P. Z. S. 1842, p. 132; B. Austr. ii. pl. 15.

Lagenoplastes ariel, Gould, Handb. B. Austr. i. p. 113.

a Australia (Cashmore) 1839.

On the label of this specimen Strickland notes that this bird may possibly be the Hirundo erythrocephala Gmelin, a name doubtfully referred by Gray to $H$. filifera, Stephens.

\section{PETROCHELIDON.}

Petrochelidon, Cabanis, Mus. Hein. i. p. 47 (1850).

\section{Petrochelidon Iunifrons.}

Hirundo lunifrons, Say, Long's Exp. ii. p. 47.

Petrochelidon lunifrons, Baird, Rev. Am. B. p. 288: Baird, Brew. \& Ridgw. N. Am. B. i. p. 334.

$a \mathrm{~N}$. America (Cashmore) 1839.

\section{Petrochelidon fulva.}

Hirundo fulva, Vieill. Ois. Am. Sept. p. 62, pl. 30.

Petrochelidon fulva, Scl. Cat. Am. B. p. 40.

Hirundo pociloma, Gosse, B. Jam. p. 64.

Petrochelidon poeciloma, Baird, Rev. Am. B. p. 292.

a Jamaica ("H. poeciloma" P. H. Gosse) 1848.

Doubtless a typical specimen. Prof. Baird keeps the Jamaican bird separate from the Cuban on account of its slightly inferior size and the deeper tint of the chestnut colour of the flanks. 


\section{AtTicora.}

Atticora, Boie, Isis, 1844, p. 172.

\section{Atticora fasciata.}

Hirundo fasciata, Gm. Syst. Nat. i. p. 1022.

Atticora fasciata, Scl. Cat. Am. B. p. 39 : Baird, Rev. Am.

B. p. 306 .

$a$ S. America (Dewgard) 1846.

\section{Atticora cyanoleuca.}

Hirundo cyanoleuca, Vieill. N. Dict. d'Hist. N. xiv. p. 509. Atticora cyanolenca, Baird, Rev. Am. B. p. 309.

$a$ S. America (N. C. Strickland) 1838. -b (Bt. at Birmingham) 1849. —c Brazil (Argent) 1851.

Psalidoprocne.

Psalidoprocne, Cabanis, Mus. Hein. i. p. 48 (185.0).

\section{Psalidoprocne holomelæna.}

Hirundo holomelas, Sundev. Öfvers. Vet. Ak. Förh. 1850, p. 108.

Psalidoprocne, Cab. l. s. c. : Sharpe, P. Z. S. 1870, p. 288. $a$ S. Africa (Dewgard) 1849.

Chelidon.

Chelidon, Boie, Isis, 1822, p. 550.

\section{Chelidon urbica.}

Hirundo urbica, Linn. Syst. Nat. i. p. 344.

Chelidon urbica, Boie, et auctt.

a Worcestershire (H. E. Strickland) 1834.

Cotyle.

Cotyle, Boie, Isis, 1822, p. 550 .

\section{Cotyle riparia.}

Hirundo riparia, Linn. Syst. Nat. i. p. 344.

Cotyle riparia, Boie, et auctt. 1840.

$a$ (Askew) 1837. - b. Worcestershire, June (T, Robinson) 


\section{Cotyle rupestris.}

Hivundo rupestris, Scop. Ann. i. Hist. Nat. p. 167.

Cotyle rupestris, Boie et auctt. : Jerd. B. Ind. i. p. 166.

$a$ Switzerland (W. Anderegg) 1836. - b Madras ("Hirundo rupestris" T. C. Jerdon) 1845.

730. Cotyle fuligula.

Hirundo fuligula, Licht. in Forst. Descr. Anim. p. 55.

Cotyle fuligula, Layard, B. S. Afr. p. 57 : Sharpe P. Z. S. 1870, p. 299.

$a$ S. Africa (Askew) 1841.

\section{Cotyle concolor.}

Hirundo concolor, Sykes, P. Z. S. 1832, p. 83.

Cotyle concolor, Jerd. B. Ind. i. p. 165.

a Madras ("Hirundo concolor" T. C. Jerdon) 1845.

\section{STELGIDOPTERYX.}

Stelgidopteryx, Baird, Rev. Am. B. p. 312 (1865).

732. Stelgidopteryx ruficollis.

Hirundo ruficollis, Vieill. N. Dict. d'Hist. N. xiv. p. 523.

Stelgidopteryx ruficollis, Baird, l. s. c. p. 315.

a Brazil (Johnson) 1837.

\section{Progne.}

Progne, Boie, Isis, 1826, p. 971.

\section{Progne purpurea.}

Hirundo purpurea, Linn. Syst. Nat. i. p. 344.

Progne purpurea, Boie, l. s. c.

Progne subis (L.), Baird, Rev. Am. B. p. 274.

$a,-b$ Brazil (Johnson) 1837. — (N. C. Strickland) 1838. -d America (N. C. Strickland) 1838. -e ô Carlisle, Pennsylvania, 8 May, 1846 (S. F. Baird) 1847.

734. Progne dominicensis.

Hirundo dominicensis, Gm. Syst. Nat. i. p. 1025.

Progne dominicensis, Gosse, B. Jam. p. 69: Baird, Rev. Am. B. p. 279.

a Jamaica (P. H. Gosse) 1848. 


\section{Progne chalybea.}

Hirundo chalybea, Gm. Syst. Nat. i. p. 1026.

Progne chalybea, Cab. J. f. Orn. 1860, p. 402.

Progne leucogaster, Baird, Rev. Am. B. p. 280.

$a$ (N. C. Strickland) 1838. - b Guatemala (J. Constancia) 1848.

\section{Progne tapera.}

Hirundo tapera, Linn. Syst. Nat. i. p. 345.

Progne tapera, Cab. Schomb. Guian. iii. p. 672 : Baird, Rev. Am. B. p. 286.

a (Johnson) 1837.

A widely-ranging South American species.

SECTION.-OSCINES TENUIROSTRES.

\section{FAMILY.-MELIPHAGIDAE.}

\section{Myzomela.}

Myzomela, Vigors \& Horsfield, Trans. Linn. Soc. xv. p. 316 (1826).

\section{Myzomela sanguinolenta.}

Certhia sanguinolenta, Lath. Ind. Orn. Suppl. p. xxxvii. Strickl. Ann. \& Mag. N. H. xi. p. 336.

Myzomela sanguinolenta, Gould, B. Austr. iv. pl. 63.

Certhia dibapha, Lath. Ind. Orn. Suppl. p. xxxvii.

a Australia (Bt. at Stevens's) 1842.

738. Myzomela rubratra.

Cinnyris rubrater, Less. Man. ii. p. 55: Kittl. Kupf. pl. 8, f.1. Myzomela rubratra, Finsch, J. Mus. Godeffr. Hft. viii. p. 16. $a$ (Burl) 1834.

A species found in the Pelew and Marianne Islands. 
ENTOMOPHILA.

Entomophila, Gould, P. Z. S. 1837, p. 154.

739. Entomophila picta.

Entomophila picta, Gould, l. s. c.: B. Austr. iv. pl. 50.

$a$ Liverpool Plains, N. S. W. (Coxen per T. C. Eyton) 1850.

Glyciphila.

Glyciphila, Sw. Classif. B. ii. p. 326 (1837).

740. Glyciphila fulvifrons.

Meliphaga fulvifrons, Vig. \& Horsf. Trans. L. S. xv. p. 317.

Glyciphila fulvifrons, Sw. l. s. c.: Gould, B. Austr. iv. pl. 28.

a Australia (Bt. at Birmingham) 1850.

\section{Glyciphila albifrons.}

Glyciphila albifrons, Gould, P. Z. S. 1840, p. 160 ; B. Austr. iv. pl. 29.

a Adelaide, S. Australia (T. C. Eyton) 1846.

Stigmatops.

Stigmatops, Gould, Handb. B. Austr. i. p. 500 (1865).

742. Stigmatops ocularis.

Glyciphila? ocularis, Gould, P. Z. S. 1837, p. 154; B. Austr. iv. pl. 31.

Stigmatops ocularis, Gould, l. s. c.

$a$ New South Wales (McDonald) 1838.

ACANTHORHYNCHUS.

Acanthorhynchus, Gould, P. Z. S. 1837, p. 24.

\section{Acanthorhynchus tenuirostris.}

Certhia temuirostris, Lath. Ind. Orn. Suppl. p. xxxvi.

Acanthorhynchus tenuirostris, Gould, l. s. c.; B. Austr. iv. pl. 61 .

Acanthorhynchus dubius, Gould, tom. cit. p. 25.

a Van Diemen's Land (Askew) 1834. - b New South Wales (N. C. Strickland) 1838. 


\section{Acanthorhynchus superciliosus.}

Acanthorhynchus superciliosus, Gould, P. Z. S. 1837, p. 24;

B. Austr. iv. pl. 62.

$a \mathrm{~W}$. Australia (Williams) 1846.

\section{XANTHOMYZa.}

Zanthomiza, Swainson, Classif. B. ii. p. 326 (1837).

Xanthomyza, Strickland, Ann. \& Mag. N. H. vi. p. 419.

\section{Xanthomyza phrygia.}

Merops phrygius, Lath. Ind. Orn. Suppl. p. xxxiv. Zanthomyza plorygia, Gould, B. Austr. iv. pl. 48. $a$ New South Wales (Askew) 1839.

\section{Ptilotis.}

Ptilotis, Swainson, Classif. B. ii. p. 326 (1837).

\section{Ptilotis lewini.}

Ptilotis lewini, Sw. l.s.c.

Ptilotis chrysotis, Gould, B. Austr. iv. pl. 32 (nec Lath.).

$a$ New South Wales (McDonald) 1838.

\section{Ptilotis leucotis.}

Turdus leucotis, Lath. Ind. Orn. Suppl. p. xliv.

Ptilotis leucotis, Gould, B. Austr. iv. pl. 36.

$a$ New South Wales ( $M^{c}$ Donald) 1838. -b Australia (Bt. at Stevens's) 1843.

\section{Ptilotis flavigula.}

Ptilotis flavigula, Gould, P. Z. S. 1838, p. 24; B. Austr. iv. pl. 35.

$a$ New South Wales (Askew) 1837. - $b$ New South Wales (N. C. Strickland) 1838.

\section{Ptilotis auricomis.}

Muscicapa auricomis, Lath. Ind. Orn. Suppl. p. xlix.

Ptilotis auricomis, Gould, B. Austr. iv. pl. 37 : Strickl. Ann. \& Mag. N. H. xi. p. 336.

$a$ New South Wales (N. C. Strickland) 1838. 


\section{Ptilotis ornata.}

Ptilotis ornata, Gould, P. Z. S. 1838, p. 24; B. Austr. iv. pl. 39.

a Swan River, W. Australia (N. C. Strickland) 1838.

\section{Ptilotis penicillata.}

Meliphaga penicillata, Gould, P. Z. S. 1836, p. 143.

Ptilotis penicillata, Gould, B. Austr. iv. pl. 43.

a Adelaide, S. Australia (T. C. Eyton) 1846.

\section{Ptilotis chrysotis.}

Certhia chrysotis, Lath. Ind. Orn. Suppl. p. xxxviii.

Ptilotis fusca, Gould, B. Austr. iv. pl. 44.

a New South Wales (N. C. Strickland) 1838.

\section{Ptilotis chrysops.}

Sylvia chrysops, Lath. Ind. Orn. Suppl. p. liv.

Ptilotis chrysops, Gould, B. Austr. iv. pl. 45.

a Australia (Mansfield) 1839.

\section{Meliphaga.}

Meliphaga, Vigors \& Horsfield Trans. L. S. xv. p. 312 (1826)".

\section{Meliphaga novæ-hollandiæ.}

Certhia novce-hollandice, Lath. Ind. Orn. p. 296.

Meliphaga novce-hollandice, Vig. \& Horsf. l. s. c.: Gould, B. Austr. iv. pl. 23.

a New Holland (N. C. Strickland) 1834. -6 New South Wales (N. C. Strickland) 1838.

\section{Meliphaga sericea.}

Meliphaga sericea, Gould, P. Z. S. 1836, p. 144; B. Austr. iv. pl. 25 .

$a,-b$ New South Wales (N. C. Strickland) 1838. $-c$ Australia (Askew) 1841. —d ("Prosthemadera sericea." E. Blyth).

1 On the use of this name as employed by Vigors and Horsfield in a restricted sense, see Strickland, Ann. \& Mag. N. H. vi. p. 419. 


\section{Meliphaga australasiana.}

Certhia australasiana, Shaw, Gen. Zool. viii. p. 226.

Meliphaga australasiana, Vig. \& Horsf. Trans. L. S. xv. p. 313 ; Gould, B. Austr. iv. pl. 27.

$a$ New South Wales (N. C. Strickland) 1838.

\section{Prosthemadera.}

Prosthemadera, Gray, List Gen. B. 1840, p. 15.

\section{5\%. Prosthemadera novæ-zealandiæ.}

Merops novce-zealandice, Gm. Syst. Nat. i. p. 464.

Prosthemadera nova-zealandia, Strickl. Ann. \& Mag. N. H. vi. p. 419 : Buller, B. N. Zeal. p. 87, pl. 10.

$a$ New Zealand (Dr Harvey) 1843.

\section{ANTHORNIS.}

Anthornis, Gray, List Gen.'B. 1840, p. 15.

758. Anthornis melanura.

Certhia melanura, Sparrm. Mus. Carls. pl. 5.

Anthornis melanura, Gray, Buller, B. N. Zeal. p. 91.

$a$ New Zealand (Gardner) 1845. - b ํํ (T. C. Eyton) 1850.

\section{ANTHOCH ÆRA.}

Anthochcera, Vigors \& Horsfield, Trans. L. S. xv. p. 320 (1826).

759. Anthochæra inauris.

Anthochcera inauris, Gould, B. Austr. iv. pl. 54.

a New Holland (Askerv) 1834.

\section{Anthochæra carunculata.}

Merops carunculata, Lath. Ind. Orn. p. 276.

Anthochcera carunculata, Gould, B. Austr. iv. pl. 55.

Anthochcera lewini, Vig. \& Horsf. Trans: L. S. xv. p. 322.

a New Holland (Askew) 1834.

761. Anthochæra mellivora.

Certhia mellivora, Lath. Ind. Orn. Suppl. p. xxxvii.

Anthochora mellivora, Vig. \& Horsf. Trans. L. S. xv. p. 321 :

Gould, B. Austr. iv. pl. 56.

$a,-b$ New Holland (A skew) 1834 . 
LEPTORNIS.

Leptornis, Hombron \& Jacquinot, Voy. au Pôle sud, Zool. iii. p. 85, Atlas, Ois. pl. 17, f. 1 (1845).

\section{Leptornis samoensis.}

Merops samoensis, Hombr. \& Jacq. Am. Sc. Nat. ser. 2 , xvi. p. 314 .

Leptornis samoensis, Hombr. \& Jacq. l.s. c.: Finsch \& Hartl. Orn. Centralpol. p. 64.

$a,-b$ Samoan Islands (T. Heath) 1846.

ACAnthogenys.

Acanthogenys, Gould, P. Z. S. 1837, p. 152.

\section{Acanthogenys rufigularis.}

Acanthogenys rufigularis, Gould, tom. cit. p. 153; B. Austr. pl. 53.

a Australia (W. Kirtland) 1846.

\section{POGONORNIS.}

Pogonornis, Gray, Gen. Birds, i. p. 123 (1846).

\section{r64. Pogonornis cincta.}

Meliphaga cincta, Du Bus, Bull. Ac. Brux. 1839, p. 295.

Pogonornis cincta, Gray, l. s. c.: Buller, B. N. Zeal. p. 98, pl. 11.

Ptilotis auritus, Lafr. Rev. Zool. 1839, p. 257.

$a$ New Zealand (T. C. Eyton) 1850.

\section{TROPIDORHYNCHUS.}

Tropidorhynchus, Vigors \& Horsfield, Trans. L. S. xv. p. 323 (1826).

\section{Tropidorhynchus corniculatus.}

Merops corniculatus, Lath. Ind. Orn. p. 276.

Tropidorhynchus corniculatus, Vig. \& Horsf. l. s. c.: Gould, B. Austr. iv. p. 324.

a New South Wales (N. C. Strickland) 1838. 


\section{Tropidorhynchus citreogularis.}

Tropidorhynchus citreogularis, Gould, P. Z. S. 1836, p. 143 ;

B. Austr. iv. pl. 60.

$a$ New South Wales ( $M^{c}$ Donald) 1838.

\section{ENTONYZA.}

Entomyza, Swainson, Classif. B. ii. p. 328 (1837).

\section{6\%. Entomyza cyanotis.}

Gracula cyanotis, Lath. Ind. Orn. Suppl. p. xxix.

Entomyza cyanotis, Sw. l. s. c:: Gould, B. Austr. iv. pl. 68. a Australia (Havell) 1839.

\section{MANORHINA.}

Manorina, Vieillot N. Dict. d'Hist. N. xix. p. 236 (1818).

\section{Manorhina melanophrys.}

Turdus melanophrys, Lath. Ind. Orn. Suppl. p. xlii.

Myzantha melanophrys, Gould, B. Austr. iv. pl. 80.

Manorina viridis, Vieill. l. s. c.; Gal. Ois. pl. 149.

a Australia (N. C. Strickland) 1838.

\section{Mrzantha.}

Myzantha, Vigors \& Horsfield, Trans. L. S. xv. p. 318 (1826).

\section{Myzantha garrula.}

Merops garrulus, Lath. Ind. Orn. Suppl. p. xxxiv.

Myzantha garrula, Vig. \& Horsf. l. s. c.: Gould, B. Austr. iv. pl. 76 : Strickl. Ann. \& Mag. N. H. vi. p. 421.

a New Holland (Askew) 1834. —b New South Wales (McDonald) 1838.

\section{MeLithreptus.}

Melithreptus, Vieillot, Anal. p. 46 (1816) ${ }^{1}$.

1 Strickland (Ann. \& Mag. N. H. vi., p. 420) considered Drepanis to be a synonym of Melithreptus and used Hamatops of Gould as the name of the present genus. As one of the species, the 'Héorotaire Fuscalbin,' included by Vieillotin Melithreptus, is the bird generally known as M. lunulatus, the use of Melithreptus as here employed seems justifiable. 


\section{7ro. Melithreptus validirostris.}

Sturnus virescens, Wagl. Syst. Av. fol. 7, p. 1 (nec Melithreptus virescens, Vieill.).

Homatops validirostris, Gould, P. Z. S. 1836, p. 144.

Melithreptus validirostris, Gould, B. Austr. iv. pl. 70.

Eidopsarus bicinctus, Sw. An. in Menag. p. 344.

a Van Diemen's Land (N. C. Strickland) 1838. -b (Dewgard), 1851.

The synonymy of this species as given above is taken from the label of specimen $a$ and from MS. notes in Strickland's copies of Wagler's Systema and Swainson's work. I have no doubt it is correctly stated. $b$ is the skin of a very young bird, apparently of this species.

\section{Melithreptus lunatus.}

Certhia lunata, Shaw, Gen. Zool. viii. p. 224.

Melithreptus lunulatus, Gould, B. Austr. iv. pl. 72: Strickl. Ann. \& Mag. N. H. xi. p. 336.

$a$ New South Wales (McDonald) 1837. $-b$ New South Wales (Askew) 1838.

\section{Melithreptus gularis.}

Hamatops gularis, Gould, P. Z. Ș. 1836, p. 144. Melithreptus gularis, Gould, B. Austr. iv. pl. 71. a New South Wales (N. C. Strickland) 1838.

A young bird with grey wings, apparently of this species.

\section{Melithreptus melanocephala.}

Melithreptus melanocephalus, Gould, P. Z. S. 1845, p. 62 ;

B. Austr. iv. pl. 75.

a Australia (Askew) 1834.

\section{FAMILY.-NECTARINIIDE.}

\section{SUB-FAMILY.-NECTARINIIN AE。}

\section{Promerops,}

Promerops, Brisson, Ornith. ii. p. 461 (1760).

\section{Promerops cafer.}

Merops cafer, Linn. Syst. Nat. i. p. 183.

S. C. 
Promerops cafer, Gray: Layard, B. S. Afr. p. 74.

$a$ Cape of Good Hope (A. Striclcland) 1832. - b, -c Cape of Good Hope (N. C. Strickland) 1838.

\section{NeCTARINIA.}

Nectarinia, Illiger, Prodr. p. 210 (1811).

\section{7\%5. Nectarinia famosa.}

Certhia famosa, Linn. Syst. Nat. i. p. 187.

Nectarinia famosa, Shelley, Mon. Cinn.

$a \hat{\jmath},-b$ o Cape of Good Hope (A. Strickland) 1832. - $c$ $\hat{\jmath}$ Cape of Good Hope (A. Strickland) 1833. —d (N. C. Strickland) 1838.

\section{Nectarinia violacea.}

Certhia violacea, Linn. Syst. Nat. i. p. 188.

Nectarinia violacea, Jard. Mon. Sun-birds, p. 200, pl. 16.

Anthobaphes violacea, Shelley, Mon. Cinn. 1832.

$a \hat{\jmath},-b$ \& Cape of Good Hope, April 16 (A. Strickland)

\section{9\%. Nectarinia pulchella.}

Certhia pulchella, Linn. Syst. Nat. i. p. 187.

Nectarinia pulchella, Strickl. P. Z. S. 1850, p. 216 (partim): Shelley, Mon. Cinn.

$a$ (Mather) 1840. -b $\hat{o}$ Kordofan 17 Aug. (J. Petherick) 1848. -c (Argent) 1851.

A second Kordofan specimen marked $N$. pulchella by Strickland seems to be an immature example of $N$. platyura.

\section{7\%8. Nectarinia metallica.}

Nectarinia metallica, Hemp. \& Ehr. Symb. Phys. Av. fol. a pl. 1 : Strickl. P. Z. S. 1850, p. 216.

Hedydipna metallica, Cab.: Shelley, Mon. Cinn. $a \hat{\delta},-b$ q Kordofan October (J. Petherick) 1848.

\section{Nectarinia platyura.}

Cinnyris platurus, Vieill. N. Dict. d'Hist. N. xxxi. p. 501.

Nectarinia cyanopygos, Licht. Verz. Doubl. p. 15. 1848.

$a$ (Mather) 1840. -b Kordofan 17 August (J. Petherick) 


\section{ETHOPYGA.}

Etthopyga, Cabanis, Mus. Hein. i. p. 103 (1850).

780. Fthopyga eximia.

Nectarinia eximia, Horsf. Trans. L. S. xiii. p. 168.

Atthopyga eximia, Wald. Ibis, 1870, p. 37.

a Java (Bt. at Dresden) 1845.

\section{1. Æethopyga nipalensis.}

Cinnyris nipalensis, Hodgs. Ind. Rev. ii. p. 273.

Athopyga nipalensis, Jerd. B. Ind. i.p. 366 : Shelley, Mon. Cinn.
a Nepal (B. H. Hodgson 523) 1845. -b (T. C. Eyton) 1849.

782. झ世thopyga miles.

Cinnyris miles, Hodgs. Ind. Rev. i. p. 273.

AEthopyga miles, Jerd. B. Ind. i. p. 262 : Wald. Ibis, 1870, p. 32.

a India (E. Blyth) 1846.

783. Aॄthopyga saturata.

Cinnyris saturatus, Hodgs. Ind. Rev. ii. p. 273.

Athopyga saturata, Jerd. B. Ind. i. p. 367: Shelley, Mon. Cinn.

a Darjeeling ("Nect. saturata," E. Blyth) 1850.

\section{CinNyRIS.}

Cinnyris, Cuvier, Règn. Anim. i. p. 411 (1817) (partim).

784. Cinnyris coccinigastra.

Certhia coccinigastra, Lath. Ind. Orn. Suppl. p. xxxv. (1802).

Certhia splendida, Shaw, Gen. Zool. viii. p. 191, pl. 26.

Nectarinia splendida, Jard. Mon. Sun-birds, 176, pl. 5.

Cinnyris splendidus, Shelley, Mon. Cinn.

a (Argent) 1851.

A West-African species.

785. Cinnyris afra.

Certhia afra, Linn. Syst. Nat. i. p. 186. 
Nectarinia afra, Jard. Mon. Sun-birds, pp. 169, 247, pl. 2. Cinnyris afra, Cuv.: Shelley, Mon. Cinn.

$a$ S. Africa (Sir W. Jardine) 1841.

\section{Cinnyris mariquensis.}

Cinnyris mariquensis, Smith, App. Rep. Exp. S. Afr. p. 53 : Shelley, Mon. Cinn.

Nectarinia bifasciata, Jard.: Strickl. \& Scl. Contr. Orn. 1852, p. 153 (nec Certhia bifasciata, Shaw).

$a,-b$ Damara-land (C.J. Andersson) 1852.

\section{Cinnyris chalybea.}

Certhia chalybea, Linn. Syst. Nat. i. p. 186.

Cinnyris chalybea, Cuv.: Shelley, Mon. Cinn.

a $\delta$ Cape of Good Hope, 18 March (A. Strickland) 1832. -b q Cape of Good Hope, 13 March (A. Strickland) 1832.

\section{Cinnyris fusca.}

Cinnyris fuscus, Vieill. N. Dict. d'Hist. N. xxxi. p. 506 : Shelley, Mon. Cinn.

Nectarinia fusca, Strickl. \& Scl. Contr. Orn. 1852, p. 153.

$a,-b$ o Damara-land (C. J. Andersson) 1852.

\section{Cinnyris amethystina.}

Certhia amethystina, Shaw, Gen. Zool. viii. p. 195.

Cinnyris amethystina, Cuv. : Shelley, Mon. Cinn.

$a,-b \operatorname{Africa}($ N. C. Strickland) 1838. - c (N. C. Strickland) 1838. -d (Bt. at Birmingham) 1850. -e (Carfrae) 1850. $-f$ (Argent) 1851.

\section{Cinnyris talatala.}

Nectarinia talatala, Smith, App. Rep. Exp. S. Afr. p. 58. Cinnyris talatala, Shelley, Mon. Cinn.

Nectarinia anderssoni, Strickl. Contr. Orn. 1852, p. 153.

a Damara-land (C. J. Andersson) 1852. - b S.-E. Africa (Argent) 1853.

$a$ is the type of Strickland's $N$. anderssoni. 


\section{Cinnyris albiventris.}

Nectarinia albiventris, Strickl. Contr. Orn. 1852, p. 42, pl. 86.

Cinnyris albiventris, Shelley, Mon. Cinn.

$a \hat{\delta},-b q$ Ras Hassoun, E. Africa (J. Daubeny) 1851.

The types of Strickland's description and the specimens from which the figures in Shelley's 'Monograph of the Cinnyridæ' were drawn.

\section{Cinnyris gutturalis.}

Certhia gutturalis, Linn. Syst. Nat. i. p. 186.

Cinnyris gutturalis, Cuv.: Shelley, Mon. Cinn.

Nectarinia senegalensis, Strickl. \& Scl. Contr. Orn. 1852, p. 152.

$a,-b$ Damara-land (C. J. Andersson) 1852.

\section{Cinnyris venusta.}

Certhia venusta, Shaw \& Nodder, Nat. Misc. x. pl. 369.

Nectarinia pusilla, Sw. B. W. Afr. ii. p. 138 (nec Linn.).

Nectarinia parvula, Jard. Mon. Sun-birds, p. 181, pl. 7.

$a$ W. Africa (Askew) 1834.

\section{Cinnyris chloropygia.}

Nectarinia chloropygia, Jard. Ann. \& Mag. N. H. x. p. 188 : Jard. \& Selb. Ill. Orn. n. s. pl. 50.

Cinnyris chloropygius, Bp. : Shelley, Mon. Cinn. a (Argent) 1851.

A West-African species.

\section{Cinnyris cyanocephala.}

Certhia cyanocephala, Vieill. N. Dict. d'Hist. N. xxxi. p. 513. Nectarinia cyanocephala, Jard. Mon. Sun-birds, p. 189, pl. 10. $a$ W. Africa (E. Brown) 1850.

\section{Cinnyris cuprea.}

Certhia cuprea, Shaw, Gen. Zool. viii. p. 201.

Nectarinia cuprea, Jard. Mon. Sun-birds, p. 254. 1851.

$a$ (Johnson) 1837. -b (N.C. Strickland) 1838. —c (Argent)

A West-African species. 


\section{Cinnyris fuliginosa.}

Certhia fuliginosa, Shaw, Gen. Zool. viii. p. 222.

Cinnyris fuliginosus, Vieill. N. Dict. d'Hist. N. xxxi. p. 497 : Shelley, Mon. Cinn.

Nectarinia fuliginosa, Jard. Mon. Sun-birds, p. 256. a Africa (N. C. Strickland) 1838.

\section{Cinnyris asiatica.}

Certhia asiatica, Lath. Ind. Orn. p. 288.

Arachnechthra asiatica, Jerd. B. Ind. i. p. 370.

Cinnyris asiatica, Shelley, Mon. Cinn.

$a$ (Askew) 1837. -b (N. C. Strickland) 1838. - c Madras (Bt. at Stevens's) 1843. - d Madras ("Nectarinia mahrattensis" T. C. Jerdon) 1845.

\section{Cinnyris lotenia.}

Certhia lotenia, Linn. Syst. Nat. i. p. 188.

A rachnechthra lotenia, Jerd. B. Ind. i. p. 272 : Wald. Ibis, 1870, p. 23.

a India (N. C. Strickland) 1838. - b Madras ("Cinnyris lotenia" T. C. Jerdon) 1845.

\section{Cinnyris flammaxillaris.}

Nectarinia flammaxillaris, Blyth, J. A. S. B. xiv. p. 557.

Arachnechthra flammaxillaris, Blyth, J.. A. S. B. xliii. extr. No. p. 141.

Cinnyris flammaxillaris, Shelley, Mon. Cinn.

a India ("Nectarinia flammaxillaris" E. Blyth) 1846. -b, —c Arakan (E. Blyth) 1847.

\section{Cinnyris jugularis.}

Certhia jugularis, Linn. Syst. Nat. i. p. 185.

A rachnechthra jugularis, Wald. Trans. Z. S. ix. p. 200.

Cinnyris jugularis, Shelley, Mon. Cinn.

$a,-b$ Mindanao, Philippine Islands, 16 May (H. Cuming) 1838. - c Philippine Islands (H. Cuming) 1839. 


\section{Cinnyris zeylonica.}

Certhia zeylonica, Linn. Syst. Nat. i. p. 188.

Leptocoma zeylonica, Jerd. B. Ind. i. p. 368.

Cinnyris zeylonica, Shelley, Mon. Cinn.

$a,-b$ India (Askew) 1837. $-c$ (Askew) 1837. $-d$ (N. C. Strickland) 1838. - e Madras (T. C. Jerdon) 1845. - $f$ India (E. Blyth) 1846. -g India ("Nectarinia zeylonica," E. Blyth) 1846.

Professor Newton's note (Ibis, 1870, p. 39) refers to specimens $a$ and $b$. The differences there mentioned between these specimens are repeated in two examples in the University collection (locality not recorded). Two others are somewhat intermediate in character, hence I doubt there being more than one species, the view taken by Capt. Shelley.

\section{Cinnyris hasselti.}

Nectarinia hasselti, Temm. PI. Col. 376, f. 3.

Nectarophila hasselti, Blyth, J. A. S. B. xliii. extr. No. p. 142.

Cinnyris hasselti, Shelley, Mon. Cinn.

Certhia brasiliana, Gm. Syst. Nat. i. p. 474.

a India ("Nect. hasseltii, Temm., N. phayrei, Bl., Certhia sperata, Raffl." E. Blyth) 1846. -b Arakan (E. Blyth) 1847.

\section{Cinnyris minima.}

Cinnyris minima, Sykes, P. Z. S. 1832, p. 99 : Shelley, Mon. Cinn.

Leptocoma minima, Jerd. B. Ind. i. p. 369.

a Madras ("Nectarinia minima" T. C. Jerdon) 1845.

\section{Anthodiata.}

Anthodiceta, Cabanis, Mus. Hein. i. p. 100 (1850).

\section{Anthodiæta collaris.}

Cinnyris collaris, Vieill. N. Dict. d'Hist. N. xxxi. p. 502.

Nectarinia collaris, Jard. Mon. Sun-birds, pp. 179, 251, pl. 6. Anthodiceta collaris, Cab.: Shelley, Mon. Cinn. $a$ S. Africa (Sir W. Jardine) 1841. 
+ ANThotreptes.

Anthreptes, Swainson, F. Bor. Am. ii. p. 495 (1831).

\section{Anthotreptes phœnicotis.}

Motacilla singalensis, Gm. Syst. Nat. i. p. 964 (nom. inept.). Chalcoparia singalensis, Cab.: Wald. Ibis, 1870, p. 48.

Nectarinia phonicotis, Temm. Pl. Col. 108, f. 1.

Anthreptes phonicotis, Shelley, Mon. Cinn.

a India ("Nectarinia phonicotis." E. Blyth) 1846. -b (Argent) 1851.

\section{0\%. Anthotreptes malaccensis.}

Certhia malaccensis, Scop. Del. Fl. et Faun. Ins. ii. p. 90.

Anthreptes malaccensis, Wald. Ibis, 1870, p. 47: Shelley, Mon. Cinn.

Nectarinia javanica, Horsf. Trans. L. S. xiii. p. 167.

a (Bt. at Edinburgh) 1852.

Apparently a Malaccan skin of this widely ranging species.

808. Anthotreptes longuemarii.

Cinnyris longuemarii, Less. Bull. Sc. xxv. p. 242 ; Zool. Ill. pl. 23.

$a$ W. Africa (G. Bell) 1842.

\section{ARACHNOTHERA.}

Arachnothera, Temm. Pl. Col. Tabl. Méth. p. 53 (1838).

809. Arachnothera affinis.

Cinnyris affinis, Horsf. Trans. L. S. xiii. p. 166.

Arachnothera affinis, Cab. Mus. Hein. i. p. 106 : Shelley, Mon. Cinn.

a ("Cinnyris affinis, E. India Comp. 1831 " Dr Horsfield).

Doubtless the true $A$. affinis of Java, being so named by Dr Horsfield.

810. Arachnothera modesta.

Anthreptes modesta, Eyton, P. Z. S. 1839, p. 105. 
Arachnothera modesta, Salvad. Ann. Mus. Genov. v. p. 183 : Shelley, Mon. Cinn.

a (E. Brown) 1850.

Apparently a Malaccan skin. The specimen is rather small for A. affinis and probably belongs to Eyton's closely allied species.

811. Arachnothera robusta.

Arachnothera robusta, Müll. \& Schl. Verh. Nat. Gesch. Ned. Overz. Bez. Zool. Aves, p. 68, pl. 11, f. 1: Salvad. Ann. Mus. Genov. v. p. 184.

Arachnoraphis robusta, Shelley, Mon. Cinn.

$a($ E. Brown $) 1850$.

Evidently a Malaccan skin.

\section{Arachnothera chrysogenys.}

Arachnothera chrysogenys, Temm. Pl. Col. 388, f. 1: Salvad. Ann. Mus. Genov. v. p. 181.

a Malacca (N. C. Strickland) 1838.

\section{Arachnothera longirostra.}

Certhia longirostra, Lath. Ind. Orn. p. 299.

Arachnothera longirostra, Salvad. Ann. Mus. Genov. v. p. 186: Wald. J. A. S. B. xliii. extr. No. p. 140.

Arachnothera affinis, Blyth, J. A. S. B. xv. p. 43 (nec Horsf.). $a$ Arakan (E. Blyth) 1847. —b (Argent) 1852. — c Malacca (E. Blyth).

\section{Arachnothera aurata.}

Arachnothera aurata, Blyth, J. A. S. B. xxiv. p. 478; xliii. extr. No. p. 140: Shelley, Mon. Cinn. a Arakan (E. Blyth) 1847.

\section{SUBFAMILY.-DIC ÆIN A巴.}

\section{DICAUM.}

Dicceum, Cuvier, Règn. Anim. i. p. 410 (1817).

815. Dicæum cruentatum.

Certhia cruentata, Linn. Syst. Nat. i. p. 187. 
Dicceum cruentatum, Cab. Mus. Hein. i. p. 98.

Dicaeum coccineum (Scop.): Jerd. B. Ind. i. p. 373.

$a$ India ("Dicceum cruentatum" E. Blyth) 1846. - b India (E. Blyth) 1846.

\section{Dicæum flammeum.}

Motacilla flammea, Sparrm. Mus. Carls. pl. 98.

Dicceum flammeum, Sundev.: Salvad. Ann. Mus. Genov. v. p. 169.

$a$ (Bt. at Dresden) 1845. -b (Gardner) 1846. -c (Bt. at Birmingham) 1849.

Found in Borneo, Java, \&c.

\section{Dicæum hirundinaceum.}

Motacilla hirundinacea, Shaw, Nat. Misc. iv. pl. 114.

Dicceum hirundinaceum, Gould, B. Austr. ii. pl. 35.

a New South Wales (N. C. Strickland) 1838.

\section{Dicæum trigonostigma.}

Certhia trigonostigma, Scop. Del. Faun. Fl. Ins. ii. p. 91.

Dicceum trigonostigma, Gray, Gen. B. i. p. 100: Salvad. Ann.

Mus. Genov. v. p. 166.

Certhia cantillans, Lath. Ind. Orn. p. 299.

Dicceum cantillans, Temm. Pl. Col. 478, f. 2.

a Malacca (T. C. Eyton) 1846.

\section{Dicæum chrysorrhæum.}

Dicceum chrysorrhceum, Temm. Pl. Col. 478, f. 1: Blyth, J. A. S. B. xliii. extr. No. p. 142: Salvad. Ann. Mus. Genov. v. p. 168. a Arakan (E. Blyth) 1847.

\section{Dicæum erythrorhynchum.}

Certhia erythrorhynchos, Lath. Ind. Orn. p. 299.

Dicaum erythrorhyncha, Blyth, J. A. S. B. xliii. extra No. p. 143.

Nectarinia minima, Tick. J. A. S. B. ii. p. 577.

Dicceum minimum, Jerd. B. Ind. i. p. 374.

Myzanthe inornata, Hodgs. Zool. Misc. p. 82.

Dicaum tickellice, Blyth, J. A. S. B. xii. p. 933. 
a Madras ("Dicaum erythrorhynchum" T. C. Jerdon) 1845. -b Nepal (B. H. Hodgson 395) 1845. —c, —d India (Dicceum tickellia E. Blyth) 1846. -e Bengal ("Dicceum minimum" $E$. Blyth) 1851.

\section{Dicæum concolor.}

Dicceum concolor, Jerd. Madr. Journ. xi. p. 227 ; Ill. Ind. Orn. pl. 39 ; B. Ind. i. p. 375.

a Madras ("Dicceum concolor" T. C. Jerdon) 1845.

Apparently a young bird thus named by Jerdon.

\section{PiPRISOMA.}

Piprisoma, Blyth, J. A. S. B. xiii. p. 394 (1844).

\section{Piprisoma agile.}

Fringilla agilis, Tick. J. A. S. B. ii. p. 578.

Piprisoma agile, Blyth, l. s. c.: Jerd. B. Ind. i. p. 376: Beavan, Ibis, 1867, p. 431, pl. 10.

$a$ (Bt. at Birmingham) 1849.

Agrees fairly with Beavan's specimens from Maunbhoom now in the University Museum.

\section{Prionochilus.}

Prionochilus, Strickland, P. Z. S. 1840, p. 29.

823. Prionochilus percussus.

Pardalotus percussus, Temm. Pl. Col. 394, f. 2.

Prionochilus percussus, Strickl. l. s. c.

$a \hat{o},-b q$ Malacca (N. C. Strickland) 1838.

\section{SUBFAMILY.-ZOSTEROPIN ÆE.}

\section{ZOSTEROPS.}

Zosterops, Vigors \& Horsfield, Trans. Linn. Soc. xv. p. 234 (1826). 
824. Zosterops lateralis.

Sylvia lateralis, Lath. Ind. Orn. Suppl. p. lv.

Zosterops dorsalis, Vig. \& Horsf. l. s. c.: Gould, B. Austr. iv. pl. 81.

a Australia (Thomas) 1842.

\section{Zosterops sp.?}

a Australia (Mansfield) 1839.

A skin in bad condition.

\section{Zosterops flava?}

Dicoeum flavum, Horsf. Trans. L. S. xiii. p. 171.

$a$ Philippine Islands (H. Cuming) 1839.

On the label of this specimen Strickland has noted 'compared with Dicceum flavum, Horsf. It is the same.' It may, however, belong to the Philippine species recently described by Lord Tweeddale as $Z$. nigrorum (P. Z. S. 1878, p. 286).

\section{Zosterops albigularis.}

Zosterops albogularis, Gould, P. Z. S. 1836, p. 75 ; B. Austr. Suppl.

a Australia (Gardner) $184 \check{.}$.

A Norfolk-Island species.

\section{Zosterops palpebrosa.}

Sylvia palpebrosa, Temm. Pl. Col. 293, f. 3.

Zosterops palpebrosa, Jerd. B. Ind. ii. p. 265.

$a$ Madras ("Zosterops maderaspatensis" and "Z. annulosa" T. C. Jerdon) 1845. - b Nepal (B. H. Hodgson 391) 1845. —c, -d (Gardner) 1846. —e Jucknie (W. J. E. Boys) 1847.

\section{Zosterops meyeni.}

Zosterops meyeni, Bp. Consp. Av. i. p. 398: Wald. Trans. Z. S. ix. p. 199.

$a$ Philippine Islands (H. Cuming) 1839.

\section{Zosterops capensis.}

Zosterops capensis, Sundev. Öfver. K. Vet. Ac. Förh. 1850, p. 102.

a S. Africa (Dewgard) 1849. 


\section{Zosterops mauritiana.}

Motacilla mauritiana, Gm. Syst. Nat. i. p. 981.

Zosterops mauritiana, Hartl. Vög. Madag. p. 96.

a Mauritius (Deyrolle) 1851.

\section{SUBFAMILY.-DREPANIN Æ.}

\section{Drepanis ${ }^{1}$.}

Drepanis, Temm.

\section{Drepanis sanguinea.}

Certhia sanguinea, Gm. Syst. Nat. i. p. 479.

Drepanis sanguinea, Gray, Gen. B. i. p. 95: Scl. Ibis, 1871, p. 358.

$a$ (Carfrae) 1840. - b Sandwich Islands (Townsend) 1841. -c (Carfrae) 1842. —d (Bt. at Edinburgh) 1852.

\section{SUBFAMILY.-PARDALOTIN $Æ$.}

\section{Pardalotus.}

Pardalotus, Vieillot, Anal. p. 31 (1816).

\section{Pardalotus punctatus.}

Pipra punctata, Lath. Ind. Orn. Suppl. p. lvi.

Pardalotus punctatus, Gould, B. Austr. ii. pl. 35.

$d$ New Holland (N. C. Strickland) 1838. $-b$ New South Wales (F. Holme) 1838.

\section{Pardalotus affinis.}

Pardalotus affinis, Gould, P. Z. S. 1837, p. 25 ; B. Austr. ii. pl. 39.

$a,-b$ Van Diemen's Land (N. C. Strickland) 1838. - c (18b) Australia (Stevens) 1842.

1 Strickland (Ann. \& Mag. N. H. vi. p. 418) considered this name equivalent to Melithreptus, Vieill. But Melithreptus, as now restricted (being originally a compound genus), having another signification, Drepanis may be retained in this its usual sense. 
835. Pardalotus melanocephalus.

Pardalotus melanocephalus, Gould, P. Z. S. 1837, p. 149;

B. Austr. ii. pl. 40.

a Australia (Carfrae) 1840.

836. Pardalotus striatus.

Pipra striata, Lath. Ind. Orn. p. 558.

Pardalotus striatus, Gould, B. Austr. ii. pl. 38.

a New Holland (N. C. Strickland) 1838.

83\%. Pardalotus quadraginta.

Pardalotus quadrigintus, Gould, P. Z. S. 1837, p. 148; B. Austr. ii. pl. 37.

a Van Diemen's Land (N. C. Strickland) 1838.

FAMILY.-CGEREBIDA.

SUBFAMILY.-DIGLOSSIN A.

Diglossa.

Diglossa, Wagler, Isis, 1832, p. 280.

838. Diglossa baritula.

Diglossa baritula, Wagl. t. s. c. p. 281 : Scl. \& Salv. Ibis, 1859, p. 144: Scl. Ibis, 1875, p. 207.

$a$ Guatemala ( $J$. Constancia) 1845. $-b,-c$ Guatemala $(J$. Constancia) 1848.

839. Diglossa sittoides.

Serrirostrum sittoides, D’Orb. \& Lafr. Syn. Av. ii. p. 25.

Diglossa sittoides, Scl. Ibis, 1875, p. 208.

a Bogota (Gardner) 1844.

840. Diglossa lafresnayi.

Uncirostrum lafresnayi, Boiss. Rev. Zool. 1840, p. 4.

Diglossa lafresnayi, Scl. Ibis, 1875, p. 214.

a Bogota (Stevens) 1844. 


\section{Diglossa humeralis.}

Agrilorhinus humeralis, Fraser, P. Z. S. 1840, p. 22.

Diglossa humeralis, Scl. Ibis, 1875, p. 215.

$a$ (Argent) 1852.

Apparently a 'Bogota' skin.

\section{Diglossa albilateralis.}

Diglossa albilateralis, Lafr. Rev. Zool. 1843, p. 98 : Scl. Ibis, 1875 , p. 216, pl. 5.

a Bogota (Bt. at Stevens's) 1852.

\section{Diglossa personata.}

Agrilorhinus personatus, Fraser, P. Z. S. 1840, p. 22.

Diglossa personata, Scl. Ibis, 1875, p. 218.

Uncirostrum cyaneum, Lafr. Rev. Zool. 1840, p. 102.

a Bogota (J. Gould) 1844.

\section{Digi،OSSOPIS.}

Diglossopis, Sclater, Ann. \& Mag. N. H. ser. 2, xvii. p. 467 (1856).

\section{Diglossopis cærulescens.}

Diglossopis coerulescens, Scl. l. s. c.; Ibis, 1875, p. 219. $a$ (Bt. at Stevens's) 1844.

A common Columbian species.

\section{SUBFAMILY.-CGEREBIN $Æ$ E.}

\section{Conirostrum.}

Conirostrum, D’Orbigny \& Lafresnaye, Syn. Av. ii. p. 25 (1838).

\section{Conirostrum sitticolor.}

Conirostrum sitticolor, Lafr. Rev. 7ool. 1840, p. 102: Scl. Cat. Am. B. p. 49.

a Bogota (J. Gould) 1844. 


\section{Conirostrum rufum.}

Conirostrum rufum, Lafr. Mag. Zool. 1843, sub tab. 35: Scl. Cat. Am. B. p. 50.

1844.

a Bogota (Bt. at Stevens's) 1844. - b Bogota (Gardner)

\section{Conirostrum albifrons.}

Conirostrum albifrons, Lafr. Rev. Zool, 1842, p. 301 ; Mag. Zool. pl. 35 : Scl. Cat. Am. B. p. 50.

$a$ Bogota (J. Gould) 1844. - b (Argent) 1852.

\section{DaCNIS.}

Dacnis, Cuvier, Règn. Anim. i. p. 395 (1817).

\section{Dacnis cayana.}

Motacilla cayana, Linn. Syst. Nat. i. p. 336.

Dacnis cayana, Strickl. Contr. Orn. 1851, p. 15: Scl. Cat. Am. B. p. 50.

$a \hat{f},-b q,-c$ Brazil (Leadbeater) 1834. $-d q$ Trinidad (Argent) 1851 .

\section{Dacnis angelica.}

Dacnis angelica, De Filippi, Bp. Atti Scienz. Ital. 1845, p. 404; Consp. i. p. 400 : De Filippi, Mus. Mediol. Aves, p. 32 (1847): Scl. Contr. Orn. 1851, p. 107.

Dacnis melanotis, Strickl. Contr. Orn. 1851, p. 16: Scl. Cat. Am. B. p. 51 : Scl. \& Salv. Nomencl. p. 16.

$a$ Demerara? (Cashmore) 1843. - b (Dewgard) 1849. -c Argent (1851).

$a$ is probably the type of $D$. melanotis, Strickl. Both $a$ and $b$ (a female) are made up in the form of Cayenne skins. $\quad c$ is probably from Bogota.

As the Cayenne and New Granadian forms of this bird seem inseparable as species, the name they should bear is $D$. angelica, and not $D$. melanotis, that adopted by $\mathrm{Mr}$ Sclater and myself in our 'Nomenclator.' 


\section{Dacnis speciosa.}

Sylvia speciosa, Max. Beitr. iii. p. 708.

Dacnis speciosa, Scl. Cat. Am. B. p. 52. 1838.

a Brazil (N. C. Strickland) 1838. -b (N. C. Stricklana)

\section{Chlorophanes.}

Chlorophanes, Reichenbach, Handb. Sp. Orn. i. Abth. 2. p. 233 (1853).

\section{Chlorophanes spiza.}

Certhia spiza, Linn. Syst. Nat. i. p. 186.

Coereba atricapilla, Vieill. N. Dict. d'Hist. N. xiv. p. 50.

Chlorophanes atricapilla, Scl. Cat. Am. B. p. 52.

$a$ (Burl) 1834. - b (Cashmore) 1839.

A widely-ranging South American species. There can be no doubt, $\mathrm{C}$ think, that this bird is the 'Green Black-cap Fly-catcher' of Edwards (Av. pl. 25. f. 1), upon which chiefly Linnæus founded his Certhia spiza.

\section{Cerreba.}

Coreba, Vieillot, Ois. Am. Sept. ii. p. 70 (1807).

\section{Cœreba cyanea.}

Certhia cyanea, Linn. Syst. Nat. i. p. 188.

Coreba cyanea, Scl. Cat. Am. B. p. 52.

$a,-b,-c,-d$ Brazil (Johnson) 1837. -e (N. C. Strickland) 1838. - $f$, $-g$ Guatemala ( $J$. Constancia) 1845. - $h$ Guatemala (J. Constancia) 1851. - i Trinidad (J. Taylor).

\section{Cœreba cærulea.}

Certhia ccerulea, Linn. Syst. Nat. i. p. 185.

Coereba ccerulea, Scl. Cat. Am. B. p. 53.

$a$ S. America (N. C. Striclland) 1838. -b (Williams) 1846. -c Trinidad (Argent) 1851.

$b$, from the shape of the skin, is evidently of Cayenne origin.

s. C. 


\section{Certhiola.}

Certhiola, Sundevall, Öfvers. Vet. Ac. Handl. 1835, p. 99.

\section{Certhiola luteola.}

Certhiola luteola, Cab. Mus. Hein. i. p. 96 : Baird, N. Am. B. i. p. 427.

$a$ Tobago (J. Kirk) 1841. - b Trinidad (Argent) 1851.

\section{Certhiola barbadensis.}

Certhiola barbadensis, Baird, N. Am. B. i. p. 428.

a (E. Brown) 1850.

Agrees best with Prof. Baird's description of the Certhiola of the island of Barbadoes.

\section{Certhiola chloropyga.}

Certhiola chloropyga, Cab. Mus. Hein. i. p. 97 : Baird, N. Am. B. i. p. 428.

a Brazil (Burl) 1834. -b Brazil (Askew) 1837. - c Brazil (G. Lloyd) 1838. —d Brazil (N. C. Strickland) 1838.

\section{GLOSSIPTILA.}

Glossiptila, Sclater, P. Z. S. 1856, p. 269.

\section{5\%. Glossiptila ruficollis.}

Tanagra ruficollis, Gm. Syst. Nat. i. p. 894.

Tanagrella ruficollis, Gosse, B. Jam. p. 236.

Glossiptila ruficollis, Scl. l.s. c.; Cat. Am. B. p. 54.

a W. Indies (N. C. Strickland) 1838.

A species peculiar to the island of Jamaica.

SECTION. OSCINES CONIROSTRES.

\section{FAMILY.-TANAGRIDÆ.}

\section{Procnias.}

Procnias, Illiger, Prodr. p. 228 (1811) (partim)1.

\section{Procnias tersa.}

Ampelis tersa, Linn. Syst. Nat. i. p. 298.

1 Concerning the application of this name, see Strickland Ann. \& Mag. N. H. vii. p. 29 , and viii. p. 373 . 
Procnias tersa, Temm. Pl. Col. Tabl. Meth. p. 16: Scl. Cat. Am. B. p. 54.

Procnias ventralis, Temm. Pl. Col. 5. 1851.

$a,-b,-c$ Brazil (N.C. Strickland) 1838. —d Brazil (Argent)

\section{Chlorophonia.}

Chlorophonia, Bonaparte, Rev. et Mag. de Zool. 1851, p. 157.

\section{Chlorophonia viridis.}

Tanagra viridis, Vieill. N. Dict. d'Hist. N. xxxii. p. 426.

Chlorophonia viridis, Bp. l. s. c.: Scl. Cat. Am. B. p. 55.

$a$ Brazil (N. C. Strickland) 1838. -b (Argent) 1851.

Euphonia.

Euphonia, Desmarest, Hist. Nat. Tang. (1805).

\section{Euphonia nigricollis.}

Tanagra nigricollis, Vieill. N. Dict. d'Hist. N. xxxii. p. 412.

Euphonia nigricollis, Scl. Cat. Am. B. p. 56.

$a$ Brazil (N. C. Strickland) 1838. - b S. America (Seaman) 1840. — B Bogota (Bt. at Stevens's) 1845.

\section{Euphonia chlorotica.}

Tanagra chlorotica, Linn. Syst. Nat. i. p. 317.

Euphonia chlorotica, Strickl. Contr. Orn. 1851, p. 72: Scl. Cat. Am. B. p. 57.

$a$ Brazil (N. C. Strickland) 1838. -b (Bt. at Birmingham) 1849. $\longrightarrow c$ (Argent) 1851. —d Brazil ? (Argent) 1852.

$c$ is a skin of Cayenne make: $d$ is probably from Bolivia.

\section{Euphonia trinitatis.}

Euphonia trinitatis, Strickl. Contr. Orn. 1851, p. 72: Scl. Cat. Am. B. p. 57.

$a$ Brazil (N. C. Strickland) 1838. - b Trinidad (P. L. Sclater) 1850.

$a$, though marked in $\mathrm{Mr}$ Sclater's handwriting ' $E$. strictifrons', is really a young male of $E$. trinitatis, the locality assigned to it being erroneous. $b$ is doubtless one of the types of the species. 
863. Euphonia minuta.

Euphonia minuta, Cab. Schomb. Guian. iii. p. 671: Scl. Cat. Am. B. p. 57.

Euphonia strictifrons, Strickl. Contr. Orn. 1851, p. 72: Scl. Contr. Orn. 1851, p. 84 .

$a(B t$. at Stevens's) 1845.

Probably one of the types of E. strictifrons. The species has a wide range over the northern portions of South America. It is also found in Central America as far north as Guatemala.

\section{Euphonia xanthogastra.}

Euphonia xanthogastra, Sundev. Vet. Ac. Handl. 1833, p. 310, pl. 10. f. 1: Strickl. Contr. Orn. 1851, p. 73: Scl. Cat. Am. B. p. 57 .

$a$ Brazil (N. C. Strickland) 1838.

\section{Euphonia chalybea.}

Tanagra chalybea, Mikan, Faun. et Fl. Bras. pl. 2. f. 1, 2.

Euphonia chalybea, Strickl. Contr. Orn. 1851, p. 71: Scl. Cat. Am. B. p. 58. 1853.

$a$ S. America (N. C. Strickland) 1838. -b Brazil (Argent)

\section{Euphonia violacea.}

Tanagra violacea, Linn. Syst. Nat. i. p. 314.

Euphonia violacea, Scl. Cat. Am. B. p. 58.

$a$ Brazil (Askew) 1837. —b, —c Trinidad (Argent) 1851.

\section{Euphonia laniirostris.}

Euphonia lanïrostris, D'Orb. \& Lafr. Syn. Av. p. 30: D'Orb. Voy. Ois. p. 266, pl. 22, f. 1: Scl. Contr. Orn. 1851, p. 86.

Euphonia crassirostris, Scl. P. Z. S. 1856, p. 277; Cat. Am. B. p. 58.

a ô Bolivia (Argent) 1852.

A recent examination of D'Orbigny's specimens in the Paris Museun convinced me that $E$. crassirostris, Scl. belongs to this species.

\section{Euphonia hirundinacea.}

Euphonia hirundinacea, Bp. P. Z. S. 1837, p. 117 : Scl. Cat. Am. B. p. 59.

$a$ Guatemala (J. Constancia) 1851. 


\section{Euphonia chrysopasta.}

Euphonia chrysopasta, Scl. \& Salv. P. Z. S. 1869, p. 438, pl. 30, f. 1, 2.

Euphonia serrirostris, D’Orb. \& Lafr. Syn. Av. p. 30 (partim?). a Brazil? (Argent) 1852.

Most probably from Bolivia. On examining D'Orbigny's specimens in the Paris Museum of his E. serrirostris, I came to the conclusion that they were females of the species described by Mr Sclater and myself from the Ucayali as $E$. chrysopasta. The bird figured by D'Orbigny (Voy. Am. Merid. Ois. pl. 21), however, represents the female of $\boldsymbol{E}$. chlorotica. I have lately received specimens from Bolivia in no way differing from the Ucayali bird.

\section{Euphonia cayana.}

Tanagra cayana, Linn. Syst. Nat. i. p. 315.

Euphonia cayana, Scl. Cat. Am. B. p. 59.

$a$ (Mansfield) 1839.

A Guiana species.

\section{Euphonia pectoralis.}

Pipra pectoralis, Lath. Ind. Orn. Suppl. p. Ivii.

Euphonia pectoralis, Scl. Cat. Am. B. p. 59.

$a$ Brazil (N. C. Strickland) 1838. - b S. America (Osman) 1846.

\section{Euphonia jamaica.}

Fringilla jamaica, Linn. Syst. Nat. i. p. 323.

Euphonia jamaica, Gosse, B. Jam. p. 238 : Scl. Cat. Am. B. p. 60 .

$a \hat{\delta},-b q$ Jamaica (P. H. Gosse) 1848.

\section{TANAGRella.}

Tanagrella, Swainson, Classif. B. ii. p. 286 (1837).

\section{Tanagrella velia.}

Motacilla velia, Linn. Syst. Nat. i. p. 336.

Tanagrella velia, Scl. Cat. Am. B. p. 60.

a (Dewgard) 1846.

A Cayenne skin. 


\section{Tanagrella cyanomelæna.}

Tanagra cyanomelas, Max. Beitr. iii. p. 453.

Tanagrella cyanomelas, Scl. Cat. Am. B. p. 60.

Tanagrella multicolor, Sw. An. in Menag. p. 313: Strickl. Ann. \& Mag. N. H. vii. p. 32.

$a$ Brazil (Askew) 1833. - b Brazil (Askew) 1837. — Brazil (N. C. Strickland) 1838.

Swainson's $T$. multicolor is identical with this species and not with T'velia as stated by Strickland $l . s . c$.

\section{Chlorochrysa.}

Chlorochrysa, Bonaparte, Compt. Rend. xxxii. p. 76 (1851).

875. Chlorochrysa phœnicotis.

Calliste phonicotis, Bp. l. s. c.

Chlorochrysa phonicotis, Scl. Contr. Orn. 1851, p. 100, pl. 73, f. 2 ; Ibis, 1875 , p. 466.

a Quito (W. Jameson) 1852.

\section{PipRIdeA.}

Pipraeidea, Swainson, Zool. Journ. iii. p. 173 (1827).

Piproidea, Strickland, Ann. \& Mag. N. H. vii. p. 29.

Pipridea, Sclater, P. Z. S. 1856, p. 265.

\section{Pipridea melanonota.}

Tanagra melanonota, Vieill. N. Dict. d'Hist. N. xxxii. p. 407.

Pipridea melanonota, Scl. Cat. Am. B. p. 61.

$a,-b$ \& Brazil (N. C. Strickland) 1838. -c Bogota? (Bt. at Stevens's) 1845.

\section{Diva.}

Diva, Sclater, Tan. Cat. Sp. p. 16 (1854).

\section{Diva vassori.}

Tanagra (Euphone?) vassori, Boiss. Rev. Zool. 1840, p. 4.

Diva vassori, Scl. Cat. Am. B. p. 62.

a Bogota (J. Gould) 1844 . 
Calliste.

Calliste, Boie, Isis, 1826, p. 974.

\section{Calliste tatao.}

Tanagra tatao, Linn. Syst. Nat. i. p. 315.

Calliste tatao, Scl. Cat. Am. B. p. 62.

a (Cashmore) 1843.

A Cayenne skin.

879. Calliste yeni.

Aglaia chilensis, Vig. P. Z. S. 1832, p. 3 (nom. inept.).

Aglaia yeni, Lafr. \& D’Orb. Syn. Av. i. p. 31.

Calliste yeni, Scl. Cat. Am. B. p. 62.

a (Argent) 1852.

A species of the Upper Amazon and Eastern slopes of the Andes.

880. Calliste tricolor.

Tanagra tricolor, Gm. Syst. Nat. i. p. 891.

Calliste tricolor, Scl. Cat. Am. B. p. 63.

$a,-b$ Brazil (G. Lloyd) 1838.

881. Calliste festiva.

Tanagra festiva, Shaw, Nat. Misc. xiii. pl. 537.

Calliste festiva, Scl. Cat. Am. B. p. 63.

a Brazil (G. Lloyd) 1838. -b Brazil (N. C. Strickland) 1838.

\section{Calliste cyaneiventris.}

Tanagra cyanoventris, Vieill. N. Dict. d'Hist. N. xxxii. p. 426.

Calliste cyaneiventris, Scl. Cat. Am. B. p. 63.

a Brazil (Askew) 1834. -b Brazil (N. C. Strickland) 1838.

883. Calliste thoracica.

Tanagra thoracica, Temm. Pl. Col. 42, f. 1.

Calliste thoracica, Scl. Cat. Am. B. p. 63.

a Brazil (N. C. Strickland) 1838.

884. Calliste schranki.

Tanagra schranki, Spix. Av. Bras. ii. p. 38, pl. 51.

Calliste schranki, Scl. Cat. Am. B. p. 64.

a Brazil (N. C. Strickland) 1838. 


\section{Calliste punctata.}

Tanagra punctata, Linn. Syst. Nat. i. p. 316.

Calliste punctata, Scl. Cat. Am. B. p. 64.

a Cayenne (P. L. Sclater) 1850.

886. Calliste guttata.

Calliste guttata, Cab. Mus. Hein. i. p. 26: Scl. Cat. Am. B. p. 64 .

Calliste chrysophrys, Scl. Contr. Orn. 1851, pp. 24, 54, pl. 69, f. 2.

$a$ S. America (T. W. Nicholls) 1841. -b Trinidad (Argent) 1851.

\section{Calliste aurulenta.}

Tanagra (Aglaia) aurulenta, Lafr. Rev. Zool. 1843, p. 290.

Calliste aurulenta, Scl. Cat. Am. B. p. 65.

a (Carfrae) 1850.

Found in Columbia and Ecuador.

888. Calliste vitriolina.

Callispiza vitriolina, Cab. Mus. Hein. i. p. 28.

Calliste vitriolina, Scl. Cat. Am. B. p. 66.

Calliste ruficapilla, Scl. Contr. Orn. 1851, p. 61.

a Bogota (Bt. at Stevens's) 1845.

889. Calliste cayana.

Tanagra cayana, Linn. Syst. Nat. i. p. 315.

Calliste cayana, Scl. Cat. Am. B. p. 66.

$a$ (Cashmore) 1843. - b Trinidad (Argent) 1851. -c (Bt. at Liverpool) 1853.

Both $a$ and $c$ are Cayenne skins.

890. Calliste cucullata.

Aglaia cucullata, Sw. Orn. Draw. pl. 7.

Callista cucullata, Scl. Mon. Call. p. 45, pl. 20.

$a$ S. America ( $F$. Holme) 1838.

Agrees with Swainson's type. The exact habitat of this species has not yet been discovered.

\section{Calliste flava.}

Tanagra flava, Gm. Syst. Nat. i. p. 896.

Calliste flava, Scl. Cat. Am. B. p. 66. 
a Brazil (Mansfield) 1832. - b, —c Brazil (Askew) 1834. -d Brazil (N. C. Strickland) 1838.

892. Calliste pretiosa.

Calliste pretiosa, Cab. Mus. Hein. i. p. 27: Scl. Cat. Am. B. p. 66 .

Calliste castanonota, Scl. Contr. Orn. 1851, p. 63. $a$ (N. C. Strickland) 1838.

A species of South Brazil.

\section{Calliste melanonota.}

Aglaia melanonota, Sw. Orn. Draw. pls. 31, 43.

Calliste melanonota, Scl. Cat. Am. B. p. 67.

$a$ (Fenton) 1850. -b (Argent) 1851.

A Brazilian species.

894. Calliste gyrola.

Tanagra gyrola, Linn. Syst. Nat. i. p. 315.

Calliste gyrola, Scl. Cat. Am. B. p. 67.

a Demerara? (Bt. at Stevens's) 1845.

A species peculiar to Guiana.

895. Calliste gyroloides.

Aglaia gyroloides, Lafr. Rev. Zool. 1847, p. 277.

Calliste gyroloides, Scl. Cat. Am. B. p. 67.

$a$ Bogota (Bt. at Stevens's) 1845. - b Brazil? (Argent) 1852.

$b$ is probably from Bolivia.

896. Calliste desmaresti.

Calliste desmaresti, Gray, Gen. B. ii. p. 366: Scl. Cat. Am. B. p. 68 .

$a$ Trinidad (Argent) 1851. $-b,-c$ Trinidad (J. Taylor).

897. Calliste brasiliensis.

Tanagra brasiliensis, Linn. Syst. Nat. i. p. 316.

Calliste brasiliensis, Scl. Cat. Am. B. p. 68.

a Brazil (Askew) 1834 .

898. Calliste vieilloti.

Calliste vieilloti, Scl. P. Z. S. 1856, p. 257 ; Cat. Am. B. p. 69.

a Trinidad (J. Taylor). 


\section{Calliste ruficervix.}

Tanagra ruficervix, Prév. \& Des Murs, Voy. Vénus. Ois. pl. 5. f. 1 .

Calliste ruficervix, Scl. Cat. Am. B. p. 69.

a Bogota (Bt. at Stevens's) 1845.

\section{Calliste nigriviridis.}

Tanagra (Aglaia) nigroviridis, Lafr. Rev. Zool. 1843, p. 69. Calliste nigriviridis, Scl. Cat. Am. B. p. 69.

a Bogota (Bt. at Stevens's) 1844.

\section{Calliste nigricincta.}

Aglaic nigrocincta, Bp. P. Z. S. 1837, p. 121.

Calliste nigricincta, Scl. Cat. Am. B. p. 70.

Calliste thalassina, Strickl. Ann. \& Mag. N. H. xiii. p. 419.

a (Thomas) 1842.

Marked as the "original type specimen" of $C$. thalassina, Strickland. The species is not uncommon in Columbia, and the countries adjoining, but it is not found in Mexico as supposed by Strickland.

902. Calliste cyaneicollis.

Aglaia cyanicollis, Lafr. \& D’Orb. Syn. Av. p. 33.

Calliste cyaneicollis, Scl. Cat. Am. B. p. 70.

$a$ (Argent) 1851. - b (Argent) 1852.

$a$ has a blue throat and belongs to the race usually found in Columbia, $b$ has the throat the same colour as the head and belongs to the Bolivian form from which country the skin probably came.

\section{Calliste labradorides.}

Tanagra (Aglaia) labradorides, Boiss. Rev. Zool. 1840, p. 67.

Calliste labradorides, Scl. Cat. Am. B. p. 70.

$a$ Bogota (J. Gould) 1844.

\section{IRIDORNIS.}

Iridosornis, Lesson, Echo du Monde Savant, 1844, p. 80.

Iridornis, Scl. P. Z. S. 1856, p. 242.

904. Iridornis dubusia.

Arremon rufivertex, Lafr. Rev. Zool. 1842, p. 335 (err. pro T. ruficervix, Prév.) $c f$. Strickl. l. $c$. 
Tanagra dubusia, Bp. Consp. i. p. 239.

Iridosornis dubusia, Strickl. Contr. Orn. 1852, p. 127, pl. 94.

Iridornis dubusia, Scl. Cat. Am. B. p. 72.

$a$ (Warwick).

A Columbian species.

\section{Pecilothraupis.}

Poecilothraupis, Cabanis, Mus. Hein. i. p. 30 (1850).

\section{Pœcilothraupis lunulata.}

Tanagra lunulata, Du Bus, Bull. Ac. Brux. vi. pt. 1. p. 439; Esq. Orn. pl. 4.

Pocilothraupis lunulata, Scl. Cat. Am. B. p. 72.

a Bogota (Bt. at Stevens's) 1845.

906. Pocilothraupis igniventris.

Aglaia igniventris, D'Orb. \& Lafr. Syn. Av. i. p. 32.

Tanagra igniventris, D'Orb. Voy. Am. Merid. Ois. p. 275, pl. 25, f. 2.

Pœcilothraupis igniventris, Scl. P. Z. S. 1856, p. 242.

a Peru (N. C. Strickland) 1840.

An original label records that this specimen was obtained in the mountains eighty leagues from Lima.

\section{STEPHANOPHORUS.}

Stephanophorus, Strickland, P. Z. S. 1841, p. 30.

\section{Stephanophorus leucocephalus.}

Tanagra leucocephala, Vieill. N. Dict. d'Hist. N. xxxii. p. 408.

Stephanophorus leucocephalus, Scl. Cat. Am. B. p. 73.

Stephanophorus cceruleus (Vieill.) Strickl. l. s. c.

a Brazil (N. C. Strickland) 1838.

Buthraupis.

Buthraupis, Cabanis, Mus. Hein. i. p. 29 (1850).

908. Buthraupis cucullata.

Tanagra cucullata, Jard. Ill. Orn. iv. pl. 43.

Buthraupis cucullata, Scl. Cat. Am. B. p. 73.

a Bogota (J. Gould) 1844. 


\section{Buthraupis eximia.}

Tanagra eximia, Boiss. Rev. Zool. 1840, p. 66.

Buthraupis eximia, Scl. Cat. Am. B. p. 73.

a Bogota (Bt. at Stevens's) 1844.

\section{Compsocoma.}

Compsocoma, Cabanis, Mus. Hein. i. p. 140 (1850).

\section{Compsocoma victorini.}

Tachyphonus victorini, Lafr. Rev. Zool. 1842, p. 336.

Compsocoma victorini, Scl. Cat. Am. B. p. 73.

a Bogota (Bt. at Stevens's) 1845.

Dubusia.

Dubusia, Bonaparte, Compt. Rend. xxxi. p. 424 (1850).

\section{Dubusia tæniata.}

Tanagra (Tachyphonus) treniata, Boiss. Rev. Zool. 1840, p. 67. Dubusia tceniata, Scl. Cat. Am. B. p. 74.

a Bogota (J. Gould) 1844.

\section{TANAGRA.}

Tanagra, Linnæus, Syst. Nat. i. p. 313 (1766) (partim).

\section{Tanagra episcopus.}

Tanagra episcopus, Linn. l. s. c. p. 316: Strickl. Ann. \& Mag. N. H. vii. p. 32: Scl. Cat. Am. B. p. 74.

$a$ S. America (N. C. Strickland) 1838. -b (Bt. at Stevens's) 1845 .

$a$ is a Cayenne skin.

\section{Tanagra cana.}

Tanagra cana, Sw. Orn. Draw. pl. 37: Scl. Cat. Am. B. p. 75.

Tanagra (Aglaia) diaconus, Less. Rev. Zool. 1842, p. 175 : Scl. \& Salv. Ibis, 1859, p. 16.

a Tobago (Sir W. Jardine) 1844. - $b,-c$ Bogota (Bt. at Stevens's) 1845. $-d$ Trinidad (Argent) 1851. $-c$ Guatemala ( $J$. ('onstancia) 1851. 


\section{Tanagra sayaca.}

Tanagra sayaca, Linn. Syst. Nat. i. p. 316: Max. Beitr. iii. p. 484.

Tanagra cyanoptera, Scl. Cat. Am. B. p. 75 (partim).

$a$ Brazil (N. C. Strickland) 1838.

\section{Tanagra cyanoptera.}

Saltator cyanopterus, Vieill. N. Dict. d'Hist. N. xiv. p. 104.

Tanagra cyanoptera, Scl. Cat. Am. B. p. 75 (partim).

$a,-b$ Brazil (N. C. Strickland) 1838.

The form of this Tanager found in South Brazil and the Argentine Republic should be called $T$. cyanoptera, whilst to the more northern bird the name $T$. sayaca seems applicable.

\section{Tanagra ornata.}

Tanagra ornata, Sparrm. Mus. Carls. No. 95: Scl. Cat. Am. B. p. 76.

Tanagra archiepiscopus, Desm. Tan. pl. 17.

a Brazil (Burl) 1834.

\section{Tanagra palmarum.}

Tanagra palmarum, Max. Reise n. Bras., ii. p. 76 ; Beitr. iii. p. 489. Scl. Cat. Am. B. p. 76.

Tanagra olivascens, Licht. Verz. Doubl. p. 32.

"Tanagra melanoptera, Hartl.," Scl. P. Z. S. 1856, p. 235.

$a,-b$ Brazil (N. C. Strickland) 1838. —c (Bt. at Stevens's) 1845. - $d$ Trinidad (Argent) 1851.

\section{Tanagra abbas.}

Tanagra abbas, Licht. Preis-Verz. Mex. Vög. p. 2; cf. Journ. f. Orn. 1863 p. 57: Scl. Cat. Am. B. p. 76.

Tanagra vicarius, Less. Cent. Zool. p. 206, pl. 68.

$a$ Bogota (J. Gould) 1844. - b Guatemala (J. Constancia) 1851.

The locality assigned to $a$ is erroneous, the species being strictly confined to Central America north of Costa Rica, and Mexico.

\section{Tanagra darwini.}

Tanagra darwini, Bp. P. Z. S. 1837, p. 121 : Scl. Cat. Am. B. p. 76 . 
$a$ Chili (N. C. Strickland) 1838.

Though found in Ecuador and Peru this species is not known to inhabit Chili.

920. Tanagra striata.

Tanagra striata, Gm. Syst. Nat. i. p. 899 : Scl. Cat. Am. B. p. 77.

a S. America (N. C. Strickland) 1838. -b (N. C. Strickland) 1838.

Found in South Brazil, Bolivia and the Argentine Republic.

\section{Tanagra cyanocephala.}

Aglaia cyanocephala, D'Orb. \& Lafr. Syn. Av. i. p. 32.

Tanagra cyanocephala, Scl. Cat. Am. B. p. 77.

$a$ Bogota (J. Gould) 1844. -b (Bt. at Birmingham) 1850.

SPINDALIS.

Spindalis, Jardine \& Selby, Ill. Orn. iv. sub pl. 9 (1836).

922. Spindalis nigricephala.

Tanagra nigricephala, Jameson, Edinb. N. Phil. Journ. xix. p. 213.

Spindalis nigricephala, Scl. Cat. Am. B. p. 77.

Spindalis bilineatus, Jard. \& Selb. Ill. Orn. iv. pl. 9.

a q W. Indies ( $N:$ C. Strickland) 1838. -b (Cashmore) 1843.

A species peculiar to the island of Jamaica.

RAMPHOCGLUS.

Ramphocelus, Desmarest, Tang. p. 5 (1805).

923. Ramphocœlus brasilius.

Tanagra brasilia, Linn. Syst. Nat. i. p. 314.

Ramphocelus brasilius, Scl. Cat. Am. B. p. 78.

a Brazil (Mansfield) 1834. - b f Brazil (Dr G. Lloyd) 1838. -c ổ Brazil (N. C. Strickland) 1838.

\section{Ramphoccelus nigrigularis.}

Tanagra nigrogularis, Spix. Av. Bras. ii. p. 35, pl. 47.

Ramphocelus nigrigularis, Scl. Cat. Am. B p. 78.

a Brazil? (Argent) 1852.

Probably a Bolivian skin. 


\section{Ramphocœlus jacapa.}

Tanagra jacapa, Linn. Syst. Nat. i. p. 313.

Ramphocelus jacapa, Scl. Cat. Am. B. p. 78.

a Brazil (N. C. Strickland) 1838. -b Brazil (Sir W. Jardine) 1844. - c Trinidad (Argent) 1851.

This species is not found south of the valley of the Amazon.

\section{Ramphocolus atrosericeus.}

Ramphocelus atrosericeus, D'Orb. \& Lafr. Syn. Av. i. p. 34; D'Orb. Voy. Am. Sept. Ois. p. 280, pl. 26, f. 1: Scl. Cat. Am. B. p. 79.

$a \hat{o},-b q$ Brazil? (Argent) 1852.

Probably both from Bolivia.

\section{2\%. Ramphocœlus dimidiatus.}

Ramphocelus dimidiatus, Lafr. Mag. Zool. 1837, cl. ii. pl. 81. Scl. Cat. Am. B. p. 79.

$a$ (Bt. at Stevens's) 1845. -b (Argent) 1853.

Both these skins are of Bogota make.

928. Ramphocœlus icteronotus.

Ramphocelus icteronotus, Bp. Rev. Zool. 1838, p. 8: Scl. Cat. Am. B. p. 80.

a Sallango (Capt. Kellett) 1850.

Obtained on the west coast of South America during the Voyage of H.M.S. 'Herald.'

\section{Pyranga.}

Pyranga, Vieillot, Analyse, p. 32 (1816).

929. Pyranga rubra.

Tanagra rubra, Linn. Syst. Nat. i. p. 314.

Pyranga rubra, Baird, Brew. \& Ridgw. N. Am. B. i. p. 435.

a N. America 1838. - $b$ \& Carlisle, Pennsylvania 13 May 1845 (S. F. Baird) 1847.

930. Pyranga æstiva.

Tanagra cestiva, Gm. Syst. Nat. i. p. 889.

Pyranga cestiva, Scl. \& Salv. Ibis, 1859, p. 15 : Scl. Cat. Am. B. p. 80 : Baird, Brew. \& Ridgw. N. Am. B. i. p. 441. 
$a,-b$ Guatemala (J. Constancia) 1845. -c Bogota (Bt. at Stevens's) 1845.

\section{Pyranga saira.}

Tanagra saira, Spix. Av. Bras. ii. p. 35, pl. 48, f. 1.

Pyranga saira, Scl. Cat. Am. B. p. 80.

$a,-b,-c$ (N. C. Strickland) 1838.

A Brazilian species.

932. Pyranga hepatica.

Pyranga hepatica, Sw. Phil. Mag. 1827, p. 438: Scl. \& Salv. Ibis, 1859, p. 15: Scl. Cat. Am. B. p. 81.

$a$ Guatemala (J. Constancia) 1848.

\section{Pyranga azaræ.}

Pyranga azarce, D’Orb. Voy. Am. Merid. Ois. p. 264. $a$ (N. C. Strickland) 1838.

A Bolivian species.

\section{Pyranga ludoviciana.}

Tanagra ludoviciana, Wils. Am. Orn. iii. p. 27, pl. 20, f. 1.

Pyranga ludoviciana, Scl. \& Salv. Ibis, 1859, p. 15: Scl. Cat. Am. B. p. 81: Baird, Brew. \& Ridgw. i. p. 437.

$a$ Guatemala (J. Constancia) 1848. - b Guatemala (J. Constancia) 1851.

\section{Pyranga erythromelæna.}

Tanagra erythromelcena, Licht. Preis-Verz. Mex. Vög. p. 2; cf. Journ. f. Orn. 1863, p. 57.

Pyranga erythromelena, Scl. \& Salv. Ibis, 1859, p. 15 : Scl. Cat. Am. B. p. 81.

Pyranga bivittata, Lafr. Rev. Zool. 1842, p. 70.

Pyranga leucoptera, Trudeau, Journ. Ac. Phil. viii. p. 160.

$a$ f Guatemala ( $J$. Constancia) 1848. $-b$ Guatemala ( $J$. Constancia) 1851. 
ORTHOGONYS.

Orthogonys, Strickland, Ann. \& Mag. N. H. xiii. p. 421 (1844).

936. Orthogonys viridis.

Tanagra viridis, Av. Bras. ii. p. 36, pl. 48, f. 2.

Orthogonys viridis, Strickl. l. s. c.: Scl. Cat. Am. B. p. 82.

a Brazil (N. C. Strickland) 1838.

\section{LAMPROTES.}

Lamprotes, Swainson, Classif. B. ii. p. 283 (1837). 93\%. Lamprotes loricatus.

Tanagra loricata, Licht, Verz. Doubl. p. 31.

Lamprotes loricatus, Scl. Cat. Am. B. p. 82.

a. Brazil (Askew) 1834. —b Brazil (N. C. Strickland) 1838.

Pheenicothraupis.

Phonicothraupis, Cabanis, Mus. Hein. p. 24 (1850).

938. Phœnicothraupis rubica.

Saltator rubicus, Vieill. N. Dict. d'Hist. N. xiv. p. 107.

Phonicothraupis rubica, Scl. Cat. Am. B. p. 82.

Tanagra flammiceps, Temm. Pl. Col. 177.

Tanagra porphyrio, Licht. Verz. Doubl. p. 31.

a Brazil (Askew) 1837. - b Brazil (G. Lloyd) 1838. —c î, -d (N. C. Strickland) 1838. - e Brazil (Arthur Strickland) 1840 .

939. Phœnicothraupis rubicoides.

Saltator rubicoides, Lafr. Rev. Zool. 1844, p. 41.

Phonicothraupis rubicoides, Scl. \& Salv. Ibis, 1859, p. 15:

Scl. Cat. Am. B. p. 83.

$a$ Guatemala (J. Constancia) 1851.

LANio.

Lanio, Vieillot, Analyse, p. 40 (1816).

940 Lanio atricapillus.

Tanagra atricapilla, Gm. Syst. Nat. i. p. 899.

Lanio atricapilla, Scl. Cat. Am. B. p. 83.

a (Cashmore) 1843. -3 (Bt. at Liverpool) 1853.

Both these skins are of Cayenne make.

S. C. 


\section{Trichothraupis.}

Trichothraupis, Cabanis, Mus. Hein. p. 23 (1850).

\section{Trichothraupis quadricolor.}

Tachyphonus quadricolor, Vieill. N. Dict. d'Hist. Nat. xxxii. p. 359.

Trichothraupis quadricolor, Scl. Cat. Am. B. p. 84. $a,-b$ Brazil (N. C. Strickland) 1838.

\section{TACHYPhONuS.}

Tachyphonus, Vieillot, Analyse, p. 33 (1816).

942. Tachyphonus melaleucus.

Oriolus melaleucus, Sparrm. Mus. Carls. fasc. ii. No. 31.

Tachyphonus melaleucus, Scl. Cat. Am. B. p. 84.

Tanagra nigerrima, Sw. Quart. Journ. Sc. xx. p. 62.

a Brazil (Askew) 1834. —b Guiana (N. C. Strickland) 1838. -c (Bt. at Stevens's) 1844 .

\section{Tachyphonus luctuosus.}

Tachyphonus luctuosus, D’Orb. \& Lafr. Syn. Av. i. p. 29: Scl. Cat. Am. B. p. 85.

$a \hat{\delta},-b q$ Brazil? (Argent) 1852. $-c$ Trinidad (P. L. Sclater).

$a$ and $b$ are probably both Bolivian skins.

\section{Tachyphonus phoniceus.}

Tachyphonus phoenicius, Sw. An. in Menag. p. 311: Scl. P. Z. S. 1856 , p. 116.

Tachyphonus saucius, Strickl. Ann. \& Mag. N. H. xiii. p. 419.

$$
\text { a (Thomas) } 1842 .
$$

A note on the label of this specimen, the type of $P$. saucius, shews that Strickland recognized the identity of his bird with Swainson's $T$. phoeniceus. The species is found in the forests of the Upper Amazon.

\section{4:5. Tachyphonus cristatus.}

Tanagra cristata, Gm. Syst. Nat. i. p. 898.

Tachyphonus cristatus, Scl. Cat. Am. B. p. 85. 
a Brazil (N. C. Strickland) 1834. -b Brazil (Askew) 1837. - c, —d Brazil (N. C. Strickland) 1838. -e S. Arnerica (Bt. at Stevens's) 1843. - $f$ (Thomas) 1845.

946. Tachyphonus surinamus.

Turdus surinamus, Linn. Syst. Nat. i. p. 297.

Tachyphonus surinamus, Scl. Cat. Am. B. p. 85.

Tanagra desmaresti, Sw. Quart. Journ. Sc. xx. p. 67.

$a$ (Cashmore) 1843.

A skin of Cayenne make.

94\%. Tachyphonus coronatus.

Agelaius coronatus, Vieill. Enc. Méth. p. 711.

Tachyphonus coronatus, Scl. Cat. Am. B. p. 85.

Tanagra coryphcea, Licht. Verz. Doubl. p. 31.

a Brazil (N. C. Stricleland) 1838. -b Brazil (G. Lloyd) 1838.

\section{Cypsnagra.}

Cypsnagra, Lesson, Traité d'Orn. p. 460 (1831).

948. Cypsnagra ruficollis.

Tanagra ruficollis, Licht. Verz. Doubl. p. 30.

Cypsnagra ruficollis, Scl. Cat. Am. B. p. 86.

$a,-b$ Brazil (N. C. Strickland) 1838.

\section{Nemosia.}

Nemosia, Vieillot, Analyse, p. 32 (1816).

949. Nemosia pileata.

Tangara à coëffe noire de Cayenne, D'Aub. Pl. Enl. 72̈0, f. 2 unde,

Tanagra pileata, Bodd. Tab. Pl. Enl. p. 45.

Nemosia pileata, Scl. Cat. Am. B. p. 86.

$a$ Brazil (Askew) 1837. - b Brazil (N. C. Strickland) 1838.

950. Nemosia guira.

Motacilla guira, Linn. Syst. Nat. i. p. 335.

Nemosia guira, Scl. Cat. Am. B. p. 87.

$a$ (N. C. Strickland) 1838. -b Brazil (G. Lloyd) 1838. 
951. Nemosia flavicollis.

Nemosia favicollis, Vieill. N. Dict. d'Hist. N. xxii. p. 491 : Scl. Cat. Am. B. p. 87.

Sylvia melanoxantha, Licht. Verz. Doubl. p. 34.

$a$ Brazil (Mansfeld) 1834. - $b$ Brazil (Askew) 1836. - c (Arthur Strickland) 1840.

952. Nemosia ruficapilla.

Nemosia ruficapilla, Vieill. N. Dict. d'Hist. N. xxii. p. 493: Scl. Cat. Am. B. p. 87.

$a,-b$ Brazil (N. C. Strickland) 1838.

953. Nemosia fulvescens.

Nemosia fulvescens, Strickl. Ann. \& Mag. N. H. xiii. p. 420: Scl. \& Salv. P. Z. S. 1866, p. 180.

$a$ (N. C. Strickland) 1838.

The type of this Brazilian species.

\section{Pyrrhocoma.}

Pyrrhocoma, Cabanis, Mus. Hein. p. 138 (1851).

954. Pyrrhocoma ruficeps.

Tachyphonus ruficeps, Strickl. Ann. \& Mag. N. H. xiii. p. 419.

Pyrrhocoma ruficeps, Scl. Cat. Am. B. p. 88.

$a$ (N. C. Strickland) 1838.

The type of this Brazilian species.

Chiorospingus.

Chlorospingus, Cabanis, Mus. Hein. i. p. 139 (1851).

955. Chlorospingus ophthalmicus.

Arremon ophthalmicus, Du Bus, Bull. Ac. Brux. xiv. pt. 2. p. 106.

Chlorospingus ophthalmicus, Scl. Cat. Am. B. p. 88.

$a$ \& S. Pedro, Mexico, Oct. 1844 (Galeotti) 1845.

956. Chlorospingus albitempora.

Tachyphonus albitempora, Lafr. Rev. Zool. 1848, p. 12.

Chlorospingus albitemporalis, Scl. Cat. Am. B. p. 89.

Chlorospingus flaviventris, Scl. P. Z. S. 1856, p. 91. 
a Brazil? (Argent) 1852.

Probably a Bolivian specimen. One of the types of his C. favieentris is stated by Mr Sclater to be in the Strickland collection. This specimen has another MS. name on its label, but I have no doubt it is the bird referred to by $\mathrm{Mr}$ Sclater.

95\%. Chlorospingus flavipectus.

Arremon flavo-pectus, Lafr. Rev. Zool. 1840, p. 227.

Chlorospingus flavipectus, Scl. Cat. Am. B. p. 89.

a Bogota (J. Gould) 1845. -b Bogota (M. Dubois) 1845.

958. Chlorospingus canigularis.

Tachyphonus canigularis, Lafr. Rev. Zool. 1848, p. 11.

Chlorospingus canigularis, Scl. Cat. Am. B. p. 89.

a Bogota (Bt. at Stevens's) 1845.

959. Chlorospingus atripileus.

Arremon atro-pileus, Lafr. Rev. Zool. 1842, p. 335.

Chlorospingus atripileus, Scl. Cat. Am. B. p. 89.

a Bogota (Bt. at Stevens's) 1844.

960. Chlorospingus rubrirostris.

Arremon rubrirostris, Lafr. Rev. Zool. 1840, p. 227.

Chlorospingus rubrirostris, Scl. Cat. Am. B. p. 89.

a Bogota (Bt. at Stevens's) 1844.

961. Chlorospingus superciliaris.

Arremon superciliaris, Lafr. Rev. Zool. 1840, p. 227.

Chlorospingus superciliaris, Scl. Cat. Am. B. p. 90.

$a,-b$ Bogota (Bt. at Stevens's) 1844.

962. Chlorospingus verticalis.

Nemosia verticalis, Lafr. Rev. Zool. 1840, p. 227.

Chlorospingus verticalis, Scl. Cat. Am. B. p. 90.

a Bogota (J. Gould) 1844.

\section{BuARremon.}

Buarremon, Bonaparte, Consp. Av. i. p. 483 (1850).

963. Buarremon assimilis.

Tanagra assimilis, Boiss. Rev. Zool. 1840, p. 67.

Buarremon assimilis, Scl. Cat. Am. B. p. 90.

a Bogota (J. Gould) 1844. 
- 964. Buarremon brunneinuchus.

Embernagra brunneinucha, Lafr. Rev. Zool. 1839, p. 97.

Buarremon brunneinuchus, Scl. Cat. Am. B. p. 90.

a Bogota (Bt. at Stevens's) 1844.

+ 965. Buarremon albinuchus.

Embernagra albinucha, D'Orb. \& Lafr. Rev. Zool. 1838, p. 165.

Buarremon albinuchus, Scl. Cat. Am. B. p. 91.

a Bogota (Gardner) 1844.

- 966. Buarremon pallidinuchus.

Tanagra (Arremon) pallidinucha, Boiss. Rev. Zool. 1840, p.69.

Buarremon pallidinuchus, Scl. Cat. Am. B. p. 92.

$a$ Bogota (J. Gould) 1844.

- 96\%. Buarremon schistaceus.

Tanagra (Arremon) schistaceus, Boiss. Rev. Zool. 1840, p. 69.

Buarremon schistaceus, Scl. Cat. Am. B. p. 92.

a Bogota (J. Gould) 1844.

\section{Phenicophilus.}

Phonicophilus, Strickland, Contr. Orn. 1851, p. 104.

968. + Phœnicophilus palmarum.

Turdus palmarum, Linn. Syst. Nat. i. p. 295.

Phoenicophilus palmarum, Strickl. l. s. c.: Scl. Cat. Am. B. p. 92.

$a$ St Domingo (P. L. Sclater) 1852.

\section{Arremon.}

Arremon, Vieillot, Analyse, p. 32 (1816).

- 969. Arremon silens.

Tangara de la Guiane, D'Aub. Pl. Enl. 742 unde,

Tanagra silens, Bodd. Tabl. Pl. Enl. p. 46.

Arremon silens, Scl. Cat. Am. B. p. 93.

$a$ Brazil (Askew) 1834. - b S. America (W. Kirtland) 1843.

$b$ is apparently a skin of Cayenne make. 


\section{- 970. Arremon semitorquatus.}

Arremon semitorquatus, Sw. An. in Menag. p. 357: Scl. Cat. Am. B. p. 93.

a ô Brazil (Burl) 1834 .

\section{Cissopis:}

Cissopis, Vieillot, Analyse, p. 40 (1816).

\section{Cissopis leveriana.}

Lanius leverianus, Gm. Syst. Nat. i. p. 302.

Cissopis leveriana, Scl. Cat. Am. B. p. 94.

a Brazil (Askew) 1833.

\section{LAMPROSPIZA.}

Lamprospiza, Cabanis, Arch. f. Naturg. 1847, p. 246.

\section{Lamprospiza melanoleuca.}

Saltator melanoleucus, Vieill. N. Dict. d'Hist. N. xiv. p. 105.

Lamprospiza melanoleuca, Scl. Cat. Am. B. p. 94.

a (Dewgard) 1846.

A skin of Cayenne make.

\section{Psittospiza.}

Psittospiza, Bonaparte, Compt. Rend. xxxi. p. 424 (1850).

\section{Psittospiza riefferi.}

Tanagra riefferi, Boiss. Rev. Zool. 1840, p. 4.

Psittospiza riefferi, Scl. Cat. Am. B. p. 94.

a Bogota (Bt. at Stevens's) 1844.

\section{Saltator.}

Saltator, Vieillot, Analyse, p. 32 (1816).

\section{Saltator atriceps.}

Tanagra (Saltator) atriceps, Less. Cent. Zool. pl. 69 : Scl. Cat. Am. B. p. 95.

$a$ (Gardner) 1845. - b Guatemala ( $J$. Constancia) 1851.

\section{Saltator magnus.}

Tanagra magna, Gm. Syst. Nat. i. p. 890.

Saltator magnus, Scl. Cat. Am. B. p. 95.

a S. America (N. C. Strickland) 1838. -b S. America (Bt. at Birmingham) 1843. 
976. Saltator similis.

Saltator similis, D’Orb. \& Lafr. Syn. Av. i. p. 36: Scl. Cat. Am. B. p. 95.

$a$ Brazil (Bt. at Aberystwith) 1833. - b Brazil (N. C. Strickland) 1838.

977. Saltator grandis.

Tanagra grandis, Licht. Preis.Verz. Mex. Vög. p. 2 ( $c f$. Journ. f. Orn. 1863, p. 57).

Saltator grandis, Scl. Cat. Am. B. p. 96.

Saltator nigrigenys, Scl. MS.

a Tepitongo, Mexico, Sept. (Galeotti) 1845. - b Guatemala (J. Constancia) 1845.

978. Saltator superciliaris.

Tanagra superciliaris, Max. Beitr. iii. p. 518.

Saltator superciliaris, Scl. \& Salv. Nomencl. p. 26.

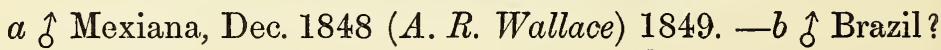
(Argent) 1852.

$b$ is probably from Bolivia.

979. Saltator orenocencis.

Saltator orenocensis, Lafr. Rev. Zool. 1846, p. 274: Scl. P.Z.S. 1856, p. 77.

$a$ Trinidad (Argent) 1851.

A Venezuelan species.

980. Saltator albicollis.

Saltator albicollis, Vieill. N. Dict. d'Hist. N. xiv. p. 107: Scl. Cat. Am. B. p. 97.

$a$ (N. C. Strickland) 1838.

Found in the northern and north-western parts of South America.

981. Saltator atricollis.

Saltator atricollis, Vieill. N. Dict. d'Hist. N. xiv. p. 104: Sc]. Cat. Am. B. p. 97.

a Brazil (N. C. Strickland) 1838. 


\section{DiUCOPIS.}

Diucopis, Bonaparte, Consp. i. p. 491 (1850).

982. Diucopis fasciata.

Tanagra fasciata, Licht. Verz. Doubl. p. 32.

Diucopis fasciata, Scl. Cat. Am. B. p. 97.

$a$ Brazil (Arthur Strickland) 1840. —b.

\section{OrChesticus.}

Orchesticus, Cabanis, Mus. Hein. i. p. 143 (1851).

983. Orchesticus abeillæi.

Pyrrhula abeillii, Less. Rev. Zool. 1839, p. 40.

Orchesticus abeillii, Scl. Cat. Am. B. p. 97.

$a$ (N. C. Strickland) 1838. - b.

A species of south-eastern Brazil.

984. Orchesticus capistratus.

Tanagra capistrata, Max. Reise N. Bras. ii. p. 179; Beitr. iii. p. 500 .

Orchesticus capistratus, Scl. Cat. Am. B. p. 98.

a Brazil (N. C. Strickland) 1832.

\section{Orchesticus ater.}

Tangara à cravatte noire de Cayenne, D'Aub. Pl. Enl. 714 f. 2 undè,

Tanagra atra, Gm. Syst. Nat. i. p. 898.

Orchesticus ater, Scl. Cat. Am. B. p. 98. $a$ (N. C. Strickland) 1838.

A widely-ranging South American species.

\section{Pitylus.}

Pitylus, Cuvier, Règn. An. i. p. 413 (1829).

\section{+986. Pitylus fuliginosus.}

Loxia fuliginosa, Daud. Orn. ii. p. 372.

Pitylus fuliginosus, Scl. Cat. Am. B. p. 98. $a,-b$ Brazil (N. C. Strickland) 1838. 


\section{8\%. Pitylus brasiliensis.}

Caryothraustes brasiliensis, Cab. Mus. Hein. i. p. 144.

Pitylus brasiliensis, Scl. Cat. Am. B. p. 99.

a Brazil (Askew) 1837. —b Brazil (G. Lloyd) 1838.

\section{FAMILY.-FRINGILLIDA.}

\section{SUBFAM. LOXIIN $Æ$.}

LOXIA.

Loxia, Linnæus, Syst. Nat. i. p. 299 (1766) (partim). 988. Loxia curvirostra.

Loxia curvirostra, Linn. l. s. c.: Newton, ed. Yarr. Brit. B. ii. p. 187 : Dresser, B. Eur.

$a \hat{\jmath},-b+$ Worcestershire, 28 November (H. E. Strickland) 1838. - c, -d (Arthur Strickland) 1840.

- 989. Loxia americana.

Curvirostra americana, Wils. Am. Orn. iv. p. 44, pl. 31, f. $1,2$.

Loxia curvirostra var. americana, Baird, Brew. \& Ridgw. N. Am. B. i. p. 484.

a Canada (Mather) 1840.

- 990. Loxia mexicana.

Loxia mexicana, Strickl. Contr. Orn. 1851, p. 43: Scl. Cat. Am. B. p. 122: Dresser, B. Eur.

a Mexico (T. Mann) 1844.

The type of the species.

991. Loxia pityopsittacus.

Loxia pityopsittacus, Bechst, Orn.Taschenb. i. p.106: Newton, ed. Yarr. Brit. B. ii. p. 207 : Dresser, B. Eur.

$a,-b$ N. Europe (J. G. Kinberg) 1843. —c (J. G. Kinberg) 1845. 


\section{- 992. Loxia leucoptera.}

Loxia leucoptera, Gm. Syst. Nat. i. p. 844: De Selys, Fauna Belge, p. 77 : Baird, Brew. \& Ridgw. N. Am. B. i. p. 488: Newton, ed. Yarr. Brit. B. ii. p. 219.

$a$ o Worcestershire (T. Robinson) 1838. -b (Bt. at Liverpool) 1852.

A North American species. There seems to be no doubt that $a$ was killed in England.

\section{Pyrrhula.}

Pyrrhula, Brisson, Ornith. iii. p. 308 (1760).

- 993. Pyrrhula europæa.

Pyrrhula europcea, Vieill. N. Dict. d'Hist. N. iv. p. 286: Newton, ed. Yarr. Brit. B. ii. p. 166: Dresser, B. Eur.

Pyrrhula vulgaris, Temm. Man. d'Orn. i. p. 338.

$a$ Oxfordshire 20 August (N. C. Strickland) 1833. $-b \hat{\delta},-c$ o Worcestershire (H. E. Strickland) 1834.

- 994. Pyrrhula major.

Loxia pyrrhula, Linn. Syst. Nat. i. p. 300.

Pyrrhula major, Brehm, Vög. Deutschl. p. 252: Desser, B. Eur.

$a$ (N. C. Strickland) 1838.

A species of Northern and Eastern Europe.

995. Pyrrhula erythrocephala.

Pyrrhula erythrocephala, Vig. P. Z. S. 1830-1, p. 174: Gould, Cent. B. Him. pl. 32: Jerd. B. Ind. ii. p. 389.

a India ("Pyrrhula erythrocephala young male," E. Blyth) 1846. - $b$ Himalaya (Stevens) 1850.

996. Pyrrhula nipalensis.

Pyrrhula nipalensis, Hodgs. As. Res. xix. p. 155: Jerd. B. Ind. ii. p. 390.

$a$ India ("Pyrrhula nipalensis female" E. Blyth) 1846. 
Pinicola.

Pinicola, Vieillot, Ois. Am. Sept. i. p. iv. pl. 1, f. 13 (1807). -99\%. Pinicola enucleator.

Loxia enucleator,Linn. Syst. Nat. i. p. 299.

Pinicola enucleator, Dresser, B. Eur.

Pyrrhula enucleator, Newton, ed. Yarr. Brit. B. ii. p. 177.

$a$ (Thomas) 1840. $-b \hat{\delta},-c$ q Government of St Petersburg (J. F. Brandt) 1841.

\section{Carpodacus.}

Carpodacus, Kaup, Skizz. Ent. Gesch. p. 161 (1829). 998. Carpodacus erythrinus.

Loxia erythrina, Pall. Nov. Comm. Petrop. xiv. p. 587, pl. 23, f. 1.

Carpodacus erythrinus, Kp. l. s. c. Jerd. B. Ind. ii. p. 398: Dresser, B. Eur.

Erythrospiza erythrina, Strickl. Ann. \& Mag. N. H. xiii. p. 38.

Pyrrhula erythrina, Newton, ed. Yarr. Brit. B. ii. p. 172.

$a$ ईิ Benares 28 February (W. J. E. Boys) 1840. -b o Siberia (J. F. Brandt) 1841. — $q$ Altai (J. F. Brandt) 1842. -d q Nepal (B. H. Hodgson, 452) 1845. - e Madras (T. C. Jerdon) 1845. $-f$ India (E. Blyth) 1846. $-g,-h \mathrm{~S}$. India (T. C. Jerdon) 1850 .

The specific identity of Indian and Siberian specimens was pointed out by Strickland, l. s. $c$.

999. Carpodacus purpureus.

Fringilla purpurea, Gm. Syst. Nat. i. p. 923.

Carpodacus purpureus, Baird, Brew. \& Ridgw. N. Am. B. i. p. 462.

a N. America (N. C. Strickland) 1838. -b (N. C. Strickland) 1838. 
1000. Carpodacus hæmorrhous.

Fringilla hoemorrhoa, Wagl. Isis. 1831, p. 525.

Carpodacus hcemorrhous, Scl. Cat. Am. B. p. 122.

a Mexico (T. Mann) 1844.

A close ally of if really different from C. frontalis, Say.

1001. Carpodacus rubicillus.

Loxia rubicilla, Güld. Nov. Comm. Petrop. xix. p. 464.

Carpodacus rubicillus, Gould, B. Asia.

a (J. F. Brandt) 1844.

Found in the Altai Mountains and in the Caucasus.

\section{Propasser.}

Propasser, Hodgson, Zool. Misc. p. 84 (1844); P. Z. S. 1845, p. 36.

1002. Propasser rhodopeplus.

Fringilla rhodopepla, Vig. P. Z. S. 1830-1, p. 23: Gould, Cent. B. Him. pl. 31, f. 1.

Propasser rhodopeplus, Jerd. B. Ind. ii. p. 400.

$a,-b$ Nepal (B. H. Hodgson, 340) 1845.

1003. Propasser pulcherrimus.

Propasser pulcherrimus, Hodgs. Zool. Misc. p. 85. Moore, P. Z. S. 1855, p. 216 : Jerd. B. Ind. ii. p. 402.

$a$ N. India (W. J. E. Boys) 1848.

\section{URAGUS.}

Uragus, Keyserling \& Blasius, Wirb. Eur. p. 158 (1840) (as a subgenus).

\section{Uragus sibiricus.}

Loxia sibirica, Pall. Reis. ii. p. 711.

Uragus sibiricus, Bp. Consp. i. p. 529.

Pyrrhula longicauda, Temm. Man. d'Orn. i. p. 340.

a ô Altai (J. F. Brandt) 1842. —b (Kinberg) 1845.

\section{ERYTHROSPIZA.}

Erythrospiza, Bonaparte, Saggio di una Distr. Met. An. Vert. p. 141 (Aggiunte, 1832). 
1005. Erythrospiza githaginea.

Fringilla githaginea, Licht. Verz. Doubl. p. 24.

Erythrospiza githaginea, Dresser, B. Eur.

a (Bt. at Dresden) 1845.

A species of North Africa extending eastwards to Sinde.

Linota.

Linota, Bonaparte, Comp. List. p. 35 (1838).

1006. Linota cannabina.

Fringilla cannabina, Linn. Syst. Nat. i. p. 322.

Linaria cannabina, Strickl. P. Z. S. 1836, p. 99.

Linota cannabina, Newton, ed. Yarr. Brit. B. ii. p. 153 : Dresser, B. Eur.

$a \hat{\delta},-b q$ Aberdovey, August (H. E. Strickland) 1833. -c $\hat{\delta}$ Worcestershire, September (H. E. Strickland) 1833. - $d$ q Smyrna, 2 December (H. E. Strickland) 1835. -e Smyrna, 12 December (H. E. Strickland) 1835. - f $\hat{\delta}$ Worcestershire, April (H. E. Strickland) 1837.

100\%. Linota flavirostris.

Fringilla flavirostris, Linn. Syst. Nat. i. p. 322.

Linota flavirostris, Newton, ed. Yarr. Brit. B. ii. p. 160: Dresser, B. Eur.

Fringilla montium, Gm. Syst. Nat. i. p. 917.

a Scotland (Dunn) 1836.

1008. Linota linaria.

Fringilla linaria, Linn. Syst. Nat. i. p. 322.

Linota linaria, Newton, ed. Yarr. Brit. B. ii. p. 133: Dresser, B. Eur.

Fringilla borealis, (Vieill.) Roux. Orn. Prov. i. p. 165, pls. 101, 102.

AEgiothus linarius, Baird, Brew. \& Ridgw. N. Am. B. i. p. 493.

$a$ (Arthur Strickland) 1840. -b Canada (Capt. Wise) 1843. -c Philadelphia (E. Wilson) 1848.

- 1009. Linota rufescens.

Linaria rufescens, Vieill. Mem. R. Ac. Sc. Tor. xxiii. p. 202. 
Linota rufescens, Newton, ed. Yarr. Brit. B. ii. p. 146: Dresser, B. Eur.

Linota linaria, auctt. plur.

$a$ o Worcestershire (H. E. Strickland) 1828. - $b$ f Worcestershire (H. E. Strickland) April 1834. -c o Worcestershire, 12 May (H. C. Strickland) 1837.

\section{-1010. Linota hornemanni.}

Linota hornemanii, Holb. Naturh. Tidsk. iv. p. 398: Dresser, B. Eur.

a (Arthur Strickland) 1840.

A specimen without locality which from its size seems referable to this northern species.

\section{SUBFAMILY.-FRINGILLIN A.}

Fringilida.

Fringilla Linnæus, Syst. Nat. i. p. 317 (1766) (partim).

\section{Fringilla colebs.}

Fringilla coelebs, Linn. l.s. c. p. 318: Strickl. P. Z. S. 1836, p. 100: Newton, ed. Yarr. Brit. B. ii. p. 68: Dresser, B. Eur.

$a \hat{\delta},-b$ \& Worcestershire (H. E. Strickland) 1833. - b 소

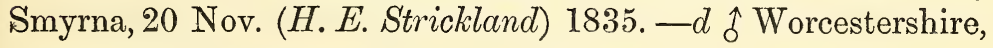
April (H. E. Strickland) 1837.

1012. Fringilla montifringilla.

Fringilla montifringilla, Linn. Syst. Nat. i. p. 318: Strickl. P. Z. S. 1836 , p. 100 : Newton, ed. Yarr, Brit. B. ii. p. 75: Dresser, B. Eur.

a Scotland (Carfrae) 1837. - b Britain (N. C. Strickland) 1838. —c Dumfriesshire, Dec. (Sir W. Jardine) 1849.

\section{MontifringiLla.}

Montifringilla, Brehm, Vog. Deutschl. p. 269 (1831).

\section{Montifringilla nivalis.}

Fringilla nivalis, Linn. Syst. Nat. i. p. 321.

Montifringilla nivalis, Dresser, B. Eur.

a Switzerland (W. Anderegg) 1836. 


\section{Fringillallauda.}

Fringalauda, Hodgson, As. Res. xix. p. 158 (1836).

1014. Fringillalauda nemoricola.

Fringalauda nemoricola, Hodgs. l. s. c.: Jerd. B. Ind.ii. p. 414. $a$ India (Bt. at Stevens's).

\section{LEUCOSTICTE.}

Leucosticte, Swainson, Faun. Bor. Am. iii. p. 493 (1831) (as a subgenus).

\section{Leucosticte griseinucha.}

Fringilla griseonucha, Brandt,Bull.Ac. St Petersb.1841, p. 36.

Leucosticte tephrocotis var. griseinucha, Baird, Brew. \& Ridgw. N. Am. B. i. p. 508.

$a$ N. W. America (J. F. Brandt) 1846.

One of Wosnessensky's skins thus named by Prof. Brandt.

\section{-1016. Leucosticte arctoa.}

Passer arctous, Pall. Zoogr. Rosso-As. ii. p. 21.

Fringilla (Linaria) gebleri, Brandt, Bull. Ac. St. Petersb. x. p. 251 (1841).

a In mont. ad f. Ob Siberiæ occidentalis (J. F. Brandt) 1844.

\section{Leucosticte brandti.}

Leucosticte gebleri, Brandt, Bull. Phys.-Math. Ac. St. Petersb. i. p. 363 .

Leucosticte brandti, Bp. Consp. i. p. 537.

$a$ W. Siberia (J. F. Brandt) 1844.

The label of this specimen is inscribed by Prof. Brandt "Fringilla (Leucosticte) gebleri nob. Fringilla gebleri nob. Siberia occidentalis." Prof. Brandt first applied this name to L. arctoa in breeding plumage, but subsequently, unlawfully, transferred it to this species. The specimen agrees with the second and not with the original description of L. gebleri.

\section{Callacanthis.}

Callacanthis, Reichenbach, Av. Syst. pl. Ixxviii. (1851). 1018. Callacanthis burtoni.

Carduelis burtoni, Gould, P. Z. S. 1837, p. 90.

Callacanthis burtoni, Jerd. B. Ind. ii. p. 407.

a Himalayas (Stevens). 


\section{LiguRinus.}

Ligurinus, Koch, Syst. d. Baierisch. Zool. p. 230 (1816).

\section{Ligurinus chloris.}

\section{Loxia chloris, Linn. Syst. Nat. i. p. 304.}

Coccothraustes chloris, Strickl. P. Z. S. 1836, p. 100: Newton, ed. Yarr. Brit. B. ii. p. 105.

Ligurinus chloris, Dresser, B. Eur.

a f Oxford, 27 August (N. C. Strickland) 1833. - b f, $\div$ f Worcestershire, January (H. E. Strickland) 1834.

\section{Ligurinus kawariba.}

Fringilla kawariba, Temm. Pl. Col. 588, f. 1.

Ligurinus kawariba, Cab. Mus. Hein. i. p. 158.

a Japan (Leadbeater).

In his list of Chinese birds (P. Z. S. 1842, p. 168) Strickland placed this name as a synonym of Carduelis sinica (Lath.).

\section{Passer.}

Passer, Brisson, Ornith. iii. p. 71 (1760).

\section{Passer domesticus.}

Fringilla domestica, Linn. Syst. Nat. i. p. 323.

Pyrgita domestica, Strickl. P. Z. S. 1836, p. 99.

Passer domesticus, Newton, ed. Yarr. Brit. B. ii. p. 89: Dresser, B. Eur.

a ô Oxfordshire, 23 October (N. C. Strickland) 1833. -b q Worcestershire June (H. E. Strickland) 1838.

Stated by Strickland to be the common House-Sparrow of the Levant. No specimens from there are in his collection.

\section{Passer indicus.}

Passer indicus, Jard. \& Selb. Ill. Orn. iii. pl. 118: Jerd. B. Ind. ii. p. 362.

a India (Lord A. Hay) 1845. - b India ("Passer domesticus? indicus, J. \& S." E. Blyth) 1846. —c, —d India (E. Blyth) 1846. -e ô India (W. J. E. Boys) 1847. - f India (E. Blyth) 1850.

S. C. 


\section{Passer italiæ.}

Fringilla italice, Vieill. N. Dict. d'Hist. N. xii. p. 199.

Passer italice, Dresser, B. Eur.

a 今, -b f Italy, July (H. E. Strickland) 1836.

\section{Passer hispaniolensis.}

Fringilla hispaniolensis, Temm. Man. d'Orn. i. p. 353.

Pyrgita hispaniolensis, Strickl. P. Z. S. 1836, p. 99.

Passer hispaniolensis, Dresser, B. Eur.

Pyrgita salicaria, Vieill., Bp. Comp. List. p. 30.

a ô Smyrna, April (H. E. Strickland) 1836.

\section{Passer arcuatus.}

Fringilla arcuata, Gm. Syst. Nat. i. p. 912.

Passer arcuatus, Layard, B. S. Afr. p. 204: Sharpe, Cat. Afr. B. p. 69.

$a,-b$ S. Africa (Dr A. Smith, 180).

\section{Passer cinnamomeus.}

Pyrgita cinnamomea, Gould, P. Z. S. 1835, p. 185.

Passer cinnamomeus, Jerd. B. Ind. ii. p. 365.

a Himalaya (N. C. Strickland) 1838. -b 今, -c q Kumaon (W. J. E. Boys) 1847.

\section{2\%. Passer montanus.}

Fringilla montana, Linn. Syst. Nat. i. p. 324.

Passer montanus, Newton, ed. Yarr. Brit. B. ii. p. 82.

a $\hat{\jmath}$ Worcestershire, 28 September (H. E. Strickland) 1833. -b $\hat{\jmath}$ Worcestershire, October (H. E. Strickland) 1833. -c Lombardy, July (H. E. Strickland) 1836.

1028. Passer flavicollis.

Fringilla flavicollis, Frankl. P. Z. S. 1831-2, p. 120.

Passer flavicollis, Jerd. B. Ind. ii. p. 368.

a (N. C. Strickland) 1838. - b India (Bt. at Stevens's) 1844. -c Madras ("Pyrgita flavicollis," T. C. Jerdon) 1845. —d Arakan (E. Blyth) 1847. - e §o Jucknie (W. J. E. Boys) 1847.

$d$ bears no original label. The species is not included in Blyth's Catalogue of the "Mammals and Birds of Burma" (J. A. S. B. xliii. extr. no.). 


\section{Passer diffusus.}

Pyrgita diffusa, Smith, Rep. S. Afr. Exp. App. p. 50.

Passer diffusus, Layard, B. S. Afr. p. 204.

a (Manchester Nat. Hist. Soc.) 1843.

A South-African species.

\section{Passer swainsoni.}

Pyrgita swainsoni, Rüpp. Neue Wirb. p. 94, pl. 33, f. 2.

Passer swainsoni, Finsch, Trans. Z. S. vii. p. 269.

Passer simplex, Strickl. P. Z. S. 185̆0, p. 218 (nec Licht.).

a $\hat{\delta}$ Kordofan, 18 July (J. Petherick) 1848. - $b$ q Kordofan 17 March (J. Petherick) 1848.

Strickland considered $P$. swainsoni to be identical with $P$. simplex, Licht (Corospiza simplex, Bp.). It is, however, a very distinct species.

\section{Petronia.}

Petronia, Kaup, Skizz. Entw. Gesch. p. 158 (1829).

\section{Petronia stulta.}

Fringilla stulta, Linn. Syst. Nat. i. p. 322.

Petronia stulta, Strickl. Ann. \& Mag. N. H. viii. p. 374.

a ô Morea, May (H. E. Strickland) 1836. - b Tuscany (C. Passerini) 1836.

\section{Coccothraustes.}

Coccothraustes, Brisson, Orn. iii. p. 218 (1760).

\section{Coccothraustes vulgaris.}

Loxia coccothraustes, Linn. Syst. Nat. i. p. 299.

Coccothraustes vulgaris, Newton, ed. Yarr. Brit. B. ii. p. 98.

a f Worcestershire, January (H. E. Strickland) 1833. -b Britain (W. Kirtland) 1847.

\section{Coccothraustes vespertinus.}

Fringilla vespertina, Cooper, Ann. Lyc. N. Y. i. p. 220.

Coccothraustes vespertinus, Scl. Cat. Am. B. p. 123.

Hesperiphona vespertina, Baird, Brew. \& Ridgw. N. Am. B. i. p. 449 .

$a,-b$ Mexico (T. Mann) 1844. 


\section{Coccothraustes carneipes.}

Coccothraustes carneipes, Hodgs. As. Res. xix. p. 151.

Mycerobas carneipes, Jerd. B. Ind. ii. p. 387.

Coccothraustes speculigerus, Brandt, Bull. Ac. Petersb. ix. p. 11.

$a f,-b$ in alp. alata ad torrentem Lepsa, Septemb. 1844 (J. F. Brandt) 1846.

Young birds from Karélin's collection (cf. Brandt $l$. s. c.). These labels bear the MS. name 'Loxia albospecularis' (cf. Gray, H.-list, ii. p. 88).

\section{Coccothraustes melanoxanthus.}

Coccothraustes melanoxanthos, Hodgs. As. Res. xix. p. 150.

Mycerobas melanoxanthos, Jerd. B. Ind. ii. p. 386.

$a$ (T. C. Eyton) 1850. - b N. India (Thomas) 1851.

\section{Coccothraustes icteroides.}

Coccothraustes icteroides, Vig. P. Z. S. 1830-1, p. 8: Gould, Cent. B. Him. pl. 45.

Hesperiphona icteroides, Jerd. B. Ind. ii. p. 384.

a N. India (W. J. E. Boys) 1848. -b Himalaya (Bt at Stevens's) 1850.

\section{Carduelis.}

Carduelis, Brisson, Ornith. iii. p. 53 (1760). 103\%. Carduelis elegans.

Fringilla carduelis, Linn. Syst. Nat. i. p. 318.

Carduelis elegans, Strickl. P. Z. S. 1836, p. 99: Newton, ed. Yarr. Brit. B. ii. p. 117: Dresser, B. Eur.

a juv. Oxfordshire, 20 August (H. E. Strickland) 1833. - \& Oxfordshire, 11 October (N. C. Strickland) 1833. -c Worcestershire, January (H. E. Strickland) 1834.

1038. Carduelis caniceps.

Carduelis caniceps, Vig. P. Z. S. 1831-2, p. 23 : Gould, Cent. B. Him. pl. 33, f. 1: Jerd. B. Ind. ii. p. 408. a. ô N. India (W. J. E. Boys). 


\section{Chrysomitris.}

Chrysomitris, Boie, Isis, 1828, p. 322.

1039. Chrysomitris citrinella.

Fringilla citrinella, Linn. Syst. Nat. i. p. 320.

Chrysomitris citrinella, Dresser, B. Eur.

Citrinella brumalis, (Gm.) Strickl. Ann. \& Mag. N. H. viii. p. 374 .

a ô S. Europe (N. C. Strickland) 1838.

\section{Chrysomitris spinus.}

Fringilla spinus, Linn. Syst. Nat. i. p. 322.

Carduelis spinus, Newton, ed. Yarr. Brit. B. ii. p. 126.

$a$ Britain? (N. C. Strickland) 1838.

\section{Chrysomitris citrinelloides.}

Serinus citrinelloides, Rüpp. Nene Wirb. p. 95, pl. 34, f. 1.

Citrinella citrinelloides, Bp. Consp. i. p. 520.

a Abyssinia (E. Verreaux) 1850.

\section{Chrysomitris pinus.}

Fringilla pinus, Wils. Am. Orn. ii. p. 133, pl. 17, f. 1.

Chrysomitris pinus, Baird, Brew. \& Ridgw. N. Am. B. i. p. 480 .

\section{$a,-b$ (N. C. Strickland) 1838.}

These birds agree best with North-American specimens of this species.

\section{-1043. Chrysomitris magellanica.}

Fringilla magellanica, Vieill. N. Dict. d'Hist. N. xii. p. 168. Fringilla barbata, Mol. Sagg. sulla St. Nat. Chili, p. 247??

Fringilla icterica, Licht. Verz. Doubl. p. 26.

Chrysomitris barbata et icterica, Scl. Cat. Am. B. p. 125.

Fringilla campestris, Spix. Av. Bras. ii. p. 48, pl. 61, f. 3.

$a$ S. America (Johnson) 1837. —b (N. C. Strickland) 1838. -c S. America (N. C. Strickland) 1838. -d, -e Brazil (N. C. Strickland) 1838.

Molina's description may be meant for this bird but the character 'alis viridibus nigro, mubroque maculatis' is in no way applicable. $b, c, d$ and $e$ all have a yellow uropygium and if separable should probably bear the name 0 . icterica (Licht.). 


\section{Chrysomitris notata.}

Carduelis notata, DuBus, Bull. Ac. Brux. xiv. pt. 2, p. 106.

Chrysomitris notata, Scl. \& Salv. Ibis, 1860, p. 275 : Scl.

Cat. Am. B. p. 124.

$a,-b$ Guatemala ( $J$. Constancia) 1848.

1045. Chrysomitris xanthogastra.

Chrysomitris xanthogastra, DuBus, Bull. Ac. Brux. xxii. pt. 1, p. 152: Scl. \& Salv. P. Z. S. 1870, p. 785.

$a$ (Bt. at Stevens's) 1845.

An Andean species found also in Costa Rica.

1046. Chrysomitris tristis.

Fringilla tristis, Linn. Syst. Nat. i. p. 320.

Chrysomitris tristis, Baird, Brew. \& Ridgw. N. Am. B. i. p. 471.

a N. America (Askew) 1834. -b (Johnson) 1837. -c (Askew) 1837. —d (Arthur Strickland) 1840. —e (Bt. at Edinburgh) 1852.

-104\%. Chrysomitris columbiana.

Chrysomitris columbiana, Lafr. Rev. Zool. 1843, p. 292: Scl, Cat. Am. B. p. 124.

a Bogota (Rogers) 184t.

\section{- 1048. Chrysomitris mexicana.}

Carduelis mexicana, Sw. Phil. Mag. 1827, p. 435.

Cirysomitris mexicana, Scl. \& Salv. Ibis, 1859, p. 19: Scl. Cat. Am. B. p. 124.

a Guatemala (J. Constancia) 1851.

1049. Chrysomitris totta.

Loxia totta, Sparrm. Mus. Carls. No. 18.

Citrinella totta, Bp. Consp. i. p. 520.

Fringilla totta, Layard, B. S. Afr. p. 202.

$a$ Cape of Good Hope (A. Strickland) 1833. $-b$ S. Africa (Dr A. Smith). 


\section{Chrysomitris spinoides.}

Carduelis spinoides, Vig. P. Z. S. 1831-2, p. 44.

Chrysomitris spinoides, Jerd. B. Ind, ii. p. 409.

$a,-b \mathrm{Nepal}$ (B. H. Hodigson 442, 443) 1845. —c f Kumaon (W. J. E. Boys) 1847. -d q N. India (W. J. E. Boys) 1848.

\section{SERINUS. ${ }^{1}$}

Serimus, Koch. Syst. d. Baierisch. Zool. i. p. 228 (1816).

1051. Serinus hortulanus.

Fringilla serinus, Linn. Syst. Nat. i. p. 320 : Strickl. P. Z. S. 1836, p. 100.

Serinus hortulanus, Koch, l. s. c., p. 229: Dresser, B. Eur.

Serinus meridionalis, Brehm. Vög. Deutschl. p. 255.

a Florence, Novernber (C. Passerini) 1834. - b f Smyrna, 19 November (H. E. Strickland) 1835.

\section{Serinus pusillus.}

Passer pusillus, Pall. Zoogr. Rosso-As. ii. p. 28, pl. 43.

Serinus pusillus, Dresser, B. Eur.

Metoponia pusilla, Jerd. B. Ind. ii. p. 410.

$a \uparrow$ W. Siberia (J. F. Brandt) 1846. - b N. India (Mather).

\section{SrCALIS.}

Sicalis, Boie, Isis, 1828, p. 324 (partim).

\section{- 1053. Sycalis flaveola.}

Fringilla flaveola, Linn. Syst. Nat. i. p. 321.

Sycalis flaveola, Scl. Ibis, 1872, p. 41.

a Brazil (G. Lloyd) 1838. -b (N. C. Strickland) 1838. -c S. America (Askew) 1838. - d S. America (Bt. at Birmingham) 1843.

\section{-1054. Sycalis pelzelni.}

Sycalis pelzelni, Scl. Ibis, 1872, p. 42.

a (N. C. Strickland) 1838.

A female agreeing best with female examples of this species, an inhabitant of South Brazil, Paraguay and the Argentine Republic.

1 According to Strickland (Ann. \& Mag. N. H. viii. p. 374), this genus should stand near Crithagra. 
- 1055. Sycalis columbiana.

Sycalis columbiana, Cab. Mus. Hein. i. p. 147: Scl. Ibis, 1872 , p. 43.

$a,-b$ Trinidad (Argent) 1851.

1056. Sycalis luteola.

Emberiza luteola, Sparrm. Mus. Carls. No. 93.

Sycalis luteola, Scl. Ibis, 1872, p. 44.

Crithagra? brevirostris, Gould, Zool. Voy. Beagle, iii. p. 88.

a Chili (N. C. Strickland) 1838. - b (Havell) 1839. - c ô Mexiana, Dec. 1848 (A. R. Wallace) 1849.

105\%. Sycalis uropygialis.

Emberiza uropygialis, D’Orb. \& Lafr. Ṡyn. Av. p. 75.

Sycalis uropygialis, Scl. Ibis, 1872, p. 47.

a Cordillera (in flocks) (N. C. Strickland) 1838.

An inhabitant of the Andes of Peru and Bolivia.

\section{CRITHAGRA.}

Crithagra, Swainson, Zool. Journ. iii. p. 348 (1827).

1058. Crithagra canicollis.

Crithagra canicollis, Sw. Classif. B. ii. p. 295: Sharpe, Cat. Afr. B. p. 67.

Fringilla canicollis, Layard, B. S. Afr. p. 201.

$a$ S. Africa (Mrs Van der Kemp) 1843. - b S. Africa (Gardner). —c S. Africa (Dewgard) 1849.

\section{Crithagra butyracea.}

Loxia butyracea, Linn. Syst. Nat. i. p. 304.

Crithagra butyracea, Layard, B. S. Afr. p. 219: Sharpe, Cat. Afr. B. p. 67.

a Cape of Good Hope, 13 March (A. Strickland) 1832. - b S. Africa (Bt. at Stevens's) 1842.

1060. Crithagra ictera.

Fringilla ictera, Vieill. N. Dict. d'Hist. N. xii. p. 170.

Serinus icterus, Bp. Consp. i. p. 523.

Crithagra chrysopyga, Sw. B. W. Afr. i. p. 206: Hartl. Beitr. Orn. Madag. p. 57 : Sharpe, Cat. Afr. B. p. 67.

$a$ (A. Strickland) 1832. - b (Argent) 1851.

A widely-distributed African species. 
1061. Crithagra flaviventris.

Loxia flaviventris, Gm. Syst. Nat. i. p. 856.

Crithagra flaviventris, Layard, B. S. Afr. p. 220. 1838.

a (A. Strickland) 1832. -b S. Africa (Capt. Alexander)

1062. Crithagra striolata.

Pyrrhula striolata, Rüpp. Neue Wirb. p. 99, pl. 37, f. 1.

Crithagra striolata, Finsch, Trans. Z. S. vii. p. 270.

a A.byssinia (E. Verreaux) 1850.

1063. Crithagra sulphurata.

Loxia sulphurata, Linn. Syst. Nat. i. p. 305.

Crithagra sulphurata, Layard, B. S. Afr. p. 218: Sharpe, Cat. Am. B. p. 67.

a (Arthur Strickland) 1840.

A South-African species.

1064. Crithagra albigularis.

Crithagra albogularis, Smith, S. Afr. Qu. Journ. i. p. 48.

Crithagra albogularis, Sharpe, Cat. Am. B. p. 67.

$a$ S. Africa ( $\operatorname{Dr}$ A. Smith).

This skin is of the same origin as the type.

Auripasser.

Auripasser, Bonaparte, Consp. Av. i. p. 519 (1850).

1065. Auripasser luteus.

Fringilla lutea, Licht. Verz. Doubl. p. 24.

Auripasser luteus, Bp. l. s. c.

Crithagra lutea, Strickl. P. Z. S. 1850, p. 218.

$a \hat{\delta},-b$ \& Kordofan, 30 April (J. Petherick) 1848.

Poliospiza.

Poliospiza, Bonaparte, Consp. Av. i. p. 519 (1850).

1066. Poliospiza gularis.

Linaria gularis, Smith, Rep. S. Afr. Exp. App. p. 49. 
Poliospiza gularis, Bp. l. s. c.: Sharpe, Cat. Afr. B. p. 68. 1842.

$a$ S. Africa (Dr A. Smith, 157). - b S. Africa (Bt. at Stevens's)

\section{SUBFAMILY.-SPERMOPHILIN $\not$ E.}

Pheucticus.

Pheucticus, Reichenbach, Av. Syst., pl. lxxviii. (1850).

106\%. Pheucticus uropygialis.

Pheucticus uropygialis, Scl. \& Salv. P.Z. S. 1870, p. 840.

$a$ (N.C. Strickland) 1838. -b (Argent) 1853.

$b$ is apparently from Bogota, whence the types of the species came.

1068. Pheucticus chrysogaster.

Pitylus chrysogaster, Less. Cent. Zool. pl. 67.

Pheucticus chrysogaster, Scl. Cat. Am. B. p. 100.

$a$ S. America (P. L. Sclater) 1848.

A species found in Venezuela and Equador.

\section{HEDYMELES.}

Hedymeles, Cabanis, Mus. Hein. i. p. 152 (1851).

\section{Hedymeles ludovicianu.s.}

Loxia ludoviciana, Linn. Syst. Nat. i. p. 306.

Hedymeles ludovicianus, Scl. Cat. Am. B. p. 100: Baird, Brew. \& Ridgw. N. Am. B. ii. 70.

$a$ N. America (N. C. Strickland) 1838. -b N. America (Carfrae) 1840. -c (Rogers) 1844. $-d$ Guatemala (J. Constancia) 1851.

\section{0\%0. Hedymeles melanocephalus.}

Guiraca melanocephala, Sw. Phil. Mag. 1827, p. 438.

Hedymeles melanocephalus, Scl. Cat. Am. B. p. 100: Baird, Brew. \& Ridgw. N. Am. B. ii. p. 73.

a Mexico (T. Mann) 1844.

\section{Cardinalis.}

Cardinalis, Bonaparte, Saggio di una Distr. Met. An. Vert. p. ว̌3 (1831).

\section{0\%1. Cardinalis virginianus.}

Loxia cardinalis, Linn. Syst. Nat. i. p. 300. 
Cardinalis virginianus, Baird, Brew. \& Ridgw. N. Am. B. ii. p. 100.

$a \hat{\jmath},-b q$ N. America (N. C. Strickland) 1838.

\section{Guiraca.}

Guiraca, Swainson, Zool. Journ. iii. p. 350 (1827). .

\section{Guiraca cærulea.}

Loxia ccerulea, Linn. Syst. Nat. i. p. 306.

Guiraca ccerulea, Baird, Brew. \& Ridgw. N. Am. B. ii. p. 77. a (N. C. Strickland) 1838.

A North-American species.

\section{- 1073. Guiraca cyanea.}

Loxia cyanea, Linn. Syst. Nat. i. p. 303.

Guiraca cyanea, Scl. Cat. Am. B. p. 101.

a (Bt. at Aberystwith) 1833. -b Brazil (Askew) 1834.

\section{Geospiza.}

Geospiza, Gould, P. Z. S. 1837, p. 5.

+ 1074. Geospiza parvula.

Geospiza parvula, Gould, l. s. c. p. 6; Zool. Voy. Beagle, iii. p. 102, pl. 39 : Salv. Trans. Z. S. ix. p. 483.

$a$ Galapagos Is. (C. Darwin).

A typical specimen of this species.

\section{LOXIGILLA,}

Loxigilla, Lesson, Traité d'Orn. p. 443 (1831).

\section{Loxigilla violacea.}

Loxia violacea, Linn. Syst. Nat. i. p. 306.

Loxigilla violacea, Scl. Cat. Am. B. p. 102.

a (N. C. Strickland) 1838.

A species peculiar to the islands of Jamaica and Hayti.

- 1076. Loxigilla anoxantha.

Spermophila anoxantha, Gosse, B. Jam. p. 247: Ill. pl. 62.

Loxigilla anoxantha, Scl. Cat. Am. B. p. 102. 1848.

$a \hat{\delta},-b$ o Jamaica ("Spermophila anoxantha," P. H. Gosse)

Typical specimens. 
ORYZOBORUS.

Oryzoborus, Cabanis, Mus. Hein. i. p. 151 (1851).

$+107 \%$. Oryzoborus torridus.

Loxia torrida, Gm. Syst. Nat. i. p. 854.

Oryzoborus torridus, Scl. Cat. Am. B. p. 102.

Loxia nasuta, Spix. Av. Bras. ii. p. 45, pl. 58.

a Brazil (Johnson) 1837. - -6 Brazil (N. C. Strickland) 1838. —c Trinidad (Argent) 1852.

+1078 . Oryzoborus maximiliani.

Oryzoborus maximiliani, Cab. Mus. Hein. i. p. 151: Scl. Cat. Am. B. p. 102.

$a$ Brazil (Argent) 1851. - b Brazil? (Argent) 1852.

$b$ is a skin of a female bird probably from Bolivia. It agrees in dimensions with $a$, a young male referable to this species.

$+10 \% 9$. Oryzoborus crassirostris.

Loxia crassirostris, Gm. Syst. Nat. i. p 862.

Sporophila crassirostris, Bp. Consp. i. p. 498.

Oryzoborus crassirostris, Scl. Cat. Am. B. p. 102. a Trinidad (Argent) 1853.

\section{Amaurospiza.}

Amaurospiza, Cabanis, Journ. f. Orn. 1861, p. 3.

- 1080. Amaurospiza unicolor.

Oryzoborus unicolor, Burm. Syst. Ueb. iii. p. 240. Amaurospiza unicolor, Scl. \& Salv. Nomencl. p. 28. a (N. C. Strickland) 1838.

A Brazilian species.

\section{SPERMOPHILA.}

Spermophila, Swainson, Zool. Journ. iii. p. 348 (1827).

1081. Spermophila minuta.

Loxia minuta, Linn. Syst. Nat. i. p. 307.

Spermophila minuta, Scl. Ibis, 1871, p. 3.

a Chili? (N. C. Strickland) 1838. - b Bogota (Bt. at Stevens's) 1845. —c Trinidad (Argent) 1852.

The locality assigned to $a$ is erroneous, the species being restricted to the northern portions of South America. 
1082. Spermophila hypoxantha.

Sporophila hypoxantha, Cab. Mus. Hein. i. p. 150.

Spermophila hypoxantha, Scl. Ibis, 1871, p. 3.

a Brazil? (Argent) 1852.

Probably a Bolivian skin.

\section{Spermophila nigro-aurantia.}

Bouvreuil de l'Isle Bourbon, D'Aub. Pl. Enl. 204, f. 1, undè,

Loxia nigro-aurantia, Bodd. Tabl. Pl. Enl. p. 12.

Spermophila nigro-aurantia, Scl. Ibis, 1871, p. 4.

Loxia brevirostris, Spix. Av. Bras. i. p. 47, pl. 59 , f. 1.

a Brazil (Johnson) 1837. -b Brazil (N. C. Strickland) 1838. -c Brazil (Argent) 1851.

\section{Spermophila castaneiventris.}

Sporophila castaneiventris, Cab. Schomb. Guian. iii. p. 679.

Spermophila castaneiventris, Scl. Ibis, 1871, p. 7.

a (Gardner) 1845.

A species of Guiana and of the valley of the Amazon.

1085. Spermophila telasco.

Spermophila telasco, Less. Voy. Coq. Zool. p. 663. Atlas, pl. 16, f. 2: Scl. Ibis, 1871, p. 7.

$a$ Chili? (N. C. Strickland) 1838.

A species peculiar to the west coast of Peru.

\section{Spermophila cucullata.}

Gros bec appellé la Nonette, D'Aub. Pl. Enl. 393, f. 3, undè, Loxia cucullata, Bodd. Tabl. Pl. Enl. p. 24.

Spermophila cucullata, Scl. Cat. Am. B. p. 103.

Loxia americana, Gm. Syst. Nat. i. p. 863.

Spermophila collaria, Scl. Ibis, 1871, p. 9 (nec Loxia collaria, Linn.).

$a$ (Arthur Strickland) 1840. -b Brazil? (Argent) 1852.

$b$ bears the label of Argent's Bolivian Collection. Judging from Linnæus's description of his Loxia collaria, I do not see how that name can, by any possibility, be associated with this species. 
108\%. Spermophila moreleti.

Spermophila moreleti, Bp. Consp. i. p. 497: Scl. Ibis, 1871, p. 1.).

a Guatemala ( $J$. Constancia) 1848. - b Guatemala (J. Constancia) 1851 .

\section{Spermophila misya.}

Pyrrhula misya, Vieill. Ois. Chant. p. 75, pl. 46.

Spermophila mysia, Bp. Consp. i. p. 496.

Loxia lineata, Gm. Syst. Nat. i. p. 858??

Spermophila lineata, Scl. Ibis, 1871, p. 11.

a (W. Kirtland) 1843. - b Tobago (Sir W. Jardine) 1844.

It appears to me that Gmelin's name $L$. lineata, based upon the Radiated Grosbeak of Latham (Syn. ii. p. 156), is most doubtfully applicable to this species.

1089. Spermophila cærulescens.

Pyrrhula ccerulescens, Vieill. Enc. Méth. p. 1023.

Spermophila ccerulescens, Scl. Ibis, 1871, p. 12.

Fringilla ornata, Licht. Verz. Doubl. p. 26.

$a,-b$ (N. C. Strickland) 1838. — $\mathrm{S}$. America (N. C. Strickland) 1838.

10\$0. Spermophila lineola.

Loxia lineola, Linn. Syst. Nat. i. p. 304.

Spermophila lineola, Scl. Ibis, 1871, p. 13.

Pyrrhula crispa, Vieill. Ois. Chant. p. 76, pl. 47. 1850 .

a (N. C. Strickland) 1838. -b (Askew) 1840. — c (Carfrae)

A species found throughout tropical South America east of the Andes.

\section{Spermophila luctuosa.}

Spermophila luctuosa, Lafr. Rev. Zool. 1843, p. 291 : Scl. Ibis, 1871, p. 15 .

a, - b Bogota (Bt at Stevens's) 1845.

1092. Spermophila gutturalis.

Fringilla gutturalis, Licht. Verz. Doubl. p. 26.

Spermophila gutturalis, Scl. Ibis, 1871, p. 15. 
$a$ S. America (N. C. Strickland) 1838. - b Trinidad (Argent) 1852 .

1093. Spermophila hypoleuca.

Fringilla hypoleuca, Licht. Verz. Doubl. p. 26.

Spermopliila hypoleuca, Scl. Ibis, 1871, p. 17.

Pyrrhula cinereola, Temm. Pl. Col. 11, f. 1.

$a$ Brazil (Arthur Strickland) 1840. -b (Thomas) 1843. -c (E. Brown) 1850.

1094. Spermophila grisea.

Loxia grisea, Gm. Syst. Nat. i. p. 857.

Spermophila grisea, Scl. Ibis, 1871, p. 18.

a Brazil (Argent) 1851.

Catamblyrhynchus.

Catamblyrhynchus, Lafresnaye, Rev. Zool. 1842, p. 301.

\section{Catamblyrhynchus diadema.}

Catamblyrhynchus diadema, Lafr. l. s. c.; Mag. Zool. 1843. Cl. ii. pl. 34: Scl. Cat. Am. B. p. 106.

a Bogota (Williams) 1846.

\section{NEORHYNCHUS.}

Neorhynchus, Sclater, P. Z. S. 1869, p. 146.

1096. Neorhynchus nasesus.

Callirhynchus nasesus, Bp. Compt. Rend. xlii. p. 822.

Neorhynchus nasesus, Scl. P. Z. S. 1869, p. 147, pl. 12. a Chili (A. Strickland) 1850.

A species of Western Peru.

\section{SUBFAMILY.-CYANOSPIZIN Æ.}

\section{Volatinia.}

Volatinia, Reichenbach, Av. Syst. pl. lxxix. (1850).

109\%. Volatinia jacarina.

Tanagra jacarina, Linn. Syst. Nat. i. p. 314.

Volatinia jacarina, Scl. Cat. Am. B. p. 106. 
$a$ S. America (Askew) 1837. -b, -c (N. C. Strickland) 1838. - $d,-e$ Guatemala (J. Constancia) 1848. - $f$ Brazil? (Argent) 1852.

\section{Phonipara.}

Phonipara, Bonaparte, Consp. Av. i. p. 494 (1850).

1098. Phonipara pusilla.

Tiaris pusilla, Sw. Phil. Mag. 1827, p. 438.

Phonipara pusilla, Scl. Cat. Am. B. p. 106.

$a$ (Bt. at Stevens's) 1845. - b Bogota (Williams) 1846.

1099. Phonipara olivacea.

Emberiza alivacea, Gm. Syst. Nat. i. p. 870.

Spermophila olivacea, Gosse, B. Jam. p. 249.

Phonipara olivacea, Scl. Cat. Am. B. p. 107.

a §̂ Jamaica (P. H. Gosse) 1848.

1100. Phonipara marchi.

Phonipara marchi, Baird, Proc. Ac. Phil. 1863, p. 297.

Spermophila bicolor, Gosse, B. Jam. p. 252; Ill. pl. 64 (nec Linn.).

a Jamaica ("Spermophila bicolor," P. H. Gosse) 1845.

Cyanospiza.

Cyanospiza, Baird, B. N. Am. p. 500 (1858).

\section{Cyanospiza cyanea.}

Tanagra cyanea, Linn. Syst. Nat. i. p. 315.

Cyanospiza cyanea, Scl. Cat. Am. B. p. 107: Baird, Brew. \& Ridgw. N. Am. B. ii. p. 82.

$a$ N. America (Askew) 1834. - b N. America (N. C. Strickland) 1838. —c Guatemala (J. Constancia) 1845. —d (Dewgard) 1849. - e Guatemala (J. Constancia) 1851.

\section{Cyanospiza versicolor.}

Spiza versicolor, Bp. P. Z. S. 1837, p. 120.

Cyanospiza versicolor, Scl. Cat. Am. B. p. 107: Baird, Brew. \& Ridgw. N. Am. B. ii. p. 86.

a Mexico (N. C. Strickland) 1838.

1103. Cyanospiza ciris.

Emberiza ciris, Linn. Syst. Nat. i. p. 313. 
Cyanospiza ciris, Scl. Cat. Am. B. p. 107: Baird, Brew. \& Ridgw. N. Am. B. ii. p. 87.

a Mexico (T. Mann) 1844. -b ㅎ, -c f Savannah 1845 (S. F. Baird) 1847.

\section{Hapalospiza.}

Hapalospiza, Cabanis, Mus. Hein. i. p. 147 (1851).

1104. Hapalospiza unicolor.

Hapalospiza unicolor, Cab. l. s. c.: Scl. Cat. Am. B. p. 108.

$a$ (Askew) 1837. -b (N. C. Strickland) 1838. - c Brazil (Argent) 1853.

\section{Paroaria.}

Paroaria, Bonaparte, Saggio di una Distr. Met. An. Vert. p. 141 (Aggiunte, 1832).

\section{Paroaria cucullata.}

Loxia cucullata, Lath. Ind. Orn. p. 378.

Paroaria cucullata, Scl. Cat. Am. B. p. 108.

a (Osborn) 1845.

A species found in Bolivia and the Argentine Republic.

\section{Paroaria dominicana.}

Loxia dominicana, Linn. Syst. Nat. i. p. 301.

Paroaria dominicana, Bp. Consp. i. p. 471.

Paroaria larvata (Bodd.): Scl. Cat. Am. B. p. 108.

$a$ S. America (Johnson) 1837. -b (N. C. Strickland) 1838.

A Brazilian species.

\section{0\%. Paroaria gularis.}

Tanagra gularis, Linn. Syst. Nat. i. p. 316.

Paroaria gularis, Scl. Cat. Am. B. p. 108.

$a$ (Dewgard) 1846.

Apparently a skin of Cayenne make.

1108. Paroaria nigrigenis.

Nemosia nigro-genis, Lafr. Rev. Zool. 1846, p. 273.

Paroaria nigrigena, Scl. Cat. Am. B. p. 108.

$a,-b$ Trinidad (Argent) 1851.

s. C. 


\section{CoRYPHOSPINGUS.}

Coryphospingus, Cabanis, Mus. Hein. i. p. 145 (1851).

1109. Coryphospingus pileatus.

Fringilla pileata, Max, Beitr. iii. p. 605.

Coryphospingus pileatus, Scl. Cat. Am. B. p. 109. 1850 .

a Brazil (N. C. Strickland) 1838. -b (Bt. at Birmingham)

\section{PORPHYRIOSPIZA.}

Porphyriospiza, Sclater \& Salvin, Nomencl. Av, Neotr. pp. 30, 155 (1873).

\section{Porphyriospiza cyanella.}

Emberiza cyanella, Sparrm. Mus. Carls. No. 42.

Cyanospiza cyanella, Pelz. Orn. Bras. p. 227.

Porphyriospiza cyanella, Scl, \& Salv. l. s. c.

a (Burl) 1834.

A Brazilian species.

\section{TIARIS.}

Tiaris, Swainson, Zool. Journ. iii. p. 351 (1827).

111. Tiaris ornata.

Fringilla ornata, Max, Reis. n. Bras. ii. p. 191; Beitr. iii. p. 610 .

Tiaris ornata, Bp. Consp. i. p. 471.

$a$, -b Brazil (N. C. Strickland) 1838. - c Brazil (Argent) 1851.

\section{Poospiza.}

Poospiza, Cabanis, Arch. f. Naturg. xiii. p. 349 (1847).

1112. Poospiza thoracica.

Fringilla thoracica, Nordm. Erm. Reis. u. d. Erde, p. 10.

Poospiza thoracica, Scl. Cat. Am. B. p. 109.

a Brazil (N. C. Strickland) 1838. -b Brazil (Osborn) 1845.

1113. Poospiza lateralis.

Fringilla lateralis, Nordm. Erm. Reis. u. d. Erde, p. 10.

Poospiza lateralis, Burm. Syst. Ueb. iii. p. 215: Pelz. Orn.

Bras. p. 228.

a Brazil (N. C. Strickland) 1838. 
1114. Poospiza torquata.

Emberiza torquata, D'Orb. \& Lafr. Syn. Av. i. p. 82.

Poospiza torquata, Scl. Cat. Am. B. p. 110.

a Brazil ? (Argent) 1852.

A Bolivian species.

1115. Poospiza cinerea.

Poospiza cinerea, Bp. Consp. Av. i. p. 473.

a (Askew) 1834.

A Brazilian species.

1116. Poospiza melanoleuca.

Emberiza melanoleuca, D’Orb. \& Lafr. Syn. Av. i. p. 82. Poospiza melanolenca, Bp. Consp. Av. i. p. 472. $a$ (N. C. Strickland) 1838. - b Brazil? (Argent) 1852.

A species found in Paraguay and the Argentine Republic.

\section{SUBFAMILY.-PASSERELLIN $刃$.}

\section{Phrygilus.}

Phrygilus, Cabanis, Arch. f. Naturg. x. p. 29 (1844).

\section{Phrygilus gayi.}

Fringilla gayi, Eyd. et Gerv. Mag. Zool. 1834, Cl. ii. pl. 23.

Phrygitus gayi, Scl. \& Salv. Ibis, 1869, p. 285.

Fringilla formosa, Gould, Zool. Voy. Beagle, iii. p. 93.

$a$ S. America (N. C. Strickland) 1838. -b Chili (T. Brown) 1842.

\section{Phrygilus caniceps.}

Phrygilus caniceps, Burm. J. f. Orn. 1860, p. 158: Durnf. Ibis, 1878, p. 393.

a Brazil? (Argent) 1852.

A Bolivian skin agreeing with examples from that country. This bird has usually been called Phrygilus atriceps (Lafr. \& D'Orb.), but it differs from that species in having a gray instead of a black head. It is, I believe, the Emberiza gayi (stirps major) of those authors. 
Bolivian examples agree fairly with others from Chupat, Patagonia named $P$. caniceps by the late Mr Henry Durnford who probably compared them with Dr Burmeister's specimens.

\section{Phrygilus unicolor.}

Emberiza unicolor, D’Orb. \& Lafr. Syn. Av. i. p. 82.

Phrygilus unicolor, Scl. Cat. Am. B. p. 110.

a (Bt. at Stevens's) 1844.

A female example of this widely-ranging Andean species.

\section{Phrygilus fruticeti.}

Fringilla fruticeti, Kittl. Kupf. p. 28, pl. 23, f. 1.

Phrygilus fruticeti, Scl. Cat. Am. B. p. 111.

$a,-b$ Coquimbo (N. C. Strickland) 1838.

\section{Phrygilus xanthogrammus.}

Chlorospiza? xanthogramma, Gray, Voy. Beagle, iii. p. 96, pl. 33.

$a$ S. America (N. C. Strickland) 1838.

\section{Diuca.}

Diuca, Reichenbach, Av. Syst. pl. lxxviii. (1850). 1122. Diuca grisea.

Fringilla diuca, Mol. Saggio, St. Nat. Chili, p. 249 (8vo. ed. 1782).

Dolichonyx grisea, Less. L'Inst. 1834, p. 316.

Diuca grisea, Scl. Cat. Am. B. p. 111.

$a$ Chili (T. Brown) 1842.

\section{Passerculus.}

Passerculus, Bonaparte, Comp. List, p. 33 (1838).

\section{Passerculus savanna.}

Fringilla savanna, Wils. Am. Orn. iii. p. 55, pl. 22, f. 2.

Passerculus savanna, Baird, Brew. \& Ridgw. N. Am. B. i. p. 534.

a Pennsylvania (S. F. Baird) 1847. 


\section{Passerculus alaudinus.}

Passerculus alaudinus, Bp. Compt. Rend. xxxvii. p. 918.

Passerculus savanna var. alaudinus, Baird, Brew. \& Ridgw.

N. Am. B. i. p. 537.

a Sitka (J. G. Kinberg) 1845.

\section{Passerculus sandwichensis.}

Emberiza sandwichensis, Gm. Syst. Nat. i. p. 875.

Passerculus savanna var. sandwichensis, Baird, Brew. \& Ridgw. N. Am. B. i. p. 538.

$a$ California (J. F. Brandt) 184.6.

This and the two preceding species are very closely allied, and may together with $P$. anthinus have ultimately to be united under one somewhat variable form which should bear the name $P$. sandwichensis.

\section{Pogecetes.}

Pooccetes, Baird, Birds of N. Am. p. 447 (1858).

1126. Poccetes gramineus.

Fringilla graminea, Gm. Syst. Nat. i. p. 922.

Pooccetes gramineus, Baird, Brew. \& Ridgw. N. Am. B. i. p. 545 .

$a,-b$ (Arthur Strickland) 1840. - o f Carlisle, Pennsylvania, May 1846 (S. F. Baird) 1847.

\section{ZONOTRICHIA.}

Zonotrichia, Swainson, Faun. Bor. Am. iii. p. 493 (1831), (as a sub-genus).

112\%. Zonotrichia leucophrys.

Emberiza leucophrys, Forst. Phil. Trans. 1xii. pp. 403, 426.

Zonotrichia leucophrys, Baird, Brew. \& Ridgw. N. Am. B. i. p. 566.

$a \mathrm{~N}$. America (Cashmore) 1839. - $b \hat{\jmath}$ Carlisle, Pennsylvania, 3 May 1844 (S. F. Baird) 1847.

1128. Zonotrichia albicollis.

Fringilla albicollis, Gm. Syst. Nat. i. p. 921.

Zonotrichia albicollis, Baird, Brew. \& Ridgw. N. Am. B. i. p. 574 . 
Fringilla pennsylvanica, Lath. Ind. Orn. p. 445.

a (N. C. Strickland) 1838. - b f̂ Carlisle, Pennsylvania, 13 April 1844 (S. F. Baird) 1847.

\section{Zonotrichia pileata.}

Bruant, du Cap de Bonne-Espérance, D'Aub. PI. Enl. 386, f. 2, undè,

Emberiza pileata, Bodd. Tab. Pl. Enl. p. 23.

Zonotrichia pileata, Scl. Cat. Am. B. p. 113.

Fringilla matutina, Licht. Verz. Doubl. p. 25.

$a$ S. America (Askew) 1834. - b Guatemala (J. Constancia) 1851.

\section{Zonotrichia strigiceps.}

Zonotrichia strigiceps, Gould, Zool. Voy. Beagle, iii. p. 92.

Zonotrichia strigiceps, Scl. Ibis, 1877, p. 47, pl. 1, f. 2.

a (Arthur Strickland) 1840.

Agrees well with the figure quoted above. The original specimens of $Z$. strigiceps were obtained by Mr Darwin at Santa Fé on the Rio Paraná.

\section{Melospiza.}

Melospiza, Baird, Birds N. Am. p. 476 (1858).

\section{Melospiza melodia.}

Fringilla melodia, Wils. Am. Orn. ii. p. 125, pl. 16, f. 4.

Melospiza melodia, Baird, Brew. \& Ridgw. N. Am. B. ii. p. 19. a (Arthur Strickland) 1840. - b Carlisle, Pennsylvania, 11 Nov. 1842 (S. F. Baird) 1847. —c (Dewgard) 1851.

\section{Melospiza insignis.}

Melospiza insignis, Baird, Trans. Chic. Ac. i. p. 319, pl. xxix. f. 2.

Melospiza melodia var. insignis, Baird, Brew. \& Ridgw. N. Am. B. ii. p. 30.

$a \hat{\delta}$ N. W. America (J. F. Brandt) 1846.

A specimen from Wosnessenski's collection referred to Fringilla unalaschensis, Gm. a name which cannot now be fixed satisfactorily to any species. The specimen agrees with the description of the autumnal plumage given in Baird, Brewer, \& Ridgway's work quoted above. 
1133. Melospiza palustris.

Fringilla palustris, Wils. Am. Orn. iii. p. 49, pl. 22, f. 1.

Melospiza palustris, Baird, Brew. \& Ridgw. N. Am. B. ii.

p. 34 .

a (Havell) 1839.

A common species in the eastern provinces of North America.

\section{Spizella.}

Spizella, Bonaparte, Comp. List, p. 33 (1838).

\section{Spizella monticola.}

Fringilla monticola, Gm. Syst. Nat. i. p. 912.

Spizella monticola, Baird, Brew. \& Ridgw. N. Am. B. ii. p. 3.

Fringilla canadensis, Lath. Ind. Orn, p. 434.

$a \mathrm{~N}$. America (Havell) 1839.

\section{Spizella socialis.}

Fringilla socialis, Wils. Am. Orn. ii. p. 127, pl. 16, f. 5 .

Spizella socialis, Baird, Brew. \& Ridgw. N. Am. B. ii. p. 7.

$a$ N. America? (Havell) 1839. - $b \hat{\jmath}$ Carlisle, Penusylvania, 25 September, 1842 (S. F. Baird) 1847.

\section{PASSerella.}

Passerella, Swainson, Classif. B. ii. p. 288 (1837).

\section{Passerella iliaca.}

Fringilla iliaca, Merrem, Beitr. z. bes. Gesch. d.Vög. ii. p. 40, pl. 10.

Passerella iliaca, Baird, Brew. \& Ridgw. N. Am. B. ii. p. 50. a N. America (N. C. Strickland) 1838.

\section{3\%. Passerella townsendi.}

Fringilla townsendi, Aud. Orn. Biogr. v. p. 236.

Passerella townsendi, Baird, Brew. \& Ridgw. N. Am. B. ii. p. 53.

$a$ N. W. America (J. F. Brandt) 1846.

A specimen from Wosnessenski's collection marked "Emberiza hyperborea, Pall." 


\section{JUNCO.}

Junco, Wagler, Isis, 1831, p. 526.

\section{+ 1138. Junco hiemalis.}

Fringilla hyemalis, Linn. Syst. Nat. i. p. 308.

Junco hyemalis, Baird, Brew. \& Ridgw. N. Am. B. i. p. 580. $a$ N. America (N. C. Strickland) 1838.

\section{Junco oregonus.}

Fringilla oregana, Towns. Journ. Ac. Phil. vii. p. 188.

Junco oregonus, Baird, Brew. \& Ridgw. N. Am. B. i. p. 584. $a \hat{o}$ N. W. America (J. F. Brandt) 1846.

A specimen from Wosnessenski's collection.

\section{Junco cinereus.}

Fringilla cinerea, Sw. Phil. Mag. 1827, p. 435.

Junco cinereus, Scl. Cat. Am. B. p. 115.

a Mexico (T. Mann) 1844.

A young bird of this Mexican species.

\section{Peucra.}

Peuccea, Audubon, Synops. B. N. Am. p. 112 (1839).

\section{Peucæa æstivalis.}

Fringilla aestivalis, Licht. Verz. Doubl. p. 25.

Peuccea cestivalis, Baird, Brew. \& Ridgw. N. Am. B. ii. p. 39. Peucaea bachmanni, Aud. l. s. c.

$a \hat{~},-b$ ก Georgia 1846 (S. F. Baird) 1847.

\section{AMmodromus.}

Ammodramus, Swainson, Zool. Journ, iii. p. 348 (1827). Ammodromus, Strickland, Ann. \& Mag. N. H. vii. p. 33.

\section{Ammodromus maritimus.}

Fringilla maritima, Wils. Am. Orn. iv. p. 68, pl. 34, f. 2.

Ammodromus maritimus, Baird, Brew. \& Ridgw. N. Am. B. i. p. 560 .

$a$ (Havell) 1839. -b N. Jersey (S. F. Baird) 1847. 


\section{CotURNiculus.}

Coturniculus, Bonaparte, Comp. List, p. 32 (1838).

\section{Coturniculus passerinus.}

Fringilla passerina, Wils. Am. Orn. iii. p. 76, pl. 24, f. 5.

Coturniculus passerinus, Scl. \& Salv. Ibis, 1859, p. 18: Baird, Brew. \& Ridgw. N. Am. B. i. p. 553.

a Guatemala (J. Constancia) 1845. - $b$ ई Carlisle, Pennsylvania, 26 May 1843 (S. F. Baird) 1847.

\section{Coturniculus manimbe.}

Fringilla manimbe, Licht. Verz. Doubl. p. 25.

Coturniculus manimbe, Scl. Cat. Am. B. p. 116.

$a$ S. America (Askew) 1837. - b S. America (N. C. Strickland) 1838.

\section{Embernagra.}

Embernagra, Lesson, Traité d'Orn. p. 465 (1831).

\section{Embernagra rufivirgata.}

Embernagra rufivirgata, Lawr. Ann. Lyc. N. Y. v. p. 112, pl. v. f. 2: Baird, Brew. \& Ridgw. N. Am. B. ii. p. 47.

$a$ (Bt. at Stevens's) 1844.

A species found in Mexico and the border State of Texas.

\section{Embernagra olivascens.}

Embernagra olivascens, D’Orb. Voy. Am. Mérid. Ois. p. 285.

Embernagra longicauda, Strickl. Ann. \& Mag. N. H. xiii. p. 420.

a (N. C. Strickland) 1838.

The type of E. longicauda, Strickl.

\section{4\%. Embernagra platensis.}

Emberiza platensis, Gm. Syst. Nat. i. p. 886.

Embernagra platensis, Strickl. Ann. \& Mag. N. H. xiii. p. 420 : Scl. Cat. Am. B. p. 117.

$a$ (Askew) 1839.

A species of Brazil and the Argentine Republic. 


\section{Emberizordes.}

Emberizoides, Temminck, Pl. Col. Livr. 19 (1824).

1148. Emberizoides sphenurus.

Passerina sphenura, Vieill. N. Dict. d'Hist. N. xxv. p. 25.

Emberizoides sphenurus, Scl. Cat. Am. B. p. 118.

$a,-b$ Brazil (N. C. Strickland) 1838.

\section{Pipilo.}

Pipilo, Vieillot, Analyse, p. 32 (1816).

1149. Pipilo erythrophthalmus.

Fringilla erythrophthalma, Linn. Syst. Nat. i. p. 318.

Pipilo erythrophthalma, Baird, Brew. \& Ridgw. N. Am. B. ii. p. 109.

$$
a,-b,-c \text { N. America (Askew) } 1834 .
$$

\section{+1150. Pipilo macronyx.}

Pipilo macronyx, Sw. Phil. Mag. 1827, p. 434: Baird, Brew. \& Ridgw. N. Am. B. ii. p. 105.

$a,-b$ Mexico (T. Mann) 1844.

Agree with Swainson's type.

\section{Pyrgisoma.}

Pyrgisoma, Bonaparte, Consp. Av. i. p. 486 (1850).

\section{Pyrgisoma biarcuatum.}

Pyrgita biarcuata, Prév. Voy. Vénus, Zool. p. 216, Atlas, pl. 6. Pyrgisoma biarcuatum, Scl. \& Salv. Ex. Orn. p. 130. a Guatemala (J. Constancia) 1845.

\section{Chondestes.}

Chondestes, Swainson, Phil. Mag. 1827, p. 435.

\section{Chondestes grammaca.}

Fringilla grammaca, Say, Long's Exp. i. p. 321 (Lond. ed. 1823).

Chondestes grammaca, Baird, Brew. \& Ridgw. N. Am. B. i. p. 562.

a f̊ Michigan (S. F. Baird) 1847. 
SUBFAMILY.-EMBERIZIN $Æ$.

EMberiza.

Emberiza, Linnæus, Syst. Nat. i. p. 308 (1766) (partim).

1153. Emberiza miliaria.

Emberiza miliaria, Linn. Syst. Nat. i. p. 308: Strickl. P. Z. S. 1836, p. 99 : Newton, ed. Yarr. Brit. B. ii. p. 38: Dresser, B. Eur.

$a \hat{\delta},-b$ o Weston, Oxfordshire, 28 February (N. C. Strickland) 1834. - c ô Smyrna, 12 November (H. E. Strickland) 1835 .

\section{Emberiza citrinella.}

Emberiza citrinella, Linn. Syst. Nat. i. p. 309: Strickl. P. Z. S. 1836, p. 99: Newton, ed. Yarr. Brit. B. ii. p. 43: Dresser, B. Eur.

$a$ o Worcestershire (H. E. Striclcland) 1833. b, -c Worcestershire (H. E. Strickland) 1834.

\section{Emberiza cirlus.}

Emberiza cirlus, Linn. Syst. Nat. i. p. 311: Strickl. P. Z. S. 1836, p. 99: Newton, ed. Yarr. Brit. B. ii. p. 50: Dresser, B. Eur.

a f Smyrna, 10 November (H. E. Strickland) 1835. - b $\hat{\delta}$ Smyrna, 11 January (H. E. Strickland) 1836. -c ô Moor, Worcestershire, 30 June (H. E. Strickland) 1838.

\section{Emberiza hortulana.}

Emberiza hortulana, Linn. Syst. Nat. i. p. 309: Strickl. P. Z. S. 1836, p. 99: Newton, ed. Yarr. Brit. B. ii. p. 57: Dresser, B. Eur.

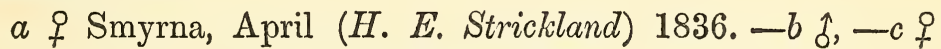
Lombardy, July (H. E. Strickland) 1836.

\section{5\%. Imberiza cinerea.}

Emberiza cinerea, Strickl. P. Z. S. 1836, p. 99 (nec Gmelin): Jard. Mem. H. E. Strickland, p. clxiii. pl. 6: Dresser, B. Eur.

a ô Smyrna, April (H. E. Strickland) 1836.

The type of the species and the specimen from which the plate in the 'Memoirs' was drawn. As E. cinerea, Gm. is not a true E'mberiza Strickland's specific name may, we hope, be allowed to stand. 
1158. Emberiza spodocephala.

Emberiza spodocephala, Pall. Zoogr. Rosso-As. ii. p. 51.

$a$ Siberia (J. F. Brandt) 1846.

1159. Emberiza fucata.

Emberiza fucata, Pall. Zoogr. Rosso-As. ii. p. 41: Strickl. Ann. \& Mag. N. H. xiii. p. 38 : Jerd. B. Ind. ii. p. 375.

a fิ Kumaon (W. J. E. Boys) 1847.

1160. Emberiza rustica.

Emberiza rustica, Pall. Zoogr. Rosso-As. ii. p. 43.

$a$ Siberia (J. F. Brandt) 1846.

1161. Emberiza cia.

Emberiza cia, Linn. Syst. Nat. i. p. 310: Strickl. P. Z. S. 1836, p. 99: Dresser, B. Eur.

a $\hat{\delta}$ Smyrna, 4 December (H. E. Strickland) 1835. -b f Smyrna, 15 December (H. E. Strickland) 1835.

1162. Emberiza leucocephala.

Emberiza leucocephalos, S. Gm. N. Comm. Petrop. xv. p. 480, pl. 23, f. 3: Dresser, B. Eur.

Emberiza pithyornus, Pall. Reis. ii. p. 710: Jerd. B. Ind. ii. p. 370.

$a$ ô Siberia (J. $F$. Brandt) 1841. - $b$ f Gungur Mt. (W. $J$. E. Boys) 1847.

\section{Emberiza cæsia.}

Emberiza ccesia, Cretzschm. Rüpp. Atlas, p. 17, pl. 10, f. b: Strickl. P. Z. S. 1836, p. 99: Dresser, B. Eur.

a ô Smyrna, April (H. E. Strickland) 1836. -b f Zante, 23 May (H. E. Strickland) 1836.

1164. Emberiza stewarti.

Euspiza stewarti, Blyth, J. A. S. B. xxiii. p. 215.

Emberiza stewarti, Jerd. B. Ind. ii. p. 374.

a (T. C. Eyton) 1850.

A species of Cashmere and the N. W. Himalayas. 
1165. Emberiza cioides.

Emberiza cioides, Brandt, Bull. Phys.-Math. Ac. St. Pétersb. i. p. 363.

a ô Altai ("Emberiza cioides, nov. sp." J. F. Brandt) 1842.

A typical specimen.

1166. Emberiza schœniclus.

Emberiza schoniclus, Linn. Syst. Nat. i. p. 311: Strickl. P. Z. S. 1836, p. 99: Newton, ed. Yarr. Brit. B. ii. p. 23.

a Oxfordshire, 11 October (N. C. Strickland) 1833. - b $\hat{0}$ Worcestershire (H. E. Strickland) 1834. $-c$ W. Siberia (J. F. Brandt) 1844 .

\section{6\%. Emberiza pyrrhuloides.}

Emberiza pyrrhuloides, Pall. Zoogr. Rosso-As. ii. p. 49; Ic. pl. 49, f. 1 : Strickl. Ann. \& Mag. N. H. xii. p. 417.

$a$ Spain (Boissoneau) 1839. Spain.

A species of South-Eastern Europe, but not known to occur in

\section{Emberiza palustris.}

Emberiza palustris, Savi, Orn. Tosc. ii. p. 91 : Strickl. P.Z. S. 1836, p. 99.

a ô Smyrna, 20th November (H. E. Strickland) 1835.

1169. Emberiza passerina.

Emberiza passerina, Pall. Reis. i. p. 456 ; Zoogr. Rosso-As. i. p. 49: Seebohm, Ibis, 1879, p. 39, pl. 1, f. 1.

a Siberia (J. F. Brandt) 1846.

\section{Euspiza.}

Euspiza, Bonaparte, Saggio di una Distr. Met. An. Vert. p. 141 (Aggiunte 1832).

\section{Euspiza americana.}

Emberiza americana, Gm. Syst. Nat. i. p. 872.

Euspiza americana, Baird, Brew. \& Ridgw. N. Am. B. ii. p. 65 .

a N. America (Askew) 1833. 


\section{Euspiza melanocephala.}

Emberiza melanocephala, Scop. Ann. i. Hist. Nat. p. 142: Dresser, B. Eur.

Euspiza melanocephala, Bp. Comp. List, p. 32.

$a$ f, - $b$ f Zante, 18 May (H. E. Strickland) 1836.

\section{Euspiza Iuteola.}

Emberiza luteola, Sparrm. Mus. Carls. No. 93.

Euspiza luteola, Jerd. B. Ind. ii. p. 378.

Emberiza icterica, Eversm. Add. ad Pall. Zoogr. Rosso-As. fasc. ii. p. 10 .

$a$ (Askew) 1837. - b ô Circa Nov. Alexandrovna, Siberia, 4. May 1840 (J. F. Brandt) 1846. - c f̂ Benares (W. J. E. Boys) 1847. - $d$ q N. India (W. J. E. Boys) 1848.

1173. Euspiza aureola.

Emberiza aureola, Pall. Reis. ii. p. 711 : Dresser, B. Eur.

Euspiza aureola, Jerd. B. Ind. ii. p. 380.

a Altai (J. F. Brandt) 1842. -b Siberia (J. F. Brandt) 1844. -c India ("Emberiza aureola, Pallas," E. Blyth) 1846.

\section{MeLOPHUS.}

Melophus, Swainson, Classif. B. ii. p. 290 (1837).

\section{Melophus melanicterus.}

Fringilla melanictera, Gm. Syst. Nat. i. p. 910.

Melophus melanicterus, Jerd. B. Ind. ii. p. 381.

Emberiza erythroptera, Jard. \& Selb. Ill. Orn. iii. pl. 132: Strickl. Ann. \& Mag. N. H. vii. p. 33.

$a$ Himalaya (N. C. Strickland) 1838. - b f Kumaon (W. J. E. Boys) 1847. —c f N. India (W. J. E. Boys) 1848.

\section{Fringillaria.}

Fringillaria, Swainson, Classif. B. ii. p. 289 (1838).

1175. Fringillaria capensis.

Emberiza capensis, Linn. Syst. Nat. i. p. 310. 
Fringillaria capensis, Strickl. Ann. \& Mag. N. H. viii. p. 374: Strickl. \& Scl. Contr. Orn. 1852, p. 151 : Layard, B. S. Afr. p. 206 : Sharpe, Cat. Afr. B. p. 70.

$a$ Simon's Bay, Cape of Good Hope, 18 March (A. Strickland) 1832. - b Cape of Good Hope (N. C. Strickland) 1838. -c Damara-land (C. J. Andersson) 1852.

\section{Fringillaria flaviventris.}

Passerina flaviventris, Vieill. Enc. Méth. p. 929.

Fringillaria flaviventris, Strickl. Ann. \& Mag. N. H. viii. p. 374: Layard, B. S. Afr. p. 206 : Sharpe, Cat. Afr. B. p. 70.

a Africa (N. C. Strickland) 1838. - b S. Africa (Bt. at Stevens's) 1842.

\section{7\%. Fringillaria septemstriata.} f. 2 .

Emberiza septemstriata, Rüpp. Neue Wirb. p. 86, pl. 30,

Emberiza striolata, Strickl. P. Z. S. 1850, p. 218 (nec Rüppell).

a f̂ Kordofan, 9 July (J. Petherick) 1848.

This skin seems to be referable to this species rather than to L. striolata.

\section{Fringillaria impetuani.}

Emberiza impetuani, Smith, Rep. S. Af. Exp. App. p. 48.

Fringillaria impetuani, Layard, B. S. Afr. p. 206.

$a$ S. Africa (Capt. Alexander) 1838.

\section{Plectrophanes.}

Plectrophanes, Meyer, Zusätze u. Bericht. z. Meyer u. Wolfs Taschenb. deutsch. Vög. p. 56 (1822).

\section{Plectrophanes nivalis.}

Emberiza nivalis, Linn. Syst. Nat. i. p. 308.

Plectrophanes nivalis, Newton, ed. Yarr. Brit. B. ii. p. 1: Dresser, B. Eur.: Baird, Brew. \& Ridgw. N. Am. B. i. p. 512.

a Britain (N. C. Strickland) 1838. - b, -c (Arthur Strickland) 1840. $-d$ Govt. of St Petersburg (J. F. Brandt) 1841. -e Canada (Barnes) 1848. 


\section{Plectrophanes lapponicus.}

Fringilla lapponica, Linn. Syst. Nat. i. p. 317.

Plectrophanes lapponicus, Newton, ed. Yarr. Brit. B. ii. p. 15: Dresser, B. Eur.

$a$ (N. C. Strickland) 1838.

A species found in the northern parts of both the old and new worlds.

\section{SUBFAMILY.-PLOCEIN AE.}

TEXTOR.

Textor, Temminck, Pl. Col. Livr. 75 (1828).

\section{Textor niger.}

Bubalornis niger, Smith, Rep. S. Afr. Expl. Exp. App. p. 52.

Textor niger, Strickl. \& Scl. Contr. Orn. 1852, p. 150.

Bubalornis erythrorhynchus, Smith, Ill. S. Afr. Zool. pl. 64: Gurney, And. B. Jamara-l. p. 165.

$a,-b$ Damara-land, (C. J. Andersson) 1852.

\section{Hyphantornis.}

Hyphantornis, G. R. Gray, Gen. B. ii. p. 369 (1849).

\section{Hyphantornis capensis.}

Oriolus capensis, Gm. Syst. Nat. i. p. 329.

Hyphantornis capensis, Sharpe, Cat. Afr. B. p. 59.

Icterus cafer, Licht. Verz. Doubl. p. 19.

Ploceus aurifrons, Temm. Pl. Col. 175, 176.

$a,-b$ (N. C. Strickland) 1838. -c S. Africa (Bt. at Stevens's) 1842.

\section{Hyphantornis cucullatus.}

Oriolus cucullatus, Müll. Syst. Nat. Suppl. p. 87.

Hyphantornis cucullatus, Gray, H-list. ii. p. 40.

Oriolus textor, Gm. Syst. Nat. i. p. 390.

a Africa (G. Lloyd) 1838. - b S. Africa (Capt. Alexander) 1838. -c (Thomas) 1842. -d (Thomas) 1845. 
1184. Hyphantornis spilonotus.

Ploceus spilonotus, Vig. P. Z. S. 1830-1, p. 92.

Hyphantornis spilonota, Sharpe, Cat. Afr. B. p. 59.

$a$ W. Africa (N. C. Strickland) 1838.

A South-African species.

\section{Hyphantornis velatus.}

Ploceus velatus, Vieill. N. Dict. d'Hist. N. xxxiv. p. 132.

Hyphantornis velatus, Finsch \& Hartl. v. d. Decken's Reise, iv. p. 391.

Ploceus mariquensis, Smith, Ill. Zool. S. Afr. pl. 103.

Hyphantornis mariquensis, Gurney, Ibis, 1868, p. 466, pl. 10.

a Cape of Good Hope (N. C. Strickland) 1838.

1186. Hyphantornis Iuteolus.

Fringilla luteola, Licht. Verz. Doubl. p. 23.

Ploceus luteolus, Strickl. P. Z. S. 1850, p. 217.

Hyphantornis luteola, Sharpe, Cat. Afr. B. p. 59.

a $\hat{o}$ Kordofan, 30 April (J. Petherick) 1848.

\section{8\%. Hyphantornis vitellinus.}

Fringilla vitellina, Licht. Verz. Doubl. p. 23.

Hyphantornis vitellina, Sharpe, Cat. Afr. B. p. 58.

$a$ Senegal (Isaacson) 1840. -b (Bt. at Stevens's) 1845.

\section{Hyphantornis personatus.}

Ploceus personatus, Vieill. Gal. des Ois. i. p. 117, pl. 84: Jard. Contr. Orn. 1849, p. 35, pl. 7.

Hyphantornis personata, Gray, Hand-1. ii. p. 42.

a (Chapman) 1849 .

Agrees with West-African specimens of this species.

1189. Hyphantornis brachypterus.

Ploceus brachypterus, Sw. B. W. Afr. i. p. 168, pl. 10.

Hyphantornis brachyptera, Sharpe, Cat. Afr. B. p. 59.

a Senegal (Isaacson) 1840.

Agrees with Swainson's type.

\section{Hyphantornis guerini.}

Ploceus melanotis, Guér. Rev. Zool. 1843, p. 321: Des Murs, Voy. Abyss. vi. p. 110 (nec Sw.).

S. C. 
Hyphantornis guerini, G. R. Gray, Gen. B. ii. p. 364.

Ploceus auricularis, Des Murs, op. cit. Album, Ois. pl. 9, f. 1. a Abyssinia (Verreaux) 1850.

\section{SYCOBRotus.}

Sycobrotus, Cabanis, Mus. Hein. i. p. 182 (1851). 1191. Sycobrotus bicolor.

Ploceus bicolor, Vieill. N. Dict. d'Hist. N. xxiv. p. 127.

Sycobrotus bicolor, Finsch \& Hartl. v. d. Decken's Reise, iv. p. 403.

$a$ W. Africa (Bt. at Birmingham) 1849.

\section{Ploceus.}

Ploceus, Cuvier, Règn. Anim. i. p. 363 (1817). 1192. Ploceus philippinus.

Loxia philippina, Linn. Syst. Nat. i. p. 305.

Ploceus philippinus, Wald. Trans. Z. S. ix. p. 209.

Ploceus baya, Blyth, J. A. S. B. xiii. p. 945 : Jerd. B. Ind. ii. p. 343.

$a \hat{\delta},-b$ 个 Madras (A. Strickland) 1833. -c Malacca (Askew) 1837. - d Malacca (Johnson) 1837. - e Malacca (N. C. Strickland) 1838. - $f$ India (Major Stacey) 1845. - g S. India ("Euplectes philippensis," T. C. Jerdon) 1850. - $h$ India (E. Blyth) 1850 .

$a, b, c, d$ and $e$ were all identified by Strickland with the Linnæan L. philippina (See Walden l. s. c.).

\section{Ploceus manyar.}

Fringilla manyar, Horsf. Trans. L. S. xiii. p. 160.

Ploceus manyar, Jerd. B. Ind. ii. p. 348.

$a$, -b India ("Ploceus manyar," E. Blyth) 1851.

\section{Ploceus bengalensis.}

Loxia bengalensis, Linn. Syst. Nat. i. p. 305.

Ploceus bengalensis, Jerd. B. Ind. ii. p. 349.

a India ("Ploceus bengalensis, non-breeding dress," E. Blyth) 1846. - b India (E. Blyth) 1850. — India (E. Blyth) 185̃1. —d India ("Ploceus bengalensis, female," E. Blyth) 1851. 


\section{Ploceus sanguinirostris.}

Loxia sanguinirostris, Linn. Syst. Nat. i. p. 303.

Ploceus sanguinirostris, Finsch \& Hartl. v. d. Decken's Reise, iv. p. 407. 1841

$a$ (Askew) 1837. -b (N. C. Strickland) 1838. -c (Askew)

All females or young birds probably belonging to this WestAfrican species.

\section{Ploceus æthiopicus.}

Ploceus sanguinirostris var. cethiopicus, Sundev. Öfv. k. Vet. Ak. Förh. 1850, p. 126.

Ploceus cethiopicus, Finsch \& Hartl. v. d. Decken's Reise, iv. p. 409 : Finsch, Trans. Z. S. vii. p. 263.

Ploceus sanguinirostris, Strickl. P. Z. S. 1850, p. 218 (nec Linn.).

$a \hat{o},-b q$ Kordofan, 15 July (J. Petherick) 1848.

\section{NigRITA.}

Ethiops, Strickland, P. Z. S. 1841, p. 30.

Nigrita, Fraser, P. Z. S. 1842, p. 145.

119\%. Nigrita canicapilla.

Ethiops canicapillus, Strickl. l. s. c.

Nigrita canicapilla, Fraser, l. s. c.; Zool. Typ. pl. 48.

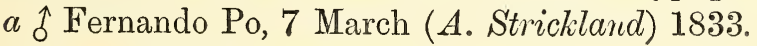

The type of this scarce species.

\section{Plocepasser.}

Plocepasser, Smith, Ill. Zool. S. Afr. Av. sub pl. 65 (1836).

\section{Plocepasser mahali.}

Plocepasser mahali, Smith, l. s. c.: Strickl. \& Scl. Contr. Orn. 1852, p. 150: Gurney, in And. B. Damara-1. p. 166.

a Damara-land (C. J. Andersson) 1852.

\section{Foudia.}

Foudia, Reichenbach, Av. Syst. pl. 1xxix. (1850).

1199. Foudia eminentissima.

Foudia eminentissima, Bp. Consp. i. p. 446 . 
Calyphantria eminentissima, Finsch \& Hartl. v. d. Decken's Reise, iv. p. 406.

$a$ E. Indies (J. Lockwood) 1837.

Agrees with Bonaparte's description of this East-African species.

Pyromel ana.

Euplectes, Swainson, Zool. Ill. ser. 2, sub pl. 37 (1830) (nec Leach): Strickl. Ann. \& Mag. N. H. vii. p. 31.

Pyromelcena, Bonaparte, Saggio di una Distr. Met. An. Vert. p. 141 (Aggiunte 1832).

\section{Pyromelæna orix.}

Emberiza orix, Linn. Syst. Nat. i. p. 309.

Pyromelana oryx, Finsch \& Hartl. v. d. Decken's Reise, iv. p. 410 . 1840.

a S. Africa (Capt. Alexander) 1838. - b Australia (Askew)

The locality assigned to $b$ is no doubt erroneous. The specimen was unnamed by Strickland who, however, suspected its African origin.

1201. Pyromelæna flammiceps.

Euplectes flammiceps, Sw. B. W. Afr. i. p. 186, pl. 13.

Pyromelana flammiceps, Finsch \& Hartl. v. d. Decken's Reise, iv. p. 414.

$a$ W. Africa (N. C. Strickland) 1838. -b (Argent) 1851.

These specimens agree with one in the Swainson collection, which is probably the type of the species.

\section{Pyromelæna franciscana.}

Loxia franciscana, Isert, Schrift. Gesellsch. Naturf. Berl. ix. p. 332 , pl. 9 .

Pyromelana franciscana, Finsch \& Hartl. v. d. Decken's Reise, iv. p. 412.

Fringilla ignicolor, Vieill. N. Dict. d'Hist. N. xii. p. 198.

Pyromelana ignicolor, Strickl. P. Z. S. 1850, p. 218.

a Cape of Good Hope (Askew) 1834. - b, —c Kordofan, September (J. Petherick) 1848.

1203. Pyromelæna taha.

Euplectes taha, Smith, Ill. Zool. S. Afr. pl. 7: Gurney, in And. B. Damara-1. p. 171. 
Ploceus abyssinicus, (Gm.) Strickl. \&Scl. Contr. Orn.1852,p.150. a Damara-land (C.J. Andersson) 1852.

\section{Pyromelæna capensis.}

Loxia capensis, Linn. Syst. Nat. i. p. 306.

Euplectes capensis, Strickl. Ann. \& Mag. N. H. vii. p. 32: Sharpe, Cat. Afr. B. p. 62.

a $f$ Cape of Good Hope, 15 April (A. Strickland) 1832. -b Africa (N. C. Strickland) 1834. — C W. Africa (Askew) 1837. —d q, - $e$ q W. Africa (N. C. Strickland) 1838. $-f$ (Bt. at Liverpool) 1853.

\section{UROBRACHYA.}

Urobrachya, Bonaparte, Consp. Av. i. p. 447 (1850).

1205. Urobrachya axillaris.

Vidua axillaris, Smith, Ill. Zool. S. Afr. Av. pl. 17.

Urobrachya axillaris, Bp. l.s. c.

a (Arthur Strickland) 1850. -b (Argent) 1851.

A South-African species.

\section{Penthetria.}

Penthetria, Cabanis, Arch. f. Naturg. xiii. p. 331 (1847).

\section{Penthetria ardens.}

Veuve à poitrine rouge, du Cap de Bonne Espérance, D’Aub. Pl. Enl. 647, undè,

Fringilla ardens, Bodd. Tab. Pl. Enl. p. 39.

Penthetria ardens, Finsch \& Hartl. v. d. Decken's Reise, iv. p. 423.

Vidua rubritorques, Sw. B. W. Afr. i. p. 174.

$a,-b$ Africa (N. C. Strickland) 1838.

\section{0\%. Penthetria macrura.}

Moineau, du Royaume de Juda, D'Aub. PI. Enl. 183, f. 1 undè, Loxia macroura, Gm. Syst. Nat. i. p. 845.

Penthetria macroura, Finsch \& Hartl. v. d. Decken's Reise, iv. p. 418.

Fringilla chrysoptera, Vieill. N. Dict. d'Hist. N. xii. p. 214.

Vidua chrysoptera, Strickl. Ann. \& Mag. N. H. vii. p. 32.

$a$ (Askew) 1834. - b, -c W. Africa (Johnson) 1837. $-d$ W. Africa (N. C. Strickland) 1838. 
PASSERES.

\section{SPOROPIPES.}

Sporopipes, Cabanis, Arch. f. Naturg. xiii. p. 332 (1847).

1208. Sporopipes frontalis.

Loxia frontalis, Lath. Ind. Orn. Suppl. p. xlvi.

Philetorus frontalis, Strickl. P. Z. S. 1850, p. 218.

Sporopipes frontalis, Cab. Mus. Hein. i. p. 179.

a (Thomas) 1842.

A species of North-east Africa.

1209. Sporopipes squamifrons.

Estrelda squamifrons, Smith, Rep. S. Afr. Exp. p. 49; Ill. Zool. S. Afr. pl. 95.

Philetcerus squamifrons, Strickl. \& Scl. Contr. Orn. 1852, p. 150.

Sporopipes squamifrons, Gurney, in And. B. Damara-l. p. 177. $a$ S. Africa (Capt. Alexander) 1838. - b Damara-land (C. J. Andersson) 1852.

\section{VIDUA.}

Vidua, Cuvier, Leçons d'An. Comp. i. Tab. ii. (1800).

\section{Vidua paradisea.}

Emberiza paradisea, Linn. Syst. Nat. i. p. 312.

Vidua paradisea, Sw. B. W. Afr. i. p. 172, pl. 11 : Strickl. P. Z. S. 1850, p. 218; Contr. Orn. 1850, p. 88. pl. 59: Finsch \& Hartl. v. d. Decken's Reise, iv. p. 424.

$a$ W. Africa (Robins) 1834. - b (N. C. Strickland) 1838. - c ¡̂ Kordofan, 17 August (J. Petherick) 1848. —d Damara-land (C. J. Andersson) 1852.

I can see no difference between the Kordofan and West-African specimens. The former have been referred to $V$. Verreauxi by Finsch \& Hartlaub.

\section{Vidua principalis.}

Emberiza principalis, Linn. Syst. Nat. i. p. 313.

Vidua principalis, Strickl. P. Z. S. 1850, p. 218: Finsch \& Hartl. v. d. Decken's Reise, iv. p. 428.

Vidua erythrorhyncha, Sw. B. W. Afr. i. p. 176, pl. 12. 
$a$ (Johnson) 1837. $-b,-c$ W. Africa (N. C. Strickland) 1838. - d (Purchased) 1839. - e, $-f$ Kordofan (J. Petherick) 1850.

\section{Chera.}

Chera, G. R. Gray, Gen. B. ii. p. 58 (1849).

\section{Chera progne.}

Veuve à ailes rouges, du Cap de Bonne Espérance, D'Aub. Pl. Enl. 635, undè,

Emberiza progne, Bodd. Tab. Pl. Enl. p. 39.

Chera progne, Layard, B. S. Afr. p. 190.

Emberiza longicauda, Gm. Syst. Nat. i. p. 884.

$a$ S. Africa (N. C. Strickland) 1838.

\section{Hypochera.}

Hypochera, Bonaparte, Consp. i. p. 450 (1850).

\section{Hypochera nitens.}

Fringilla nitens, Gm. Syst. Nat. i. p. 909.

Philetcerus nitens, Strickl. P. Z. S. 1850, p. 218.

Hypochera nitens, Finsch \& Hartl. v. d. Decken's Reise, iv. p. 430 .

a Africa (Johnson) 1837. - b $\hat{o}$ Kartoum, October (J. Petherick) 1848 .

\section{SPERMOSPIZA.}

Spermospiza, G. R. Gray, List Gen. B. p. 43 (1840).

\section{Spermospiza hæmatina.}

Loxia homatina, Vieill. Enc. Méth. pp. 1007, 1016.

Spermospiza hcematina, Sharpe, Cat. Afr. B. p. 68.

a Gambia (N. C. Strickland) 1838.

\section{SPERMESTES.}

Spermestes, Swainson, Classif. B. ii. p. 280 (1837).

\section{Spermestes cucullatus.}

Spermestes cucullata, Sw. B. W. Afr. i. p. 201: Sharpe, Cat. Afr. B. p. 64.

$a \mathrm{~W}$. Africa (Askew) 1837.

Agrees with Swainson's type. 


\section{Amadina.}

Amadina, Swainson, Zool. Journ. iii. p. 349 (1827).

1216. Amadina erythrocephala.

Loxia erythrocephala, Gm. Syst. Nat. i. p. 849.

Amadina erythrocephala, Layard, B. S. Afr. p. 200.

a $\hat{\delta},-b$ \& S. Africa (Capt. Alexander) 1838.

121\%. Amadina fasciata.

Loxia fasciata, Gm. Syst. Nat. i. p. 859.

Amadina fasciata, Strickl. P. Z. S. 1850, p. 218.

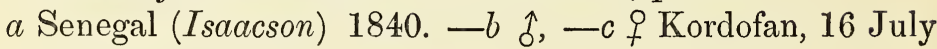
(J. Petherick) 1848.

\section{HABROPYGA.}

Habropyga, Cabanis, Arch. f. Naturg. xiii. p. 331 (1847).

1218. Habropyga cærulescens.

Fringilla coerulescens, Vieill. N. Dict. d'Hist. N. xii. p. 176.

Estrelda ccerulescens, Sw. B. W. Afr. i. p. 195.

a (N. C. Strickland) 1838.

A West-African species.

\section{Pytelia.}

Pytelia, Swainson, Classif. B. ii. p. 280 (1837).

1219. Pytelia melba.

Fringilla melba, Linn. Syst. Nat. i. p. 319.

Pytelia melba, Strickl. \& Scl. Contr. Orn. 1852, p. 150.

a (Mather) 1840. -b Damara-land (C. J. Andersson) 1852.

1220. Pytelia citerior.

Pytelia elegans, Strickl. P. Z. S. 1850, p. 218 (nec Gm.).

Pytelia citerior, Strickl. Contr. Orn. 1852, p. 151.

$a \hat{\delta},-b q$ Kordofan, August (J. Petherick) 1848.

The types of this species.

LAGONOSTICTA.

Lagonosticta, Cabanis, Mus. Hein. i. p. 171 (1851).

1221. Lagonosticta rubricata.

Fingilla rubricata, Licht. Verz. Doubl. p. 27. 
Estrelda rubricata, Layard, B. S. Afr. p. 197.

a S. E. Africa (Argent) 1853.

\section{SPERMophaGa.}

Spermophaga, Swainson, Classif. B. ii. p. 277 (1837).

\section{Spermophaga margaritata.}

Spermophaga margaritata, Strickl. Ann. \& Mag. N. H. xiii. p. 418, pl. 10.

Spermophaga verreauxi, Des Murs, Icon. Orn. pl. 64.

a Madagascar (Mrs Van der Kemp) 1843.

The specimen described and figured by Des Murs was shot by Jules Verreaux at the Cape of Good Hope, so that in all probability Strickland was misinformed as to the Madagascan origin of his type.

\section{ESTRILDA.}

Estrilda, Swainson, Zool. Journ. iii. p. 349 (1827).

\section{Estrilda erythronota.}

Fringilla erythronotos, Vieill. N. Dict. d'Hist. N. xii. p. 182; Ois. Chant. pl. 14.

Estrelda erythronota, Gurney, in And. B. Damara-l. p. 178.

Estrelda lipiniana, Smith, Rep. S. Afr. Exp. App. p. 49: Strickl. \& Scl. Contr. Orn. 1852, p. 150.

$a$ S. Africa (Capt. Alexander) 1838. -b Damara-land (C. J. Andersson) 1852.

\section{Estrilda dufresnii.}

Fringilla dufresnii, Vieill. N. Dict. d'Hist. N. xii. p. 181.

Estrilda dufresnii, Layard, B. S. Afr. p. 197.

$a,-b$ S. Africa ( $D r A$. Smith).

\section{Estrilda granatina.}

Fringilla granatina, Linn. Syst. Nat. i. p. 319.

Estrilda granatina, Strickl. \& Scl. Contr. Orn. 1852, p. 150: Layard, B. S. Afr. p. 199.

$a$ (Askew) 1840. -b (Argent) 1851.

A South-African species. 
1226. Estrilda bengala.

Fringilla bengalus, et $F$. angolensis, Linn. Syst. Nat. i. p. 323.

Estrelda benghala, Layard, B. S. Afr. p. 199.

Estrelda phoenicotis, Sw. B. W. Afr. i. p. 192, pl. 14.

$a$ S. Africa (Capt. Alexander) 1838.

122\%. Estrilda cinerea.

Fringilla cinerea, Vieill. N. Dict. d'Hist. N. xii. p. 176.

Estrelda cinerea, Sharpe, Cat. Afr. B. p. 65.

$a$ (F. D. Cartwright) 1846.

A West-African species.

1228. Estrilda astrild.

Loxia astrild, Linn. Syst. Nat. i. p. 303.

Estrilda astrild, Strickl. \& Scl. Contr. Orn. 1852, p. 150.

$a,-b$ Mauritius (A. Striclland) 1832. $\longrightarrow$ Damara-land (C. J. Andersson) 1852.

\section{Estrilda amandava.}

Fringilla amandava, Linn. Syst. Nat. i. p. 319.

Estrilda amandava, Strickl. Ann. \& Mag. N. H. xiii. p. 37.

Estrelda amandava, Jerd. B. Ind. ii. p. 359.

$a$ Mauritius (Mrs Telfair) 1832. —b (Johnson) 1837. -c, -d (N. C. Strickland) 1838. - e Madras ("Estrelda amandava" T. C. Jerdon) 1845. - $f$ q Benares (W. J. E. Boys) 1847. -g (Mrs Acland) 1847.

\section{Estrilda bella.}

Loxia bella, Lath. Ind. Orn. Suppl. p. xlvi.

Estrilda bella, Strickl. Ann. \& Mag. N. H. xi. p. 337 : Gould, B. Austr. iii. pl. 78.

$a,-b$ Australia (N. C. Strickland) 1838. -c.

\section{Estrilda temporalis.}

Fringilla temporalis, Lath. Ind. Orn. Suppl. p. xlviii.

Estrelda temporalis, Gould, B. Austr. iii. pl. 82.

a New South Wales (N. C. Strickland) 1838. 


\section{StAgONOPLEURA.}

Stagonopleura, Reichenbach, Av. Syst. pl. lxxv. (1850).

1232. Stagonopleura guttata.

Loxia guttata, Shaw, Mus. Lev. ii. p. 47, pl. 12.

Stagonopleura guttata, Cab. Mus. Hein. i. p. 172: Gould, Handb. B. Austr. i. p. 417.

Fringilla lathami, Vig. \& Horsf. Trans. L. S. xv. p. 256.

Amadina lathami, Gould, B. Austr. iii. pl. 86.

$a,-b$ New South Wales (N. C. Strickland) 1838.

\section{TANIOPYGIA.}

Taniopygia, Reichenbach, Singvög. p. 26.

1233. Tæniopygia castanotis.

A madina castanotis, Gould, P. Z. S. 1836, p. 105; B. Austr. iii. pl. 87.

Tceniopygia castanotis, Gould, Handb. B. Austr. i. p. 419.

a f Yarrundi, Upper Hunter, Australia, 20 March, 1839 (T. C. Eyton) 1846. -b (Dewgard) 1851.

Poephila.

Poephila, Gould, P. Z. S. 1842, p. 18.

\section{Poephila cincta.}

Amadina cincta, Gould, P. Z. S. 1836, p. 105; B. Austr. iii. pl. 93.

Poephila cincta, Gould, Handb. B. Austr. i. p. 425.

a New South Wales (N. C. Strickland) 1838.

\section{PADDA.}

Padda, Reichenbach, Av. Syst. pl. 1xxvi. (1850).

1235. Padda oryzivora.

Loxia oryzivora, Linn. Syst. Nat. i. p. 302.

Amadina oryzivora, Strickl. P. Z. S. 1842, p. 167.

Padda oryzivora, Salvad. Ann. Mus. Genov. v. p. 263.

$a$ E. Indies (Purchased) 1839.

\section{Munia.}

Munia, Hodgson, As. Res. xix. p. 153 (1836).

1236. Munia maja.

Loxia maja, Linn. Syst. Nat. i. p. 301. 
Munia maja, Salvad. Ann. Mus. Genov. v. p. 264. $a$ (Thomas) 1842. - b (Bt. at Stevens's) 1845.

A species of Malacca and the large neighbouring islands.

\section{3\%. Munia flaviprymna?}

Donacola flaviprymna, Gould, P. Z. S. 1845, p. 80; B. Austr. iii. pl. 96 .

Munia flaviprymna, Gould, Handb. B. Austr. i. p. 428.

a Cape Upstart, Australia, 20 May (Argent) 1848.

A female or young bird possibly belonging to this species.

1238. Munia malacca.

Loxia malacca, Linn. Syst. Nat. i. p. 302.

Munia malacca, Jerd. B. Ind. ii. p. 352.

a Madras ("Amadina malacca," T. C. Jerdon) 1845. - b S. India ("A madina malacca," T. C. Jerdon) 1850.

1239. Munia rubronigra.

Munia rubroniger, Hodgs. As. Res. xix. p. 153: Jerd. B. Ind. ii. p. 353 .

a $\hat{\delta}$ (Bt. at Stevens's) 1845. -b (Bt. at Dresden) 1845.

\section{Munia malabarica.}

Loxia malabarica, Linn. Syst. Nat. i. p. 305.

Munia malabarica, Jerd. B. Ind. ii. p. 357.

Lonchura cheet, Sykes, P. Z. S. 1832, p. 95.

$a$ India (Askew) 1837. - b India (N. C. Strickland) 1838. -c India ("Amadina malabarica, Lonchura cheet, Sykes, L. bicolor, Tickell." E. Blyth) 1846. —d $q$ Benares (W. J.E. Boys) 1847.

\section{Munia acuticauda.}

Munia acuticauda, Hodgs. As. Res. xix. p. 153: Strickl. P. Z. S. 1846, p. 103: Jerd. B. Ind. ii. p. 356.

a Malacca (Bt. at Stevens's) 1844.

\section{Munia striata.}

Loxia striata, Linn. Syst. Nat. i. p. 306. Amadina striata, Strickl. P. Z. S. 1846, p. 103.

Munia striata, Jerd. B. Ind. ii. p. 356.

a (N. C. Strickland) 1838.

A species of Southern India. 


\section{Munia punctulata.}

Loxia punctulata, Linn. Syst. Nat. i. p. 302.

A madina punctulata, Strickl. Ann. \& Mag. N. H. xiii. p. 38.

Loxia undulata, Lath. Ind. Orn. p. 387.

Munia undulata, Jerd. B. Ind. ii. p. 354.

a Mauritius, 14 June (A. Strickland) 1832. - b (Mrs Telfair) 1832. —c, - d (Askew) 1837. - e Madras " Amadina punctularia," T. C. Jerdon) 1845. $-f \hat{\delta},-g$ q, $-h$ q Kumaon (W. J. E. Boys) 1847.

\section{ORTYGOSPIZA.}

Ortygospiza, Sundevall, Öfvers. k. Vet. Ak. Förh. 1850, p. 98.

\section{Ortygospiza polyzona.}

Fringilla polyzona, Temm. Pl. Col. 221, f. 3.

Ortygospiza polyzona, Sundev. l. s. c.

a W. Africa (N. C. Strickland) 1838.

\section{UROLONCHA.}

Uroloncha, Cabanis, Mus. Hein. i. p. 173 (1851).

\section{Uroloncha cantans.}

Loxia cantans, Gm. Syst. Nat. i. p. 859.

Amadina cantans, Strickl. P. Z. S. 1850, p. 218.

Uroloncha cantans, Cab. l. s. c.

$a$ (Thomas) 1842. -b (Bt. at Stevens's) 1845. —c Kordofan (J. Petherick) 1848.

\section{Uroloncha jagori.}

Oxycerca jagori, Cab. J. f. Orn. 1872, p. 317: Twedd. Trans. Z. S. ix. p. 208.

a Philippine Islands, 13 May (H. Cuming) 1840. 


\section{FAMILY.-PARADOXORNITHIDAE.}

PARAdoxornis ${ }^{1}$.

Paradoxornis, Gould, P. Z. S. 1836, p. 17.

124\%. Paradoxornis ruficeps.

Paradoxornis ruficeps, Blyth, J. A. S. B. xi. p. 177; J. A. S. B. xliii. extr. No. p. 117 : Jerd. B. Ind. ii. p. 5.

a Arakan (E. Blyth) 1847. - b India (" Paradoxornis ruficeps," E. Blyth) 1851.

\section{FAMILY.-ALAUDIDE.}

\section{Alauda.}

Alauda, Linnæus, Syst. Nat. i. p. 287 (1766) (partim).

1248. Alauda arvensis.

Alauda arvensis, Linn. Syst. Nat. i. p. 287 : Strickl. P. Z. S. 1836, p. 99: Newton, ed. Yarr. Brit. B. i. p. 614: Dresser, B. Eur.

a $\hat{o}$ juv. Worcestershire (H. E. Strickland) 1833. - - $\hat{\delta}$ Worcestershire, January (H. E. Strickland) 1834. —c f Smyrna, 28 December (H. E. Strickland) 1835.

\section{Alauda gulgula.}

Alauda gulgula, Frankl. P. Z. S. 1830-1, p. 119: Blyth, J. A. S. B. xiii. p. 961: Jerd. B. Ind. ii. p. 434.

Alauda gracilis, Blyth, J. A. S. B. xi. p. 201.

a India (Askew) 1837. - b India ("Alauda gulgula, Bengal var." E. Blyth) 1846. -c Madras ("Alauda gulgula," T. C. Jerdon) 1850. —d India ("Alauda gulgula," E. Blyth) 1851. - e India (E. Blyth) 1851.

1 The right position of this genus has been and still is a matter of doubt. Various places have been assigned to it, such as the families Timeliidæ, Paridæ, and in the neighbourhood of the Buntings. A close relationship to the genus Panurus has also been suggested. 
1250. Alauda arborea.

Alauda arborea, Linn. Syst. Nat. i. p. 287 : Strickl. P. Z. S. 1836, p. 99: Newton, ed. Yarr. Brit. B. i. p. 625: Dresser, B. Eur.

a $\hat{\delta}$ juv. Aberdovey, August (H. E. Strickland) 1833. - $b$ ô Smyrna, 12 November (H. E. Strickland) 1835. —c (Bt. at Stevens's) 1845.

\section{Pyrrhulauda.}

Pyrrhulauda, Gray, Gen. B. ii. p. 281 (1844).

\section{Pyrrhulauda grisea.}

Alauda grisea, Scop. Del. Faun. et Fl. Insubr. ii. p. 95.

Pyrrhulauda grisea, Strickl. Ann. \& Mag. N. H. xiii. p. 37 : Jerd. B. Ind. ii. p. 424.

Alauda gingica, Gm. Syst. Nat. i. p. 795.

Pyrrhulauda gingica, Strickl. P. Z. S. 1842, p. 167.

a India (N. C. Strickland) 1838. - b (N. C. Strickland) 1838. - c India (Bt. at Stevens's) 1845. —d Madras ("Pyrrhulauda gingica," T. C. Jerdon) 1845. - e o Marwar (W. J. E. Boys) 1847. - f o Jucknie (W. J. E. Boys) 1847. -g S. India ("Pyrrhulauda gingica," T. C. Jerdon) 1850. - h (Bt. at Edinburgh) 1850.

\section{Calendula.}

Calendula, Swainson, Classif. B. ii. p. 292 (1837).

1252. Calendula crassirostris.

Alauda crassirostris, Vieill. N. Dict. d'Hist. N. i. p. 373.

Calendula crassirostris, Sharpe, P. Z. S. 1874, p. 636.

a S. Africa (Bt. at Aberystwith) 1833. -b (Askew) 1834.

\section{Melanocorypha.}

Melanocorypha, Boie, Isis, 1828, p. 322.

1253. Melanocorypha yeltoniensis.

Alauda yeltoniensis, Forst. Phil. Trans. lvii. p. 350.

Melanocorypha yeltoniensis, Dresser, B. Eur.

Alauda tartarica, Pall. Reise, ii. p. 707.

$a$ Govt. of Orenburg (J. F. Brandt) 1841. 


\section{Melanocorypha calandra.}

Alauda calandra, Linn. Syst. Nat. i. p. 288: Strickl. P. Z. S. 1836, p. 99.

Melanocorypha calandra, Dresser, B. Eur.

$a$ f, - $b$ \& Smyrna, 2 \& 11 January (H. E. Strickland) 1836.

\section{Calandrella.}

Calandrella, Kaup. Naturl. Syst. p. 39 (1829).

\section{Calandrella brachydactyla.}

Alauda brachydactyla, Leisl. Wett. Annal. iii. p. 357, pl. 19 : Newton, ed. Yarr. Brit. B. i. p. 637.

Calandrella brachydactyla, Jerd. B. Ind. ii. p. 426: Dresser, B. Eur.

Emberiza baghaira, Frankl. P. Z. S. 1830-1, p. 119.

Coryphidea bagheira, Blyth, J. A. S. B. xiii. p. 961.

Alauda dukhunensis, Sykes, P. Z. S. 1832, p. 93.

a Athens, April 1839 (Bt. at Dresden) 1845. - b Madras ("Alauda dukhunensis (bagheira)," T. C. Jerdon) 1845. — India ("Coryphidea bagheira," E. Blyth) 1846.

\section{Calandrella cinerea.}

Alauda cinerea, Gm. Syst. Nat. i. p. 798.

Tephrocorys cinereus, Sharpe, P. Z. S. 1874, p. 633.

Alauda spleniata, Strickl. Contr. Orn. 1852, p. 152.

a (N. C. Strickland) 1838. - b S. Africa (Dr A. Smith, No. 121). $-c$ (Bt. at Stevens's) 1843. - d Damara-land (C. J. Andersson) 1852.

$d$ is the type of $A$. spleniata, Strickland.

\section{Alaudula.}

Alaudala, Horsfield \& Moore, Cat. B. E. I. Mus. ii. p. 471 (1856).

\section{5\%. Alaudula raytal.}

Alauda raytal, Blyth, J. A. S. B. xiii. p. 962.

Alaudala raytal, Jerd. B. Ind. ii. p. 428.

a $q$ (W. J. E. Boys) 1847. -b India (Major Stacey). 


\section{Spizalauda.}

Spizalauda, Blyth, J. A. S. B. xxiv. p. 258 (1855).

1258. Spizalauda deva.

Alauda deva, Sykes, P. Z. S. 1832, p. 92.

Spizalauda deva, Jerd. B. Ind. ii. p. 432.

Mirafra hayi, Blyth, J. A. S. B. xiii. p. 959.

a Madras (T. C. Jerdon) 1845. - b S. India ("Mirafra hayi," T. C. Jerdon) 1850.

\section{Galerita.}

Galerida, Boie, Isis, 1828, p. 321.

1259. Galerita cristata.

Alauda cristata, Linn. Syst. Nat. i. p. 288: Strickl. P. Z. S. 1836, p. 99.

Galerita cristata, Dresser, B. Eur.

Galerida cristata, Jerd. B. Ind. ii. p. 436.

a ô Smyrna, 4 December (H. E. Strickland) 1835. - b (Askew) 1837. — $\mathrm{S}$ S. India (Bt. at Stevens's) 1843. —d Madras (T. C. Jerdon) 1845. - e ô N. India (W. J. E. Boys) 1847.

Both $b$ and $d$ are in bad condition and $\mathrm{I}$ am doubtful if they are correctly referred to $G$. cristata.

1260. Galerita isabellina.

Alauda isabellina, Temm. Pl. Col. 244, f. 2.

Galerida cristata? Strickl. P. Z. S. 1850, p. 218.

a ô Kartoum, October (J. Petherick) 1848.

\section{Certhilauda.}

Certhilauda, Swainson, Zool. Journ. iii. p. 344 (1827).

1261. Certhilauda capensis.

Sirli du Cap de Bonne Espérance, D'Aub. Pl. Enl. 712, undè, Alauda capensis, Bodd. Tabl. Pl. Enl. p. 45.

Certhilauda capensis, Sharpe, P. Z. S. 1874, p. 615.

Alauda africana, Gm. Syst. Nat. i. p. 798.

a $\hat{\delta}$ Cape of Good Hope (A. Strickland) 1833.

s. C. 
ALAMMON.

Alcemon, Keyserling \& Blasius, Wirb. Eur. pp. xxxvi。94 (1840).

\section{Alæmon semitorquata.}

Certhilauda semitorquata, Smith, Rep. S. Afr. Exp. p. 47.

Alomon semitorquata, Sharpe, P. Z. S. 1874, p. 620.

Certhilauda rufopalliata, Lafr. Mag. Zool. 1836, Cl. ii. pl. 59. $a$ S. Africa (Bt. at Stevens's) 1842.

\section{Alæmon nivosa.}

Certhilauda nivosa, Sw. B. W. Afr. i. p. 213.

Alcemon nivosa, Sharpe, P. Z. S. 1874, p. 623.

Alauda lagepa, Smith, Ill. Zool. S. Afr. pl. 87, f. 2.

a $\hat{\delta}$ S. Africa (Dr A. Smith, 368).

\section{Ammomanes.}

Ammomanes, Cabanis, Mus. Hein. i. p. 125 (1851).

1264. Ammomanes phœnicurus.

Mirafra phœnicura, Frankl. P. Z. S. 1830-1, p. 119.

Ammomanes phonicura, Jerd. B. Ind. ii. p. 421.

a (N. C. Strickland) 1838. -b Assa (W. J. E. Boys) 1847. -c S. India (T. C. Jerdon) 1850.

1265. Ammomanes erythropygia.

Alauda erythropygia, Strickl. P. Z. S. 1850, p. 219, pl. 24. a $q$ Kordofan, 9 July (J. Petherick) 1848.

The type of this species.

\section{Ammomanes erythroclamys.}

Alauda erythroclamys, Strickl. Contr. Orn. 1852, p. 151. Ammomanes erythroclamys, Sharpe, P. Z. S. 1874, p. 628. a Damara-land (C.J. Andersson) 1852.

The type of this species.

\section{Mirafra.}

Mirafra, Horsfield, Trans. Linn. Soc. xiii. p. 159 (1820).

\section{6\%. Mirafra assamica.}

Mirafra assamica, Horsf. P. Z. S. 1839, p. 162: Jerd. B. Ind. ii. p. 416. 
a (N. C. Strickland) 1838. - b India ("Mirafra assamica," E. Blyth) 1846. -c, -d India (E. Blyth) 1846. -e India ("Mirafra assamica," E. Blyth) 1851.

1268. Mirafra affinis.

Mirafra affinis, Blyth, J. A. S. B. xiii. p. 959 : Jerd. B. Ind. ii. p. 417 : Blyth, J. A. S. B. xliii. extra No. p. 95.

a Madras, September (A. Strickland) 1833. - b Madras ("Mirafra affinis, malabarica?" T. C. Jerdon) 1845. —c Arakan (E. Blyth) 1847.

$a$ was referred by Strickland to Alauda malabarica of Scopoli, a name of doubtful application which has been associated with several different species.

1269. Mirafra erythroptera.

Mirafra erythroptera, Blyth, J. A. S. B. xiii. p. 958: Jerd. Ill. Ind. Orn. pl. 38; B. Ind. ii. p. 418.

$a$ (Askew) 1837. -b ô N. India (W. J. E. Boys) 1848. -c S. India ("Mirafra erythroptera," T. C. Jerdon) 1850.

$b$ has a small bill but otherwise resembles $c$. It is apparently an immature bird.

\section{2\%0. Mirafra cantillans.}

Mirafra cantillans, Blyth, J. A. S. B. xiii. p. 960: Jerd. B. Ind. ii. p. 420.

a Madras (T. C. Jerdon) 1845. -b S. India ("Mirafra cantillans," T. C. Jerdon) 1850.

1271. Mirafra africana.

Mirafra africana, Smith, Rep. S. Afr. Exp. App. p. 47: Sharpe, P. Z. S. 1874, p. 642.

$a($ Askew) 1834. - b S. Africa (Dr A. Smith, 137).

1272. Mirafra apiata.

Alauda apiata, Vieill. N. Dict. d'Hist. N. i. p. 342.

Mirafra apiata, Sharpe, P. Z. S. 1874, p. 638.

$a$ (Gardner).

A South-African species. 
1273. Mirafra sabota.

Mirafra sabota, Smith, Rep. S. Afr. Exp. App. p. 47: Sharpe, P. Z. S. 1874, p. 645.

Megalophonus chineanus, Gray, Hand-1. B. ii. p. 122 (nec Smith).

a S. Africa (Askew) 1840.

12\%4. Mirafra nævia.

Alauda noevia, Strickl. Contr. Orn. 1852, p. 152.

Mirafra navia, Sharpe, P. Z. S. 1874, p. 646.

a Damara-land (C. J. Andersson) 1852.

The type of this species.

\section{OTOCORYS.}

Otocoris, Bonaparte, Faun. Ital. Ucc. fol. **** (1839).

1275. Otocorys alpestris.

Alauda alpestris, Linn. Syst. Nat. i. p. 289.

Otocorys alpestris, Newton, ed. Yarr. Brit. B. i. p. 604: Dresser, B. Eur.

Alauda cornuta, Wils. Am. Orn. i. p. 85, pl. 5, f. 4. 1846.

$a$ N. America (Thomas) 1843. - b Siberia (J. F. Brandt)

\section{Otocorys penicillata.}

Alauda penicillata, Gould, P. Z. S. 1837, p. 126.

Otocorys penicillata, Dresser, B. Eur.

Otocoris longirostris, Moore, P. Z. S. 1855, p. 215, pl. 111.

$a$ N. India (Bt. at Stevens's). - $b$ $\hat{o}$ circa Nov. Alexandrovna, 4 Mai 1840 (J. F. Brandt) 1846.

$b$ is marked by Prof. Brandt "Alauda alpestris, Linn. (A. nivalis, Pall.)."

SECTION.-OSCINES CULTRIROSTRES.

FAMILY.-ICTERIDAE.

Ostinops.

Ostinops, Cabanis, Mus. Hein. i. p. 187 (1851).

127\%. Ostinops decumanus.

Xanthornus decumanus, Pall. Spic. Zool. vi. p. 1, pl. 1. 
Ostinops decumanus, Salv. \& Godm. Ibis, 1879, p. 200.

Oriolus cristatus, Gm. Syst. Nat. i. p. 387.

Ostinops cristatus, Scl. Cat. Am. B. p. 127.

a Brazil (Askew) 1837. -b Brazil (N. C. Strickland) 1838.

1278. Ostinops viridis.

Oriolus viridis, Müll. Syst. Nat. Suppl. p. 87.

Cassicus viridis, Vieill. N. Dict. d'Hist. N. v. p. 364.

Ostinops viridis, Scl. Cat. Am. B. p. 128.

a Guiana (Schomburgk).

\section{Cacicus.}

Cacicus, Daudin, Traité d’Orn. ii. p. 322 (1800).

1279. Cacicus persicus.

Oriolus persicus, Linn. Syst. Nat. i. p. 161.

Cassicus persicus, Scl. Cat. Am. B. p. 128.

$a$ S. America (Askew) 1834. -b Brazil (W. Kirtland) 1838.

1280. Cacicus hæmorrhous.

Oriolus homorrhous, Linn. Syst. Nat. i. p. 161 (partim).

Cacicus hoemorrhous, Daud. Traité d'Orn. ii. p. 328: Scl. Cat. Am. B. p. 129.

a Brazil (Bt. at Aberystwith) 1833. -b Brazil (Askew) 1834. —c (Askew) 1834. —d Brazil (N.C. Strickland) 1838. —e Brazil (G. Lloyd) 1838.

\section{Cacicus leucorhamphus.}

Xanthornus leucorhamphus, Bp. Atti Sc. Ital. 1845, p. 405.

Cassiculus leucorhamphus, Scl. Cat. Am. B. p. 129.

Cassicus leucorhamphus, Scl. \& Salv. Nomencl. p. 36.

$a$ (N.C.Strickland) 1838. $-b,-c$ Bogota (Stevens) 1845.

\section{Cacicus albirostris.}

Cassicus albirostris, Vieill. N. Dict. d'Hist. N. v. p. 364.

Cassiculus albirostris, Scl. Cat. Am. B. p. 129.

Xanthornus chrysopterus, Vig. Zool. Journ. ii. p. 190, suppl. pl. 9.

a (Askew) 1834. 


\section{Cacicus holosericeus.}

Sturnus holosericeus, Licht. Preis. Verz. Mex. Vög. p. 1; J. f. Orn. 1863, p. 55.

Amblyrhamphus prevosti, Less. Cent. Zool. p. 159, pl. 54.

Cassiculus prevosti, Scl. Cat. Am. B. p. 129.

a Guatemala ( $J$. Constancia) 1845.

Lichtenstein's name I have no doubt applies to this bird.

\section{Cacicus solitarius.}

Cassicus solitarius, Vieill. N. Dict. d'Hist. N. v. p. 364.

Cassiculus solitarius, Scl. Cat. Am. B. p. 130.

a Brazil (Osborn) 1845.

\section{ICTERUS.}

Icterus, Brisson, Orn. ii. p. 85 (1760).

\section{Icterus baltimore.}

Oriolus baltimore, Linn. Syst. Nat. i. p. 162.

Icterus baltimore, Scl. Cat. Am. B. p. 130: Baird, Brew. \& Ridgw. N. Am. B. ii. p. 195.

$a$ N. America (Askew) 1833. $-b,-c$ N. America (N. C. Strickland) 1838. - $d$ f Carlisle, Pennsylvania (S. F. Baird) 1847. - e Guatemala (J. Constancia) 1848.

\section{Icterus abeillæi.}

Xanthornus abeillei, Less. Rev. Zool. 1839, p. 101.

Icterus abeillcei, Scl. \& Salv. Ex. Orn. p. 187, pl. 94.

a Mexico (T. Mann) 1844.

\section{8\%. Icterus spurius.}

Oriolus spurius, Linn. Syst. Nat. i. p. 162.

Icterus spurius, et I. affinis, Scl. Cat. Am. B. p. 130.

Icterus spurius, Baird, Brew. \& Ridgw. N. Am. B. ii. p. 190.

a N. America (Askew) 1834. -b N. America (Johnson) 1837. --c N. America (N. C. Strickland) 1838. $-d$ N. America (Askew) 1838. - e $\hat{\delta}$ Mexico (Galeotti) 1845. $-f,-g$ Guatemala (J. Constancia) 1845. - $h$ Guatemala (J. Constancia) 1848. -i Guatemala ( $J$. Constancia) 1850. 
1288. Icterus chrysocephalus.

Oriolus chrysocephalus, Linn. Syst. Nat. i. p. 164.

Icterus chrysocephalus, Cab. Schomb. Guian. iii. p. 680: Scl. Cat. Am. B. p. 131.

a $\hat{o}$ Guiana (Schomburgk).

1289. Icterus cayennensis.

Oriolus cayennensis, Linn. Syst. Nat. i. p. 163.

Icterus cayennensis, Scl. Cat. Am. B. p. 131.

a Brazil (N. C. Strickland) 1838.

This specimen having black thighs agrees with the Guianan species of this form.

\section{Icterus tibialis.}

Icterus tibialis, Sw. An. in Menag. p. 302.

a Brazil (Askew) 1834. - b Brazil (Burl) 1834. - c Brazil (N. C. Strickland) 1838.

\section{Icterus wagleri.}

Icterus wagleri, Scl. P. Z. S. 1857, p. 7; Cat. Am. B. p. 131. $a,-b$ Guatemala ( $J$. Constancia) 1845.

$a$ is the specimen referred to by Strickland (Contr. Orn. 1850, p. 120) as probably a more adult example of the species he there described as $I$. prosthemelas.

\section{Icterus auricapillus.}

Icterus auricapillus, Cassin, Journ. Ac. Phil. ser. 2, i. pl. 16, f. 2: Scl. Cat. Am. B. p. 132.

$a$ (N. C. Strickland) 1838. - b Bogota (Bt. at Stevens's) 1845.

+1293. Icterus giraudi.

Icterus giraudii, Cassin, Journ. Ac. Phil. ser. 2, i. p. 138: Scl. Cat. Am. B. p. 133.

$a,-b$ Guatemala (J. Constancia) 1848. - c (Bt. at Birmingham) 1850.

$c$ is a skin of Bogota make.

\section{Icterus xanthornus.}

Oriolus xanthornus, Gm. Syst. Nat. i. p. 391.

Icterus xanthornus, Scl. Cat. Am. B. p. 133. 
a West Indies ( $N$. C. Strickland) 1834. - - b Trinidad (Staunton) 1843. —c, —d Trinidad (Argent) 1851.

\section{Icterus vulgaris.}

Oriolus icterus, Linn. Syst. Nat. i. p. 161.

Icterus vulgaris, Daud. Traité d'Orn. ii. p. 340: Scl. Cat. Am. B. p. 143.

a (Arthur Strickland) 1840.

A Venezuelan species.

1296. Icterus jamacaii.

Oriolus jamacaii, Gm. Syst. Nat. i. p. 391.

Icterus jamacaii, Scl. Cat. Am. B. p. 133.

a Bahia (S. Dutton) 1846.

\section{9\%. Icterus pectoralis.}

Psarocolius pectoralis, Wagl. Isis, 1829, p. 755.

Icterus pectoralis, Scl. Cat. Am. B. p. 134.

$a$ Guatemala (J. Constancia) 1845.

1298. Icterus pustulatus.

Psarocolius pustulatus, Wagl. Isis, 1829, p. 757.

Icterus pustulatus, Scl. \& Salv. Ex. Orn. p. 47, pl. 24.

a f Mexico (Galeotti) 1845.

\section{Icterus leucopteryz.}

Psarocolius leucopteryx, Wagl. Syst. Av. fol. 22, p. 10.

Icterus leucopteryx, Gosse, B. Jam. p. 226: Scl. Cat. Am. B. p. 134.

a §̊ Jamaica (P. H. Gosse) 1848.

\section{DoLICHONYX.}

Dolichonyx, Swainson, Zool. Journ. iii. p. 351 (1827). 1300. Dolichonyz oryzivorus.

Emberiza oryzivora, Linn. Syst. Nat. i. p. 311.

Dolichonyx oryzivora, Scl. Cat. Am. B. p. 134: Baird, Brew. \& Ridgw. N. Am. B. ii. p. 149.

$a$ N. America (N. C. Strickland) 1838. -b N. America, 1838. -c N. America (Askew) 1838. —d (Havell) 1839. - $e,-f$ Bolivia (Argent) 1852. 


\section{Molothrus.}

Molothrus, Swainson, Faun. Bor. Am. Birds, p. 494 (1831).

1301. Molothrus pecoris.

Fringilla pecoris, Gm. Syst. Nat. i. p. 910.

Molothrus pecoris, Baird, Brew. \& Ridgw. N. Am. B. ii. p. 154. $a$ §ิ N.America (N. C. Strickland) 1838.

\section{Molothrus æneus.}

Psarocolius ceneus, Wagl. Isis, 1829, p. 758.

Molothrus ceneus, Scl. Cat. Am. B. p. 135.

$a$ Guatemala ( $J$. Constancia) 1845. $-b,-c$ Guatemala (J. Constancia) 1848.

\section{Molothrus bonariensis.}

Tanagra bonariensis, Gm. Syst. Nat. i. p. 898.

Molothrus bonariensis, Cab. Mus. Hein. i. p. 193.

Icterus sericeus, Licht. Verz. Doubl. p. 19.

Molothrus sericeus, Scl. Cat. Am. B. p. 135.

$a$ S. America (Askew) 1834. - b Brazil (Johnson) 1837. -c Brazil (Askew) 1837. -d, -e (N. C. Strickland) 1838. - $f$ (Askew) 1840. $-g$ (Thomas) 1842.

\section{Molothrus atronitens.}

Molothrus atronitens, Cab. Schomb. Guian. iii. p. 682.

a Bogota? (Bt. at Stevens's) 1845.

Agrees with specimens of this small race of $M$. bonariensis found in Guiana. The habitat 'Bogota' assigned to it is probably erroneous.

\section{AGEL AUS.}

Agelaius, Vieillot, Anal. p. 33 (1816).

\section{Agelæus phœnicæus.}

Oriolus phonicceus, Linn. Syst. Nat. i. p. 161.

Agelceus phønicceus, Scl. Cat. Am. B. p. 135 : Baird, Brew. \& Ridgw. N. Am. B. ii. p. 159.

a N. America (N. C. Strickland) 1829. -b N. America (Johnson). $-c,-d,-e$ N. America (Askew) 1834. - $f$ Guatemala ( $J$. Constancia) 1848. $-g$ N. America. 


\section{Agelæus thilius.}

Turdus thilius, Mol. Sagg. sulla St. Nat. Chili (8vo. ed.) p. 250.

Agelasticus thilius, Scl. Cat. Am. B. p. 136.

Xanthornus chrysocarpus, Vig. P. Z. S. 1832, p. 3.

$a,-b$ Chili (Tucker) 1838.

\section{0\%. Agelæus cyanopus.}

Agelaius cyanopus, Vieill. N. Dict. d'Hist. N. xxxiv. p. 552. Lampropsar cyanopus, Cab. Mus. Hein. i. p. 194. a (Gardner) 1845.

A Brazilian species.

\section{XANTHOCEPHALUS.}

Xanthocephalus, Bonaparte, Consp. Av. i. p. 431 (1850).

1308. Xanthocephalus icterocephalus.

Icterus icterocephalus, Bp. Am. Orn. i. p. 27, pl. 3, f. 1, 2.

Xanthocephalus icterocephalus, Scl. Cat. Am. B. p. 136: Baird, Brew. \& Ridgw. N. Am. B. ii. p. 167.

Icterus xanthocephalus, Bp. Journ. Ac. Phil. v. p. 222.

a (N.C. Strickland) 1838.

A North-American species.

\section{Xanthosonus.}

Xanthosomus, Cabanis, Mus. Hein. i. p. 189 (1851).

\section{Xanthosomus frontalis.}

Agelaius frontalis, Vieill. N. Dict. d'Hist. N. xxxiv. p. 545.

Xanthosomus frontalis, Scl. Cat. Am. B. p. 136.

a (N. C. Strickland) 1839. -b (Thomas) 1842.

\section{Xanthosomus icterocephalus.}

Oriolus icterocephalus, Linn. Syst. Nat. i. p. 163.

Xanthosomus icterocephalus, Scl. Cat. Am. B. p. 136.

a Cayenne (N. C. Strickland) 1838. -b (Thomas) 1840. -c (Stevens) 1845. $-d$ Trinidad (Argent) 1851. -e (Argent) 1851. 


\section{Xanthosomus flavus.}

Oriolus flavus, Gm. Syst. Nat. i. p. 389.

Xanthosomus flavus, Scl. Cat. Am. B. p. 137.

a (Thomas) 1842.

A species of Southern Brazil and the Argentine Republic.

\section{Amblyrhamphus.}

Amblyrhamphus, Leach, Zool. Misc. i. p. 81 (1814).

1312. Amblyrhamphus holosericeus.

Xanthornus holosericeus, Scop. Del. Faun. et Fl. Insubr. ii. p. 88.

Amblyrhamphus holosericeus, Scl. Cat. Am. B. p. 137.

Oriolus ruber, Gm. Syst. Nat. i. p. 388.

a Brazil (N. C. Strickland) 1838.

\section{Grmnomystax.}

Gymnomystax, Reichenbach, Av. Syst. pl. lxxiii. (1850).

1313. Gymnomystax melanicterus.

Agelaius melanicterus, Vieill. N. Dict. d'Hist. N. xxxvi. p. 536.

Gymnomystax melanicterus, Scl. Cat. Am. B. p. 137.

a Trinidad (Argent) 1852.

\section{Pseudoleistes.}

Pseudoleistes, Sclater, Cat. Am. B. p. 137 (1862).

\section{Pseudoleistes viridis.}

Oriolus viridis, Gm. Syst. Nat. i. p. 395.

Pseudoleistes viridis, Scl. Cat. Am. B. p. 137.

Agelaius guirahuro, Vieill. N. Dict. d'Hist. N. xxxiv. p. 545 , a Brazil (N. C. Strickland) 1838.

\section{LEISTES.}

Leistes, Vigors, Zool. Journ. ii. p. 191 (1825).

1315. Leistes guianensis.

Oriolus guianensis, Linn. Syst. Nat. i. p. 162. 
Leistes guianensis, Scl. Cat. Am. B. p. 138.

Tanagra militaris, Linn. Syst. Nat. i. p. 316.

$a,-b,-c$ (Tucker) 1838. —d (N. C. Strickland) 1838.

A species of the Northern parts of South-America. $\quad a$ and $b$ are both skins of Cayenne make.

\section{STURNELLA.}

Sturnella, Vieillot, Anal. p. 34 (1816).

1316. Sturnella militaris.

Sturnus militaris, Gm. Syst. Nat. i. p. 803.

Sturnella militaris, Scl. Cat. Am. B. p. 138.

a S. America (N. C. Strickland) 1838. - b Falkland Is. (Capt. McMurdo) 1845.

\section{1\%. Sturnella ludoviciana.}

Sturnus ludovicianus, Linn. Syst. Nat. i. p. 290.

Sturnella ludoviciana, Strickl. Ann. \& Mag. N. H. vii. p. 31: Scl. Cat. Am. B. p. 139.

a N. America (Askew) 1834. -b N. America (N. C. Strickland) 1838. $\_$Guatemala ( $J$. Constancia) 1848. $\_d$ Trinidad (Argent) 1851.

\section{Curaus.}

Curceus, Sclater, Cat. Am. B. p. 139 (1862).

\section{Curæus aterrimus.}

Turdus curceus, Mol. Sagg. sulla St. Nat. Chili (8vo. ed.) p. 252.

Curceus aterrimus, Scl. Cat. Am. B. p. 139.

a Chili (N. C. Strickland) 1838.

\section{SCOLECOPHAGUS.}

Scolecophagus, Swainson, Faun. Bor. Am. Birds, p. 494 (1831).

1319. Scolecophagus ferrugineus.

Oriolus ferrugineus, Gm. Syst. Nat. i. p. 393.

Scolecophagus ferrugineus, Scl. Cat. Am. B. p. 140. 
$a$ N. America (N. C. Strickland) 1833. $-b$ N. America ( $N$. C. Strickland) 1838. —c Prince Edward's Island (Bt. at Stevens's) 1843.

\section{Scolecophagus cyanocephalus.}

Psarocolius cyanocephalus, Wagl. Isis, 1829, p. 758.

Scolecophagus cyanocephalus, Scl. Cat. Am. B. p. 140.

Scolecophagus mexicanus, Sw. An. in Menag. p. 302.

a Mexico (Askew) 1839.

LAMPROPSAR.

Lampropsar, Cabanis, Arch. f. Naturg. xiii. p. 333 (1847).

\section{Lampropsar guianensis.}

Lampropsar guianensis, Cab. Schomb. Guian. iii. p. 682. $a$ Brazil? (Argent) 1852.

Probably a Bolivian skin. It agrees with Guiana specimens referable to this species.

\section{Lampropsar warszewiezi.}

Lampropsar warszewiezi, Cab. Journ. f. Orn. 1861, p. 83.

a Brazil? (N. C. Strickland) 1838.

This skin probably came from Peru.

\section{Lampropsar dives.}

Lampropsar dives, Bp. Consp. i. p. 425: Cab. Mus. Hein. i. p. 194.

Quiscalus sumichrasti, Scl. Cat. Am. B. p. 140.

a Mexico (Brandt, Hamburg) 1845.

\section{Quiscalus.}

Quiscalus, Vieillot, Anal. p. 36 (1816).

\section{Quiscalus versicolor.}

Quiscalus versicolor, Vieill. N. Dict. d'Hist. N. xxviii. p. 488: Strickl. Ann. \& Mag. N. H. vii. p. 31 : Scl. Cat. Am. B. p. 140.

$a$ N. America (N. C. Strickland) 1838. - b America ( $W$. Kirtland) 1838. —c (Mansfield) 1839. —d (Arthur Strickland) 1840. -e (Askew) 1841. $-f$ (Gardner) 1845. $-g$ Illinois ( $T$. Dunn) 1851. 


\section{Quiscalus macrurus.}

Quiscalus macrourus, Sw. An. in Menag. p. 299.

Quiscalus macrurus, Scl. Cat. Am. B. p. 141.

$a,-b,-c$ Guatemala (J. Constancia) 1845.

1326. Quiscalus assimilis.

Quiscalus assimilis, Scl. Cat. Am. B. p. 141.

a Columbia (Galeotti) 1845.

132\%. Quiscalus tenuirostris.

Quiscalus tenuirostris, Sw. An. in Menag. p. 299: Cassin, Pr. Ac. Phil. 1866, p. 411.

$a$ (Thomas) 1840. —b Mexico (Brandt, Hamburg) 1845.

1328. Quiscalus lugubris.

Quiscalus lugubris, Sw. An. in Menag. p. 299: Scl. Cat. Am. B. p. 141.

$a$ (Arthur Strickland) 1840. - b Brazil (Bt. at Stevens's) 1844. —c (Bt. at Stevens's) 1845. -d, - $\mathrm{f}$ Trinidad (Argent) 1851.

1329. Quiscalus crassirostris.

Quiscalus crassirostris, Sw. An. in Menag. p. 355: Gosse, B. Jam. p. 217.

a Jamaica (J.Gould) 1846. —b Jamaica (P. H. Gosse) 1848.

1330. Quiscalus subalaris.

Quiscalus subalaris, Boiss. Rev. Zool. 1840, p. 70: Scl. Cat. Am. B. p. 141.

$a$ (Bt. at Stevens's) 1844 .

A species found in Columbia.

\section{APHOBUs:}

Aphobus, Cabanis, Mus. Hein. i. p. 194 (1851).

1331. Aphobus chopi.

Agelaius chopi, Vieill. N. Dict. d'Hist. N. xxxiv. p. 537. Aphobus chopi, Scl. Cat. Am. B. p. 141.

Icterus sulcirostris, Spix, Av. Bras. i. p. 67, pl. 64, f. 2. $a$ S. America (Bt. at Stevens's) 1843. 


\section{CASSIDIX.}

Cassidix, Lesson, Traité d’Orn. p. 433 (1831).

\section{Cassidix oryzivora.}

Oriolus oryzivorus, Gm. Syst. Nat. i. p. 386.

Cassidix oryzivora, Scl. Cat. Am. B. p. 142.

a Brazil (N. C. Strickland) 1838. -b Brazil (N. C. Strickland) 1838. —c (Stevens) 1844. —d Bogota (Gardner) 1845.

\section{FAMILY.-STURNID正。}

\section{SUBFAMILY.-STURNIN $\mathbb{E}$.}

\section{Gracula.}

Gracula, Linnæus, Syst. Nat. i. p. 164 (1766) (partim).

1333. Gracula religiosa.

Gracula religiosa, Linn. l. s. c.

Eulabes religiosa, Jerd. B. Ind. ii. p. 337.

Eulabes indicus, Cuv. Règne An. i. p. 377.

$a$ (Askew) 1834. - b India (Bt. at Stevens's) 1843. - $\mathrm{C}$. India ("Gracula religiosa," T. C. Jerdon) 1850.

\section{Gracula intermedia.}

Gracula intermedia, Hay, Madr. Journ. xiii. p. 156 : Jerd. B. Ind. ii. p. 339.

Eulabes vel Gracula religiosa, Hodgs. Zool. Misc. p. 84 (nec Linn.).

Gracula religiosa, Strickl. Ann. \& Mag. N. H. vii. p. 30. 1845 .

a Malacca (W. Kirtland) 1843. - b Nepal (B.H.Hodgson)

SARCOPS.

Sarcops, Walden, Trans. Z. S. ix. p. 205 (1875) vice Gymnops auctt. nec Spix.

1335. Sarcops calvus.

Gracula calva, Linn. Syst. Nat. i. p. 164.

Sarcops calvus, Wald. l. s. c.

$a, \ldots b$ Philippine Islands (J. Gould) 1846. 


\section{PASTOR.}

Pastor, Temminck, Man. d'Orn. p. 136 (1815).

1336. Pastor roseus.

Turdus roseus, Linn. Syst. Nat. i. p. 294.

Pastor roseus, Jerd. B. Ind. ii. p. 333 : Newton, ed. Yarr. Brit. B. ii. p. 243: Dresser, B. Eur.

a Madras (Stuchbury) 1836. - b Orkney (Forbes) 1837. - c Malacca (N. C. Strickland) 1838. - d Madras (T. C. Jerdon) 1845. - e S. India (T. C. Jerdon) 1850. $-f$ (Bt. at Liverpool) 1852.

\section{ACRIDOTHERES.}

Acridotheres, Vieillot, Anal. p. 42 (1816).

133\%. Acridotheres tristis.

Paradisea tristis, Linn. Syst. Nat. i. p. 167.

Pastor tristis, Hodgs. Zool. Misc. p. 84.

Acridotheres tristis, Jerd. B. Ind. ii. p. 325.

a Mauritius (A. Strickland) 1832. $-b,-c$ Ceylon (Capt. Pearson) 1841. - d Nepal (B. H. Hodgson, 303) 1845. - e (Bt. at Liverpool) 1853.

\section{Acridotheres cristatellus.}

Gracula cristatella, Linn. Syst. Nat. i. p. 164.

Acridotheres cristatellus, Strickl. P. Z. S. 1842, p. 167 :

Swinh. P. Z. S. 1871, p. 384: Wald. Trans. Z. S. ix. p. 202.

a Chusan, China (P. L. Sclater) 1847.

\section{Acridotheres ginginianus.}

Turdus ginginianus, Lath. Ind. Orn. p. 362.

Acridotheres ginginianus, Je d. B. Ind. ii. p. 326.

a India ("Acridotheres ginginianus," E. Blyth) 1846. - b $\hat{\delta}$ Kumaon (W. J. E. Boys) 1847.

\section{Acridotheres fuscus.}

Pastor fuscus, Wagl. Syst. Av. fol. 6, p. 8.

Acridotheres fuscus, Jerd. B. Ind. ii. p. 327.

Maina cristatelloides, Hodgs. J. A. S. B. v. p. 773.

a Nepal (B. H. Hodgson, 304) 1845. -b ^ิ Kumaon (W. J. E. Boys) 1847. 


\section{Temenuchus.}

Temenuchus, Cabanis, Mus. Hein. i. p. 204 (1851).

1311. Temenuchus pagodarum.

Turdus pagodarum, Gm. Syst. Nat. i. p. 816.

Temenuchus pagodarum, Jerd. B. Ind. ii. p. 329.

a E. Indies (N. C. Strickland) 18:38. - b (Bt. at Stevens's) 1844 .

1342. Temenuchus malabaricus.

Turdus malabaricus, Gm. Syst. Nat. i. p. 816.

Pastor malabaricus, Strickl. Ann. \& Mag. N. H. xiii. p. 35 .

Acridotheres malabaricus, Strickl. P. Z. S. 1842, p. 167.

Temenuchus malabaricus, Jerd. B. Ind. ii. p. 330.

Pastor malabaricus? v. caniceps, Hodgs. Zool. Misc. p. 84.

a Nepal (B. H. Hodgson, 271) 1845. - b, -c India ("Sturnia. malabarica," E. Blyth) 1846. -d q Rohilkund (W. J. E. Boys) 1847. -e India (Major Stacy).

1343. Temenuchus blythi.

Pastor blythi, Jerdon, Madras Journ. xiii. p. 133; Ill. Ind. Orn. pl. 22.

Temenuchus blythi, Jerd. B. Ind. ii. p. 331.

a India (F'enton) 1844.

Sturnia.

Sturnia, Lesson, Compl. Buff. ix. p. 53 (1837). 1344. Sturnia sturnina.

Gracula sturnina, Pall. Reis. iii. p. 695 : Wald. Trans. Z. S. ix. p. 204.

$a,-b$ Malacca (N. C. Strickland) 1838.

\section{Dilophus.}

Dilophus, Vieillot, Anal. p. 34 (1816).

1345. Dilophus carunculatus.

Gracula carunculata, Gm. Syst. Nat. i. p. 399.

Dilophus carunculatus, Strickl. \& Scl. Contr. Orn. 1852, p. 149: Layard, B. S. Afr. p. 177.

S. C, 

1838.

$a$ (Askew) 1834. - $b$ q Cape of Good Hope (N. C. Strickland) Sturnus.

Sturnus, Linnæus, Syst. Nat. i. p. 290 (1766) (partim).

1346. Sturnus vulgaris.

Sturnus vulgaris, Linn. l. s. c.: Strickl. P. Z. S. 1836, p. 100 : Jerd. B. Ind. ii. p. 321 : Newton, ed. Yarr. Brit. B. ii. p. 228, Dresser, B. Eur.

Sturnus indicus, Hodgs. Zool. Misc. p. 84.

$a,-b$ Worcestershire (H. E. Strickland) 1830. —c Oxfordshire, 17 September (N. C. Strickland) 1833. - d young Worcestershire, 20 June (H. E. Strickland) 1837. -e Nepal (B. II. Hodgson, 306) 1845. - $f$ India ("Sturnus indicus," E. Blyth) 1846.

\section{4\%. Sturnus unicolor.}

Sturnus unicolor, Temm. Man. d'Orn. i. p. 133 : Dresser, B. Eur.

a Egypt? (N. C. Strickland) 1838.

\section{STURNOPASTOR.}

Sturnopastor, Hodgson, Zool. Misc. p. 84 (1844).

1348. Sturnopastor jalla.

Pastor jalla, Horsf. Trans. L. S. xiii. p. 155.

Sturnopastor jalla, Bp. Consp. i. p. 420.

a $\hat{o},-b$ Java (N. C. Strickland) 1838.

\section{SUBFAMILY.-LAMPROTORNITHIN AE.}

\section{LAMPROTORNIS.}

Lamprotornis, Temminck, Man. d'Orn. i. p. Iv. (1820).

1349. Lamprotornis æneus.

Turdus ceneus, Gm. Syst. Nat. i. p. 818.

Lamprotornis ceneus, Hartl. Abh. Nat. Ver. Bremen, iv. p. 44. Lamprotornis longicauda, Sw. Orn. W. Afr. i. p. 148, pl. 7.

$a$ Senegal (N. C. Strickland) 1838. 
1350. Lamprotornis australis.

Megalopterus australis, Smith, Rep. S. Afr. Exp. App. p. 52. Juida australis, Strickl. \& Scl. Contr. Orn. 1852, p. 149: Gurney, in And. B. Damara-l. p. 158.

Lamprotornis burchelli, Smith, l. s. c.: Hartl. Abh. Nat. Ver. Bremen, iv. p. 50.

a Damara-land (C. J. Andersson) 1852.

\section{LAMPROCOLIUS.}

Lamprocolius, Sundevall, K. Vet. Ac. Handl. 1835, p. 104.

\section{Lamprocolius splendidus.}

Turdus splendidus, Vieill. N. Dict. d'Hist. N. xx. p. 252.

Lamprocolius splendidus, Hartl. Abh. Nat. Ver. Bremen, iv. p. 54 .

Lamprotornis chrysonotis, Sw. Orn. W. Afr. i. p. 143, pl. 6. $a$ (Arthur Strickland) 1850.

A West-African species.

1352. Lamprocolius auratus.

Merle violet, du Royaume de Juda, D'Aub. Pl. Enl. 540, undè, Turdus auratus, Gm. Syst. Nat. i. p. 819.

Lamprocolius auratus, Hartl. Abh. Nat. Ver. Bremen, iv. p. 57.

Turdus splendens, Leach, Zool. Misc. ii. p. 30, pl. 71.

$a$ (Kirtland) 1838. - $b$ Senegal? (Weaver) 1843.

A West-African species.

1353. Lamprocolius chalybeius.

Lamprotornis chalybcea, Hempr. \& Ehr. Symb. Phys. Aves, fol. $y$, pl. 10.

Juida chalybea, Strickl. P. Z. S. 1850, p. 217.

Lamprocolius chalybceus, Hartl. Abh. Nat. Ver. Bremen, iv. p. 61.

$a$ W. Africa (Mather) 1840. - b Africa (Bt. at Stevens's) 1843. -c Kordofan, 20 June (J. Petherick) 1848.

1354. Lamprocolius chloropterus.

Lamprotornis chloropterus, Sw. An. in Menag. p. 359. 
Lamprocolius chloropterus, Hartl. Abh. Nat. Ver. Bremen, vi. p. 64 .

a Senegal? (Weaver) 1843.

A West-African species.

1355. Lamprocolius phcenicopterus.

Lamprotornis phonicopterus, Sw. An. in Menag. p. 360.

Lamprocolius phonicopterus, Gurney, in And. B. Damara-l. p. 160: Hartl. Abh. Nat. Ver. Bremen, iv. p. 67.

Spreo bispecularis, Strickl. Contr. Orn. 1852, p. 149.

a Cape of Good Hope (Bt. at Stevens's) 1843. - b, -c (Arthur Strickland) 1850. -d Damara-land (C.J. Andersson) 1852.

$d$ is the type of S. bispecularis, Strickl., and is smaller than is usual in specimens of $L$. phoenicopterus. But as the difference is slight and apparently not constant, I think Mr Gurney (l. s. c.) right in recognizing only a single species.

1356. Lamprocolius melanogaster.

Lamprotornis melanogaster, Sw. An. in Menag. p. 297.

Lamprocolius melanogaster, Hartl. Abh. Nat. Ver. Bremen, iv. p. 72.

$a$ South Africa (Gardner) 1845.

\section{Pholidauges.}

Pholidauges, Cabanis, Mus. Hein. i. p. 198 (1851).

135\%. Pholidauges leucogaster.

Turdus leucogaster, Gm. Syst. Nat. i. p. 819.

Pholidauges leucogaster, Cab. l. s. c.: Finsch \& Hartl. v. d. Decken's Reise, iv. p. 376 : Hartl. Abh. Nat. Ver. Bremen, iv. p. 76.

Lamprotornis leucogaster, Sw. B. W. Afr. i. p. 152, pl. 8. 1838.

a W. Africa (W. Kirtland) 1838. - $b$ q Senegal (G. Lloyd)

\section{Spreo.}

Spreo, Lesson, Traité d'Orn. p. 407 (1831), as a subgenus. 1358. Spreo bicolor.

Turdus bicolor, Gm. Syst. Nat. i. p. 835. 
Notanges bicolor, Hartl. Abh. Nat. Ver. Bremen, iv. p. 83. a Cape of Good Hope (Askew) 1834.

\section{Spreo pulchra.}

Merle à ventre orangé, du Sénégal, D'Aub. Pl. Enl. 358, undè, Turdus pulcher, Müll. Natursyst. Suppl. p. 139.

Spreo pulchra, Gray, Hand-1. ii. p. 25.

Lamprotornis rufiventris, Rüpp. Neue Wirb. Vög. p. 27, pl. 11, f. 1.

Juida rufiventris, Strickl. P. Z. S. 1850, p. 217.

Notanges chrysogaster, Gm.: Hartl. Abh. Nat. Ver. Bremen, iv. p. 81. 1848.

$a$ Africa (Thomas) 1840. - b $\hat{\delta}$ Kordofan (J. Petherick)

\section{Amydrus.}

Amydrus, Cabanis, Mus. Hein. i. p. 201 (1851).

1360. Amydrus morio.

Turdus morio, Linn. Syst. Nat. i. p. 297.

Amydrus morio, Cab. l. s. c.: Hartl. Abh. Nat. Ver. Bremen, iv. p. 89 .

$a$ Africa (Askew) 1833. -b Africa (Askew) 1834. -c Cape of Good Hope (N. C. Strickland) 1838.

Pyrrhocheira.

Pyrrhocheira, Reichenbach, Av. Syst. pl. 53 (1850).

1361. Pyrrhocheira caffra.

Coracias caffra, Linn. Syst. Nat. i. p. 159.

Pyrrhocheira caffra, Hartl. Abh. Nat. Ver. Bremen, iv. p. 96.

Spreo nabouroup (Daud.), Strickl. \& Scl. Contr. Orn. 1852, p. 149.

Amydrus caffer, Gurney, in And. B. Damara-1. p. 162.

$a$ Damara-land (C. J. Andersson) 1852.

APLONIS.

Aplonis, Gould, P. Z. S. 1836, p. 73.

1362. Aplonis obscura.

Lamprotornis obscurus, Du Bus, Bull. Ac. Brux. 1839, i. p. 297; Esq. Orn. pl. 12. 
$a$ (Bt. at Stevens's) 1845 .

Agrees with a Norfolk-Island specimen in the British Museum.

\section{Aplonis brevirostris.}

Lamprotornis brevirostris, Peale, U. S. Expl. Exp. viii. p. 111 (1848).

Aplonis brevirostris, Cassin, U. S. Expl. Exp. viii. p. 126, pl. 7, f. 2: Finsch \& Hartl. Faun. Centralpol. p. 105, pl. 10, f. 3.

a Samoan Islands (Rev. T. Heath) 1846.

A skin in bad condition probably referable to this species.

Calornis.

Calornis, G. R. Gray, List Gen. B. p. 53 (1841).

\section{Calornis panayensis.}

Muscicapa panayensis, Scop. Del. Faun. et Fl. Insubr. ii. p. 96 .

Calornis panayensis, Wald. Trans. Z. S. ix. p. 205.

Turdus columbinus, Gm. Syst. Nat. i. p. 836.

a Luzon, 14 June (H. Cuming) 1839. - $b$ Philippine Islands, 13 May (H. Cuming) 1840. — (Carfrae) 1840. -d Philippine Islands (J. Gould) 1846.

\section{Calornis chalybeius.}

Turdus chalybeus, Horsf. Trans. L. S. xiii. p. 148.

Calornis chalybcea, Salvad. Ann. Mus. Genov. v. p. 271.

a Malacc (Johnson) 1837. -b Malacca (Capt. Nash) 1837. -c E. Indies (Dr Horsfield) 1838.

\section{SARAGLOSSA.}

Saraglossa, Hodgson, Zool. Misc. p. 84 (1844).

1366. Saraglossa spiloptera.

Lamprotornis spilopterus, Vig. P. Z. S. 1830-1, p. 35: Gould, Cent. B. Him. pl. 34.

Saraglossa spiloptera, Jerd. B. Ind. ii. p. 336.

a Himalaya (N. C. Strickland) 1838. - b ô Kumaon (W. $J$. E. Boys) 1847. - f India (W. J. E. Boys) 1848. 


\section{SUBFAMILY.-BUPHAGIN 㘴.}

Buphaga.

Buphaga, Linnæus, Syst. Nat. i. p. 154 (1766).

136\%. Buphaga africana.

Buphaga africana, Linn. l. s. c.: Strickl. \& Scl. Contr. Orn. 1852, p. 149: Gurney, in And. B. Damara-1. p. 163.

$a$ W. Africa (N. C. Strickland) 1838. - b Damara-land (C.J. Andersson) 1852.

\section{FAMILY.-PARADISEIDE. SUBFAMILY.-EPIMACHIN $\approx$ 。 \\ Ptilorhis.}

Ptiloris, Swainson, Zool. Journ. i. p. 481 (1825). 1368. Ptilorhis paradisea.

Ptiloris paradisea, Sw. l. s. c.: Gould, B. Austr. iv. pl. 100. Ptilorhis paradiseus, Sharpe, Cat. B. Brit. Mus. iii. p. 154. a Australia (Bt. at Birmingham) 1834. -b (Argent) 1851.

SUBFAMILY.-PARADISEIN AE.

\section{Minucodia.}

Manucodia, Boddaert, Tabl. Pl. Enl. p. 39 (1783).

1369. ILanucodia chalybeia.

Le chalybé, de la Nouvelle Guinée, D’Aub. Pl. Enl. 634, unde, Manucodia chalybea, Bodd. l. s. c.: Elliot, Mon. Parad. pl. 6. a (Brandt) 1845 .

A New-Guinea species.

\section{Sericulus.}

Sericulus, Swainson, Zool. Journ. i. p. 476 (1825). 1370. Sericulus melinus.

Turdus melinus, Lith. Ind. Orn. Suppl. p. xliv.

Sericulus melinus, Elliot, Mon. Parad. pl. 27.

Sericulus chrysocephalus, Sw. l. s. c.: Gould, B. Austr. iv. pl. 12.

a (N. C. Strickland) 1838. - b.

An Australian species. 


\section{SUBFAMILY.-PTILONORHYNCHIN $\mathbb{E}$.}

\section{Ptilorhynchus.}

Ptilonorhynchus, Kuhl. Beitr. z. Zool. p. 150 (1820).

\section{Ptilorhynchus violaceus.}

Pyrrhocorax violaceus, Vieill. N. Dict. d'Hist. N. vi. p. 569.

Ptiloris violaceus, Elliot, Mon. Par. pl. 25.

Ptilonorhynchus holosericeus, Kuhl. l. s. c., et auctt.

a New South Wales (Askew) 1833. - $b$ New Holland (N. C. Strickland) 1838.

\section{Chlamydodera.}

Chlamydera, Gould, B. Austr. Pt. 1 (1837) fide Gould, B Austr. iv. pl. 8 (1841).

\section{Chlamydodera maculata.}

Calodera maculata, Gould, P. Z. S. 1836, p. 106.

Chlamydera maculata, Gould, B. Austr. iv. pl. 8.

Chlamydodera maculata, Cab. Mus. Hein. i. p. 212.

a New Holland (N. C. Strickland) 1838.

\section{AEIUREDUS.}

Ailurcedus, Cabanis, Mus. Hein. i. p. 213 (1851).

1373. Allurœdus crassirostris.

Lanius crassirostris, Payk. N. Act. Ac. Upsal. vii. p. 282, pl. 10.

Alluroedus crassirosiris, Elliot, Mon. Par. pl. 34.

Ptilonorhynchus smithii, Vig. \& Horsf. Trans. L. S. xv. p. 264. a Australia (Askew) 1840.

\section{FAMILY.-GYMNORHINIDE.}

\section{STrREPERA.}

Strepera, Lesson, Traité D’Orn. p. 329 (1831), as a subgenus. 1374. Strepera graculina.

Corvus graculinus, White, Voy. N. S. W. App. p. 25̃1, pl. 35. Strepera graculina, Gould, B. Austr. ii. pl. 42: Sharpe, Cat. B. Brit. Mus. iii. p. 57. a Australia (Gardner) 1846. 
1375. Strepera arguta.

Strepera arguta, Gould, P. Z. S. 1846, p. 19; B. Austr. ii. pl. 44: Sharpe, Cat. B. Brit. Mus. iii. p. 59. a Australia (N. C. Strickland) 1838.

\section{Strepera cuneicaudata.}

Cracticus cuneicaudatus, Vieill. N. Dict. d'Hist. N. v. p. 356. Strepera cuneicaudata, Sharpe, Cat. B. Brit. Mus. iii. p. 60. Barita anaphonensis, Temm. Pl. Col. sub tab. 273. Strepera anaphonensis, Gould, B. Austr. ii. pl. 45. a Australia (Ware) 1846.

\section{3\%\%. Strepera fuliginosa.}

Cracticus fuliginosus, Gould, P. Z. S. 1836, p. 106. Strepera fuliginosa, Gould, B. Austr. ii. pl. 43. a (Askew) 1841.

A Tasmanian species.

\section{Cracticus.}

Cracticus, Vieillot, Anal. p. 37 (1816).

\section{Cracticus torquatus.}

Lanius torquatus, Lath. Ind. Orn. Suppl. p. xviii.

Cracticus torquatus, Gould, Handb. B. Austr. i. p. 184.

Vanga destructor, Temm. Man. D’Orn. i. p. lix.

Cracticus destructor, Gould, B. Austr. ii. pl. 52.

a New South Wales (N. C. Strickland) 1838. - - N New Holland (N. C. Strickland) 1838. —c Australia (Bt. at Stevens's) 1843. - d (Arthur Strickland) 1850.

\section{Gymnorhina.}

Gymnorhina, G. R. Gray, List Gen. B. p. 37 (1840).

1379. Gymnorhina tibicen.

Coracias tibicen, Lath. Ind. Orn. Suppl. p. xxvii.

Gymnorhina tibicen, Gould, BB. Austr. ii. pl. 46 .

a Australia (J. Robinson) 1842.

1380. Gymnorhina organicum.

Cracticus hypoleucus, Gould, P. Z. S. 1836, p. 106 (? err. pro hypomelas). 
Gymnorhina organicum, Gould, B. Austr. ii. pl. 48.

a Australia (Askew) 1834. - b Van Diemen's Land (N. C. Strickland) 1838.

\section{Grallina}

Grallina, Vieillot, Anal. p. 42 (1816).

\section{Grallina picata.}

Gracula picata, Lath. Ind. Orn. Suppl. p. xxix.

Grallina picata, Strickl. Ann. \& Mag. N. H. xi. p. 335.

Grallina australis, Gould, B. Austr. ii. pl. 54.

$a$ New South Wales ( $M^{c}$ Donald) 1838. - $b$ New South Wales (N. C. Strickland) 1838.

\section{FAMILY.-ARTAMIDA. \\ Artamus.}

Artamus, Vieillot, Anal. p. 41 (1816).

1382. Artamus leucorhinus.

Lanius leucorynus, Linn. Mantiss. Plant. p. 524.

Artamus lencorynus, Wald. Trans. Z. S. ix. p. 174.

$a$ Philippine Islands (Cuming) 1840.

1383. Artamus superciliosus.

Ocypterus superciliosus, Gould, P. Z. S. 1836, p. 142.

Artamus superciliosus, Gould, B. Austr. ii. pl. 32.

$a$ New South Wales (McDonald) 1838.

1384. Artamus sordidus.

Turdus sordidus, Lath. Ind. Orn. Suppl. p. xliii.

Artamus sordidus, Gould, B. Austr. ii. pl. 27.

$a,-b$ New South Wales (N. C. Strickland) 1838. -c New South Wales (McDonald) 1838.

1385. Artamus fuscus.

Artamus fuscus, Vieill. N. Dict. d'Hist. N. xvii. p. 297 : Jerd. B. Ind. i. p. 441.

Ocypterus rufiventer, Val. Mem. Mus. vi. p. 25, pl. 7, f. 1.

a (Askew) 1834. - b Nepal (B. H. Hodgson, 741) 1840. —c India ("Artamus rufiventer," $E$. Blyth) $18 \pm 6$.

1 An anomalons genus the right position of which has yet to be determined. 


\section{FAMILY.CORVID $\mathbb{E}$.}

Convus.

Carvus, Linnæus, Syst. Nat. i. p. 155 (1766) (partim).

1386. Corvus corax.

Corvus corax, Linn. l. s. c.: Strickl. P. Z. S. 1836, p. 100 : Newton, ed. Yarr. Brit. B. ii. p. 259: Sharpe, Cat. B. Brit. Mus. iii. p. 14.

a Britain (N. C. Strickland) 1838. - b United States (J. J. Audubon) 1846. -c ô India (W. J. E. Boys) 1847.

138\%. Corvus australis.

Corvus australis, Gm. Syst. Nat. i. p. 365: Gould, Hand-b. B. Austr. i. p. 475.

Corvus coronoides, Vig. \& Horsf. Trans. L. S. xv. p. 261: Gould, B. Austr. iv. pl. 18.

$a$ (N. C. Strickland) 1838. -b Australia (Isaacson) 1840.

\section{Corvus americanus.}

Corvus americanus, Aud. Orn. Biogr. ii. p. 317: Baird, Brew. \& Ridgw. N. Am. B. ii. p. 243.

$a$ United States (J. J. Audubon).

1389. Corvus culminatus.

Corvus culminatus, Sykes, P. Z. S. 1832, p. 96: Sharpe, Cat. B. Brit. Mus. iii. p. 20.

a India (E. Blyth) 1846.

\section{Corvus corone.}

Corvus corone, Linn. Syst. Nat. i. p. 155.

Corone corone, Sharpe, Cat. B. Brit. Mus. iii. p. 36.

a $\hat{\delta}$ Worcestershire, January (H. E. Strickland) 1838. - - b (N. C. Stricliland) 1838.

\section{Corvus cornix.}

Corvus cornix, Linn. Syst. Nat. i. p. 156: Strickl. P.Z. S. 1836, p. 100.

Corone cornix, Sharpe, Cat. B. Brit. Mus. iii. p. 31.

a Yorkshire, March (H. E. Strickland) 1833. 


\section{Corvus splendens.}

Corvus splendens, Vieill. N. Dict. d'Hist. N. viii. p. 44: Strickl. Ann. \& Mag. N. H. xiii. p. 35: Jerd. B. Ind. ii. p. 298. Corone splendens, Sharpe, Cat. B. Brit. Mus. iii. p. 33. a E. Indies (Havell) 1839.

\section{Corvus umbrinus.}

Corvus umbrinus, Sundev. K. Vet. Ac. Handl. 1838, p. 198 : Strickl. P. Z. S. 1850, p. 217: Sharpe, Cat. B. Brit. M. iii. p. 17.

a ô Kordofan (J. Petherick) 1848.

\section{Corvus enca.}

Fregilus enca, Horsf. Trans. L. S. xiii. p. 164.

Corone enca, Sharpe, Cat. B. Brit. Mus. iii. p. 43.

a Malacca (Havell) 1839.

Apparently a specimen of this species, though its Malaccan origin is perhaps doubtful.

\section{Corvus frugilegus.}

Corvus frugilegus, Linn. Syst. Nat, i. p. 156: Newton, ed. Yarr. Brit. B. ii. p. 289.

Trypanocorax frugilegus, Sharpe, Cat. B. Brit. Mus. iii. p. 9. $a \hat{\delta},-b$ young Worcestershire, January (H. E. Strickland) 1838.

\section{Corvus scapulatus.}

Corvus scapulatus, Daud. Traité ii. p. 232: Strickl. P. Z. S. 1850, p. 217: Sharpe, Cat. B. Brit. Mus. iii. p. 22.

Corvus leuconotus, Sw. B. W. Afr. i. p. 133, pl. v.

a Senegal (N. C. Strickland) 1838. - 6 q Kordofan (J. Petherick) 1848.

\section{Corvus monedula.}

Corvus monedula, Linn. Syst. Nat. i. p. 156: Strickl. P.Z. S. 1836, p. 100 : Newton, ed. Yarr. Brit. B. ii. p. 305: Dresser, B.

Eur. Colous monedula, Sharpe, Cat. B. Brit. Mus. iii. p. 26.

a Worcestershire, February (T'. Robinson) 1841. 
1398. Corvus collaris.

Corvus collaris, Drummond, Ann. \& Mag. N. H. xviii. p. 11. Colous collaris, Sharpe, Cat. B. Brit. Mus. iii. p. 27.

a Macedonia (II. M. Drummond) 1846.

A typical specimen.

\section{Corvultur.}

Corvultur, Lesson, Traité d'Orn. p. 327 (1831) as a subgenus. 1399. Corvultur albicollis.

Corvus albicollis, Lath. Ind. Orn. p. 151 : Layard, B. S. Afr. p. 167.

Corvultur albicollis, Sharpe, Cat. B. Brit. Mus. iii. p. 24. a S. Africa (N. C. Strickland) 1838.

\section{NUCIFRAGA.}

Nucifraga, Brisson, Ornith. ii. p. 58 (1760). 1400. Nucifraga caryocatactes.

Corvus caryocatactes, Linn. Syst. Nat. i. p. 157.

Nucifraga caryocatactes, Sharpe, Cat. B. Brit. Mus. iii. p. 53. a Switzerland (W. Anderegg) 1836. $-b$ N. Europe (J.G. Kinberg) 1845. —c (J. F. Brandt) 1846.

1401. Nucifraga hemispila.

Nucifraga hemispila, Vig. P. Z. S. 1830-31, p. 8: Gould, Cent. B. Him. pl. 36: Jerd. B. Ind, ii. p. 304: Sharpe, Cat. B. Brit. Mus. iii. p. 54 .

a Nepal (B. H. Hodgson) 184วّ.

1402. Hucifraga multipunctata.

Nucifraga multipunctata, Gould, P. Z. S. 1849, p. 23: Sharpe, Cat. B. Brit. Mns. iii. p. 55.

a Himalaya (N. C. Strickland) 1838.

\section{PyrRHOCORAX.}

Pyrrhocorax, Vieillot, Anal. p. 36 (1816). 1403. Pyrrhocorax graculus.

Corvus graculus, Linn. Syst. Nat. i. p. 158. 
Pyrrhocorax graculus, Newton, ed. Yarr. Brit. B. ii. p. 252: Dresser, B. Eur.

a Wales (T. Robinson) 1835. - b Siberia (J. F. Brandt) 1841. —c Himalaya (Stevens) 184ら.

1404. Pyrrhocorax alpinus.

Pyrrhocorax alpinus, Vieill. N. Dict. d'Hist. N. vi. p. 568: Sharpe, Cat. B. Brit. Mus. iii. p. 148.

a Switzerland (Etienne Elaerts) 1836. - b Siberia (J. F. Brandt) 1841.

Corcorax.

Corcorax, Lesson, Traité d'Orn. p. 325 (1831).

1405. Corcorax melanorhamphus.

Coracia melanorhamphos, Vieill. N. Dict. d'Hist. N. viii. p. 2.

Cocorax melanorhamphus, Sharpe, Cat. B. Brit. Mus. iii. p. 149.

Cocorax leucopterus (Temm.), Gould, B. Austr. iv. pl. 16. a Australia (Arthur Strickland) 1840.

PICA.

Pica, Brisson, Orn. ii. p. 35 (1760).

1406. Pica rustica.

Corvus pica, Linn. Syst. Nat. i. p. 157.

Corvus rusticus, Scop. Ann. I. Hist. Nat. p. 38.

Pica rustica, Dresser, B. Eur.: Newton, ed. Yarr. Brit. B. ii. p. 312.

Pica caudata, Ray, Strickl. P. Z. S. 1836, p. 100.

Pica pica, Sharpe, Cat. B. Brit. Mus. iii. p. 62.

a f Worcestershire (H. E. Strickland) 1838.

140\%. Pica nuttalli.

Corvus nuttalli, Aud. Orn. Biogr. iv. p. 450.

Pica nuttalli, Sharpe, Cat. B. Brit. Mus. iii. p. 66.

Pica caudata var. nuttalli, Baird, Brew. \& Ridgw. N. Am. B. ii. p. 270.

a Kodjak (Wosnessenski) 1846. 


\section{UROCISSA.}

Urocissa, Cabanis, Mus. Hein. i. p. 87 (1850). 1408. Urocissa occipitalis.

Psilorhinus occipitalis, Blyth, J. A. S. B. xv. p. 27.

Urocissa occipitalis, Cab. Mus. Hein. i. p. 87: Sharpe, Cat. B. Brit. Mus. iii. p. 70.

Pica erythrorhyncha, Vig. P. Z. S. 1830-1, p. 173 (nec Gmelin). a Himalaya (Fenton) 1844. - b Nepal (B. H. Hodgson) 1845.

CISsA.

Cissa, Boie, Isis, 1826, p. 975.

1409. Cissa chinensis.

Rollier, de la Chine, D'Aub. Pl. Enl. 620, undè,

Coracias chinensis, Bodd. Tabl. Pl. Enl. p. 38.

Cissa chinensis, Sharpe, Cat. B. Brit. Mus. iii. p. 85.

Cissa sinensis, Jerd. B. Ind. ii. p. 312.

$a,-b$ India (E. Blyth) 1846.

DENDROCITTA.

Dendrocitta, Gould, P. Z. S. 1833 , p. 57.

1410. Dendrocitta rufa.

La Pie rousse de la Chine, Sonn. Voy. Ind. ii. p. 186, pl. 106, undè,

Lanius rufus, Scop. Del. Faun. et Flor. Insubr. ii. p. 86.

Dendrocitta rufa, Jerd. B. Ind. ii. p. 314: Sharpe, Cat. B. Brit. Mus. iii. p. 76.

Pica vagabunda, Vieill. N. Dict. d'Hist. N. xxvi. p. 131.

a India (N. C. Strickland) 1834. -b India (N. C. Strickland) 1838.

1411. Dendrocitta frontalis.

Dendrocitta frontalis, McClell. P. Z. S. 1839, p. 163: Jerd. B. Ind. ii. p. 317: Sharpe, Cat. B. Brit. Mus. iii. p. 78.

a Darjeeling (A. D. Bartlett). 1412. Dendrocitta leucogastra.

Dendrocitta leucogastra, Gould, P. Z. S. 1833, p. 57; Trans. Z. S. i. p. 89, pl. 12: Jerd. B. Ind. ii. p. 317: Sharpe, Cat. B. Brit. Mus. iii. p. 79.

a E. Indies (N. C. Strickland) 1838. 
1413. Dendrocitta himalayensis.

Dendrocitta sinensiș, Gould, P. Z. S. 1833, p. 57; Trans. Z. S. i. p. 89 (nec Latham).

Dendrocitta himalayensis, Blyth, Ibis, 1865, p. 45: Sharpe, Cat. B. Brit. Mus. iii. p. 79.

a Nepal (B. H. Hodgson, 190) 1845.

\section{Cryptorhina.}

Cryptorhina, Wagler, Syst. Av. fol. 8, p. 16 (1827).

1414. Cryptorhina afra.

Corvus afer, Linn. Syst. Nat. i. p. 157.

Cryptorhina afra, Sharpe, Cat. B. Brit. Mus. iii. p. 75.

Piilostomus senegalensis (L.), Sw. B. W. Afr. i. p. 135.

Ptilostomus pœcilorhynchus (Wagl.), Gray, Gen. B. ii. p. 311. a Senegal (N. C. Strickland) 1838. -b Africa (Thomas) 1842.

\section{Crypsirhina.}

Crypsirina, Vieillot, Anal. p. 36 (1816).

Crypsirhina, Strickland, Ann. \& Mag. N. H. vii. p. 30.

\section{Crypsirhina varians.}

Corvus varians, Lath. Ind. Orn. Suppl. p. xxvi.

Crypsirhina varians, Vieill. l. s. c.: Sharpe, Cat. B. Brit. Mus. iii. p. 83.

a Indian Archipelago (Askew) 1840.

Temnurus.

Temnurus, Lesson, Traité d'Orn. p. 341 (1831).

1416. Temnurus leucopterus.

Glaucopis leucopterus, Temm. Pl. Col. 265.

Temnurus leucopterus, Less. l. s. c.

Platysmurus leucopterus, Sharpe, Cat. B. Brit. Mus. iii. p. 90. a Malacca (Capt. Nash) 1837. - b Malacca (Mather) 1840.

\section{Garrulus.}

Garrulus, Brisson, Orn. ii. p. 46 (1860).

\section{1\%. Garurlus glandarivs.}

Corvus glandarius, Linn. Syst. Nat. i. p. 156. 
Garrulus glandarius, Dresser, B. Eur.: Sharpe, Cat. B. Brit. Mus. iii. p. 93.

a $\hat{\delta}$ Worcestershire, March (H. E. Strickland) 1838.

1418. Garrulus japonicus.

Garrulus glandarius, japonicus, Schl. Faun. Jap. p. 83, pl. 43. Garrulus japonicus, Bp. Consp. i. p. 375 : Sharpe, Cat. B. Brit. Mus. iii. p. 95.

a Japan (P. L. Sclater).

\section{Garrulus brandti.}

Garrulus brandtii, Eversm. Add. ad Pall. Zoogr. Rosso-As. fasc. iii. p. 8: Dresser, B. Eur.: Sharpe, Cat. B. Brit. Mus. iii. p. 96.

a Altai Mts. (J. F. Brandt) 1844.

\section{Garrulus krynikii.}

Garrulus krynikii, Kaleniczenko, Bull. Ac. Mosc. 1839, p. 319 pl. 9: Dresser, B. Eur.: Sharpe, Cat. B. Brit. Mus. iii. p. 99.

Garrulus melanocephalus, Strickl. P. Z. S. 1836, p. 100; Ann. \& Mag. N. H. xii. p. 414 (nec Géné).

a ô Smyrna, 24 November (H. E. Strickland) 183 .

\section{Garrulus bispecularis.}

Garrulus bispecularis, Vig. P. Z. S. 1830-1, p. 7: Gould, Cent. B. Him. pl. 38: Jerd. B. Ind. ii. p. 307: Sharpe, Cat. B. Brit. Mus. iii. p. 100.

a Nepal (B. H. Hodgson, 206) 1845.

\section{Garrulus lanceolatus.}

Garrulus lanceolatus, Vig. P. Z. S. 1830-1, p. 7: Gould, Cent. B. Him. pls. 39, 40: Jerd. B. Ind. ii. p. 308: Sharpe, Cat. B. Brit. Mus. iii. p. 101.

a Himalaya (Fenwick) 1844. -b Himalaya (E. Brown) 1850.

\section{Perisoreus.}

Perisoreus, Bonaparte, Saggio di una Distr. Met. An. Vert. p. 43 (1831) as a subgenus.

\section{Perisoreus infaustus.}

Lanius infaustus, Linn. Syst. Nat. i. p. 138.

S. C. 
Perisoreus infaustus, Bp. l. s. c.: Sharpe, Cat. B. Brit. Mus. iii. p. 103.

$a$ Govt. of St Petersburg (J. F. Brandt) 1841. -b (J. F. Brandt) 1846. - c Norway (Arthur Strickland) 1850.

\section{Perisoreus canadensis.}

Corvus canadensis, Linn. Syst. Nat. i. p. 158.

Perisoreus canadensis, Baird, Brew. \& Ridgw. N. Am. B. ii. p. 299.

$a$ California (J. F. Brandt) 1844. $-b-c$ Nova Scotia (Askew) 1839.

\section{Cyanocitta.}

Cyanocitta, Strickland, Ann. \& Mag. N. H.xv. p. 261 (1845).

\section{Cyanocitta cristata.}

Corvus cristatus, Linn. Syst. Nat. i. p. 157.

Cyanocitta cristata, Strickl. l. s. c.: Sharpe, Cat. B. Brit. Mus. iii. p. 107.

Cyanura cristata, Baird, Brew. \& Ridgw. N. Am. B. ii. p. 273.

$a$ N. America (Arthur Strickland) 1834. -b (A. Strickland) 1850. —c Illinois (T. Dunn) 1851.

1426. Cyanocitta stelleri.

Corvus stelleri, Gm. Syst. Nat. i. p. 370.

Cyanocitta stelleri, Strickl. Ann. \& Mag. N. H. xv. p. 261: Sharpe, Cat. B. Brit. Mus. iii. p. 108.

Cyanura stelleri, Baird, Brew. \& Ridgw. N. Am. B. ii. p. 277. $a$ Sitka (J. F. Brandt) 1844.

142\%. Cyanocitta coronata.

Garrulus coronatus, Sw. Phil. Mag. 1827, p. 437.

Cyanocitta coronata, Strickl. Ann. \& Mag. N. H. xv. p. 261: Sharpe, Cat. B. Brit. Mus. iii. p. 111.

a Mexico (T. Mann) 1844. - b Guatemala (J. Constancia) 1848. 
1428. Cyanocitta beecheii.

Pica beecheii, Vig. Zool. Journ. iv. p. 353: Zool. Beech. Voy. p. 22 , pl. 6.

Cyanocitta beecheii, Scl. \& Salv. P. Z. S. 1876, p. 270.

Xanthura beechei, Sharpe, Cat. B. Brit. Mus. iii. p. 133.

Cyanocorax geoffroyi, Bp. Compt. Rend. xxxi. p. 564.

$a$ Chili or Central America (Miss Ball) 1844.

On a label is the following note written in Prince Bonaparte's handwriting "Cyanocorax geoffroyi, Bp. \& Comptes rendus, if not a new sp." The species is an inhabitant of North-Western Mexico.

1429. Cyanocitta armillata.

Cyanocorax armillatus, Gray \& Mitch. Gen. B. ii. p. 7, pl. 3.

Cyanocitta armillata, Scl. Cat. Am. B. p. 144.

Xanthura armillata, Sharpe, Cat. B. Brit. Mus. iii. p. 136.

$a$ (Carfrae) 1850.

A Colombian species common in Bogota Collections.

1430. Cyanocitta melanocyanea.

Garrulus (Cyanocorax) melanocyaneus, Hartl. Rev. Zool. 1844, p. 215.

Cyanocitta melanocyanea, Sel. \& Salv. Ibis, 1859, p. 21.

Xanthura melanocyanea, Sharpe, Cat. B. Brit. Mus. iii. p. 134, pl. 6.

$a$ Guatemala (J. Constancia) 1845.

\section{Cyanocitta californica.}

Garrulus californicus, Vig. Zool, Beechey's Voy. p. 21, pl. 5.

Cyanocitta californica, Strickl. Ann. \& Mag, N. H. xv, p. 343: Baird, Brew. \& Ridgw. N. Am. B. ii. p. 288.

Cyanocitta superciliosa, Strickl. l. s. c. p. 260.

a California (J. F. Brandt) 1844.

The type of $C$. superciliosa, Strickland.

\section{Cyanocitta sordida.}

Garrulus sordidus, Sw. Phil. Mag. 1827, p. 437; Zool. Ill. ser. 2, pl. 86 . 
Cyanocitta sordida, Scl. Cat. Am. B. p. 143.

Aphelocoma sordida, Sharpe, Cat. B. Brit. Mus. iii. p. 116.

Cyanocitta ultramarina, Strickl. Ann. \& Mag. N. H. xv. p. 260.

a Mexico (Arthur Strickland) 1840. —b Mexico (T. Mann) 1844.

$b$ agrees with Swainson's type; $a$ has the top and sides of the head rather bluer but does not otherwise differ from it.

\section{Cyanocitta pumilo.}

Cyanocorax pumilo, Strickl. Contr. Orn. 1849, p. 122, pl. 33 : Sharpe, Cat. B. Brit. Mus. iii. p. 127.

$a$ Guatemala ( $J$. Constancia) 1848.

The type of this species.

\section{Cyanocorax.}

Cyanocorax, Boie, Isis, 1826, p. 975.

\section{Cyanocorax cæruleus.}

Pica ccerulea, Vieill. N. Dict. d'Hist. N. xxvi. p. 126.

Cyanocorax cceruleus, Sharpe, Cat. B. Brit. Mus. iii. p. 126. Corvus azureus, Temm. Pl. Col. 168.

Cyanocorax azureus, Strickl. Ann. \& Mag. N. H. xv. p. 261. a Brazil (N. C. Strickland) 1838.

\section{Cyanocorax cyanopogon.}

Corvus cyanopogon, Max. Beitr. iii. p. 1247.

Cyanocorax cyanopogon, Strickl. Ann. \& Mag. N. H. xv. p. 261 : Sharpe, Cat. B. Brit. Mus. iii. p. 123.

$a$ Brazil (N. C. Strickland) 1838. - b Brazil (Argent) 1851. $a$ is marked "Uroleuca cyanopogon, Bp. ex Wied" in Bonaparte's handwriting.

\section{Cyanocorax cyanoleucus.}

Corvus cyanoleucus, Max, Reis. ii. p. 190.

Cyanocorax cyanoleucus, Pelz. Orn. Bras. p. 189.

Uroleuca cyanoleuca, Sharpe, Cat. B. Brit. Mus. iii. p. 137. Corvus cristatellus, Temm. Pl. Col. 193. 
Cyanocorax cristatellus, Strickl. Ann. \& Mag. N. H. xv. p. 261.

a Brazil (N. C. Strickland) 1838.

Marked "Urolenca cyanoleuca, Bp. ex Wied" in Bonaparte's handwriting.

\section{Cyanocorax chrysops.}

Pica chrysops, Vieill. N. Dict. d'Hist. N. xxvi. p. 124.

Cyánocorax chrysops, Sharpe, Cat. B. Brit. Mus. iii. p. 120.

Corvus pileatus, Temm. Pl. Col. 58.

Cyanocorax pileatus, Strickl. Ann. \& Mag. N. H. xv. p. 261. a Brazil (N. C. Strickland) 1838.

Marked "Uroleuca pileata, Bp." in Bonaparte's handwriting.

\section{Cyanocorax affinis.}

Cyanocorax affinis, Pelz. Sitz. Ak. Wien, xx. p. 164: Scl. Cat. Am. B. p. 145: Sharpe, Cat. B. Brit. Mus. iii. p. 121. a (liogers) 1846.

A bad skin apparently of this Colombian species.

1439. Cyanocorax incas.

Geai du Pérou, D'Aub. Pl. Enl. 625, undè,

Corvus yncas, Bodd. Tabl. Pl. Enl. p. 38.

Xanthura yncas, Sharpe, Cat. B. Brit. Mus. iii. p. 129.

Corvus peruvianus, Gm. Syst. Nat. i. p. 373.

Cyanocorax peruvianus, Strickl. Ann. \& Mag. N. H. xv. p. 261.

a Bogota (Stevens) 1844.

\section{Calocitta.}

Calocitta, Gray, Gen. B. p. 50 (1841).

\section{Calocitta formosa.}

Pica formosa, Sw. Phil. Mag. 1827, p. 437.

Calocitta formosa, Scl. \& Salv. Ibis, 1859, p. 22: Sharpe, Cat.

B. Brit. Mus. iii. p. 88.

Garrula gubernatrix, Temm. Pl. Col. 436.

Calocitta bullocki, Strickl. Ann. \& Mag. N. H. xv. p. 261.

$a$ (Dewgard) 1846. —b Guatemala (J. Constancia) 1851. 


\section{Struthidea.}

Struthidea, Gould, P. Z. S. 1836, p. 143.

\section{Struthidea cinerea.}

Struthidea cinerea, Gould, P. Z. S. 1836, p. 143; B. Austr. iv. pl. 17 : Sharpe, Cat. B. Brit. Mus. iii. p. 140.

a New Zealand (T. C. Eyton) 1850.

An Australian species.

\section{Glaucopis.}

Glaucopis, Gmelin, Syst. Nat. i. p. 363 (1788).

1442. Glaucopis cinerea.

Glaucopis cinerea, Gm. l. s. c.: Buller, B. N. Zeal. p. 155, pl. 15, f. 2: Sharpe, Cat. B. Brit. Mus. iii. p. 142.

a §ิ Banks Penins. S. Isl. New Zealand ( $F$. Strange) 1850.

\section{LOPHOCITTA.}

Platylophus, Swainson, Faun. Bor. Am. ii. p. 482 (1831). Lophocitta, G. R. Gray, List. Gen. B. p. 50 (1841).

1443. Lophocitta galericulata.

Garrulus galericulatus, Cuv. Règn. Anim. i. 399 (1817).

Platylophus galericulatus, Sw. l. s. c.: Elliot, Ibis, 1878, p. 54. Lanius scapulatus, Licht. Verz. Doubl. p. 49. $a,-b$ E. Indies (N. C. Strickland) 1838.

\section{FAMILY.-ORTHONYCIDA.}

\section{ORTHONYX ${ }^{1}$.}

Orthonyx, Temminck, Pl. Col. Livr. 72 (1827).

\section{Orthonyx spinicauda.}

Orthonyx spinicauda, Temm. l. s. c. Pl. 428, 429: Gould, B. Austr. iv. pl. 99.

Orthonyx temmincki, Vig. \& Horsf. Trans. L. S. xv. p. 294: Strickl. Ann. \& Mag. N. H. vi. p. 421.

a New South Wales ( $M^{c}$ Donald) 1838.

1 The position of this genus has not, so far as I am aware, been satisfactorily determined, nor can it be until its internal organization has been thoroughly examined. Strickland (l.c.p. 420) suggested that it should be placed near Menura. 


\section{FAMILY.-MENURID压。}

Menura.

Menura, Davies, Trans. Linn. Soc. vi. p. 207 (1800); Garrod, P. Z. S. 1876 , p. 514 .

1445. Menura superba.

Menura superba, Davies, l. s. c. pl. 22: Gould, B. Austr. iii. pl. 14: Strickl. Ann. \& Mag. N. H. vi. p. 420; vii. p. 39.

$a$ Australia (T. Robinson) 1842. -b (tail) N. Australia ( $J$. Gould) 1850.

$b$ is labelled? $M$. alberti, but as that species has no rufous marks on the outer tail feathers (well shewn in this specimen) I conclude that it should be referred to $M$. superba.

\section{Menura alberti.}

Menura alberti, Gould, P. L. S. ii. p. 67; B. Austr. Suppl. $a$ (tail)

A tail of a Menura without rufous marks on the outer feathers but with both upper and under coverts strongly tinged with that colour. Both characters are found in $M$. alberti.

SUBORDER II.-OLIGOMYODE.

FAMILY.-OXYRHAMPHID $\mathbb{E}^{1}$.

OXYRHAMPHUS.

Oxyrhynchus, Temminck, Pl. Col. Livr. 21, 1823 (nec Leach).

Oxyrhamphus, Strickland, Ann. \& Mag. N. H. vi. p. 420.

\section{4\%. Oxyrhamphus flammiceps.}

Oxyrhynchus flammiceps, Temm. l. s. c. pl. 125: Scl. Cat. Am. B. p. 160.

Oxyrhamphus flammiceps, Scl. \& Salv. Nomencl. Av. Neotr. p. 41. 1838.

a Brazil (N. C. Strickland) 1838. - b Brazil (W. Kirtland) 
PASSERES.

\section{FAMILY.-TYRANNID㤅. \\ SUBFAMILY.-TANIOPTERIN AE。}

AGRIORNIS.

Agriornis, Gould, Zool. Voy. Beagle, iii. p. 56 (1841).

1448. Agriornis livida.

Thamnophilus lividus, Kittl. Mém. prés. Ac. Pétersb. ii. p. 465, pl. 1 (1834).

Agriornis livida, Bp. Consp. p. 197 : Scl. Cat. Am. B. p. 196. a Chili (Capt. Brown) 1842.

\section{TANIOPTERA.}

Tcenioptera, Bonaparte, Journ. Ac. Phil. iv. p. 380 (1825), as a subgenus.

\section{Tænioptera nengeta.}

Lanius nengeta, Linn. Syst. Nat. i. p. 135.

Tcenioptera nengeta, Cab. \& Hein. Mus. Hein. ii. p. 44: Scl. Cat. Am. B. p. 197.

Muscicapa polyglotta, Licht. Verz. Doubl. p. 54.

Xolmis polyglotta, Strickl. Ann. \& Mag. N. H. vii. p. 28. a Brazil (N. C. Strickland) 1838.

1450. Tænioptera velata.

Muscicapa velata, Licht. Verz. Doubl. p. 54.

Tcenioptera velata, Scl. Cat. Am. B. p. 197.

a Brazil (N. C'. Strickland) 1838.

1451. Tænioptera irupero.

Tyrannus irupero, Vieill. Encl. Méth. Ois. p. 856.

Tonioptera irupero, Scl. Cat. Am. B. p. 198.

a (Argent) 1852.

Probably a Bolivian specimeu.

1452. Tænioptera pyrope.

Muscicapa pyrope, Kittl. Mém. prés. Ac. Pétersb. i. p. 191, pl. 10 (1831).

I'cenioptera pyrope, Scl. Cat. Am. B. p. 198.

a Chili (Capt. Brown) 1842. 


\section{Fluvicola.}

Fluvicola, Swainson, Zool. Journ. iii. p. 172 (1827).

\section{Fluvicola pica.}

Gobe-mouche pie, de Cayenne, D'Aub. Pl. Enl. 675, f. 1, undè, Muscicapa pica, Bodd. Tabl. Pl. Enl. p. 42.

Fluvicola pica, Scl. Cat. Am. B. p. 200.

$a$ (Cashmore) 1839. -b (Carfrae) 1840. $-c$ Trinidad ( $J$. Taylor).

\section{Fluvicola albiventris.}

Muscicapa albiventer, Spix, Av. Bras. ii. p. 21, pl. 30, f. 1, 2.

Fluvicola albiventris, Scl. Cat. Am. B. p. 200.

a Brazil (N. C. Strickland) 1838.

\section{Fluvicola climacura.}

Enanthe climazura, Vieill. Gal. Ois. p. 255, pl. 157.

Fluvicola climacura, Cab. \& Hein. Mus. Hein. ii. p. 40: Scl. Cat. Am. B. p. 201.

Muscicapa mystacea, Spix, Av. Bras. ii. p. 22, pl. 31 a, f. 2. Fluvicola mystacea, Strickl. Ann. \& Mag. N. H. vii. p. 28. a S. America (N. C. Strickland) 1838.

\section{Arundinicola.}

Arundinicola, d'Orbigny, Voy. Am. Mérid. Ois. p. 334 (1839). 1456. Arundinicola leucocephala.

Pipra leucocephala, Linn. Syst. Nat. i. p. 340.

Arundinicola leucocephala, Scl. Cat. Am. B. p. 201.

a Brazil (N. C. Strickland) 1838. -b (Arthur Strickland) 1840. $-c$ Trinidad (Col. J. Taylor).

\section{Alectorurus.}

Alectrurus, Vieillot, Anal. p. 39 (1816).

\section{5\%. Alectorurus tricolor.}

Alectrurus tricolor, Vieill. l.s. c.

Alectorurus tricolor, Scl. Cat. Am. B. p. 201.

a Paraguay (T. Holme) 1838. - b Paraguay (N. C. Strickland) 1838. -c Brazil (Arthur Strickland) 1840. 
Cybernetes.

Gubernetes, Such, Zool. Journ. ii. p. 114 (1825).

Cybernetes, Cabanis \& Heine, Mus. Hein. ii. p. 42 (1859).

\section{Cybernetes yetapa.}

Tyrannus yetapa, Vieill. N. Dict. d'Hist. N. xxi. p. 460.

Cybernetes yetapa, Scl. Cat. Am. B. p. 203.

Muscicapa yiperu, Licht. Verz. Doubl. p. 52.

Gubernetes yiperu, Strickl. Ann. \& Mag. N. H. vii. p. 28.

Gubernetes cunninghami, Such, Zool. Journ. ii. p. 114, pl. 4. a Brazil (N. C. Strickland) 1838.

\section{SISOPYGIS.}

Sisopygis, Cabanis \& Heine, Mus. Hein. ii. p. 46 (1859).

\section{Sisopygis icterophrys.}

Muscicapa icterophrys, Vieill. N. D. d'Hist. Nat. xxi. p. 458. Suiriri? icterophrys, Strickl. Ann. \& Mag. N. H. xiii. p. 414, pl. 12.

Sisopygis icterophrys, Scl. Cat. Am. B. p. 202.

a Brazil (Askew) 1834.

\section{Chipolegus.}

Knipolegus, Boie, Isis, 1826, p. 973.

Cnipolegus, Strickland, Ann. \& Mag. N. H. vii. p. 28.

\section{Cnipolegus comatus.}

Muscicapa comata, Licht. Verz. Doubl. p. 55.

Cnipolegus comatus, Bp. Consp. p. 195; Scl. Cat. Am. B. p. 202.

a Paraguay (Askew) 1833.

\section{Cnipolegus nigerrimus.}

Muscicapa nigerrima, Vieill. N. Dict. d'Hist. N. xxi. p. 453.

Cnipolegus nigerrimus, Heine, J. F. Orn. 1859, p. 332 : Scl. Cat. Am. B. p. 202.

a S. America (N. C. Strickland) 1838.

A Brazilian species. 


\section{Cnipolegus cyanirostris.}

Muscicapa cyanirostris, Vieill. N. Dict. d'Hist. N. xxi. p. 447. Cnipolegus cyanirostris, Boie, Isis, 1826, p. 973 : 'Scl. Cat. Am. B. p. 203.

a Brazil ? (Askew) 1834.

\section{LiCHENOPS.}

"Perspicillarius, nictitarius, Lichenops; Clignot," Commerson in Buff. Hist. Nat. Ois. v. p. 235: Sonn. Ed. Ois. xv. p. 291 (1799).

Lichenops, Commerson, Sundevall, K. Vet. Ac. Handl. 1835, p. 88.

\section{Lichenops perspicillata.}

Motacilla perspicillata, Gm. Syst. Nat. i. p. 969.

Lichenops perspicillata, Scl. Cat. Am. B. p. 202.

Lichenops erythroptera, Gould, Voy. Beagle, iii. p. 51, pl. 9.

a South Brazil (N. C. Strickland) 1838. - b Chili? (N. C. Strickland) 1838. — - S. America (N. C. Strickland) 1838.

\section{Muscipipra.}

Muscipipra, Lesson, Traité d'Orn. p. 387 (1831).

\section{Muscipipra vetula.}

Muscicapa vetula, Licht. Verz. Doubl. p. 53: Strickl. Ann. \& Mag. N. H. vii. p. 28.

Muscipipra vetula, Scl. Cat. Am. B. p. 204.

a Brazil (N. C. Strickland) 1838.

\section{Copurus.}

Copurus, Strickland, P. Z. S. 1841, p. 28.

\section{Copurus colonus.}

Muscicapa colonus, Vieill. N. Dict. d'Hist. N. xxi. p. 448.

Copurus colonus, Cab. \& Hein. Mus. Hein. ii. p. 41 : Scl. Cat. Am. B. p. 204.

Platyrhynchus filicauda, Spix, Av. Bras. ii. p. 12, pl. 14.

Copurus filicaudus, Strickl. l. s. c.

Muscipeta leucocilla, Hahn, Ausl. Vög. Lief. ix. pl. 2.

a Brazil (N. C. Strickland) 1838. -b, -c, -d Brazil ( $G$. Lloyd) 1838. 


\section{MaCHETORNIS.}

Machetornis, G. R. Gray, List Gen. B. 41 (1841).

1466. Machetornis rixosa.

Tyrannus rixosa, Vieill. Nouv. Dict. d'Hist. N. xxxv. p. 85.

Machetornis rixosus, Burm. Syst. Ueb. ii. p. 514: Scl. Cat. Am. B. p. 204.

$a$ Brazil (Graham) 1849. —b, -c Trinidad (Argent) 1851. -d ô Bolivia (Argent) 1852.

\section{Muscisaxicola.}

Muscisaxicola, d'Orbigny \& Lafresnaye, Syn. Av. i. p. 65 (1837).

\section{6\%. Muscisaxicola alpina.}

Toenioptera alpina, Jard. Contr. Orn. 1849, p. 47, pl. 21.

Muscisaxicola alpina, Scl. \& Salv. P. Z. S. 1867, p. 986.

Muscisaxicola albifrons, Scl. Cat. Am. B. p. 205 (nec Tschudi).

a Andes of Quito (Sir W. Jardine) 1849.

This is one of the original specimens sent to Sir W. Jardine by William Jameson who long resided in Quito. The label bears the following note: "This species occurs on the highest ledges of the Andes rarely descending below the snow line."

\section{Muscisaxicola mentalis.}

Muscisaxicola mentalis, d'Orb. \& Lafr. Syn. Av. i. p. 66 : d’Orb. Voy. Am. Mérid. Ois. p. 355, pl. 41, f. 1 : Scl. Cat. Am. B. p. 205.

$a$ Chili (N. C. Strickland) 1838.

\section{Centrites.}

Centrites, Cabanis, Arch. f. Naturg. xiii. p. 256 (1847). 1469. Centrites niger.

Alouette noire, de la Encenada, D'Aub. Pl. Enl. 738, f. 2, undè, Alauda nigra, Bodd. Tab. Pl. Enl. p. 46.

Centrites niger, Cab. \& Hein. Nus. Hein. ii. p. 48: Scl. Cat. Am. B. p. 206. 
Alauda rufa, Gm. Syst. Nat. i. p. 792.

a S. America (N. C. Strickland) 1838. - b Chili (N.C. Strickland) 1838.

\section{SUBFAMILY.-PLATYRHYNCHIN $Æ$.}

\section{Platyrhynchus.}

Platyrhynchus, Desmarest, Hist. Nat. Tod. Sub. pl. 72 (1805).

\section{Platyrhynchus rostratus.}

Todus rostratus, Pall. Spic. Zool. fasc. vi. p. 19, pl. 3.

Platyrhynchus rostratus, Bp. Consp. p. 183: Scl. Cat. Am. B. p. 206.

a Brazil (Argent) 1851.

\section{Platyrhynchus mystaceus.}

Platyrhynchus mystaceus, Vieill. N. Dict. d'Hist. N. xxvii. p. 14: Scl. Cat. Am. B. p. 207. 1838.

a Brazil (Johnson) 1837. - b Brazil ( $N$. C. Strickland)

\section{Platyrhynchus coronatus.}

Platyrhynchus coronatus, Scl. P. Z. S. 1858, p. 71 ; Cat. Am. B. p. 207.

a (Dewgard) 18.51.

Apparently a Cayenne skin agreeing with Mr Sclater's type.

\section{TODIROSTRUM.}

Todirostrum, Lesson, Traité d'Orn. p. 384 (1831) as a subgenus.

\section{Todirostrum cinereum.}

Todus cinereus, Linn. Syst. Nat. i. p. 178.

Todirostrum cinereum, Less. l. s. c.: Scl. Cat. Am. B. p. 207.

$a$ (Bt at Dresden) 1845. -b Guatemala (J. Constancia) 1851. —c Trinidad (Argent) 1852. —d Brazil (Argent) 1853. 


\section{Todirostrum poliocephalum.}

Todus poliocephalus, Max. Beitr. iii. p. 964.

Todirostrum poliocephalum, Scl. P. Z. S. 1857, p. 84; Cat. Am. B. p. 208.

$a$ (N. C. Strickland) 1838.

A Brazilian species.

\section{Todirostrum ruficeps.}

Todirostrum ruficeps, Kaup. P. Z. S. 1851, p. 52 : Scl. Cat. Am. B. p. 207.

Todirostrum multicolor, Strickl. Contr. Orn. 1852, p. 41, pl. 85, f. 2.

a Bogota (Bt at Stevens's) 1845.

The type of T. multicolor, Strickland.

\section{Todirostrum maculatum.}

Todus maculatus, Desm. Tod. pl. 70.

Todirostrum maculatum, Less. Traité d'Orn. p. 384: Scl. Cat. Am. B. p. 208.

a Para (Stevens) 1849.

This specimen bears Mr Wallace's label which is thus inscribed " 127 Mexiana o Dec. 48 A. W." The species is not included in the List of Birds collected by Mr Wallace in the Lower Amazons and Rio Negro (P. Z. S. 1868, p. 566 et seq.).

\section{EusCARTHMUS.}

Euscarthmus, Maximilian, Pr. v. Wied, Beitr. Nat. Bras. iii. p. 945 (1831).

147\%. Euscarthmus nidipendulus.

Euscarthmus nidipendulus, Max. l. s. c. p. 950: Scl. Cat. Am. B. p. 208 .

a (N. C. Strickland) 1838.

A Brazilian species.

1478. Euscarthmus gularis.

Muscicapa gularis, Temm. Pl. Col. 167, f. 1.

Todirostrum gulare, Strickl. Contr. Orn. 1852, p. 41. 
Euscarthmus gularis, Scl. Cat. Am. B. p. 209.

a Brazil (Manchester Nat. Hist. Soc.) 1843.

Agrees with Bolivian examples.

1479. Euscarthmus granadensis.

Todirostrum granadense, Hartl. Rev. Zool. 1843, p. 289 ; et Contr. Orn. 1852, p. 41, pl. 85, f. 1.

Euscarthmus granadensis, Scl. Cat. Am. B. p. 209.

a (Argent) 1851.

Has the appearance of a Bogota skin.

\section{Euscarthmus orbitatus.}

Euscarthmus orbitatus, Max. Beitr. iii. p. 958: Scl. Cat. Am. B. p. 209 .

a (Gardner) 1846.

Agrees with Bahia examples.

\section{OrCHILUS.}

Orchilus, Cabanis, Tschudi's Fauna Per. p. 164 (1845).

1481. Orchilus auricularis.

Platyrhynchus auricularis, Vieill. N. Dict. d'Hist. N. xxvii. p. 16.

Orchilus auricularis, Cab. \& Hein. Mus. Hein. ii. p. 51: Scl. Cat. Am. B. p. 209.

a Brazil (Manchester Nat. Hist. Soc.) 1843.

\section{HeMitriccus.}

Hemitriccus, Cabanis \& Heine, Mus. Hein. ii. p. 52 (1859).

1482. Hemitriccus diops.

Muscicapa diops, Temm. Pl. Col. 144, f. 1.

Todirostrum diops, Strickl. Contr. Orn. 1852, p. 41.

Hemitriccus diops, Cab. \& Hein. l.s. c.: Scl. Cat. Am. B. p. 210.

$a,-b$ (N. C. Strickland) 1838. $-c$ Brazil (Manchester Nat. Hist. Soc.) 1843. 


\section{Phylloscartes.}

Phylloscartes, Cabanis \& Heine, Mus. Hein. ii. p. 52 (1859).

\section{Phylloscartes ventralis.}

Muscicapa ventralis, Temm. Pl. Col. 275, f. 2.

Phylloscartes ventralis, Cab. \& Hein. l. s. c.: Scl. \& Salv. P. Z. S. 1867, p. 577.

a Rio Tocantins (A. R. Wallace) 1849.

\section{LEPTOTRICCUS.}

Leptotriccus, Cabanis \& Heine, Mus. Hein. ii. p. 54 (1859).

\section{Leptotriccus sylviola.}

Leptotriccus sylviola, Cab. \& Hein. Mus. Hein. ii. p. 54: Scl. Cat. Am. B. p. 211.

a Brazil (N. C. Strickland) 1838.

\section{SERPHOPHAGA.}

Serpophaga, Gould, Zool. Voy. Beagle, iii. p. 49 (1841).

\section{Serphophaga cinerea.}

Euscarthmus cinereus, Strickl. Ann. \& Mag. N. H. xiii. p. 414 .

Serphophaga cinerea, Cab. \& Hein. Mus. Hein. ii. p. 53: Scl. Cat. Am. B. p. 211.

$a$ Chili? (Tucker) 1838.

The type of this Western American species.

\section{Serphophaga nigricans.}

Sylvia nigricans, Vieill. N. Dict. d'Hist. Nat. xi. p. 204. Serphophaga nigricans, Gould, Zool. Voy. Beagle, iii. p. 50. Serphophaga nigricans, Scl. Cat. Am. B. p. 211. a Brazil (Argent) 1853.

A species found in the valley of the La Plata. 


\section{8\%. Serphophaga parvirostris.}

Myiobius parvirostris, Gould, Zool. Voy. Beagle, iii. p. 48. Serphophaga parvirostris, Scl. Cat. Am. B. p. 212. a Chili (Capt. Brown) 1842.

\section{ANERETES.}

Anairetes, Reichenbach, Av. Syst. pl. lxvi. (1850). 1488. Anæretes parulus.

Muscicapa parulus, Kittl. Mém. prés. Ac. Pétersb. i. p. 190, pl. 9 (1831).

Euscarthmus parulus, Strickl. Ann. \& Mag. N. H. xiii. p. 415.

Anceretes parulus, Cab. \& Hein. Mus. Hein, ii. p. 54: Scl. Cat. Am. B. p. 212.

a Chili (Tucker) 1838.

\section{Anæretes fernandezianus.}

Culicivora fernandeziana, Philippi, Arch. f. Naturg. xxiii. pt. 1, p. 265.

Anceretes fernandezianus, Scl. Ibis, 1871, p. 179, pl. vii. f. 1. $a$ Chili (Tucker) 1838.

Agrees with specimens from the island of Juan Fernandez.

1490. Anæretes agilis.

Euscarthmus agilis, Scl. P. Z. S. 1856, p. 28, pl. 118.

Ancretes agilis, Cab. \& Hein. Mus. Hein. ii. p. 54: Scl. Cat. Am. B. p. 212.

a Bogota? (Bt at Stevens's) 1845.

Cyanotis ${ }^{1}$.

Cyanotis, Swainson, Classif. B. ii. p. 24:3 (1837).

1491. Cyanotis azaræ.

Regulus azarce, Naum. Vög. Deutschl. iii. p. 966.

1 Considered by Strickland (Ann. \& Mag. N. H. vi. p. 421) to come next to Regulus (Sylviidæ), to the members of which genus C. azarce has a superficial resemblance.

S. C. 
Cyanotis azarce, Cab. \& Hein. Mus. Hein. ii. p. 54: Scl. Cat. Am. B. p. 212.

Tachyuris omnicolor, d'Orb. \& Lafr. Syn. Av. i. p. 55.

a Brazil (N. C. Strickland) 1838.

A species found in Chili and the Argentine Republic.

\section{SUBFAMILY.-ELAINEIN E.}

\section{Mionectes.}

Mionectes, Cabanis, Tschudi's Fauna Per. p. 147 (1845).

+ 1492. Mionectes olivaceus.

Mionectes olivaceus, Lawr. Ann. Lyc. N. Y. ix. p. 111.

$a$ Brazil (Argent) 1853.

Agrees best with Central American specimens of this species.

+1493. Mionectes oleagineus.

Muscicapa oleaginea, Licht. Verz. Doubl. p. 55.

Mionectes oleagineus, Cab. in Tsch. Fauna Per. p. 148: Scl. Cat. Am. B. p. 213. 1840.

$a,-b$ (N. C. Strickland) 1838. $\longrightarrow$ (Arthur Strickland)

A Brazilian species.

LEPTOPOGON.

Leptopogon, Cabanis, Tschudi's Fauna Per. p. 161 (1845). 1494. Leptopogon pæcilotis.

Leptopogon pcecilotis, Scl. P. Z. S. 1862, p. 111.

a Bogota (Bt at Stevens's) 1845.

1495. Leptopogon tristis.

Leptopogon tristis, Scl. \& Salv. P. Z. S. 1876, p. 254.

a (Mansfield) 1852.

Agrees with the types of this Bolivian species.

\section{CAPSIEMPIS.}

Capsiempis, Cabanis \& Heine, Mus. Hein. ii. p. 56 (1859).

+ 1496. Capsiempsis flaveola.

Muscicapa flaveola, Licht. Verz. Doubl. p. 56. 
Capsiempis flaveola, Cab. \& Hein. l. s. c.: Scl. Cat. Am. B. p. 214.

$a$ Brazil (N. C. Strickland) 1838.

\section{Phyllomyias.}

Phyllomyias, Cabanis \& Heine, Mus. Hein。 ii. p. 57 (1859). 149\%. Phyllomyias brevirostris.

Platyrhynchus brevirostris, Spix, Av. Bras. ii. p. 13, pl. 15, f. 2.

Phyllomyias brevirostris, Cab. \& Hein. Mus. Hein. ii. p. 57: Scl. Cat. Am. B. p. 214 .

a (N. C. Strickland) 1838.

A Brazilian species.

1498. Phyllomyias griseicapilla.

Phyllomyias griseicapilla, Scl. P. Z. S. 1861, p. 382, pl.36, f. 2 ; Cat. Am. B. p. 214.

$a$ (Argent) 1852.

A Brazilian species.

\section{ORNITHION.}

Ornithion, Hartl. J. f. Orn. 1853, p. 35.

1499. Ornithion imberbe.

Camptostoma imberbe, Scl. P. Z. S. 1857, p. 203 ; Cat. Am. B. p. 215 .

Ornithion incanescens, Scl. P. Z. S. 1873, p. 577 (nec Max.) $c f$. Lawr. Ibis, 1876, p. 497.

$a$ Chili? (N. C. Strickland) 1838.

Agrees with a specimen in Mr Sclater's collection from Lagoa Santa.

\section{Tyrannulus.}

Tyrannulus, Vieillot, Anal. p. 31 (1816). 1500. Tyrannulus elatus.

Sylvia elata, Lath. Ind. Orn. ii. p. 549.

Tyrannulus elatus, Scl. Cat. Am. B. p. 215.

a Demarara? (Cashmore) 1843. 
Elainea.

Elcenia, Sundevall, K. Vet. Ac. Handl. 1835, p. 89.

1501. Elainea pagana.

Muscicapa pagana, Licht. Verz. Doubl. p. 54.

Elaenea pagana, Cab. Schomb. Guian. iii. p. 701.

a Brazil (N. C. Strickland) 1838. —b (Bt. at Stevens's) 1845.

1502. Elainea placens.

Elainia placens, Scl. P. Z. S. 1859, p. 46; Cat. Am. B. p. 217. a (Thomas) 1843.

This specimen agrees fairly with examples of this widely-ranging Central American species but may perhaps prove to be distinct.

1503. Elainea elegans.

Elainea elegans, Pelz. Orn. Bras. pp. 107, 179.

a (Askew) 1840.

1504. Elainea obscura.

Elcenia obscura, Cab. Tsch. Faun. Per. Orn. p. 158.

Elainea rustica, Scl. Cat. Am. B. p. 218.

a (Bt at Aberystwith) 1833.

A Brazilian species.

1505. Elainea mesoleuca.

Elainea mesoleuca, Cab. \& Hein. Mus. Hein. ii. p. 60. a (Bt at Stevens's) 1844.

Agrees with examples from Bahia, Brazil.

\section{LEGATUS.}

Legatus, Sclater, P. Z. S. 1859, p. 46.

+ 1506. Legatus albicollis.

Tyrannus albicollis, Vieill. Nouv. Dict. d'Hist. N. xxxv. p. 89.

Legatus albicollis, Cab. Hein. Mus. Hein. ii. p. 60: Scl. Cat. Am. B. p. 218.

a (Arthur Strickland) 1840.

A widely-ranging South-American species. 


\section{MYiozeteTES.}

Myiozetetes, Scl. P. Z. S. 1859, p. 46, ex Myiozeta, Bonaparte. 150\%. Myiozetetes cayennensis.

Muscicapa cayennensis, Linn. Syst. Nat. i. p. 327.

Myiozetetes cayennensis, Scl. P. Z. S. 1871, p. 752.

$a$ Trinidad (Argent) 1851. - b 个. Bolivia (Argent) 1852.

1508. Myiozetetes similis.

Muscicapa similis, Spix, Av. Bras. ii. p. 18, pl. 25.

Myiozetetes similis, Scl. P. Z. S. 1871, p. 753.

a Brazil (N. C. Strickland) 1838.

1509. Myiozetetes texensis.

Muscicapa texensis, Giraud, B. Texas, pl. 1.

Myiozetetes texensis, Scl. Cat. Am. B. p. 219; P. Z. S. 1871, p. 753.

$a$ Guatemala (J. Constancia) 1848.

\section{RHyNChOCYCLUS.}

Rhynchocyclus, Cabanis \& Heine, Mus. Hein. ii. p. 56 (1859). +1510. Rhynchocyclus olivaceus.

Platyrhynchos olivaceus, Temm. Pl. Col. 12, f. 1.

Rhynchocyclus olivaceus, Cab. \& Hein. Mus. Hein. ii. p. 56 : Scl. Cat. Am. B. p. 220.

$a$ S. America 1842.

1511. Rhynchocyclus sulphurescens.

Platyrhynchus sulphurescens, Spix, Av. Bras. ii. p. 10, pl. 12, f. 1.

Rhynchocyclus sulphurescens, Scl. Cat. Am. B. p. 220.

$a$ (Askew) 1840.

A widely-ranging South-American species.

1512. Rhynchocyclus flaviventris.

Platyrhynchus flaviventris, Spix, Av. Bras. ii. p. 12, pl. 15, f. 1 . 
Rhynchocyclus flaviventer, Cab. \& Hein. Mus. Hein. ii. p. 56: Scl. Cat. Am. B. p. 221.

a Bahia (S. Dutton) 1845. -b (Argent) 1851.

\section{Conopias.}

Conopias, Cabanis \& Heine, Mus. Hein. ii. p. 62 (1859). 1513. Conopias trivirgata.

Muscicapa trivirgata, Max. Beitr. iii. p. 871.

Conopias trivirgata, Scl. P. Z. S. 1871, p. 755.

Conopias superciliosa, Scl. Cat. Am. B. p. 221.

a Brazil (N. C. Strickland) 1838.

\section{Conopias inornata.}

Myiozetetes inornata, Lawr. Ann. Lyc. N. Y. ix. p. 268.

Conopias inornata, Scl. P. Z. S. 1871, p. 756.

a Trinidad (Argent) 1851. Brazil.

A little known species allied to the commoner C. trivirgata of

\section{Pitangus.}

Pitangus, Swainson, Zool. Journ. iii. p. 165 (1827).

\section{Pitangus lictor.}

Lanius lictor, Licht. Verz. Doubl. p. 49.

Pitangus lictor, Scl. Cat. Am. B. p. 221.

a Brazil (Graham) 1849. -b Trinidad (Argent) 1851.

+ 1516. Pitangus parvus.

Pelz. Orn. Bras. pp. 111, 181.

a (Cashmore) 1843.

This little known species was originally described from a single skin obtained by Natterer at Marabitanas in Brazil. Mr Sclater has a specimen from Oyapoc in French Guiana with which the present example agrees.

\section{1\%. Pitangus sulphuratus.}

Lanius sulphuratus, Linn. Syst. Nat. i. p. 137.

Pitangus sulphuratus, Strickl. Ann. \& Mag. N. H. viii. p. 372 : Scl. Cat. Am. B. p. 222.

$a,-b$ Brazil (G. Lloyd) 1838. — Brazil (N. C. Strickland) 1838. 


\section{Pitangus caudifasciatus.}

Tyrannus caudifasciatus, d'Orb: La Sagra's Cuba, Av. p. 70, t. 12 : Gosse, B. Jam. p. 177, et Ill. pl. 44.

Pitangus caudifasciatus, Scl. Cat. Am. B. p. 222.

a Jamaica (J. Gould) 1846. — b Jamaica (P. H. Gosov) 1 148.

\section{Sirystes.}

Sirystes, Cabanis \& Heine, Mus. Hein. ii. p. 75 (1859).

\section{Sirystes sibilator.}

Muscicapa sibilator, Vieill. N. Dict. d'H. N. xxi. p. 457.

Sirystes sibilator, Cab. \& Hein. Mus. Hein. ii. p. 75 : Scl. Cat. Am. B. p. 222.

a Brazil (J. F. Brandt) 1845.

\section{Mriodynastes.}

Myiodynastes, Bonaparte, Compt. Rend. xxxviii. p. 657 (1856). 1520. Myiodynastes audax.

Muscicapa audax, Gm. Syst. Nat. i. p. 934.

Myiodynastes audax, Scl. P. Z. S. 1859, p. 43; et Cat. Am. B. p. 223.

a Brazil (Arthur Strickland) 1834.

\section{SUBFAMILY._TYRANNIN Æ.}

\section{MegarhynChus.}

Megarhynchus, Thunberg, Diss. de Gen. Megarh. Upsala (1824) $c f$. ; J. f. Orn. 1859, p. 337.

\section{Megarhynchus pitangua.}

Lanius pitangua, Linn. Syst. Nat. i. p. 136.

Megarhynchus pitangua, Cab. \& Hein. Mus. Hein. ii. p. 64: Scl. Cat. Am. B. p. 224.

Scaphorhynchus piiangua, Strickl. Ann. \& Mag. N. H. viii. p. 372.

$a,-b,-c$ Brazil (N. C. Strickland) 1838. —d Guatemala (J. Constancia) 1845. —e Guatemala (J. Constancia) 1848. $-f$ Trinidad (Argent) 1851. - g Trinidad (Argent) 1852. 
Muscivora.

Muscivora, Cuvier, Leç. Anat. Comp. i. Tab. 2 (1800).

\section{Muscivora mexicana.}

Muscivora mexicana, Scl. P. Z. S. 1856, p. 295.

a Coban (L. L. Dillwyn) 1849.

\section{HIRUNDINEA.}

Hirurdinea, d'Orbigny \& Lafresnaye, Syn. Av. i. p. 46 (1837). 1523. Hirundinea rupestris.

Platyrhynchus rupestris, Max. Beitr. iii. p. 977.

Hirundinea rupestris, Scl. Ibis, 1869, p. 198, pl. 5, f. 3.

a Brazil (N. C. Strickland) 1838.

\section{Myiobius.}

Myiobius, G. R. Gray, List Gen. B. p. 30 (1840).

\section{Myiobius barbatus.}

Le Barbichon de Cayenne, D'Aub. Pl. Enl. 830, f. 1, undè,

Muscicapa barbata, Gm. Syst. Nat. i. p. 933 : Strickl. Ann. \& Mag. N. H. vii. p. 28.

Myiobius barbatus, Scl. Cat. Am. B. p. 225.

$a \mathrm{~S}$. America (Bt at Aberystwith) 1833. - b S. America ( $N$. C. Strickland) 1838.

\section{Myiobius cinnamomeus.}

Muscipeta cinnamomea, d'Orb. \& Lafr. Syn. Av. i. p. 49.

Myiobius cinnamomeus, Scl. Cat. Am. B. p. 226.

a Bogota (Gardner) 1845.

1526. Myiobius ornatus.

Tyrannula ornata, Lafr. Rev. Zool. 1853, p. 57 : Scl. P. Z. S. 1854 , p. 113, pl. 66 , f. 2.

Myiobius ornatus, Scl. Cat. Am. B. p. 226.

a Bogota (Bt at Stevens's) 1845.

\section{+1527. Myiobius nævius.}

Gobe-mouche à poitrine tachetée, de Cayenne, D'Aub. Pl. Enl. 574, f. 3, undè,

Muscicapa ncevia, Bodd. Tabl. Pl. Enl. p. 34. 
Myiobius ncevius, Scl. Cat. Am. B. p. 227.

$a,-b$ Brazil (N. C. Strickland) 1838. — (Mather) 1840. -d Brazil (Argent) 1851.

\section{Pyrocephalus.}

Pyrocephalus, Gould, Zool. Voy. Beagle iii. p. 44 (1841). +1528. Pyrocephalus rubineus.

Gobe-mouche rouge hupé, D'Aub. Pl. Enl. 675, f. 2, undè, Muscicapa rubinus, Bodd. Tabl. Pl. Enl. p. 42.

Pyrocephalus rubineus, Cab. \& Hein. Mus. Hein. ii. p. 67: Scl. Cat. Am. B. p. 227.

Pyrocephalus obscurus, Gould, Zool. Voy. Beagle iii. p. 45.

$a$ S. America (N. C. Strickland) 1838. - b Chili ? (Tucker) 1838. $-c$ (Gardner) 1845. $-d$, —e Trinidad (Argent) 1851. $-f$ Bolivia (Argent) 1852.

\section{Mitrephorus.}

Mitrephorus, Sclater, P. Z. S. 1859, p. 44.

\section{Mitrephorus phæocercus.}

Mitrephorus phceocercus, Scl. l. s. c.; Cat. Am. B. p. 228.

a Guatemala (J. Constancia) 1848.

\section{Empidonax.}

Empidonax, Cabanis, Journ. f. Orn. 1855, p. 480.

\section{Empidonax trailli.}

Muscicapa traillii, Aud. Orn. Biogr. i. p. 236.

Empidonax trailli, Baird, Birds N. Am. p. 193.

a Carlisle, Pennsylvania, 30 May 1843 (S. F. Baird) 1847. -b Pennsylvania (S. F. Baird) 1847.

\section{Empidonax minimus.}

Tyrannula minima, W. M. \& S. F. Baird, Proc. Ac. Phil. 1843 , p. 284.

Empidonax minimus, Baird, Birds N. Am. p. 195: Scl. Cat. Am. B. p. 229.

a 1 Carlisle, Pennsylvania, 30 April 1846 (S. F. Baird) 1847. -b f Carlisle, Pennsylvania, 7 May 1847 (S. F. Baird) 1847. 


\section{Empidonax acadicus.}

Muscicapa acadica, Gm. Syst. Nat. i. p. 947.

Empidonax acadicus, Baird, B. N. Am. p. 197.

a Pennsylvania, 22 December 1842 (S. F. Baird) 1847.

+ 1533. Empidonax flaviventris.

Tyrannula flaviventris, W. M. \& S. F. Baird, Proc. Ac. Phil. 1843, p. 283.

Empidonax flaviventris, Baird, Birds N. Am. p. 198: Scl. Cat. Am. B. p. 229.

a $\hat{o}$ Carlisle, Pennsylvanja, 8 May 1847 (S. F. Baird) 1847. -b (Dewgard) 1849.

\section{+ 1534. Empidonax affinis.}

Tyrannula affinis, Swains. Phil. Mag. 1827, p. 367.

Empidonax fulvipectus, Lawr. Ann. Lyc. N. Y.x. p. 11 : Salv. Ibis, 1874, p. 310.

a $q$ Cinco Señores, Mexico, Sep. 1844 (Galeotti) 1845.

This specimen agrees accurately with Swainson's type example now in the University Museum.

\section{Contopus.}

Contopus, Cabanis, Journ. f. Orn. 1855, p. 479.

\section{Contopus pertinax.}

Contopus pertinax, Cab. \& Hein. Mus. Hein. ii. p. 72 : Scl. Cat. Am. B. p. 231.

a Mexico (T. Mann) 1844. -b ô Sta Gertrudis, Mexico, August (Galeotti) 1845.

\section{+ 1536. Contopus virens.}

Muscicapa virens, Linn. Syst. Nat. i. p. 327.

Contopus virens, Baird, Birds N. Am. p. 190.

$a$ New York (J. F. Brandt) 1845. - $b$ $\hat{o}$ Carlisle, Pennsylvania, 13 May 1847 (S. F. Baird) 1847.

\section{$+153 \%$. Contopus brachytarsus.}

Empidonax brachytarsus, Scl. Ibis, 1859, p. 441.

Contopus brachytarsus, Salv. Ibis, 1861, p. 364: Scl. Cat. Am. B. p. 231 : Scl. \& Salv. P. Z. S. 1867, p. 578.

a ô Mexiana, November 1848 (A. R. Wallace) 1849. 


\section{+1538. Contopus pallidus.}

Myiobius pallidus, Gosse, B. Jam. p. 166 ; Ill. pl. 40.

Contopus pallidus, Scl. Cat. Am. B. p. 231.

a Jamaica (P. H. Gosse) 1848.

One of $\mathrm{Mr}$ Gosse's typical specimens.

\section{MYIOCHANES.}

Myiochanes, Cabanis \& Heine, Mus. Hein. ii. p. 71 (1859).

\section{Myiochanes cinereus.}

Platyrhynchus cinereus, Spix, Av. Bras. ii. p. 11, pl. 13, f. 2. Myiochanes cinereus, Cab. \& Hein. Mus. Hein. ii. p. 71: Scl. Cat. Am. B. p. 232.

$a,-b$ Brazil (N. C. Strickland) 1838.

\section{Mriarchus.}

Myiarchus, Cabanis, Tschudi's Fauna Per. p. 152 (1845). 1540. Myiarchus validus.

Myiarchus validus, Cab. Arch. f. Naturg. xiii. p. 351: Scl. Cat. Am. B. p. 232.

Tyrannus crinitus, Gosse, B. Jam. p. 186 (nec Linn.).

a Jamaica (P. H. Gosse) 1848.

1541. Myiarchus crinitus.

Muscicapa crinita, Linn. Syst. Nat. i. p. 325.

Myiarchus crinitus, Cab. J. f. Orn. 1855, p. 479; Scl. Cat. Am. B. p. 232.

$a$ N. America (Havell) 1839. -b Mexico (Galeotti) 1845. -c ô Carlisle, Pennsylvania, May 1847 (S. F. Baird) 1847.

\section{Myiarchus tyrannulus.}

Muscicapa tyrannula, Müll. Syst. Nat. Suppl. p. 169.

Myiarchus tyrannulus, Cassin, Pr. Ac. Phil. 1864, p. 255.

Myiarchus ferox, (Gm.) Scl. Cat. Am. B. p. 233.

$a$ S. America (N. C. Strickland) 1838. —b (Cashmore) 1843. -c Tobago (Sir W. Jardine) 1844. 


\section{+1543. Myiarchus lawrencii.}

\section{Tyrannula lawrencii, Giraud, B. Texas, pl. 2.}

Myiarchus lawrencii, Baird, B. N. Am. p. 181: Scl. Cat. Am. B. p. 233.

a f Comaltepec, Mexico, December 1843 (Galeotti) 1845.

+1544. Myiarchus nigriceps.

Myiarchus nigriceps, Scl. P. Z. S. 1860, p. 68; Cat. Am. B. p. 234 .

a (Bt at Birmingham) 1849.

Agrees fairly with Sclater's Ecuador specimens of this species.

\section{Myiarchus stolidus.}

Myiobius stolidus, Gosse, B. Jam p. 168 ; Ill. pl. 42.

Myiarchus stolidus, Cab. J. f. Orn. 1855, p. 479; Scl. Cat. Am. B. p. 234.

$a$ (N. C. Strickland) 1838. - b Jamaica (P. H. Gosse) 1848.

$a$ is a faded specimen possibly of this species; $b$ is one of $\mathrm{Mr}$ Gosse's typical examples.

\section{Blacicus.}

Blacicus, Cabanis, Journ. f. Orn. 1855, p. 480.

\section{+1546. Blacicus barbirostris.}

Tyrannula barbirostris, Swains. Phil. Mag. 1827, p. 367.

Blacicus barbirostris, Scl. P. Z. S. 1871, p. 85.

Myiobius tristis, Gosse, B. Jam. p. 167 ; Ill. pl. 41.

a Jamaica (P. H. Gosse) 1848. - b (Thomas) 1843.

$a$ is a typical specimen of $\mathrm{Mr}$ Gosse's $M$. tristis, agreeing with Swainson's type of his Tyrannula barbirostris.

\section{Empidias.}

Empidias, Cabanis \& Heine, Mus. Hein. ii. p. 69 (1859).

154\%. Empidias fuscus.

Muscicapa fusca, Gm. Syst. Nat. i. p. 931.

Empidias fuscus, Scl. Cat. Am. B. p. 234.

$a$ (Askew) 1837. - b §o Carlisle, Pennsylvania, 23 April 1845 (S. F. Baird) 1847. -c. 


\section{Empidonomus.}

Empidonomus, Cabanis \& Heine, Mus. Hein. ii. p. 76 (1859). 1548. Impidonomus varius.

Muscicapa varia, Vieill. N. Dict. d'Hist. N. xxi. p. 458. Empidonomus varius, Cab. \& Hein. Mus. Hein. ii. p. 76. $a$ S. America (Mather) 1840. - b (Thomas) 1843.

\section{Tyrannus.}

Tyrannus, Cuvier, Leç. Anat. Comp. i. Tab. 2 (1800).

\section{Tyrannus pipiri.}

Lanius tyrannus $\gamma$ carolinensis, Gm. Syst. Nat. i. p. 302 : undè "Tyrannus carolinensis, Gm." Temm. et Auctt. Strickl. Ann. \& Mag. N. H. viii. p. 372.

Tyrannus pipiri, Vieill. Ois. Am. Sept. i. p. 73, t. 44: Scl. Cat. Am. B. p. 236.

$a$ (Askew) 1834. - b Brazil? (Argent) 1852.

$b$ is probably from Bolivia.

1550. Tyrannus griseus.

Tyrannus griseus, Vieill. Ois. Am. Sept. i. p. 76, t. 46: Scl. Cat. Am. B. p. 236.

Tyrannus tyrannus $\beta$ dominicensis, Gm. Syst. Nat. i. p. 302. Tyrannus dominicensis, Gosse, B. Jam. p. 169: Strickl. Ann. \& Mag. N. H. viii. p. 372.

a Jamaica (P. H. Gosse) 184.8.

\section{Tyrannus melancholicus.}

Tyrannus melancholicus, Vieill. N. Dict. d'Hist. N. xxxv. p. 84: Scl. Cat. Am. B. p. 235.

a Brazil (Askew) 1834. —b, —c Brazil (G. Lloyd) 1838. —d Tobago (Sir W. Jardine) 1844. - e Bogota? (Bt at Stevens's) 1844. - $f$ Mexico (Galeotti) 1845. - $g$, - $h$ Guayaquil (Capt. Kellett \& Lt. Wood) 1850. -i Guatemala ( $J$. Constancia) 1851. -j Trinidad (Argent) 1852. 


\section{Tyrannus aurantio-atro-cristatus.}

Tyrannus aurantio-atro-cristatus, d'Orb. \& Lafr. Syn. Av. i. p. 45 : Scl. \& Salv. P. Z. S. 1866, p. 190.

Tyrannus inca, Scl. P. Z. S. 1861, p. 383: Cat. Am. B. p. 237. a Brazil? (Argent) 1852.

Probably from Bolivia.

\section{Milvulus.}

Milvulus, Swainson, Zool. Journ. iii. p. 165 (1827).

\section{Milvulus tyrannus.}

Muscicapa tyrannus, Linn. Syst. Nat. i. p. 325.

Milvulus tyrannus, Cab. Schomb. Guian. iii. p. 699: Strickl. Ann. \& Mag. N. H. viii. p. 373: Scl. Cat. Am. B. p. 237.

a Brazil (N. C. Strickland) 1834. -b Brazil (Johnson) 1837.

\section{FAMILY.-PIPRIDAE.}

PIPRITES.

Piprites, Cabanis, Arch. f. Naturg. xiii. p. 234 (1847).

1554. Piprites chloris.

Pipra chloris, Temm. Pl. Col. 172, f. 2.

Piprites chloris, Scl. Cat. Am. B. p. 246.

a Brazil (G. Lloyd) 1838.

\section{PIPRA.}

Pipra, Linnæus, Syst. Nat. i. p. 338 (1766) (partim).

\section{Pipra filicauda.}

Pipra filicauda, Spix, Av. Bras. ii. p. 6, pl. 8, f. 1, 2: Scl. Cat. Am. B. p. 247.

a Amazons (E. Wilson) 1850.

\section{Pipra aureola.}

Pipra aureola, Linn. Syst. Nat. i. p. 339: Scl. Cat. Am. B. p. 248.

$$
a \text { (J. Stevens) 1829. -b (Cashmore) } 1843 .
$$

A Guiana species. 
155\%. Pipra fasciata.

Pipra fasciata, d'Orb. \& Lafr. Syn. Av. i. p. 38: d'Orb. Voy. Am. Mérid. Ois. p. 295, pl. 30, f. 1: Scl. Cat. Am. B. p. 248.

$a \hat{\delta},-b q$ (Argent) 1852.

Both probably from Bolivia.

\section{Pipra rubricapilla.}

Pipra rubricapilla, Temm. Pl. Col. 54, f. 3: Scl. Cat. Am. B. p. 248.

Pipra erythrocephala, $\beta$, Linn. Syst. Nat. i. p. 339.

a Brazil (Arthur Strickland) 18:2. - b Brazil (Askew) 1837.

\section{Pipra chloromerus.}

Pipra chloromeros, Tsch. Arch. f. Naturg. x. p. 271; et Fauna Per. p. 144: Scl. Cat. Am. B. p. 248.

$a \hat{\jmath},-b q$ Brazil? (Argent) 1852.

\section{Pipra auricapilla.}

Pipra auricapilla, Licht. Verz. Doubl. p. 29 : Scl. Cat. Am. B. p. 249 .

$a$ S. America (Robins) 1834. - b S. America (N.C. Strickland) 1838. — c (Bt at Stevens's) 1845. —d Trinidad (Argent) 1851.

1561. Pipra leucocilla.

Pipra leucocilla, Linn. Syst. Nat. i. p. 340: Scl. Cat. Am. B. p. 249.

a Brazil (Askew) 1834. -b Brazil (Askew) 1834. - c f Brazil (Askew) 1834.

\section{Pipra serena.}

Pipra serena, Linn. Syst. Nat. i. p. 340: Scl. Cat. Am. B. p. 249.

$a$ (Thomas) 1843.

A Guiana species.

1563. Pipra gutturalis.

Pipra gutturalis, Linn. Syst. Nat. i. p. 340: Scl. Cat. Am. B. p. 250.

$a$ Int. of Guiana (G. Bell) 1842. -b (Argent) 1851.

$b$ is a skin of Cayenne make. 


\section{MACHAROPTERUS.}

Machceropterus, Bonaparte, Consp. Vol. Anis. p. 6 (1854). 1564. Machæropterus regulus.

Pipra regulus, Hahn, Ausl. Vög. Liefr. iv. pl. 4, ff. $a, b$. Machceropterus regulus, Scl. Cat. Am. B. p. 250.

Pipra strigilata, Max. Reise n. Bras. i. p. 187; Beitr. iii. p. 430.

$a,-b$ Brazil (Askew) 1834. - c Brazil (Askew) 1837.

1565. Machæropterus striolatus.

Pipra striolata, Bp. P. Z. S. 1837, p. 122.

Machceropterus striolatus, Scl. Cat. Am. B. p. 250.

a (Argent) 1853.

A skin of Bogota make.

\section{HeILICURA。}

Ilicura, Reichenbach, Av. Syst. pl. 1xiii. (1850).

1566. Heilicura militaris.

Pipra militaris, Shaw, Nat. Misc. xx. pl. 849.

Ilicura militaris, Scl. Cat. Am. B. p. 250.

$a$ (T. Holme) 1838. —b, —c Brazil (N. C. Strickland) 1838.

\section{Chiroxiphia.}

Chiroxiphia, Cabanis, Arch. f. Naturg. xiii. p. 235 (1847).

156\%. Chiroxiphia pareola.

Pipra pareola, Linn. Syst. Nat. i. p. 339.

Chiroxiphia pareola, Scl. Cat. Am. B. p. 251.

$a$ Brazil (Askew) 1833. - b Brazil (Mansfield) 1834. -c Brazil (Askew) 1837. —d (N. C. Strickland) 1838.

+ 1568. Chiroxiphia lanceolata.

Pipra lanceolata, Wagl. Isis, 1830, p. 931.

Chiroxiphia lanceolata, Scl. Cat. Am. B. p. 251.

Pipra pareolides, Lafr. \& d'Orb. Rev. Zool. 1838, p. 165.

a Trinidad (Argent) 1851. 
1569. Chiroxiphia linearis.

Pipra linearis, Bp. P. Z. S. 1837, p. 113.

Chiroxiphia linearis, Scl. Cat. Am. B. p. 251.

$a,-b$ Guatemala (J. Constancia) 1851.

1570. Chiroxiphia caudata.

Pipra caudata, Shaw, Nat. Misc. v. pl. 153.

Chiroxiphia caudata, Scl. Cat. Am. B. p. 251.

a Brazil (Mansfield) 1834. $-b,-c,-d$ Brazil (N. C. Strickland) 1848.

Metopia.

Metopia, Swainson, Classif. B. ii. p. 254 (1837).

15\%1. Metopia galeata.

Pipra galeata, Licht. Verz. Doubl. p. 28.

Metopia galeata, Sw. l. s. c.: Scl. Cat. Am. B. p. 252.

$a$ (Brandt) 1845.

A Brazilian species.

\section{Chiromach eris.}

Chiromacharis, Cabanis, Arch. f. Naturg. xiii. p. 235 (1847). 1572. Chiromachæris manacus.

Pipra manacus, Linn. Syst. Nat. i. p. 340.

Chiromachoeris manacus, Scl. Cat. Am. B. p. 252.

a Trinidad (Argent) 1851.

1573. Chiromachæris gutturosa.

Pipra gutturosa, Desm. Tang. pl. 58.

Chiromachceris gutturosx, Scl. Cat. Am. B. p. 252.

a Brazil (Askew) 1834.

Heteropelma.

Heteropelma, Bonaparte, Consp. Vol. Anis. p. 4 (Ateneo Ital. agosto 1854).

\section{Heteropelma turdinum.}

Muscicapa turdina, Max. Beitr. iii. p. 817.

Heteropelma turdinum, Scl. Cat. Am. B. p. 244.

Ptilochloris rufo-olivaceus, Lafr. Rev. Zool. 1838, p. 238.

$a$ (Thomas) 1843. — b Bahia (S. Dutton) 1846.

s. C. 


\section{Heteropelma flavicapillum.}

Heteropelma flavicapillum, Scl. P. Z. S. 1860, p. 466.

$a$ (Arthur Strickland) 1840. -b (Bt at Birmingham) 1849.

These specimens agree with Mr Sclater's types from Brazil.

\section{Heteropelma igniceps.}

Heteropelma igniceps, Scl. P. Z. S. 1871, p. 750.

$a$ Brazil (Argent) 1853.

This specimen agrees fairly with Mr Sclater's type of this Cayenne species of which it is probably a female or young example, as it does not possess the bright-coloured crown of the adult male bird.

\section{7\%. Heteropelma aurifrons.}

Muscicapa aurifrons, Max. Beitr. iii. p. 829.

Heteropelma aurifrons, Scl. Cat. Am. B. p. 245.

$a$ (N. C. Strickland) 1838.

A Brazilian species.

\section{FAMILY.-COTINGIDAE.}

\section{Tityra.}

Tityra, Vieillot, Anal. p. 39 (1816).

\section{Tityra cayana.}

Lanius cayanus, Linn. Syst. Nat. i. p. 137 : Strickl. Ann. \& Mag. N. H. vii. p. 28.

Tityra cayana, Scl. Cat. Am. B. p. 238.

$a$ S. America (Askew) 1834. - $b$ S. America (N. C. Strickland) 1838. $-c$ (Dewgard) 1846.

\section{Tityra brasiliensis.}

Psaris brasiliensis, Sw. Anim. in Menag. p. 286.

Tityra brasiliensis, Scl. Cat. Am. B. p. 238.

$a$ S. America (Askew) 1834. — b Brazil (Askew) 1834. —c, -d (A. Strickland) 1850.

\section{+1580. Tityra semifasciata.}

Pachyrhynchus semifasciatus, Spix, Av. Bras. ii. p. 32, pl. 44, f. 2 .

Tityra semifasciata, Scl. Cat. Am. B. p. 238. 
a f Brazil? (Argent) 1852. - b (Bt at Liverpool) 1853.

$a$ is probably a Bolivian specimen.

1581. Tityra inquisitor.

Lanius inquisitor, Licht. Verz. Doubl. p. 50.

Tityra inquisitor, Scl. Cat. Am. B. p. 239. 1838 .

$a$ (N. C. Strickland) 1838. - b S. America (N. C. Strickland)

\section{Hadrostomus.}

Hadrostomus, Cabanis \& Heine, Mus. Hein. ii. p. 84 (1859).

\section{Hadrostomus atricapillus.}

Tityra atricapilla, Vieill. N. Dict. d'Hist. N. iii. p. 347.

Hadrosiomus atricapillus, Scl. Cat. Am. B. p. 239.

$a,-b$ Brazil (N. C. Strickland) 1838. — Brazil (Argent) 1851.

1583. Hadrostomus niger.

Lanius niger, Gm. Syst. Nat. i. p. 301.

Hadrostomus niger, Scl. Cat. Am. B. p. 239.

Tityra leuconotus, Gosse, B. Jam. p. 187.

$a \hat{\jmath},-b$ q Jamaica (P. H. Gosse) 1848.

1584. Hadrostomus aglaiæ.

Pachyrhynchus aglaice, Lafr. Rev. Zool. 1839, p. 98.

Hadrostomus aglaice, Scl. Cat. Am. B. p. 240.

$a,-b$ Guatemala (Constancia) 1845. —c Guatemala (Constancia) 1851.

\section{PaChyrhamphus.}

Pachyrhamphus, Gray, List Gen. B. p. 31 (1840).

1585. Pachyrhamphus viridis.

Tityra viridis, Vieill. N. Dict. d'Hist. N. iii. p. 348.

Pachyrhamphus viridis, Scl. Cat. Am. B. p. 240.

a Brazil (Johnson) 1837. - b S. America (Thomas) 1842. —c

S. America (Osman) 1846. —d (Mansfield) 1849.

1586. Pachyrhamphus surinamus.

Muscicapa surinama, Linn. Syst. Nat. i. p. 325. 
Pachyrhamphus surinamus, Scl. Cat. Am. B. p. 241. $a$ (Dewgard) 1851.

A skin of Cayenne make.

+ 158\%. Pachyrhamphus cinereus.

Manakin cendré, de Cayenne, D’Aub. Pl. Enl. 687, f. 1, undè, Pipra cinerea, Bodd. Tabl. Pl. Enl. p. 43.

Pachyrhamphus cinereus, Scl. Cat. Am. B. p. 241.

$a$ Cayenne (Dewgard) 1846.

1588. Pachyrhamphus cinereiventris.

Pachyrhamphus cinereiventris, Scl. Cat. Am. B. p. 242.

$a$ (N. C. Strickland) 1838. - b (Bt at Stevens's) 1845.

A species of the north-western parts of South America.

\section{Pachyrhamphus polychropterus.}

Platyrhynchos polychropterus, Vieill. N. Dict. d'Hist. N. xxvii. p. 10.

Pachyrhamphus polychropterus, Scl. Cat. Am. B. p. 242.

a (E. Brown) 1850.

A Brazilian species.

1590. Pachyrhamphus niger.

Pachyrhamphus niger, Spix, Av. Bras. ii. p. 33, pl. 45, f. 1: Scl. Cat. Am. B. p. 241.

a (Argent) 1851.

A species of the northern parts of South America.

+1591. Pachyrhamphus atricapillus.

Lanius atricapillus, Gm. Syst. Nat. i. p. 302.

Pachyrhamphus atricapillus, Scl. Cat. Am. B. p. 242.

$a$ (N. C. Strickland) 1838. - b Brazil (Gardner) 1845. -c (Bt at Birmingham) 1849. -d $q$.

+ 1592. Pachyrhamphus versicolor.

Vireo versicolor, Hartl. Rev. Zool. 1843, p. 289.

Pachyrhamphus versicolor, Scl. Cat. Am. B. p. 243.

a (Argent) 1852.

A Columbian species. 


\section{SUBFAMILY.-LIPAUGIN Æ.}

\section{LATHRIA.}

Lathria, Swainson, Classif. B. ii. p. 255 (1837).

\section{Lathria fuscocinerea.}

Querula fuscocinerea, Lafr. Rev. Zool. 1843, p. 291.

Lipaugus fuscocinereus, Scl. Cat. Am. B. p. 243.

a Bogota? (Stevens's sale) 1845.

\section{Lathria plumbea.}

Muscicapa plumbea, Licht. Verz. Doubl. p. 53.

Lipaugus plumbeus, Scl. Cat. Am. B. p. 243.

a Brazil (N. C. Strickland) 1838.

\section{Lathria cinerea.}

Ampelis cinerea, Vieill. N. Dict. d'Hist. N. viii. p. 162 (nec Latham).

Lipaugus cineraceus, Scl. Cat. Am. B. p. 243.

a Para, August 1848 (S. Stevens) 1849.

Ampelis cinerea Lath. is a synonym of Lanius nengeta, $\mathrm{L} .=$ Toenioptera nengeta, Auctt.

$a$ is doubtless a specimen from Mr Wallace's collection.

\section{Aulia.}

Aulea, Bonaparte, Consp. Vol. Anis. p. 4 (Ateneo Ital. agosto 1854).

1596. Aulia hypopyrrha.

Ampelis hypopyrra, Vieill. N. Dict. d'Hist. N. viii. p. 164. Aulia hypopyrrha, Cab. \& Hein. Mus. Hein. ii. p. 101.

Lipaugus hypopyrrhus, Scl. Cat. Am. B. p. 244 : Scl. \& Salv. Ex. Orn. p. 6.

$a$ (Seaman) 1840. - b Brazil (Thomas) 1842.

The name $A$. hypopyrrha was bestowed by Vieillot upon a Guiana bird and it is questionable if it ought to be applied to the Brazilian species. A comparison of Guianan and Brazilian skins can alone decide this point. 


\section{LIPAUGUS.}

Lipangus, Boie, Isis, 1828, p. 318: Strickl. Ann. \& Mag. N. H. vii. p. 27.

159\%. Lipaugu simplex.

Muscicapa simplex, Licht. Verz. Doubl. p. 53.

Lipaugus simplex, Scl. \& Salv. Ex. Orn. p. 6.

a Brazil (N. C. Strickland) 1838. -b (A. Strickland) 1840.

LANIISOMA.

Laniisoma, Swainson, Faun. Bor. Am. ii. p. 492 (1831): Strickl. Ann. \& Mag. N. H. vii. p. 29.

Ptilochloris, Swainson, Classif. B. ii. p. 250 (1837).

\section{Laniisoma squamatum.}

Muscicapa squamata, Max. Beitr. iii. p. 814.

Ptilochloris squamata, Scl. Cat. Am. B. p. 246.

Lanius arcuatus, Geoffr. St.- Hil. Mag. Zool. 1833, cl. ii. pl. 12. $a$ Brazil (Seaman) 1840. -b (Argent) 1852.

\section{SUBFAMILY.-ATTILIN $\nexists$ E.}

\section{AtTila.}

Attila, Lesson, Traité d'Orn. p. 360 (1831).

\section{Attila brasiliensis.}

Attila brasiliensis, Less. l. s. c.: Scl. Cat. Am. B. p. 195.

$a$ (Seaman) 1840. -b (Thomas) 1843.

A Brazilian species.

1600. Attila cinereus.

Muscicapa cinerea, Gm. Syst. Nat. i. p. 933: Strickl. Ann. \& Mag. N. H. vi. p. 423.

Attila cinereus, Scl. P. Z. S. 1859, p. 41; Cat. Am. B. p. 194. a Brazil (Murray) 1834. - b Brazil (N. C. Strickland) 1838.

\section{Attila spadaceus.}

Muscicapa spadacea, Gm. Syst. Nat. i. p. 937.

Attila spadaceus, Lafr. Rev. Zool. 1848, p. 46: Scl. Cat. Am. B. p. 195.

a (E. Brown) 1850.

A Guiana species. 


\section{Attila thamnophiloides.}

Muscicapa thamnophiloides, Spix, Av. Bras. ii. p. 19, pl. 26, f. 1 .

Attila thamnophiloides, Scl. P. Z. S. 1859 , p. 41; Cat. Am. B. p. 195 .

a Brazil? (Argent) 1852.

\section{SUBFAMILY.-RUPICOLIN Æ.}

Rupicola.

Rupicola, Brisson, Ornith. iv. p. $4: 37$ (1760).

\section{Rupicola crocea.}

Pipra rupicola, Linn. Syst. Nat. i. p. 338.

Rupicola crocea, Bonn. Tabl. Enc. Méth. Orn. p. 266 (1790):

Scl. Cat. Am. B. p. 253.

Rupicola aurantia, Vieill. N. Dict. d'Hist. N. xxix. p. 551. $a$ S. America (Thomas) 1842.

A Guiana species.

\section{Phanicocercus.}

Phœenicircus, Swainson, Faun. Bor. Am. ii. p. 491 (1831).

Phoenicocercus, Cabanis, Arch. f. Naturg. xiii. p. 236 (1847).

1604. Phœnicocercus carnifex.

Ampelis carnifex, Linn. Syst. Nat. i. p. 298.

Phonicocercus carnifex, Scl. Cat. Am. B. p. 253.

Ampelis cuprea, Vieill. N. Dict. d'Hist. N. viii. p. 163. $a$ (N. C. Strickland) 1838.

A Guiana species.

SUBFAMILY.—COTINGIN Æ.

TiJuca.

Tijuca, Lesson, Cent. Zool. p. 30, pl. 6 (1830).

1605. Tijuca nigra.

Tijuca nigra, Less. l. s. c.: Scl. Cat. Am. B. p. 254.

a Chili (N. C. Strickland) 1838.

A Brazilian species. 


\section{Phibalura.}

Phibalura, Vieillot, Anal. p. 31 (1816).

\section{Phibalura flavirostris.}

Phibalura flavirostris, Vieill. l. s. c.: Scl. Cat. Am. B. p. 254. $a,-b$ S. America (N. C. Strickland) 1838.

\section{PiPREOLA.}

Pipreola, Swainson, An, in Menag. p. 357 (1838).

\section{Pipreola riefferi.}

Ampelis riefferi, Boiss. Rev. Zool. 1840, p. 3.

Pipreola riefferi, ${ }_{\text {, Scl. Ibis, }}$ 1878, p. 166.

Pyrrhorhynchus riefferi, Bp. Consp. p. 177. 1845 .

$a$ Bogota (Bt at Stevens's) 1844. - b Bogota (Bt at Stevens's)

\section{Ampelion.}

Ampelion, Cabanis, in Tsch. Faun. Per. p. 137 (1845).

1608. Ampelion cucullatus.

Procnias cucullata, Sw. Zool. Ill. i. pl. 37.

Ampelio cucullatus, Scl. Cat. Am. B. p. 255.

Carpornis cucullata, Bp. Consp. p. 176.

a (Carfrae) 1850.

A Brazilian species.

1609. Ampelion melanocephalus.

Procnias melanocephalus, Sw. Zool. Ill. i. pl. 25.

Ampelio melanocephalus, Scl. Cat. Am. B. p. 255.

$a$ Brazil (Johnson) 1837. -b (N. C. Strickland) 1838:

\section{HeLIOCHERA.}

Heliochera, de Filippi, Cat. Mus. Mediol. Av. p. 31 (1847).

\section{Heliochera rubrocristata.}

Ampelis rubrocristatus, d'Orb. \& Lafr. Syn. Av. i. p. 39. Heliochera rubrocristata, Scl. Cat. Am. B. p. 255.

a Bogota (Williams) 1848. —b Bogota (Dewgard) 1849. 


\section{Cotinga.}

Cotinga, Brisson, Ornith. ii. p. 339 (1760).

\section{Cotinga cincta.}

Ampelis cotinga, Linn. Syst. Nat. i. p. 298.

Ampelis superbus, Shaw, Nat. Misc. xix. pl. 821.

Ampelis cincta, Kuhl, Buff. \& D’Aub. Fig. Av. Nom. Syst. p. 4: Strickl. Ann. \& Mag. N. H. viii. p. 373.

Cotinga cincta, Scl. Cat. Am. B. p. 256.

$a$ S. America (Thomas). $-b$ (N. C. Strickland). $-c$ (Argent) 1851.

The name Ampelis cincta is usually attributed to Boddaert who, however, proposed no such title. The next in priority is $A$. superbus of Shaw, but as this name was suggested solely on account of certain orange-coloured spots produced by the application of heat to the purple under-plumage of a bird, which that author a few pages before rightly considered to be $A$. cotinga Linn., I think we may fairly be dispensed from the necessity of calling this species Cotinga superba. Kuhl's title is applied to Le Cotinga du Brésil of D'Aubenton (Pl. Enl. 188).

\section{Cotinga cærulea.}

Le Cotinga, D'Aub. Pl. Enl. 186, undè, Ampelis carulea, Vieill. N. Dict. d'Hist. N. viii. p. 161.

Cotinga ccerulea, Scl. Cat. Am. B. p. 256.

$a$ (Argent) 1852.

A skin of Cayenne make.

\section{Cotinga cayana.}

Ampelis cayana, Linn. Syst. Nat. i. p. 298.

Cotinga cayana, Scl. Cat. Am. B. p. 256.

a (Argent) 1852.

A Guiana species found also in the Upper Amazon and Colombia.

\section{Xipholena.}

Xipholena, Gloger, Handb. p. 320 (1842).

\section{Xipholena pompadora.}

Ampelis pompadora, Linn. Syst. Nat. i. p. 298.

Xipholena pompadora, Scl. Cat. Am. B. p. 256.

$a$ (Bt at Stevens's) 1845. - b (Dewgard) 1846.

A Guiana species. 
1615. Xipholena atropurpurea.

Ampelis atropurpurea, Max. Reise n. Bras. i. pp. 262, 275.

Xipholena atropurpurea, Scl. Cat. Am. B. p. 257: Scl. \& Salv. Ex. Orn. p. 9, pl. 5.

a Brazil (Johnson) 1837. $-b,-c$ Guiana (N. C. Strickland) 1838.

A purely Brazilian species.

\section{IODOPLEURA.}

Iodopleura, Lesson, Rev. Zool. 1839, p. 45.

1616. Iodopleura pipra.

Pardalotus pipra, Less. Cent. Zool. p. 81, pl. 26.

Iodopleura pipra, Scl. Cat. Am. B. p. 246.

$a$ (N. C. Strickland) 1838. -b Brazil (Fenton) 1850.

161\%. Iodopleura fusca.

Ampelis fusca, Vieill. N. Dict. d'Hist. N. viii. p. 162.

Pipra laplacii, Gerv. Mag. Zool. 1836, cl. ii. pl. 68.

a (Bt at Liverpool) 1853.

A Guiana species.

\section{Calyptura.}

Calyptura, Swainson, Faun. Bor. Am. ii. p. 491 (1831).

\section{Calyptura cristata.}

Pardalotus cristatus, Vieill. N. Dict. d'Hist. N. xxiv. p. 528. Calyptura cristata, Scl. Cat. Am. B. p. 247.

a Brazil (N. C. Strickland) 1838.

\section{SUBFAMILY.-GYMNODERIN $A$.}

\section{Querula.}

Querula, Vieillot, Anal. p. 37 (1816): Strickl. Ann. \& Mag. N. H. vii. p. 27.

\section{Querula cruenta.}

Gobe-Mouche noir à gorge pourpre, de Cayenne, D'Aub. Pl. Enl. 381, undè,

Muscicapa cruenta, Bodd. Tab. Pl. Enl. p. 23.

Querula cruenta, Scl. Cat. Am. B. p. 257. 
$a$ S. America (Mansfield) 1839. —b Demarara (Cashmore) 1843.

\section{Chasmorhynchus.}

Chasmorhynchos, Temminck, Pl. Col. Livr. 9 (1823): Strickl. Ann. \& Mag. N. H. viii. p. 373.

\section{Chasmorhynchus nudicollis.}

Ampelis nudicollis, Vieill. N. Dict. d'Hist. N. viii. p. 164.

Chasmorhynchus nudicollis, Scl. Cat. Am. B. p. 258: Salv. Ibis, 1865 , p. 91 .

$a,-b$ Brazil (N. C. Strickland) 1838.

\section{Chasmorhynchus variegatus.}

Ampelis variegatus, Gm. Syst. Nat. i. p. 841.

Chasmorhynchus variegatus, Scl. Cat. Am. B. p. 257: Salv. Ibis, 1865, p. 91 .

a Brazil (N. C. Strickland) 1838. - b S. America (Thomas) 1842. - c Trinidad (Col. J. Taylor).

The true habitat of this species is Guiana, Venezuela and Trinidad.

\section{Grmnocephalus.}

Gymnocephalus, Geoffroy Saint-Hilaire, Ann. du Mus. xiii. p. 237 (1809).

\section{Gymnocephalus calvus.}

Corvus calvus, Gm. Syst. Nat. i. p. 372.

Gymnocephalus calvus, Scl. Cat. Am. B. p. 258.

$a$ S. America (Williams) 1846.

\section{Pyroderus.}

Coracina, Temminck, Pl. Col. Livr. 7 (1823) (nec Vieillot). Pyroderus, G. R. Gray, List Gen. B. p. 38 (1840): Strickl. Ann. \& Mag. N. H. vii. p. 27.

\section{Pyroderus scutatus.}

Coracias scutata, Shaw, Mus. Lev. p. 199.

Pyroderus scutatus, Scl. Cat. Am. B. p. 259.

a Brazil (N. C. Strickland) 1838. 


\section{FAMILY.-PHYTOTOMIDA.}

\section{Рнутотома ${ }^{1}$.}

Phytotoma, Molina, Sagg. sulla St. Nat. Chili, p. 254 (1782). 1624. Phytotoma rara.

Phytotoma rara, Mol. l. s. c.: Scl. Cat. Am. B. p. 260. $a$ V.alparaiso (Capt. Brown) 1842.

\section{SUBORDER III.-TRACHEOPHONA. \\ FAMILY.-DENDROCOLAPTIDA. \\ SUBFAMILY._FURNARIIN Æ. \\ Geobates.}

Geobates, Swainson, An. in Menag. p. 322 (1838).

1625. Geobates pœcilopterus.

Anthus poecilopterus, Max. Beitr. iii. p. 633.

Geobates pocilopterus, Scl. P. Z. S. 1866, p. 204, pl. 21.

Geobates brevicauda, Sw. l. s. c.

a (Arthur Strickland) 1840.

A Brazilian species.

Geositta.

Geositta, Swainson, Classif. B. ii. p. 317 (1837).

1626. Geositta cunicularia.

Alauda cunicularia, Vieill. N. Dict. d'Hist. N. i. p. 369.

Geositta cunicularia, Scl. Cat. Am. B. p. 146.

$a$ (N. C. Strickland) 1838. -b Chili (Capt. Brown) 1842.

Furnarius.

Furnarius, Vieillot, Anal. p. 47 (1816).

1627. Furnarius figulus.

Turdus figulus, Licht. Verz. Doubl. p. 40.

Furnarius figulus, Scl. Cat. Am. B. p. 147.

a (N. C. Strickland) 1838.

\section{UPUCERTHIA.}

Upucerthia, I. Geoffroy Saint-Hilaire, Nouv. Ann. du Mus. i. p. 393 (1832).

1 Considered by Strickland (Ann. \& Mag. N. H. vii. p. 33, \& Contr. Orn. 1850$, p. 49$)$, to belong to the Subfamily Tanagrinæ. 
1628. Upucerthia dumetoria.

Upucerthia dumetoria, Geoffr. \& d'Orb. Ann. du Mus. i. p. 394: Scl. Cat. Am. B. p. 147.

a S. America (N. C. Strickland) 1838.

\section{Cinclodes.}

Cinclodes, G. R. Gray, List Gen. B. p. 16 (1840).

\section{Cinclodes nigrifumosus.}

Upucerthia nigrifumosa, d'Orb. \& Lafr. Syn. Av. ii. p. 23: d'Orb. Voy. Am. Mérid. Ois. p. 372, pl. 57, f. 2.

Cinclodes nigrifumosus, Scl. Cat. Am. B. p. 148.

a Chili (Capt. Brown) 1842.

\section{Cinclodes patagonicus.}

Motacilla patagonica, Gm. Syst. Nat. i. p. 957.

Cinclodes patagonicus, Scl. Cat. Am. B. p. 148.

a (N. C. Strickland) 1838.

A Chilian species.

\section{LOCHMias.}

Lochmias, Swainson, Zool. Journ. iii. p. 355 (1827).

1631. Iochmias nematura.

Myiothera nematura, Licht. Verz. Doubl. p. 43.

Lochmias nematura, Scl. Cat. Am. B. p. 149.

Lochmias squamatula, Sw. Orn. Draw. pl. 33.

a (N. C. Strickland) 1837.

A Brazilian species.

\section{Sclerurus.}

Sclerurus, Swainson, Zool. Journ. iii. p. 356 (1827).

1632. Sclerurus umbretta.

Myiothera umbretta, Licht. Verz. Doubl. p. 43.

Sclerurus umbretta, Scl. \& Salv. P. Z. S. 1867, p. 574.

$a$ (Thomas) 1843. — b Brazil (Argent) 1851.

1633. Sclerurus caudacutus.

Thamnophilus caudacutus, Vieill. N. Dict. d'Hist. N. iii. p. 310. 
Sclerurus caudacutus, Scl. \& Salv. P. Z. S. 1867, p. 574.

$a$ (Mather) 1840.

A Guiana species.

\section{Oxyurus.}

Oxyurus, Swainson, Zool. Journ. iii. p. 354 (1827).

\section{Oxyurus spinicauda.}

Motacilla spinicauda, Gm. Syst. Nat. i. p. 978.

Oxyurus spinicauda, Scl. Cat. Am. B. p. 150.

Synallaxis tupinieri, Less. Voy. Coq. Zool. i. p. 665. Atlas, pl. 29, f. 1 .

$a$ Chili (Capt. Brown) 1842.

\section{Phleocryptes.}

Phleocryptes, Cabanis \& Hein. Mus. Hein. ii. p. 26 (1859).

\section{Phleocryptes melanops.}

Sylvia melanops, Vieill. N. Dict. d'Hist. N. xi. p. 232.

Phleocryptes melanops, Scl. Cat. Am. B. p. 150.

a (N. C. Strickland) 1838.

A Chilian species.

\section{LEPTASTHENURA.}

Leptasthenura, Reichenbach, Scansoriæ p. 160 (1853).

\section{Leptasthenura ægithaloides.}

Synallaxis agithaloides, Kittl. Mém. prés. à l'Ac. Pétersb., i. p. 187, pl. 7 (1831).

Leptasthenura cegithaloides, Scl. Cat. Am. B. p. 150.

a. Juan Fernandez (Tucker) 1838.

This species has not been included in recent collections made in Juan Fernandez. (Cf. Sclater, Ibis, 1871, p. 178; \& Salvin, Ibis, 1875, p. 370.)

SyNallaxis.

Synallaxis, Vieillot, N. Dict. d'Hist. N. xxxii. p. 309 (1819). 163\%. Synallaxis frontalis.

Synallaxis frontalis, Pelz. Sitz. Ak. Wien, xxxiv. p. 117: Scl. P. Z. S. 1874, p. 8.

a Brazil (N. C. Strickland) 1838. -b Bogota? (Gardner) 1844. $-c$ (Dewgard) 1851. 


\section{Synallaxis spixi.}

Synallaxis spixi, Scl. P. Z. S. 1856, p. 98; P. Z. S. 1874, p. 9. a Brazil (N. C. Strickland) 1838.

\section{Synallaxis hypospodia.}

Synallaxis hypospodia, Scl. P. Z. S. 1874, p. 10.

a (Gardner) 1846.

A Brazilian species.

\section{Synallaxis cinnamomea.}

Certhia cinnamomea, Gm. Syst. Nat. i. p. 480.

Synallaxis cinnamomea, Scl. P. Z. S. 1874, p. 13.

Synallaxis ruficauda, Vieill. N. Dict. d'Hist. N. xxxii. p. 310. a Brazil (N. C. Strickland) 1838. - b Brazil (Argent) 1851.

1641. Synallaxis terrestris.

Synallaxis terrestris, Jard. Ann. \& Mag. N. H. xix. p. 80: Scl. P. Z. S. 1874, p. 16.

a Bogota? (Bt at Stevens's) 1844.

A species of Tobago and Venezuela.

\section{Synallaxis erythrothorax.}

Synallaxis erythrothorax, Scl. P. Z. S. 1855, p. 75, pl. 86; P. Z. S. 1874 , p. 17.

$a$ Guatemala (J. Constancia) 1851.

1643. Synallaxis phryganophila.

Sylvia phryganophila, Vieill. N. Dict. d'Hist. N. xi. p. 207.

Synallaxis phryganophila, Scl. P. Z. S. 1874, p. 17.

a Brazil (N. C. Strickland) 1838.

\section{Synallaxis pallida.}

Synallaxis pallidus, Max. Beitr. iii. p. 690 : Scl. P. Z. S. 1874, p. 18.

a Brazil (N. C. Strickland) 1838.

1645. Synallaxis humicola.

Synallaxis humicola, Kittl. Mém. prés. à l'Ac. Pétersb. i. p. 185, pl. 6 (1831): Scl. P. Z. S. 1874, p. 22.

a Chili (Capt. Brown) 1842. 


\section{Phacelodomus.}

Phacellodomus, Reichenbach, Scansoriæ p. 169 (1853).

\section{Phacelodomus frontalis.}

Sphenura frontalis, Licht. Verz. Doubl. p. 42.

Phacellodomus frontalis, Scl. Cat. Am. B. p. 154.

Anabates rufifrons, Spix, Av. Bras. i. p. 84, pl. 85, f. 1.

$a$ Bahia (S. Dutton) 1846.

\section{Thripophaga.}

Thripophaga, Cabanis, Arch. f. Naturg. xiii. p. 338 (1847).

\section{Thripophaga erythrophthalma.}

Anabates erythrophthalma, Max. Beitr. iii. p. 1175.

Thripophaga erythrophthalma, Scl. Cat. Am. B. p. 156.

a (Thomas) 1840.

A Brazilian species.

\section{Thripophaga striolata.}

Sphenura striolata, Licht. Verz. Doubl. p. 42.

Thripophaga striolata, Scl. Cat. Am. B. p. 156. a (Bt at Birmingham) 1850.

A Brazilian species.

\section{Pseudocolaptes.}

Pseudocolaptes, Reichenbach, Scansoriæ p. 209 (1853).

\section{Pseudocolaptes boissoneauti.}

Anabates boissoneauti, Lafr. Rev. Zool. 1840, p. 104.

Pseudocolaptes boissoneauti, Scl. Cat. Am. B. p. 156.

Anabates auritus, Tsch. Arch. f. Naturg.

$a$ Bogota (J. Gould) 1844. - b Bogota (Bt at Stevens's) 1844.

—c (Dewgard) 1849. —d (Argent) 1852.

\section{Homorus.}

Homorus, Reichenbach, Scansoriæ p. 172 (1853).

\section{Homorus cristatus.}

Anabates cristatus, Spix, Av. Bras. i. p. 83, pl. 84. 
Homorus cristatus, Scl. Cat. Am. B. p. 157. $a$ Interior of Bolivia (T. C. Eyton) 1850.

\section{Automolus.}

Automolus, Reichenbach, Scansores, p. 174 (1853).

\section{Automolus ferruginolentus.}

Anabates ferruginolentus, Max. Beitr. iii. p. 1166. Automolus ferruginolentus, Scl. Cat. Am. B. p. 157. Anabates leucophrus, Jard. \& Selb. Ill. Orn. ii. pl. 93. $a$ (N. C. Strickland) 1838.

A Brazilian species.

\section{Automolus rubiginosus.}

Anabates rubiginosus, Scl. P. Z. S. 1856, p. 288. Automolus rubiginosus, Scl. Cat. Am. B. p. 157. a Guatemala (J. Constancia) 1851.

1653. Automolus sulphurascens.

Sphenura sulphurascens, Licht. Verz. Doubl. p. 41. Automolus sulphurascens, Scl. Cat. Am. B. p. 157. Philydor albogularis, Spix, Av. Bras. i. p. 74, pl. 74, f. 1. $a$ (N. C. Strickland) 1834. -6 (Gardner) 1845.

\section{Automolus striaticeps.}

Automolus striaticeps, Scl. \& Salv. P. Z. S. 1875, p. 37. a Bogota (Bt at Stevens's) 1845.

\section{PhILydor.}

Philydor, Spix, Av. Bras. i. p. 73 (1824).

\section{Philydor rufus.}

Dendrocopus rufus, Vieill. N. Dict. d'Hist. N. xxvi. p. 119. Philydor rufus, Scl. Cat. Am. B. p. 155. a (N. C. Strickland) 1838.

A Brazilian species.

S. C. 
1656. Philydor amaurotis.

Anabates amaurotis, Temm. Pl. Col. 238, f. 2.

Philydor amaurotis, Scl. Cat. Am. B. p. 155.

$a$ (Mansfield) 1834.

A Brazilian species.

165\%. Philydor columbianus.

Philydor columbianus, Cab. \& Hein. Mus. Hein. ii. p. 29.

a Brazil (N. C. Strickland) 1838.

Originally described from Venezuelan specimens.

1658. Philydor montanus.

Anabates montanus, Tsch. Faun. Per. Av. p. 241, pl. 20, f. 1. Philydor montanus, Scl. P. Z. S. 1871, p. 86.

a Bogota (Bt at Stevens's) 1845.

1659. Philydor superciliaris.

Sphenura superciliaris, Licht. Verz. Doubl. p. 41.

Philydor superciliaris, Scl. Cat. Am. B. p. 154.

Anabates atricapillus, Max. Beitr. iii. p. 1187.

$a$ Brazil (Arthur Strickland) 1834. -b S. America (Mather) 1840. $-c$ Brazil (Argent) 1851.

\section{Anabatoides.}

Anabatoides, Burmeister, Syst. Ueb. iii. p. 23 (1856).

1660. Anabatoides fuscus.

Sitta fusca, Vieill. Anal. p. 68.

Anabatoides fuscus, Scl. Cat. Am. B. p. 158.

Sphenura albicollis, Licht. Verz. Doubl. p. 41.

$a$ (N. C. Strickland) 1838.

A Brazilian species.

\section{ANabazenops.}

Anabazenops, Lafresnaye, Dict. Univ. d'Hist. N. i. p. 411 (1841).

\section{Anabazenops rufosuperciliatus.}

Xenops rufosuperciliatus, Lafr. Mag. Zool. 1832, Cl. ii. pl. 7. Anabazenops rufosuperciliatus, Scl. Cat. Am. B. p. 158.

$a$ (N. C. Strickland) 1838.

A Brazilian species. 
1662. Anabazenops subalaris.

Anabates subalaris, Scl. P. Z. S. 1859, p. 141.

Anabazenops subalaris, Scl. Cat. Am. B. p. 158.

a Bogota (Bt at Stevens's) 1845.

\section{Xenops.}

Xenops, Illiger, Prod. p. 213 (1811).

\section{Xenops genibarbis.}

Xenops genibarbis, Ill. l. s. c.: Strickl. Ann. \& Mag. N. H. vi. p. 420: Scl. Cat. Am. B. p. 159.

a Brazil (Askew) 1834. -b (N. C. Strickland) 1838.

\section{Xenops rutilus.}

Xenops rutilus, Licht. Verz. Doubl. p. 17: Strickl. Ann. \& Mag. N. H. vi. p. 420: Scl. Cat. Am. B. p. 159.

a Brazil (N. C. Strickland) 1838.

\section{Sittasomus.}

Sittasomus, Swainson, Zool. Journ. iii. p. 355 (1827). 1665. Sittasomus erithacus.

Dendrocolaptes erithacus, Licht. Abh. k. Ak. Berl. 1820, p. 266, pl. 1, f. 2.

Sittasomus erithacus, Strickl. Ann. \& Mag. N. H. viii. p. 371: Scl. Cat. Am. B. p. 160.

$a$ (N. C. Strickland) 1838. -b (N. C. Strickland) 1838.

\section{Sittasomus olivaceus.}

Sittasomus olivaceus, Max. Beitr. iii. p. 1146 : Scl. \& Salv. P. Z. S. 1868, p. 630.

Sittasomus griseus, Jard. Ann. \& Mag. N. H. xix. p. 82. a Bogota (Bt at Stevens's) 1844.

\section{Margarornis.}

Margarornis, Reichenbach, Scansores, i. p. 179 (1853). 166\%. Margarornis perlata.

Sittasomus perlatus, Less. Echo du Monde Savant, 1844, p. 275 . 
Margarornis perlata, Salv. Ibis, 1874, p. 323.

a Bogota (Bt at Stevens's) 1844.

GLYPHORHYNCHUS.

Glyphorynchus, Maximilian Prinz zu Wied, Beitr. iii. p. 1149 (1830).

1668. Glyphorhynchus cuneatus.

Dendrocolaptes cuneatus, Licht. Abh. d. Ak. d. Wiss. 1818, p. 204, pl. 3, f. 2 .

Glyphorhynchus cuneatus, Strickl. P. Z. S. 1841, p. 28 : Scl. Cat. Am. B. p. 161.

a Brazil (Arthur Strickland) 1840.

Dendrocincla.

Dendrocincla, G. R. Gray, List Gen. B. p. 18 (1840).

1669. Dendrocincla atrirostris.

Dendrocolaptes atrirostris, d'Orb. \& Lafr. Syn. Av. ii. p. 12.

Dendrocincla atrirostris, Scl. Cat. Am. B. p. 162.

$a$ Columbia (Gardner) 1845.

\section{6\%0. Dendrocincla turdina.}

Dendrocolaptes turdinus, Licht. Abh. d. Ak. d. Wiss. 1818, p. 204, pl. 2, f. 1.

Dendrocincla turdina, Scl. \& Salv. P. Z. S. 1868, p. 54.

a Brazil (N. C. Strickland) 1838. - b Brazil (Sir W. Jardine) $184 \check{.}$

\section{Dendrocolaptes.}

Dendrocolaptes, Hermann, Obs. Zool. p. 135 (1804).

1671. Dendrocolaptes picumnus.

Dendrocolaptes picumnus, Licht. Abh. d. Ak. d. Wiss. 1818, p. 202 : Scl. Cat. Am. B. p. 162.

Dendrocolaptes platyrostris, Spix, Av. Bras. i. p. 87, pl. 89.

$a,-b$ Brazil (N. C. Strickland) 1838. - c Bogota (Bt at Stevens's) 1844.

16r2. Dendrocolaptes certhia.

Le Picucule, de Cayenne, D’Aub. Pl. Enl. 621, undè, 
Picus certhia, Bodd. Tabl. Pl. Enl. p. 38.

Dendrocolaptes certhia, Scl. \& Salv. P. Z. S. 1868, p. 56.

Gracula cayennensis, Gm. Syst. Nat. i. p. 3:99: Strickl. Ann. \& Mag. N. H. vi. p. 420.

$a($ W. Kirtland $) \mathbf{1 8 4 3}$.

A Guiana species.

\section{NASICA.}

Nasica, Lesson, Traité d'Orn. p. 311 (1831).

1673. Nasica longirostris.

Dendrocolaptes longirostris, Licht. Abh. d. Ak. d. Wiss. 1818, p. 200.

Nasica longirostris, Scl. Cat. Am. B. p. 163.

a Brazil (T. C. Eyton) 1850.

A Guiana and Amazonian species.

\section{XiPHOCOLAPTES.}

Xiphocolaptes, Lesson, Rev. Zool. 1840, p. 269.

\section{Xiphocolaptes albicollis.}

Dendrocopus albicollis, Vieill. N. Dict. d'Hist. N. xxvi. p. 117.

Xiphocolaptes albicollis, Scl. Cat. Am. B. p. 163.

$a$ (N. C. Strickland) 1838. -b (Williams) 1848. -c (Mansfield) 1849.

A species of South-East Brazil.

1675. Xiphocolaptes promeropirhynchus.

Dendrocolaptes promeropirhynchus, Less. Rev. Zool. 1840, p. 270.

Xiphocolaptes promeropirhynchus, Scl. Cat. Am. B. p. 163.

$a$ Bogota (Bt at Stevens's) 1845. - b S. America.

\section{Xiphocolaptes emigrans.}

Xiphocolaptes emigrans, Scl. \& Salv. Ibis, 1859, p. 118.

$a$ Guatemala (J. Constancia) 1848.

Dendrornis.

Dendrornis, Eyton, Contr. Orn. 1852, p. 23. 
1677. Dendrornis guttatus.

Dendrocolaptes guttatus, Licht. Abh. d. Ak. d. Wiss. 1820, p. 264.

Dendrornis guttatus, Scl. Cat. Am. B. p. 164.

$a$ (N. C. Strickland) 1838. - b (Thomas) 1843. — c (Thomas).

A Brazilian species.

1678. Dendrornis triangularis.

Dendrocolaptes triangularis, Lafr. Rev. Zool. 1842, p. 134.

Dendrornis triangularis, Scl. Cat. Am. B. p. 165.

a Bogota (J. Gould) 1844.

\section{Dendrornis susurrans.}

Dendrocolaptes susurrans, Jard. Ann. \& Mag. N. H. xix. p. 81.

Dendrornis susurrans, Scl. Cat. Am. B. p. 165.

a Tobago (Kirk) 1844. - b Bogota? (Gardner) 1844.

$a$ is one of the typical specimens of this species.

\section{Dendrornis multiguttatus.}

Dendrocolaptes multiguttatus, Lafr. Rev. Zool. 1850, p. 417.

Dendrornis multiguttatus, Scl. Cat. Am. B. p. 164.

$a$ (Bt at Birmingham) 1850.

A Guiana species.

\section{Dendroplex.}

Dendroplex, Swainson, Zool. Journ. iii. p. 354 (1827).

\section{Dendroplex picus.}

Le Talapiot, de Cayenne, D’Aub. Pl. Enl. 605, undè,

Oriolus picus, Gm. Syst. Nat. i. p. 384.

Dendroplex picus, Scl. Cat. Am. B. p. 165: Scl. \& Salv. P. Z. S. 1867, p. 575 .

a Brazil (N. C. Striclland) 1838. - b Cayenne (P. L. Sclater) 1846. - C Mexiana, Para, November, 1848 (A. R. Wallace) 1849. 


\section{Picolaptes.}

Picolaptes, Lesson, Traité d'Orn. p. 313 (1831).

\section{Picolaptes lacrymiger.}

Dendrocolaptes lacrymiger, Des Murs, Icon. Orn. pl. 70.

Picolaptes lacrymiger, Scl. Cat. Am. B. p. 166.

a Bogota (Bt at Stevens's) 1844. -b Bogota (J. Gould) 1844.

1683. Picolaptes compressus.

Thripobrotus compressus, Cab. J. f. Orn. 1861, p. 243.

a Guatemala ( $J$. Constancia) 1851.

\section{Picolaptes tenuirostris.}

Dendrocolaptes tenuirostris, Licht. Abh. d. Ak. d. Wiss. 1818, p. 202.

Picolaptes tenuirostris, Scl. Cat. Am. B. p. 167.

$a$ (N. C. Strickland) 1838. -b (Arthur Strickland) 1840.

A species of South-East Brazil.

1685. Picolaptes squamatus.

Dendrocolaptes squamatus, Licht. Abh. d. Ak. d. Wiss. 1820, p. 265 , pl. 2 , f. 1.

Thripobrotus squamatus, Cab. \& Hein. Mus, Hein. ii. p. 37.

Dendrocolaptes wagleri, Spix, Av. Bras. i. p. 88, pl. 90, f. 2.

a Brazil (Askew) 1837. - b Brazil (Brandt) 1845.

\section{XIPHORHYNCHUS.}

Xiphorhynchus, Swainson, Zool. Journ. iii. p. 354 (1827).

\section{Xiphorhynchus trochilirostris.}

Dendrocolaptes trochilirostris, Licht. Abh. d. Ak. d. Wiss. 1818, p. 207, pl. 3.

Xiphorhynchus trochilirostris, Strickl. Ann. \& Mag. N. H. viii. p. 371 : Scl. Cat. Am. B. p. 167.

a Bahia, S. America (Thomas) 1843. -b Bolivia (Argent) 1852. 
FAMILY.-FORMICARIID E.

SUBFAMILY.-THAMNOPHILIN $Æ$.

Batara.

Batara, Lesson, Traité d'Orn. i. p. 347 (1831).

168\%. Batara cinerea.

Thamnophilus cinereus, Vieill. N. Dict. d'Hist. N. xxxv. p. 200.

Batara cinerea, Scl. P. Z. S. 1858, p. 207 ; Cat. Am. B. p. 170.

Lanius undulatus, Mikan, Del. Faun. Bras. pl. 2.

$a$ f, - $b$ Brazil (N. C. Strickland) 1838.

\section{Thamnophilus.}

Thamnophilus, Vieillot, Anal. p. 40 (1816).

1688. Thamnophilus leachi.

Thamnophilus leachi, Such, Zool. Journ. i. p. 558: Scl. P. Z.S. 1858, p. 207.

a Brazil (N. C. Strickland) 1838. - b (N. C. Strickland) 1838.

1689. Thamnophilus severus.

Lanius severus, Licht. Verz. Doubl. p. 45.

Thamnophilus severus, Scl. P. Z. S. 1858, p. 208.

Thamnophilus niger, Such, Zool. Journ. i. p. 559.

Thamnophilus swainsoni, Such, l. c. p. 556.

$a,-b$ Brazil (N. C. Strickland) 1838. — Brazil (G. Lloyd) 1838.

1690. Thamnophilus guttatus.

Thamnophilus guttatus, Vieill. N. Dict. d'Hist. N. iii. p. 315: Scl. P. Z. S. 1858, p. 208.

a Brazil (N. C. Strickland) 1838. 


\section{Thamnophilus major.}

Thamnophilus major, Vieill. N. Dict. d'Hist. N. iii. p. 313: Scl. P. Z. S. 1858, p. 209.

$a,-b$ Brazil (Askew) 1834. - c (N. C. Strickland) 1838. —d Trinidad (Argent) 1851.

\section{Thamnophilus cinereoniger.}

Thamnophilus cinereo-niger, Pelz. Orn. Bras. pp. 76, 143.

a Para (S. Stevens) 1849.

Evidently one of Mr Wallace's specimens but without his label. The species, however, is not included in the list of Mr Wallace's Amazon Collection (P. Z. S. 1867).

\section{Thamnophilus ambiguus.}

Thamnophilus ambiguus, Sw. Zool. Journ. ii. p. 91: Scl. P.Z. S. 1858, p. 213.

a Brazil (Arthur Strickland) 1834. —b Brazil (Askew) 1837. $-c,-d,-e$ Brazil (N. C. Strickland) 1838. $-f$ Brazil (G. Lloyd) 1838. - $g,-h$ Brazil? (Argent) 1852.

\section{Thamnophilus cærulescens.}

Thamnophilus ccerulescens, Vieill. N. Dict. d'Hist. N. iii. p. 311: Scl. Cat. Am. B. p. 174.

$a$ Brazil (N. C. Strickland) 1838.

\section{Thamnophilus atricapillus.}

Lanius atricapillus, Gm. Syst. Nat. i. p. 302.

Thamnophilus atricapillus, Scl. P. Z. S. 1858, p. 215; Cat. Am. B. p. 174.

$a$ (Bt at Stevens's) 1845. —b (Dewgard) 1849. —c, —d Trinidad (Argent) 1851.

\section{Thamnophilus doliatus.}

Lanius doliatus, Linn. Syst. Nat. i. p. 136.

Thamnophilus doliatus, Scl. P. Z. S. 1858, p. 217.

$a,-b$ (N. C. Strickland) 1838. — $\mathrm{S}$. America (N. C. Strickland) 1838. - d, - e Guatemala (J. Constancia) 1845. $\rightarrow f$ Guatemala (J. Constancia) 1851. -g Trinidad (Argent) 1851. 
169\%. Thamnophilus multistriatus.

Thamnophilus multistriatus, Lafr. Rev. Zool. 1844, p. 82 : Scl. P. Z. S. 1858, p. 219.

$a$ (Bt at Stevens's) 1845. - b Bogota (Bt at Stevens's) 1845. 1698. Thamnophilus palliatus.

Thamnophilus palliatus, Licht. Verz. Doubl. p. 46: Scl. P.Z. S. 1858, p. 219.

a Brazil (Askew) 1834. —b Brazil (Askew) 1837. —c (Arthur Strickland) 1850.

1699. Thamnophilus torquatus.

Thamnophilus torquatus, Sw. Zool. Journ. ii. p. 89: Scl. P. Z. S. 1858, p. 220.

$a$ (N. C. Strickland). - b Brazil (N. C. Strickland) 1838. -c S. America (Mather) 1840. —d, -e Brazil (Argent) 1851.

\section{Dysithamnus.}

Dysithamnus, Cabanis, Arch. f. Naturg. xiii. p. 223 (1847). 1700. Dysithamnus guttulatus.

Lanius guttulatus, Licht. Verz. Doubl. p. 46.

Dysithamnus guttulatus, Scl. P. Z. S. 1858, p. 221.

Myothera stictothorax, Temm. Pl. Col. 179, f. 1, 2.

a Brazil (Burl) 1834. —b (Arthur Strickland) 1850.

\section{1\%01. Dysithamnus mentalis.}

Myiothera mentalis, Temm. Pl. Col. 179, f. 3.

Dysithamnus mentalis, Scl. P. Z. S. 1858, p. 221.

a (N. C. Strickland) 1838. -b Brazil? (Argent) 1852.

$b$ is probably from Bolivia.

1702. Dysithamnus semicinereus.

Dysithamnus semicinereus, Scl. P. Z. S. 1855, p. 90, pl. 97; 1858, p. 222.

a Bogota (Bt at Stevens's) 1845. - b (Bt at Stevens's) 1845. 1703. Dysithamnus xanthopterus.

Dysithamnus xanthopterus, Burm. Syst. Ueb. iii. p. 81: Scl. P. Z. S. 1858, p. 222.

a Brazil (Argent) 1853. 


\section{Thamnomanes.}

Thamnomanes, Cabanis, Arch. f. Naturg. xiii. p. 230 (1847). 1704. Thamnomanes cæsius.

Lanius ccesius, Licht. Verz. Doubl. p. 46.

Thamnomanes ccesius, Scl. P. Z. S. 1858, p. 223.

$a$ (N. C. Strickland) 1838. -b (Thomas) 1842.

A species of South-eastern Brazil and Bolivia.

1705. Thamnomanes glaucus.

Thamnomanes glaucus, Cab. Arch. f. Naturg. xiii. p. 230 : Scl. P. Z. S. 1858, p. 223.

$a$ (Bt at Stevens's) 1845.

A Guiana species.

\section{SUB-FAMILY.-FORMICIVORIN $Æ$.}

\section{HERPSILOCHMUS.}

Herpsilochmus, Cabanis, Arch. f. Naturg. xiii. p. 224 (1847).

\section{Herpsilochmus pileatus.}

Myiothera pileata, Licht. Verz. Doubl. p. 44.

Herpsilochmus pileatus, Scl. P. Z. S. 1858, p. 233.

$a$ (N. C. Strickland) 1838.

Originally described from Bahia specimens.

170\%. Herpsilochmus rufomarginatus.

Myiothera rufimarginata, Temm. Pl. Col. 132, f. 1.

Herpsilochmus rufomarginatus, Scl. P. Z. S. 1858, p. 233.

$a$ Brazil (N. C. Striclland) 1838. —b Brazil (Arthur Strickland) 1840.

\section{Myrmotherula.}

Myrmotherula, Sclater, P. Z. S. 1858, p. 234.

1708. Myrmotherula surinamensis.

Sitta surinamensis, Gm. Syst. Nat. i. p. 442.

Myrmotherula surinamensis, Scl. l. s. c. pl. 141, f. 1.

$a$ S. America (Gardner) 1844. 
1709. Myrmotherula erythronota.

Formicivora erythronota, Hartl. Rev. Zool. 1853, p. 4.

Myrmotherula erythronota, Scl. P. Z. S. 1858, p. 236.

a Brazil (Argent) 1851. —b Brazil (Argent) 1853.

1710. Myrmotherula melanogaster.

Thamnophilus melanogaster, Spix, Av. Bras. ii. p. 31, pl. 43, f. 1.

Myrmotherula melanogastra, Scl. Cat. Am. B. p. 180.

a (N. C. Strickland) 1838.

A Brazilian species.

1711. Myrmotherula ménétriési.

Myrmothera menetriesii, d'Orb. Voy. Ois. p. 184.

Myrmotherula menetriesi, Scl. Cat. Am. B. p. 180.

a Brazil (Bt at Stevens's) 1845.

A species of the Upper Amazon spreading into Central America.

1712. Myrmotherula brevicauda.

Formicivora brevicauda, Sw. Zool. Journ. ii. p. 148.

Myrmotherula brevicauda, Scl. P. Z. S. 1858, p. 237.

a Brazil (N. C. Strickland) 1838.

FormicIVORA.

Formicivora, Swainson, Zool. Journ, ii. p. 145 (1825).

1713. Formicivora grisea.

Le Grisin, de Cayenne, D’Aub. Pl. Enl. 643, f. 1, undè,

Turdus griseus, Bodd. Tabl. Pl. Enl. p. 39.

Formicivora grisea, Scl. P. Z. S. 1858, p. 238.

a Brazil (Askew) 1834. —b Brazil (N. C. Strickland) 1838.

In his commentary on Gray's Genera of Birds (Ann. \& Mag. N. H. vi. p. 423) Strickland points out that Formicivora nigricollis = Myiothera superciliaris, Licht. $=$ Motacilla grisea, $\mathrm{Gm}$.

\section{Formicivora rufatra.}

Thamnophilus rufater, d’Orb. \& Lafr. Syn. Av. i. p. 12.

Formicivora rufatra, Scl. P. Z. S. 1858, p. 239.

Thamnophilus griseus $q$, Spix, Av. Bras. ii. p. 29, pl. 40, f. 1 (nec Bodd.).

$a$ (Thomas) 1843.

A Brazilian and Bolivian species. 
1715. Formicivora ferruginea.

Myiothera ferruginea, Licht. Verz. Doubl. p. 44.

Formicivora ferruginea, Ménétr. Mém. de l'Ac. imp. Sc. St Pét. Ser. 6. Sc. Nat. 1, p. 488: Scl. P. Z. S. 1858, p. 239.

Drymophila variegata, Such, Zool. Journ. i. p. 559.

$a$ o Brazil (N. C. Strickland) 1838. -b (Argent) 1852.

\section{Formicivora striata.}

Thamnophilus striatus, Spix, Av. Bras. ii. p. 29, pl. 40, f. 2.

Formicivora striata, Scl. Cat. Am. B. p. 182.

$a$ S. America (Osman) 1846.

\section{1\%. Formicivora squamata.}

Myiothera squamata, Licht. Verz. Doubl. p. 44.

Formicivora squamata, Scl. P. Z. S. 1858, p. 241.

a Brazil (Mansfield) 1834. —b Brazil (Askew) 1837. —c Brazil (N.C. Strickland) 1838.

\section{Terenura.}

Terenura, Cabanis \& Heine, Mus. Hein. ii. p. 11 (1859).

\section{Terenura maculata.}

Myiothera maculata, Max. Beitr. iii. p. 1088.

Terenura maculata, Scl. Cat. Am. B. p. 183.

$a$ S. America (N. C. Strickland) 1838.

\section{RHAMPHOCGENUS.}

Ramphocœenus, Vieillot, N. Dict. d'Hist. N. xxix. p. 5 (1819). 1719. Rhamphocœnus melanurus.

Ramphocœenus melanurus, Vieill. l: s. c. p. 6.

Rhamphocoenus melanurus, Scl. P. Z. S. 1858, p. 243.

a Brazil (N. C. Strickland) 1838.

\section{Pyriglena.}

Pyriglena, Cabanis, Arch. f. Naturg. xiii. p. 211 (1847). 1720. Pyriglena leucoptera.

Turdus leucopterus, Vieill. N. Dict. d'Hist. N. xx. p. 272. 
Pyriglena leucoptera, Scl. P. Z. S. 1858, p. 246.

Myrmeciza melanura, Strickl. Ann. N. H. xiii. p. 417.

Lanius domicella, Licht. Verz. Doubl. p. 47.

a Brazil (Askew) 1834. - b (N. C. Strickland) 1838. - c Brazil (N. C. Strickland) 1838. —d Brazil (G. Lloyd) 1838.

$b$ is doubtless the type of Myrmeciza melanura $l . s$. $c$. which Strickland appears from a note on the label to have discovered to be the female of domicella, i.e. leucoptera, Vieill.

\section{Pyriglena atra.}

Drymophila atra, Sw. Zool. Journ. ii. p. 153.

Pyriglena atra, Scl. P. Z. S. 1858, p. 246.

$a$ S. America (Seaman) 1840.

\section{Perchostola.}

Percnostola, Cabanis \& Heine, Mus. Hein. ii. p. 10 (1859).

\section{Percnostola funebris.}

Lanius funebris, Licht. Verz. Doubl. p. 47.

Percnostola funebris, Scl. Cat. Am. B. p. 185.

a (Dewgard) 1851.

Apparently a skin of Cayenne make.

\section{Heterochemis.}

Holocnemis, Strickl. Ann. N. H. xiii. p. 415 (1844) (nom. proeoc.).

Heterocnemis, Scl. P. Z. S. 1855, p. 146.

\section{Heterocnemis nævia.}

Sitta ncevia, Gm. Syst. Nat. i. p. 442.

Holocnemis noevia, Strickl. Contr. Orn. 1849, p. 34, pl. 18.

Heterocnemis ncevia, Scl. Cat. Am. B. p. 186.

Holocnemis flammata, Strickl. Ann. N. H. xiii. p. 415, pl. 13.

a (Bt at Plymouth) 1841.

The type of this Guiana genus and species described as Holocnemis flammata l. s. c. Strickland afterwards discovered that the bird was the same as the "Wall-creeper of Surinam" of Edwards and thus 
should bear Gmelin's name ncevius. The name Holocnemis having been previously used for a genus of Coleoptera Mr Sclater changed it to Heterocnemis in 1855.

\section{Mrrmeciza.}

Myrmeciza, G. R. Gray, List Gen. B. p. 34 (1841).

1724. Myrmeciza cinnamomea.

Turdus cinnamomeus, Gm. Syst. Nat. i. p. 825.

Holocnemis cinnamomea, Strickl. Ann. \& Mag. N. H. xiii. p. 416.

Myrmeciza cinnamomea, Scl. P. Z. S. 1858, p. 249.

$a$ (Cashmore) 1843.

A Guiana species placed by Strickland in his genus Holocnemis (Heterocnemis, Scl.). It belongs rather to Myrmeciza, into which genus it was removed by Sclater in his monograph of Formicariidæ.

1725. Myrmeciza ruficauda.

Myiothera ruficauda, Max. Beitr. iii. p. 1060. Myrmeciza ruficauda, Scl. Cat. Am. B. p. 186.

$a$ Brazil (Bt at Leeds) 1849. - b Brazil (Argent) 1851.

1726. IMyrmeciza squamosa.

Myrmeciza squamosa, Pelz. Orn. Bras. p. 87.

a (Arthur Strickland) 1840.

A Brazilian species.

172\%. Myrmeciza loricata.

Myiothera loricata, Licht. Verz. Doubl. p. 44.

Myrmeciza loricata, Scl. Cat. Am. B. p. 186.

$a$ (Arthur Strickland) 1840. —b (Bt at Dresden) 184.5.

\section{SUBFAMILY.-FORMICARIIN $\nexists$.}

\section{Pithys.}

Pithys, Vieillot, N. Dict. d'Hist. N. xxvi. p. 520 (1818).

1728. Pithys albifrons.

Pipra albifrons, Gm. Syst. Nat. i. p. 1000.

Pithys albifrons, Scl. Cat. Am. B. p. 189.

a Guiana (Askew) 1839. 


\section{Pithys leucaspis.}

Myrmeciza leucaspis, Scl. P. Z. S. 1854, p. 253, pl. 70.

Pithys leucaspis, Scl. Cat. Am. B. p. 189.

a (Thomas) 1842.

A Columbian and Amazonian species.

\section{RHOPOTERPE.}

Rhopoterpe, Cabanis, Arch. f. Naturg. xiii. p. 227 (1847).

\section{Rhopoterpe torquata.}

Le Fourmillier, de Cayenne, D’Aub. Pl. Enl. 700, f. 1, undè, Formicarius torquatus, Bodd. Tabl. Pl. Enl. p. 43.

Rhopoterpe torquata, Scl. Cat. Am. B. p. 190.

Turdus formicivorus, Gm. Syst. Nat. i. p. 828.

$a$ (Mansfield) 1839. —b (Dewgard) 1846.

$b$ is a skin of Cayenne make.

\section{Formicarius.}

Formicarius, Boddaert, Tabl. Pl. Enl. p. 50 (1783) (partim).

1731. Formicarius cayennensis.

Le Tétema, de Cayenne, D’Aub. Pl. Enl. 821, undè,

Formicarius cayennensis, Bodd. Tabl. Pl. Enl. p. 50; Scl. P. Z. S. 1858, p. 277 ; Cat. Am. B. p. 190.

a Brazil (Murray) 1834.

1732. Formicarius hoffmanni.

Myiothera hoffmanni, Cab. J. f. Orn. 1861, p. 96.

Formicarius hoffmanni, Salv. P. Z. S. 1866, p. 75 : Finsch, P. Z. S. 1870 , p. 568.

$a$ (Mather) 1840. —b (Williams) 1846.

$a$ is a skin of Cayenne make agreeing best with this species of the northern parts of South America.

\section{Cham}

Chamceza, Vigors, Zool. Journ. ii. p. 395 (1826).

1733. Chamæza brevicauda.

Turdus brevicaudus, Vieill. N. Dict. d'Hist. N. xx. p. 239. 
Chamceza brevicauda, Scl. P. Z. S. 1858, p. 278 : et Cat. Am. B. p. 191.

Chamceza meruloides, Vig. l.s. c.

$a$ S. America (Arthur Strickland) 1840.

1734. Chamæza olivacea.

Chamoeza olivacea, Tsch. Arch. f. Naturg. x. p. 279 : Scl. Cat. Am. B. p. 191.

$a$ Brazil (Argent) 1853.

The locality assigned to this specimen is perhaps erroneous, as the species is an inhabitant of the more northern parts of South America, Venezuela, \&c.

\section{Grallaria.}

Grallaria, Vieillot, N. Dict. d'Hist. N. xiii. p. 400 (1817).

\section{Grallaria imperator.}

Grallaria imperator, Lafr. Rev.Zool. 1842, p. 333 : Scl. Cat. Am. B. p. 191; Scl. Ibis, 1877, p. 443.

Myioturdus rex, Max. Beitr. iii. p. 1027 (nec Gm.).

$a$ (Mather) 1840. —b Brazil (Seaman) 1840.

\section{SUBFAMILY.—CONOPOPHA GIN Æ.}

CONOPOPHAGa.

Conopophaga, Vieillot, Anal. p. 39 (1816).

\section{Conopophaga melanops.}

Platyrhynchos melanops, Vieill. N. Dict. d'Hist. N. xxvii. p. 14 .

Conopophaga melanops, Scl. Cat. Am. B. p. 194.

Myiothera perspicillata, Licht. Verz. Doubl. p. 43.

$a$ S. America (Mather) 1840.

\section{Conopophaga lineata.}

Myiagrus lineatus, Max. Beitr. iii. p. 1046.

Conopophaga lineata, Cab. Arch. f. Naturg. xiii. p. 215.

Conopophaga vulgaris, Ménétr. Mém. de l’Ac. imp. Sc. St. Pét. Ser. 6. Sc. Nat. i. p. 534, pl. 14, f. 1.

$a,-b$ (N. C. Strickland) 1838.

A species of South-eastern Brazil.

S. C. 
PASSERES.

\section{CORYTHOPIS.}

Corythopis, Sundevall, K. Vet. Ac. Handl. 1835, p. 93.

1738. Corythopis anthoides.

Muscicapa anthoides, Cuv. Puch. Arch. du Mus. vii. p. 334.

Corythopis anthoides, Scl. P. Z S. 1858, p. 288 ; et Cat. Am. B. p. 194 .

$a$ S. America (Williams) 1846.

\section{FAMILY.PTEROPTOCHID E.}

\section{Merulaxis.}

Merulaxis, Lesson, Cent. Zool. p. 88 (1830) : Strickl. Ann. \& Mag. N. H. vi. p. 421.

1739. Merulaxis rhinolophus.

- Myiothera rhynolopha, Max. Beitr. iii. p. 1051.

Merulaxis rhinolophus, Scl. Ibis, 1874, p. 197.

Merulaxis ater, Less. Cent. Zool. p. 88.

$a,-b$ Brazil (Argent) 1851.

\section{PTERoptochus.}

Pteroptochos, Kittl. Mém. prés. Ac. Sc. Pétersb. 1831, p. 178. 1740. Pteroptochus rubecula.

Pteroptochos rubecula, Kittl. l. s. c. p. 179, pl. 2.

Pteroptochus rubecula, Scl. Ibis, 1874, p. 201.

a (Gardner) 1844.

A Chilian species.

\section{Pteroptochus albicollis.}

Pteroptochos albicollis, Kittl. l. s. c. p. 180, pl. 3.

Pteroptochus albicollis, Scl. Ibis, 1874, p. 201. a Chili (Capt. Brown) 1842. 


\section{Hylactes.}

Hylactes, King, P. Z. S. 1830-1, p. 15.

\section{Hylactes megapodius.}

Pteroptochos megapodius, Kittl. Mém. prés. Ac. Sc. Pétersb. 1831, p. 182, pl. 4.

Pteroptochus megapodius, Scl. Ibis, 1874, p. 203.

$a$ Chili (Capt. Brown) 1842. -b (Gardner) 1846.

\section{FAMILY.-PITTID正.}

\section{PITTA.}

Pitta, Vieillot, Anal. p. 42 (1816), Cf. Ibis, 1877, p. 260.

\section{Pitta cærulea.}

Myiothera coerulea, Raff. Trans. Linn. Soc. xiii. p. 301.

Pitta ccerulea, Vig. Mem. Raffl. App. p. 659.

Brachyurus cceruleus, Elliot, Mon. Pitt. pl. 1.

a Malacca (Stevens) 1844.

The recognized habitat of this species is Sumatra.

\section{Pitta cyanea.}

Pitta cyanea, Blyth, J. A. S. B. xii. p. 1008.

Brachyurus cyaneus, Elliot, Mon. Pitt. pl, 13 : Blyth, J. A. S. B. xliii. Extr. No. p. 98.

a

A specimen without any indication of its origin. The species was originally described from Arakan examples.

\section{Pitta cyanoptera.}

Pitta cyanoptera, Temm. Pl. Col. 218 : Salvad. Ann. Mus. Genov. v. p. 235.

? Corvus brachyurus, Linn. Syst. Nat. i. p. 158.

Pitta brachyura, Strickl. Ann. \& Mag. N. H. xiii. p. 37 ; xiv. p. 47.

Turdus moluccensis, P. L. S. Mïll. Naturs. Suppl. p. 144 (nom. inept.).

Brachyurus moluccensis, Elliot, Ibis, 1870, p. 413.

a Malacca (Capt. Nash) 1837. 


\section{Pitta angolensis.}

Pitta angolensis, Hartl. Orn. W. Afr. p. 74.

Brachyurus angolensis, Elliot, Mon. Pitt. pl. う.

a (E. Brown) 1850.

A West African species.

\section{Pitta coronata.}

Merle, de Bengal, D’Aub. Pl. Enl. 258, undè,

Turdus coronatus, P. L. S. Müll. Naturs. Suppl. p. 144.

Corvus brachyurus $\gamma$ C. bengalensis, Gm. Syst. Nat. i. p. 375.

Pitta bengalensis, Jerd. B. Ind. i. p. 503.

$a$ (Askew) 1833. - b Madras (T. C. Jerdon) 1845.

$a$ is probably the bird referred to $P$. abdominalis, Wagl. by Strickland in his notes on birds from Calcutta (Ann. \& Mag. N. H. xiii. p. 37) and again as $P$. malaccensis (Scop.) (l. c. xiv. p. 47, note).

1748. Pitta strepitans.

Pitta strepitans, Temm. Pl. Col. 333 : Gould, B. Austr. iv. pl. 1.

Pitta versicolor, Sw. Zool. Journ. i. p. 468.

a Australia (Warwick Museum) 1841.

\section{Pitta coccinea.}

Pitta coccinea, Eyt. P. Z. S. 1839, p. 104: Tweedd. Ibis, 1872 , p. 374.

$a$ (Argent) 1851.

This specimen, which is of Malaccan make, has the narrow black frontal band which distinguishes, with other characters, the Malaccan bird from the Bornean P. granatina.

\section{Pitta cucullata.}

Pitta cucullata, Hartl. Rev. Zool. 1843, p. 65 : Strickl. Ann. \& Mag. N. H. xiii. p. 410, pl. 11.

Brachyurus cucullatus, Elliot, Mon. Pitt. pl. 28.

a Malacca (Capt. Nash) 1837. 


\section{FAMILY.-EURYLAMIDA ${ }^{1}$.}

Calyptomena.

Calyptomena, Raffles, Trans. Linn. Soc. xiii. p. 295 (1822). 1751. Calyptomena viridis.

Calyptomena viridis, Raffl. l.s. c.: Salvad. Ann. Mus. Genov. v. p. 106.

a Malacca (N. C. Strickland) 1838. - b Malacca (Sir W. Jardine) 1845.

\section{EURYLAMUS.}

Eurylaimus, Horsfield, Trans. Linn. Soc. xiii. p. 170 (1822). Eurylcemus, Strickl. Ann. \& Mag. N. H. viii. p. 544.

1752. Eurylæmus javanicus.

Eurylaimus javanicus, Horsf. l.s.c.

Eurylcemus javanicus, Salvad. Ann. Mus. Genov, v. p. 107. a Malacca (Williams) 1847.

1753. Eurylæmus ochromelas.

Eurylaimus ochromalus, Raffl. Trans. Linn. Soc. xiii. p. 297. Eurylcemus ochromelas, Salvad. Ann. Mus. Genov. v. p. 108. a Sumatra (Dr Prattington) 183-.

\section{Cymborhynchus.}

Cymbirhynchus, Vigors, Mem. Raffl. App. p. 654 (1830).

1754. Cymborhynchus macrorhynchus.

Todus macrorhynchus, Gm. Syst. Nat. i. p. 446.

Cymborhynchus macrorhynchus, Salvad. Ann. Mus. Genov. v. p. 109.

Todus nasutus, Lath. Ind. Orn. i. p. 268.

a Malacca (Capt. Nash) 1837. - b Malacca (E. Blyth) 1847.

1 Cf. Garrod, P. Z. S. 1877, p. 447 et seq. 
1755. Cymborhynchus affinis.

Cymbirhynchus affinis, Blyth, J. A. S. B. xv. p. 312 ; xliii. Extr. No. p. 126.

a Arakan (E. Blyth) 1847.

$\mathrm{Mr}$ Gould's figure of this species in the fifth part of the 'Birds of Asia' was taken from this specimen.

\section{Corydon.}

Corydon, Lesson, Man. d'Orn. i. p. 177 (1828).

1756. Corydon sumatranus.

Coracias sumatranus, Raff. Trans. Linn. Soc. xiii. p. 303.

Corydon sumatranus, Strickl. Ann. \& Mag. N. H. vi. p. 417 : Salvad. Ann. Mus. Genov. v. p. 111.

a Sumatra (J. W. Lockwood) 1837.

\section{Psarisomus.}

Psarisomus, Swainson, Classif. B. ii. p. 261 (1837).

175\%. Psarisomus dalhousiæ.

Eurylcemus dalhousice, Jameson, Ed. New. Phil. Mag. xviii. p. 261.

Psarisomus dalhousice, Gould, B. As. a (T. C. Eyton) 1849.

A species of Northern India and Burma. 


\section{ORDER II.-MACROCHIRES.}

\section{FAMILY.-TROCHILIDE.}

GLAUCIS.

Glaucis, Boie, Isis, 1831, p. 545.

1758. Glaucis hirsuta.

Trochilus hirsutus, Gm. Syst. Nat. i. p. 490.

Glaucis hirsuta, Gould, Mon. Troch. i. pl. 5 : Salv. \& Elliot, Ibis, 1873, p. 276.

$a$ (Bt at Leeds) 1840. - b Trinidad (J. Taylor).

A species of wide range, being found from Costa Rica to Rio de Janeiro.

\section{Phaethornis.}

Phcethornis, Swainson, Zool. Journ. iii. p. 357 (1827).

1759. Phaethornis eurynome.

Trochilus eurynome, Less. Troch. p. 91, pl. 31.

Phaethornis eurynome, Gould, Mon. Troch. i. pl. 16 : Salv. \& Elliot, Ibis, 1873, p. 8. a (F. Holme) 1838.

A species of South-eastern Brazil.

1760. Phaethornis pretrii.

Trochilus pretrei, Less. \& Delattre, Rev. Zool. 1839, p. 20.

Phaethornis pretrii, Gould, Mon. Troch. i. pl. 28: Salv. \& Elliot, Ibis, 1873, p. 11.

$a$ (Osman) 1846.

A Brazilian species.

1761. Phaethornis guyi.

Trochilus guyi, Less. Troch. p. 119, pl. 44.

Phaethornis guyi, Gould, Mon. Troch. i. pl. 26 : Salv. \& Elliot, Ibis, 1873, p. 12.

a Trinidad (J. Taylor). 


\section{Eupetomena.}

Eupetomena, Gould, Mon. Troch. Part vi. (1853).

\section{Eupetomena macrura.}

Trochilus macrourus, Gm. Syst. Nat. i. p. 487.

Eupetomena macrura, Elliot, Syn. Troch. p. 21.

a S. America (Leadbeater) 1834. - b S. America (Bt at Stevens's) 1846. —c S. America (Dewgard) 1847.

A Brazilian and Guiana species.

\section{CAMpylopterus.}

Campylopterus, Swainson, Zool. Journ. iii. p. 358 (1827). 1763. Campylopterus rufus.

Campylopterus rufus, Less. Rev. Zool. 1840, p. 73 : Gould, Mon. Troch. ii. pl. 50: Muls. Hist. Nat. Ois. Mouch. i. p. 133.

$a$ Guatemala ( $J$. Constancia) 1845.

\section{Aphantochroa.}

Aphantochroa, Gould, Mon. Troch. pt. vi. (1853).

\section{Aphantochroa cirrhochloris.}

Trochilus cirrochloris, Vieill. N. Dict. d'Hist. N. xxiii. p. 430 .

Aphantochroa cirrochloris, Gould, Mon. Troch. i. pl. 54. $a,-b$ (Osman) 1846 .

A Brazilian species.

\section{BASILINNA.}

Basilinna, Boie, Isis, 1831, p. 546.

\section{Basilinna leucotis.}

Trochilus leucotis, Vieill. N. Dict. d'Hist. N. xxiii. p. 428.

Basilinna leucotis, Elliot, Syn. Troch. p. 227.

Heliopcedica melanotis, Gould, Mon. Troch. ii. pl. 64.

a Mexico (T. Mann) 1844. —b, —c Guatemala (J. Constancia) 1845. 


\section{Topaza.}

Topaza, G. R. Gray, List Gen. B. p. 13 (1840).

1766. Topaza pella.

Trochilus pella, Linn. Syst. Nat. i. p. 189.

Topaza pella, Gould, Mon. Troch. ii. pl. 66.

$a$ (Bt at Stevens's) 1845.

A species found in the North-eastern parts of South America.

\section{OREOTROCHILUS.}

Oreotrochilus, Gould, P. Z. S. 1847, p. 9.

\section{6\%. Oreotrochilus pichincha.}

Trochilus pichincha, Bourc. \& Muls. Mém. de l'Ac. Roy. de Lyon. Sect. Sc. ii. 1849, p. 427.

Oreotrochilus pichincha, Gould, Mon. Troch. ii. pl. 69.

Oreotrochilus jamesoni, Jard. Contr. Orn. 1849, p. 67 ; 1850, p. 27, pl. 43.

a Quito (W. Jameson) 1850.

Found in the higher parts of the volcanoes of Pichincha and Cotopaxi in Ecuador.

\section{Oreotrochilus leucopleurus.}

Oreotrochilus leucopleurus, Gould, P. Z. S. 1847, p. 10; Mon. Troch. ii. pl. 71.

a (T. C. Eyton) 1849.

A species found in the Andes of Chili.

\section{LAMPORNIS.}

Lampornis, Swainson, Zool. Journ. iii. p. 358 (1827) partim.

\section{Lampornis violicauda.}

Colibri à queue violette, D'Aub. Pl. Enl. 671, f. 2, undè,

Trochilus violicauda, Bodd. Tabl. Pl. Enl. p. 41.

Lampornis violicauda, Elliot, Syn. Troch. p. 38.

Lampornis mango, Auctt. plurr. (nec Linn.).

$a$ (Leadbeater) 1834. - b (Bt at Stevens's) 1845. -c, -d Trinidad (J. Taylor). 


\section{7\%0. Lampornis prevosti.}

Trochilus prevosti, Less. Col. p. 87, pl. 24.

Lampornis prevosti, Gould, Mon. Troch. ii. pl. 75.

a Guatemala (J. Constancia) 1845.

\section{7'11. Lampornis gramineus.}

Trochilus gramineus, Gm. Syst. Nat. i. p. 488.

Lampornis gramineus, Gould, Mon. Troch. ii. pl. 77.

a (Bt at Edinburgh) 1852.

Found in Guiana and Venezuela.

\section{EULAMPIS.}

Eulampis, Boie, Isis, 1831, p. 547.

\section{Eulampis holosericeus.}

Trochilus holosericeus, Linn. Syst. Nat. i. p. 191.

Eulampis holosericeus, Gould, Mon. Troch. ii. pl. 83 : Elliot, Syn. Troch. p. 42.

$a$ (Carfrae) 1850.

A species of the Lesser Antilles.

\section{AITHURUS.}

Aithurus, Cabanis \& Heine, Mus. Hein. iii. p. 50 (1860).

1719. Aithurus polytmus.

Trochilus polytmus, Linn. Syst. Nat. i. p. 189: Gosse, B. Jam. p. 97 ; Ill. pls. 19, 20 : Gould, Mon. Troch. ii. pl. 98.

Aithurus polymus, Elliot, Syn. Troch. p. 96.

a ô Jamaica (P. H. Gosse) 1848.

\section{Thalurania.}

Thalurania, Gould, P. Z. S. 1848, p. 13.

17'74. Thalurania glaucopis.

Trochilus glaucopis, Gm. Syst. Nat. i. p. 497.

Thalurania glaucopis, Gould, Mon. Troch. ii. pl. 99 : Salv. \& Elliot, Ibis, 1873, p. 355.

$a,-b$ (Leadbeater) 1834. — Brazil (Manchester Nat. Hist. Soc.) 1843. - d (Bt at Stevens's) 1849. - - (Bt at Stevens's) 1850. 


\section{Thalurania furcata.}

Trochilus furcatus, Gm. Syst. Nat. i. p. 486.

Thalurania furcata, Gould, Mon. Troch. ii. pl. 101 : Salv. \& Elliot, Ibis, 1873, p. 356.

$a$ (Argent) 1852.

A skin of Cayenne make.

\section{Panoplites.}

Panoplites, Gould, Mon. Troch. pt. viii. (1854).

1776. Panoplites jardinii.

Trochilus jardinii, Bourc. Compt. Rend. xxii. p. 187.

Panoplites jardini, Gould, Mon. Troch. ii. pl. 110.

a Quito (W. Jameson) 1852.

\section{Florisuga.}

Florisuga, Bonaparte, Compt. Rend. xxx. p. 382 (1850).

17\%1\%. Florisuga mellivora.

Trochilus mellivorus, Linn. Syst. Nat. i. p. 193.

Florisuga mellivora, Gould, Mon. Troch. ii. pl. 113.

$a$ (Osman) 1846. - b Trinidad (J. Taylor).

\section{9\%8. Florisuga fusca.}

Trochilus fuscus, Vieill. N. Dict. d'Hist. N. vii. p. 348.

Florisuga fusca, Elliot, Syn. Troch. p. 48.

Florisuga atra, Gould, Mon. Troch. ii. pl. 115.

$a$ (Leadbeater) 1834. - b (Bt at Birmingham) 1840. -c (Thomas) 1843.

A common Brazilian species.

\section{LOPHORNIS.}

Lophornis, Lesson, Ois. Mouch. p. xxxvii. (1829).

1779. Lophornis ornatus.

Le Huppe colibri, D'Aub. Pl. Enl. 640, f. 3, undè,

Trochilus ornatus, Bodd. Tabl. Pl. Enl. p. 39.

Lophornis ornatus, Gould, Mon. Troch. iii. pl. 117. 
a Brazil (Manchester Nat. Hist. Soc.) 1843. $-b$ ई, $-c$ ई juv., $-d$ o Trinidad (J. Taylor).

$a$ is a young bird most probably of this species, the locality Brazil assigned to it being erroneous.

\section{Discura.}

Discura, Reichenbach, Aufz. d. Col. p. 8 (1853).

1780. Discura longicauda.

Trochilus longicaudus, Gm. Syst. Nat. i. p. 498.

Discura longicauda, Gould, Mon. Troch. iii. p. 126.

$a$ (Bt at Stevens's) 1846. -b (Graham) 1849.

A Brazilian species.

\section{Trochilus.}

Trochilus, Linnæus, Syst. Nat. i. p. 189 (1766) partim.

1781. Trochilus colubris.

Trochilus colubris, Linn. l. s. c. p. 191 : Gould, Mon. Troch. iii. pl. 131.

$a,-b,-c,-d$ Guatemala (J. Constancia) 1845. $-e,-f$ Canada (Barnes) 1848.

\section{SELASPHORUS.}

Selasphorus, Swainson, Faun. Bor. Am. ii. p. 496 (1831). 1782. Selasphorus platycercus.

Trochilus platycercus, Sw. Phil. Mag. New Ser. i. p. 441. Selasphorus platycercus, Gould, Mon. Troch. iii. pl. 140. a Mexico (T. Mann) 1844.

\section{Calothorax.}

Calothorax, G. R. Gray, List Gen. B. p. 13 (1840). 1783. Calothorax lucifer.

Cynanthus lucifer, Sw. Phil. Mag. New Ser. i. p. 442.

Calothorax lucifer, Elliot, Syn. Troch. p. 118.

Calothorax cyanopogon, Gould, Mon. Troch. iii. pl. 143. a Mexico (T. Mann) 1844. 


\section{DORICHA.}

Doricha, Reichenbach, Aufz. d. Col. p. 12 (1853).

\section{Doricha henicura.}

Trochilus enicurus, Vieill. N. Dict. d'Hist. N. xxiii. p. 429.

Thaumastura enicura, Gould, Mon. Troch. iii. pl. 157.

Doricha enicura, Elliot, Syn. Troch. p. 124.

Trochilus swainsoni, Less. Troch. p. 167, pl. 66.

$a,-b,-c,-d$ Guatemala (J. Constancia) 1845.

\section{Calliphlox.}

Calliphlox, Boie, Isis, 1831, p. 544.

\section{Calliphlox amethystina.}

Trochilus amethystinus, Gm. Syst. Nat. i. p. 496.

Calliphlox amethystina, Gould, Mon. Troch. iii. pl. 159.

$a,-b,-c,-d$ Brazil (Manchester Nat. Hist. Soc.) 1843.

\section{Chrysolampis.}

Chrysolampis, Boie, Isis, 1831, p. 546.

+ 1786. Chrysolampis moschitus.

Trochilus moschitus, Linn. Syst. Nat. i. p. 192.

Chrysolampis moschitus, Gould, Mon. Troch. iv. pl. 204.

$a,-b,-c$ Brazil (Leadbeater) 1834. $-d,-e,-f$ Trinidad (J. Taylor).

\section{Cephalolepis.}

Cephallepis, Loddiges, P. Z. S. 1830-1, p. 12.

Cephalolepis, Cabanis \& Heine, Mus. Hein. iii. p. 61. 178\%. Cephalolepis delalandii.

Trochilus lalandi, Vieill. N. Dict. d'Hist. N. xxiii. p. 427.

Cephalepis lalandi, Gould, Mon. Troch. iv. pl. 208.

$a$ S. America (G. Bell-Whitehouse) 1842. — b Brazil (Manchester Nat. Hist. Soc.) 1843. —c (Osman) 1846. 


\section{HELIOTHRIX.}

Heliothrix, Boie, Isis, 1831, p. 547.

1788. Heliothrix auritus.

Trochilus auritus, Gm. Syst. Nat. i. p. 493.

Heliothrix auritus, Gould, Mon. Troch. iv. pl. 213 : Elliot, Syn. Troch. p. 174.

a (Bt at Edinburgh) 1852.

Found in the northern parts of South America, Guiana, \&c.

Petasophora.

Petasophora, G. R. Gray, List Gen. B. p. 13 (1840).

1789. Petasophora serrirostris.

Trochilus serrirostris, Vieill. N. Dict. d'Hist. N. vii. p. 359.

Petasophora serrirostris, Gould, Mon. Troch. iv. pl. 223.

$a$ (Leadbeater) 1834. -b (Osman) 1846.

A Brazilian species.

\section{Polytmus.}

Polytmus, Brisson, Orn. iii. p. 666 (1760).

1790. Polytmus thaumantias.

Trochilus thaumantias, Linn. Syst. Nat. i. p. 190.

Polytmus thaumantias, Cab. \& Hein. Mus. Hein. iii. p. 5.

Chrysobronchus virescens, Gould, Mon. Troch. iv. pl. 230.

$a$ (Bt at Stevens's) 1846.

A species of Brazil, Venezuela, \&c.

\section{Patagona.}

Patagona, G. R. Gray, List Gen. B. p. 14 (1840).

1791. Patagona gigas.

Trochilus gigas, Vieill. Gal. Ois. i. p. 296, pl. 180.

Patagona gigas, Gould, Mon. Troch. iv. pl. 232.

a Chili (Capt. Brown) 1842.

\section{Helianthea.}

Helianthea, Gould, P. Z. S. 1848, p. 11.

1792. Helianthea typica.

Ornismya helianthea, Less. Rev. Zool. 1838, p. 314. 
Helianthea typica, Gould, Mon. Troch. iv. pl. 235.

a (Graham) 1849.

A Colombian species.

\section{Helianthea bonapartii.}

Ornismya bonapartei, Boiss. Rev. Zool. 1840, p. 6.

Helianthea bonapartei, Gould, Mon. Troch. iv. pl. 236.

$a$ (Dewgard) 1847.

A Colombian species.

\section{Clytolema.}

Clytolcema, Gould, Mon. Troch. Pt. vi. (1853).

1794. Clytolæma rubinea.

Trochilus rubineus, Gm. Syst. Nat. i. p. 493.

Clytoloema rubinea, Gould, Mon. Troch. iv. pl. 249.

a (W. Kirtland) 1838. -b Brazil (Manchester Nat. Hist. Soc.) 1843. -c, -d (Osman) 1846. - e (T. C. Eyton) 1853.

\section{Heliomaster.}

Heliomaster, Bonaparte, Compt, Rend. xxx. p. 382 (1850). 1795. Heliomaster pallidiceps.

Heliomaster pallidiceps, Gould, Intr. Mon. Troch. i. p. xcii. : Salv. P. Z. S. 1867, p. 155.

a Guatemala ( $J$. Constancia) 1845.

1796. Heliomaster constanti.

Trochilus constanti, DeLattre, Echo du Monde Sav. 1843, p. 1069.

Heliomaster constanti, Gould, Mon. Troch. iv. pl. 260.

a Guatemala (J. Constancia) 1845.

\section{Eustephanus.}

Eustephanus, Reichenbach, Aufz. d. Col. p. 14 (1853). 179\%. Eustephanus galeritus.

Trochilus galeritus, Mol. Saggio sulla St. Nat. Chili, p. 247. Eustephanus galeritus, Gould, Mon. Troch. iv. pl. 265.

a Chili (Capt. Brown) 1842. 


\section{Cyanomyia.}

Cyanomyia, Bonaparte, Rev. Zool. 1854, p. 254.

1798. Cyanomyia cyanocephala.

Ornismya cyanocephala, Less. Suppl. Ois. Mouch. p. 134, pl. 18.

Cyanomyia cyanocephala, Gould, Mon. Troch. v. pl. 286.

$a$ Guatemala (J. Constancia) 1845.

\section{LEUCOCHLORIS.}

Leucochloris, Reichenbach, Aufz. d. Col. p. 10 (1853). 1799. Leucochloris albicollis.

Trochilus albicollis, Vieill. N. Dict. d'Hist. N. xxiii. p. 426.

Leucochloris albicollis, Gould, Mon. Troch. v. pl. 291.

$a$ (Leadbeater) 1834. — b (Osman) 1846.

A common Brazilian species.

\section{AgYrTRia.}

Agyrtria, Reichenbach, Aufz. d. Col. p. 6 (1853). 1800. Agyrtria niveipectus.

Agyrtria niveipectus, Cab. \& Hein. Mus. Hein. iii. p. 33 : Elliot, Syn. Troch. p. 202.

Thaumatias chionopectus, Gould, Mon. Troch. v. pl. 293.

$a,-b$ Trinidad (J. Taylor).

\section{Agyrtria tephrocephala.} 430.

Trochilus tephrocephalus, Vieill. N. Dict. d'Hist. N. xxiii. p.

Agyrtria tephrocephala, Elliot, Syn. Troch. p. 206.

Thaumatias albiventris, Gould, Mon. Troch. v. pl. 301.

a Brazil (Manchester Nat. Hist. Soc.) 1843.

1802. Agyrtria nitidifrons.

Thaumatias nitidifrons, Gould, P. Z. S. 1860, p. 308; Mon. Troch. v. p. 297.

Agyrtria nitidifrons, Elliot, Syn. Troch. p. 20 ๖.

a (Robins) 1834.

This specimen agrees fairly with descriptions of $A$. nitidifrons but has the forehead alone and not the crown shining green. 


\section{Amazilia.}

Amazilia, Lesson, Troch. p. xxvii (1832).

1803. Amazilia riefferi.

Trochilus riefferi, Bourc. Rev. Zool. 1843, p. 103.

Amazilia riefferi, Gould, Mon. Troch. v. pl. 311.

a (Bt at Birmingham) 1850.

Apparently a skin of Bogota make.

\section{Amazilia devillii.}

Trochilus devillei, Bourc. Rev. Zool. 1848, p. 272.

Amazilia devillei, Gould, Mon. Troch. v. pl. 313.

$a,-b$ Guatemala ( $J$. Constancia) 1845.

1805. Amazilia erythronota.

Ornismya erythronotos, Less. Ois. Mouch. p. 181, pl. 61.

A mazilia erythronota, Elliot, Syn. Troch. p. 224.

Erythronota antiqua, Gould, Mon. Troch. v. pl. 316.

$a$ (Leadbeater) 1824. $-b, \longrightarrow c$ Trinidad (J. Taylor).

\section{Eucephala.}

Eucephala, Reichenbach, Aufz. d. Col. p. 10 (1853), as a subgenus.

1806. Eucephala cærulea.

Trochilus caruleus, Vieill. N. Dict. d'Hist. N. vii. p. 361 .

Eucephala ccerulea, Gould, Mon. Troch. v. pl. 335 : Elliot, Syn. Troch. p. 230.

a. Trinidad (J. Taylor).

\section{HYLOCHARIS.}

Hylocharis, Boie, Isis, 1831, p. 546.

\section{0\%. Hylocharis sapphirina.}

Trochilus sapphirinus, Gm. Syst. Nat. i. p. 496.

Hylocharis sapphirinus, Gould, Mon. Troch. v. p. 342.

$a($ W. Kirtland) 1838. $-b,-c$ (Osman) 1846.

A Brazilian species.

S. C. 
Chlorostilbon.

Chlorostilbon, Gould, Mon. Troch. pt. v. (1853).

1808. Chlorostilbon caniveti.

Ornismya caniveti, Less. Suppl. Ois. Mouch. pp. 174, 177, pls. $37,38$.

Chlorostilbon caniveti, Elliot, Syn. Troch, p. 243.

Chlorostilbon osberti, Gould, Mon. Troch. v. pl. 352.

a Guatemala (J. Constancia) 1845.

1809. Chlorostilbon pucherani.

Trochilus pucherani, Bourc. \& Muls. Rev. Zool. 1848, p. 271.

Chlorostilbon pucherani, Elliot, Syn. Troch. p. 244.

Chlorostilbon prasinus, Gould, Mon. Troch. v. pl. 355.

$a,-b,-c,-d,-e$ Brazil (Manchester Nat. Hist. Soc.) 1843. $-f,-g$ (Osman) 1846.

\section{FAMILY.-CYPSELID咂.}

\section{SUBFAMILY.-CYPSELIN $A$.}

\section{Cypselus.}

Cypselus, Illiger, Prodr. p. 229 (1811).

1810. Cypselus melba.

Hirundo melba, Linn. Syst. Nat. i. p. 345.

Cypselus melba, Scl. P. Z. S. 1865, p. 598 : Dresser, B. Eur.

a Tuscany (E. Passerini) 1835.

\section{Cypselus apus.}

Hirundo apus, Linn. Syst. Nat. i. p. 344.

Cypselus apus, Scl. P. Z. S. 1865, p. 598 : Dresser, B. Eur. $a$ Middlesex (H. E. Strickland) 1827. - b South Africa ( $N$. C. Strickland) 1838.

\section{Cypselus parvus.}

Cypselus parvus, Licht. Verz. Doubl. p. 58 : Strickland, P. Z. S. 1844, p. 99 : Scl. P. Z. S. 1865, p. 601.

a West Africa (Sir W. Jardine) 1845.

Recorded from Accra in Strickland's notes on a collection of birds made by I. Fraser in W. Africa. 


\section{Cypselus batassiensis.}

Cypselus balasiensis, Gray, Cuv. Anim. Kingd. Griffith's Ed. Aves, ii. p. 60.

Cypselus batassienis, Jerd. B. Ind. i. p. 180 : Scl. P. Z. S. 1865, p. 602.

Cypselus palmarum, Gray, Ill. Ind. Zool. pl. 35, f. 1.

a Madras (T. C. Jerdon) 1845. — b India ("Cypselus palmarum," E. Blyth) 1846.

\section{Cypselus affinis.}

Cypselus affinis, Gray, Ill. Ind. Zool. pl. 35, f. 2 : Jerd. B. Ind. i. p. 177 : Scl. P. Z. S. 1865, p. 603.

a India (N. C. Strickland) 1838. - b India ("Cypselus affinis, Gray," E. Blyth) 1844.

Strickland's note (P. Z. S. 1846, p. 99) respecting the occurrence of this species in Malacca refers to Cypselus subfurcatus, Blyth.

\section{Cypselus phœnicobius.}

Tachornis phonicobius, Gosse, B. of Jamaica, p. 58, et Ill. B. Jam. pl. 9.

Cypselus phonicobius, Scl. P. Z. S. 1865, p. 604, a Jamaica (P. H. Gosse) 1848.

A typical specimen named in Mr Gosse's handwriting Tachornis phoenicobia. In Strickland's copy of the 'Birds of Jamaica' I find the following pencil note "a typical Cypselus." This accords with $\mathrm{Mr}$ Sclater's view (l.s. c.).

\section{Panyptila,}

Panyptila, Cabanis, Arch. f. Naturg. xiii. p. 34 (1847). 1816. Panyptila cayennensis.

Hirundo cayennensis, Gm. Syst. Nat. i. p. 1024.

Panyptila cayennensis, Cab. l. s. c.: Scl.P. Z. S. 1865, p. 606. a (Bt at Birmingham) 1849.

A species widely distributed in Tropical America. 


\section{SUBFAMILY.-CH $\not$ ETURIN $Æ$.}

Ch жTURA.

Chcetura, Stephens, in Shaw's Zool. xiii. pt. 2, p. 76 (1825).

181\%. Chætura caudacuta.

Hirundo caudacuta, Lath. Ind. Orn. Suppl. p. lvii.

Chcetura caudacuta, Scl. P. Z. S. 1865, p. 607.

a Australia? (N. C. Strickland) 1838.

In his remarks on the Earl of Derby's Collection of Australian Drawings (Ann. \& Mag. N. H. xi. p. 337) Strickland gives as synonyms of $H$. caudacuta Lath. Chcetura australis Steph., Hirundo fusca Steph., and Chcetura macroptera Sw.

\section{Chætura zonarius.}

Hirundo zonaris, Shaw, Cim. Phys. p. 100, pl. 55.

Choetura zonaris, Scl. P. Z. S. 1865, p. 609.

$a$ Brazil (N. C. Strickland) 1838. -b (Argent) 1851.

\section{Chætura pelasgia.}

Hirundo pelasgia, Linn. Syst. Nat. i. p. 34ว.

Choetura pelasgia, Steph. Shaw's Zool. xiii. pt. 2, p. 76 : Scl. P. Z. S. 1865, p. 610 : Baird, Brew. \& Ridgw. N. Am. B. ii. p. 432. 1844.

a N. America (F. Holme) 1838. - b Bogota? (Bt at Stevens's)

This species has as yet not been observed south of Mexico. The locality doubtfully ascribed to specimen $b$ is erroneous.

1820. Chætura cinereiventris.

Chcetura cinereiventris, Scl. Cat. Am. B. p. 283 ; P. Z. S. 1863, p. 101, pl. 24, f. 1 ; 1865, p. 612.

a Brazil (Argent) 18.51 .

\section{Collocalia.}

Collocalia, G. R. Gray, List Gen. B. p. 8 (1840). 1821. Collocalia francica.

Hirundo francica, Gm. Syst. Nat. i. p. 1017.

Collocalia francica, Walden, Ibis, 1874, p. 132.

a Mauritius (Mrs Telfair) 1833. 
SUBFAMILY.-MACROPTERIGIN AE.

\section{MACROPTERYX.}

Macropteryx, Swainson, Zool. Ill. pl. 47 (1831).

Dendrochelidon, F. Boie, Isis, 1844, p. 165.

\section{Macropteryx longipennis.}

Hirundo longipennis, Rafin. Bull. Soc. Philom. iii. p. 153 (1804).

Dendrochelidon longipennis, Salvad. Ann. Mus. Genov. v. p. 122.

Hirundo klecho, Horsf. Trans. L. S. xiii. p. 143.

a (N. C. Strickland) 1838.

A species found in Java, Borneo, Malacca, \&c.

\section{Macropteryx coronatus.}

Hirundo coronata, Tickell, J. A. S. B. ii. p. 580.

Dendrochelidon coronata, Gould, B. of Asia : Jerd. B. Ind. i. p. 185.

a Madras ("Macropteryx klecho" T. C. Jerdon) 1845. -b S. India (T. C. Jerdon) 1845. -c India (W. J. E. Boys) 1848.

1824. Macropteryx comatus.

Cypselus comatus, 'Temm. Pl. Col. 268.

Dendrochelidon comata, Gould, B. of Asia: Salvad. Ann. Mus. Genov. v. p. 123.

a (Gardner) 1847.

A species of Sumatra, Borneo, \&c.

\section{FAMILY. - CAPRIMULGIDAE.}

\section{SUBFAMILY.-PODARGIN E.}

\section{Podargus.}

Podargus, Cuvier, Règn. Anim, i. p. 398 (1829). 1825. Podargus strigoides.

Caprimulgus strigoides, Lath. Ind. Orn. Suppl. p. lviii.

Podargus strigoides, G. R. Gray, Ann. \& Mag. N. H. xi. p. 194. 
Podargus humeralis, Vig. \& Horsf. Trans. L. S. xv. p. 198 : Gould, B. Austr. ii. pl. 3.

$a$ New South Wales (McDonald) 1838 . - b Australia (Arthur Strickland) 1850.

\section{Podargus cuvieri.}

Podargus cuvieri, Vig. \& Horsf. Trans. Linn. Soc. xv. p. 200 : Gould, B. of Austr. ii. pl. 4.

a New South Wales (N.C. Strickland) 1838.

\section{Batrachostomus.}

Batrachostomus, Gould, Icon. Av. Pt. ii. (1838).

1827. Batrachostomus auritus.

Podargus auritus, Vig. Cuv. Anim. Kingd. Griffith's Ed. Aves, ii. p. 114, pl. 4.

Batrachostomus auritus, Gould, l.s.c. pl. 7.

a (Bt. at Liverpool) 1853.

Found in Malacca, Borneo and Sumatra.

1828. Batrachostomus stellatus.

Podargus stellatus, Gould, P. Z. S. 1837, p. 43.

Batrachostomus stellatus, Tweedd. P. Z. S. 1877, p. 436, pl. 47 .

$a$ (Argent) 1851.

A species found in Malacca, Sumatra, and Borneo. The specimen, which appears to be of Malacca make, agrees with the plate quoted above.

\section{SUBFAMILY.-NYCTIBIIN $Æ$.}

\section{Nyctibius.}

Nyctibius, Vieillot, Anal. p. 38 (1816).

1829. Nyctibius æthereus.

Caprimulgus athereus, Max. Beitr. iii. p. 303.

Nyctibius cethereus, Scl. P.Z. S. 1866, p. 129.

a (Dewgard) 1849.

A species of South-eastern Brazil. 


\section{Nyctibius jamaicensis.}

Caprimulgus jamaicensis, Gm. Syst. Nat. i. p. 1029.

Nyctibius jamaicensis, Scl. P.Z. S. 1866, p. 129.

a (Mather), 1840.

A species found in Jamaica and having a wide range in South America.

\section{SUBFAMILY.-CAPRIMULGIN $Æ$.}

\section{PodAgER.}

Podager, Wagler, Isis, 1832, p. 277.

\section{Podager nacunda.}

Caprimulgus nacunda, Vieill. N. Dict. d'Hist. N. x. p. 240.

Podager nacunda, Scl. P. Z. S. 1866, p. 132.

a Brazil (Thomas) 1840.

\section{LUROCALIS.}

Lurocalis, Cassin, Pr. Ac. Phil. 1851, p. 189.

\section{Luracalis nattereri.}

Caprimulgus nattereri, Temm. Pl. Col. 107.

Lurocalis nattereri, Scl. P. Z. S. 1866, p. 132. a (N. C. Strickland) 1838.

A South Brazilian species.

\section{Chordeiles.}

Chordeiles, Swainson, Fauna Bor. Am. ii. p. 496 (1831), as a subgenus.

\section{Chordeiles popetue.}

Caprimulgus popetue, Vieill. Ois. Am. Sept. i. p. 56, pl. 24.

Chordeiles popetue var. popetue, Baird, Brew. \& Ridgw. N. Am. B. ii. p. 401. 1837.

$a,-b$ N. America? (Askew) 1834. - b N. America? (Askew)

\section{Chordeiles texensis.}

Chordeiles texensis, Lawr. Ann. Lyc. N. York, vi. p. 167 : Scl. P. Z. S. 1866, p. 134.

$a$ (N. C. Strickland) 1838. -b (Thomas) 1840.

A species of the South-western States of America and also found in Central America. 


\section{Caprimulgus.}

Caprimulgus, Linnæus, Syst. Nat. i. p. 346 (1766).

1835. Caprimulgus europæus.

Caprimulgus europceus, Linn. l. s. c.: Dresser, B. Eur.

a Worcestershire, August (H. E. Strickland) 1833. -b W. Africa (N. C. Strickland) 1838. —c (Arthur Strickland) 1840. -d ₹ Worcestershire, June (H. E. Strickland) 1841. - e Gloucestershire, June (H. E. Strickland) 1849.

\section{Caprimulgus nubicus.}

Caprimulgus nubicus, Licht. Verz. Doubl. p. 59.

Caprimulgus infuscatus, Cretzschm. in Rüpp. Zool. Atl. Vög. pl. 6.

a Kartoum, 25 September J. Petherick) 1848.

183\%. Caprimulgus ruficollis.

Caprimulgus ruficollis, Temm. Man. Orn. i. p. 438 : Dresser, B. Eur.

$a$ Tangier (M. Favier) 1847. -b Tangier (Favier) 1851.

1838. Caprimulgus indicus.

Caprimulgus indicus, Lath. Ind. Orn. p. 588: Jerd. B. Ind. i. p. 192.

Caprimulgus saturatior, Hodgs. Zool. Misc. p. 82.

a India ("Caprimulgus indicus," E. Blyth) 1846.

1839. Caprimulgus kelaarti.

Caprimulgus kelaarti, Blyth, J. A. S. B. xx. p. 175 : Jerd. B. Ind. i. p. 193.

$a$ S. India ("Caprimulgus indicus," T. C. Jerdon) $18 \check{0}$.

1840. Caprimulgus albonotatus.

Caprimulgus albonotatus, Tick. J. A. S. B. ii. p. 580 : Strickl. P. Z. S. 1846, p. 99 : Jerd. B. Ind. i. p. 194.

Caprimulgus gagateus, Strick. MS. $c f$. Blyth, J. A. S. B. xiv. p. 205.

$a$ Himalaya Mts. (N. C. Strickland) 1838. - b India (Bt. at Stevens's) 1844. — - India ("Caprimulgus albonotatus," E. Blyth) 1846. 


\section{Caprimulgus macrurus.}

Caprimulgus macrurus, Horsf. Trans. Linn. Soc. xiii. p. 142 : Strickl. P. Z. S. 1846, p. 99 : Jerd. B. Ind. i. p. 195. 1848.

a Malacca (W. Kirtland) 1843. - b Malacca (Williams)

\section{Caprimulgus atripennis.}

Caprimulgus atripennis, Jerd. Ill. Ind. Orn. sub. pl. 24; B. Ind. i. p. 196.

Caprimulgus mahrattensis, Blyth, et Strickland (nec Sykes).

a Madras ("Caprimulgus atripennis, Jerd. mahrattensis, Sykes??", T. C. Jerdon) 1845. - b S. India ("Caprimulgus atripennis, Jerd. mahrattensis, Sykes??" T. C. Jerdon) 1850.

\section{Caprimulgus asiaticus.}

Caprimulgus asiaticus, Lath. Ind. Orn. p. 588 : Jerd. B. Ind. i. p. 197.

a India ? (N. C. Strickland) 1838. - b Madras "Caprimulgus asiaticus," T. C. Jerdon) 1845. —c India ("Caprimulgus asiaticus," E. Blyth) 1846. —d (Bt. at Birmingham) 1849. -e (Dewgard) 1851.

\section{Caprimulgus monticolus.}

Caprimulgus monticolus, Frankl. P. Z. S. 1830-1, p. 116 : Jerd. B. Ind. i. p. 198.

$a$ (Bt. at Stevens's) 1844. - b, —c India ("Caprimulgus monticolus," E. Blyth) 1846. —d N. India (W. J. E. Boys) 1848. -e, - $f$ S. India ("Caprimulgus monticolus," T. C. Jerdon) 1850.

\section{Caprimulgus rufigena.}

Caprimulgus rufigena, Smith, Ill. Zool. S. Afr. Birds, pl. 100: Gurney, in And. B. Damaral. p. 44.

Caprimulgus damarensis, Strickl. Contr. Orn. 1852, p. 143.

a Damara, S. Africa (C. J. Andersson) 1852.

The type of Strickland's $C$. damarensis.

1846. Caprimulgus pectoralis.

Caprimulgus pectoralis, Cuv. Règn. Anim. i. p. 376 (1817).

Caprimulgus atrorarius, Sundev. Öfvers. Vet. Ak.1851, p.128. 
a (N. C. Strickland) 1838.

This specimen bears Sundevall's name. Mr Sharpe considers it to belong to the true $C$. pectoralis, Cuv. [ $C f$. Layard's B. of S. Afr. Ed. 2, i. p. 84.]

\section{4\%. Caprimulgus furvidus.}

Caprimulgus furvidus, Sharpe, Layard's B. S. Afr. Ed. 2, i. p. 85.

Caprimulgus pectoralis, Strickl. \& Scl. Contr. Orn. 1852, p. 143 ; Gurney, in And. B. Damaral. p. 45.

a Damara, S. Africa (C.J. Andersson) 1852.

The specimen referred to $C$. pectoralis by Strickland and Sclater l.s.c.

\section{Caprimulgus lentiginosus.}

Caprimulgus lentiginosus, Smith, Ill. Zool. S. Afr. Birds, pl. 101 : Strickl. \& Sclater, Contr. Orn. 1852, p. 143: Gurney, in And. B. Damaral. p. 45.

a Damara, S. Africa (C. J. Andersson) 1852.

\section{SCOTORNIS.}

Scortornis, Swainson, Classif. B. ii. p. 339 (1837).

\section{Scotornis longicauda.}

Caprimulgus longicauda, Drap. Dict. Class. d'Hist. N. vi. p. 169.

Scotornis longicauda, Cass. Cat. Capr. Ac. Nat. Sc. Phil. p. 4. Scotornis climacurus, Strickl. P. Z. S. 1850, p. 216. a Kordofan (j. Petherick) 1848.

\section{MACRODIPTERYX.}

Macrodipteryx, Swainson, Classif. B. ii. p. 339. (1837).

1850. Macrodipteryx longipennis.

Caprimulgus longipennis, Shaw, Nat. Misc. viii. pl. 265.

Macrodipteryx longipennis, Gray, List Gen. B. p. 8 (1840).

Caprimulgus infuscatus, Strickl. P. Z. S. 1850, p. 216 (nec Cretzschm.).

$a$ (W. Kirtland) 1843. -b Kordofan (J. Petherick) 1848. -c (Seeman) 1853. 


\section{Antrostomus.}

Antrostomus, Gould, Icon. Avium, Part ii. (1838).

\section{Antrostomus carolinensis.}

Caprimulgus carolinensis, Gm. Syst. Nat. i. p. 1028.

Antrostomus carolinensis, Scl. P. Z. S. 1866, p. 136 : Baird, Brew. \& Ridgw. N. Am. B. ii. p. 410.

$a$ (N. C. Strickland) 1838.

A common North American species.

\section{Antrostomus vociferus.}

Caprimulgus vociferus, Wils. Am. Orn. v. p. 71, pl. 41, f. 1, $2,3$. Antrostomus vociferus, Scl. P. Z. S. 1866, p. 137 : Baird, Brew. \& Ridgw. N. Am. B. ii. p. 413.

$a$ Guatemala ( $J$. Constancia) 1851.

\section{Antrostomus macromystax.}

Caprimulgus macromystax, Wagl. Isis, 1831, p. 533.

Antrostomus macromystax, Cass. Proc. Ac. Phil. 1851, p. 183 : Scl. P. Z. S. 1866, p. 137.

a Mexico, October, 1844 (Galeotti) 1845.

\section{Antrostomus Ocellatus.}

Caprimulgus ocellatus, Tsch. Arch. f. Naturg. 1844, p. 268, et Fauna Per. pl. 5, f. 2.

Antrostomus ocellatus, Scl. P. Z. S. 1866, p. 137.

a (Seeman) 1853.

A Brazilian species.

\section{Antrostomus parvulus.}

Caprimulgus parvulus, Gould, P.Z. S. 1837, p. 22. Antrostomus parvulus, Scl. P.Z. S. 1866, p. 138, pl. 13. $a$ (N. C. Strickland) 1838. —b Bahia (S. Dutton) 1846.

\section{Hydropsalis.}

Hydropsalis, Wagl. Isis, 1832, p. 1222.

1856. Hydropsalis torquata.

Caprimulgus torquatus, Gm. Syst. Nat. i. p. 1032. 
Hydropsalis torquata, Cass. Journ. Ac. Phil. ii. p. 114 : Scl. P. Z. S. 1866, p. 142.

a (Bt at Edinburgh) 1852.

A species of South-eastern Brazil.

185\%. Hydropsalis forcipata.

Caprimulgus forcipatus, Nitzsch, Pter. p. 125.

Hydropsalis forcipata, Burm. Syst. Übers. ii. p. 380 : Scl. P. Z. S. 1866, p. 143.

a Brazil (N. C. Strickland) 1838.

\section{Nyctidromus.}

Nyctidromus, Gould, Icon. Avium, Part ii. (1838).

\section{Nyctidromus albicollis.}

Caprimulgus albicollis, Gm. Syst. Nat. i. p. 1030.

Nyctidromus albicollis, Scl. P.Z. S. 1866, p. 144.

$a$ (Thomas) 1843. - b Bahia (S. Dutton) 1846. -c Guatemala(J. Constancia) 1848. - $d$ Brazil? (Argent) 1852.-e (Argent) 1852. $-f$ (Bt. at Liverpool) 1853.

\section{FAMILY.-STEATORNITHIDA.}

\section{Steatornis.}

Steatornis, Humboldt, Bull. Soc. Philom. 1817, p. 51.

\section{Steatornis caripensis.}

Steatornis caripensis, Humb. l. s. c.: Scl. P. Z. S. 1866, p. 130.

a Colombia (Galeotti) 1845. 


\section{ORDER III.-PICI.}

\section{FAMILY.-PICIDA.}

\section{SUBFAMILY.-IYNGIN Æ世.}

\section{Irnx.}

Yunx, Linnæus, Syst. Nat. i. p. 172 (1766).

Iynx, Sundevall, Consp. Av. Pic. p. 107.

\section{Iynx torquilla.}

Yunx torquilla, Linn. l. s. c.: Strickl. P. Z. S. 1842, p. 168 ; 1850, p. 219 : Jerd. B. Ind. i. p. 303: Dresser, B. Eur.

$a$ Britain (Askew) 1838. - b Altai (T. F. Brandt) 1842. - c Madras ("Junx torquilla," T. C. Jerdon) 1845. —d ई Malwa (W. J. E. Boys) 1847. - e ô Kordofan, March (J. Petherick) 1848.

\section{Iynx pectoralis.}

Yunx pectoralis, Vigors, P. Z. S. 1830-1, p. 93: Layard, B. S. Afr. p. 240.

$a$ S. Africa (Mrs Van der Kemp) 1843.

\section{Picumnus.}

Picumnus, Temminck, Pl. Col. Livr. 62 (1825).

\section{Picumnus cirratus.}

Picumnus cirratus, Temm. Pl. Col. 371: Sundev. Consp. Av. Pic. p. 98.

$a$ (N. C. Strickland) 1838. - b S. America (N. C. Strickland) 1838. - c Brazil (N. C. Strickland) 1838.

\section{Picumnus sp.?}

a Brazil (Johnson) 1837.

A young bird, supposed by Strickland to be $P$. minutus, but apparently not belonging to that species. I have not materials to determine it satisfactorily. 
1864. Picumnus minutus.

Pipra minuta, Linn. Syst. Nat. i. p. 240.

Picumnus minutus, Sundev. Consp. Av. Pic. p. 98.

a Brazil (Askew) 1834.

\section{Picumnus pygmæus.}

Picus pygmceus, Licht. Verz. Doubl. p. 12.

Picumnus pygmceus, Malh. Mon. Pic. ii. p. 280, iv. pl. 115, ff. 3, 4 : Cab. \& Hein. Mus. Hein. iv. p. 23.

$a$ Brazil (Johnson) 1837. -b (Bt at Birmingham) 1849.

\section{Picumnus lepidotus.}

Picumnus lepidotus, Cab. \& Hein. Mus. Hein. iv. p. 14: Sundev. Consp. Av. Pic. p. 102.

$a$ (N. C. Strickland) 1838.

The male specimen of this species in the Stockholm museum is said to have come from Surinam.

\section{Picumnus albosquamatus?}

Picumnus albosquamatus, d’Orb. Voy. Am. Mér. Ois. pp. 330, 380, pl. 64, f. 2.

$a,-b$ Brazil ? (Argent) 1852.

These are most probably Bolivian specimens, perhaps belonging to this species. They differ, however, from d'Orbigny's figure in having the feathers of the undersurface edged with black and in the back being spotted with indistinct white spots.

\section{SASIA.}

Sasia, Hodgson, J. A. S. B. v. p. 778 (1836).

1868. Sasia ochracea.

Sasia ochracea, Hodgson, l. s. c.; Jerd. B. Ind. i. p. 301 ; Ibis, 1872, p. 10: Blyth, J. A. S. B. xliii. Extr. No. p. 78. a Arakan (E. Blyth) 1847. 


\section{SUBFAMILY.-PICIN E.}

Campephilus.

Campephilus, G. R. Gray, List Gen. B. p. 54 (1840). 1869. Campephilus melanoleucus.

Picus melanoleucos, Gm. Syst. Nat. i. p. 426: Sundev. Consp. Av. Picin. p. 5.

Picus albirostris, Vieill. N. Dict. d'Hist. N. xxvi. p. 69.

Megapicus albirostris, Malh. Mon. Pic. i. p. 17, iii. pl. 4.

a f Brazil (N. C. Strickland) 1838.

\section{Campephilus malherbii.}

Campephilus malherbii, Gray \& Mitch. Gen. B. pl. 108: Scl. Cat. Am. B. p. 331.

Megapicus malherbii, Malh. Mon. Pic. i. p. 15, iii. pl. 6.

a Bogota (Williams) 1846.

1871. Campephilus rubricollis.

Picus rubricollis, Gm. Syst. Nat. i. p. 426: Sundev. Consp. Av. Picin. p. 6.

Campephilus rubricollis, Scl. Cat. Am. B. p. 332.

Megapicus rubricollis, Malh. Mon. Pic. i. p. 25, iii. pl. 8, ff. 6,7 .

a Demarara (T. C. Eyton) 1846.

\section{Campephilus trachelopyrus.}

Megapicus trachelopyrus, Malh. Mém. Soc. N. H. Moselle, 1857, p. 1 ; Mon. Pic. i. p. 26, pl. 8, ff. 2, 3.

Campephilus trachelopyrus, Scl. Cat. Am. B. p. 332.

a (Thomas) 1843.

A species of the Upper Amazon, Peru, \&c.

1873. Campephilus robustus.

Picus robustus, Licht. Verz. Doubl. p. 10: Sundev. Consp. Av. Picin. p. 6.

Megapicus robustus, Malh. Mon. Pic. i. p. 23, iii. pl. 3, ff. 4, 5. $a \hat{b},-b$ o Brazil (N. C. Strickland) 1838. -c f Brazil (G. Lloyd) 1838. 


\section{Campephilus validus.}

Picus validus, Temm. Pl. Col. 378, 402; Sundev. Consp. Av. Picin. p. 7.

Megapicus validus, Malh. Mon. Pic. i. p. 28, iii. pl. 9, ff. 5, 6. a Malacca (Mather) 1840.

\section{Dryocopus.}

Dryocopus, Boie, Isis, 1826, p. 977.

\section{Dryocopus martius.}

Picus martius, Linn. Syst. Nat. i. p. 173 : Sundev. Consp. Av. Picin. p. 9.

Dryopicus martius, Malh. Mon. Pic. i. p. 34. iii. pl. 10, ff. $5,6,7$.

Dryocopus martius, Strickl. P. Z. S. 1836, p. 100 ; Ann. \& Mag. N. H. vii. p. 37 : Dresser, B. Eur.

$a$ Switzerland (W. Andercgg) 1836. - b N. Europe $(J . G$. Kinberg) 1843.

Included by Strickland in his list of the birds of Asia Minor, a specimen having been seen by him at Broussa which had been shot in the pine forests of Mount Olympus.

\section{8\%6. Dryocopus pileatus.}

Picus pileatus, Linn. Syst. Nat. i. p. 173 : Sundev. Consp. Av. Picin. p. 8.

Dryopicus pileatus, Malh. Mon. Pic. i. p. 34, iii. pl. 11.

Hylotomus pileatus, Baird, Brew. \& Ridgw. N. Am. B. ii, p. 550 .

a. $\delta$ N. America (N. C. Strickland) 1838. - $b$ q N. America.

\section{7\%. Dryocopus lineatus.}

Picus lineatus, Linn. Syst. Nat. i. p. 174: Sundev. Consp. Av. Picin. p. 7.

Dryopicus lineatus, Malh. Mon. Pic. i. p. 39, iii. pl. 12, ff. $4,5,6,7$.

Dryocopus lineatus, Scl. Cat. Am. B. p. 332.

$a$ Brazil (Askew) 1834. $-b \hat{f},-c$ Brazil (N. C. Strickland) 1838. - d (Bt at Stevens's) 1845. 


\section{Dryocopus scapularis.}

Picus scapularis, Vig. Zool. Journ. iv. p. 354.

Dryopicus scapularis, Malh. Mon. Pic. i. p. 44, iii. pl. 10, f. $1,2,3$.

Dryocopus scapularis, Scl. Cat. Am. B. p. 333.

$a$ Guatemala ( $J$. Constancia) 1845.

\section{Dryocopus erythrops.}

Picus erythrops, Valenc. Dict. Sc. Nat. xl. p. 178.

Dryopicus erythrops, Malh. Mon. Pic. i. p. 42, iii. pl. 12, f. 1,2 . 1838.

$a$ \& Brazil (N. C. Strickland) 1838. -b (N. C. Strickland)

\section{Dryocopus leucogaster.}

Picus javensis, Horsf. Trans. L. S. xiii. p. 175 (nec Ljungh).

Thriponax javensis, Cab. \& Hein. Mus. Hein. iv. p. 105.

Picus leucogaster, Temm. Pl. Col. 501: Sundev. Consp. Av. Picin. p. 9.

Dryopicus leucogaster, Malh. Mon. Pic. i. p. 47, iii. pl. 13, f. 4, 5. a Java (N. C. Strickland) 1838.

\section{ALOPHONERPES.}

Alophonerpes, Reichenbach, Handb. p. 385 (1854).

Mulleripicus, Bonaparte.

\section{Alophonerpes pulverulentus.}

Picus pulverulentus, Temm. Pl. Col. 389.

Mulleripicus pulverulentus, Wald. Ibis, 1871, p. 164.

Picus gutturalis, Valenc. Dict. Sc. Nat. xl. p. 178.

Alophonerpes gutturalis, Cab. \& Hein. Mus. Hein. iv. p. 106.

a Java (N. C. Strickland) 1838. - b (Mansfield) 1849.

\section{PICUs.}

Picus, Linnæus, Syst. Nat. i. p. 173 (1766) partim.

\section{Picus major.}

Picus major, Linn. l. s. c.: Dresser B. Eur.

a $\hat{\delta}$ Worcestershire (H. E. Strickland) 1834. $-b \hat{\jmath}$ Worcestershire (T. Robinson) 1838. -c f Gloucestershire, January (H. E. Strickland) 1850.

S. C. 


\section{Picus syriacus.}

Picus syriacus, Hempr. \& Ehr. Symb. Phys. Av. fol. $r$ : Dresser, B. Eur.

Picus major, Strickl. P. Z. S. 1836, p. 100 (nec Linn.).

a f Smyrna (H. E. Strickland) 1836.

1884. Picus numidicus.

Picus numidicus, Malh. Mém. Ac. Metz, ii. p. 242 : Strickl. Ann. \& Mag. N. H. xiv. p. 433: Dresser, B. Eur.

a Tangier (Favier) 1848.

\section{Picus himalayanus.}

Picus himalayanus, Jard. \& Selb. Ill. Orn. iii. pl. 116: Malh. Mon. Pic. i. p. 67, iii. pl. 19 : Jerd. B. Ind. i. p. 269.

$a,-b$ Himalaya (N. C. Strickland) 1838. - c (Bt at Stevens's) 1843. —d India ("Picus himalayanus," E. Blyth) 1851.

1886. Picus medius.

Picus medius, Linn. Syst. Nat. i. p. 176: Dresser, B. Eur. a Europe (Askew) 1836.

\section{8\%. Picus leuconotus.}

Picus leuconotus, Bechst. Naturg. Deutschl. ii. p. 1034: Strickl. Ann. \& Mag. N. H. xiv. p. 349: Dresser, B. Eur.

$a \hat{\jmath},-b q$ Norway (N. C. Strickland) 1838.

\section{Picus minor.}

Picus minor, Linn. Syst. Nat. i. p. 176: Dresser, B. Eur.

$a \hat{\delta}$ Worcestershire (T. Robinson) 1838. - b N. Europe ( $J$. G. Kinberg) 1843. - c $\hat{o}$ Gloucestershire, January (H. E. Strickland) 1850.

1889. Picus pubescens.

Picus pubescens, Linn. Syst. Nat. i. p. 175: Malh. Mon. Pic. i. p. 119, iii. pl. 29: Baird, Brew. \& Ridgw. N. Am. B. ii. p. 509.

$a \hat{\delta}$ N. America (Audubon) 1834. - $b$ o N. America (Arthur Strickland) 1834. -c q (N. C. Strickland) 1838.

1890. Picus borealis.

Picus borealis, Vieill. Ois. Am. Sept. ii. p. 66, pl. 122 : Baird, Brew. \& Ridgw. N. Am. B. ii. p. 524. 
Picus querulus, Malh. Mon. Pic. i. p. 134, iii. pl. 31.

a ô Georgia, 1846 (S. F. Baird) 1847.

\section{Picus villosus.}

Picus villosus, Linn. Syst. Nat. i. p. 175: Malh. Mon. Pic. i. p. 75, iii. pl. 21 : Sundev. Consp. Av. Picin. p. 16: Baird, Brew. \& Ridgw. N. Am. B. ii. p. 503.

a \& N. America (Arthur Strickland) 1840.

\section{Picus jardinii.}

Picus jardinii, Malh. Rev. Zool. 1845, p. 374; Mon. Pic. i. p. 103, iii. pl. 25, f. 4, 5: Scl. Cat. Am. B. p. 334.

a Mexico (T. Mann) 1844.

1893. Picus scalaris.

Picus scalaris, Wagl. Isis, 1829, p. 511: Malh. Mon. Pic. i. p. 116, iii. pl. 17 : Scl. Cat. Am. B. p. 333.

$a$ (Arthur Strickland) 1850.

A Mexican species.

1894. Picus stricklandi.

Picus stricklandi, Malh. Rev. Zool. 1845, p. 373; Mon. Pic. i. p. 108, iii. pl. 28, f. 4, 5, 6: Scl. Cat. Am. B. p. 334.

a Mexico (T. Mann) 1844.

\section{Picus lignarius.}

Picus lignarius, Mol. Sagg. sulla St. Nat. Chili, p. 215: Malh. Mon. Pic. i. p. 109, iii. pl. 26, f. 9, 10: Scl. Cat. Am. B. p. 334.

Picus melanocephalus, King, P. Z. S. 1830-1, p. 14.

$a$ S. America (N. C. Strickland) 1838.

A Chilian species.

1896. Picus darjilenensis.

Picus majoroides, Hodgs. Zool. Misc. p. 85 (descr. nulla): Jerd. B. Ind. i. p. 270.

Picus darjellensis, Blyth, J. A. S. B. xiv. p. 196.

a Nepal (B. H. Hodgson, 156) 1845.

189'. Picus macæi.

Picus macei, Vieill. N. Dict. d'Hist. N. xxvi. p. 80: Jerd. B. Ind. i. p. 272.

$a$ q Himalaya (Askew) 1839. - b Darjeeling (E. Blyth) 1850. 


\section{Picus auriceps.}

Picus auriceps, Vig. P. Z. S. 1830-1, p. 44: Sundev. Consp. Av. Picin. p. 30.

Picus brunneifrons, Gould, Cent. B. Him. pl. 52: Jerd. B. Ind. i. p. 273: Malh. Mon. Pic. i. p. 107, iii. pl. 18, f. 5, 6.

a ồ Himalaya (N. C. Strickland) 1838.

1899. Picus mahrattensis.

Picus mahrattensis, Lath. Ind. Orn. Suppl. p. xxxi.: Malh. Mon. Pic. i. p. 10こ, iii. pl. 28, f. 1, 2: Jerd. B. Ind. i. p. 274.

$a$ (Fenwick) 1844. - b Madras ("Picus mahrattensis," T. C. Jerdon) 1845 .

1900. Picus hyperythrus.

Picus hyperythrus, Vig. P. Z. S. 1830-1, p. 23: Gould, Cent. B. Him. pl. 50: Malh. Mon. Pic. i. p. 130, iii. pl. 30, f. 4, 5.

Hypopicus hyperythrus, Jerd. B. Ind. i. p. 276.

$a$ Nepal (B. H. Hodgson, 151) 1845.

\section{Picus semicoronatus.}

Picus semicoronatus, Malh. Bull. Soc. d'Hist. Nat. Metz, 1848, p. 21; Mon. Pic. i. p. 148, iii. pl. 34, f. 8: Jerd. Ibis, 1872, p. 8.

Picus rubricatus, Blyth, Cat. B. Asiat. Soc. p. 63.

Yungipicus rubricatus, Jerd. B. Ind. i. p. 276.

a Darjeeling ("Picus rubricatus," E. Blyth) 1850.

\section{Picus pygmæus.}

Picus pygmceus, Vig. P. Z. S. 1830-1, p. 44: Malh. Mon. Pic. i. p. 147 , iii. pl. 34 , f. 5, 6,7 .

Yungipicus pygmaeus, Jerd. B. Ind. i. p. 277.

a (Bt at Stevens's) 1844.

A Himalayan species.

\section{Picus nanus.}

Picus nanus, Vig. P. Z. S. 1830-1, p. 172: Malh. Mon. Pic. i. p. 145 , iii. pl. 33 , f. $1-7$.

Picus Dendrocopus hardwickii, Jerd. Madr. Journ. xiii. pt. 2, p. 138.

Yungipicus hardwickii, Jerd. B. Ind. i. p. 278.

Picus moluccensis, Gray, Ill. Ind. Zool. i. pl. 33, f. 1 (nec Gm.). 
$a$ Madras ("Picus moluccensis," T. C. Jerdon) 1845. - b Travancore ("Dendrocopus moluccensis," T. C. Jerdon) 1848.

\section{SPHYROPICUS.}

Sphyrapicus, Baird, B. N. Am. p. 101 (1858).

\section{Sphyropicus varius.}

Picus varius, Linn. Syst. Nat. i. p. 176.

Sphyrapicus, Baird, l. s. c.: Malh. Mon. Pic. i. p. 157, iii. pl. 37, f. 2-5: Scl. Cat. Am. B. p. 335.

$a$ N. America (Askew) 1833. - b N. America (Arthur Strickland) 1834. —c Guatemala (J. Constancia) 1845. —d Guatemala (J. Constancia) 1848.

\section{Picoides.}

Picoides, Lacépède, Mém. de l'Inst. iii. p. 509 (1801).

\section{Picoides tridactylus.}

Picus tridactylus, Linn. Syst. Nat. i. p. 177.

Picoides tridactylus, Dresser, B. Eur.

Apternus crissoleucus, Bp. Consp. Vol. Zyg. p. 9 (ex Brandt M.S.).

Picus crissoleucus, Sundev. Consp. Av. Picin. p. 14.

$a \hat{\delta},-b$ O Switzerland ( $W$. Anderegg) 1836. $-c,-d$ Siberia (J. F. Brandt) 1846.

$c$ and $d$ belong to the race prevalent in Siberia called P. crissoleucus by Brandt, but which Mr Dresser does not consider specifically distinct from $P$. tridactylus.

\section{Gecinus.}

Gecinus, Boie, Isis, 1831, p. 542.

\section{Gecinus viridis.}

Picus viridis, Linn. Syst. Nat. i. p. 175 : Sundev. Consp. Av. Picin. p. 60.

Gecinus viridis, Dresser, B. Eur.

Chloropicus viridis, Malh. Mon. Pic. ii. p. 118, iv. pl. 79, f. $1-4$.

$a$ Worcestershire (H. E. Strickland) 1830. -b (N. C. Strickland) 1838. 
190\%. Gecinus canus.

Picus canus, Gm. Syst. Nat. i. p. 434: Sundev. Consp. Av. Picin. p. 61.

Gecinus canus, Dresser, B. Eur.

Chloropicus canus, Malh. Mon. Pic. ii. p. 124, iv. pl. 81. 1838.

a Switzerland (W. Anderegg) 1836. -b (N.C. Strickland)

\section{Gecinus occipitalis.}

Picus occipitalis, Vig. P. Z. S. 1830-1, p. 8: Gould, Cent. B. Him. pl. 47 : Sundev. Consp. Av. Picin. p. 61.

Gecinus occipitalis, Jerd. B. Ind. i. p. 287.

Chloropicus occipitalis, Malh. Mon. Pic. ii. p. 129, iv. pl. 77. $a \hat{\delta},-b$ q Himalaya (N. C. Strickland) 1838. -c Nepal (B. H. Hodgson, 149) 1845. - $d$ I India ("Gecinus barbatus (Gray), occipitalis (Vig.), affinis? (Raffl.)," E. Blyth) 1847.

1909. Gecinus viridanus.

Picus viridanus, Blyth, J. A. S. B. xii. p. 1000.

Gecinus viridanus, Blyth, J. A. S. B. xliii. extr. No. p. 76. a Arakan (E. Blyth) 1847.

Hardly separable from $G$. vittatus.

\section{Gecinus vittatus.}

Picus vittatus, Vieill. N. Dict. d'Hist. N. xxvi. p. 91: Sundev. Consp. Av. Picin. p. 59.

Gecinus vittatus, Salvad. Ann. Mus. Genov. v. p. 51.

Chloropicus dimidiatus, Malh. Mon. Pic. ii. p. 132, iv. pl. 76. $a$ (Arthur Strickland) 1840. -b Himalaya (Askew) 1840.

This species is from Java, Borneo, \&c. but not from India.

\section{Gecinus striolatus.}

Gecinus striolatus, Blyth, J. A. S. B. xii. p. 1000: Jerd. B. Ind. i. p. 287.

Picus striolatus, Sundev. Consp. Av. Picin. p. 60.

Chloropicus striolatus, Malh. Mon. Pic. ii. p. 134, iv. pl. 77.

$a$ (Gardner) 1845. -b Madras ("Picus viridanus, Bl.," T. C. Jerdon) 1845. — India ("Gecinus striolatus," E. Blyth) 1846. 
1912. Gecinus squamatus.

Picus squamatus, Vig. P. Z. S. 1830-1, p. 8: Gould, Cent. B. Him. pl. 48: Sundev. Consp. Av. Picin. p. 59.

Gecinus squamatus, Jerd. B. Ind. i. p. 286.

Chloropicus squamatus, Malh. Mon. Pic. ii. p. 131, iv. pl. 78. a Himalaya (Bt at Stevens's) 1843.

Chrysophlegma.

Chrysophlegma, Gould, B. Asia, Part I. (1849).

1913. Chrysophlegma flavinucha.

Picus flavinucha, Gould, P. Z. S. 1833, p. 120: Sundev. Consp. Av. Picin. p. 57.

Chrysophlegma flavinucha, Jerd. B. Ind. i. p. 289: Blyth, J. A. S. B. xliii. extr. No. p. 76.

Chloropicus flavinucha, Malh. Mon. Pic. ii. p. 106, iv. pl. 73. $a$ India (E. Blyth) 1846. - b, —c Arakan (E. Blyth) 1847.

\section{Chrysophlegma chlorolophus.}

Picus chlorolophus, Vieill. N. Dict. d'Hist. N. xxvi. p. 78: Sundev. Consp. Av. Picin. p. 58.

Chrysophlegma chlorolophus, Jerd. B. Ind. i. p. 289.

Chloropicus chlorolophus, Malh. Mon. Pic. ii. p. 108, iv. pl. 74.

Picus nipalensis, Gray, Ill. Ind. Zool. i. pl. 31, f. 1.

Brachylophus sericeicollis, Hodgs. Zool. Misc. p. 85.

a India (Gardner) 1845. -b, - c Nepal (B. H. Hodgson, 146) 1845. —d India ("Gecinus chloropus (Vieill.), nipalensis (Gray)," E. Blyth) 1846.

\section{Chrysophlegma xanthoderus.}

Chloropicus xanthoderus, Malh. Rev. Zool. 1845, p. 402; Mon. Pic. ii. p. 114, iv. pl. 75.

Picus xanthoderus, Sundev. Consp. Av. Picin. p. 58.

Picus chlorigaster, Jerd. Madr. Journ. xiii. p. 138.

Chrysophlegma chlorophanes, Jerd. B. Ind. i. p. 290 (nec Vieill.).

a Madras (Bt at Stevens's) 1843.

1916. Chrysophlegma mentalis.

Picus mentalis, Temm. Pl. Col. 384: Sundev. Consp. Av. Picin. p. 58. 
Brachylophus mentalis, Strickl. P. Z. S. 1841, p. 31.

Chloropicus mentalis, Malh. Mon. Pic. ii. p. 112, iv. pl. 75.

Callolophus mentalis, Salvad. Ann. Mus. Genov. v. p. 49. a Malacca (Seaman) 1840.

\section{Chrysophlegma malaccensis.}

Picus malaccensis, Lath. Ind. Orn. i. p. 241.

Venilia malaccensis, Scl. P. Z. S. 1863, p. 211.

Callolophus malaccensis, Salvad. Ann. Mus. Genov. v. p. 50.

Chloropicus miniatus, Malh. Mon. Pic. iii. p. 116, iv. pl. 76 (nec Forster).

Brachylophus miniatus, Strickl. P. Z. S. 1841, p. 31.

$a,-b$ Malacca (N. C. Strickland) 1838.

\section{GauropicoIdes.}

Gauropicoides, Malherbe, Mon. Pic. i. p. liii. (1861).

1918. Gauropicoides rafflesi.

Picus rafflesi, Vig. Mem. Raffl. App. p. 669: Strickl. P. Z. S. 1846, p. 103: Sundev. Consp. Av. Picin. p. 57.

Tiga rafflesi, Blyth, J. A. S. B. xv. p. 16.

Gauropicoides rafflesi, Malh. l. s. c.: Salvad. Ann. Mus. Genov. v. p. 54.

$a(E$. Brown $) 1850$.

A Malacca skin.

Dendrobates.

Dendrobates, Swainson, Classif. B. ii. p. 306 (1837).

\section{Dendrobates namaquus.}

Picus namaquus, Licht. Cat. Hamb. p. 17 (fide Cabanis): Sundev. Consp. Av. Picin. p. 42.

Dendrobates namaquus, Strickl. \& Scl. Contr. Orn. 1852,p. 155 .

Thripias namaquus, Cab. \& Hein. Mus. Hein. iv. p. 121 : Gurney, in And. B. Damara-l. p. 219.

Dendropicus biarmicus, Malh. Mon. Pic. i. p. 193, iii. pl. 42.

$a$ \& S. Africa (N. C. Strickland) 1838. - b Damara-land ( $C$. J. Andersson) 1852.

\section{Dendrobates cardinalis.}

Picus cardinalis, Gm. Syst. Nat. i. p. 438: Sundev. Consp. Av. Picin. p. 42. 
Dendropicus cardinalis, Gurney, in And. B. Damara-l. p. 220.

Dendrobates fuscescens, Vieill. N. Dict. d'Hist. N. xxvi. p. 86 : Strickl. \& Scl. Contr. Orn. 1852, p. 155.

Picus fulviscapus, Licht. Verz. Doubl. p. 11.

Dendropicus fulviscapus, Malh. Mon. Pic. i. p. 196, iii. pl. 43.

$a$ S. Africa (N. C. Strickland) 1838. - b Cape of Good Hope (N. C. Strickland) 1838. — D Damara-land (C. J. Andersson) 1852.

\section{Dendrobates goertan.}

Picus goertan, Gm. Syst. Nat. i. p. 434: Sundev. Consp. Av. Picin. p. 45.

Mesopicus goertan, Malh. Mon. Pic. ii. p. 45, iv. pl. 63.

Scolecotheres goertan, Cab. \& Hein. Mus. Hein. iv. p. 136.

$a$ W. Africa (N. C. Strickland) 1838. -b Gambia (N. C. Strickland) 1838. —c (Thomas) 1840.

\section{Dendrobates spodocephalus.}

Dendrobates spodocephalus, Bp. Consp. Av. i. p. 125.

Mesopicus spodocephalus, Malh. Mon. Pic. ii. p. 49, iv. pl. 63.

Scolecotheres spodocephalus, Cab. \& Hein. Mus. Hein. iv. p. 137.

a Abyssinia (E. Verreaux) 1850.

\section{Dendrobates menstruus.}

Picus menstruus, Scop. Del. Faun. et Fl. Ins. ii. p. 89: Sundev. Consp. Av. Picin. p. 45.

Picus capensis, Gm. Syst. Nat. i. p. 430.

Mesopicus capensis, Malh. Mon. Pic. ii. p. 42, iv. pl. 62.

$a,-b$ o S. Africa ( $N$. C. Strickland) 1838. — (Dewgard) 1849.

\section{CAMPethera.}

Campethera, Gray, List. Gen. B. p. 70 (1841).

1924. Campothera notata.

Picus notatus, Licht. Verz. Doubl. p. 11: Sundev. Consp. Av. Picin. p. 66.

Chrysopicus notatus, Malh. Mon. Pic. ii. p. 162, iv. pl. 95. 
Ipagrus notatus, Cab. \& Hein. Mus. Hein. iv. p. 127. a S. Africa (N. C. Strickland) 1838.

\section{Campothera capricorni.}

Campethera capricorni, Strickl. \& Scl. Contr. Orn. 1852, p. 155: Newton, Ibis, 1869, p. 323, pl. 9.

Ipagrus capricorni, Gurney, in And. B. Damara-1. p. 221.

a Damara-land (C. J. Andersson) 1852.

The type of this species.

1926. Campothera punctata.

Picus punctatus, Valenc. Dict. Sc. Nat. xl. p. 171: Sundev. Consp. Av. Picin. p. 67.

Ipagrus punctatus, Cab. \& Hein. Mus. Hein. iv. p. 124.

Picus punctuligerus, Wagl. Syst. Av. fol. 2, p. 11.

Chrysopicus punctuligerus, Malh. Mon. Pic. ii. p. 164, iv. pl. 92.

Picus nubicus, Licht. Verz. Doubl. p. 11 (nec Gmelin).

a $\hat{\delta}$ W. Africa (N. C. Strickland) 1838.

\section{ChLORONERPES.}

Chloronerpes, Swainson, Classif. B. ii. p. 307 (1837).

\section{2\%. Chloronerpes fumigatus.}

Picus fumigatus, d’Orb. Voy. Am. Mérid. Ois. p. 380, pl. 65, f. 1.

Chloronerpes fumigatus, Scl. Cat. Am. B. p. 337.

Mesopicus fumigatus, Malh. Mon. Pic. ii. p. 51, iv. pl. 57, f. 3, 4. a Bogota? (Gardner) 184.5.

1928. Chloronerpes sanguineus.

Picus sanguineus, Licht. Cat. Hamb. p. 17, fide Wagl. Syst. Av. fol. 3, p. 15.

Mesopicus sanguineus, Malh. Mon. Pic. ii. p. 74, iii. pl. 60, f. 4,5 .

$a$ (T. Stevens) 1829.

A Guiana species.

1929. Chloronerpes affinis.

Picus affinis, Sw. Zool. Ill. pl. 78: Sundev. Consp. Av. Pic. p. 37. 
Mesopicus affinis, Malh. Mon. Pic. ii. p. 56, iv. pl. 61. Campias affinis, Cab. \& Hein. Mus. Hein. iv. p. 151. a (N. C. Strickland) 1838. - b (Bt at Stevens's) 1843.

A Brazilian species.

\section{Chloronerpes selysi.}

Mesopicus selysi, Malh. Mon. Pic. ii. p. 67, iv. pl. 62, f. 1, 2. Chloronerpes selysi, Scl. Cat. Am. B. p. 337. a f Brazil (Askew) 1834. -b ô Brazil (Askew) 1837.

\section{Chloronerpes tephrodops.}

Picus tephrodops, Wagl. Syst. Av. fol. 2, p. 15.

Chloronerpes tephrodops, Scl. Cat. Am. B. p. 337.

Campias tephrodops, Cab. \& Hein. Mus. Hein. iv. p. 145.

a (W. Kirtland) 1843.

Apparently a skin of Cayenne make.

1932. Chloronerpes olivinus.

Picus olivinus, Malh. Mém. Soc. Liége, 1845, p. 67 (ex Natt. MS.): Sundev. Consp. Av. Picin. p. 40.

Mesopicus olivinus, Malh. Mon. Pic. ii. p. 65, iv. pl. 59.

Campias olivinus, Pelz. Orn. Bras. p. 246.

a Brazil (N. C. Strickland) 1838. —b Brazil? (Argent) 1852.

\section{Chloronerpes erythropis.}

Picus erythropis, Vieill: N. Dict. d'Hist. N. xxvi. p. 98.

Chloropicus erythropsis, Malh. Mon. Pic. ii. p. 143, pl. 87, f. 1,2 .

Chloronerpes erythropis, Scl. Cat. Am. B. p. 338.

$a \hat{\jmath},-b$ ô Brazil (Askew) 1837.

\section{Chloronerpes flavigula.}

Pic d gorge jaune, de Cayenne, D’Aub. Pl. Enl. 784, undè, Picus flavigula, Bodd. Tabl. Pl. Enl. p. 49.

Chloronerpes flavigularis, Scl. Cat. Am. B. p. 339.

Chloropicus chlorocephalus, Malh. Mon. Pic. ii. p. 144, pl. 86, f. $4,5,6$.

$a$ (Gardner) 1845. 


\section{Chloronerpes polyzonus.}

Picus polyzonus, Valenc. Dict. Sc. Nat. xl. p. 170: Sundev. Consp. Av. Picin. p. 12.

Chloropicus polyzonus, Malh. Mon. Pic. ii. p. 136, iv. pl. 83, f. $1,2$.
$a$ (Arthur Strickland) 1840.

A Brazilian species.

\section{Chloronerpes yucatanensis.}

Picus yucatanensis, Cabot. Bost. Journ. N. H. v. p. 92.

Chloronerpes yucatanensis, Scl. Cat. Am. B. p. 339. $a$ Guatemala (J. Constancia) 1851.

193\%. Chloronerpes rubiginosus.

Picus rubiginosus, Sw. Zool. Ill. pl. 14.

Chloronerpes rubiginosus, Scl. Cat. Am. B. p. 339.

Chrysopicus rubiginosus, Malh. Mon. Pic. ii. p. 174, iv. pl. 89, f. 4,5 .

$a$ (N. C. Strickland) 1838. - b Bogota? (Bt at Stevens's) 1845. $\longrightarrow c$ Trinidad (Argent) 1851.

\section{Chrysoptilus.}

Chrysoptilus, Swainson, Faun. Bor. Am. Birds, p. 300 (1831). 1938. Chrysoptilus melanochlorus.

Grand Pic rayé, de Cayenne, D'Aub. Pl. Enl. 719 undè, Picus melanochlorus, Gm. Syst. Nat. i. p. 427: Sundev. Consp. Av. Picin. p. 75.

Chrysopicus melanochlorus, Malh. Mon. Pic. ii. p. 187, iv. pl. 87 bis.

Chrysoptilus melanochlorus, Cab. \& Hein. Mus. Hein. iv. p. 164.

$a$ S. America (N. C. Strickland) 1838.

Agrees with Rio specimens.

\section{Chrysoptilus flavilumbis.}

Picus flavilumbis, Sundev. Consp. Av. Picin. p. 74.

a interior of Bolivia (T. C. Eyton) 1850.

Agrees with Bahia specimens, apparently belonging to this form of C. chrysomelas. 


\section{Chrysoptilus punctigula.}

Pic rayé, de Cayenne, D’Aub. Pl. Enl. 613 undè,

Picus punctigula, Bodd. Tabl. Pl. Enl. p. 37 : Sundev. Consp. Av. Picin. p. 73.

Chrysoptilus punctigula, Cab. \& Hein. Mus. Hein. iv. p. 162. a (Thomas) 1843.

A skin of Cayenne make.

\section{Chrysoptilus punctipectus.}

Chrysoptilus punctipectus, Cab. \& Hein. Mus. Hein. iv. p. 163. $a,-b$ Trinidad (Argent) 1851.

A close ally of $C$. punctigula and perhaps barely separable.

\section{Melanerpes.}

Melanerpes, Swainson, Faun. Bor. Am. Birds, p. 300 (1831). 1942. Melanerpes torquatus.

Picus torquatus, Wils. Am. Orn. iii. p. 31, pl. 20, f. 3 : Sundev. Consp. Av. Picin. p. 51.

Melampicus torquatus, Malh. Mon. Pic. ii. p. 214, iv. pl. 96. Melanerpes torquatus, Baird, Brew. \& Ridgw. N. Am. B. ii. p. 561 .

$a \mathrm{~N}$. W. America (Wosnessenski) 1846.

\section{Melanerpes erythrocephalus.}

Picus erythrocephalus, Linn. Syst. Nat. i. p. 174: Sundev. Consp. Av. Picin. p. 50.

Melanerpes erythrocephalus, Baird, Brew.\& Ridgw. N. Am. B. ii. p. 564 .

Melampicus erythrocephalus, Malh. Mon. Pic. ii. p. 210, iv. pl. 97.

$a$ N. America (Askew) 1834. - b N. America (Mansfield) 1834.

\section{Melanerpes formicivorus.}

Picus formicivorus, Sw. Phil. Mag. New Ser. i. p. 439.

Melanerpes formicivorus, Baird, Brew. \& Ridgw. N. Am. B. ii. p. 566 .

Melampicus formicivorus, Malh. Mon. Pic. ii. p. 199, iv. pl. 99.

Picus melanopogon, Temm. Pl. Col. 451: Sundev. Consp. Av. Picin. p. 51. 
a Mexico (Arthur Strickland) 1840. - b Guatemala (J. Constancia) 1845. -c N. W. America (Wosnessenski) 1846. -d Guatemala ( $J$. Constancia) 1848.

\section{Melanerpes flavigula.}

Melampicus flavigula, Malh. Rev. Zool. 1849, p. 542: Mon. Pic. ii. p. 202, iv. pl. 99.

Melanerpes favigularis, Scl. Cat. Am. B. p. 341.

a Bogota (Bt at Stevens's) 1846.

\section{Melanerpes flavifrons.}

Picus flavifrons, Vieill. N. Dict. d'Hist. N. xxvi. p. 75: Sundev. Consp. Av. Picin. p. 50.

Melanerpes flavifrons, Scl. Cat. Am. B. p. 341.

Melampicus flavifrons, Malh. Mon. Pic. ii. p. 207, iv. pl. 100. $a \hat{\delta},-b$ f Brazil (N. C. Strickland) 1838.

\section{Melanerpes cruentatus.}

Petit Pic noir, de Cayenne, D'Aub. Pl. Enl. 694, f. 2 undè, Picus cruentatus, Bodd. Tabl. Pl. Enl. p. 43.

Melanerpes cruentatus, Scl. Cat. Am. B. p. 341. Picus hirundinaceus, Gm. Syst. Nat. i. p. 426. $a$ Guatemala (Bt at Stevens's) 1848.

This is a Guiana species and is not known in Central America.

\section{LEUCONERPES.}

Leuconerpes, Swainson, Classif. B. ii. p. 310 (1837).

1948. Leuconerpes candidus.

Picus candidus, Otto, Buff. Vög. Ueb. xxiii. p. 251.

Leuconerpes candidus, Scl. Cat. Am. B. p. 342.

Picus dominicanus, Vieill. N. Dict. d'Hist. N. xxvi. p. 72.

Melampicus dominicanus, Malh. Mon. Pic. ii. p. 217, iv. pl. 101.

$a$ (N. C. Strickland) 1838.

A Brazilian species.

\section{Hypoxanthus.}

Hypoxanthus, Bonaparte, Consp. Vol. Zyg. p. 11 (1854). 
1949. Hypoxanthus rivolii.

Picus rivolii, Boiss. Rev. Zool. 1840, p. 36: Sundev. Consp. Av. Picin. p. 71.

Geopicus rivolii, Malh. Mon. Pic. ii. p. 267, iv. pl. 112.

Colaptes rivolii, Scl. Cat. Am. B. p. 344.

$a,-b$ Bogota (Bt at Stevens's) 1845.

\section{Centurus.}

Centurus, Swainson, Classif. B. ii. p. 310 (1837).

1950. Centurus carolinus.

Picus carolinus, Linn. Syst. Nat. i. p. 174: Sundev. Consp. Av. Picin. p. 53.

Zebrapicus carolinus, Malh. Mon. Pic. ii. p. 234, iv. pl. 103.

Centurus carolinus, Baird, Brew. \& Ridgw. N. Am. B. ii. p. 554. a (Askew) 1834.

A North American species.

1951. Centurus radiolatus.

Picus radiolatus, Wagl. Syst. Av. fol. 2, p. 12: Sundev. Consp. Av. Picin. p. 52.

Centurus radiolatus, Gosse, B. Jam. p. 271.

a f Jamaica (P. H. Gosse) 1848.

1952. Centurus albifrons.

Picus albifrons, Sw. Phil. Mag. New Ser. i. p. 439.

Centurus santacruzi, Bp. P. Z. S. 1837, p. 116: Scl. Cat. Am. B. p. 343.

Zebrapicus santacruzi, Malh. Mon. Pic. ii. p. 241, iv. pl. 105.

$a$ Guatemala ( $J$. Constancia) 1845. - b Guatemala (J. Constancia) 1848.

1953. Centurus elegans.

Picus elegans, Sw. Phil. Mag. New Ser. i. p. 439: Sundev. Consp. Av. Picin. p. 55.

Centurus elegans, Scl. Cat. Am. B. p. 342.

Zebrapicus elegans, Malh. Mon. Pic. ii., p. 225, iv. pl. 102. $a,-b$ (N. C. Strickland) 1838.

A Mexican species. 
1954. Centurus tricolor.

Picus tricolor, Wagl. Isis, 1829, p. 512 (nec Gm.): Sundev. Consp. Av. Picin. p. 54.

Centurus tricolor, Scl. Cat. Am. B. p. 343.

Zebrapicus tricolor, Malh. Mon. Pic. ii. p. 247, iv. pl. 106.

$a$ (Isaacson) 1840. — b, —c Trinidad (Argent) 1851.

\section{Colaptes.}

Colaptes, Swainson, Zool. Journ. iii. p. 353 (1827).

\section{Colaptes auratus.}

Picus auratus, Linn. Syst. Nat. i. p. 174: Sundev. Consp. Av. Picin. p. 71.

Colaptes auratus, Scl. Cat. Am. B. p. 344: Baird, Brew. \& Ridgw. N. Am. B. ii. p. 575.

Geopicus auratus, Malh. Mon. Pic. ii. p. 25̌, iv. pl. 109.

a o N. America (Arthur Strickland) 1832. - $b$ ô N. America (N. C. Strickland) 1838.

1956. Colaptes mexicanus.

Colaptes mexicanus, Sw. Phil. Mag. New Ser. i. p. 440: Scl. Cat. Am. B. p. 344.

Geopicus mexicanus, Malh. Mon. Pic. ii. p. 262, iv. pl. 110. $a,-b$ (N. C. Strickland) 1838. - c Mexico (T. Mann) 1844.

\section{5\%. Colaptes mexicanoides.}

Colaptes mexicanoides, Lafr. Rev. Zool. 1844, p. 42 : Scl. Cat. Am. B. p. 344 .

Geopicus rubrieatus, Malh. Mon. Pic. ii. p. 265, iv. pl. 110.

$a$ Guatemala (J. Constancia) 1848. - b Guatemala (J. Constancia) 1851.

\section{Colaptes pitius.}

Picus pitius, Mol. Saggio sulla St. Nat. Chili, p. 236.

Colaptes pitius, Scl. Cat. Am. B. p. 344.

Geopicus chilensis, Malh. Mon. Pic. ii. p. 269, iv. pl. 111.

a (T. C. Eyton) 1846.

A Chilian species. 


\section{Colaptes campestris.}

Picus campestris, Licht. Verz. Doubl. p. 10.

Colaptes campestris, Scl. Cat. Am. B. p. 343.

Geopicus campestris, Malh. Mon. Pic. ii. p. 251, iv. pl. 108.

a Brazil (Murray) 1834.

\section{Colaptes agricola.}

Geopicus agricola, Malh. Mém. Ac. Metz, 1849, p. 359; Mon. Pic. ii. p. 254, iv. pl. 108.

Colaptes agricola, Scl. Cat. Am. B. p. 343.

a (Thomas) 1842.

A Brazilian species.

Geocolaptes.

Geocolaptes, Swainson, Classif. B. ii. p. 309 (1837).

\section{Geocolaptes olivaceus.}

Picus olivaceus, Gm. Syst. Nat. i. p. 431.

Picus arator, Cuv. Reg. An. i. p. 459 (1829): Sundev. Consp. Av. Picin. p. 68.

Geopicus arator, Malh. Mon. Pic. ii. p. 274, iv. pl. 111.

$a$ (N. C. Strickland) 1838. - b S. Africa (Askew) 1841.

\section{HeMicercus.}

Hemicercus, Swainson, Classif. B. ii. p. 306 (1837).

1962. Hemicercus sordidus.

Dendrocopus sordidus, Eyton, Ann. \& Mag. N. H. xvi. p. 229.

Hemicercus sordidus, Salvad. Ann. Mus. Genov. v. p. 46.

Hemicercus concretus, Strickl. P. Z. S. 1846, p. 104 (nec Temm.).

a Malacca (Mather) 1840. - b Malacca (Bt at Stevens's) 1844. 1963. Hemicercus canente.

Picus canente, Less. Cent. Zool. p. 215, pl. 73 : Sundev. Consp. Av. Picin. p. 11.

Micropicus canente, Malh. Mon. Pic. i. p. 190, iii. pl. 42.

Hemicercus canente, Jerd. B. Ind. i. p. 280.

Picus caudatus, Jerd. Madr. Journ. xi. p. 211.

$a,-b$ Madras ("Picus caudatus, Jerd." T. C. Jerden) 1845.

S. C. 
PICI.

\section{Venilia.}

Venilia, Bonaparte, Consp. Av. i. p. 128 (1850).

\section{Venilia rubiginosa.}

Hemicercus rubiginosus, Sw. B. W. Afr. ii. p. 1ృ0: Strickl. P. Z. S. 1846 , p. 104 .

Celeopicus porphyromelas, Malh. Mon. Pic. ii. p. 39, iii. pl. 49 (e.x Boie).

Picus porphyromelas, Sundev. Consp. Av. Picin. p. 47.

a Malacca (Thomas) 1840.

Meiglyptes.

Meiglyptes, Swainson, Classif. B. ii. p. 309 (1837).

\section{Meiglyptes brunneus.}

Hemicercus brunneus, Eyt. P. Z. S. 1839, p. 106.

Picus brunneus, Sundev. Consp. Av. Picin. p. 91.

Meiglyptes tukki, Salvad. Ann. Mus. Genov, v. p. 57 (ex Lesson).

Phaiopicus pectoralis, Malh. Mon. Pic. ii. p. 8, iii. pl. 47.

a Malacca (N. C. Strickland) 1838, -b Malacca (Mather) 1840. -c Malacca (Carfrae) 1840.

\section{Meiglyptes tristis.}

Picus tristis, Horsf. Trans. L. S. xiii. p. 177: Sundev. Consp. Av. Picin. p. 91.

Meiglyptes tristis, Salvad. Ann. Mus. Genov. v. p. 56.

Phaiopicus grammithorax, Malh. Mon. Pic. ii. p. 12, iii. pl. 48. a Malacca (N. C. Strickland) 1838.

Micropternus.

Micropternus, Blyth, J. A. S. B. xiv. p. 194 (1845).

196\%. Micropternus phaioceps.

Micropternus phaioceps, Blyth, l. s. c.: Jerd. B. Ind. i. p. 294. Phaiopicus rufinotus, Malh. Mon. Pic. ii. p. 1, iii. pl. 46.

Picus rufinotus, Sundev. Consp. Av. Picin. p. 88.

a Malacca? (N. C. Striclland) 1838. - b India ("Micropternus phaioceps," E. Blyth) 1846. 


\section{Micropternus gularis.}

Picus (micropternus) gularis, Jerd. Madr. Journ. xiii. pt. ii. p. 139.

Micropternus gularis, Jerd. B. Ind. i. p. 294.

Picus gularis, Sundev. Consp. Av. Picin. p. 88.

Phaiopicus jerdoni, Malh. Mon. Pic. ii. p. 3, iii. pl. 47. a Madras ("Picus gularis," T. C. Jerdon) 1845.

A young bird very similar in plumage to the preceding species.

\section{Celeus.}

Celeus, Bois, Isis, 1831, p. 542.

\section{Celeus flavus.}

Pic jaune, de Cayenne, D’Aub. Pl. Enl. 509 undè,

Picus flavus, Müll. Naturs. Suppl. p. 91.

Celeus citrinus, Scl. Cat. Am. B. p. 336 (ex Bodd.).

Picus exalbidus, Gm. Syst. Nat. i. p. 428: Sundev. Consp. Av. Picin. p. 85.

Celeopicus exalbidus, Malh. Mon. Pic. ii. p. 30, iii. pl. 55.

$a$ (E. Brown) 1850. -b (Carfrae) 1850. —c Brazil (Argent) 1851. $-d,-e,-f$ Trinidad (Argent) 1851.

$a$ and $b$ are skins of Cayenne make.

\section{Celeus flavescens.}

Picus flavescens, Gm. Syst. Nat. i. p. 427: Sundev. Consp. Av. Picin. p. 84.

Celeopicus flavescens, Malh. Mon. Pic. ii. p. 21, iii. pl. 53.

Celeus flavescens, Scl. Cat. Am. B. p. 336.

$a \hat{b},-b$ o Brazil (N. C. Strickland) 1838.

\section{Celeus elegans.}

Picus elegans, Müll. Naturs. Suppl. p. 92.

Picus cinnamomeus, Gm. Syst. Nat. i. p. 428: Sundev. Consp. Av. Picin. p. 85.

Celeopicus cinnamomeus, Malh. Mon. Pic. ii. p. 32, iii. pl. 56. $a \hat{\delta},-b$ o Guiana (N. C. Strickland) 1838. — Demarara (Cashmore) 1843.

\section{Chrysocolaptes.}

Chrysocolaptes, Blyth, J. A. S. B. xii. p. 1004 (1843). 


\section{Chrysocolaptes sultaneus.}

Picus sultaneus, Hodgs. J. A. S. B. vi. p. 105: Sundev. Consp. Av. Picin. p. 79.

Indopicus sultaneus, Malh. Mon. Pic. ii. p. 77, iv. pl. 64.

Chrysocolaptes sultaneus, Jerd. B. Ind. i. p. 281.

a Madras ("Picus strictus," T. C. Jerdon) 1845. - b Madras ("Picus sultaneus, Hodgson, P. strenuus, Gould; a small specimen but made to look too small, male. E. Blyth, MS." T. $C$. Jerdon) 1845. —c India (Lord A. Hay) 1845. —d India ( $E$. Blyth) 1846. -e f Kumaon (W. J. E. Boys) 1847.

\section{Chrysocolaptes festivus.}

Pic verd, de Goa, D'Aub. Pl. Enl. 696 undè,

Picus festivus, Bodd. Tabl. Pl. Enl. p. 43: Sundev. Consp. Av. Picin. p. 80.

Picus goensis, Gm. Syst. Nat. i. p. 434 .

Indopicus goensis, Malh. Mon. Pic. ii. p. 82, iv. pl. 66 .

Chrysocolaptes goensis, Jerd. B. Ind. i. p. 282.

Brachypternus goensis, Strickl. Ann. \& Mag. N. H. vii. p. 35. $a,-b$ Madras (T. C. Jerdon) 1845.

\section{Chrysocolaptes hæmatribon.}

Picus hcematribon, Wagl. Syst. Av. fol. 3, p. 14: Sundev. Consp. Av. Picin. p. 80.

Indopicus hcematribon, Malh. Mon. Pic. ii. p. 84, iv. pl. 68. Chrysocolaptes hoematribon, Wald. Trans. Z. S. ix. p. 147.

Brachypternus hcematribon, Strickl. Ann. \& Mag. N. H. vii. p. 35 .

a Philippine Islands (J. Gould) 1846.

\section{BRACHYPTERNUS.}

Brachypternus, Strickland, P. Z. S. 1841, p. 31.

\section{Brachypternus aurantius.}

Picus aurantius, Linn. Syst. Nat. i. p. 174.

Brachypternus aurantius, Strickl. Ann. \& Mag. N. H. vii. p. 35 : Jerd. B. Ind. i. p. 295.

Brahmapicus bengalensis, Malh. Mon. Pic. ii. p. 93, iv. pl. 69 (ex Linn.). 
Picus bengalensis, Sundev. Consp. Av. Picin. p. 81.

$a \hat{\delta}$ E. Indies (Major Stacy) 1836. $-b$ o Bengal ( $N$. $C$. Strickland) 1838. - e f Bengal ("Brachypternus aurantius," $E$. Blyth) 1846.

1976. Brachypternus chrysonotus.

Picus chrysonotus, Less. Traité d'Orn. p. 220.

Brachypternus chrysonotus, Jerd. B. Ind. i. p. 296.

Brahmapicus puncticollis, Malh. Mon. Pic. ii. p. 92, iv. pl. 70. $a,-b$ India (Bt at Stevens's) 1843.

\section{7\%. Brachypternus ceylonus.}

Picus ceylonus, Forst. Naturf. xiii. p. 14, pl. 4.

Brachypternus ceylonus, Cab. \& Hein. Mus. Hein. iv. p. 171. Picus erithronothos, Vieill. N. Dict. d'Hist. N. xxvi. p. 73.

Brahmapicus erythronotus, Malh. Mon. Pic. ii. p. 90, iv. pl. 69. Brachypternus erythronotus, Strickl. Ann. \& Mag. N. H. vii. p. 35 .

a Ceylon ("Brachypternus ceylonus," E. Blyth) 1850.

\section{Tiga.}

Tiga, Kaup. Thierr. ii. p. 37 (1836); Strickland, Ann. \& Mag. N. H. vii. p. 35.

Chrysonotus, Swainson, Classif. B. ii. p. 309 (1837).

\section{9'78. Tiga javanensis.}

Picus javanensis, Ljungh. Act. Stockh. xviii. p. 134: Wald. Ibis, 1871, p. 164.

Chrysonotus javanensis, Cab. \& Hein. Mus. Hein. iv. p. 172.

Tiga javanensis, Blyth, J. A. S. B. xliii. extr. No. p. 75: Salvad. Ann. Mus. Genov. v. p. 54.

Tiga intermedia, Blyth, J. A. S. B. xiv. p. 193.

$a$ (Arthur Strickland) 1840. -b Madras ("Picus tiga," T. C. Jerdon) 1845. —c, —d Arakan (E. Blyth) 1847. 


\section{ORDER IV.-COCCYGES.}

\section{SUBORDER.-COCCYGES ANOMALODACTYLAE.}

\section{FAMILY.-COLIID $\mathbb{E}^{1}$.}

\section{Colius.}

Colius, Brisson, Orn. iii. p. 304 (1760): Murie, Ibis, 1872 , p. 262.

\section{Colius capensis.}

Colius capensis, Gm. Syst. Nat. i. p. 842: Gurney, in And. B. Damara-1. p. 202.

Colius erythropus, Gm. l. s. c.: Strickl. \& Scl. Contr. Orn. 1852, p. 151.

$a$ Cape of Good Hope (N. C. Strickland) 1838. - b Damaraland (C.J.Andersson) 1852.

1980. Colius erythromelon.

Colius erythromelon, Vieill. N. Dict. d'Hist. N. vii. p. 378: Gurney, in And. B. Damara-1. p. 203.

Colius macrurus, Strickl. \&. Scl. Contr. Orn. 1852, p. 151 (nec Linn.).

$a$ Cape of Good Hope (N. C. Strickland) 1838. - b Damaraland (C. J. Andersson) 1852.

\section{Colius macrurus.}

Lanius macrurus, Linn. Syst. Nat. i. p. 134.

Colius macrurus, Strickl. P. Z. S. 1850, p. 219.

a $\hat{\delta}$ Kartoum, October (J. Petherick) 1848.

\section{Colius striatus.}

Colius striatus, Gm. Syst. Nat. i. p. 843: Layard, B. S. Afr. p. 222.

$a$ Cape of Good Hope (Askew) 1834.

* Cf. Strickl. Ann. \& Mag. N. H. vii. p. 33. 
UPUPIDE.

\section{SUBORDER.-COCCYGES ANISODACTYLAE.}

\section{FAMILY.-UPUPIDA.}

Upupa, Linnæus, Syst. Nat. i. p. 183 (1766) partim: Strickl. Ann. \& Mag. N. H. xii. p. 239.

\section{Upupa epops.}

Upupa epops, Linn. l. s. c.: Strickl. P. Z. S. 1836, p. 100; Ann. \& Mag. N. H. xiv. p. 349: Jerd. B. Ind. i. p. 390: Dresser, B. Eur.

a Himalaya (Askew) 1836. - b S. India ("Upupa minor," T. C. Jerdon) 1844. -c Madras (T. C. Jerdon) 1845. -d, -e India ("Upupa epops," E. Blyth) 1846.

1984. Upupa minor.

Upupa minor, Shaw, Gen. Zool. viii. p. 139: Strickl. \& Scl. Contr. Orn. 1852, p. 155 : Layard, B. S. Afr. p. 72.

$a$ (N. C. Strickland) 1838. - b Cape of Good Hope (N. C. Strickland) 1838.

\section{IRRISOR.}

Irrisor, Lesson, Traité d'Orn. p. 239 (1831): Strickl. Ann. \& Mag. N. H. xii. p. 239.

\section{Irrisor erythrorhynchus.}

Upupa erythrorhynchus, Lath. Ind. Orn. p. 280.

Irrisor erythrorhynchus, Strickl. \& Scl. Contr. Orn. 1852, p. 154: Andersson, Ibis, 1865, p. 549.

Falcinellus senegalensis, Vieill. Enc. Méth. p. 577.

Irrisor senegalensis? Strickl. P. Z. S. 1850, p. 216.

$a$ (Johnson) 1837. - b, -c Cape of Good Hope (N. C. Strickland) 1838. -d, -e (N. C. Strickland) 1838. -f, -g (Bt at Birmingham) 1843. - $h$ Africa (Osman) 1846. -i Kordofan, 23 March (J. Petherick) 1848. - j Damara-land (C. J. Andersson) 1852.

1986. Irrisor aterrimus.

Promerops aterrimus, Steph. in Shaw's Gen. Zool. xiv. p. 257. 
Irrisor aterrimus, Sharpe, Cat. Afr. B. p. 10.

Promerops pusillus, Sw. B. W. Afr. ii. p. 120.

a Africa (Bt at Stevens's) 1845.

\section{8\%. Irrisor cyanomelas.}

Falcinellus cyanomelas, Vieill. N. Dict. d'Hist. N. xxviii. p. 164.

Irrisor cyanomelas, Sharpe, Cat. Afr. B. p. 10: Gurney, in And. B. Damara-1. p. 67.

Rhinopomastes cyanomelas, Strickl. \& Scl. Contr. Orn. 1852, p. 155 .

$a,-b$ Damara-land (C. J. Andersson) 1852.

\section{FAMILY.-MEROPID $\$$.}

\section{Merops.}

Merops, Linnæus, Syst. Nat. i. p. 182 (1766).

\section{Merops apiaster.}

Merops apiaster, Linn. l. s. c.: Dresser, B. Eur.

$a$ Tuscany (C. Passerini) 1835. - b (Arthur Striclkland) 1850.

\section{Merops ægyptius.}

Merops agyptius, Forsk. Fauna Arab. No. 2.

$a,-b \operatorname{Egypt}(J$. F. Brandi) 1842. — River Gambia (Arthur Strickland) 1850. -d (Arthur Strickland) 1850.

\section{Merops philippinus.}

Merops philippinus, Linn. Syst. Nat. i. p. 183: Strickl. P. Z. S. 1842, p. 167: Wald. Trans. Z. S. ix. p. 149.

Merops philippensis, Jerd. B. Ind. i. p. 207.

a Indian Archipelago (Askew) 1833. - b q Nepal (W. J. E. Boys) 1848. —c (Arthur Strickland) 1850.

\section{Merops sumatranus.}

Merops sumatranus, Raffl. Trans. L. S. xiii. p. 294: Wald. Trans. Z. S. ix. p. 151, pl. 26, f. 2.

Merops bicolor, Gm. Auctt. partim.

$a,-b$ Malacca (Capt. Nash) 1837. 


\section{Merops ornatus.}

Merops ornatus, Lath. Ind. Orn. Suppl. p. xxxv.: Gould, B. Austr. ii. pl. 16.

$a$ New South Wales (McDonald) 1838. -b, -c (Arthur Strickland) 1850.

\section{Merops quinticolor.}

Merops quinticolor, Vieill. N. Dict. d'Hist. N. xiv. p. 21 : Jerd. B. Ind. i. p. 208. 1847.

$a$ S. India (Gardner) 1844. -b Kumaon (W.J.E. Boys)

\section{Merops albicollis.}

Merops albicollis, Vieill. N. Dict. d'Hist. N. xiv. p. 15: Strickl. P. Z. S. 1850 , p. 216.

$a$ W. Africa (Thomas) 1840. - $b$ 个 Kordofan, 12 June (J. Petherick) 1848.

\section{Merops nubicus.}

Merops nubicus, Gm. Syst. Nat. i. p. 464: Strickl. P. Z. S. 1850, p. 216.

a Africa (Gardner) 1844. - b ô Kordofan, 1 July (J. Petherick) 1848.

1996. Merops viridis.

Merops viridis, Linn. Syst. Nat. i. p. 182: Strickl. P. Z. S. 1842 , p. 167; Ann. \& Mag. N. H. xiii. p. 34 : Jerd. B. Ind. i. p. 205.

$a($ Askew) 1834. - b (Askew) 1837. — $c$ Northern India (Bt at Stevens's) 1843. - d Malwa (W. J. E. Boys) 1847. - e, -f, (Arthur Strickland) 1850.

\section{9\%. Merops viridissimus.}

Merops viridissimus, Sw. B. W. Afr. ii. p. 82.

Merops lamarki, Strickl. P. Z. S. 1850, p. 216 (nec Cuv.?).

a o Kordofan, February (J. Petherick) 1848. - b (Arthur Strickland) 1850. 


\section{Merops lafresnayii.}

Merops lafresnayii, Guér. Rev. Zool. 1843, p. 322: Finsch, Trans. Z. S. vii. p. 225.

a Abyssinia (Verreaux) 1850.

Considered by Strickland to be the same as $M$. variegatus Vieill. of W. Africa ( $C f$. Contr. Orn. 1851, pp. 135, 162).

\section{Merops hirundinaceus.}

Merops hirundinaceus, Vieill. N. Dict. d'Hist. N. xiv. p. 21.

Melittophagus hirundinaceus, Strickl. \& Scl. Contr. Orn. 1852 , p. 154.

Dicrocercus hirundinaceus, Gurney, in And. B. Damara-l. p. 63.

Merops furcatus, Stanley in Salt's Trav. App. p. 27.

$a$ W. Africa (Johnson) 1837. - b. Damara-land (C. J. Andersson) 1852.

\section{Merops erythropterus.}

Merops erythropterus, Gm. Syst. Nat. i. p. 464: Strickl. P. Z. S. 1850, p, 216.

$a$ W. Africa (Johnson) 1837. $-b$ \& Kordofan, 28 April (J. Petherick) 1848. -c (Arthur Strickland) 1850.

\section{1: Merops bullocki.}

Merops bullocki, Vieill. N. Dict. d'Hist. N. xiv. p. 13. Spheconax bullocki, Cab. \& Hein. Mus. Hein. ii. p. 134. $a$ S. Africa (Havell) 1839. - b (Arthur Strickland) 1850.

A West-African species.

\section{NyCTIORNIS.}

Nyctiornis, Swainson, Zool. Ill. ser. 2, ii. pl. 56 (1831).

\section{Nyctiornis amictus.}

Merops amictus, Temm. PI. Col. 310.

Nyctiornis amictus, Sw. l. s. c.: Salvad. Ann. Mus. Genov. v. p. 91.

$a$ (Arthur Strickland) 185̃0. - b Malacca (Argent) 1851. 
2003. Nyctiornis athertoni.

Merops athertoni, Jard. \& Selb. Ill. Orn. i. pl. 58.

Nyctiornis athertoni, McClelland, P. Z. S. 1839, p. 155: Cab. \& Hein. Mus. Hein. ii. p. 132: Jerd. B. Ind. i. p. 211.

a Madras (T. C. Jerdon) 1845.

\section{FAMILY.-CORACIIDA.}

Coracias.

Coracias, Linnæus, Syst. Nat. i. p. 159 (1766).

2004. Coracias garrulus.

Coracias garrula, Linn. l. s. c.

Coracias garrula, Sharpe, \& Dresser, B. Eur.

a Spain (Boissoneau) 1839.

2005. Coracias indicus.

Coracias indica, Linn. Syst. Nat. i. p. 159: Jerd. B. Ind. i. p. 214.

$a$ India (N. C. Strickland) 1838.

2006. Coracias affinis.

Coracias affinis, MClelland, P. Z. S. 1839, p. 164: Jerd. B. Ind. i. p. 217.

$a$ India (Lord A. Hay) 1845.

200\%. Coracias nævius.

Coracias ncevia, Daud. Traité d'Orn. ii. p. 258: Strickl. P. Z. S. 1850, p. 216: Sharpe, Ibis, 1871, p. 190.

Coracias ptilosa, Lath. Ind. Orn. Suppl. p. xxvii.

$a$ Africa (N. C. Strickland) 1838. -b $q$ Kordofan, 8 July (J. Petherick) 1848. - c Damara-land (C. J. Andersson) 1852.

2008. Coracias caudatus.

Coracias caudata, Linn. Syst. Nat. i. p. 160: Strickl. \& Scl. Contr. Orn. 1852, p. 154: Sharpe, Ibis, 1871, p. 194.

a Damara-land (C. J. Andersson) 1852.

2009. Coracias abyssinicus.

Rollier d'Abyssinie, D'Aub. Pl. Enl. 626, undè, 
Coracias abyssinica, Bodd. Tabl. Pl. Enl. p. 38: Strickl. P. Z. S. 1850, p. 216: Sharpe, Ibis, 1871, p. 197.

a Africa (N. C. Strickland) 1838. - b ํ Kordofan, February (J. Petherick) 1848.

\section{EURYSTOMUS.}

Eurystomus, Vieill. Anal. p. 37 (1816).

2010. Eurystomus orientalis.

Coracias orientalis, Linn. Syst. Nat. i. p. 159.

Eurystomus orientalis, Salvad. Ann. Mus. Genov. v. p. 105: Wald. Trans. Z. S. ix. p. 152.

a Malacca (Capt. Nash) 1837. -b (N. C. Strickland) 1838. -c Philippine Islands (J. Gould) 1846. $\_d$ Malacca (Williams) 1847.

\section{Eurystomus pacificus.}

Coracias pacifica, Lath. Ind. Orn. Suppl. p. xxvii.

Eurystomus pacificus, Gould, Handb. B. Austr. i. p. 119.

Eurystomus australis, Vig. \& Horsf. Trans. L. S. xv. p. 202 : Gould, B. Austr. ii. pl. 17.

$a$ New South Wales (McDonald) 1838.

\section{Eurystomus afer.}

Coracias afra, Lath. Ind. Orn. i. p. 172.

Eurystomus afer, Strickl. P. Z. S. 1850, p. 216: Sharpe, Ibis, 1871, p. 274.

a Africa (Johnson) 1837. -b (N. C. Strickland) 1838. -c $\hat{\delta}$ Kordofan, 9 July (J. Petherick) 1848.

\section{FAMILY.-MOMOTID无.}

\section{Momotus.}

Momotus, Brisson, Ornith. iv. p. 465 (1760).

\section{Momotus brasiliensis.}

Ramphastos momota, Linn. Syst. Nat. i. p. 152.

Momotus brasiliensis, Lath. Ind. Orn. i. p. 140: Scl. Cat. Am. B. p. 261.

a Brazil (Thomas) 1842.

A skin of Cayenne make. 
2014. Momotus swainsoni.

Prionites bahamensis, Sw. An. in. Menag. p. 332 (nom. inept).

Momotus swainsoni, Scl. Cat. Am. B. p. 261.

$a$ Trinidad (Argent) 1851. - $b$ Trinidad (J. Taylor).

2015. Momotus lessoni.

Momotus lessoni, Less. Rev. Zool. 1842, p. 174: Scl. Cat. Am. B. p. 262.

a Guatemala (J. Constancia) 1845. - b Guatemala (J. Constancia) 1848.

\section{Momotus mexicanus.}

Momotus mexicanus, Sw. Phil. Mag. New Ser. i. p. 442: Scl. Cat. Am. B. p. 262.

a Mexico (Galeotti) 1845.

\section{BARYPHTHENGUS.}

Baryphthengus, Cabanis \& Heine, Mus. Hein. ii. p. 114 (1860).

\section{Baryphthengus ruficapillus.}

Baryphonus ruficapillus, Vieill. N. Dict. d'Hist. N. xxi. p. 315 .

Baryphthengus ruficapillus, Cab. \& Hein. l. s. c.: Scl. Cat. Am. B. p. 262.

a S. America (N. C. Strickland) 1838.

Eumomota.

Eumomota, Sclater, P. Z. S. 1857, p. 257.

\section{Eumomota superciliaris.}

Prionites (Crypticus) superciliaris, Jard. \& Selb. Ill. Orn. iv. pl. 18 (ex Sandb. MSS.).

Eumomota superciliaris, Scl. Cat. Am. B. p. 263.

a Guatemala (Bt at Stevens's) 1848.

\section{Hylomanes.}

Hylomanes, Lichtenstein, Abh. Ak. Berl. 1838, p. 449. 


\section{Hylomanes gularis.}

Prionites gularis, Lafr. Rev. Zool. 1840, p. 130.

Momotus gularis, Strickl. Contr. Orn. 1849, p. 33, pl. 5. $a$ Guatemala (J. Constancia) 1848.

\section{FAMILY.-TODID AE.}

\section{Todus.}

Todus, Linnæus, Syst. Nat. i. p. 178 (1766), partim.

\section{Todus viridis.}

Todus viridis, Linn. l. s. c.: Sharpe, Ibis, 1874, p. 349.

$a \mathrm{~W}$. Indies (Askew) 1810. —b (Bt at Edinburgh) 1852.

A species peculiar to the island of Jamaica.

\section{FAMILY.-ALCEDINID Æ.}

\section{SUBFAMILY.-ALCEDINID $Æ$.}

\section{Alcedo.}

Alcedo, Linnæus, Syst. Nat. i. p. 178 (1766), partim. 2021. Alcedo ispida.

Alcedo ispida, Linn. l. s. c.: Strickl. P. Z. S. 1836, p. 100: Sharpe, Mon. Alced. p. 1. pl. 1.

a Lincolnshire (H. E Strickland) 1827. - b f Smyrna 30 November (H. E. Strickland) 1835.

\section{Alcedo bengalensis.}

Alcedo bengalensis, Gm. Syst. Nat. i. p. 450: Jerd. B. Ind. i. p. 230 : Sharpe, Mon. Alced. p. 11, pl. 2.

$a \hat{\jmath},-b$ o India (N. C. Strickland) 1838. $-c$ Nepal (B. H. Hodgson, 606) 1845. —d h Malwa (W. J. E. Boys) 1847.

\section{Alcedo quadribrachys.}

Alcedo quadribrachys, Bp. Consp. i. p. 158: Strickl. Contr. Orn. 1851, p. 134, pl. 79: Sharpe, Mon. Alced. p. 25, pl. 6.

$a$ W. Africa (N. C. Strickland) 1838. 
2024. Alcedo semitorquata.

Alcedo semitorquata, Swains. Zool. Ill. iii. pl. 151: Sharpe, Mon. Alced. p. 27, pl. 7.

$a$ Africa? (N. C. Strickland) 1838. -b (Dewgard) 1851.

A widely-ranging African species.

2025. Alcedo beryllina.

Alcedo beryllina, Vieill. N. Dict. d'Hist. N. xix. p. 414: Sharpe, Mon. Alced. p. 31, pl. 9.

Alcedo biru, Horsf. Trans. L. S. xiii. p. 172.

a Java (N. C. Strickland) 1838.

Corythornts.

Corythornis, Kaup. Fam. Eisv. p. 10 (1848).

2026. Corythornis cristatus.

Alcedo cristata, Linn. Syst. Nat. i. p. 178.

Corythornis cristata, Sharpe, Mon. Alced. p. 35, pl. 11.

a $\hat{\jmath}$ Cape of Good Hope (A. Strickland) 1833.

\section{ALCYone.}

Alcyone, Swainson, Classif. B. ii. p. 336 (1837).

\section{Alcyone azurea.}

Alcedo azurea, Lath. Ind. Orn. Suppl. p. xxxii.

Alcyone azurea, Gould, B. Austr. ii. pl. 25: Sharpe, Mon. Alced. p. 41, pl. 13.

$a$ New South Wales ( $M^{c}$ Donald) 1838. - b Cape York, 15 October 1848 (Argent) 1852.

\section{Ceryle.}

Ceryle, Boie, Isis, 1828, p. 316.

\section{Ceryle guttata.}

Alcedo guttatus, Vig. P. Z. S. 1830-1, p. 22: Gould, Cent. B. Him. pl. 5.

Ceryle guttata, Jerd. B. Ind. i. p. 234: Sharpe, Mon. Alced. p. 57, pl. 18.

a ôे Kumaon (W. J. E. Boys) 1847. 


\section{Ceryle rudis.}

Alcedo rudis, Linn. Syst. Nat. i. p. 181: Strickl. P. Z. S. 1836, p. 100; Ann. \& Mag. N. H. vi. p. 418.

Ceryle rudis, Strickl. Ann. \& Mag. N. H. ix. p. 442; xiii. p. 34 ; xviii. p. 405 ; P. Z. S. 1842 , p. 167 ; 1850, p. 216 : Sharpe, Mon. Alced. p. 61, pl. 19.

Ispida bicincta, Sw. B. W. Afr. ii. p. 95; Strickl. Ann. \& Mag. N. H. vi. p. 418; xiii. p. 34; P. Z. S. 1850, p. 216.

Ceryle varia, Strickl. Ann. \& Mag. N. H. iv. p. 418; xiii. p. 34; xviii. p. 405 ; P. Z. S. 1842, p. 167.

$a$ Calcutta (S. P. Stacy) 1833. - $b$ ô Smyrna, 13 December (H. E. Strickland) 1835. -c o Smyrna, 2 January (H. E. Strickland) 1836. - d W. Africa (N. C. Strickland) 1838. -e Madras ("Ispida varia, Strickl." T. C. Jerdon). $-f$ o India (W. J. E. Boys) 1847. - g, - h Kordofan (J. Petherick) 1848. $-i$ India. $-j,-k \mathrm{~S}$. Africa (Dr A Smith).

2030. Ceryle torquata.

Alcedo torquata, Linn. Syst. Nat. i. p. 180.

Ceryle torquata, Sharpe, Mon. Alced. p. 73, pl. 22.

$a$ o S. America (N. C. Strickland) 1838. $-b$ Trinidad (Argent) 1851.

\section{Ceryle alcyon.}

Alcedo alcyon, Linn. Syst. Nat. i. p. 180.

Ceryle alcyon, Sharpe, Mou. Alced. p. 79, pl. 23.

$a$ o N. America (Askew) 1834. - b ô N. America (N. C. Strickland) 1834. —c Mexico (I'. Mann) 1844. —d Sitka, N. W. America (J. F. Brandt) 1844. - e N. W. America (Wosnessenski) 1846. - $f$ Jamaica (P. H. Goose) 1846.

\section{Ceryle amazona.}

Alcedo amazona, Lath. Ind. Orn. i. p. 255.

Ceryle amazonia, Sharpe, Mon. Alced. p. 83, pl. 24.

$a$ (N. C. Strickland) 1838. - $b$ Guatemala (J. Constancia) 1848.

\section{Ceryle americana.}

Alcedo americana, Gm. Syst. Nat. i. p. 451. 
Ceryle americana, Sharpe, Mon. Alced. p. 89, pl. 26.

$a,-b,-c$ Brazil (Askew) 1837. $-d$ S. America (N. C. Strickland) 1838. - e Trinidad (Argent) 1851. $-f$.

2034. Ceryle inda.

Alcedo inda, Linn. Syst. Nat. i. p. 179.

Ceryle inda, Sharpe, Mon. Alced. p. 91, pl. 27.

$a,-b$ S. America (N. C. Strickland) 1838.

\section{Ceryle superciliosa.}

Alcedo superciliosa, Linn. Syst. Nat. i. p. 179.

Ceryle superciliosa, Sharpe, Mon. Alced. p. 93, pl. 28.

$a$ (N. C. Strickland) 1838. - b S. America (N. C. Strickland) 1838. -c (Argent) 1851.

$c$ is a skin of Cayenne make.

\section{PELARGOPSIS.}

T'elargopsis, Gloger, Handb. p. 338 (1842).

2036. Pelargopsis amauroptera.

IIalcyon amauropterus, Pears. J. A. S. B. x. p. 635.

Pelargopsis amauroptera, Sharpe, Mon. Alced. p. 97, pl. 30.

a India ("Pelargopsis amauropterus" E. Blyth) 1846.

203\%. Pelargopsis leucocephala.

Alcedo leucocephala, Gm. Syst. Nat. i. p. 456.

I'elargopsis leucocephala, Sharpe, Mon. Alced. p. 99, pl. 31.

$a$ E. Indies (Dr Horsficld) 1838.

\section{Pelargopsis gurial.}

Halcyon gurial, Pears. J. A. S. B. x. p. 633.

Pelargopsis gurial, Sharpe, Mon. Alced. p. 105, pl. 34.

Halcyon leucocephalus, Jerd. B. Ind. i. p. 222 (nec Gm.).

$a$ Ceylon (Bt at Stevens's) 1843.

\section{Pelargopsis malaccensis.}

Pelargypsis malaccensis, Sharpe, P. Z. S. 1870, p. 67; Mon. Alced. p. 107.

a India ("Halcyon capensis" E. Blyth) 1846.

A skin agreeing with the description of this Malaccan race.

S. C. 
COCCYGES。

SUBFAMILY.-DACELONIN A.

Cery.

Ceyx, Lacépède, Mém. de l'Inst. 1801, p. 511.

\section{Ceyz rufidorsa.}

Ceyx rufidorsa, Strickl. P. Z. S. 1846, p. 99: Sharpe, Mon. Alced. p. 121, pl. 41: P. Z. S. 1869, p. 507.

a Malacca (Sir W. Jardine) 1842.

Though not marked as belonging to his species this specimen is evidently typical of $C$. rufidorsa, Strickl. and is probably of the same origin as the bird figured in the Illustrations of Ornithology by Sir W. Jardine. The specimen is fully described by Mr Sharpe.

\section{ISPIDINA.}

Ispidina, Kaup. Fam. Eisv. p. 10 (1848).

\section{Ispidina picta.}

Todier bleu à ventre Orangé, D’Aub. Pl. Enl. 783, f. 1, undè, Todus pictus, Bodd. Tabl. Pl. Enl. p. 49.

Ispidina picta, Sharpe, Mon. Alced. p. 141, pl. 51.

a (Bt at Birmingham) 1850.

A West-African species.

2042. Ispidina natalensis.

Alcedo natalensis, Smith, S. Afr. Quart. Journ. ii. p. 14. Ispidina natalensis, Sharpe, Mon. Alced. p. 145, pl. 52.

$a$ S. E. Africa (Argent) 1853.

\section{HALCYON.}

Halcyon, Swainson, Zool. Ill. i. pl. 27, text (1820).

\section{Halcyon coromanda.}

Alcedo coromanda, Lath. Ind. Orn. i. p. 252.

Halcyon coromandelianus, Jerd. B. Ind. i. p. 227.

Halcyon coromanda, Sharpe, Mon. Alced. p. 155, pl. 57. a India (Lord A. Hay) 1845.

2044. Halcyon gularis.

Grand martin-pêcheur de Madagascar, D'Aub. Pl. Enl. 232, undè, 
Alcedo gularis, Kuhl, Buff. et D'Aub. Fig. Av. Col. Nom. Syst. p. 4.

Halcyon gularis, Strickl. Ann. \& Mag. N. H. xiii. p. 34 Sharpe, Mon. Alced. p. 165, pl. 60.

a Luzon 14 June (H. Cuming) 1839.

\section{Halcyon smyrnensis.}

Alcedo smyrnensis, Linn. Syst. Nat. i. p. 181.

Halcyon smyrnensis, Strickl. Brit. Assoc. Rep. 1842, p. 70 : Ann. \& Mag. N. H. ix. p. 441; xiii. p. 34: Sharpe, Mon. Alced. p. 161, pl. 59 .

$a,-b$ Ceylon (Capt. Pearson) 1841. — Macri, Asia Minor, February (Capt. T. Graves) 1842. - d Nepal (B. H. Hodgson, 590) 1845.

2046. Halcyon pileata.

Martin-pêcheur de la Chine, D’Aub. Pl. Enl. 673, undè, Alcedo pileata, Bodd. Tabl. Pl. Enl. p. 41.

Halcyon pileata, Sharpe, Mon. Alced. p. 169, pl. 62.

a India ("Halcyon atricapillus, $H$. albiventris Scop." $E$. Blyth) 1846.

\section{4\%. Halcyon semicærulea.}

Alcedo semiccerulea, Forsk. Descr. Anim. p. 2.

Halcyon semiccerulea, Sharpe, Mon. Alced. p. 173, pl. 64.

Dacelo actcoon, Less. Traité d'Orn. p. 247.

a (Mather) 1840.

A species of N. E. Africa.

2048. Halcyon albiventris.

Alcedo albiventris, Scop. Del. Faun. et Fl. Ins. ii. p. 90.

Halcyon albiventris, Sharpe, Mon. Alced. p. 177, pl. 65.

Dacelo fuscicapilla, Lafr. Mag. Zool. 1833, Cl. ii. pl. 18.

$a$ Cape of Good Hope ( $\operatorname{Dr} A$. Smith) 1829. - b Africa $(N$. C. Strickland) 1838. -c, -d (N. C. Strickland) 1838.

2049. Halcyon chelicutensis.

Alcedo chelicuti, Stanley in Salt's Trav. App. p. lvi.

Halcyon chelicutensis, Sharpe, Mon. Alced. p. 183, pl. 67. 
Halcyon damarensis, Strickl. Contr. Orn. 1852, p. 153.

$a$ (Johnson) 1837. - b Africa (Mather) 1840. - c Sierra Leone (Dr F'erguson) 1845. —d Damara-land (C.J. Andersson) 1852.

$d$ is the type of Halcyon damarensis, a much larger bird than any of the other three specimens, but not specifically distinct according to Mr Sharpe.

\section{Halcyon senegalensis.}

Alcedo senegalensis, Linn. Syst. Nat. i. p. 180.

Halcyon senegalensis, Sharpe, Mon. Alced. p. 191, pl. 70.

a W. Africa (Johnson) 1837.

\section{Halcyon malimbica.}

Alcedo malimbica, Shaw, Gen. Zool. viii. p. 66.

Halcyon malimbica, Sharpe, Mon. Alced. p. 195, pl. 72.

$a$ W. Africa (N. C. Strickland) 1838.

\section{Halcyon pyrrhopygia.}

Halcyon pyrrhopygia, Gould, P. Z. S. 1840, p. 113: Sharpe, Mon. Alced. p. 211, pl. 79.

a Australia (Bt at Liverpool) 1853.

\section{Halcyon chloris.}

Martin-pêcheur à tête verte du Cap de Bonne Esperance, D'Aub. Pl. Enl. 783, undè,

Alcedo chloris, Bodd. Tabl. Pl. Enl. p. 49.

Halcyon chloris, Sharpe, Mon. Alced. p. 229, pl. 87.

Alcedo chlorocephalus, Gm. Syst. Nat. i. p. 454.

a Mindanan, 16 May (H. Cuming) 1839. - b India ("Todirhamphus collaris, Scop. T. chlorocephalus, Gm." E. Blyth) 1846.

\section{Halcyon sanctus.}

Halcyon sanctus, Vig. \& Horsf. Trans. L. S. xv. p. 206 : Sharpe, Mon. Alced. p. 239, pl. 91.

a Australia (Burl) 1834. - b, -c Australia (N. C. Strickland) 1838. - d (Dewgard) 1851. 
Todirhamphus.

Todirhamphus, Lesson, Mém. Soc. d'Hist. Nat. iii. p. 420 (1828).

2055. Todirhamphus veneratus.

Alcedo venerata, Gm. Syst. Nat. i. p. 453.

Todirhamphus veneratus, Sharpe, Mon. Alced. p. 245 , pl. 93. a (Bt at Edinburgh) 1852.

A species found in many of the islands of the South Pacific.

\section{DACELO.}

Dacelo, Leach, Zool. Misc. ii. p. 125 (1815).

2056. Dacelo gigas.

Martin-pêcheur de la Nouvelle Guinée, D’Aub. Pl. Enl. 663, undè,

Alcedo gigas, Bodd. Tabl. Pl. Enl. p. 40.

Dacelo gigas, Sharpe, Mon. Alced. p. 285, pl. 112.

Alcedo gigantea, Gm. Syst. Nat. i. p. 454.

$a,-b$ New South Wales (McDonald) 1838.

\section{Carcineutes.}

Carcineutes, Cabanis \& Heine, Mus. Hein. ii. p. 163 (1860). 205\%. Carcineutes pulchellus.

Dacelo pulchella, Horsf. Trans. L. S. xiii. p. 175.

Carcineutes pulchellus, Sharpe, Mon. Alced. p. 251, pl. 96.

a Java (G. Bell) 1842. - b Malacca (Williams) 1848.

Both these skins are of Malacca make.

\section{FAMILY.-BUCEROTIDE.}

\section{RHINOPLAX.}

Rhinoplax, Gloger, Handb. p. 335 (1842).

\section{Rhinoplax scutatus.}

Calao à Casque rond, D'Aub. Pl. Enl. 933, undè,

Buceros scutatus, Bodd. Tabl. Pl. Enl. p. 55 .

Buceros galeatus, Gm. Syst. Nat. i. p. 360. 
Rhinoplax vigil, Elliot, Mon. Bucer. (ex Forst.). a (Arthur Strickland) 1850.

A species of the Malay peninsula, Borneo, \&c.

ANTHRACOCERos.

Anthracoceros, Reichenbach, Av. Syst. pl. xlix. (1849).

2059. Anthracoceros malabaricus.

Buceros malabaricus, Gm. Syst. Nat. i. p. 359 : Strickl. Ann. \& Mag. N. H. xiii. p. 205.

Anthracoceros malabaricus, Elliot, Mon. Bucer.

$a$ (Askew) 1841. - b Malacca (Bt at Stevens's) 1843.

$b$ has the casque not fully developed and the tail wanting, so that its determination is uncertain.

\section{Anthracoceros coronatus.}

Calao des Philippines, D'Aub. Pl. Enl. 873, undè

Buceros coronatus, Bodd. Tabl. Pl. Enl. p. 53.

Anthracoceros coronatus, Elliot, Mon. Bucer.

a (Bt at Stevens's) 1843.

A species of the peninsula of India.

\section{Penelopides.}

Penelopides, Reichenbach, Av. Syst. pl. xlix. (1849).

2061. Penelopides manillæ.

Calao de Manille, D’Aub. Pl. Enl. 891, undè,

Buceros manillce, Bodd. Tabl. Pl. Enl. p. 54.

Penelopides manillce, Wald. Trans. Z. S. ix. p. 168: Elliot, Mon. Bucer.

a Philippine Islands (J. Gould) 1846. —b (Gardner) 1847.

\section{Toccus.}

Tockus, Lesson, Traité d'Orn. p. 252 (1831).

Toccus, Strickland, Ann. \& Mag. N. H. vii. p. 33 (1811).

2062. Toccus erythrorhynchus.

Calao à bec rouge, du Sénégal, D’Aub. Pl. Enl. 260, undè,

Buceros erythrorhynchus, Kuhl, Buff. et D'Aub. Fig. Av. Col. Nom. Syst. p. 5. 
Tockus erythrorhynchus, Strickl. P. Z. S. 1850, p. 219 : Elliot, Mon. Bucer.

a † Kordofan, February (J. Petherick) 1848.

2063. Toccus griseus.

Buceros griseus, Lath Ind. Orn. i. p. 147.

Tockus griseus, Jerd. Ibis, 1872, p. õ: Elliot, Mon. Bucer.

Tockus gingalensis, Jerd. B. Ind. i. p. 250 (nec Shaw).

a Madras ("Buceros gingalensis," T. C. Jerdon) 1845. -6

S. India ("Buceros gingalensis," T. C. Jerdon) 1850.

\section{LOPHOCEROS.}

Lophoceros, Ehrenberg, Symb. Phys. Aves, fol. Z (1831).

2064. Lophoceros nasutus.

Buceros nasutus, Linn. Syst. Nat. i. p. 154: Strickl. \& Scl. Contr. Orn. 1852, p. 155.

Tockus nasutus, Gurney, in And. B. Damara-1. p. 206.

Lophoceros nasutus, Elliot, Mon. Bucer.

a Damara-land (C. J. Andersson) 1852.

\section{Lophoceros birostris.}

Buceros birostris, Scop. Del. Faun. et Fl. Ins. ii. p. 87.

Lophoceros birostris, Elliot, Mon. Bucer.

Meniceros bicornis, Jerd. B. Ind. i. p. 248.

a Madras ("Buceros ginginianus," T. C. Jerdon) 1845. - b Madras ("Buceros ginginianus," T. C. Jerdon) 1850.

\section{Lophoceros melanoleucus.}

Buceros melanoleucus, Licht. Cat. Hamb. p. 8, (fide, Bechst. Syst. Ueb. ii. p. 362).

Lophoceros melanoleucus, Cab. \& Hein. Mus. Hein. ii. p. 168. Buceros coronatus, Shaw, Gen. Zool. viii. p. 35. $a$ (Gardner) 1845. - b (Arthur Strickland) 1850.

A South-African species. 


\section{FAMILY.-MUSOPHAGIDA.}

\section{Corythaix.}

Corythaix, Illiger, Prodr. p. 202 (1811).

\section{Corythaix albocristatus.}

Turacus albocristatus, Strickl. Ann. \& Mag. N. H. vii. p. 33; Contr. Orn. 1851, p. 161.

Corythaix albocristatus, Cab. \& Hein. Mus. Hein. iii. p. 98.

Turacus persa, Layard, B. S. Afr. p. 223 (nec Linn.).

a (Arthur Strickland) 1840.

This specimen was unnamed by Strickland but is probably the type of this South-African species.

\section{Corythaix buffoni.}

Opcethus buffoni, Vieill. N. Dict. d'Hist. N. xxxiv. p. 304. Turacus buffoni, Strickl. Contr. Orn. 1851, p. 161.

Corythaix buffoni, Cab. \& Hein. Mus. Hein. iii. p. 98. Corythaix senegalensis, Sw. B. W. Afr. i. p. 225, pl. 21. $a$ W. Africa (N. C. Strickland) 1838.

\section{Musophaga.}

Musophaga, Isert, Schrift. Berl. Ges. ix. p. 16, pl. 1 (1789). 2069. Musophaga violacea.

Musophaga violacea, Isert, l. s. c.: Cab. \& Hein. Mus. Hein. iii. p. 100.

a Africa ( $W$. Kirtland) 1838.

\section{ScHIZORHIS.}

Chizaerhis, Wagler, Syst. Av. fol. 7, p. 15 (1827).

Schizorhis, Wagler, Isis, 1829, p. 656.

\section{0\%0. Schizorhis africana.}

Phasianus africanus, Lath. Ind. Orn. ii. p. 631.

Schizorhis africana, Cab. \& Hein. Mus. Hein. iii. p. 100. $a$ W. Africa (N. C. Strickland) 1838. 
SUBORDER.-COCCYGES HETERODACTYL王。

\section{FAMILY.-TROGONIDA.}

Trogon.

Trogon, Linnæus, Syst. Nat. i. p. 167 (1766).

20\%1. Trogon mexicanus.

Trogon mexicanus, Sw. Phil. Mag. New Ser. i. p. 440 : Gould, Mon. Trog. Ed. 2, pl. 15.

$a,-b$ Mexico (T. Mann) 1844. —c Guatemala (J. Constancia) 1845 .

\section{0'72. Trogon collaris.}

Trogon collaris, Vieill. N. Dict. d'Hist. N. viii. p. 320; Gould, Mon. Trog. Ed. 2, pl. 5.

a Brazil (N. C. Strickland) 1838. - b Trinidad (Col. J. Taylor).

\section{Trogon personatus.}

Trogon personatus, Gould, Ann. \& Mag. N. H. ix. p. 237; Mon. Trog. Ed. 2, pl. 10.

a (Argent) 1852.

A species found in the Andes from Colombia to Bolivia.

20\%4. Trogon viridis.

Trogon viridis, Linn. Syst. Nat. i. p. 167: Gould, Mon. Trog. Ed. 2, pl. 10.

a Brazil (N. C. Strickland) 1838. - b S. America (W. Kirtland) 1838. -c (N. C. Strickland) 1838.

2075. Trogon aurantius.

Trogon aurantius, Spix. Av. Bras. i. p. 47, pl. 36: Gould, Mon. Trog. Ed. 2, pl. 14.

a Brazil? (Argent) 1852.

20\%6. Trogon surucura.

Trogon surucura, Vieill. N. Dict. d'Hist. N. viii. p. 322: Gould, Mon. Trog. Ed. 2, pl. 25.

$a$ (N. C. Striclcland) 1838.

A species of Southern Brazil and Paraguay. 
207\%. Trogon melanurus.

Trogon melanurus, Sw. An. in Menag. p. 329: Gould, Mon. Trog. Ed. 2, pl. 18.

$a$ (Gardner) 1846. -b (Argent) 1852.

A species of Guiana and the Upper Amazon.

Pharomacrus.

Pharomacrus, De la Llave, Registro Trimestre I. p. 48 (1832).

20'78. Pharomacrus auriceps.

Calurus auriceps, Gould, Ann. \& Mag. N. H. ix. p. 238. Pharomacrus auriceps, Gould, Mon. Trog. Ed. 2, pl. 4. a (Argent) 1852.

A species of Colombia and Ecuador.

HARPACTES.

Harpactes, Swainson, Classif. B. ii. p. 337 (1837).

20\%9. Harpactes rutilus.

Trogon rutilus, Vieill. N. Dict. d'Hist. N. viii. p. 313.

Harpactes rutilus, Gould, Mon. Trog. Ed. 2, pl. 41.

Pyrotrogon orrhophceus, Cab. \& Hein. Mus. Hein. iv. p. 156. $a,-b$ Malacca (N. C. Strickland) 1838.

2080. Harpactes diardi.

Trogon diardi, Temm. Pl. Col. 541.

Harpactes diardi, Gould, Mon. Trog. Ed. 2, pl. 30.

a Malacca (Williams) 1848.

2081. Harpactes fasciatus.

Trogon fasciatus, Gm. Syst. Nat. i. p. 405.

Harpactes fasciatus, Gould, Mon. Trog. Ed. 2, pl. 31. $a,-b$ (Gardner) 1846. — (P. L. Sclater) 1847.

A species found in the island of Ceylon.

2082. Harpactes kasumba.

Trogon kasumba, Raffles, Trans. L. S. xiii. p. 282.

Harpactes kasumba, Gould, Mon. Trog. Ed. 2, pl. 37.

$a,-b$ Malacca (Capt. Nash) 1837. — c Malacca (Fenwick) 1844. - d Malacca (T. C. Eyton). 


\section{Harpactes hodgsoni.}

Harpactes hodgsoni, Gould, Mon. Trog. Ed. 1, pl. 34; Ed. 2, pl. 42.

$a$ (Kirtland) 1838. -b, —c Nepal (B. H. Hodgson) 1845.

2084. Harpactes orescius.

Trogon oreskios, Steph. in Shaw's Gen. Zool. xiv. p. 220: Temm. Pl. Col. 181.

Harpactes oreskios, Gould, Mon. Trog. Ed. 1, pl. 36; Ed. 2, pl. 46.

a Arakan (E. Blyth) 1847.

\section{Hapaloderma.}

Apaloderma, Swainson, Classif. B. ii. p. 337 (1837).

\section{Hapaloderma narina.}

Trogon narina, Vieill. N. Dict. d'Hist. N. viii. p. 318.

Hapaloderma narina, Swains. l. s. c.; Gould, Mon. Trog. Ed. 2, pl. 34 .

$a,-b$ S. Africa (N. C. Strickland) 1838. - c Africa (N.C. Strickland) 1838.

\section{SUBORDER.-COCCYGES ZYGODACTYL杘.}

FAMILY.-GALBULID AE.

\section{Galbula.}

Galbula, Brisson, Ornith. iv. p. 86 (1760).

2086. Galbula viridis.

Alcedo galbula, Linn. Syst. Nat. i. p. 459.

Galbula viridis, Scl. Mon. Galb. et Bucc. p. 7, pl. 2.

a Brazil (Johnson) 1837. - b (Bt at Liverpool) 1853.

This is a Guiana species, $b$ being a skin of Cayenne make. 208\%. Galbula rufoviridis.

Galbula rufoviridis, Cab. Ersch. \& Grub. Enc. sec. 1. lii. p. 308: Scl. Mon. Galb. et Bucc. p. 11, pl. 3.

Galbula maculicauda, Scl. Contr. Orn. 1852, p. 29.

a Brazil (Askew) 1837. —b Brazil (Johnson) 1837. 
2088. Galbula ruficauda.

Galbula ruficauda, Cuv. Règn. Anim. i. p. 420: Scl. Mon. Galb. et Bucc. p. 15, pl. 4 .

a Trinidad (Argent) 1851.

2089. Galbula melanogenia.

Galbula melanogenia, Scl. Contr. Orn. 1852, pp. 61, 93, pl. 90 ; Mon. Galb. et Bucc. p. 19, pl. 5.

$a$ (Argent) 1852.

A Central American species.

2090. Galbula albirostris.

Galbula albirostris, Lath. Ind. Orn. i. p. 245: Scl. Mon. Galb. et Bucc. p. 27, pl. 7.

Galbula chalcocephala, Deville, Rev. Zool. 1849, p. 55.

$a$ Brazil ? (Argent) 1852. - b (Bt at Liverpool) 1853.

$a$ is probably from Bolivia. $b$ is a skin of Cayenne make.

2091. Galbula leucogastra.

Galbula leucogastra, Vieill. N. Dict. d'Hist. N. xxvi. p. 444: Scl. Mon. Galb. et Bucc. p. 33, pl. 9.

a Guiana (N. C. Strickland) 1838.

URogalba.

Urogalba, Bonaparte, Consp. Vol. Zyg. p. 13 (1854).

2092. Urogalba paradisea.

Alcedo paradisea, Linn. Syst. Nat. i. p. 181.

Urogalba paradisea, Scl. Mon. Galb. et Bucc. p. 1, pl. 1. a (Askew) 1839.

A skin of Cayenne make.

JACAMEROPS.

Jacamerops, Cuvier, Règn. Anim. i. p. 420 (1817).

2093. Jacamerops grandis.

Alcedo grandis, Gm. Syst. Nat. i. p. 458.

Jacamerops grandis, Scl. Mon. Galb. et Bucc. p. 57, pl. 18.

a Bolivia (Argent) 1852. 


\section{JACAMARALCYON.}

Jacamaralcyon, Cuvier, Règn. Anim. i. p. 448 (1829).

\section{Jacamaralcyon tridactyla.}

Alcedo tridactyla, Pall. Spic. Zool. vi. p. 10.

Jacamaralcyon tridactyla, Strickl. Ann. \& Mag. N. H. vi. p. 418: Scl. Mon. Galb. et Bucc. p. 49, pl. 16. $a,-b$ Brazil (N. C. Strickland) 1838.

\section{FAMILY.-BUCCONIDE.}

\section{Bucco.}

Bucco, Linnæus, Syst. Nat. i. p. 168 (1766): Strickl. Ann. \& Mag. N. H. viii. p. 371.

\section{Bucco dysoni.}

Bucco dysoni, Scl. P. Z. S. 1855, p. 193 (ex Gray MS.). Notharchus dysoni, Cab. \& Hein. Mus. Hein. iv. p. 149. $a$ Guatemala (Bt at Stevens's) 1848.

\section{Bucco swainsoni.}

Tamatia macrorhynchus, Sw. Zool. Ill. ii. pl. 99 (nec Gm.). Bucco swainsoni, Gray, List Gen. B. i. p. 74: Cab. \& Hein. Mus. Hein. iv. p. 151.

$a$ (N. C. Strickland) 1838. -b (Argent) 1852 .

A Brazilian species.

\section{9\%. Bucco tectus.}

Plus petit Barbu à poitrine noire, de Cayenne, D'Aub. $\mathrm{Pl}$. Enl. 688, f. 2, undè,

Bucco tectus, Bodd. Tabl. Pl. Enl. p. 43.

Nothriscus tectus, Cab. \& Hein. Mus. Hein. iv. p. 146.

$a$ S. America (Cushmore) 1843.

Apparently a skin of Cayenne make.

2098. Bucco tamatia.

Barbu à ventre tacheté, de Cayenne, D'Aub. Pl. Enl. 756, undè, Bucco tamatia, Gm. Syst. Nat. i. p. 405. 
Chaunornis tamatia, Cab. \& Hein. Mus Hein. iv. p. 144. a (Bt at Birmingham) 1850.

A Guiana species.

2099. Bucco bicinctus.

Tamatia bicinctus, Gould, P. Z. S. 1836, p. 80.

Hypnelus bicinctus, Cab. \& Hein. Mus. Hein. iv. p. 143.

$a$ Trinidad (Argent) 1851.

2100. Bucco chacuru.

Bucco chacuru, Vieill. N. Dict. d'Hist. N. iii. p. 239.

Nystalus chacuru, Cab. \& Hein. Mus. Hein. iv. p. 141.

$a,-b$ Brazil (N. C. Strickland) 1838.

2101. Bucco striatipectus.

Bucco striatipectus, Scl. P. Z. S. 1853, p. 125.

a ô Bolivia (Argent) 1852.

\section{MaLACOPTILA.}

Malacoptila, G. R. Gray, List Gen. B. p. 13 (1841).

2102. Malacoptila torquata.

Bucco torquatus, Hahn, Vög. aus As. Afr. \&c. Liefr. 13, p. 3, pl. 5 (ex Wagl.).

Monasa torquata, Strickl. Contr. Orn. 1852, p. 43.

Malacoptila torquata, Cab. \& Hein. Mus. Hein. iv. p. 130. 1838.

a Brazil (Arthur Strickland) 1834. -b Brazil (G. Lloyd)

\section{NonNula.}

Nonnula, Sclater, P. Z. S. 1853, p. 124.

2103. Nonnula rubecula.

Bucco rubecula, Spix, Av. Bras. i. p. 51, pl. 39, f. 1.

Nonnula rubecula, Cab. \& Hein. Mus. Hein. iv. p. 129.

$a,-b$ Brazil (N. C. Strickland). 


\section{MONASA.}

Monasa, Vieillot, Anal. p. 29 (1816).

2104. Monasa atra.

Coucou noire de Cayenne, D'Aub. Pl. Enl. 512, undè,

Cuculus ater, Bodd. Tabl. Pl. Enl. p. 30.

Monasta atra, Cab. \& Hein. Mus. Hein. iv. p. 126.

$a$ Cayenne (N. C. Strickland) 1838. - b Trinidad (Argent) 1851.

\section{Monasa morphea.}

Bucco morphceus, Hahn, Vög. aus As. Afr. \&c. Liefr. 14, p. 1, pl. 2 (ex Wagl.).

Monasta morphea, Cab. \& Hein. Mus. Hein. iv. p. 127.

a f Brazil (N. C. Strickland) 1838.

\section{CHELIDOPTERA.}

Chelidoptera, Gould, P. Z. S. 1836, p. 81.

2106. Chelidoptera tenebrosa.

Cuculus tenebrosus, Pall. Neue Nord. Beitr. iii. p. 3, pl. 1, f. 1. Chelidoptera tenebrosa, Cab. \& Hein. Mus. Hein. iv. p. 124. a Brazil (Askew) 1834. - b Trinidad (Argent) 1851. $a$ is greyer beneath than $b$, but they are of the same size.

\section{FAMILY.-CUCULID AE.}

\section{SUBFAMILY.-CUCULIN AE.}

\section{Cuculus.}

Cuculus, Linnæus, Syst. Nat. i. p. 168 (1766) partim.

\section{0\%. Cuculus canorus.}

Cuculus canorus, Linn. l. s. c.: Strickl. P. Z. S. 1836, p. 100: Jerd. B. Ind. i. p. 322: Sharpe, P. Z. S. 1873, p. 580.

a Smyrna, April (H. E. Strickland) 1834. -b (Askew) 1834. -c $\hat{o}$ Worcestershire, June (H. E. Strickland) 1839. -d f, -e Worcestershire, August (T. Robinson) 1839. - $f$ (Bt at Stevens's) 1844. - $g$ (Bt at Stevens's) 1845. - $h$ Gloucestershire, July ( $H$. E. Strickland) 1853. -i f Rohilcund (W. J. E. Boys) 1847. 


\section{Cuculus micropterus.}

Cuculus micropterus, Gould, P. Z. S. 1837, p. 137: Jerd. B. Ind. i. p. 326.

a Himalaya (L. Fraser) 1845.-b Madras ("Cuculus micropterus," T. C. Jerdon) 1845.

\section{Cuculus solitarius.}

Cuculus solitarius, Steph. in Shaw's Gen. Zool. ix. pt. 1, p. 84, pl. 18: Sharpe, P. Z S. 1873, p. 582.

Cuculus rubiculus, Sw. B. W. Afr. ii. p. 181.

Cuculus flaviventris, Strickl. J. A. S. B. xiii. p. 390, ex Scop. Cf. Wald. Trans. Z. S. ix. p. 161.

a Malacca (W. Kirtland) 1843.

This specimen, referred by Strickl. l. s. c. to C. flaviventris, Scopoli, agrees closely with Swainson's type of $C$. rubiculus, a name, according to Mr Sharpe, synonymous with $C$. solitarius, a Cuckoo found in West and South Africa. The Malaccan origin of this skin is doubtless erroneous. C. flaviventris, Scopoli, probably cannot now be satisfactorily identified.

\section{Cuculus sonnerati.}

Cuculus sonnerati, Lath. Ind. Orn. i. p. 215: Strickl. P. Z. S. 1846, p. 104: Jerd. B. Ind. i. p. 325.

a India (Bt at Stevens's) 1843. - b Madras ("Cuculus sonnerati," T. C. Jerdon) 1846.

\section{Cuculus pravatus.}

Cuculus pravatus, Horsf. Trans. L. S. xiii. p. 179.

Penthoceryx pravatus, Salvad. Ann. Mus. Genov. v. p. 63.

a Malacca (N. C. Strickland) 1838.

\section{Cuculus pallidus.}

Columba pallida, Lath. Ind. Orn. Suppl. p. Ix.

Caccomantis pallidus, Gould, Handb. B. Austr. i. p. 615.

Cuculus inornatus, Vig. \& Horsf. Trans. L. S. xv. p. 297 : Gould, B. Austr. iv. pl. 85.

$a$ New South Wales ( $M^{c}$ Donald) 1838. - b Australia (N. C. Strickland) 1838. —c (Bt at Stevens's) 1843. 


\section{Cuculus poliocephalus.}

Cuculus poliocephalus, Lath. Ind. Orn. i. p. 214: Jerd. B. Ind. i. p. 324 .

a Madras ("Cuculus poliocephalus," T. C. Jerdon) 1845. —b, —c India ("Cuculus poliocephalus," E. Blyth) 1851.

\section{Cuculus clamosus.}

Cuculus clamosus, Lath. Ind. Orn. Suppl. p. xxx.: Sharpe, P. Z. S. $187 \AA$, p. 587 .

Cuculus nigricans, Sw. Zool. Ill. 2nd Ser. i. pl. 7.

$a$ W. Africa (N. C. Strickland) 1838. -b (N. C. Strickland). 1838.

\section{HiEROCOCCYX.}

Hierococcyx, Müller, Verh. Nat. Gesch. nederl. overz. Bezitt. p. 117 (1842).

\section{Hierococcyz varius.}

Cuculus varius, Vahl. Skrivt. Nat. Selsk. iv. p. 60 (1797): Strickl. Ann. \& Mag. N. H. xviii. p. 399.

Hiracococcyx varius, Cab. \& Heine, Mus. Hein. iv. p. 29 : Jerd. B. Ind. i. p. 329.

$a$ India (Bt at Stevens's) 1844. - b Madras (T. C. Jerdon) 1845.

\section{Hierococcyx fugax.}

Cuculus fugax, Horsf. Trans. L. S. xiii. p. 178.

Hieracococcyx fugax, Salvad. Ann. Mus. Genov. v. p. 65. a Malacca (N. C. Strickland) 1838.

\section{1\%. Hierococcyx sparverioides.}

Cuculus sparverioides, Vig. P. Z. S. 1830-1, p. 173: Gould, Cent. B. Him. pl. 53.

Hierococcyx sparverioides, Jerd. B. Ind. i. 331.

a India (Askew) 1840.

\section{Cacomantis.}

Cacomantis, Müller, Verh. Nat. Gesch. nederl, overz. Bezitt. p. 117 (1842).

S. C. 


\section{Cacomantis merulinus.}

Cuculus merulinus, Scop. Del. Faun. et Fl. Ins. ii. p. 89 . Cacomantis merulinus, Wald. Trans Z. S. ix. p. 160. a Philippine Islands, 13 May (J. Cuming) 1840.

\section{Cacomantis flabelliformis.}

Cuculus fabelliformis, Lath. Ind. Orn. Suppl. p. xxx.

Cacomantis fabelliformis, Gould, Handb. B. Austr.i. p. 618.

Cuculus cineraceus, Vig. \& Horsf. Trans. L. S. xv. p. 298: Gould, B. Austr. iv. pl. 86.

a Australia (Askew) 1834. -b (Askew) 1837. - c Australia (N. C. Strickland) 1838. -d New South Wales (MDonald) 1838.

2120. Cacomantis threnodes.

Cacomantis threnodes, Cab. \& Hein. Mus. Hein. iv. p. 19: Wald. Trans. Z. S. viii. p. 54.

$a$ (Aslew) 1837. - b Malacca? (N. C. Strickland) 1838.

These specimens agree best with Malaccan skins.

\section{Cacomantis passerinus.}

Cuculus passerinus, Vahl. Skrivt. Nat. Selsk. iv. p. 57.

Cacomantis passerinus, Cab. \& Hein. Mus. Hein. iv. p. 18.

Polyphasia passerina, Jerd. Ibis, 1872, p. 14, pl. 1.

Cuculus tenuirostris, Gray, Ill. Ind. Zool. ii. pl. 34, f. 1: cf. Strickl. Ann. \& Mag. N. H. xiii. p. 34.

$a$ (Askew) 1834. -b (Askew) 1837. -c (N. C. Strickland) 1838. - $d$ S. India ("Cuculus flavus, adult," T. C. Jerdon) 1844. -e S. India ("Cuculus flavus, young female," T. C. Jerdon) 1844 .

\section{Cacomantis rufiventris.}

Polyphasia rufiventris, Jerd. Ibis, 1872, p. 15.

Polyphasia tenuirostris, Jerd. B. Ind. i. p. 335 (nec Gray). 1846.

$a$ (Askew) 1833. - b India ("Cuculus tenuirostris," E. Blyth)

Two young birds which should probably bear this name.

Surniculus.

Surniculus, Lesson, Traité d'Orn. p. 151 (1831). 


\section{Surniculus lugubris.}

Cuculus lugubris, Horsf. Trans. L. S. xiii. p. 175.

Surniculus lugubris, Salvad. Ann. Mus. Genov. v. p. 63.

a Malacca (Capt. Nash) 1837. - b Indian Archipelago ( $N$. C. Strickland) 1838. — Malacca (Bt at Stevens's) 1844.

\section{Surniculus dicruroides.}

Pseudornis dicruroides, Hodgs. J. A. S. B. viii. p. 136.

Surniculus dicruroides, Jerd. B. Ind. i. p. 336.

a Nepal (B. H. Hodgson, 502) 1845.

\section{Mesocalius.}

Mesocalius, Cabanis \& Heine, Mus. Hein. iv. p. 16 (1862).

\section{Mesocalius osculans.}

Chalcites osculans, Gould, P. Z. S. 1847, p. 32; B. Austr. iv. pl. 88.

Mesocalius osculans, Gould, Handb. B. Austr. i. p. 621.

$a$ New South Wales $\left(M^{\circ}\right.$ Donald) 1838.

\section{CHRysococcyx.}

Chrysococcyx, Boie, Isis, 1826, p. 997.

\section{Chrysococcyx lucidus.}

Cuculus lucidus, Gm. Syst. Nat. i. p. 421: cf. Strickl. Ann. \& Mag. N. H. xi. p. 337.

Chrysoccocyx lucidus, Gould, B. Austr. iv. p. 89.

Lamprococcyx lucidus, Cab. \& Hein. Mus. Hein. iv. p. 14.

$a$ (Askew) 1837. -b, -c New South Wales (N. C. Strickland) 1838. -d (Chapman) 1849. —e (Argent) 1851.

\section{2\%. Chrysococcyx smaragdinus.}

Chrysococcyx smaragdinus, Blyth, J. A. S. B. xv. p. 53.

Chrysococcyx hodgsoni, Horsf. \& Moore, Cat. B. E. I. Co. Mus. ii. p. 70 5 : Jerd. B. Ind. i. p. 338.

$a($ Bt at Stevens's) 1844. - b Ceylon (E. Blyth) 1850.

2128. Chrysococcyx smaragdineus.

Chalcites smaragdineus, Sw. B. W. Afr. ii. p. 191.

Chrysococcyx smaragdineus, Strickl. Contr. Orn. 1851, pp. $135,162$. 
Cuculus smaragdineus, Sharpe, P. Z. S. 1873, p. 589.

$a,-b$ Cape of Good Hope (N. C. Strickland) 1838.

\section{Chrysococcyx cupreus.}

Coucou vert du Cap de Bonne Espérance, D'Aub. Pl. Enl. 657, undè,

Cuculus cupreus, Bodd. Tabl. Pl. Enl. p. 40: Sharpe, P. Z. S. 1873, p. 591.

Cuculus auratus, Gm. Syst. Nat. i. p. 421.

a W. Africa (N. C. Strickland) 1838.

\section{Chrysococcyx klaasi.}

Cuculus klaasi, Steph. in Shaw's Gen. Zool. ix. pt. i. p. 129: Sharpe, P. Z. S. 1873, p. 592.

$a,-b$ S. Africa (N. C. Strickland) 1838. -c, -d (N.C. Strickland) 1838.

\section{Coccystes.}

Coccystes, Gloger, Handb. i. p. 449 (1834).

\section{Coccystes glandarius.}

Cuculus glandarius, Linn. Syst. Nat. i. p. 169.

Coccystes glandarius, Cab. \& Hein. Mus. Hein. iv. p. 44: Sharpe, P. Z. S. 1873 , p. 595.

Oxylophus glandarius, Strickl. P. Z. S. 1850, p. 219.

$a$ (N. C. Strickland) 1838. - b $\hat{o}$ Kordofan, 10th August (J. Petherick) 1848.

\section{Coccystes cafer.}

Cuculus cafer, Licht. Cat. Hamb. p. 14.

Coccystes cafer, Sharpe, P. Z. S. 1873, p. 596.

Cuculus afer, Leach, Zool. Misc. i. p. 71, pl. 31.

Oxylophus afer, Strickl. P. Z. S. 1850, p. 219.

a Africa (N. C. Strickland) 1838.

\section{Coccystes jacobinus.}

Coucou truppé de la côte de Coromandel, D’Aub. Pl. Enl. 872 , undè,

Cuculus jacobinus, Bodd. Tabl. Pl. Enl. p. 53: Strickl. P. Z. S. 185 0, p. 219.

Coccystes jacobinus, Sharpe, P. Z. S. 1873, p. 598. 
Cuculus melanoleucus, Gm. Syst. Nat. i. p. 416.

$a$ India (Askew) 1834. -b (N. C. Strickland) 1838.

\section{Coccystes serratus.}

Cuculus serratus, Sparrm. Mus. Carls. i. pl. 3.

Oxylophus serratus, Strickl. P. Z. S. 1850, p. 219.

Coccystes serratus, Sharpe,.P. Z. S. 1873, p. 599.

$a$ S. Africa (Askew) 1834. - b S. Africa (Bt at Stevens's) 1843. —c o Kordofan, 9th July ( $J$. Petherick) 1848. —d S. Africa (Dewgard) 1849.

\section{Coccystes coromandus.}

Cuculus coromandus, Linn. Syst. Nat. i. p. 171.

Coccystes coromandus, Cab. \& Hein. Mus. Hein. iv. p. 45; Salvad. Ann. Mus. Genov. v. p. 67.

$a(N$. C. Strickland) 1838.

A common Malaccan species also found in India, \&c.

\section{Eudynamis.}

Eudynamis, Vigors \& Horsfield, Trans. L. S. xv. p. 303 (1826).

\section{Eudynamis malayana.}

Eudynamis malayana, Cab. \& Hein. Mus. Hein. iv. p. 52: Wald. Ibis, 1869, p. 339.

$a$ E. Indies (Arthur Strickland) 1834. $-b+$ E. Indies (Dr Horsfield) 1837. —c E. Indies ("Cuculus orientalis," Dr Horsfield) 1837. —d (Bt at Stevens's) 1843.—e India (Bt at Stevens's) 1843.

\section{3\%. Eudynamis mindanensis.}

Cuculus mindanensis, Linn. Syst. Nat. i. p. 169.

Eudynamis mindanensis, Wald. Ibis, 1869, p. 340: Trans.

Z. S. ix. p. 162.

a Philippine Islands (J. Gould) 1846.

\section{Eudynamis cyanocephala.}

Cuculus cyanocephalus, Lath. Ind. Orn. Suppl. p. xxx.

Eudynamis cyanocephala, Wald. Ibis, 1869, p. 340.

Cuculus findersi, Lath. Gen. Hist. iii. p. 308. 
Eudynamis australis, Sw. An. in Menag. p. 344.

$a$ New South Wales ( $M^{c}$ Donald) 1838. - b Australia (Bt at Birmingham) 1843.

\section{SCYTHROPS.}

Scythrops, Latham, Ind. Orn. i. p. 141 (1790): Strickl. Ann. \& Mag. N. H. vii. p. 33; xii. p. 240.

\section{Scythrops novæ-hollandiæ.}

Scythrops novce hollandice, Lath. l. s. c.; Gould, B. Austr. iv. pl. 90: Cab. \& Hein. Mus. Hein. iv. p. 57.

$a$ New South Wales $\left(M^{c}\right.$ Donald $) 1838$.

\section{SUBFAMILY.-PHENICOPHAIN E.}

TACCOCUA.

Taccocua, Lesson, Traité d'Orn. p. 143 (1831).

2140. Taccocua leschnaulti.

Taccocua leschnaulti, Less. l. s. c., Jerd. B. Ind. i. p. 352.

Zanclostomus sirkee, Jerd. (olim nec Gray).

a Madras ("Zanclostomus sirkee," T. C. Jerdon) 1845.

RHINORTHA.

Rhinortha, Vigors, Mem. Raffles, p. 671 (1830).

\section{Rhinortha chlorophæa.}

Cuculus chlorophceus, Raffl. Trans. L. S. xiii. p. 288.

Rhinortha chlorophcea, Salvad. Ann. Mus. Genov. v. p. 69.

Rhinortha caniceps, Vig. l. s. c.

$a,-b$ Malacca (Capt. Nash) 1837. -c Malacca (N.C. Strickland) 1838.

\section{RHOPODYTES.}

Rhopodytes, Cab. \& Hein. Mus. Hein. iv. p. 61 (1862).

\section{Rhopodytes diardi.}

Melias diardi, Less. Traité d'Orn. p. 132.

Rhopodytes diardi, Cab. \& Hein. Mus. Hein. iv. p. 61.

a Malacca (Capt. Nash) 1837. - b Malacca (Major Stacey). 


\section{Rhopodytes viridirostris.}

Zanclostomus viridirostris, Jerd. Madr. Journ. xi. p. 223; B. Ind. i. p. 346.

Rhopodytes viridirostris, Cab. \& Hein. Mus. Hein. iv. p. 63.

$a$ India (Askew) 1837. - $b$ S. India (Zanclostomus viridirostris, T. C. Jerdon) 1850.

\section{Rhopodytes tristis.}

Melias tristis, Less. Traité d'Orn. p. 132.

Zanclostomus tristis, Jerd. B. Ind. i. p. 345.

Rhopodytes tristis, Cab. \& Hein. Mus. Hein. iv. p. 64.

a India (Lord A. Hay) 1845. - b India (Zanclostomus tristis, E. Blyth) 1846.

\section{Rhopodytes erythrognathus.}

Cuculus melanognathus, Raff. Trans. L. S. xiii. p. 287 (nec Horsf.).

Phoenicophoeus erythrognathus, Hartl. Verz. Mus. Brem. p. 95. Rhamphococcyx erythrognathus, Cab. \& Hein. Mus. Hein. iv. p. 67.

a Malacca (Capt. Nash) 1837.

RHINococcyx.

Rhinococcyx, Sharpe, P. Z. S. 1873, p. 604.

2146. Rhinococcyx curvirostris.

Cuculus curvirostris, Shaw, Nat. Misc. xxi. pl. 905.

Rhamphococcyx curvirostris, Cab. \& Hein. Mus. Hein. iv. p. 66 .

Phcenicophceus tricolor, Steph. in Shaw's Gen. Zool. ix. p. 61, pl. 14.

a Malacca (N. C. Strickland) 1838. -b (Bt at Birmingham) 1849.

\section{ZaNCLOSTOMUS.}

Zanclostomus, Swainson, Classif. B. ii. p. 123 (1837).

214\%. Zanclostomus javanicus.

Phœnicophceus javanicus, Horsf. Trans. L. S. xiii. p. 178.

Zanclostomus javanicus, Cab. \& Hein. Mus. Hein. iv. p. 64.

a Malacca.(N. C. Strickland) 1838. 
Poliococcyx.

Poliococcyx, Sharpe, P. Z. S. 1873, p. 606.

\section{Poliococcyx sumatranus.}

Cuculus sumatranus, Raff. Trans. L. S. xiii. p. 287.

Rhopodytes sumatranus, Cab. \& Hein. Mus. Hein. iv. p. 62.

Poliococcyx sumatranus, Sharpe, $l$. s. c.

a Malacca (Thomas) 1840.

\section{SUBFAMILY.-COCCYGIN $Æ$.}

Coccygus.

Coccyzus, Vieillot, Anal. p. 28 (1816).

\section{Coccygus americanus.}

Cuculus americanus, Linn. Syst. Nat. i. p. 170.

Coccygus americanus, Cab. \& Hein. Mus. Hein. iv. p. 75 : Baird, Brew. \& Ridgw. N. Am. B. p. 477. 1848.

\section{Coccygus minor.}

Cuculus minor, Gm. Syst. Nat. i. p. 411.

Cuculus seniculus, Lath. Ind. Orn. i. p. 219.

Coccyzus seniculus, Gosse, B. Jam. p. 281.

a Jamaica (P. H. Gosse) 1846.

\section{Coccygus erythrophthalmus.}

Cuculus erythrophthalmus, Wils. Am. Orn. iv. p. 16, pl. 28, f. 2 .

Coccygus erythrophthalmus, Cab. \& Hein. Mus. Hein. iv. p. 76: Baird, Brew. \& Ridgw. N. Am. B. ii. p. 484.

a ई̊ Carlisle, Pennsylvania, 13 May 1846 (S. F. Baird) 1847.

\section{Coccygus melanocoryphus.}

Coccyzus melacoryphus, Vieill. N. Dict. d'Hist. N. viii.p. 271. Coccyzus melanocoryphus, Scl. Cat. Am. B. p. 323.

$a$ Chili? ( $N$. C. Strickland) 1838. -b (Arthur Strickland) 1840.

A common South-American Cuckoo, but not found in Chili. 


\section{Coccygus pumilus.}

Coccyzus pumilus, Strickl. Contr. Orn. 1852, p. 28, pl. 82.

$a$ Trinidad (Argent) 1851.

The type of this species.

\section{Hyetornis.}

Hyetornis, Sclater, Cat. Am. B. p. 321 (1862).

\section{Hyetornis pluvialis.}

Cuculus pluvialis, Gm. Syst. Nat. i. p. 411.

Piaya pluvialis, Gosse, B. Jam. p. 277.

Hyetornis pluvialis, Scl. l. s. c.

$a$ Gulf of Mexico (N. C. Strickland) 1838.

A species peculiar to the island of Jamaica.

\section{SAUROTHERA.}

Saurothera, Vieillot, Anal. p. 28 (1816).

2155. Saurothera vetula.

Cuculus vetula, Linn. Syst. Nat. i. p. 169.

Saurothera vetula, Gosse, B. Jam. p. 273; Ill. pl. 74: Cab. \& Hein. Mus. Hein. iv. p. 90.

a Jamaica (J. Gould) 1846. -b (Bt at Edinburgh) 1852.

\section{Pinya.}

Piaya, Lesson, Traité d'Orn. p. 139 (1831).

\section{Piaya cayana.}

Cuculus cayanus, Linn. Syst. Nat. i. p. 170.

Piaya circe Bp., P. cayana (L.), P. nigricrissa Scl., P. mexicana Sw., P. mehleri Bp., Scl. Cat. Am. B. pp. 321, 322.

$a$ S. America (Askew) 1834. - b Brazil (Askew) 1834. —c Guatemala (J. Constancia) 1848.

\section{5\%. Piaya minuta.}

Cuculus rutilus, Ill. Abhandl. Berl. Ak. Wissensch. 1812, p. 224.

Pyrrhococcyx rutilus, Cab. \& Hein. Mus. Hein. iv. p. 88.

Cuculus minutus et $C$. rutilus, Vieill. N. Dict. d'Hist. N. viii. pp. $275,277$. 
Coccycua monacha, Less. Traité d'Orn. p. 142.

a (N. C. Strickland) 1838. - b S. America (Arthur Strickland) 1840.

\section{DiPLOPTERUS.}

Diplopterus, Boie, Isis, 1826, p. 977.

\section{Diplopterus nævius.}

Cuculus ncevius, Linn. Syst. Nat. i. p. 170.

Diplopterus novius, Cab. \& Hein. Mus. Hein. iv. p. 93. 1851.

$a,-b,-c$ (N. C. Strickland) 1838. —d Trinidad (Argent)

\section{Dromococcyx.}

Dromococcyx, Wied, Beitr. Naturg. Bras. iv. p. 353 (1832).

\section{Dromococcyx phasianellus.}

Macropus phasianellus, Spix, Av. Bras. i. p. 53, pl. 42.

Dromococcyx phasianellus, Cab, \& Hein. Mus. Hein. iv. p. 95. a Trinidad (Argent) 1851.

\section{GeOCOCCYX.}

Geococcyx, Wagler, Isis, 1831, p. 524.

\section{Geococcyx mexicanus.}

Phasianus mexicanus, Gm. Syst. Nat. i. p. 741.

Geococcyx mexicanus, Strickl. Ann. \& Mag. N. H. viii. p. 544: Scl. Cat. Am. B. p. 324.

a Mexico (T. Mann) 1844.

\section{Geococcyx affinis.}

Geococcyx affinis, Hartl. Rev. Zool. 1844, p. 215: Scl. Cat. Am. B. p. 324.

$a$ (Dewgard) 1846. - b Guatemala (J. Constancia) 1848.

\section{SUBFAMILY.-CROTOPHAGIN AA.}

Gurra.

Guira, Lesson, Traité d'Orn. p. 149 (1831).

\section{Guira piririgua.}

Cuculus guira, Gm. Syst. Nat. i. p. 414.

Octopteryx guira, Cab. \& Hein. Mus. Hein. iv. p. 98. 
Crotophaga piririgua, Vieill. N. Dict. d'Hist. N. i. p. 549. Guira piririgua, Strickl. Ann. \& Mag. N. H. vii. p. 36. a Brazil (Askew) 1833.

\section{Crotophaga.}

Crotophaga, Linnæus, Syst. Nat. i. p. 154 (1766).

\section{Crotophaga ani.}

Crotophaga ani, Linn. l. s. c.: Scl. Cat. Am. B. p. 320.

a Brazil (Askew) 1834. — b S. America.

\section{Crotophaga sulcirostris.}

Crotophaga sulcirostris, Sw. Phil. Mag. New Ser. i. p. 440: Scl. Cat. Am. B. p. 320.

$a$ (Thomas) 1840. - b, —c Guatemala (J. Constancia).

\section{Crotophaga major.}

Crotophaga major, Gm. Syst. Nat. i. p. 363: Scl. Cat. Am. B. p. 320.

$a$ S. America (Bt at Aberystwith) 1833. - $b$ Trinidad (Argent) 1851 .

\section{SUBFAMILY.-CENTROPIN A.}

\section{Centropus.}

Centropus, Illiger, Prodr. p. 205 (1811).

2166. Centropus bengalensis.

Cuculus bengalensis, Gm. Syst. Nat. i. p. 412.

Centrococcyx bengalensis, Cab. \& Hein. Mus. Hein. iv. p. 111.

Centropus philippensis, Jerd. B. Ind. i. p. 348.

$a,-b$ (N. C. Strickland) 1838. - c Malacca (N. C. Strickland) 1838. - d (Bt at Stevens's) 1844. —e Madras ("Centropus philippensis," T. C. Jerdon) 1845. $-f,-g$ India ("Centropus philippensis," E. Blyth) 1846.

\section{Centropus viridis.}

Cuculus viridis, Scop. Del. Faun. et Fl. In. ii. p. 89.

Centrococcyx viridis, Wald. Trans. Z. S. ix. p. 163.

Corydonis pyrrhopterus, Vieill. Enc. Méth. p. 1353.

a Philippine Islands (J. Gould) 1846. 


\section{Centropus affinis.}

Centropus affinis, Horsf. Trans. L. S. xiii. p. 180.

Centrococcyx affinis, Wald. Trans. Z. S. viii. p. 56.

$a$ (N. C. Strickland) 1838.

Agrees with a specimen from Dr Horsfield in the University collection marked 'Centropus affinis.' The species is found in Java.

2169. Centropus javanensis.

Cuculus javanensis, Dumont, Dict. Sc. Nat. xi. p. 144.

Centrococcyx javanensis, Wald. Trans. Z. S. viii. p. 60.

Centropus lepidus, Horsf. Trans. L. S. xiii. p. 180.

a Malacca (N.C. Strickland) 1838. - b Malacca (W. Kirtland) 1843.

\section{1\%0. Centropus phasianinus.}

Cuculus phasianinus, Lath. Ind. Orn. Suppl. p. xxx.

Centropus plıasianinus, Strickl. Ann. \& Mag. N. H. xi. p. 335.

Centropuis phasianus, Gould, B. Austr. iv. pl. 92 ; Handb. B. Austr. i. p. 634 .

a Australia (Sir W. Jardine) 1845. - b

21\%1. Centropus monachus.

Centropus monachus, Rïpp. Neue Wirb. Vög. p. 57, pl. 21, f. 2 ; Sharpe, P. Z. S. 1873 , p. 620.

a Senegal (Askew) 1837. - $b$ Cape of Good Hope (N. C. Strickland) 1838.

\section{FAMILY.-INDICATORIDE.}

\section{INDICATOR.}

Indicator, Vieillot, Anal. p. 28 (1816).

2172. Indicator minor.

Indicator minor, Steph. in Shaw's Gen. Zool. ix. p. 140: Cab. \& Hein. Mus. Hein. iv. p. 3.

$a$ Africa (Askew) 1834.

\section{Indicator sparrmani.}

Indicator sparrmani, Steph. in Shaw's Gen. Zool. ix. p. 138: Cab. \& Hein. Mus. Hein. iv. p. 6.

Indicator leucotis, Sw. B. W. Afr. ii. p. 193.

$a$ Africa (N. C. Strickland) 1838. - b S. Africa (Bt at Stevens's) 1842. 


\section{FAMILY.-CAPITONIDA.}

PogONORHYNCHUS.

Pogonorhynchus, Van der Hoven, Handb. ii. p. 461 (1835).

2174. Pogonorhynchus dubius.

Bucco dubius, Gm. Syst. Nat. i. p. 109.

Pogonorhynchus dubius, Marsh. Mon. Cap. p. 7, pl. 4.

Pogonia sulcirostris, Leach, Zool. Misc. ii. p. 46, pl. 76.

a W. Africa (W. Kirtland) 1838.

\section{Pogonorhynchus torquatus.}

Bucco torquatus, Dum. Dict. Sc. Nat. iv. p. 56.

Pogonorhynchus torquatus, Marsh. Mon. Cap. p. 19, pl. 10.

$a$ S. Africa (N. C. Strickland) 1838.

2176. Pogonorhynchus vieilloti.

Pogonius vieilloti, Leach, Zool. Misc. ii. p. 104, pl. 97: Strickl. P. Z. S. 1850, p. 219.

Pogonorhynchus vieilloti, Marsh. Mon. Cap. p. 21, pl. 11.

Pogonias senegalensis, Licht. Verz. Doubl. p. 9.

$a$ W. Africa (Johnson) 1837. - $b$ \& Kordofan, 8 July ( $J$. Petherick) 1846.

\section{7\%. Pogonorhynchus leucomelas.}

Le Barbu à plastron noir, D'Aub. Pl. Enl. 688, f. 1, undè, Bucco leucomelas, Bodd. Tabl. Pl. Enl. p. 43.

Pogonias leucomelas, Strickl. \& Scl. Contr. Orn. 1852, p. 155.

Pogonorhynchus leucomelas, Marsh. Mon. Cap. p. 23, pl. 12.

$a$ S. Africa (N. C. Strickland) 1838. - b Damara-land (C.J. Andersson) 1852.

\section{Megalmina.}

Megalaima, G. R. Gray, List Gen. B. App. p. 12 (1842).

\section{Megalæma virens.}

Le grand Barbu, D'Aub. Pl. Enl. 871, undè,

Bucco virens, Bodd. Tabl. Pl. Enl. p. 53.

Megalaema virens, Marsh. Mon. Cap. p. 33, pl. 16.

Bucco grandis, Gm. Syst. Nat. i. p. 408.

a India (Fenwick) 1844. 


\section{Megalæma chrysopogon.}

Bucco chrysopogon, Temm. Pl. Col. 285.

Megalcema chrysopogon, Marsh. Mon. Cap. p. 39, pl. 18.

a Malacca (Capt. Nash) 1837.

\section{Megalæma mystacophonus.}

Bucco mystacophonus, Temm. Pl. Col. 31 .

Megalcema mystacophonus, Marsh. Mon. Cap. p. 41, pl. 19.

a Malacca (Capt. Nash) 1837.

\section{Megalæma versicolor.}

Bucco versicolor, Raffl. Trans. L. S. xiii. p. 284.

Megalcema versicolor, Marsh. Mon. Cap. p. 47, pl. 22.

a Malacca (Capt. Nash) 1837.

\section{Megalæma corvina.}

Bucco corvinus, Temm. Pl. Col. 522 (ex Reinw.).

Megalcema corvina, Marsh. Mon. Cap. p. 49, pl. 23.

a Malacca (N. C. Strickland) 1838.

\section{Megalæma franklini.}

Bucco franklini, Blyth, J. A. S. B. xi. p. 167.

Megalcema franklini, Marsh. Mon. Cap. p. 51, pl. 24.

a Darjuling (E. Blyth) 1850.

\section{Megalæma asiatica.}

Trogon asiaticus, Lath. Ind. Orn. p. 201.

Megalcema asiatica, Marsh. Mon. Cap. p. 63, pl. 29.

Capito cyaneicollis, Vieill. N. Dict. d'Hist. N. p. 498.

Bucco cyanops, Cuv. Règn. An. i. p. 428.

Bucco cceruleigula, Hodgs. Zool. Misc. p. 85.

$a,-b$ Nepal (B. H. Hodgson) 1845. — (Gardner) 1845.

-d India ("Bucco cyanops, Cùv." E. Blyth) 1846. -e India (Major Stacey).

\section{Megalæma henrici.}

Bucco henrici, Temm. Pl. Col. 524 (ex Boie).

Megalcema henrici, Marsh. Mon. Cap. p. 71, pl. 31.

$a,-b$ Malacca (Capt. Nash) 1837. 


\section{Megalæma duvauceli.}

Bucco duvaucelii, Less. Traité d'Orn. p. 164.

Megalcema duvancelii, Marsh. Mon. Cap. p. 75, pl. 33.

Bucco frontalis, Temm. Pl. Col. 536, f. 1.

a Malacca (Dr Prattington) 183- - - b Sumatra (Askew) 1833. — Malacca (Capt. Nash) 1837. —d (T. C. Eyton) 1846.

\section{8\%. Megalæma hodgsoni.}

Megalcema hodgsoni, Bp. Consp. Av. i. p. 144: Marsh. Mon. Cap. p. 83, pl. 36 .

a Nepal (B. H. Hodgson, 170) 1845. - b Himalaya Mts. (Bt at Stevens's) 1848.

\section{Megalæma viridis.}

Barbu verd, D'Aub. Pl. Enl. 870, undè,

Bucco viridis, Bodd. Tabl. Pl. Enl. p. 53: Strickl. Ann. \& Mag. N. H. xiii. p. 34.

Megalcema viridis, Marsh. Mon. Cap. p. 81, pl. 35.

$a$ (Askew) 1833. - b (Bt at Stevens's) 1843.

A species of Southern India.

\section{Megalæma caniceps.}

Bucco caniceps, Frankl. P. Z. S. 1830-31, p. 121: Strickl. Ann. \& Mag. N. H. xiii. p. 34.

Megalcema caniceps, Marsh. Mon. Cap. p. 91, pl. 39.

a Madras (T. C. Jerdon) 1845. - b Madras ("Bucco zeylanicus," T. C. Jerdon) 1845. - c India ("Bucco zeylanicus, Gm., caniceps, Frankl.," E. Blyth) 1846. -d India (E. Blyth) 1847. -e ô Benares (W. J. E. Boys) 1847. - $f$ Ceylon ("Bucco zeylonicus, Yellow-cheeked Barbet, Brown," (E. Blyth) 1850.

$e$ and $f$ are almost exactly alike, the origin of the latter being perhaps incorrectly stated.

\section{XANTHOL שMA.}

Xantholcema, Bonaparte, Ann. Sci. Nat. 1854, p. 130.

2190. Xantholæma hæmacephala.

Barbu des Philippines, D'Aub. Pl. Enl. 331, undè, Bucco hcemacephalus, Müll. Syst. Nat. Suppl. p. 88. 
Xantholcema hoemacephala, Marsh. Mon. Cap. p. 101, pl. 42: Wald. Trans. Z. S. ix. p. 156.

$a$ Philippine Islands, 18 May (H. Cuming) 1840. - b Malacca.

\section{Xantholæma rosea.}

Bucco roseus, Dumont, Dict. Sc. Nat. iv. p. 52.

Xantholcema rosea, Marsh. Mon. Cap. p. 107, pl. 43.

a (N. C. Strickland) 1838.

A Javan species.

\section{Xantholæma rubricapilla.}

Bucco rubricapillus, Gm. Syst. Nat. i. p. 408.

Xantholcema rubricapilla, Marsh. Mon. Cap. p. 109, pl. 44.

Bucco barbiculus, Cuv. Règn. An. i. p. 428. $C f$. Wald. Trans.

Z. S. ix. p. 157.

a Ceylon (Capt. Pearson) 1840.

\section{Xantholæma malabarica.}

Bucco malabaricus, Blyth, J. A. S. B. xvi. pp. $386,465$.

Xantholcema malabarica, Marsh. Mon. Cap. p. 111, pl. 45.

a Madras ("Bucco indicus," T. C. Jerdon) 1845. - b Madras ("Bucco barbiculus," T. C. Jerdon) 1846.

\section{BARBATULA.}

Barbatula, Lesson, Compl. Buff. ix. p. 292 (1837).

\section{Barbatula pusilla.}

Bucco pusillus, Dumont, Dict. Sc. Nat. iv. p. 50.

Barbatula pusilla, Marsh. Mon. Cap. p. 117, pl. 48.

$a$ S. Africa (Bt at Stevens's) 1842.

\section{Barbatula chrysocoma.}

Bucco chrysocomus, Temm. Pl. Col. 536, f. 2.

Barbatula chrysocoma, Marsh. Mon. Cap. p. 119, pl. 49. $a,-b$ Senegal (N. C. Strickland) 1838.

\section{TRACHYPHONUS.}

Trachyphonus, Ranzani, Elem. Orn. p. 157 (1823).

2196. Trachyphonus margaritatus.

Bucco margaritatus, Rüpp. Atlas, Vög. p. 30. 
Trachyphonus margaritatus, Strickl. P. Z. S. 1850, p. 219: Marsh. Mon. Cap. p. 143, pl. 57.

$a \hat{\delta},-b$ q Kordofan, 28 April (J. Petherick) 1848.

Capito.

Capito, Vieillot, Anal. p. 27 (1816).

\section{Capito niger.}

Barbu de Cayenne, D'Aub. Pl. Enl. 206, undè,

Bucco niger, Müll. Syst. Nat. Suppl. p. 89.

Capito niger, Marsh. Mon. Cap. p. 157, pl. 63.

Bucco cayennensis, Gm. Syst. Nat. i. p. 405.

Capito cayennensis, Cab. in Schomb. Guiana, iii. p. 720.

$a$ S. America (N. C. Strickland) 1838. - b $\hat{\delta}$ Demarara (R. Schomburgk) 183-.

\section{Capito bourcieri.}

Micropogon bourcieri, Lafr. Rev. Zool. 1845, p. 179.

Capito bourcieri, Marsh. Mon. Cap. p. 165, pl. 66.

Micropogon hartlaubi, Lafr. l. s. c.

a (Argent) 1852.

A skin of Bogota make.

\section{Calorhamphus.}

Calorhamphus, Lesson, Rev. Zool. 1839, p. 138.

\section{Calorhamphus lathami.}

Bucco lathami, Raff. Trans. L. S. xiii. p. 284: Strickl. Ann. \& Mag. N. H. viii. p. 374.

Calorhamphus lathami, Marsh. Mon. Cap. p. 179, pl. 72.

a Malacca (Capt. Nash) 1837. - b Malacca (N. C. Strickland) 1838.

\section{FAMILY.-RHAMPHASTID $\mathbb{E}$.}

\section{Rhamphastos.}

Rhamphastos, Linnæus, Syst. Nat. i. p. 150 (1766).

2200. Rhamphastos toco.

Rhamphastos toco, Gm. Syst. Nat. i. p. 356: Gould, Mon. Rhamph. ed. 2, pl. 1.

S. C. 
$a$ (Arthur Strickland) 1850.

A Guiana species.

2201. Rhamphastos carinatus.

Rhamphastos carinatus, Sw. Zool. Ill. i. pl. 45: Gould, Mon. Rhamph. ed. 2, pl. 2.

$a$ (Arthur Strickland) 1850.

A Central-American species.

2202. Rhamphastos erythrorhynchus.

Rhamphastos erythrorhynchus, Gm. Syst. Nat. i. p. 355: Gould, Mon. Rhamph. ed. 2, pl. 6.

$a$ Guiana (E. Brown) 1850.

2203. Rhamphastos cuvieri.

Rhamphastos cuvieri, Wagl. Syst. Av. fol. 1, p. 3: Gould, Mon. Rhamph. ed. 2, pl. 8.

a S. America (Thomas) 1845.

An Amazon species.

2204. Rhamphastos ariel.

Rhamphastos ariel, Vig. Zool. Journ. ii. p. 466: Gould, Mon. Rhamph. ed. 2. pl. 12.

$a$ S. America (Askew) 1839.

A. Brazilian species.

2205. Rhamphastos vitellinus.

Rhamphastos vitellinus, Licht. Verz. Doubl. p. 7: Gould, Mon. Rhamph. ed. 2, pl. 13.

$a$ (Arthur Strickland) 1840.

A Guiana species also found in Venezuela and Trinidad.

2206. Rhamphastos dicolorus.

Rhamphastos dicolorus, Linn. Syst. Nat. i. p. 152: Gould, Mon. Rhamph. ed. 2, pl. 14.

$a$ (Thomas) 1842. -b (Arthur Strickland) 1850.

A Brazilian species. 


\section{Pteroglossus.}

Pteroglossus, Illiger, Prodr. p. 202 (1811).

\section{0\%. Pteroglossus wiedi.}

Pteroglossus wiedi, Sturm, Mon. Rhamph. pt. iv: Gould, Mon. Rhamph. ed. 2, pl. 16.

$a$ S. America (Thomas) 1843.

A Brazilian species.

\section{Pteroglossus viridis.}

Rhamphastos viridis, Linn. Syst. Nat. i. p. 150.

Pteroglossus viridis, Gould, Mon. Rhamph. ed. 2, pl. 24.

$a$ (E. Brown) 1850.

A skin of Cayenne make.

\section{Pteroglossus beauharnaisi.}

Pteroglossus beauharnaisi, Wagl. Isis, 1832, p. 279: Gould, Mon. Rhamph. ed. 2, pl. 25.

a Amazons (E. Wilson) 1850.

\section{Selenidera.}

Selenidera, Gould, Mon. Rhamph. p. 33 (1854).

\section{Selenidera maculirostris.}

Pteroglossus maculirostris, Licht. Verz. Doubl. p. 7.

Selenidera maculirostris, Gould, Mon. Rhamph. ed. 2, pl. 31. $a$ (Askew) 1839. - b, -c (Arthur Strickland) 1850.

\section{Selenidera piperivora.}

Rhamphastos piperivorus, Linn. Syst. Nat. i. p. 150.

Selenidera piperivora, Gould, Mon. Rhamph. ed. 2, pl. 36. a S. America (Thomas) 1843.

A skin of Cayenne make.

\section{Andigena.}

Andigena, Gould, P. Z. S. 1850, p. 93.

2212. Andigena nigrirostris.

Pteroglossus nigrirostris, Waterh. P. Z. S. 1839, p. 111. 
Andigena nigrirostris, Gould, Mon. Rhamph. ed. 2, pl. 39. a (Dewgard) 1849.

A skin of Bogota make.

\section{Andigena bailloni.}

Rhamphastos bailloni, Vieill. N. Dict. d'Hist. N. xxxiv, p. 283.

Pteroglossus bailloni, Gould, Mon. Rhamph. ed. 2, pl. 41. a Brazil (Cashmore). -b (Arthur Strickland) 1850.

\section{AUlaCorhampHus.}

Aulacorhamphus, Gould, Mon. Rhamph. p. 24 (1854).

\section{Aulacorhamphus albivitta.}

Pteroglossus albivitta, Boiss. Rev. Zool. 1840, p. 70. Aulacorhamphus albivitta, Gould, Mon. Rhamph. ed. 2, pl. 49.

a Bogota (Bt at Stevens's) 1844. 


\section{ORDER V.-PSITTACI.}

\section{FAMILY.PSITTACID正。}

\section{SUBFAMILY.-CACATUIN ג。}

\section{Cacatua.}

Cacatua, Vieillot, N. Dict. d'Hist. N. xvii. p. 6 (1817).

\section{Cacatua galerita.}

Psittacus galeritus, Lath. Ind. Orn. p. 109.

Cacatua galerita, Gould, B. Austr. v. pl. 1.

Plictolophus galeritus, Finsch, Papag. i. p. 286.

a Australia (Ashmolean Museum) 1845.

\section{Cacatua hæmaturopygia.}

Petit Kakatoes, des Philippines, D'Aub. P1. Enl. 191.

Psittacus homaturopygius, Müll. Syst. Nat. Suppl. p. 77.

Cacatua homaturopygia, Wald. Trans. Z. S. ix. p. 132. Plictolophus philippinarum, Finsch, Papag. i. p. 310 (ex Gm.). a (Gardner) 1847.

A species of the Philippine Islands.

\section{1\%. Cacatua roseicapilla.}

Cacatua roseicapilla, Vieill. N. Dict. d'Hist. N. xvii. p. 12. Plictolophus roseicapillus, Finsch, Papag. i. p. 313.

Cacatua eos, Gould, B. Austr. v. pl. 4 (ex Kuhl). a Australia (Cashmore) 1849. 


\section{Callocephalus.}

Callocephalon, Lesson, Voy. Thétis, p. 311 ; Compl. Buff. ix. p. 195 (1837).

\section{Callocephalus galeatus.}

Psittacus galeatus, Lath. Ind. Orn. Suppl. p. xxiii. Callocephalon galeatum, Gould, B. Austr. v. pl. 14. Calyptorhynchus galeatus, Finsch, Papag. i. p. 337. a Australia (A. Stricklund) 1850.

\section{Calyptorhynchus.}

Calyptorhynchus, Vigors \& Horsfield, Trans. L. S. xv. p. 269 (1826).

\section{Calyptorhynchus banksi.}

Psittacus banksi, Lath. Ind. Orn. p. 107.

Culyptorhynchus banksi, Gould, B. Austr. v. pl. 7 : Finsch, Papag. i. p. 345 .

a New South Wales (Arthur Strickland) 1850.

\section{Calyptorhynchus solandri.}

Psittacus solandri, Temm. Trans. L. S. xiii. p. 113.

Calyptorhynchus solandri, Finsch, Papag. i. p. 340.

Calyptorhynchus leachii, Gould, B. Austr. v. pl. 10.

$a,-b$ Australia (W. Kirtland) 1846.

\section{Calyptorhynchus funereus.}

Psittacus funereus, Shaw, Nat. Misc. vi. pl. 186.

Calyptorhynchus funereus, Gould, B. Austr. v. pl. 11 : Finsch, Papag. i. p. 357.

a Australia (Holden) 1834. -b Australia (Arthur Strickland) 18.50.

\section{Calopsittacus.}

Calopsitta, Less. Ill. Zool. pl. 49 (1832).

\section{Calopsittacus novæ-hollandiæ.}

Psittacus novce-hollandice, Gm. Syst. Nat. i. p. 328.

Nymphicus novce-hollandie, Gould, B. Austr. v. pl. 45. 
Callipsittacus novo-hollandice, Finsch, Papag. i. p. 260.

$a,-b$ New South Wales ( $M^{c}$ Donald) 1838.

\section{SUBFAMILY._LORIIN $Æ$.}

\section{LORIUS.}

Lorius, Vigors, Zool. Journ. ii. p. 400 (1826).

\section{Lorius lori.}

Psittacus lori, Linn. Syst. Nat. i. p. 145.

Domicella lori, Finsch, Papag. ii. p. 769.

a (Arthur Strickland) 1850.

A species of New Guinea.

Eos.

Eos, Wagler, Münch. Abh. 1829-30, p. 494.

\section{Eos rubra.}

Psittacus ruber, Gm. Syst. Nat. i. p. 335.

Domicella rubra, Finsch, Papag. ii. p. 786.

a (Gardner) 1845 .

A species of Ceram, \&c.

\section{Trichoglossus.}

Trichoglossus, Vigors \& Horsfield, Trans. L. S. xv. p. 287 (1826).

\section{Trichoglossus novæ-hollandiæ.}

Psittacus novce-hollandice, Gm. Syst. Nat. i. p. 316.

Trichoglossus novce-hollandiae, Finsch, Papag. ii. p. 820.

Trichoglossus swainsonii, Gould, B. Austr. v. pl. 48 (ex Jard. \& Selb.).

$a$ New South Wales (Mc Donald) 1838. - b Australia (Arthur Strickland) 1850.

\section{Trichoglossus chlorolepidotus.}

Psittacus chlorolepidotus, Kuhl, Consp. Psitt. p. 48.

Trichoglossus chlorolepidotus, Gould, B. Austr. v. pl. 50: Finsch, Papag. ii. p. 847.

a Australia (Mansfield) 1849. 


\section{2\%. Trichoglossus versicolor.}

Trichoglossus versicolor, Lear, Psitt. pl. 36: Gould, B. Aust. v. pl. 51: Finsch, Papag. ii. p. 853.

$a$ N. Australia (Argent) 1851.

\section{Trichoglossus concinnus.}

Psittacus concinnus, Shaw, Nat. Misc. iii. pl. 87.

Trichoglossus concinnus, Gould, B. Austr. v. pl. 52: Finsch, Papag. ii. p. 857.

a New South Wales ( $M^{c}$ Donald) 1838. $-b$ Australia (Arthur Strickland) 1850.

\section{Trichoglossus porphyrocephalus.}

Trichoglossus porphyrocephalus, Dietr. Trans. L. S. xvii. p. 553: Gould, B. Austr. v. pl. 53: Finsch, Papag. ii. p. 862.

a Adelaide, Australia (T. C. Eyton) 1846. -b Australia (Dewgard) 1847.

\section{Trichoglossus pusillus.}

Psittacus pusillus, White, Voy. p. 262, pl. 48.

Trichoglossus pusillus, Gould, B. Austr. v. pl. 54: Finsch, Papag. ii. p. 860.

a Australia (Thomas) 1843. - b Australia (Arthur Strickland) 1850 .

\section{CoRipHILUs.}

Coriphilus, Wagler, Münch. Abh. 1829-30, p. 494 (1832).

\section{Coriphilus ultramarinus.}

Psittacus ultramarinus, Kuhl, Consp. Psitt. p. 49.

Domicella smaragdina, Finsch, Papag. ii. p. 745 (ex Homb. \& Jacq.).

$$
a \text { (Argent) } 1851 .
$$

A species of the Marquesas Islands.

\section{Coriphilus fringillaceus.}

Psittacus fringillaceus, Gm. Syst. Nat. i. p. 337.

Domicella fringillacea, Finsch, Papag. ii. p. 747.

Psittacus porphyrocephalus, Shaw, Nat. Misc. i. pl. 1. a (Argent) 1852.

A species of the Society Islands. 


\section{SUBFAMILY.-PALEEORNITHINA.}

PALARORNIS.

Palceornis, Vigors, Zool. Journ. ii. p. 46 (1825).

2233. Palæornis torquatus.

La Perruche à collier, D’Aub. Pl. Enl. 551, undè,

Psittacus torquatus, Bodd. Tabl. Pl. Enl. p. 32.

Palceornis torquatus, Strickl. P. Z. S. 1850, p. 219 : Jerd. B. Ind. i. p. 258: Finsch, Papag. ii. p. 17.

Psittacus docilis, Vieill. N. Dict. d'Hist. N. xxv. p. 343.

$a,-b$ Madras (A. Strickland) 1833. -c (Askew) 1833. -d Senegal (Isaacson) 1840. -e Nepal (B. H. Hodgson, 701) 1845. - $f \hat{\delta}$ Kordofan, 8 July (J. Petherick) 1848.

\section{Palæornis rosa.}

La Perruche, de Mahé, D’Aub. Pl. Enl. 888, undè,

Psittacus rosa, Bodd. Tabl. Pl. Enl. p. 53.

Palceornis rosa, Jerd. B. Ind. i. p. 259; Ibis, 1872, p. 6.

Palcoornis cyanocephalus, Strickl. Ann. \& Mag. N. H. xiii. p. 33 : Finsch, Papag. ii. p. 40 (ex Linn.).

$a,-b$ Madras (A. Strickland) 1833. -c India (Askew) 1839. - $d$, - e Madras (Bt at Stevens's) 1843. $-f$ Nepal (B. H. Hodgson, 709) 1845. -g (Arthur Strickland) 1850 .

\section{Palæornis melanorhynchus.}

Palceornis melanorhynchus, Wagl. Münch. Abh. 1829-30, p. 511: Finsch, Papag. ii. p. 70: Wald. Ibis, 1873, p. 297.

Palceornis lathami, Finsch, Papag. ii. p. 66. a (Carfrae) 1837. -b Nepal (B. H. Hodgson, 695) 1845.

\section{Palæornis columboides.}

Palceornis columboides, Vig. Zool. Journ. v. p. 274: Jerd. B. Ind. i. p. 261.

Palceornis peristerodes, Finsch, Papag. ii. p. 74.

$a$ S. India (Chapman) 1849 .

\section{3\%. Palæornis schisticeps.}

Palceornis schisticeps, Hodgs. As. Res. xix. p. 178: Jerd. B. Ind. i. p. 261. 
Palceornis hodgsoni, Finsch, Papag. ii. p. 50.

a f̂ Kumaon (W. J. E. Boys) 1847.

\section{Psittinus.}

Psittinus, Blyth, J. A. S. B. xi. p. 789 (1842).

\section{Psittinus incertus.}

Psittacus incertus, Shaw, Nat. Misc. xviii. pl. 769.

Psittinus incertus, Salvad. Ann. Mus. Genov. v. p. 25.

Psittacus malaccensis, Lath. Ind. Orn. p. 130.

$a,-b$ Malacca (Capt. Nash) 1837. —c (Arthur Strickland) 1850. - d (Argent) 1851.

\section{ECLECTUS.}

Eclectus, Wagler, Münch. Abh. 1829-30, p. 495.

\section{Eclectus cardinalis.}

Lory d'A mboine, D'Aub. Pl. Enl. 518, undè, Psittacus cardinalis, Bodd. Tabl. Pl. Enl. p. 30. Eclectus cardinalis, Finsch, Papag. ii. p. 344. a (Arthur Strickland) 1850.

A male probably of this species which is found in Ceram and the adjacent islands.

\section{Polytelis.}

Polytelis, Wagler, Münch. Abh. 1829-30, p. 489.

\section{Polytelis barrabandi.}

Psittacus barrabandi, Sw. Zool. Ill. i. pl. 59.

Polytelis barrabandi, Gould, B. Austr. v. pl. 15.

Platycercus barrabandi, Finsch, Papag. ii. p. 259.

$a$ New South Wales (Bt at Birmingham) 1850.

\section{Polytelis melanura.}

Palceornis melanura, Lear, Ill. Parr. pl. 28.

Polytelis melanura, Gould, B. Austr. v. pl. 16.

Platycercus melanurus, Finsch, Papag. ii. p. 256.

a Australia (Bt at Leeds) 1849.

Aprosmictus.

Aprosmictus, Gould, P. Z. S. 1842, p. 111. 


\section{Aprosmictus cyanopygius.}

Psittacus cyanopygius, Vieill. N. Dict. d'Iist. N. xxv. p. 339. Platycercus cyanopygus, Finsch, Papag. ii. p. 245.

Aprosmictus scapulatus, Gould, B. Austr. v. pl. 17 (ex Kuhl). a Australia (Arthur Strickland) 1840.

\section{Aprosmictus erythropterus.}

Psittacus erythropterus, Gm. Syst. Nat. i. p. 343. Aprosmictus erythropterus, Gould, B. Austr. v. pl. 18. Platycercus erythropterus, Finsch, Papag. ii. p. 262. a Australia (Bt at Birmingham) 1850.

\section{LORICULUS.}

Loriculus, Blyth, Cat. B. As. Soc. p. 9 (1849).

\section{Loriculus philippensis.}

Psittacus philippensis, Müll. Syst. Nat. Suppl. p. 80 (ex Buffon).

Loriculus philippensis, Wald. Trans. Z. S. ix. p. 135.

a (Gardner) 1847.

A species of the Philippine Islands.

\section{Loriculus vernalis.}

Psittacus vernalis, Sparrm. Mus. Carls. no. 29.

Loriculus vernalis, Jerd. B. Ind. i. p. 265.

Coryllis vernalis, Finsch, Papag. ii. p. 721.

a Madras ("Psittaculus vernalis" T. C. Jerdon) 1845.

\section{Loriculus galgulus.}

Psittacus galgulus, Linn. Syst. Nat. i. p. 150 .

Psittaculus galgulus, Strickl. Ann. \& Mag. N. H. vii. p. 34.

Coryllis galgulus, Finsch, Papag. ii. p. 699.

a Malacca (G. B. M. Whitehaven) 1842.

EupHeMa.

Euphema, Wagler, Münch. Abh. 1829-30, p. 492.

\section{4\%. Euphema pulchella.}

Psittacus pulchellus, Shaw, Nat. Misc. iii. pl. 96. 
Euphema pulchella, Gould, B. Austr. v. pl. 41 : Finsch, Papag. ii. p. 161.

a Australia (Thomas) 1843.

\section{Euphema chrysostoma.}

Psittacus chrysostomus, Kuhl, Consp. Psitt. p. 50. pl. 1.

Euphema chrysostoma, Gould, B. Austr. v. pl. 37.

Euphema venusta, Finsch, Papag. ii. p. 163 (ex Temm.).

a Australia (Thomas) 1843. - b Australia (Dewgard) 1846.

-c, -d (Arthur Strickland) 1850.

\section{Melopsittacus.}

Melopsittacus, Gould, B. Austr. v. pl. 44 (1840).

\section{Melopsittacus undulatus.}

Psittacus undulatus, Shaw, Nat. Misc. xvi. pl. 673.

Melopsittacus undulatus, Gould, B. Austr. v. pl. 44. a Australia (Fenwick) 1844.

\section{Pezoporus.}

Pezoporus, Illiger, Prodr. p. 201 (1811).

\section{Pezoporus formosus.}

Psittacus formosus, Lath. Ind. Orn. p. 103.

Pezoporus formosus, Gould, B. Austr. v. pl. 46: Finsch, Papag. ii. p. 148.

a (Dewgard) 1851.

An Australian species.

\section{SUBFAMILY.-AGAPORNITHIN $\nexists$.}

\section{AGAPORNIS.}

Agapornis, Selby, Nat. Libr. Parrots, p. 117 (1836).

\section{Agapornis pullaria.}

Psittacus pullarius, Linn. Syst. Nat. i. p. 149.

Psittacula pullaria, Finsch, Papag. ii. p. 636.

a (Argent) 1851.

A common African species. 


\section{Agapornis roseicollis.}

Psittacus roseicollis, Vieill. N. Dict. d'Hist. N. xxv. p. 377.

Agapornis roseicollis, Strickl. \& Scl. Contr. Orn. 1852, p. 156.

Psittacula roseicollis, Finsch, Papag. ii. p. 640.

a Damara-land (C. J. Andersson) 1852.

\section{Agapornis cana.}

Psittacus canus, Gm. Syst. Nat. i. p. 350.

Psittacula cana, Finsch, Papag. ii. p. 643.

a Mauritius (A. Strickland) 1832.

\section{SUBFAMILY.-PLATYCERCIN $Æ$.}

Platycercus.

Platycercus, Vigors, Zool. Journ. i. p. 527 (1825).

\section{Platycercus pennanti.}

Psittacus pennanti, Lath. Ind. Orn. p. 90.

Platycercus pennanti, Gould, B. Austr. v. pl. 23: Finsch, Papag. ii. p. 180.

a New South Wales (McDonald) 1838. - b Australia (Arthur Strickland) 1850.

\section{Platycercus flaveolus.}

Platycercus flaveolus, Gould, P. Z. S. 1837, p. 26; B. Austr. v. pl. 25: Finsch, Papag. ii. p. 185.

a S. Australia (Cashmore) 1849.

\section{Platycercus icterotis.}

Psittacus icterotis, Temm. Trans. L. S. xiii. p. 120.

Platycercus icterotis, Gould, B. Austr. v. pl. 29: Finsch, Papag. ii. p. 186.

a (Bt at Edinburgh) 1852.

A vird of Western Australia.

\section{5\%. Platycercus eximius.}

Psittacus eximius, Shaw, Nat. Misc. iii. pl. 93.

Platycercus eximius, Gould, B. Austr. v. pl. 27: Finsch, Papag. ii. p. 190.

a Australia (Askew) 1833. -b (Dewgard) 1851. 
2258. Platycercus flaviventris.

Psittacus flavigaster, Temm. Trans. L. S. xiii. p. 116.

Platycercus flaviventris, Gould, B. Austr. v. pl. 24: Finsch, Papag. ii. p. 204.

$a$ Van Diemen's Land (Arthur Strickland) 1850.

2259. Platycercus barnardi.

Platycercus barnardi, Vig. \& Horsf. Trans. L. S. xv. p. 283: Gould, B. Austr. v. pl. 21: Finsch, Papag. ii. p. 207.

a Australia (Thomas) 1842.

Psephotus.

Psephotus, Gould, B. Austr. v. pl. 36 (1845).

2260. Psephotus hæmatonotus.

Platycercus hcematonotus, Gould, P. Z. S. 1837, p. 151: Finsch, Papag. ii. p. 219.

Psephotus hcematonotus, Gould, B. Austr. v. pl. 36.

a Australia (Osborn) 1845. —b Australia (Williams) 1846. -c Australia (Williams) 1848.

\section{Lathamus.}

Lathamus, Lesson, Traité d'Orn. p. 205 (1831) as a subgenus.

\section{Lathamus discolor.}

Psittacus discolor, White, Voy. p. 263, pl. 49.

Lathamus discolor, Gould, B. Austr. v. pl. 47.

Trichoglossus discolor, Finsch, Papag. ii. p. 863.

a Australia (W. N. H. Soc.) 1843. - b Australia (Arthur Strickland) 1850. -c (Arthur Strickland) 1850.

\section{SUBFAMILY.-PSITTACULIN A.}

\section{Psittacula.}

Psittacula, Kuhl, Consp. Psitt. p. 8 (1821).

2262. Psittacula passerina.

Psittacus passerinus, Linn. Syst. Nat. i. p. 150.

Psittacula passerina, Scl. Cat. Am. B. p. 357.

$a$ Brazil (G. Lloyd) 1828. -b, -c (Arthur Strickland) 1850. 


\section{Psittacula cyanoptera.}

Petite Perruche, du Cap de Bonne-Esperance, D'Aub. Pl. Enl. 455 f. 1, undè,

Psittacus cyanopterus, Bodd. Tabl. Pl. Enl. p. 27.

Psittacula cyanoptera, Scl. Cat. Am. B. p. 357.

a Brazil (Askew) 1834. - b Trinidad (Argent) 1851.

\section{SUBFAMILY.-PSITTACIN $Æ$ E.}

\section{Pegocephalus.}

Poicephalus, Swainson, Classif. B. ii. p. 301 (1837).

Poocephalus, Strickl. Ann. and Mag. W. H. vii. p. 34.

\section{Pœocephalus robustus.}

Psittacus robustus, Gm. Syst. Nat. i. p. 344.

Pionias robustus, Finsch, Papag. ii. p. 475.

$a$ (S. Africa) 1842. -b (Arthur Strickland) 1850.

2265. Pœocephalus senegalus.

Psittacus senegalus, Linn. Syst. Nat. i. p. 149.

Pionias senegalus, Finsch, Papag. ii. p. 489.

$a$ W. Africa (Gardner) 1845. -b (Arthur Strickland) 1850.

\section{Pœocephalus meyeri.}

Psittacus meyeri, Cretzschm. in Rüpp. Alt. Vög. p. 18, pl. 11.

Pcocephalus meyeri, Strickl. \& Scl. Contr. Orn. 1852, p. 156.

Pionias meyeri, Finsch, Papag. ii. p. 494.

a Damara-land (C. J. Andersson) 1852.

\section{6\%. Pœocephalus rueppelli.}

Psittacus rueppelli, Gray, P. Z. S. 1848, p. 125, Aves, pl. 5.

Pcocephalus rüppelli, Strickl. \& Scl. Contr. Orn. 1852, p. 156 .

Pionias rueppelli, Finsch, Papag. ii. p. 498.

$a,-b$ q Damara-land (C. J. Andersson) 1852.

SUBFAMILY.-NESTORIN A.

NESTOR.

Nestor, Wagler, Münch. Abh. 1829-30, p. 505.

2268. Nestor productus.

Plyctolophus productus, Gould, P. Z. S. 1836, p. 19.

Nestor productus, Gould, B. Austr. v. pl. 6: Finsch, Papag. ii. p. 888 . 
a (Bt at Edinburgh) 1852.

This bird, which is now extinct, was found on Phillip Island, off the east coast of Australia.

\section{Nestor meridionalis.}

Psittacus meridionalis, Gm. Syst. Nat. i. p. 333: Strickl. Ann. \& Mag. N. H. vii. p. 34.

Nestor meridionalis, Buller, B. N. Z. p. 39, pl. 6.

a New Zealand (T. C. Eyton) 1846.

\section{SUBFAMILY.-ARIN Æ.}

\section{ArA.}

Ara, Cuvier, Leç. d'Anat. Comp. Tab. ii. (1799).

\section{2\%0. Ara nobilis.}

Psittacus nobilis, Linn. Syst. Nat. i. p. 140.

Sittace nobilis, Finsch, Papag. i. p. 424.

a (Arthur Strickland) 1850.

A Brazilian species.

\section{2\%1. Ara severa.}

Psittacus severus, Linn. Syst. Nat. i. p. 140.

Sittace severa, Finsch, Papag. i. p. 417.

a Brazil (Argent) 1853.

\section{RHYNChOPSTTTA.}

Rhynchopsitta, Bonaparte, Rev. Znol. 1854, p. 149.

2272. Rhynchopsitta pachyrhyncha.

Macrocercus pachyrhynchus, Sw. Phil. Mag. New Ser. i. p. 439.

Sittace pachyrhyncha, Finsch, Papag. i. p. 428.

a Mexico (T. Mann) 1844.

\section{Henicognathus.}

Enicognathus, Gray, List Gen. B. p. 51 (1840).

2273. Henicognathus leptorhynchus.

Psittacara leptorhyncha, King, P. Z. S. 1830-1, p. 14. 
Henicognathus leptorhynichus, Finsch, Papag. i. p. 432. a Chili (Thomas) 1842.

\section{Conurus.}

Conurus, Kuhl, Consp. Psitt. p. 4 (1820), partim. 22\%4. Conurus patagonus.

Psittacus patagonus, Vieill. N. Dict. d'Hist. N. xxv. p. 367. Conurus patagonus, Finsch, Papag. i. p. 444.

a (T. C. Eyton) 1845 .

A Chilian species.

22\%5. Conurus holochlorus.

Conurus holochlorus, Scl. Ann. \& Mag. N. H. ser. 3, iv. p. 224: Finsch, Papag. i. p. 466.

$a$ Guatemala (J. Constancia) 1845. — $b$ Guatemala (J. Constancia) 1851.

22\%6. Conurus carolinensis.

Psittacus carolinensis, Linn. Syst. Nat. i. p. 141.

Conurus carolinensis, Finsch, Papag. i. p. 478.

a (Arthur Strickland) 1850.

A North-American species.

22\%\%. Conurus jendaya.

Psittacus jendaya, Gm. Syst. Nat. i. p. 319.

Conurus jendaya, Finsch, Papag. i. p. 495.

a Brazil (Johnson) 1837. —b Brazil (T. C. Eyton) 1846. -c (Arthur Strickland) 1850.

\section{Conurus aureus.}

Psittacus aureus, Gm. Syst. Nat. i. p. 329.

Conurus aureus, Finsch, Papag. i. p. 499.

$a$ (Askew) 1839. —b S. America (Dewgard) 1849. — (Arthur Strickland) 1850.

A Brazilian species.

\section{Conurus æruginosus.}

Psittacus ceruginosus, Linn. Syst. Nat. i. p. 142.

Conurus pertinax, Finsch, Papag. i.p. 506, partim. a 'Trinidad (Argent) 1851.

S. C. 


\section{Conurus nanus.}

Psittacara nanus, Vig. Zool. Journ. v. p. 273.

Conurus nanus, Finsch, Papag. i. p. 520.

Conurus flaviventer, Gosse, B. Jam. p. 263.

a Jamaica (P. H. Gosse) 1848.

A typical specimen of $C$. flaviventer.

2281. Conurus cruentatus.

Psittacus cruentatus, Wied, Reise, i. pp. 53, 72 ; ii. pp. 147, 341 ; Beitr. iv. p. 183.

Conurus cruentatus, Finsch, Papag. i. 527.

$a,-b$ (Arthur Strickland) 1850.

A Brazilian species.

2282. Conurus vittatus.

Psittacus vittatus, Shaw, Gen. Zool. viii. p. 404.

Conurus vittatus, Finsch, Papag. i. p. 530.

a (Arthur Strickland) 1850.

A Brazilian species.

\section{Conurus leucotis.}

Psittacus leucotis, Kuhl, Consp. Psitt. p. 21.

Conurus leucotis, Finsch, Papag. i. p. 535.

$a$ (Fenwick) 1844.

A Brazilian species.

\section{Conurus cyanopterus.}

Perruche à gorge tachetée, de Cayenne, D'Aub. Pl. Enl. 144, undè,

Psittacus cyanopterus, Bodd. Tabl. Pl. Enl. p. 9.

Conurus cyanopterus, Finsch, Papag. i. p. 538.

$a$ Trinidad (Argent) 1851.

\section{BOLBORHYNCHUS.}

Bolborhynchus, Bonaparte, Compt. Rend. xliv. p. 596 (1857).

\section{Bolborhynchus aymara.}

Arara amayra, d'Orb. Voy. Am. Mérid. ii. p. 376.

Bolborhynchus aymara, Finsch, Papag. ii. p. 123.

a Brazil ? (Argent) 1852.

Probably a Bolivian skin. 


\section{Chrysotis.}

Chrysotis, Swainson, Classif. B. ii. p. 300 (1837).

\section{Chrysotis guildingi.}

Psittacus guildingi, Vig. P. Z. S. 1836, p. 80.

Chrysotis guildingi, Finsch, Papag. ii. p. 559.

$a$ West Indies (Sir W. Jardine) 1845.

A species peculiar to the West Indian island of St Vincent.

228\%. Chrysotis auripalliata.

Psittacus (amazona) auropalliatus, Less. Rev. Zool. 1842, p. 135.

Chrysotis auripalliatus, Finsch, Papag. ii. p. 568.

a Guatemala (J. Constancia) 1845.

2288. Chrysotis æstiva.

Psittacus cestivus, Linn. Syst. Nat. i. p. 146.

Chrysotis cestiva, Scl. Cat. Am. B. p. 353.

$a$ (Thomas) 1842.

A Brazilian species.

\section{TRICLARIA。}

Triclaria, Wagler, Münch. Abh. 1829-30, p. 499.

2289. Triclaria cyanogastra.

Psittacus cyanogaster, Max. Reise n. Bras. i. p. 263 ; Beitr. iv. p. 202.

Triclaria cyanogastra, Scl. Cat. Am. B. p. 354.

Pionias cyanogaster, Finsch, Papag. ii. p. 407. $a,-b$ Brazil (Argent) 1853.

\section{Pronus.}

Pionus, Wagler, Münch. Abh. 1829-30, p. 497. 2290. Pionus menstruus.

Psittacus menstruus, Linn. Syst. Nat. i. p. 148.

Pionius menstruus, Finsch, Papag. ii. p. 441.

$a$ Brazil (Askew) 1839. -b (Arthur Strickland) 1850. 


\section{Pionus maximilliani.}

Psittacus maximilliani, Kuhl, Consp. Psitt. p. 72.

Pionius maximilliani, Finsch, Papag. ii. p. 448.

$a$ (Dewgard) 1851. - b Brazil? (Argent) 1852.

$b$ is probably from Bolivia.

2292. Pionus chalcopterus.

Psittacus chalcopterus, Fraser, P. Z. S. 1843, p. 59.

Pionius chalcopterus, Finsch, Papag. ii. p. 462.

$a$ (Gardner) 1845.

A Colombian species.

2293. Pionus violaceus.

Perroquet varié, de Cayenne, D'Aub. Pl. Enl. 408, undè,

Psittacus violaceus, Bodd. Tabl. Pl. Enl. p. 25.

Pionias violaceus, Finsch, Papag. ii. p. 463.

Psittacus purpureus, Gm. Syst. Nat. i. p. 346.

a (Askew) 1839.

A Guianan species.

\section{CAICA.}

Caica, Lesson, Traité d'Orn. p. 198 (1831).

2294. Caica melanocephala.

Psittacus melanocephahus, Linn. Syst. Nat. i. p. 149.

Pionias melanocephalus, Finsch, Papag. ii. p. 432.

$a$ (Dewgard) 1846. - b Demarara (Bt at Liverpool) 1854.

$a$ is a skin of Cayenne make.

\section{Pionopsittacus.}

Pionopsitta, Bonaparte, Rev. Zool. 1854, p. 152.

\section{Pionopsittacus pileatus.}

Psittacus pileatus, Scop. Ann. I. Hist. Nat. p. 32.

Pionopsitta pileata, Scl. Cat. Am. B. p. 356.

Pionias mitratus, Finsch, Papag. ii. p. 413 (ex Neuwied).

$a$ S. America (G. Bell Whitehouse) 1842. -b (Arthur Strickland) 1850. 


\section{UROCHROMA.}

Urochroma, Bonaparte, Naum. 1856, inter pp. 352-353.

2296. Urochroma surda.

Psittacus surdus, Kuhl, Consp. Psitt. p. 59.

Urochroma surda, Scl. Cat. Am. B. p. 356.

Psittacula surda, Finsch, Papag. ii. p. 672.

a Brazil (Johnson) 1837. - b Brazil (Seaman) 1840. -c (Arthur Strickland) 1850.

229\%. Urochroma melanonota.

Psittacus melanonotus, Kuhl, Consp. Psitt. p. 59.

Urochroma melanonota, Scl. Cat. Am. B. p. 356.

Psittacula melanonota, Finsch, Papag. ii. p. 675.

a (Bt at Liverpool) 1853.

A Brazilian species.

2298. Urochroma cingulata.

Psittacus cingulatus, Scop. Del. Faun. et Fl. Ins. ii. p. 86.

Psittacula cingulata, Finsch, Papag. ii. p. 677.

a Trinidad (Argent) 1851.

\section{Urochroma hueti.}

Psittacus hueti, Temm. Pl. Col. 491.

Psittacula hueti, Finsch, Papag. ii. p. 684.

$a$ (Bt at Bristol) 1852. -b (T. C. Eyton) 1853.

A species of Trinidad, Venezuela, \&c.

\section{BROTOGERYS.}

Brotogeris, Vigors, Zool. Journ. ii. p. 400.

2300. Brotogerys tiriacula.

Petite Perruche appellée, la petite jaseuse, D’Aub. Pl. Enl. 837, unde,

Psittacus tiriacula, Bodd. Tabl. Pl. Enl. p. 51.

Conurus tiriacula, Scl. Cat. Am. B. p. 351.

Brotogerys tirica, Finsch, Papag. ii. p. 89 (ex Gmelin).

a (Arthur Strickland) 1850.

A Brazilian species. 
2301. Brotogorys tui.

Psittacus tui, Gm. Syst. Nat. i. p. 352.

Brotogerys tui, Finsch, Papag. ii. p. 108.

a (E. Brown) 1850.

An Amazon species.

2302. Brotogerys chrysoptera.

Psittacus chrysopterus, Linn. Syst. Nat. i. p. 149.

Brotogerys tuipara, Finsch, Papag. ii. p. 103 (ex Gmelin).

a S. America (Bt at Stevens's) 1845. - b Demarara (T. C. Eyton) 1846.

2303. Brotogerys tovi.

Psittacus tovi, Gm. Syst. Nat. i. p. 351.

Brotogerys tovi, Finsch, Papag. ii. p. 99.

$a$ S. America (Bt at Stevens's) 1845.

2304. Brotogerys xanthoptera.

Aratinga xanthopterus, Spix, Av. Bras. i. p. 31, pl. 15, f. 2.

Brotogerys xanthoptera, Finsch, Papag. ii. p. 92.

a Brazil? (Argent) 1852.

Probably a Bolivian skin. 


\section{ORDER VI.-STRIGES.}

\section{FAMILY.-STRIGIDA.}

\section{STRIX.}

Strix, Linnæus, Syst. Nat. i. p. 131 (1766) partim. 2305. Strix flammea.

Strix flammea, Linn. l. s. c.: Strickl. Orn. Syn. p. 178: Sharpe, Cat. B. ii. p. 291.

Strix javanica, Strickl. Orn. Syn. p. 180 (ex Gmelin).

Strix delicatula, Strickl. Orn. Syn. p. 180 (ex Gould).

a Worcestershire (H. E. Strickland) 1830. -b Australia (Askew) 1839. -c Australia (Askew) 1841. —d Madras (T. C. Jerdon) 145. -e India (E. Blyth) 1846.

\section{Strix novæ-hollandiæ.}

Strix novce-hollandice, Steph. in Shaw's Gen. Zool. xiii. Pt. 2. p. 61: Strickl. Orn. Syn. p. 181: Sharpe, Cat. B. ii. p. 303.

a Australia (Arthur Strickland).

\section{0\%. Strix castanops.}

Strix castanops, Gould, P. Z. S. 1836, p. 140; B. Austr. i. pl. 28: Strickl. Orn. Syn. p. 181 : Sharpe, Cat. B. ii. p. 304.

a Para (Stevens) 1849.

This specimen has been called S. punctatissima in the Orn. Syn. (p. 182) a species to which it does not belong. I cannot distinguish it from the Tasmanian S. castanops and have no doubt the locality 'Para' has been wrongly assigned to it. This specimen is not from Mr A. R. Wallace's collection as its origin and date might lead one to infer. 


\section{Strix candida.}

Strix candida, Tick. J. A. S. B. ii. p. 572: Strickl. Orn. Syn. p. 181: Sharpe, Cat. B. ii. p. 308.

a (T. Robinson) 1842 .

An Indian species.

\section{FAMILY.-BUBONIDAE.}

Ketupa.

Ketupa, Lesson, Traité d'Orn. p. 114 (1831).

2309. Ketupa ceylonensis.

Strix zeylonensis, Gm. Syst. Nat. i. p. 287.

Ketupa ceylonensis, Strickl. Orn. Syn. p. 196: Sharpe, Cat. B. ii. p. 4.

$a$ India (E. Blyth) 1846.

2310. Ketupa javanensis.

Ketupa javanensis, Less. Traité d'Orn. p. 114: Strickl. Orn. Syn. p. 195: Sharpe, Cat. B. ii. p. 8.

a Malacca (Thomas) 1842.

\section{BuBo.}

Bubo, Duméril, Zool. Anal. p. 34 (1806).

2311. Bubo ignavus.

Strix bubo, Linn. Syst. Nat. i. p. 131.

Bubo ignavus, Forst. Syst. Cat. Brit. B. p. 3: Sharpe, Cat. B. ii. p. 14 .

Bubo maximus, Strickl. P. Z. S. 1836, p. 97; Orn. Syn. p. 212 (ex Sibbald).

a Smyrna, 15 November (H. E. Strickland) 1835.

2312. Bubo virginianus.

Strix virginiana, Gm. Syst. Nat. i. p. 287.

Bubo virginianus, Strickl. Orn. Syn. p. 213: Sharpe, Cat. B. ii. p. 19 .

$a$ Guatemala ( J. Constancia) 1851. - b (Arthur Strickland). 


\section{Bubo bengalensis.}

Otus bengalensis, Frankl. P. Z. S. 1830-1, p. 115.

Bubo bengalensis, Strickl. Orn. Syn. p. 216: Sharpe, Cat. B. ii. p. 25.

Urrua bengalensis, Jerd. B. Ind. i. p. 128.

a (T. C. Jerdon) 1845.

\section{Bubo maculosus.}

Strix maculosa, Vieill. N. Dict. d'Hist. N. vii. p. 44.

Bubo maculosus, Strickl. Orn. Syn. p. 215: Sharpe, Cat. B. ii. p. 30.

a Cape of Good Hope, June (A. Strickland) 1833.

\section{Bubo coromandus.}

Strix coromanda, Lath. Ind. Orn. i. p. 53.

Urrua coromanda, Strickl. Orn. Syn. p. 218: Jerd. B. Ind. i. p. 130.

Bubo coromandus, Sharpe, Cat. B. ii. p. 35.

a Madras (T. C. Jerdon) 1845. —b India (E. Blyth) 1846.

\section{SCOPS.}

Scops, Savigny, Syst. des Ois. de l'Egypte, p. 47 (1810).

2316. Scops giu.

Strix scops, Linn. Syst. Nat. i. p. 132.

Scops giu, Sharpe, Cat. B. ii. p. 47 (ex Scopoli).

Scops zorca, Strickl. Orn. Syn. p. 197 (ex Gmelin).

a Italy (C. Passerini) 1836.

\section{1\%. Scops capensis.}

Scops capensis, Smith, S. Afr. Quart. Journ. ii. p. 314: Sharpe, Cat. B. ii. p. 52.

Scops senegalensis, Strickl. \& Scl. Contr. Orn. 1852, p. 142: Strickl. Orn. Syn. p. 199 (nec Swainson).

a Damara-land (C. J. Andersson) 1852.

A smaller bird than $S$. giv, but hardly specifically distinct.

2318. Scops spilocephalus.

Ephialtes spilocephalus, Blyth, J. A. S. B. xv. p. 8.

Scops spilocephalus, Sharpe, Cat. B. ii. p. 63.

a Kumaon (W. J. E. Boys) 1847. 


\section{Scops sunia.}

Scops sunia, Hodgs. As. Res. xix. p. 175 : Sharpe, Cat. B. ii. p. 67 .

$a,-b$ Madras (T. C. Jerdon) 184.5.

\section{Scops lempiji.}

Strix lempiji, Horsf. Trans. Linn. Soc. xiii. p. 140.

Scops lempiji, Strickl. Orn. Syn. p. 200: Sharpe, Cat. B. ii. p. 91 .

$a$ (Askew). - b Java (Arthur Strickland). $\rightarrow$ S. India (T. C. Jerdon) 1850.

\section{Scops leucotis.}

Strix leucotis, Temm. Pl. Col. 16.

Scops leucotis, Strickl. P. Z. S. 1850, p. 216: Orn. Syn. p. 202 : Strickl. \& Scl. Contr. Orn。1852, p. 142: Sharpe, Cat. B. ii. p. 97.

$a$ Kordofan, 13 March (J. Petherick) 1848, - b Damara-land (C. J. Andersson) 1852.

\section{Scops sagittatus.}

Ephialtes sagittatus, Cassin, Journ. Ac. Phil. Ser. 2, fig. 2, ii. p. 96, pl. 12 .

Scops sagittatus, Sharpe, Cat. B. ii. p. 98.

Scops rufescens, Strickl. Orn. Syn. p. 201 (nec Horsfield). a India (Arthur Strickland).

A Malaccan species.

\section{Scops asio.}

Strix asio, Linn. Syst. Nat. i. p. 132.

Scops asio, Strickl. Orn. Syn. p. 199 : Sharpe, Cat. B. ii. p. 114. land).

$a,-b$ N. America (Arthur Strickland). -c (Arthur Strick-

\section{LOPHOSTRIX.}

Lophostrix, Lesson, Compl. Buff. vii. p. 261 (1836). 2324. Lophostrix stricklandi.

Scops cristatus, var. Strickl. Contr. Orn. 1848, p. 60, pl. 8.

Lophostrix stricklandi, Scl. \& Salv. Ibis, 1859, p. 221.

Scops stricklandi, Sharpe, Cat. B. ii. p. 124.

a Coban (L. L. Dillwyn) 1848. 


\section{NyCTEA.}

Nyctea, Stephens in Shaw's Gen. Zool. xiii. pt. 2, p. 62 (1826).

2325. Nyctea scandiaca.

Strix scandiaca, Linn. Syst. Nat. i. p. 132.

Nyctea scandiaca, Sharpe, Cat. B. ii. p. 125.

Nyctea nivea, Strickl. Orn. Syn. p. 194 (ex Daudin).

$a$ Sweden (J. G. Kinberg) 1844.

\section{SURNIA.}

Surnia, Duméril, Zool. Anal. p. 34 (1806).

2326. Surnia ulula.

Strix ulula, Linn. Syst. Nat. i. p. 133.

Surnia ulula, Sharpe, Cat. B. ii. p. 129.

Surnia funerea, Strickl. Orn. Syn. p. 183 (partim).

$a$ St. Petersburg, Russia (J. F. Brandt) 1841.

\section{2\%. Surnia funerea.}

Strix funerea, Linn. Syst. Nat. i. p. 133.

Surnia funerea, Strickl. Orn. Syn. p. 183 (partim): Sharpe, Cat. B. ii. p. 131.

$a$ (Askew) 1840. -b Toronto, Canada (P. L. Sclater) 1847.

\section{Ciccaba.}

Ciccaba, Wagler, Isis, 1832, p. 1222.

\section{Ciccaba hulula.}

Strix hulula, Daud. Traité ii. p. 190.

Ciccaba hulula, Strickl. Orn. Syn. p. 174.

Syonium hululum, Sharpe, Cat. B. ii. p. 275.

a Brazil (Arthur Strickland).

A Guianan and Amazonian species.

2329. Ciccaba melanonota.

Noctua melanonota, Tsch. Arch. f. Naturg. x. i. p. 266.

Athene melanota, Strickl. Orn. Syn. p. 172. 
Syrnium melanonotum, Sharpe, Cat. B. ii. p. 280. Syrnium hylophilus, Strickl. Orn. Syn. p. 192 (partim). a (Arthur Strickland).

A Peruvian and Brazilian species.

\section{Athene.}

Athene, Boie, Isis, 1822 , p. 549 .

2330. Athene glaux.

Noctua glaux, Savign. Syst. des Ois. de l'Egypte, p. 45.

Carine glaux, Sharpe, Cat. B. ii. p. 135.

Noctua nudipes, Strickl. P. Z. S. 1836, p. 97 (nec Nilsson).

Athene noctua, Strickl. Orn. Syn. p. 159 (partim).

a Smyrna, 30 November (H. E. Striclland) 1835. -b Smyrna, 28 December (H. E. Strickland) 1835.

\section{Athene brama.}

Strix brama, Temm. Pl. Col. 68.

Athene brama, Strickl. Orn. Syn. p. 166.

Carine brama, Sharpe, Cat. B. ii. p. 138.

a India (E. Blyth) 1840.

\section{Speotyto.}

Speotyto, Gloger, Handb. Naturg. p. 226 (1842).

2332. Speotyto cunicularia.

Strix cunicularia, Mol. Sagg. sulla St. Nat. p. 34 s.

Athene cunicularia, Strickl. Orn. Syn. p. 160.

Speotyto cunicularia, Sharpe, Cat. B. ii. p. 142.

a Chili (Capt. Brown) 1842.

\section{Ninox.}

Ninox, Hodgson, Madr. Journ. v. p. 23 (1837).

\section{Ninox lugubris.}

Strix lugubris, Tick. J. A. S. B. ii. p. 572.

Ninox lugubris, Strickl. Ann. \& Mag. N. H. xiii. p. 40: Sharpe, Cat. B. ii. p. 154.

a India (Askew) 1834. —b India (Havell) 1839. 
2334. Ninox scutulata.

Strix scutulata, Raffl. Trans. L. S. xiii. p. 280.

Athene scutulata, Strickl. P. Z. S. 1846, p. 99; Orn. Syn.

p. 170 (partim).

Ninox scutulata, Sharpe, Cat. B. ii. p. 156.

a Malacca (W. Kirtland) 1843.

2335. Ninox boobook.

Strix boobook, Lath. Ind. Orn. Suppl. p. xv.

Athene boobook, Strickl. Orn. Syn. p. 165.

Ninox boobook, Sharpe, Cat. B. ii. p. 168.

a Australia (Arthur Strickland).

2336. Ninox connivens.

Falco connivens, Lath. Ind. Orn. Suppl. p. xii.

Athene connivens, Strickl. Orn. Syn. p. 164.

Ninox connivens, Sharpe, Cat. B. ii. p. 175.

$a$ New South Wales (Isaac) 1842. - $b$ Australia (Arthur Strickland).

\section{GLaucidium.}

Glaucidium, Boie, Isis, 1826, p. 970.

233\%. Glaucidium passerinum.

Strix passerina, Linn. Syst. Nat. i. p. 13 s.

Glaucidium passerinum, Strickl. Orn. Syn. p. 158: Sharpe, Cat. B. ii. p. 191.

a Stockholm, Sweden, November 1832 (J. G. Kinberg) 1845. - b Sweden, March, 1845 (J. G. Kinberg) $184 \check{.}$.

2338. Glaucidium nanum.

Strix nana, King, Zool. Journ. iii. p. 427.

Athene nana, Strickl. Orn. Syn. p. 170.

Glaucidium nanum, Sharpe, Cat. B. ii. p. 190.

a Chili (Capt. Brown) 1842.

2339. Glaucidium pumilum.

Strix pumila, Temm. Pl. Col. 39.

Glaucidium pumilum, Sharpe, Cat. B. ii. p. 198.

Athene ferox, Strickl. Orn. Syn. p. 162 (nec Vieillot).

a South America (Thomas) 1840. 


\section{Glaucidium phalænoides.}

Strix phalcenoides, Daud. Traité ii. p. 206.

Glaucidium ferox, G. phalcenoides et G. ridgwayi, Sharpe,

Cat. B. p. 200 et seq.

Athene ferruginea, Strickl. Orn. Syn. p. 162 (ex Wied).

Athene infuscata, Strickl. Orn. Syn. p. 163 (ex Temm.).

a Brazil (Thomas) 1842. - b Trinidad (Argent) 1852. - c, -d Brazil (Arthur Strickland).

\section{Glaucidium perlatum.}

Strix perlata, Vieill. N. Dict. d'Hist. N. vii. p. 26.

Athene perlata, Strickl. Orn. Syn. p. 163.

Glaucidium perlatum, Sharpe, Cat. B. ii. p. 209.

Athene licua, Strickl. \& Scl. Contr. Orn. 1852, p. 142: Strickl.

Orn. Syn. p. 164 (ex Licht.).

a Damara-land (C. J. Andersson) 1852.

\section{Glaucidium brodiei.}

Noctua brodiei, Burton, P. Z. S. 1835, p. 152.

Athene brodiei, Strickl. Orn. Syn. p. 169.

Glaucidium brodiei, Sharpe, Cat. B. ii. p. 212.

a (W. J. E. Boys) 1847.

An Indian species.

\section{Glaucidium radiatum.}

Strix radiata, Tick. J. A. S. B. ii. p. 572.

Athene radiata, Strickl. Orn. Syn. p. 167.

Glaucidium radiatum, Sharpe, Cat. B. ii. p. 217.

a (T. C. Jerdon) 1845. - b Benares (W. J. E. Boys) 1847.

-c India (Arthur Strickland).

\section{Glaucidium cuculoides.}

Noctua cuculoides, Gould, Cent. B. Him. pl. 4.

Athene cuculoides, Strickl. Orn. Syn. p. 167.

Glaucidium cuculoides, Sharpe, Cat. B. ii. p. 219.

a India (Askew) 1840. -b India (Lord A. Hay) 1845. -c (T. C. Eyton) 1853.

Asso.

Asio, Brisson, Ornith. i. p. 28 (1760): Strickl. Ann. \& Mag. N. H. viii. p. 371. 
2345. Asio otus.

Strix otus, Linn. Syst. Nat. i. p. 132.

Asio otus, Strickl. l. s. c.; Orn. Syn. p. 205 : Sharpe, Cat. B. ii. p. 227.

a Britain (H. E. Strickland) 1831.

2346. Asio americanus.

Strix americana, Gm. Syst. Nat. i. p. 288.

Asio americanus, Sharpe, Cat. B. ii. p. 229.

Asio perigrinator, Strickl. Orn. Syn. p. 207 (ex Bartram).

$a$ (Arthur Strickland).

Referred to $A$. otus in the "Ornithological Synonyms," but the specimen agrees best with the North-American bird.

234\%. Asio mexicanus.

Strix mexicana, Gm. Syst. Nat. i. p. 288.

Asio mexicanus, Strickl. Orn. Syn. p. 208: Sharpe, Cat. B. ii. p. 231.

$a$ South America (Arthur Strickland).

2348. Asio accipitrinus.

Stryx accipitrina, Pall. Reis. i. p. 455.

Asio accipitrina, Sharpe, Cat. B. ii. p. 234.

Asio brachyotus, Strickl. P. Z. S. 1836, p. 97 ; 1842, p. 168;

Orn. Syn. p. 209 (ex Gmelin).

a Madras (T. C. Jerdon) 1845.

234.9. Asio capensis.

Otus capensis, Smith, S. Afr. Quart. Journ. ii. p. 316.

Asio capensis, Strickl. Orn. Syn. p. 211 : Sharpe, Cat. B. ii. p. 239.

a South Africa (Robinson) 1835. - b Morocco (J. G. Kinberg) 1844. —c Tangier (Favier) 1848.

$b$ is called Bubo ascalaphus in the "Ornithological Synonyms" (p. 215).

SYRNIUM.

Syrnium, Savigny, Syst. des Ois. de l'Egypte p. 51 (1810). 2350. Syrnium aluco.

Strix aluco, Linn. Syst. Nat. i. p. 132. 
Syrnium aluco, Strickl. Orn. Syn. p. 186: Sharpe, Cat. B. ii. p. 247.

Ulula stredula, Strickl. P. Z. S. 1836, p. 97 (ex Selby).

a Worcestershire (H. E. Strickland) 1831. - b Smyrna, 12 December (H. E. Strickland) 1835.

2351. Syrnium lapponicum.

Strix lapponica; Retz. Faun. Suec. p. 79.

Syrnium lapponicum, Strickl. Orn. Syn. p. 188: Sharpe, Cat. B. ii. p. 254.

a Lapland (J. G. Kinberg) 1844.

2352. Syrnium uralense.

Stryx uralensis, Pall. Reis. i. p. 455.

Syrnium uralense, Strickl. Orn. Syn. p. 189: Sharpe, Cat. B. ii. p. 255 .

a North Europe (J. G. Kinberg) 1843.

2353. Syrnium nebulosum.

Strix nebulosa, Forst. Phil. Trans. xxii. pp. 386, 424.

Ulula nebulosa, Strickl. Ann. \& Mag. N. H. vi. p. 417.

Syrnium nebulosum, Strickl. Orn. Syn. p. 189: Sharpe, Cat. B. ii. p. 257.

a (Arthur Strickland).

A species of North America.

2354. Syrnium fulvescens.

Syrnium fulvescens, Scl. \& Salv. P. Z. S. 1868, p. 58: Sharpe, Cat. B. ii. p. 258.

a Guatemala (J. Constancia) 1845.

2355. Syrnium ocellatum.

Syrnium ocellatum, Less. Rev. Zool. 1839, p. 289: Sharpe, Cat. B. ii. p. 263.

Syrnium sinense, Strickl. Orn. Syn. p. 190: Jerd. B. Ind. i. p. 123 (nec Latham).

a S. India (T. C. Jerdon) 1850.

2356. Syrnium woodfordi.

Noctua woodfordi, Smith, S. Afr. Quart. Journ. ii. p. 312. 
Athene woodfordi, Strickl. Orn. Syn. p. 170.

Syrnium woodfordi, Sharpe, Cat. B. ii. p. 267.

a (Arthur Strickland).

A South-African species.

\section{Phodilus.}

Phodilus, I. St. Hilaire, Ann. Sc. Nat. xxi. p. 200 (1830): A. Milne-Edwards, Compt. Rend. Ixxxv. p. 1173 (1877).

\section{5\%. Phodilus badius.}

Strix badia, Horsf. Zool. Res. pl. 37.

Phodilus badius, Strickl. Orn. Syn. p. 183: Sharpe, Cat. B. ii. p. 309.

a Java (Arthur Strickland).

Prof. A. Milne-Edwards has recently shewn $l$. $s$. c. that the resemblance of Phodilus to Strix is quite superficial its real relationship being with Syrnium.

\section{Nyctala.}

Nyctala, Brehm, Isis, 1828, p. 1271.

\section{Nyctala tengmalmi.}

Strix tengmalmi, Gm. Syst. Nat. i. p. 291.

Nyctale tengmalmi, Strickl. Ann. \& Mag. N. H. vi. p. 417; Orn. Syn. p. 175.

Nyctala tengmalmi, Sharpe, Cat. B。 ii. p. 284.

$a$ Switzerland (W. Anderegg) 1836. 


\section{ORDER VII-ACCIPITRES.}

\section{FAMILY.-FALCONID丑。}

\section{SUBFAMILY.-PANDIONIN E.}

\section{Pandion.}

Pandion, Savigny, Syst. des Ois. de l'Egypte, p. 35 (1809). 2359. Pandion haliaetus.

Falco haliaetus, Linn. Syst. Nat. i. p. 129.

Pandion haliaetus, Strickl. Orn. Syn. p. 63: Sharpe, Cat. B. i. p. 449 .

$a$ Orkney (Forbes) 1837. - b India (E. Blyth) 1846.

\section{SUBFAMILY.-CIRCIN $\nexists$ E.}

\section{Circus.}

Circus, Lacépède, Mém. de l’Inst. iii. p. 506 (1801). 2360. Circus æruginosus.

Falco ceruginosus, Linn. Syst. Nat. i. p. 130.

Circus ceruginosus, Strickl. Orn. Syn. p. 145: Sharpe, Cat. B. i. p. 69.

Circus rufus, Strickl. P. Z. S. 1836, p. 97 (ex Brisson).

a f Smyrna, 12 January (H. E. Strickland).

\section{Circus cyaneus.}

Falco cyaneus, Linn. Syst. Nat. i. p. 126.

Circus cyaneus, Strickl. P. Z. S. 1836, p. 97; Orn. Syn. p. 147: Sharpe, Cat. B. i. p. 52.

a Smyrna, 3 January (H. E. Strickland) 1836. - $b$ Worcestershire, March (H. E. Strickland) 1842. 


\section{Circus hudsonius.}

Falco hudsonius, Linn. Syst. Nat. i. p. 128.

Circus hudsonius, Strickl. Orn. Syn. p. 150: Sharpe, Cat. B. i. p. 55 .

$a$ Mexico (T. Mann) 1844. - b, -c Guatemala (J. Constancia) 1851.

$a$ was referred in the 'Ornithological Synonyms' to $C$. cinereus but it is the skin of an adult male of this species.

\section{Circus macruru3.}

Accipiter macrourus, S. G. Gmelin, N. Comm. Petrop. xv. p. 4.39.

Circus macrurus, Sharpe, Cat. B. i. p. 67.

Circus pallidus, Strickl. P. Z. S. 18っ0, p. 216 (ex Sykes).

Circus swainsoni, Strickl. Orn. Syn. p. 151 (ex Smith).

$a$ Madras (T. C. Jerdon) 1845. - b Khartoum (J. Petherick) 1848. —c S. India (T. C. Jerdon) 1850.

$c$ is referred in the 'Ornithological Synonyms' to C. cyaneus but it really belongs here.

\section{Circus cineraceus.}

Falco cineraceus, Mont. Orn. Dict. i. sign. K. p. 4.

Circus cineraceus, Newton, ed. Yarr. Brit. B. i. p. 138.

Circus cinerarius, Strickl. Orn. Syn. p. 152 (ex Mont. Trans. L. S.).

Circus pygargus, Sharpe, Cat. B. i. p. 64 (ex Linn.). a (Askew) 1836.

A species of wide range in Europe, Africa and India.

\section{Circus maurus.}

Falco maurus, Temm. Pl. Col. 461.

Circus maurus, Strickl. Orn. Syn. p. 153: Sharpe, Cat. B. i. p. 60 .

$a$ (Arthur Strickland).

A South-African species.

\section{Circus melanoleucus.}

Falco melanoleucos, Gm. Syst. Nat. i. p. 274.

Circus nelanoleucus, Strickl. Orn. Syn. p. 154: Sharpe, Cat. B. i. p. 61 . 
a Madras (T. C. Jerdon) 1845. -b India (E. Blyth) 1846. —c India ("Circus swainsoni" E. Blyth) 1846.

$c$ seems certainly to belong to this species and not to $C$. swainsoni (sive macrurus) under which it is placed in the 'Ornithological Synonyms.' .

\section{Circus assimilis.}

Circus assimilis, Jard. \& Selby, 11l. Orn. i. pl. 51: Strickl. Orn. Syn. p. 155: Sharpe, Cat. B. i. p. 63.

$a,-b$ New South Wales ( $M^{c}$ Donald) 1838. - c Australia (Arthur Strickland).

$a$ stands in the 'Ornithological Synonyms' as $C$.jardinii a name synonymous with $C$. assimilis.

\section{Circus maculosu.}

Aquila maculosa, Vieill. Ois. Am. Sept. i. p. 28*, pl. 3 bis.

Circus maculosus, Strickl. Orn. Syn. p. 155: Sharpe, Cat. B. i. p. 62.

a Brazil (Arthur Strickland).

2369. Circus cinereus.

Circus cinereus, Vieill. N. Dict. d'Hist. N. iv. p. 454: Strickl. Orn. Syn. p. 156: Sharpe, Cat. B. i. p. 56.

a Chili (Arthur Strickland).

\section{SUBFAMILY.-BUTEONIN AE.}

\section{Asturina.}

Asturina, Vieillot, Anal. p. 24 (1816).

\section{Asturina nitida.}

Falco nitidus, Lath. Ind. Orn. p. 41.

Asturina nitida, Strickl. Orn. Syn. p. 41: Scl. \& Salv. P. Z. S. 1869, p. 130 : Sharpe, Cat. B. i. p. 203.

$a$ Brazil ? (Argent) 1852.

\section{Asturina plagiata.}

Asturina plagiata, Schl. Mus. d. Pays-Bas, ii. Asturince, p. 1 : Scl. \& Salv. Ex. Orn. p. 179, pl. 90; Sharpe, Cat. B. i. p. 204. 
a Guatemala (J. Constancia) 1845. - b Guatemala (J. Constancia) 1851.

$a$ is referred in the 'Ornithological Synonyms' (p. 32) to Buteo pennsylvanicus and $b$ to Asturina nitida (p. 41).

\section{Asturina magnirostris.}

Falco magnirostris, Gm. Syst. Nat. i. p. 282.

Astur magnirostris, Strickl. Orn. Syn. p. 120.

Asturina magnirostris, Scl. \& Salv. P. Z. S. 1869, p. 131: Sharpe, Cat. B. i. p. 207.

$a$ (Gardner) 1846. -b Mexiana, 31 January 1849, A. W. (S. Stevens) 1849.

$b$ is a specimen from $\mathrm{Mr}$ A. R. Wallace's collection.

\section{3'33. Asturina nattoreri.}

Asturina nattereri, Scl. \& Salv. P. Z. S. 1869, p. 132; Ex. Orn. p. 173, pl. 87 : Sharpe, Cat. B. i. p. 208.

$a$ South America (Askew) 1840. -b Brazil (Arthur Strickland).

Both these specimens are referred in the 'Ornithological Synonyms' (p. 120) to Astur magnirostris.

\section{Buteola.}

Buteola, Bonaparte, Compt. Rend. xli. p. 651 (1855).

\section{Buteola brachyura.}

Buteo brachyurus, Vieill. N. Dict. d'Hist. N. iv. p. 477.

Buteola brachyura, Sharpe, Cat. B. i. p. 201.

$a$ (Bt at Stevens's) 1843.

A South-American species. In the 'Ornithological Synonyms' (p. 120) this specimen is referred to the South-African Astur melanoleucus.

\section{Bu'TEO.}

Buteo, Cuvier, Leç. Anat. Comp. i. tab. ii. (1800).

\section{Buteo vulgaris.}

Falco buteo, Linn. Syst. Nat. i. p. 127.

Buteo vulgaris, Leach, Syst. Cat. Mamm. \& B. Brit. Mus. p. 10 : Strickl. P. Z. S. 1836, p. 97; Orn. Syu. p. 27: Sharpe, Cat. B. i. p. 180.

a Smyrna, 30 November (H. E. Strickland) 1835. 


\section{3'76. Buteo desertorum.}

Falco desertorum, Daud. Traité, ii. p. 162.

Buteo desertorum, Strickl. Orn. Syn. p. 33: Sharpe, Cat. B. i. p. 179.

Buteo tachardus, Strickl. Orn. Syn. p. 33 (ex Daudin). a Africa (Arthur Strickland).

\section{7\%. Buteo borealis.}

Falco borealis, Gm. Syst. Nat. i. p. 266.

Buteo borealis, Strickl. Orn. Syn. p. 29: Sharpe, Cat. B. i. p. 188.

$a$ Guatemala ( $J$. Constancia) 1845. - b o J Jamaica (J. Gould) 1846. $-c$ N. America (Arthur Strickland).

\section{Buteo lineatus.}

Falco lineatus, Gm. Syst. Nat. i. p. 268.

Buteo lineatus, Strickl. Orn. Syn. p. 31: Sharpe, Cat. B. i. p. 191.

$a$ N. America (Arthur Strickland). -b (Arthur Strickland).

\section{Buteo pennsylvanicus.}

Falco pennsylvanicus, Wils. Am. Orn. vi. p. 92, pl. 54, f. 1.

Buteo pennsylvanicus, Strickl. Orn. Syn. p. 32.

Buteo latissimus, Sharpe, Cat. B. i. p. 193 (ex Wilson).

a Columbia, Pennsylvania (S. F. Baird) 1847.

\section{Buteo abbreviatus.}

Buteo abbreviatus, Cab. in Schomb. Guian. iii. p. 739. Tachytriorchis abbreviatus, Sharpe, Cat. B. i. p. 163. Buteo albonotatus, Strickl. Orn. Syn. p. 31 (ex Gray). $a$ Guatemala (J. Constancia) 1851.

\section{Buteo albicaudatus.}

Buteo albicaudatus, Vieill. N. Dict. d'Hist. N. iv. p. 477 : Strickl. Orn. Syn. p. 35.

Tachytriorchis albicaudatus, Sharpe, Cat. B. i. p. 162. $a,-b$ Brazil (Arthur Strickland). 


\section{Asturinula.}

Asturinula, Finsch \& Hartlaub, Vög. Ost-Afr., p. 59 (1870). 2382. Asturinula monogrammica.

Falco monogrammicus, Temm. Pl. Col. 314.

Astur monogrammicus, Strickl. Orn. Syn. p. 121.

Asturinula monogrammica, Sharpe, Cat. B. i. p. 275.

a West Africa (Arthur Strickland).

\section{Archibuteo.}

Archibuteo, Brehm, Isis, 1828, p. 1269.

\section{Archibuteo lagopus.}

\section{Falco lagopus, Gm. Syst. Nat. i. p. 260.}

Archibuteo lagopus, Strickl. Orn. Syn. p. 38: Sharpe, Cat. B. i. p. 196.

a Britain (Arthur Strickland).

\section{Archibuteo sanctijohannis.}

Falco sanctijohannis, Gm. Syst. Nat. i. p. 273.

Archibuteo sanctijohannis, Strickl. Orn. Syn. p. 40: Sharpe, Cat. B. i. p. 197.

$a$ North America (Arthur Strickland):

\section{Busarellus.}

Busarellus, Lafresnaye, Dict. Univ. d'Hist. N. ii. p. 785 (1844). 2385. Busarellus nigricollis.

Falco nigricollis, Lath. Ind. Orn. p. 35.

Buteogallus nigricollis, Strickl. Orn. Syn. p. 43.

Busarellus nigricollis, Sharpe, Cat. B. i. p. 211.

$a$ Cayenne (Arthur Strickland).

\section{URUBITINGA.}

Urubitinga, Lesson, Rev. Zool. 1839, p. 132.

\section{Urubitinga zonura.}

Falco urubitinga, Gm. Syst. Nat. i. p. 265.

Falco zonurus, Shaw, Gen. Zool. vii. p. 62.

Urubitinga zonura, Sharpe, Cat. B. i. p. 213.

Morphnus brasiliensis, Strickl. Orn. Syn. p. 24 (ex Brisson).

a Brazil (Arihur Strickland). -b (Arthur Strickland). 


\section{Urubitinga anthracina.}

Falco anthracinus, Nitzsch, Pteryl. p. 83.

Morphnus anthracinus, Strickl. Orn. Syn. p. 25.

Urubitinga anthracina, Sharpe, Cat, B. i. p. 215.

$a$ Guatemala (J. Constancia) 1845.

\section{Urubitinga meridionalis.}

Falco meridionalis, Lath. Ind. Orn. p. 36.

Heterospizias meridionalis, Sharpe, Cat. B. i. p. 160.

Astur rufulus, Strickl. Orn. Syn. p. 221 (ex Vieillot).

$a$ South America (Arthur Strickland).

\section{LEUCOPTERNIS.}

Leucopternis, Kaup. Isis, 1847, p. 210.

2389. Leucopternis melanops.

Falco melanops, Lath. Ind. Orn. p. 37.

Leucopternis melanops, Strickl. Orn. Syn. p. 36.

Urubitinga melanops, Sharpe, Cat. B. i. p. 220.

$a$ (E. Brown) 1850.

A Guiana species.

\section{Leucopternis albicollis.}

Falco albicollis, Lath. Ind. Orn, p. 36.

Leucopternis albicollis, Strickl. Orn. Syn. p. 37.

Urubitinga albicollis, Sharpe, Cat. B. i. p. 216.

$a$ (Arthur Strickland).

\section{Geranoaetus.}

Geranoaetus, Kaup. Class. Säug. u. Vög. p. 122 (1844).

\section{Geranoaetus melanoleucus.}

Spizaetus melanoleucus, Vieill. N. Dict. d'Hist. N. xxxii. p. 57.

Geranoaetus melanoleucus, Strickl. Orn. Syn. p. 54.

Buteo melanoleucus, Sharpe, Cat. B. i. p. 168.

$a$ South America (Arthur Strickland). 


\section{HARPYHALIAETUS.}

Harpyhaliaetus, Lafresnaye, Rev. Zool. 1842, p. 173.

2392. Harpyhaliaetus coronatus.

Harpyia coronata, Vieill. N. Dict. d'Hist. N. xiv, p. 237.

Circaetus coronatus, Strickl. Orn. Syn. p, 47.

Harpyhaliaetus coronatus, Sharpe, Cat. B. i. p. 221 : Gurney, Ibis, 1876, p. 492.

a i (Arthur Strickland).

A South-American species. This specimen stands in the 'Ornithological Synonyms' under Circaetus pectoralis. It was subsequently described by Mr Gurney, l. s. $c$.

\section{MoRphnus.}

Morphnus, Cuvier, Règn. Anim. i. p. 318 (1817).

\section{Morphnus guianensis.}

Falco guianensis, Daud. Traité, ii, p. 78.

Morphnus guianensis, Strickl. Orn. Syn. p. 24: Sharpe, Cat. B. i. p. 222.

$a$ (Arthur Strickland) 1850.

A South-American species.

\section{Helotarsus.}

Helotarsus, Smith, S. Afr. Quart. Journ. i. p. 110 (1830).

\section{Helotarsus ecaudatus.}

Falco ecaudatus, Daud. Traité, ii. p. 54.

Helotarsus ecaudatus, Strickl. P. Z. S. 1850, p. 215; Orn. Syn. p. 76: Sharpe, Cat. B. i. p. 300.

a Africa (Arthur Strickland).

\section{Helotarsus leuconotus.}

Helotarsus leuconotus, Rüpp. Syst. Ueb. p. 10: Sharpe, Cat. B. i. p. 301.

$a$ South Africa (Arthur Strickland). 


\section{Aquila.}

Aquila, Brisson, Orn. i. p. 419 (1760).

\section{Aquila rapax.}

Falco rapax, Temm. Pl. Col. 455.

Aquila rapax, Sharpe, Cat. B. i. p. 242.

Aquila ncevia, Strickl. P. Z. S. 1850, p. 215.

Aquila navioides, Strickl. Orn. Syn. p. 59 (ex Cuvier).

a Kordofan, 20 June (J. Petherick) 1848. -b India (Arthur Strickland).

The origin of $b$ is questionable.

\section{Aquila vindhiana.}

Aquila vindhiana, Frankl. P. Z. S. 1830-1, p. 114: Strickl. Orn. Syn. p. 59 : Sharpe, Cat. B. i. p. 243.

$a \hat{\delta},-b$ India (E. Blyth) 1846.

\section{Aquila clanga.}

Aquila clanga, Pall. Zoogr. Rosso-Asiat. i. p. 351: Sharpe, Cat. B. i. p. 248.

$a$ India $(E . B l y t h) 1846$.

\section{Nisaetus.}

Nisaetus, Hodgson, J. A. S. B. v. p. 227 (1836).

2399. Nisaetus fasciatus.

Aquila fasciata, Vieill. Mem. Linn. Soc. Paris, 1822, p. 152 (fide Strickland).

Aquila fasciata, Strickl. Orn. Syn. p. 61.

Nisaetus fasciatus, Sharpe, Cat. B. i. p. 250.

Aquila bonellii, Strickl. Ann. \& Mag. N. H. xv. p. 275.

$a$ (Arthur Strickland). -b S. India (T. C. Jerdon) 1850.

\section{NEOPUS.}

Neopus, Hodgson, Zool. Misc. p. 81 (1844).

2400. Neopus malayensis.

Falco malayensis, Temm. Pl. Col. 117.

Aquila malayensis, Strickl. Orn. Syn. pp. 60, 220.

Neopus malayensis, Sharpe, Cat. B. i. p. 257.

a India (E. Blyth) 1846. 


\section{Haliaetus.}

Halicetus, Savigny, Syst. des Ois. de l'Egypte, p. 25 (1809). 2401. Haliaetus albicilla.

Vultur albicilla, Linn. Syst. Nat. i. p. 123.

Haliaetus albicilla, Strickl. Orn. Syn. p. 48: Sharpe, Cat. B. i. p. 302 .
$e$ (Arthur Strickland). World.

A species of wide range in the northern portions of the Old

\section{Haliaetus leucocephalus.}

Falco leucocephalus, Linn. Syst. Nat. i. p. 124.

Haliaetus leucocephalus, Strickl. Orn. Syn. p. 49: Sharpe, Cat. B. i. p. 304.

$a \mathrm{~N}$. W. America, 1841 (Wosnessenski) 1846.

\section{Haliaetus leucoryphus.}

Aquila leucorypha, Pall. Reis. i. p. 454.

Haliaetus leucoryphus, Strickl. Orn. Syn. p. 52 : Sharpe, Cat. B. i. p. 308 .

Haliaetus macei, Strickl. Orn. Syn. p. 52 (ex Temminck). a India (E. Blyth) 1846.

\section{Haliaetus leucogaster.}

Falco leucogaster, Gm. Syst. Nat. i. p. 257.

Haliaetus leucogaster, Strickl. Orn. Syn. p. 53: Sharpe, Cat. B. i. p. 307.

a Polynesia (Arthur Strickland). -b India (E. Blyth) 1846.

\section{Poliohetus.}

Polioaetus, Kaup, Contr. Orn. 1850, p. 72.

\section{Polioaetus ichthyaetus.}

Falco ichthycetus, Horsf. Trans. L. S. xiii. p. 136.

Polioaetus ichthyaetus, Sharpe, Cat. B. i. p. 452.

Ichthyaetus bicolor, Strickl. Orn. Syn. p. 66 (ex Gray).

$a,-b$ India (E. Blyth) 1846.

$b$, the skin of a young bird, was referred in the 'Ornithological Synonyms' (p. 52) to Haliaetus leucoryphus. 


\section{Spizaktus.}

Spizaetus, Vieillot, Anal. p. 24 (1816).

2406. Spizaetus cirrhatus.

Falco cirrhatus, Gm. Syst. Nat. i. p. 274.

Spizaetus cirrhatus, Strickl. Orn. Syn. p. 69: Sharpe, Cat. B. i. p. 269.

$a,-b$ India (E. Blyth) 1846.

\section{Spizaetus alboniger.}

Nisaetus alboniger, Blyth, J. A. S. B. xiv. p. 173.

Spizaetus alboniger, Strickl. Orn. Syn. p. 71: Sharpe, Cat. B. i. p. 271.

$a$ (E. Brown).

A Bornean species.

\section{Spizaetus limnaetus.}

Falco limncetus, Horsf. Trans. L. S. xiii. p. 138.

Spizaetus limnaetus, Strickl. Orn. Syn. p. 71 : Sharpe, Cat. B. i. p. 272.

a Java (Arthur Strickland).

\section{Circaetus.}

Circaetus, Vieillot, Anal. p. 23 (1826).

2409. Circaetus gallicus.

Falco gallicus, Gm. Syst. Nat. i. p. 259.

Circaetus gallicus, Strickl. Orn. Syn. p. 45: Sharpe, Cat. B. i. p. 280.

Circaetus brachydactylus, Strickl. P. Z. S. 1850, p. 215 (ex Temminck).

a Madras (T. C. Jerdion) 1845.

\section{SPILORNIS.}

Spilornis, Gray, List. Gen. B. p. 3 (1840).

2410. Spilornis bido.

Falco bido, Horsf. Trans. L. S. xiii. p. 137.

Spilornis bido, Gurney, Ibis, 1878, p. 100.

Spilornis bacha, Strickl. Orn. Syn. p. 44: Sharpe, Cat. B. i. p. 290 (nec Daudin). 
a (Arthur Strickland).

Agrees with one of Horsfield's Javan specimens in the University Museum.

2111. Spilornis cheela.

Falco cheela, Lath. Ind. Orn. i. p. 14.

Spilornis albidus, Wald. Ibis, 1873, p. 298 (ex Temm.): Gurney, Ibis, 1878, p. 90.

Spilornis melanotis, Sharpe, Cat. B. i. p. 289 (ex Jerdon). $a,-b$ India (E. Blyth) 1845. —c (Arthur Strickland).

\section{BUTASTUR.}

Butastur, Hodgson, J. A. S. B. xii. p. 311 (1843).

\section{Butastur teesa.}

Circus teesa, Frankl. P. Z. S. 1830-1, p. 115.

Poliornis teesa, StrickJ. Orn. Syn. p. 125.

Butastur teesa, Sharpe, Cat. B. i. p. 295.

a Benares (W. J. E. Boys) 1847.

\section{Butastur rufipennis.}

Buteo rufipennis, Strickl. P. Z. S. 1850, p. 214, pl. 22; Orn. Syn. p. 36.

Butastur rufipennis, Sharpe, Cat. B. i. p. 299.

a ô Kordofan, 14 August (e. Petherick) 1848.

The type of Strickland's description and figure.

\section{SUBFAMILY.-ACCIPITRIN $\mathbb{A}$.}

\section{Astur.}

A stur, Lacépède, Mém. de l'Inst. iii. p. 506 (1801).

2414. Astur palumbarius.

Falco palumbarius, Linn. Syst. Nat. i. p. 130.

Astur palumbarius, Strickl. Orn. Syn. p. 116: Sharpe, Cat. B. i. p. 95 .

a.

A European species.

2415. Astur novæ-hollandiæ.

Falco novce-hollandice, Gm. Syst. Nat. i. p. 264. 
Astur novce-hollandice, Strickl. Orn. Syn. p. 118: Sharpe, Cat. B. i. p. 118.

Falco clarus, Lath. Ind. Orn. Suppl. p. xiii.: Strickl. Ann. \& Mag. N. H. xi. p. 338.

a New Holland (T. C. Eyton) 1843. - b New South Wales (Arthur Strickland).

\section{Astur radiatus.}

Falco radiatus, Lath. Ind. Orn. Suppl. p. xii.

Astur radiatus, Strickl. Orn. Syn. p. 119.

Urospizias radiatus, Sharpe, Cat. B. i. p. 159.

a Australia (Arthur Strickland) 1840.

\section{ACCIPITER.}

Accipiter, Brisson, Ornith. i. p. 310 (1760): Strickl. Ann. \& Mag. N. H. vi. p. 416.

\section{1\%. Accipiter approximans.}

Astur approximans, Vig. \& Horsf. Trans. L. S. xv. p. 181: Sharpe, Cat. B. i. p. 126.

Accipiter approximans, Strickl. Ann. \& Mag. N. H. xi. p. 335 ; xiii. p. 33; Orn. Syn. p. 111.

$a$ New South Wales (McDonald) 1838. - b, -c Australia (Arthur Strickland).

\section{Accipiter pileatus.}

Falco pileatus, Temm. Pl. Col. 20 5.

Accipiter pileatus, Strickl. Orn. Syn. p. 109: Sharpe, Cat. B. i. p. 153 .

$a$ Brazil (Arthur Strickland). -b (Arthur Strickland).

$b$, a bird in immature plumage, is assigned to $A$. tachiro in the 'Ornithological Synonyms' (p. 114).

\section{Accipiter cooperi.}

Falco cooperi, Bp. Am. Orn. ii. p. 1, pl. 10, f. 1. Accipiter cooperi, Sharpe, Cat. B. i. p. 137.

$a$ Guatemala (J. Constancia) 18š1. - b (Arthur Strickland). In the 'Ornithological Synonyms' $b$ was placed under A. tachiro. 


\section{Accipiter nisus.}

Falco nisus, Linn. Syst. Nat. i. p. 130.

Accipiter nisus, Strickl. Orn. Syn. p. 104: Sharpe, Cat. B. i. p. 132.

Accipiter fringillaria, Strickl. P. Z. S. 1836, p. 97 (ex Ray). Accipiter nisosimilis, Strickl. Orn. Syn. p. 105 (ex Tickell).

$a$ o Smyrna, 1 December (H. E. Strickland) 1835. -b (Sir W. Jardine) 1844. —c Madras (T. C. Jerdon) 1845. -d $q$ Dumfriesshire (H. E. Strickland) 1845. -e.

\section{Accipiter fuscus.}

Falco fuscus, Gm. Syst. Nat. i. p. 280.

Accipiter fuscus, Strickl. Orn. Syn. p. 108: Sharpe, Cat. B i. p. 135 .

$a$ f̂ Carlisle, Pennsylvania (S. F. Baird) 1847. - b.

\section{Accipiter chionogaster.}

Nisus (seu Accipiter) chionogaster, Kp. P. Z. S. 1851, p. 41. Accipiter chionogaster, Sharpe, Cat. B. i. p. 148.

a (P. L. Sclater) 1848.

This specimen agrees best with the Venezuelan form of this species. In the 'Ornithological Synonyms' (p. 110) it stands as A. exilis, Temm. (=A. rufiventris, Smith.)

\section{Accipiter rufiventris.}

Accipiter rufiventris, Smith, S. Afr. Quart. Journ. i. p. 231: Sharpe, Cat. B. i. p. 148.

Accipiter exilis, Strickl. Orn. Syn. p. 110 (ex Temm.).

a (Arthur Strickland).

A South-African species.

\section{Accipiter cirrhocephalus.}

Sparvius cirrhocephalus, Vieill. N. Dict. d'Hist. N. x. p. 329. Astur cirrhocephalus, Sharpe, Cat. B. i. p. 141.

Falco melanops, Lath. Ind. Orn. Suppl. p. xii. (nom. inept. $c f$. Strickl. Ann. \& Mag. N. H. xi. p. 338).

Accipiter melanops, Strickl. Orn. Syn. p. 111.

a New South Wales (McDonald) 1838, -b Australia (Arthur Strickland). 


\section{Accipiter superciliosus.}

Falco superciliosus, Linn. Syst. Nat. i. p. 128.

Accipiter superciliosus, Strickl. Orn. Syn. p. 115.

Falco tinus, Lath. Ind. Orn. p. 50.

Accipiter tinus, Gray, Gen. B. i. pl. 10: Sharpe, Cat. B. i. p. 139.

Falco ferrugineus, Nordm. in Erm. Reise, p. 16.

Accipiter ferrugineus, Strickl. Orn. Syn. p. 109.

a Brazil (Arthur Strickland).

\section{Accipiter minullus.}

Falco minullus, Daud. Traité d'Orn. ii. p. 88.

Accipiter minullus, Strickl. Orn. Syn. p. 113: Sharpe, Cat.

B. i. p. 140.

a (Arthur Strickland).

A South-African species.

\section{2\%. Accipiter virgatus.}

Falco virgatus, Temm. Pl. Col. 109.

Accipiter virgatus, Strickl. Orn. Syn. p. 106: Sharpe, Cat. B. i. p. 150 .

Accipiter besra, Jerd. cf. Strickl. Ann. \& Mag. N. H. xv. p. 275.

a Madras (T. C. Jerdon) 184.5.

\section{Accipiter badius.}

Falco badius, Gm. Syst. Nat. i. p. 280.

Accipiter badius, Strickl. Ann. \& Mag. N. H. xiii. p. 33;

Orn. Syn. p. 106.

Astur badius, Sharpe, Cat. B. i. p. 109.

a India (Askew) 1839. - b Madras (T. C. Jerdon) 1845. —c.

\section{Accipiter soloensis.}

Falco soloensis, Horsf. Trans. L. S. xiii. p. 137.

Accipiter soloensis, Strickl. Orn. Syn. p. 107.

Astur soloensis, Sharpe, Cat. B. i. p. 114.

a Malacca (W. Kirtland) 1843. 


\section{Accipiter niger.}

Sparvius niger, Vieill. Enc. Méth. iii. p. 1269.

Accipiter niger, Strickl. \& Scl. Contr. Orn. 1852, p. 142 : Strickl. Orn. Syn. p. 113.

Melierax niger, Sharpe, Cat. B. i. p. 91.

Accipiter carbonarius, Strickl. P. Z. S. 1850, p. 215 (ex Licht.).

a f̂ Kordofan, 19 July (J. Petherick) 1848.

\section{Accipiter gabar.}

Falco gabar, Daud. Traité d’Orn. ii. p. 87.

Melierax gabar, Strickl. P. Z. S. 1850, p. 215: Sharpe, Cat. B. i. p. 89 .

Accipiter gabar, Strickl. \& Scl. Contr. Orn. 1852, p. 142: Strickl. Orn. Syn. p. 112.

Accipiter sphenurus?, Strickl. P. Z. S. 1850, p. 215 (nec Rüpp.).

a $q$ Kordofan, 6 August (J. Petherick) 1850. - $b$ \& Kordofan, 25 August (J. Petherick) 1848.

\section{MicRasteur.}

Micrastur, Gray, List Gen. B. p. 6 (1841).

\section{Micrastur semitorquatus.}

Sparvius semitorquatus, Vieill. N. Dict. d'Hist. N. x. p. 322. Micrastur semitorquatus, Strickl. Orn. Syn. p. 122: Sharpe, Cat. B. i. p. 75.

$a$ Guatemala ( $J$. Constancia) 1851.

\section{Micrastur ruficollis.}

Sparvius ruficollis, Vieill. N. Dict. d'Hist. N. x. p. 322.

Micrastur ruficollis, Strickl. Orn. Syn. p. 1亡2: Sharpe, Cat. B. i. p. 76 .

\section{$a$ (Thomas) 1840. -b Brazil (Arthur Strickland).}

$b$ stands in the 'Ornithological Synonyms' (p. 123) as M. leucauchen, Temm. a probable synonym of $M$. ruficollis.

\section{Micrastur gilvicollis.}

Sparvius gilvicollis, Vieill. N. Dict. d'Hist. N. x. p. 323.

Micrastur gilvicollis, Scl. \& Salv. P. Z. S. 1869, p. 368: Sharpe, Cat. B. i. p. 78.

$$
\text { s. C. }
$$


Micrastur concentricus, Strickl, Orn. Syn. p. 123 (ex Lesson). a Trinidad (Argent) 1851.

\section{GERANOSPIZIAS.}

Ischnosceles, Strickland, Ann. \& Mag. N. H. xiii. p. 409 (1844, nec Burmeister).

Geranospiza (rectius Geranospizias, Sundev.), Kaup, Isis, 1847, p. 183.

\section{Geranospizias cærulescens.}

Sparvius ccerulescens, Vieill. N. Dict. d'Hist. N. x. p. 318.

Geranospizias ccerulescens, Sharpe, Cat. B. i. p. 81.

Ischnosceles gracilis, Strickl. Ann. \& Mag. N. H. xiii. p. 409; Orn. Syn. p. 124 (ex Temm.)

$a$ (Arthur Strickland).

A species of wide range in South America.

Polyboroides.

Polyboroides, Smith, S. Afr. Quart. Journ. i. p. 106 (1830). 2436. Polyboroides typicus.

Polyboroides typicus, Smith, S. Afr. Quart. Journ. i. p. 107: Sharpe, Cat. B. i. p. 48.

Polyboroides radiatus, Strick1. P. Z. S. 1850, p. 216; Orn. Syn. p. 143 (partim, nec Scopoli).

a $q$ Kordofan, 15 July (J. Petherick) 1848.

\section{Melierax.}

Melierax, Gray, List Gen. B. p. 5 (1840).

\section{3\%. Melierax canorus.}

Falco canorus, Rislach, in Thunb. Diss. Ac. iii. p. 264.

Melierax canorus, Strickl. P. Z.S. 1850, p. 215: Sharpe, Cat. B. i. p. 87.

Melierax musicus, Strickl. Orn. Syn. p. 142 (nec Daudin). a ô (Arthur Strickland).

A South-African species. 


\section{Melierax polyzonus.}

Falco (Nisus) polyzonus, Rüpp. Neue Wirb. p. 36, pl. 15.

Melierax polyzonus, Strickl. P. Z. S. 1850, p. 215; Orn. Syn. p. 143 : Sharpe, Cat. B. i. p. 88.

a $\hat{\delta}$ Kordofan, 20 June (J. Petherick) 1848.

\section{SUBFAMILY.-FALCONIN 无.}

\section{FALCO.}

Falco, Linnæus, Syst. Nat. i. p. 124 (1766) (partim). 2439. Falco islandus.

Falco islandus, Gm. Syst. Nat. i. p. 271: Strickl. Orn. Syn. p. 77.

Hierofalco islandus, Sharpe, Cat. B. i. p. 414.

a Iceland (J. G. Kinberg) 1843.

A young bird referred in the 'Ornithological Synonyms' to $F$. gyrfalco.

2440. Falco sacer.

Falco sacer, Gm. Syst. Nat. i. p. 273 (nec Forster): Strickl. Orn. Syn. p. 79.

Hierofalco saker, Sharpe, Cat. B. i. p. 417.

Falco biarmicus, Strickl. P. Z. S. 1850, p. 215 (partim, nec Temminck).

a ô Kordofan, 16 March (J. Petherick) 1848.

\section{Falco peregrinus.}

Falco peregrinus, Tunst. Orn. Brit. p. 1: Strickl. Orn. Syn. p. 81.

Falco communis, Sharpe, Cat. B. i. p. 376 (ex Gmelin). Falco anatum, Strickl. Orn. Syn. p. 83 (ex Bonap.).

$a$ Chili (Capt, Brown) 1842. - $b$ S. India ( $T . C$. Jerdon) 1850.

$a$ is in the dark-coloured plumage described by Mr Sharpe under the name Falco cassini (Cat. B. i. p. 384). 


\section{Falco melanogenys.}

Falco melanogenys, Gould, P. Z. S. 1837, p. 139; B. Austr. i. pl. 8: Strickl. Orn. Syn. p. 84: Sharpe, Cat. B. i. p. 385.

$a$ New South Wales ( $M^{c}$ Donald) 1838.

\section{Falco biarmicus.}

Falco biarmicus, Temm. Pl. Col. 324: Strickl. P. Z. S. 1850, p. 215; Orn. Syn. p. 79: Sharpe, Cat. B. i. p. 391. a ô Kordofan, 10 July (J. Petherick) 1848.

Some writers separate the north and south African Lanners and if their views be correct this bird should probably bear the name Falco tanypterus, Schl. Strickland's note (P. Z. S. 1850, p. 215) refers to $F$. sacer.

\section{Falco jugger.}

Falco jugger, J. E. Gray, Ill. Ind. Zool. ii. pl. 26: Strickl. Orn. Syn. p. 79 : Sharpe, Cat. B. i. p. 393.

a India (W. J. E. Boys) 1847. -b Southern India (T. C. Jerdon) 1850.

\section{Falco æsalon.}

Falco cesalon, Tunst. Orn. Brit. p. 1: Strickl. P. Z. S. 1836, p. 97 ; Orn. Syn. p. 90.

Falco regulus, Sharpe, Cat. B. i. p. 406 (ex Pallas).

a ô Smyrna, 24 November (H. E. Strickland) 1835. -b (Bt at Birmingham) 1843.

\section{Falco columbarius.}

Falco columbarius, Linn. Syst. Nat. i. p. 128: Strickl. Orn. Syn. p. 92: Sharpe, Cat. B. i. p. 408.

$a$ N. America (J. G. Kinberg) 1845. - b Jamaica (J. Gould) 1846.

\section{4\%. Falco chiquera.}

Falco chiquera, Daud. Traité d'Orn. ii. p. 121: Strickl. Orn. Syn. p. 87: Sharpe, Cat. B. i. p. 403. 1846 .

$a,-b$ Madras (T. C. Jerdon) 1845. —c India (E. Blyth) 


\section{Falco fusco-cærulescens.}

Falco fusco-ccerulescens, Vieill. N. Dict. d'Hist. N. xi. p. 90: Sharpe, Cat. B. i. p. 400.

Falco femoralis, Strickl. Orn. Syn. p. 88 (ex Temminck).

$a,-b$ (Arthur Strickland).

A South-American species.

\section{Falco rufigularis.}

Falco rufigularis, Daud. Traité d'Orn. ii. p. 131: Strickl. Orn. Syn. p. 88.

Falco albigularis, Sharpe, Cat. B. i. p. 401 (ex Daudin).

$a$ Guatemala (J. Constancia) 1851. -b (Arthur Strickland).

\section{Falco concolor.}

Falco concolor, Temm. Pl. Col. 330 : Sharpe, Cat. B. i. p. 405.

Falco peregrinator, Strickl. Ann. \& Mag. N. H. xviii. p. 455 (nec Sundevall).

$a$ Lat.-Long. (Arthur Strickland).

This specimen labelled "Falco peregrinator" is doubtless the one referred to in Strickland's note appended to the translation of Sundevall's paper on the Birds of Calcutta. He there states that he possessed a specimen which he referred to $F$. peregrinator procured in 1833 on board ship between Mauritius and Madagascar. Other examples, with which this one agrees, have been procured in the same seas under similar circumstances ( $f$. Gurney, Ibis, 1869, p. 445).

\section{Falco subbuteo.}

Falco subbuteo, Linn. Syst. Nat. i. p. 127: Strickl. Orn. Syn. p. 85: Sharpe, Cat. B. i. p. 395.

a (Arthur Strickland) 1850.

A common species in the northern portions of the old world. This specimen (a skin of a young bird) is referred in the 'Ornithological Synonyms' (p. 90) to F'alco cesalon.

\section{Tinnunculus.}

Tinnunculus, Vieillot, Ois. Am. Sept. i. p. 39 (1807). 2452. Tinnunculus alaudarius.

Falco tinnunculus, Linn. Syst. Nat. i. p. 127 : Strickl. P. Z. S. 1836, p. $97 ; 1850$, p. 215. 
Tinnunculus alaudarius, Strickl. Orn. Syn. p. 95 (ex Gmelin).

Cerchneis tinnuncula, Sharpe, Cat. B. i. p. 425.

$a$ Smyrna, 10 December (H. E. Strickland) 1835. - b Britain (Askew) 1838. — Madras (T. C. Jerdon) 1845. —d India ( $E$. Blyth) 1846. - e Furrudpore (W. J. E. Boys) 1847. - $f$ Kordofan, March (J. Petherick) 1848. -g (Arthur Strickland).

\section{Tinnunculus tinnunculoides.}

Falco tinnunculus, Temm. Man, d'Orn, i. p. 31 (1820): Strickl. P. Z. S. 1836, p. 97.

Falco cenchris, Strickl. Orn. Syn. p. 97 (ex Naumann).

Falco naumanni, Sharpe, Cat. B. i. p. 435 (ex Fleisch).

a Azani in Mysia, April (H. E. Strickland) 1836.

\section{Tinnunculus rupicola.}

Falco rupicolus, Daud, Traité d'Orn. ii. p. 135.

Tinnunculus rupicolus, Strickl. Orn. Syn. p. 97.

Cerchneis rupicola, Sharpe, Cat. B. i. p. 429.

$a,-b$ Cape of Good Hope (A. Strickland) 1833.

\section{Tinnunculus rupicoloides.}

Falco rupicoloides, Smith, S. Afr. Quart. Journ. i. p. 238.

Tinnunculus rupicoloides, Strickl. Orn. Syn. p. 98.

Cerchneis rupicoloides, Sharpe, Cat. B, i. p. 432.

a (Arthur Strickland).

A South-African species.

\section{Tinnunculus punctatus.}

Falco punctatus, Temm. Pl. Col. 45.

Tinnunculus punctatus, Strickl. Orn. Syn. p. 98. Cerchneis punctata, Sharpe, Cat. B. i. p. 434.

a Mauritius (Arthur Strickland) 1832.

\section{5\%. Tinnunculus cenchroides.}

Falco cenchroides, Vig. \& Horsf. Trans. L. S. xv. p. 183.

I'innunculus cenchroides, Strickl. Orn. Syn. p. 98.

Cerchneis cenchroides, Sharpe, Cat. B. i. p. 431.

$a,-b$ New South Wales ( $M^{c}$ Donald) 1838. 
2458. Tinnunculus sparverius.

Falco sparverius, Linn. Syst. Nat. i. p. 128.

Tinnunculus sparverius, Strickl. Orn. Syn. p. 99.

Cerchneis sparveria, Sharpe, Cat. B. i. p. 437.

$a \mathrm{~N}$. America (Askew) 1834. -b Mexico (T. Mann) 1844. -c Guatemala (J. Constancia) 1845. —d Canada (Barnes) 1848. - e (Arthur Strickland).

\section{Tinnunculus cinnamominus.}

Falco cinnamominus, Sw. Anim. in Menag. p. 281.

Tinnunculus cinnamominus, Strickl. Orn. Syn. p. 100.

Cerchneis cinnamomina, Sharpe, Cat. B. i. p. 439.

$a,-b$ (Arthur Strickland).

A South-American race of T'. sparverius. In the 'Ornithological Synonyms' $b$ is referred to $T$. dominicensis.

\section{Tinnunculus isabellinus.}

Falco isabellinus, Sw. Anim. in Menag. p. 281.

Cerchneis isabellina, Sharpe, Cat. B. i. p. 441.

a Trinidad (Argent) 1851.

This specimen is referred in the 'Ornithological Synonyms' to T. dominicensis.

\section{Tinnunculus ardesiacus.}

Falco ardosiacus, Vieill. Enc. Méth. i. p. 1238: Strickl. Orn. Syn. p. 90.

Cerchneis ardesiaca, Sharpe, Cat. B. i. p. 446.

a (Arthur Strickland).

A species of Western and North-Western Africa.

\section{HIERACIDEA.}

Ieracidea, Gould, P. Z. S. 1837, p. 140.

2462. Hieracidea berigora.

Falco berigora, Vig. \& Horsf. Trans. L. S. xv. p. 184.

Hieracidea berigora, Strickl. Orn. Syn. p. 101: Sharpe, Cat. B. i. p. 421 .

$a,-b$ Australia (Arthur Strickland). 


\section{SUBFAMILY.-MILVIN E.}

\section{Milvus.}

Milvus, Cuvier, Leç. d'Anat. Comp. i. tab. 2 (1800).

\section{Milvus ictinus.}

Falco milvus, Linn. Syst. Nat. i. p. 126.

Milvus ictinus, Savign. Syst. des Ois. de l'Egypte, p. 28: Sharpe, Cat. B. i. p. 319.

Milvus regalis, Strickl. Orn. Syn. p. 131.

$a$ Switzerland (L. Coulon) 1836.

\section{Milvus ægyptius.}

Falco cegyptius, Gm. Syst. Nat. i. p. 261.

Milvus agyptius, Sharpe, Cat. B. i. p. 320.

Milvus forskåhli, Strickl. Orn. Syn. p. 124 (ex Gmelin).

Milvus parasiticus, Strickl. \& Scl. Contr. Orn. 1852, p. 142 (ex Daudin).

a Africa (Arthur Strickland).

\section{Milvus govinda.}

Milvus govinda, Sykes, P. Z. S. 1832, p. 81: Sharpe, Cat, B. i. p. 325 .

Milvus ater, Strickl. Ann. \& Mag. N. H. xiii. p. 204 (nec Gmelin).

Milvus migrans, Strickl. Orn. Syn. p. 133 partim (nec Boddaert).

a Madras (T. C. Jerdon) 1845. - b Australia (Arthur Strickland).

The origin given of $b$ is doubtless erroneous; the specimen (a young one) has the base of the primaries mottled with white as in the Indian bird.

\section{Milvus isurus.}

Milvus isurus, Gould, P. Z. S. 1837, p. 140.

Lophoictinia isura, Sharpe, Cat. B. i. p. 326.

Falco pacificus, Strickl. Ann. \& Mag. N. H. xi. p. 338; Orn. Syn. p. 135 (ex Latham).

a Australia (Arthur Strickland).

Latham's name has not a very satisfactory basis. 


\section{Pernis.}

Pernis, Cuvier, Règn. Anim. i. p. 322 (1817).

2467. Pernis ptilonorhynchus.

Falco ptilorhynchus, Temm. Pl. Col. 44.

Pernis ptilonorhynchus, Sharpe, Cat. B. i. p. 347.

Pernis cristata, Strickl. Orn. Syn. p. 131 (ex Cuvier). a S. India (T. C. Jerdon) 1850.

\section{Haliastur.}

Haliastur, Selby, Cat. Gen. p. 3 (1840): Strickl. Ann. \& Mag. N. H. vi. p. 416.

\section{Haliastur indus.}

Aigle des grandes Indes, D’Aub. Pl. Enl. 416, undè,

Falco indus, Bodd. Tab. Pl. Enl. p. 25.

Haliastur indus, Strickl. Orn. Syn. p. 73: Sharpe, Cat. B. i. p. 313.

a India (Havell) 1839, - b India ("Haliastur pondicerianus second plumage which is very variable being Milvus rotundicaudatus, Hodgs.," E. Blyth) 1846. —c Philippine Islands (J. Gould) 1846.

$c$ is probably referable to $H$. intermedius if really a recognizable race (cf. Sharpe, l.s. c. p. 314).

\section{BAZA.}

Baza, Hodgson, J. A. S. B. v. p. 777 (1836): Strickl. Ann. \& Mag. N. H. xiii. p. 33.

\section{Baza subcristata.}

Lepidogenys subcristatus, Gould, P. Z. S. 1837, p. 140.

Baza subcristata, Strickl. Orn. Syn. p. 127 : Sharpe, Cat. B. i. p. 357 .

a Australia (Bt at Birmingham) 1843.

\section{LEPTODON.}

Leptodon, Sundevall, K. Vet. Ac. Handl. 1835, p. 114. 24\%0. Leptodon cayennensis.

Falco cayennensis, Gm. Syst. Nat. i. p. 263. 
Cymindis cayennensis, Strickl. Orn. Syn. p. 128 (ex Cuvier). Leptodon cayennensis, Sharpe, Cat. B. i. p. 333. $a$ Guatemala (J. Constancia) 1851.

\section{Leptodon uncinatus.}

Falco uncinatus, Temm. Pl. Col. 103, 104, 105.

Rostrhamus uncinatus, Strickl. Orn. Syn. p. 136.

Leptodon uncinatus, Sharpe, Cat. B. i. p. 330.

$a$ South America (Mather) 1840.

\section{Harpagus.}

Harpagus, Vigors, Zool. Journ. i. p. 338 (1824).

2472. Harpagus bidentatus.

Falco bidentatus, Lath. Ind. Orn. i. p. 38.

Harpagus bidentatus, Strickl. Orn. Syn. p. 101 : Sharpe, Cat. B. i. p. 362 .

$a$ S. America (Thomas) 1842.

\section{GampsonyX.}

Gampsonyx, Vigors, Zool. Journ. ii. p. 69 (1825): Strickl. Ann. \& Mag. N. H. vi. p. 416.

\section{4'73. Gampsonyx swainsoni.}

Gampsonyx swainsoni, Vig. l. s. c.: Strickl. Orn. Syn. p. 137: Sharpe, Cat. B. i. p. 340.

a Trinidad (Argent) 1851. - b Brazil (Arthur Strickland).

\section{Microhierax.}

Ierax, Vigors, Zool. Journ. i. p. 339 (1824) (nec Leach). Microhierax, Sharpe, Cat. B. i. p. 366 (1874).

\section{Microhierax fringillarius.}

Falco fringillarius, Drap. Dict. Class. d'Hist. Nat. vi. p. 412, t. 5 .

Microhierax fringillarius, Sharpe, l. s. c. p. 367.

Hierax malayensis, Strickl. Ann. \& Mag. N. H. xiii. p. 33; Orn. Syn. p. 103.

a East Indies (East India Co.) 1831.-b Malacca (Capt. Nash) 1837.

The types, probably, of Strickland's note $l_{0} s . c$. 


\section{Elanus.}

Elanus, Savigny, Syst. des Ois. de l'Egypte, p. 37 (1809).

\section{Elanus cæruleus.}

Falco cceruleus, Desf. Hist. Ac. R. des Sc. 1787, p. 503, pl. 6. Elanus cceruleus, Strickl, Orn. Syn. p. 137: Sharpe, Cat. B. i. p. 336 .

Elanus melanopterus, Blyth, Ann. \& Mag. N. H. xiv. p. 38 (ex Daudin): Strickl. Ann, \& Mag. N. H. xviii. p. 456.

$a$ (Askew) 1838. —b India (E. Blyth) 1846.

\section{Elanus leucurus.}

Milvus leucurus, Vieill. N. Dict. d'Hist. N. xx. p. 563.

Elanus leucurus, Strickl. Orn. Syn. p. 138: Sharpe, Cat. B. i. p. 339.

Elanus dispar, Strickl. Ann. \& Mag. N. H. xviii. p. 456 (ex Bonaparte).

a Chili (Arthur Strickland).

\section{4\%\%. Elanus axillaris.}

Falco axillaris, Lath. Ind. Orn. Suppl. p. ix.

Elanus axillaris, Strickl. Orn. Syn. p. 139: Sharpe, Cat. B. i. p. 338 .

a Australia (Arthur Strickland).

\section{ICTINIA.}

Ictinia, Vieillot, Anal, p. 24 (1816).

\section{Ictinia plumbea.}

Falco plumbeus, Gm. Syst. Nat. i. p. 283.

Ictinia plumbea, Strickl. Orn, Syn. p. 140 : Sharpe, Cat. B. i. p. 364 .

a Brazil (Arthur Strickland).

\section{Elanoides.}

Elanoides, Vieillot, N. Dict. d'Hist. Nat. xxiv. p. 101 (1818). 2479. Elanoides riocouri.

Elanoides riocouri, Vieill. Gal. Ois. i. p. 43, pl. 16: Strickl. Orn. Syn. p. 142. 
Nauclerus riocouri, Strickl. P. Z. S. 1850, p. 215: Sharpe, Cat. B. i. p. 318.

a ईิ Kordofan, 5 August (J. Petherick) 1848.

\section{SUBFAMILY.-HERPETOTHERIN $Æ$.}

HeRpetotheres.

Herpetotheres, Vieillot, N. Dict. d'Hist. N. xviii. p. 317 (1817) (cf. Strickl. Ann. \& Mag. N. H. vi. p. 415).

2480. Herpetotheres cachinnans.

Falco cachinnans, Linn. Syst. Nat. i. p. 128.

Herpetotheres cachinnans, Strickl. Orn. Syn. p. 75: Sharpe, Cat. B. i. p. 278.

a Island of Mexiana, November (A. W.) 1848.

One of Mr A. R. Wallace's specimens.

\section{SUBFAMILY.-POLYBORIN AE.}

\section{IBYCTER.}

Ibycter, Vieillot, Anal. p. 22 (1816).

\section{Ibycter americanus.}

Aigle d'Amérique, D'Aub. Pl. Enl. 417, undè,

Falco americanus, Bodd. Tabl. Pl. Enl. p. 25.

Ibycter americanus, Strickl. Orn. Syn. p. 22: Sharpe, Cat. B. i. p. 35 .

a Guatemala (J. Constancia) 1851.

\section{Milvago.}

Milvago, Spix, Av. Bras. i. p. 12 (1824).

\section{Milvago chimachima.}

Polyborus chimachima, Vieill. N. Dict. d'Hist. N. v. p. 259. Milvago chimachima, Strickl. Orn. Syn. p. 19.

Ibycter chimachima, Sharpe, Cat. B. i. p. 39.

$a$ Brazil (Bt at Birmingham) 1844. -b (Arthur Strickland). 
Polyborus.

Polyborus, Vieillot, Anal. p. 22 (1816).

2483. Polyborus tharus.

Falco tharus, Mol. Saggio sulla St. Nat. p. 264.

Polyborus tharus, Strickl. Orn. Syn. p. 18: Sharpe, Cat. B. i. p. 31.

a Brazil (Arthur Strickland).

2484. Polyborus cheriway.

Falco cheriway, Jacq. Beitr. p. 17, pl. 4.

Polyborus cheriway, Sharpe, Cat. B. i. p. 33.

$a$ Guatemala (J. Constancia) 1851.

\section{FAMILY.-CATHARTIDE.}

\section{Cathartes.}

Cathartes, Illiger, Prodr. p. 236 (1811) (partim).

\section{Cathartes aura.}

Vultur aura, Linn. Syst. Nat. i. p. 122.

Cathartes aura, Strickl. Orn. Syn. p. 1.

Enops aura, Sharpe, Cat. B. i. p. 25.

a Chili (T. C. Eyton) 1846.

\section{Sarcorhamphus.}

Sarcorhamphus, Duméril, Zool. Anal. p. 32 (1806).

2486. Sarcorhamphus gryphus.

Vultur gryphus, Linn. Syst. Nat. i. p. 121.

Sarcorhamphus gryphus, Strickl. Orn. Syn. p. 4: Sharpe, Cat. B. i. p. 20 .

a (Arthur Strickland) 1850.

A species of the Chilian Andes, Patagonia, \&c.

Gypagus.

Gypagus, Vieillot, Anal. p. 21 (1816).

248\%. Gypagus papa.

Vultur papa, Linn. Syst. Nat. i. p. 122. 
Sarcorhamphus papa, Strickl. Orn. Syn. p. 5.

Cathartes papa, Sharpe, Cat. B. i. p. 22.

a Guatemala (J. Constancia) 1851.

\section{FAMILY.VULTURID}

GYPS.

Gyps, Savigny, Syst. des Ois. de l'Egypte, p. 11 (1809).

2488. Gyps indicus.

Vultur indicus, Scop. Del. Faun. et Fl. Insubr. ii. p. 85.

Gyps indicus, Strickl. Orn. Syn. p. 10: Sharpe, Cat. B. 1. p. 10.

$a$ India $(E$. Blyth) 1846.

\section{Pseudogyps.}

Pseudogyps, Sharpe, Ann. \& Mag. N. H. Ser. 4, xi. p. 133 (1873).

\section{Pseudogyps bengalensis.}

Vultur bengalensis, Gm. Syst. Nat. i. p. 24 .

Gyps bengalensis, Strickl. Orn. Syn. p. 11.

Pseudogyps bengalensis, Sharpe, Cat. B. i. p. 11.

$a,-b$ India (E. Blyth) 1846 .

\section{OTOGYPS.}

Otogyps, Gray, List Gen. B. p. 2 (1841).

2490. Otogyps calvus.

Vultur calvus, Scop. Del. Faun. et Fl. Insubr. ii. p. 85 .

Otogyps calvus, Strickl. Orn. Syn. p. 8: Sharpe, Cat. B. is p. 14.

$a \mathrm{~S}$. India $\left(T . C_{\mathrm{o}}\right.$ Jerdon) $1850_{\mathrm{s}}$ 


\section{ORDER VIII.-STEGANOPODES.}

\section{FAMILY.-PHAETHONTID AE。}

\section{Phatethon.}

Phaeton, Linnæus, Syst. Nat. i. p. 219 (1766) (partim). 2491. Phaethon rubricauda.

Paille en queue de l'Ile de France, D'Aub. Pl. Enl. 979, undè, Phaeton rubricauda, Bodd. Tabl. Pl. Enl. p. 57.

Phaeton phoenicurus, Gould, B. Austr. vii. pl. 73 (ex Gmelin). $a$ New South Wales (McDonald) 1838, - $b$.

\section{Phaethon æthereus.}

Phaeton cethereus, Linn. Syst. Nat. i. p. 219: Penrose, Ibis, 1879, p. 276.

a Ascension I. December (A. Strickland) 1833. - -b (Arthur Strickland) 1850 .

\section{Phaethon flavirostris.}

Phaeton flavirostris, Brandt, Bull. Ac. St. Petersb. 1837, p. 349: Penrose, Ibis, 1879, p. 277.

$a$ Mauritius, 13 November (A. Strickland) 1833, - - b Ascension I. December (A. Strickland) 1833. - c.

\section{FAMILY.-PELECANIDE.}

\section{Pelecanus.}

Pelecanus, Linnæus, Syst. Nat. i. p. 215 (1766) (partim). 2494. Pelecanus rufescens.

Pelecanus rufescens, Gm. Syst. Nat. i. p. 571 : Elliot, P. Z, S. 1869 , p. 583. 
Pelecanus philippensis, Jerd. B. Ind. iii. p. 858 (apud Elliot). $a$ India (E. Blyth) 1845. -b India (E. Blyth) 1846. -c (Arthur Strickland) 1850.

\section{SULA.}

Sula, Brisson, Orn. vi. p. 494 (1760).

2495. Sula bassana.

Pelecanus bassana, Linn. Syst. Nat. i. p. 217.

Sula bassana, Strickl. Ann. \& Mag. N. H. viii. p. 39 J̆: Dresser, B. Eur.

a Bass Rock, 3 August (H. E. Strickland) 1850.

\section{Sula cyanops.}

Dysporus cyanops, Sundev. Physiogr. Sällsk. Tidskr. i. p. 218, pl. 5: Ann. \& Mag. N. H. xix. p. 236.

Sula cyanops, Penrose, Ibis, 1879, p. 281.

a Ascension I. (A. Strickland) 1833.

\section{9\%. Sula piscator,}

Pelecanus piscator, Linn. Syst. Nat. i. p. 217.

Sula piscator, Gould, B. Austr. vii. p. 79: Legge, B. Ceylon, p. 1180.

a Ceylon, 19 October (A. Strickland) 1833. -b (Arthur Strickland) 1850.

2498. Sula leucogastra.

Le Fou, de Cayenne, D'Aub. Pl. Enl. 973, undè,

Pelecanus leucogaster, Bodd. Tabl. Pl. Enl. p. 57.

Sula leucogastra, Salv. Trans. Z. S. ix. p. 496: Penrose, Ibis, 1879, p. 281.

$a$ Prince's Island, March (A. Strickland) 1833. - b Ascension I. (A. Strickland) 1833.

\section{FAMILY.-PHALACROCORACIDA.}

\section{Phalacrocorax.}

Phalacrocorax, Brisson, Orn. vi. p. 511. (1760). 2499. Phalacrocorax carbo.

Pelecanus carbo, Linn. Syst. Nat. i. p. 216. 
Phalacrocorax carbo, Strickl. P. Z. S. 1836, p. 102 : Dresser, B. Eur.

a Constantinople, March (H. E. Strickland) 1836. - b Britain (N. C. Strickland) 1838.

\section{Phalacrocorax graculus.}

Pelecanus graculus, Linn. Syst. Nat. i. p. 217.

Phalacrocorax graculus, Dresser, B. Eur.

a Orkney, August (H. E. Strickland) 1837.

\section{Phalacrocorax capensis.}

Pelecanus capensis, Sparrm. Mus. Carls. pl. 61.

Graculus capensis, Schl. Mus. Pays-Bas, vi. Pelec. p. 19.

$a$ Cape of Good Hope, April (A. Strickland) 1831. -b (A. Strickland) 1850.

\section{Phalacrocorax sinensis.}

Phalacrocorax sinensis, Lath. Ind. Orn. Suppl. p. lxx.

Graculus sinensis, Jerd. B. Ind. iii. p. 862.

$a,-b$ Madras (T. C. Jerdon) 1845.

\section{Phalacrocorax dilophus.}

Pelecanus (Carbo) dilophus, Sw. Faun. Bor. Am. ii. p. 473.

Graculus dilophus, Baird, B. N. Am. p. 877.

$a$ (Thomas) 1842.

Agrees fairly with Hepburn's Californian examples.

2504. Phalacrocorax melanoleucus.

Hydrocorax melanoleucus, Vieill. N. Dict. D'Hist. N. viii. p. 88.

Phalacrocorax melanoleucus, Gould, B. Austr. vii. pl. 70.

$a$ New South Wales (McDonald) 1838.

\section{Phalacrocorax pygmæus.}

Phalacrocorax pygmceus, Pall. Zoogr. Rosso-As. ii. p. 300, pl. 74, f. 1 : Strickl. P. Z. S. 1836, p. 102: Dresser, B. Eur.

a Smyrna, 9 December (H. E. Strickland) 1835.

s. C. 
2506. Phalacrocorax javanicus.

Carbo javanicus, Horsf. Trans. L. S. xiii. p. 197.

Graculus javanicus, Jerd. B. Ind. iii. p. 863.

Carbo melanognathos, Brandt, Bull. Sc. Ac. Petersb. iii. p. 56.

$a$ (Stevens) 1844. - b Madras (T. C. Jerdon) 1845. -c, -d India (E. Blyth) 1846. -e (Gardner) 1847.

\section{FAMILY.-PLOTIDE.}

\section{Plotus.}

Plotus, Linnæus, Syst. Nat. i. p. 218 (1766).

250\%. Plotus melanogaster.

Plotus melanogaster, Pennant vel Forster, Zool. Ind. pl. 12: Jerd. B. Ind. iii. p. 865.

a Madras (T. C. Jerdon) 1845. -b India (E. Blyth) 1846. $\longrightarrow$ (A. Strickland) 1850. 


\section{ORDER IX.-HERODIONES.}

\section{FAMILY.-ARDEIDE.}

ARDEA.

Ardea, Linnæus, Syst. Nat. i. p. 233 (1766) (partim).

2508. Ardea cinerea.

Ardea cinerea, Linn. Syst. Nat. i. p. 236: Dresser, B. Eur.: Reich. J. f. Orn. 1877, p. 265.

$a$ Britain (W. Kirtland) 1843. - b Britain.

\section{Ardea melanocephala.}

Ardea melanocephala, Vig. \& Childr. Denham \& Clapperton's Narr. Disc. in N. \& Centr. Afr. App. p .201: Reich. J.f. Orn. 1877, p. 264.

a (Arthur Strickland) 1850.

An African species.

\section{Ardea pacifica.}

Ardea pacifica, Lath. Ind. Orn. Suppl. p. 1xv.: Gould, B. Austr. vi. p. 52: Reich. J. f. Orn. 1877, p. 263.

a Australia (W. Kirtland) 1846.

\section{Ardea purpurea.}

Ardea purpurea, Linn. Syst. Nat. i. p. 236: Dresser, B. Eur. : Reich. J. f. Orn. 1877, p. 266.

a Malacca (Havell) 1839. - b.

\section{Ardea herodias.}

Ardea herodias, Linn. Syst. Nat. i. p. 237.

Ardea lessoni, Reich. J. f. Orn. 1877, p. 266 (ex Wagler). 1851

$a$ (Arthur Strickland) 1850. - b Guatemala (J. Constancia) 


\section{Ardea alba.}

Ardea alba, Linn. Syst. Nat. i. p. 239: Dresser, B. Eur.: Reich. J. f. Orn. 1877, p. 272.

Ardea egretta, Strickl. P. Z. S. 1836, p. 101 (nec Linnæus).

a $\hat{\delta}$ Smyrna, 6 January (H. E. Strickland) 1836. -b New South Wales (McDonald) 1838. - c India ("Herodias alba," $E$. Blyth) 1846. - d, -e India (E. Blyth).-f.

\section{Ardea egretta.}

Ardea egretta, Gm. Syst. Nat. i. p. 629.

Herodias egretta, Baird, B. N. Am. p. 666.

Ardea egretta, subsp. galatea, Reich. J. f. Orn. 1877, p. 272 (ex Molina).

$a$ Guatemala ( $J$. Constancia) 18ə̌1. - $b$ N. America.

\section{Ardea intermedia.}

Ardea intermedia, Wagl. Isis, 1829, p. 659 : Reich. J. f. Orn. 1877, p. 273.

Herodias egrettoides, Jerd. B. Ind. iii. p. 745 (ex Temminck).

a Madras (T. C. Jerdon) 1845. - b India ("Herodias intermedia," E. Blyth) 1846. —c India (E. Blyth). —d (Arthur Strickland) 1850.

\section{Ardea garzetta.}

Ardea garzetta, Linn. Syst. Nat. i. p. 237 : Dresser, B. Eur. Herodias garzetta, Strickl. P. Z. S. 1842, p. 168: Jerd. B. Ind. iii. p. 746 .

Ardea nivea, Reich. J. f. Orn. 1877, p. 271 (ex Gmelin).

a Malacca (Thomas) 1842. -b India ("Herodias garzetta," E. Blyth) 1846 .

\section{Ardea candidissima.}

Ardea candidissima, Gm. Syst. Nat. i. p. 633: Reich. J. f. Orn. 1877, p. 273.

$a \mathrm{~N}$. America (Askew) 1836. - $b$.

\section{Ardea novæ-hollandiæ.}

Ardea novo-hollandice, Lath. Ind. Orn. p. 701: Gould, B. Austr. vi. pl. 53: Reich. J. f. Orn. p. 263.

$a$ New South Wales $\left(M^{c}\right.$ Donald) 1838. -b. 


\section{Ardea asha.}

Ardea asha, Sykes, P. Z. S. 1832, p. 157.

Demiegretta asha, Jerd. B. Ind. iii. p. 747.

Ardea jugularis, Reich. J. f. Orn. 1877, p. 261 (ex Forster).

$a$ New South Wales ( $M^{c}$ Donald) 1838. - $b$ Madras (T. $C$. Jerdon) 1845.

2520. Ardea cærulea.

Ardea ccerulea, Linn. Syst. Nat. i. p. 238: Reich. J. f. Orn. 1877, p. 263.

a N. America (de Rham) 1838. -b (Arthur Strickland) 1850. $-c,-d$ Guatemala (J. Constancia) 1851.

\section{Ardea ludoviciana.}

Ardea ludoviciana, Wils. Am. Orn. viii. p. 13, pl. 16, f. 1.

Demiegretta ludoviciana, Baird, B. N. Am. p. 663.

Ardea leucogaster, var. Reich. J. f. Orn. 1877, p. 269 (ex Boddaert).

a (Arthur Strickland) 1850.

A North-American species.

2522. Ardea coromanda.

Crabier, de la côte de Coromandel, D'Aub. Pl. Enl. 910, undè, Cancroma coromanda, Bodd. Tabl. P1. Enl. p. 54. Ardeola coromanda, Strickl. P. Z. S. 1850, p. 221. Ardea coromanda, Reich. J. f. Orn. 1877, p. 259.

$a$ India (Bt at Stevens's) 1843. - b (Bt at Stevens's) 1843. - c f Benzert, Tunis, April (L. Fraser) 1846. - $q$ \% Kordofan, 16 July (J. Petherick) 1848.

2523. Ardea ralloides.

Ardea ralloides, Scop. Ann. i. Hist. Nat. p. 8s: Reich. J. f. Orn. 1877, p. 256.

a Italy (Sign. Riccioli) 1831.

\section{Ardea leucoptera.}

Crabier de Malac, D’Aub. Pl. Enl. 911, unde, Ardea leucoptera, Bodd. Tabl. Pl. Enl. p. 54: Reich. J. f. Orn. 1877, p. 257.

a Malacca (Bt at Stevens's) 1843. -b. 


\section{Ardea grayi.}

Ardea grayi, Sykes, P. Z. S. 1832, p. 158: Reich. J. f. Orn. 1877, p. 257.

Ardeola leucoptera, Jerd. B. Ind. iii. p. 751 (nec Boddaert).

a Madras (T. C. Jerdon) 1845. - b India ("Ardeola malaccensis," E. Blyth) 1846. — c Benares (W. J. E. Boys) 1847. —d Ceylon (Capt. Pearson) 1849. - e (Arthur Strickland) 1850.

\section{Butorides.}

Butorides, Blyth, Cat. B. As. Soc. p. 281 (1849).

\section{Butorides virescens.}

Ardea virescens, Linn. Syst. Nat. i, p. 238 : Reich. J. f. Orn. 1877, p. 255.

Butorides virescens, Baird, B. N. Am. p. 676.

a America (Askew) 1834. -b (Arthur Strickland) 1850. -c Guatemala (J. Constancia) 1851.

\section{2\%. Butorides javanicus.}

Ardea javanica, Horsf. Trans, L. S. xiii. p. 190.

Butorides javanica, Jerd. B. Ind. iii. p. 752.

a Madagascar (E. Verreaux) 1833. - b Malacca (Askew) 1834. — c (Bt at Stevens's) 1845. — d, - e India ("Butorides javanicus," E. Blyth) 1846. - $f$.

\section{Butorides macrorhynchus.}

Ardetta macrorhyncha, Gould, P. Z. S. 1848, p. 39; B. Austr. vi. pl. 66 .

$a$ (Arthur Strickland) 1850. - b.

An Australian species.

\section{Ardetta.}

A ${ }^{2}$ detta, Gray, List Sp. B. B. M. Part iii. p. 83 (1844).

\section{Ardetta cinnamomea.}

Ardea cinnamomea, Gm. Syst. Nat. i. p. 643.

Ardetta cinnamomea, Jerd. B. Ind. iii. p. 755: Wald. Trans. Z. S. ix. p. 237.

Botaurus cinnamomeus, Reich. J. f. Orn. 1877, p. 245.

a Madras (T. C. Jerdon) 1845. - b Philippine Islands ( $J$. Gould) 1846. 


\section{Ardetta sinensis.}

Ardea sinensis, Gm. Syst. Nat. i. p. 642.

Ardetta sinensis, Jerd. B. Ind. iii. p. 755.

Botaurus sinensis, Reich. J. f. Orn. 1877, p. 244.

$a$ Madras (T. C. Jerdon) 1845. -b (Arthur Strickland) 1850.

\section{Ardetta exilis.}

Ardea exilis, Gm. Syst. Nat. i. p. 645.

Ardetta exilis, Baird, B. N. Am. p. 673.

Botaurus exilis, Reich. J. f. Orn. 1877, p. 244.

a f Carlisle, Pennsylvania, 25 May 1844 (S. E. Baird) 1849. -b Guatemala ( $J$. Constancia) 1850. $\longrightarrow$ Illinois (T. Dunn) 1851.

\section{Botaurus.}

Botaurus, Stephens in Shaw's Gen. Zool. xi. p. 592 (1819).

\section{Botaurus stellaris.}

Ardea stellaris, Linn. Syst. Nat. i. p. 239.

Botaurus stellaris, Strickl. P. Z. S. 1836, p. 101; 1850, p. 221: Dresser, B. Eur.: Reich. J. f. Orn. 1877, p. 248.

a Oxfordshire (H. E. Strickland) 1831. - b $\hat{\delta}$ Smyrna, 28 December (H. E. Strickland) 1835. — Kordofan (J. Petherick) 1848.

\section{Botaurus poeciloptilus.}

Ardea pociloptila, Wagl. Syst. Av. fol. 12, p. 9.

Botaurus poeciloptilus, Reich. J. f. Orn. 1877, p. 249.

Botaurus australis, Gould, B. Austr. vi. pl. 64.

a Australia (Bt at Birmingham) 1843.

\section{Botaurus lentiginosus.}

Ardea lentiginosa, Mont. Orn. Dict. Suppl.

Botaurus lentiginosus, Baird, B. N. Am. p. 674: Reich. J. f. Orn. 1877, p. 248.

$a \mathrm{~N}$. America (Koch) 1842. -b. 


\section{Botaurus pinnatus.}

Ardea pinnata, Wagl. Isis, 1829, p. 662.

Botaurus pinnatus, Scl. \& Salv. Ex. Orn. p. 181, pl. 91: Reich. J. f. Orn. 1877, p. 249.

$a$ (Arthur Stricliland) 1850.

A species of wide range in South America.

\section{Tigrisoma.}

Tigrisoma, Sw. Classif. B. ii. p. 354 (1837).

\section{Tigrisoma brasiliense.}

Ardea brasiliensis, Linn. Syst. Nat. i. p. 239.

Tigrisoma brasiliense, Strickl. Ann. \& Mag. N. H. viii. p. 545 : Scl. \& Salv. Ex. Orn. p. 184.

Botaurus brasiliensis, Reich. J. f. Orn. 1877, p. 250. $a,-b$ (Arthur Strickland) 1850.

A species of wide range in South America.

\section{Tigrisoma cabanisi.}

Tigrisoma cabanisi, Heine, J. f. Orn. 1859, p. 107 : Scl. \& Salv. Ex. Orn. p. 95, pl. 48.

Botaurus cabanisi, Reich. J. f. Orn. 1877, p. 251.

a Panama Bay (Capt. líellett \& Lieut. Wood) 1850.

\section{Zebrilus.}

Zebrilus, Bonaparte, Consp. Av. ii. p. 138 (1857).

\section{Zebrilus pumilus.}

Crabier, des Philippines, D'Aub. Pl. Enl. 898, undè, Ardea pumila, Bodd. Tabl. Pl. Enl. p. 54.

Botaurus pumilus, Reich. J. f. Orn. 1877, p. 249.

Ardea philippensis, Gm. Syst. Nat. i. p. 644.

a (Bt at Birmingham) 1853.

A skin of Cayenne make. 


\section{NyCticorax.}

Nycticorax, Stephens in Shaw's Gen. Zool. xi. p. 608 (1819). 2539. Nycticorax griseus.

Ardea nycticorax, Linn. Syst. Nat. i. p. 235.

Ardea grisea, Linn. Syst. Nat. i. p. 239.

Nycticorax griseus, Strickl. Ann. \& Mag. N. H. viii. p. 545: Jerd. B. Ind. iii. p. 758: Dresser, B. Eur.: Reich. J. f. Orn. 1877, p. 237.

a Zante, May (H. E. Strickland) 1834. - 6 Benares (W. J. E. Boys) 1847 .

\section{Nycticorax gardeni.}

Ardea gardeni, Gm. Syst. Nat. i. p. 645.

Nyctiardea gardeni, Baird, B. N. Am. p. 678.

a Illinois (T. Dunn) 1851.

\section{Nycticorax obscurus.}

Nycticorax obscurus, Bp. Consp. ii. p. 141 (ex Licht.) : Schl. Mus. d. Pays-Bas, v. Ardece, p. 58.

a Chili (T. C. Eyton) 1845.

\section{Nycticorax caledonicus.}

Ardea caledonica, Gm. Syst. Nat. i. p. 626.

Nycticorax caledonicus, Gould, B. Austr. vi. pl. 63: Strickl. Ann. \& Mag. N. H. xi. p. 338: Reich. J. f. Orn. 1877, p. 238.

a Australia (Warwick Museum) 18t1. - b Australia (Bt at Birmingham) 1843.

\section{Nycticorax pileatus.}

Heron blanc, hupé de Cayenne, D'Aub. Pl. Enl. 907, undè, Ardea pileata, Bodd. Tabl. Pl. Enl. p. 54.

Nycticorax pileatus, Reich. J. f. Orn. 1877, p. 240.

$a$ (Gardner) 1846.

A widely ranging South-American species.

\section{Nyctherodius.}

Nyctherodius, Reichenbach, Syst. Av. p. xvi. (1852-3).

\section{Nyctherodius violaceus.}

Ardea violacea, Linn. Syst. Nat. i. p. 238. 
Nyctherodius violaceus, Baird, B. N. Am. p. 679.

Nycticorax cayennensis, Reich. J. f. Orn. 1877, p. 239 (ex Gmelin).

$a$ Mexico (T. Mann) 1844. - b South America (Osman) 1846. - c Jamaica (J. Gould) 1846. -d Guatemala (J. Constancia) 1851.

\section{Cancroma.}

Cancroma, Linnæus, Syst. Nat. i. p. 233 (1766).

2545. Cancroma cochlearia.

Cancroma cochlearia, Linn. Syst. Nat. i. p. 233.

Nycticorax cancrophagus, Reich. J. f. Orn. 1877, p. 236 (ex Linnæus).

$a$ South America (Bt at Birmingham) 1844. - b (Arthur Strickland) 1850.

\section{FAMILY.-SCOPIDAE。}

Scopus.

Scopus, Brisson, Orn. v. p. 503 (1760).

2546. Scopus umbretta.

Scopus umbretta, Gm. Syst. Nat. i. p. 618: Reich. J. f. Orn. 1877, p. 231.

$a$ (Kirtland) 1838. - b.

A South-African species.

\section{FAMILY.-CICONIIDA.}

\section{Ciconia.}

Ciconia, Brisson, Orn. v. p. 361 (1760).

254\%. Ciconia alba.

Ardea ciconia, Linn. Syst. Nat. i. p. 235.

Ciconia alba, Bechst. Gem. Naturg. Deutschl. iv. p. 82: Strickl. P. Z. S. 1836, p. 101; 1850, p. 221: Reich. J. f. Orn. 1877, p. 170.

a Smyrna, May (H. E. Strickland) 1836. 


\section{Ciconia episcopus.}

Heron, de la côte de Coromandel, D'Aub. Pl. Enl. 906, undè, Ardea episcopus, Bodd. Tabl. Pl. Enl. p. 54.

Ciconia leucocephala, Jerd. B. Ind. iii. p. 737 (ex Gmelin). $a$ Madras ("Ciconia leucocephala," E. Blyth) 1845. —b.

\section{MyCTERIA.}

Mycteria, Linnæus, Syst. Nat. i. p. 232 (1766).

\section{Mycteria australis.}

Mycteria australis, Shaw, Trans. L. S. v. p. 32: Strickl. Ann. \& Mag. N. H. xiii. p. 39; xiv. p. 119: Jerd. B. Ind. iii. p. 734. $a$ India (E. Blyth) 1846.

\section{Anaśtonus.}

Anastomus, Bonnaterre, Enc. Méth. Ornith. p. xciii. (1790).

\section{Anastomus oscitans.}

Le Bec ounert de Pondichery, D’Aub. P1. Enl. 932, undè, Ardea oscitans, Bodd. Tabl. Pl. Enl. p. 55.

Anastomus oscitans, Jerd. B. Ind. iii. p. 765.

a India ("Anastomus oscitans, adult," E. Blyth) 1846. - India ("Anastomus oscitans, young," E. Blyth) 1846.

\section{Leptoptilus.}

Leptoptilus, Lesson, Traité d'Orn. p. 583 (1831).

\section{Leptoptilus dubius.}

Ardea dubia, Gm. Syst. Nat. i. p. 624 (partim).

Leptoptilus dubius, Reich. J. f. Orn. 1877, p. 165.

Leptoptilus argala, Strickl. Ann. \& Mag. N. H. vii. p. 38: Jerd. B. Ind. iii. p. 730 (ex Linnæus!).

$a$ S. India (T. C. Jerdon) 1850.

\section{Tantalus.}

Tantalus, Linnæus, Syst. Nat. i. p. 240 (1766) (partim).

\section{Tantalus leucocephalus.}

Tantalus leucocephalus, Gm. Syst. Nat. i. p. 649: Jerd. B. Ind. iii. p. 761: Reich. J. f. Orn. 1877, p. 162. 
$a$ (Arthur Strickland) 1850. - b.

A widely ranging Asiatic species.

\section{Tantalus ibis.}

Tantalus ibis, Linn. Syst. Nat. i. p. 241: Reich. J. f. Orn. 1877, p. 162.

$a$ (Arthur Strickland) 1850.

A widely ranging African species.

\section{FAMILY.-PLATALEIDE.}

\section{SUBFAMILY.-IBIDIN $Æ$.}

IBIS.

Ibis, Lacépède, Mém. de l'Inst. Sc. Math. et Phys. iii. p. 518 (1801) : Savigny, Descr. de l'Egypte, xxiii. p. 392 (1828).

\section{Ibis æthiopica.}

Tantalus athiopicus, Lath. Ind. Orn. p. 706.

Ibis cethiopica, Strickl. P. Z. S. 1850, p. 221: Elliot, P. Z. S. 1877, p. 485 (partin).

$a$ (Arthur Strickland) 1850.

An African species.

\section{Ibis melanocephalus.}

Tantalus melanocephalus, Lath. Ind. Orn. p. 709.

Threskiornis melanocephalus, Jerd. B. Ind. iii. p. 768.

Ibis melanocephahus, Strickl. Ann. \& Mag. N. H. xiii. p. 39 : Elliot, P. Z. S. 1877, p. 488.

$a$ India ("Threskiornis melanocephala," $E$. Blyth) 1845. -b India ("Threskiornis melanocephala," E. Blyth) 1846.

\section{Carphibis.}

Carphibis, Reichenbach, Syst. Av. p. xiv. (1852-3).

\section{Carphibis spinicollis.}

Ibis spinicollis, Jameson, Mem. Wern. N. H. Soc. vii. p. 485; Edinb. New Phil. Journ. xix. p. 213.

Geronticus spinicollis, Gould, B. Austr. vi. pl. 45.

Carphibis spinicollis, Elliot, P. Z. S. 1877, p. 491. $a$ New South Wales (McDonald) 1838. 


\section{INOCOTIS.}

Inocotis, Reichenbach, Syst. Av. p. xiv. (1852-3).

\section{Inocotis papillosus.}

Ibis papillosa, Temm. Pl. Col. 304.

Geronticus papillosus, Jerd. B. Ind. iii. p. 769.

Inocotis papillosus, Elliot, P. Z. S. 1877, p. 492.

$a$ S. India (T. C. Jerdon) 1850.

\section{Theristicus.}

Theristicus, Wagler, Isis, 1832, p. 1231.

\section{Theristicus caudatus.}

Courly à col blanc, de Cayenne, D'Aub. Pl. Enl. 976, undè, Scolopax caudatus, Bodd. Tabl. Pl. Enl. p. 57.

Theristicus caudatus, Elliot, P. Z. S. 1877, p. 498.

Theristicus melanopis, auctt. plur. (ex Gmelin).

a Chili (Capt. Brown) 1842. -b (Arthur Strickland) 1850. $-c$.

\section{HAGEDASHIA.}

Hagedashia, Bonaparte, Consp. Av. ii. p. 152 (1857).

\section{Hagedashia caffrensis.}

Ibis caffrensis, Licht. Cat. Hamb.

Hagedashia caffrensis, Gurney, in And. B. Damaral. p. 298.

Hagedashia chalcoptera, Elliot, P. Z. S. 1877, p. 500 (ex Vieillot).

$a$ (Arthur Strickland) 1850.

An African species of wide range.

\section{HARPIPRION.}

Harpiprion, Wagler, Isis, 1832, p. 1232.

\section{Harpiprion cayennensis.}

Courly verd, de Cayenne, D'Aub. P1. Enl. 820, undè, Tantalus cayennensis, Gm. Syst. Nat. i. p. 652.

Harpiprion cayennensis, Elliot, P. Z. S. 1877, p. 502. a (Arthur Stricleland) 1850 .

A species of wide range in the northern parts of South America. 


\section{Plegadis.}

Plegadis, Kaup, Skizz. Entw. Gesch. p. 82 (1829). 2561. Plegadis falcinellus.

Tantalus falcinellus, Linn. Syst. Nat. i. p. 241: Strickl. Ann. \& Mag. N. H. vii. p. 38.

Falcinellus igneus, Elliot, P. Z. S. 1877, p. 503 (ex Gmelin). $a$ (Askew) 1840.

A species of very wide range extending over the Eastern Hemisphere and North America.

\section{Plegadis ridgwayi.}

Falcinellus ridgwayi, Allen, Bull. Mus. Comp. Zool. Cambr. iii. p. 355: Eiliot, P. Z. S. 1877, p. 506.

a Chili (Capt. Brown) 1842.

\section{Eudocimus.}

Eudocimus, Wagler, Isis, 1832, p. 1232.

2563. Eudocimus ruber.

Tantalus ruber, Linn. Syst. Nat. i. p. 241.

Eudocimus ruber, Elliot, P. Z. S. 1877, p. 509.

a (Arthur Strickland) 1850.

A Guiana species.

\section{SUBFAMILY.-PLATALEIN $\nexists$.}

\section{Platalea.}

Platalea, Linn. Syst. Nat. i. p. 231 (1766) (partim).

\section{Platalea leucorodia.}

Platalea leucorodia, Linn. l. s. c.: Reich. J. f. Orn. 1877, p. 159.

Platea leucorodia, Dresser, B. Eur.

a Morocco (J. G. Kinberg) 1844.

2565. Platalea cristata.

Platalea cristata, Scop. Del. Faun. et Fl. Ins. ii. p. 92: Reich. J. f. Orn. 1877, p. 158. 
Platalea tenuirostris, Temm. Man. d'Orn. p. ciii. (1820). $a,-b$ (Arthur Strickland) 1850.

An African species.

2566. Platalea ajaja.

Platalea ajaja, Linn. Syst. Nat. i. p. 231.

Platalea rosea, Reich. J. f. Orn. 1877, p. 157 (ex Brisson). a Chili (Capt. Brown) 1842.

\section{FAMILY.-PHENICOPTERID $\mathbb{E}^{3}$.}

\section{Phenicopterus.}

Phœnicopterus, Linnæus, Syst. Nat. i. p. 230 (1766). 256\%. Phœnicopterus ignipalliatus.

Phœnicopterus ignipalliatus, Geoffr. \& d'Orb. Mag. Zool. 1832, Cl. ii. pl. 2.

Phœenicopterus chilensis, Reich. J. f. Orn. 1877, p. 228 (ex Molina).

a Chili (Capt. Brown) 1842.

1 Strickl. Ann. \& Mag. N. H. vii. p. 39. 


\section{ORDER X.-ANSERES.}

\section{FAMILY.-ANATID $A$.}

\section{SUBFAMILY.-ANSERANATIN E.}

\section{ANSERANAS.}

Anseranas, Lesson, Man. d'Orn. ii. p. 418 (1828) as a subgenus.

2568. Anseranas melanoleucus.

Anas melanoleucus, Lath. Ind. Orn. Suppl. p. Ixix.

Anseranas melanoleucus, Gould, B. Austr. vii. pl. 2.

a Australia (Arthur Strickland) 1850.

\section{SUBFAMILY.-ANSERIN Æ.}

Anser.

Anser, Brisson, Orn, vi. p. 261 (1760).

2569. Anser segetum.

Anas segetum, Gm. Syst. Nat. i. p. 512.

Anser segetum, Dresser, B. Eur.

a Worcestershire, January (H. E. Strickland) 1838.

\section{5\%0. Anser albifrons.}

Branta albifrons, Scop. Ann. I. Hist. Nat. p. 69.

Anser albifrons, Strickl. P. Z. S. 1842, p. 167 : Dresser, B. Eur.

a $q$ Oxfordshire, December (H. E. Strickland) 1846.

\section{5\%1. Anser indicus.}

Anas indica, Lath. Ind. Orn. p. 839.

Anser indicus, Jerd. B. Ind. iii. p. 782.

a India ("Anser indicus," E. Blyth) 1846. 
2572. Anser hyperboreus.

Anser hyperboreus, Pall. Spicil. Zool. vi. p. 25: Baird, B. N. Am. p. 760.

a.

A North-American species.

\section{Anser cærulescens.}

A nas ccerulescens, Linn. Syst. Nat. i. p. 198. Anser corrulescens, Cones, Birds of the N. W. p. 553.

$a \mathrm{~N}$. W. America (J. F. Brandt) 1844.

\section{Bernicla.}

Bernicla, Stephens, in Shaw's Gen. Zool. xii. pt. 2, p. 45 (1824).

\section{Bernicla leucopsis.}

Anser leucopsis, Bechst. Naturg. Deutschl. iv. p. 921.

Bernicla leucopsis, Dresser, B. Eur. vi. p. 397, pl. 415.

a Orkney (Forbes) 1837.

\section{5\%5. Bernicla brenta.}

Anser brenta, Pall. Zoogr. Rosso-As. ii. p. 229.

Bernicla brenta, Dresser, B. Eur. vi. p. 389, pl. 415.

$a$ Oxfordshire (H. E. Strickland) 1831.

2576. Bernicla jubata.

Anas jubata, Lath. Ind. Orn. Suppl. p. 1xix.

Bernicla jubata, Gould, B. Austr. vii. pl. 3.

$a \hat{\delta},-b \neq$ New South Wales (McDonald) 1838. - c.

\section{5\%7. Bernicla sandvicensis.}

Bernicla sandvicensis, Vig. P. Z. S. 1833, p. 65: Jard. \& Selby, Ill. Orn. iv. pl. 8.

a "Domestic" (T. Goatley) 1847.

A species peculiar to the Sandwich Islands.

\section{NetTAPUS.}

Nettapus, Brandt, Descr. et Icon. An. Ross. Nov. p. 5 (1836). 2578. Nettapus coromandelianus.

Anas coromandeliana, Gm. Syst. Nat. i. p. 522.

S. C. 
Nettapus coromandelianus, Jerd. B. Ind. ii. p. 786. 1846.

a Madras (T. C. Jerdon) 1845. -b, -c India (E. Blyth)

\section{Nettapus albipennis.}

Nettapus albipennis, Gould, B. Austr. vii. pl. 5.

$a$ (Arthur Strickland) 1850. - b New Holland (Bt at Liverpool) 1852.

Rather larger than the Indian bird but very doubtfully distinct.

\section{SUBFAMILY.-ANATIN $\approx$.}

\section{Dendrocygna.}

Dendrocygna, Swainson, Classif. B. ii. p. 365 (1837).

2580. Dendrocygna viduata.

Anas viduata, Linn. Syst. Nat. i. p. 205.

Dendrocygna viduata, Strickl. P. Z. S. 1850, p. 221 : Scl. \& Salv. P. Z. S. 1876 , p. 376.

a $\hat{\delta}$ Kordofan, 15 September (J. Petherick) 1848. - b.

\section{Dendrocygna autumnalis.}

Anas autumnalis, Linn. Syst. Nat. i. p. 205.

Dendrocygna autumnalis, Scl. \& Salv. P. Z. S. 1876, p. 373. a Guatemala (J. Constancia) 1851.

\section{Dendrocygna discolor.}

Dendrocygna discolor, Scl. \& Salv. Nomencl. Av. Neotr. p. 161 ; P. Z. S. 1876 , p. 375.

a (Arthur Strickland) 1850.

The South-American representative of the preceding species.

2583. Dendrocygna arborea.

Anas arborea, Linn. Syst. Nat. i. p. 207.

Dendrocygna arborea, Gosse, B. Jam. p. 395: Scl. \& Salv. P. Z. S. 1876 , p. 375.

a Jamaica (P. H. Gosse) 1848. 


\section{Dendrocygna fulva.}

Anas fulva, Gm. Syst. Nat. i. p. 530.

Dendrocygna fulva, Scl. \& Salv. P. Z. S. 1876, p. 372.

Dendrocygna major, Jerd. B. Ind. ii. p. 790.

a Madras (T. C. Jerdon) 1845. - b India (E. Blyth) 1846.

\section{Dendrocygna arcuata.}

Anas javanica, Horsf. Trans. Linn. Soc. xiii. p. 199.

Anas arcuata, Horsf. Zool. Res. Java.

Dendrocygna arcuata, Scl. P. Z. S. 1864, p. 300.

Dendrocygna awsuree, Jerd. B. Ind. ii. p. 789 (ex Sykes).

a Madras (T. C. Jerdon) 1845. - - India ("Dendrocygna arcuata," E. Blyth) 1846. —c India (E. Blyth) 1846. —d.

\section{Dendrocygna vagans.}

Dendrocygna vagans, Fraser, Zool. Typ. pl. 68 (ex Eyton, MS.): Wald. Trans. Z. S. ix. p. 242.

a Luzon, 14 June (J. Cuming) 1839.

\section{SARCIDIORNIS.}

Sarcidiornis, Eyton, Mon. Anat. p. 20 (1838).

258\%. Sarcidiornis melanonotus.

Oye de la côte de Coromandel, D'Aub. Pl. Enl. 937, undè

Anas melanonotos, Forst. aut Penn. Zool. Ind. p. 42, pl. 11.

Sarcidiornis melanonotus, Jerd. B. Ind. ii. p. 785 : Scl. P. Z. S. 1876, p. 695 , pl. 67.

a Madras (E. Blyth) 1845. -b India ("Sarcidiornis regius," E. Blyth) 1846 .

TADORNa.

Tadorna, Fleming, Phil. Zool. ii. p. 260 (1822). 2588. Tadorna cornuta.

Anas tadorna, Linn. Syst. Nat. i. p. 195.

Tadorna vulpanser, Strickl. P. Z. S. 1836, p. 102 : Scl. P. Z. S. 1864, p. 189 (ex Fleming).

Tadorna cornuta, Dresser, B. Eur. vi. p. 451, pl. 420 (ex S. G. Gmelin).

a ô Orkney (E. Forbes) 1837. 


\section{Tadorna radjah.}

Anas radjah, Less. Voy. Coq. Zool. p. 602, pl. 49.

Tadorna radjah, Gould, B. Austr. vii. pl. 8: Scl. P. Z. S. 1864, p. 190.

a Australia (Worcester Nat. Hist. Soc.).

\section{Tadorna casarca.}

Anas casarca, Linn. Syst. Nat. iii. App. p. 224.

Tadorna casarca, Dresser, B. Eur. vi. p. 461, pl. 421.

Anas rutila, Pall. Nov. Comm. Petrop. xiv. pt. 1, p. 579, pl. 22 , f. 1.

Tadorna rutila, Strickl. P. Z. S. 1836, p. 102: Scl. P. Z. S. 1864, p. 190.

a W. Siberia (J. F. Brandt) 1844. -b, -c India ("Casarca rutila," E. Blyth) 1846.

2591. Tadorna cana.

Anas cana, Gm. Syst. Nat. i. p. 510.

Tadorna cana, Scl. P. Z. S. 1864, p. 190.

a Cape of Good Hope (Mrs Van der Kemp) 1813.

\section{Arx.}

Aix, Boie, Isis, 1828, p. 329.

\section{Aix sponsa.}

Anas sponsa, Linn. Syst. Nat. i. p. 207.

Aix sponsa, Scl. \& Salv. P. Z. S. 1876, p. 397.

a Canada (Barnes) 1848.

\section{MARECA.}

Mareca, Stephens, in Shaw's Gen. Zool. xii. pt. 2, p. 130 (1824).

\section{Mareca penelope.}

Anas penelope, Linn. Syst. Nat. i. p. 202.

Mareca penelope, Strickl. P. Z. S. 1836, p. 102; 1842, p. 168: Jerd. B. Ind. ii. p. 804: Dresser, B. Eur. vi. p. 541, pls. 432, 433.

$a$ (N. C. Strickland) 1838. - b Rohilkund (W. J. E. Boys) 1847. 
2594. Mareca americana.

Anas americana, Gm. Syst. Nat. i. p. 526.

Mareca americana, Scl. \& Salv. P. Z. S. 1876, p. 394.

a f Carlisle, Pennsylvania, 10 April, 1841 (S. F. Baird) 1847.

- $b$ Canada (Barnes) 1848.

\section{DAFILA.}

Dafila, Swainson, Classif. B. ii. p. 367 (1837).

\section{Dafila acuta,}

Anas acuta, Linn. Syst. Nat. i. p. 202.

Querquedula acuta, Strickl. P. Z. S. 1836, p. 102.

Dafila acuta, Scl. \& Salv. P. Z. S. 1876, p. 391: Dresser, B. Eur. vi. p. 531, pls. 430, 431.

a Rohilkund (W. J. E. Boys) 1847.

\section{Dafila exythrorhyncha.}

Anas erythrorhyncha, Gm. Syst. Nat. i. p. 517.

Dafila erythrorhyncha, Scl. P. Z. S. 1880, p. 515.

a Cape of Good Hope (Arthur Strickland) 1850.

\section{9\%. Dafila spinicauda.}

Anas spinicauda, Vieill. N. Dict. d'Hist. N. v. p. 135.

Dafila spinicauda, Scl. \& Salv. P. Z. S. 1876, p. 392.

$a$ Chili (T. C. Eyton) 1846. -b (Arthur. Strickland) 1850.

2598. Dafila bahamensis.

Anas bahamensis, Linn. Syst. Nat. i. p. 199.

Dafila bahamensis, Scl. \& Salv. P. Z. S. 1876, p. 393.

a (Gardner) 1846.

A species of wide range in South America.

\section{Anas.}

Anas, Linnæus, Syst. Nat. i. p. 194 (1766) (partim).

\section{Anas boscas.}

Anas boschas, Linn. l. s. c. p. 205: Strickl. P. Z. S. 1836 , p. 102 : Scl. \& Salv. P. Z. S. 1876, p. 380 : Dresser, B. Eur. vi. p. 469 , pl. 422 .

a $\hat{\delta}$ Oxfordshire, 6 January (N. C. Strickland) 1832. - b ई Orknoy (E. Forbes) 1837. —c Canada (Barnes) 1848. 
2600. Anas obscura.

Anas obscura, Gm. Syst. Nat. i. p. 541: Scl. \& Salv. P. Z. S. 1876 , p. 380.

a $\hat{\jmath}$ Massachusetts (J. G. Kinberg) 1845.

\section{Anas pocilorhyncha.}

Anas pocilorhyncha, Forst. aut Penn. Ind. Zool. p. 23, pl. 13, f. 1: Jerd. B. Ind. ii。 p. 799.

a Madras ("Anas poecilorhyncha," E. Blyth) 1845.

\section{Anas capensis.}

Anas capensis, Gm. Syst. Nat. i. p. 527.

Querquedula capensis, Smith, Ill. Zool. S. Afr. pl. 98.

a Muysenberg, Cape of Good Hope, June (Arthur Strickland) 1833. -b (Arthur Strickland) 1850.

\section{Anas castanea.}

Marea castanea, Eyton, Mon. Anat. p. 119, pl. 22.

Anas punctata, Gould, B. Austr. vii. pl. 11.

a Australia ( $W$. Kirtland) 1846. - b.

\section{MarmaronetTa.}

Marmaronetta, Reichenbach, Syst. Av. p. ix. (1852). 2604. Marmaronetta angustirostris.

Anas angustirostris, Ménétr. Cat. Rais. p. 58: Dresser, B. Eur. vi. p. 479, pl. 423.

Marmaronetta angustirostris, Scl. P. Z. S. 1880, p. 520.

a Tangier (Favier) 1848.

\section{Chaulelasmus.}

Chaulelasmus, Gray, List. Gen. B. p. 74 (1840).

\section{Chaulelasmus streperus.}

Anas strepera, Linn. Syst. Nat. i. p. 200 : Dresser, B. Eur. vi. p. 487 , pl. 424 .

Chaulelasmus streperus, Scl. P. Z. S. 1880, p. 519.

a ô Britain, November (II. E. Strickland) 1836. 


\section{RHODONESSA.}

Rhodonessa, Reichenbach, Syst. Av. p. ix. pl. 85 (185̌2). 2606. Rhodonessa caryophyllacea.

Anas caryophyllacea, Lath. Ind. Orn. p. 866 : Jerd. B. Ind. ii. p. 800 .

Rhodonessa caryophyllacea, Scl. P. Z. S. 1880, p. 520.

a India ("Anas caryoplyyllacea," E. Blyth) 1846.

\section{QUERQUEDULA.}

Querquedula, Stephens in Shaw's Gen. Zool. xii. pt. 2, p. 142 (1824).

\section{0\%. Querquedula circia.}

Anas circia, Linn. Syst. Nat. i. p. 204.

Querquedula circia, Strickl. Ann. \& Mag. N. H. viii. p. 545 : Jerd. B. Ind. ii. p. 807: Dresser, B. Eur. vi. p. 513, pl. 427.

a ô Britain (N.C. Strickland) 1838. - b Deysalpore (W. J. E. Boys) 1847.

\section{Querquedula discors.}

Anas discors, Linn. Syst. Nat. i. p. 205.

Querquedula discors, Scl. \& Salv. P. Z. S. 1876, p. 383.

a, -b Jamaica (J. Gould) 1846. —c Canada (Barnes) 1848. -d Jamaica (Arthur Strickland) 1850. —e Guatemala (J. Constancia) 1851.

\section{Querquedula crecca.}

Anas crecca, Linn. Syst. Nat. i. p. 204.

Querquedula crecca, Strickl. P. Z. S. 1836, p. 102: Jerd. B. Ind. ii. p. 806 : Dresser, B. Eur. vi. p. 507, pl. 426.

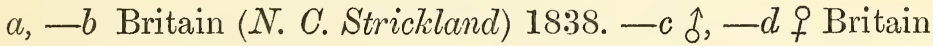
(N. C. Strickland) 1838. - e Nepal (B. H. Hodgson, 681) 1845. - $f$ o Malwa (W. J. E. Boys) 1847.

\section{Querquedula carolinensis.}

Anas carolinensis, Gm. Syst. Nat. i. p. 533.

Querquedula carolinensis, Scl. \& Salv. P. Z. S. 1876, p. 385. a Canada (Barnes) 1848. 
2611. Querquedula formosa.

Anas formosa, Georgi, Reise, i. p. 168.

Querquedula formosa, Strickl. P. Z. S. 1842, p. 168: Dresser, B. Eur. vi. p. 521, pl. 428 .

$a,-b$ Siberia (J. F. Brandt) 1846. —c (Arthur Strickland) 1850.

\section{Querquedula cyanoptera.}

Anas cyanoptera, Vieill. N. Dict. d'Hist. N. v. p. 104. Querquedula cyanoptera, Scl. \& Salv. P. Z. S. 1876, p. 384. a (Arthur Strickland) 1850.

An American species of wide range.

2613. Querquedula brasiliensis.

Anas brasiliensis, Gm. Syst. Nat. i. p. 517.

Querquedula brasiliensis, Scl. \& Salv. P. Z. S. 1876, p. 390. $a$ (Gardner) 1846. -b (Arthur Strickland) 1850.

A South-American species.

\section{Spatula.}

Spatula, Boie, Isis, 1822, p. 564.

\section{Spatula clypeata.}

Anas clypeata, Linn. Syst. Nat. i. p. 200.

Rhynchaspis clypeata, Strickl. P. Z. S. 1836, p. 102.

Spatula clypeata, Scl. \& Salv. P. Z. S. 1876, p. 396 : Dresser, B. Eur. vi. p. 497 , pl. 425.

a Oxford, 6 January 1832 (N. C. Strickland) 1838. -b f., -c f̂ Smyrna, 8 January (H. E. Strickland) 1836. -d Illinois (T. Dunn) 1851.

\section{Spatula rhynchotis.}

Anas rhynchotis, Lath. Ind. Orn. Suppl. p. 1xx. Spatula rhynchotis, Gould, B. Austr. vii. pl. 12. a Australia (Arthur Strickland) 1850.

\section{Spatula platalea.}

Anas platalea, Vieill. N. Dict. d'Hist. N. v. p. 157. Spatula platalea, Scl. \& Salv. P. Z. S. 1876, p. 396. a (T. C. Eyton) 1846.

A species of the southern parts of South America. 


\section{Malacorhynchus.}

Malacorhynchus, Swainson, Journ. Roy. Inst. ii. p. 18 (1831).

\section{Malacorhynchus membranaceus.}

Anas membranacea, Lath. Ind. Orn. Suppl. p. Ixix.

Malacorhynchus membranaceus, Gould, B. Austr. vii. pl. 13.

a New South Wales (McDonald) 1838. - b (Arthur Strickland) 1850 .

\section{SUBFAMILY.-FULIGULIN $A$.}

\section{Fuligula.}

Fuligula, Stephens in Shaw's Gen. Zool. xii. pt. 2, p. 187 (1824).

\section{Fuligula rufina.}

Anas rufina, Pall. Reise, ii. p. 713.

Branta rufina, Jerd. B. Ind. ii. p. 811.

Fuligula rufina, Dresser, B. Eur. vi. p. 559, pl. 435.

a (Bt at_Stevens's) 1844. - b India ("Fuligula rufina," E. Blyth) 1846.

\section{Fuligula affinis.}

Fuligula affinis, Eyton, Mon. Anat. p. 157: Scl. \& Salv. P.Z.S. 1876, p. 399.

a ईิ Carlisle, Pennsylvania, April, 1841 (S. F. Baird) 1847.

\section{Fuligula collaris.}

Anas collaris, Donov. Brit. B. vi. pl. 147.

Fuligula collaris, Scl. \& Salv. P. Z. S. 1876, p. 400.

Fuligula rufitorques, Gosse, B. Jam. p. 408.

a Canada (Barnes) 1848.

\section{Fuligula ferina.}

Anas ferina, Linn. Syst. Nat. i. p. 203.

Fuligula ferina, Strickl. P. Z. S. 1836, p. 102: Dresser, B. Eur. vi. p. 551, pl. 434.

a ô Britain (W. Kirtland) 1832. 


\section{Fuligula nyroca.}

Anas nyroca, Güld. Nov. Comm. Petr. xiv. pt. 1, p. 403.

Aythya nyroca, Jerd. B. Ind. ii. p. 813.

Nyroca ferruginea, Dresser, B. Eur. vi. p. 581, pl. 438 (ex Gmelin).

a Holland, March (H. E. Strickland) 1844. -b Rohilkund (W. J. E. Boys) 1847.

\section{Fuligula australis.}

Nyroca australis, Gould, B. Austr. vii. pl. 16.

$a$ New South Wales ( $M^{c}$ Donald) 1838. - b.

\section{Clangula.}

Clangula, Fleming, Phil. Zool. ii. p. 260 (1822).

\section{Clangula glaucion.}

Anas glaucion, Linn. Syst. Nat. i. p. 201.

Clangula glaucion, Dresser, B. Eur. vi. p. 595, pl. 440.

Clangula vulgaris, Strickl. P. Z. S. 1836, p. 102 (ex Fleming). a ô Britain (H. E. Strickland) 1830.

2625. Clangula islandica.

Anas islandica, Gm. Syst. Nat. i. p. 541.

Clangula islandica, Dresser, B. Eur. vi. p. 603, pl. 441.

Clangula barrovii, Sw. Faun. Bor. Am. Birds, p. 456, pl. 70. a Iceland (Thomas) 1842.

2626. Clangula albeola.

Anas albeola, Linn. Syst. Nat. i. p. 199.

Bucephala albeola, Baird, B. N. Am. p. 797.

Clangula albeola, Dresser, B. Eur. vi. p. 589, pl. 439.

$a \hat{\delta},-b q$ Kodiak, N. W. America (Wosnessensky) 1846.

\section{Cosmonetta.}

Cosmonetta, Kaup, Skizz. Ent. gesch. p. 196 (1829).

262\%. Cosmonetta histrionica.

Anas histrionica, Linn. Syst. Nat. i. p. 204.

Cosmonetta histrionica, Dresser, B. Eur. vi. p. 609, pl. 422.

a Iceland (Thomas) 1842. 
HARELDA.

Harelda, Stephens, in Shaw's Gen. Zool. xii. pt. 2, p. 174 (1824).

2628. Harelda glacialis.

Anas glacialis, Linn. Syst. Nat. i. p. 203.

Harelda glacialis, Dresser, B. Eur. vi. p. 617, pls. 443, 444 .

$a$ Orkney (E. Forbes) 1837. -b Britain (N. C. Strickland) 1838. -c (N. C. Strickland) 1838. —d Iceland (Thomas) 1842.

\section{HeniconetTa.}

Eniconetta, Gray, List Gen. B. p. 95 (1841).

2629. Heniconetta stelleri.

Anas stelleri, Pall. Spic. Zool. vi. p. 35, pl. 5.

Heniconetta stelleri, Gould, B. Gt. Brit. v. pl. 25.

Somateria stelleri, Dresser, B. Eur. vi. p. 649, pl. 447.

a (J. G. Kinberg) 1845.

A species of the northern parts of Europe and America.

\section{CAMptol}

Camptolcemus, Gray, List Gen. B. iii. p. 622 (1844).

2630. Camptolæmus labradorus.

Anas labradora, Gm. Syst. Nat. i. p. 537.

Somateria labradora, Rowley, Orn. Misc. ii. p. 205, pl. 55.

a (Arthur Strickland) 1850.

A species of N.E. America, probably extinct.

\section{SOMATERIA.}

Somateria, Boie, Isis, 1822 , p. 564.

2631. Somateria mollissima.

Anas mollissima, Linn. Syst. Nat. i. p. 198.

Somateria mollissima, Gould, B. Gt. Brit. v. pl. 26: Dresser, B. Eur. vi. p. 629, pl. 445.

a f Bergen, Norway, 6 September (N. C. Strickland) 1831. -b Britain (N. C. Strickland) 1838. 
2632. Somateria spectabilis.

Anas spectabilis, Linn. Syst. Nat. i. p. 195.

Somateria spectabilis, Gould, B. Gt. Brit. v. pl. 27 : Dresser,

B. Eur. vi. p. 643 , pl. 446 .

a Iceland (J. G. Kinberg) 1844.

\section{CEdemia.}

Oidemia, Fleming, Phil. Zool. ii. p. 260 (1822).

\section{Edemia nigra.}

Anas nigra, Linn. Syst. Nat. i. p. 196.

Oidemia nigra, Gould, B. Gt. Brit. v. pl. 28.

Edemia nigra, Dresser, B. Eur. vi. p. 663, pl. 449.

$a$ Pennsylvania (S. F. Baird) 1847. -b 'Tangier (Favier) 1848. -c (Arthur Strickland) 1850.

2634. @idemia fusca.

Anas fusca, Linn. Syst. Nat. i. p. 196.

Oidemia fusca, Gould, B. Gt. Brit. v. pl. 29.

Edemia fusca, Dresser, B. Eur. vi. p. 657, pl. 448.

a $\hat{\delta}$ juv. Britain, November (H. E. Strickland) 1836. -b, -c Britain (N. C. Strickland) 1838.

\section{Wdemia perspicillata.}

Anas perspicillata, Linn. Syst. Nat. i. p. 201.

Oidemia perspicillata, Gould, B. Gt. Brit. v. pl. 30.

Edemia perspicillata, Dresser, B. Eur. vi. p. 669, pl. 450.

a Canada (Barnes) 1848.

\section{SUBFAMILY.-ERISMATURIN AE.}

\section{Biziura.}

Biziura, Stephens in Shaw's Gen. Zool. xii. pt. 2, p. 221 (1824).

\section{Biziura lobata.}

Anas lobata, Shaw, Nat. Misc. viii. pl. 265.

Biziura lobata, Gould, B. Austr. vii. pl. 18.

a Australia (Arthur Strickland) 1850. -6 (Bt at Birming. ham) 1850 . 
Erismatura.

Erismatura, Bonaparte, Sagg. Dist. Met. An. Vert. Agg. \& Corr. p. 143 (1832).

\section{Erismatura leucocephala.}

Anas leucocephala, Scop. Ann. I. Hist. N. p. 65: Strickl. Ann. \& Mag. N. H, viii. p. 545.

Erismatura leucocephala, Dresser, B. Eur. vi. p. 677, pl. 451. a Africa (J. G. Kinberg) 1844. - b Tangier (Favier) 1848. 2638. Erismatura dominica.

Anas dominica, Linn. Syst. Nat. i. p. 201.

Erismatura dominica, Scl. \& Salv. P. Z. S. 1876, p. 405.

$a$ (E. Brown 1850.

A South-American species.

\section{SUBFAMILY.-MERGIN $\nexists$.}

Mergus.

Mergus, Linnæus, Syst. Nat. i. p. 207 (1766).

\section{Mergus merganser.}

Mergus merganser, Linn. l. s. c. p. 208: Strickl. Ann. \& Mag. N. H. viii. p. 545: Dresser, B. Eur. vi. p. 685, pl. 452.

a Canada (Barnes) 1848.

\section{Mergus serrator.}

Mergus serrator, Linn. Syst. Nat. i. p. 208: Gould, B. Gt. Brit. v. pl. 35 : Dresser, B. Eur. vi. p. 693, pl. 453.

$a$ ô Britain ( $N . C$ Strickland) 1838. - - b, —c Canada (Barnes) 1848.

\section{Mergus albellus.}

Mergus albellus, Linn. Syst. Nat. i. p. 209: Strickl. P. Z. S. 1836, p. 102: Gould, B. Gt. Brit. v. pl. 37 : Dresser, B. Eur. vi. p. 699 , pls. 454,455 .

a $\hat{o}$ Smyrna, 6 January (H. E. Strickland) 1836. - - $q$ Smyrna, February (H. E. Strickland) 1836. 


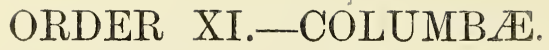

\section{FAMILY.-COLUMBIDE.}

\section{SUB-FAMILY.-COLUMBIN $Æ$.}

\section{Columba。}

Columba, Linnæus, Syst. Nat. i. p. 279 (1766) (partim).

\section{Columba palumbus.}

Columba palumbus, Linn. l. s. c. p. 282 : Strickl. P. Z. S. 1836, p. 100 ; Ann. \& Mag. N. H. xix. p. 106 : Dresser, B. Eur. vii. p. 3, pl. 456 .

a (N. C. Strickland) 1838.

A common British and European species.

\section{Columba fasciata.}

Columba fasciata, Say in Long's Exped. ii. p. 10 : Baird, B. N. Am. p. 597.

a Mexico (T. Mann) 1844.

2644. Columba leucocephala.

Columba leucocephala, Linn. Syst. Nat. i. p. 281 : Gosse, B. Jam. p. 299.

a Jamaica (J. Gould) 1846.

\section{Columba corensis.}

Columba corensis, Gm. Syst. Nat. i. p. 783.

Columba portoricensis, Knip et Temm. Pig. i. Columb. p. 41, pl. 15.

$$
a \text { (Gardner) } 1845 .
$$

An Antillean species. 
2646. Columba elphinstoni.

Ptilinopus elphinstonii, Sykes P. Z. S. 1832, p. 149.

Columba elphinstonii, Strickl. Ann. \& Mag. N. H. xix. p. 106. Palumbus elphinstonei, Jerd. B. Ind. ii. p. 465.

$a$ S. India (Havell) 1839.

\section{4\%. Columba punicea.}

Alsocomus puniceus, Tick. J. A. S. B. xi. p. 461 ; Jerd. B. Ind. ii. p. 462.

a India ("Columba punicea, Tickell," E. Blyth) 1846.

\section{Columba arquatrix.}

Columba arquatrix, Knip et Temm. Pig. i. Columb. p. 11, pl. 5 : Layard. B. S. Afr. p. 257.

$a$ S. Africa (Bt at Stevens's) 1842. -b (Arthur Strickland) 18.0 .

\section{Columba guinea.}

Columba guinea, Gm. Syst. Nat. i. p. 774 : Strickl. P. Z. S. 1850, p. 219 : Strickl. and Scl. Contr. Orn. 1852, p. 156.

a 令 Kordofan, February (J. Petherick) 1848.

\section{Columba speciosa.}

Columba speciosa, Gm. Syst. Nat. i. p. 783 : Knip et Temm. Pig. i. Columb. p. 39, pl. 14.

$a$ (Mansfield) 1839. -b (Arthur Strickland) 1850.

A South and Central American species of wide range.

2651. Columba rufina.

Columba rufina, Knip et Temm. Pig. i. Columb. p. 59, pl. 24. $a$ Cayenne (Mather) 1840. -b Cayenne (Gardner) 1846.

\section{Columba inornata.}

Columba inornata, Vig. Zool. Journ. iii. p. 446.

Columba rufina, Gosse, B. Jam. p. 296 (nec Temm.).

a Jamaica (P. H. Gosse) 1848.

\section{Columba plumbea.}

Columba plumbea, Vieill. N. Dict. d'Hist. N. xxvi. p. 358.

Columba locutrix, Knip et Prév. Pig. ii. p. 80, pl. 44 (ex Wied.).

$a$ (Bt at Stevens's) 1843. -b (Arthur Strickland) 1850.

A common South American species. 


\section{Columba gymnophthalma.}

Columba gymnophthalma, Knip et Temm. Pig. i. Columb. p. 48, pl. 18.

$a$ (Bt at Liverpool) 1853.

A South Brazilian species.

2655. Columba leuconota.

Columba leuconota, Vig. P. Z. S. 1830-1, p. 23 : Gould, B. Cent. Him. pl. 59 : Jerd. B. Ind. ii. p. 471.

a (T. C. Eyton) 1850.

A species of the North-western Himalayas.

\section{Columba rupestris.}

Columba cenas var. rupestris, Pall. Zoogr. Rosso-As. i. p. 560, pl. 35 .

Columba rupestris, Jerd. B. Ind. ii. p. 470.

$a q$ (Gardner) 1845 .

From Capt. Boys's Indian collection.

\section{5\%. Columba œnas.}

Columba œnas, Linn. Syst. Nat. i. p. 279 : Strickl. P. Z. S. 1836, p. 100 : Dresser, B. Eur. vii. p. 23, pl. 458.

a $\hat{o}$ Buckinghamshire, February (H. E. Strickland) 1848.

2658. Columba intermedia.

Columba intermedia, Strickl. Ann. \& Mag. N. H. xiii. p. 39 : Jerd. B. Ind. ii. p. 469.

a India (Bt at Stevens's) 1845.

Probably Strickland's type of this species.

2659. Columba livia.

Columba livia, Bonn. Tabl. Enc. Méth. p. 227.

Columba livia, Dresser, B. Eur. vii. p. 11, pl. 457.

$a$ Britain (N. C. Strickland) 1838.

\section{TURTUR.}

Turtur, Selby, Nat. Libr. Orn. v. p. 169 (1835). 2660. Turtur vulgaris.

Columba turtur, Linn. Syst. Nat. i. p. 284: Strickl. P. Z. S. 1836, p. 100. 
Turtur vulgaris, Dresser, B. Eur. vii. p. 39, pl. 462 (ex Eyton).

a Smyrna, April (H. E. Strickland) 1836. -b Britain (W. Kirtland).

2661. Turtur orientalis.

Columba orientalis, Lath. Ind. Orn. p. 606.

Turtur orientalis, Dresser, B. Eur. vii. p. 45, pl. 463.

Turtur rupicolus, Jerd. B. Ind. ii. p. 476 (ex Pallas).

a India (Askew) 1839. - b o Benares (W. J. E. Boys) 1847.

\section{Turtur meena.}

Columba meena, Sykes, P. Z. S. 1832, p. 149.

Turtur meena, Jerd. B. Ind. ii. p. 476.

a Madras (T. C. Jerdon) 1845. -b India ("Turtur meena, Sykes," E. Blyth) 1846.

\section{Turtur suratensis.}

Columba suratensis, Gm. Syst. Nat. i. p. 778.

Turtur suratensis, Strickl. P. Z. S. 1842, p. 168: Jerd. B. Ind. ii. p. 479.

a E. Indies (N. C. Strickland) 1838.

2664. Turtur tigrinus.

Columba tigrina, Temm. Pig. et Gall. i. p. 317.

Turtur tigrinus, Schl. Mus. des Pays-Bas, Columbæ, p. 127.

a Malacca (Capt. Nash) 1837. - b (Arthur Strickland) 1850.

2665. Turtur cambayensis.

Columba cambayensis, Gm. Syst. Nat. i. p. 779.

Turtur cambayensis, Jerd. B. Ind. ii. p. 478.

a Madras ("Turtur cambayensis," T. C. Jerdon) 1845.

2666. Turtur senegalensis.

Columba senegalensis, Linn. Syst. Nat. i. p. 283.

Turtur senegalensis, Layard, B. S. Afr. p. 261 : Dresser, B. Eur. vii. p. 55, pl. 465.

Turtur cambayensis, Strickl. P. Z. S. 1836, p. 100.

a Constantinople, March (H. E. Strickland) 1836. -b S. Africa (Mrs Van der Kemp) 1843.

S. C. 


\section{6\%. Turtur tranquebarica.}

Turtur tranquebarica, Herm. Obs. Zool. p. 200: Wald. Trans. Z. S. ix. p. 219.

Turtur humilis, Jerd. B. Ind. ii. p. 482 (nec Temminck).

a Malacca (Cashmore) 1839. - b Madras ("Turtur humilis," T. C. Jerdon) 1845.

2668. Turtur risorius.

Columba risoria, Linn. Syst. Nat. i. p. 285.

Turtur risorius, Strickl. Ann. \& Mag. N. H. xiii. p. 38 ; xiv. p. 117 : Jerd. B. Ind. ii. p. 481.

a $\hat{o}$ India (Major Stacy).

2669. Turtur semitorquatus.

Turtur semitorquatus, Sw. B. W. Afr. ii. p. 208: Strickl. Ann. \& Mag. N. H. xix. p. 182 : Layard, B. S. Afr. p. 260.

a Veyge Kraal, Cape of Good Hope, July (A. Strickland) 1833. - b S. Africa (Bt at Stevens's) 1842.

26\%0. Turtur bitorquatus.

Columba bitorquata, Knip et Temm. Pig. i. Columb. p. 86, pl. 40 .

Turtur bitorquatus, Schl. Mus. des Pays-Bas, Columb. p. 121. a Java (Bt at Birmingham) 1849.

26\%1. Turtur vinaceus.

Columba vinacea, Gm. Syst. Nat. i. p. 782.

Turtur vinaceus, Strickl. Ann. \& Mag. N. H. xiii. p. 39 ; xix. p. 182 : Strickl. \& Scl. Contr. Orn. 1852, p. 157 : Layard, B. S. Afr. p. 259 : Schl. Mus. des Pays-Bas, Columbæ, p. 123. $a$ (E. Brown) 1850.

An African species.

\section{MaCROPYGIA.}

Macropygia, Swainson, Classif. B. ii. p. 348 (1837). 2672. Macropygia tenuirostris.

Macropygia tenuirostris, G. R. Gray, List B. Brit. Mus. Columbx, p. 39: Wald. Trans. Z. S. ix. p. 218.

a Philippine Islands ( $J$. Gould) 1846. 
2673. Macropygia tusalia.

Coccyzura tusalia, Hodgs. J. A. S. B. xii. p. 937.

Macropygia tusalia, Jerd. B. Ind. ii. p. 473.

$a$ Darjeeling (E. Blyth) 1850.

Chamepelia.

Chamcepelia, Swainson, Zool. Journ. iii. p. 361 (1827).

2674. Chamæpelia passerina.

Columba passerina, Linn. Syst. Nat. i. p. 285.

Chamcepelia passerina, Baird, B. N. Am. p. 606.

$a \mathrm{~N}$. America (Askew) 1837. $-b$ Guatemala (J. Constancia) 1845.

\section{Chamæpelia griseola.}

Columbina griseola, Spix, Av. Bras. ii. p. 58, pl. 75 a, fig. 2. Chamcepelia griseola, Bp. Consp. Av. ii. p. 78.

a ô (Arthur Strickland) 1850.

A South-American species of wide range.

26'6. Chamæpelia talpacoti.

Columba talpacoti, Knip et Temm. Pig. i. Colo-Gall. p. 22, pl. 12.

Talpacotia cinnamomea, Bp. Consp. Av, ii. p. 79 (ex Swainson).

$a$ Brazil (Askew) 1839.

\section{SCARDAFELIA.}

Scardafella, Bonaparte, Consp. Av. ii. p. 85 (1854).

\section{7\%. Scardafella squamosa.}

Columba squamosa, Knip et Temm. Pig. i. Columb. p. 127, pl. 59.

Scardafella squamosa, Bp. Consp. Av. ii. p. 85.

$a,-b$ (Arthur Strickland) 1850. — $c$ Trinidad (Argent) 1851. 2678. Scardafella inca.

Chamcepelia inca, Less. Descr. Mamm. et Ois. p. 211.

Scardafella inca, Bp. Consp. Av. ii. p. 85. 1848.

$a$ S. Africa (Askew) 1837. - b Guatemala (J. Constancia)

A species peculiar to Mexico and Central America. 
Gymiopelia.

Gymnopelia, Scl. \& Salv. Nomencl. Av. Am. p. 156 (1873). 2679. Gymnopelia erythrothorax.

Columba erythrothorax, Meyen, Nov. Ac. Leop. xvi. Suppl. p. 98 , pl. 26.

Columbula erythrothorax, Bp. Consp. Av. ii. p. 80. a (Askew) 1837.

A species of Western South America.

ZENAIDA.

Zenaida, Bonaparte, Comp. List, p. 41 (1838). 2680. Zenaida amabilis.

Columba zenaida, Bp. Journ. Ac. Phil. v. p. 30.

Zenaida amabilis, Gosse, B. Jam. p. 307.

a Jamaica (P. H. Gosse) 1848.

\section{Zenaida maculata.}

Columba maculata, Vieill. Enc. Méth. i. p. 376.

Zenaida maculata, Bp. Consp. Av. ii. p. 85.

a Chili (Capt. Brown) 1842. -b (Arthur Strickland) 1850. $-c$ (E. Brown) 1850 .

\section{Zenaidura.}

Zenaidura, Bonaparte, Consp. Av. ii. p. 84 (1854). 2682. Zenaidura carolinensis.

Columba carolinensis, linn. Syst. Nat. i. p. 286.

Zenaidura carolinensis, Baird, B. N. Am. p. 604.

a N. America (Havell) 1839. -b Mexico (T. Mann) 1844. -c (Arthur Strickland) 1850.

\section{MELOPELIA.}

Melopelia, Bonaparte, Consp. Av. ii. p. 81 (1854).

2683. Melopelia leucoptera.

Columba leucoptera, Linn. Syst. Nat. i. p. 281.

Turtur leucopterus, Gosse, B. Jam. p. 304.

Melopelia leucoptera, Baird, B. N. Am. p. 603.

a Jamaica (P. H. Gosse) 1848. - b Guatemala (J. Constancia) 1851. 


\section{Metriopelta.}

Metriopelia, Reichenbach, Tauben, p. 17 (1853).

2684. Metriopelia melanoptera.

Columba melanoptera, Mol. Saggio sulla St. Nat. Chili, p. 236. Metropelia melanoptera, Bp. Consp. Av. ii. p. 75.

$a$ (Arthur Strickland) 1850.

A species of Western South America.

\section{Peristera.}

Peristera, Swainson, Zool. Journ. iii. po 360 (1827).

\section{Peristera cinerea.}

Columba cinerea, Knip et Temm. Pig. i. Columb. p. 126, pl. 58.

Peristera cinerea, Bp. Consp. Av. ii. p. 75.

$a,-b,-c q,-d q$ Brazil (G. Lloyd) 1838. - e Guatemala (J. Constancia) 1851.

\section{Peristera geoffroyi.}

Columba godefrida, Knip et Temm. Pig. i. Columb. p. 125, pl. 57.

Peristera geoffroyi, Schl. Mus. des Pays-Bas, Columbæ, p. 139.

$a f(G$. Lloyd $) 1838$.

A Brazilian species.

\section{HAPLOPELIA.}

Haplopeleia, Reichenbach, Tauben, p. 76 (1853).

268\%. Haplopelia larvata.

Columba larvata, Knip et Temm. Pig. i. Columb. p. 71, pl. 31.

Aplopelia larvata, Bp. Consp. Av. ii. p. 66.

Peristera larvata, Layard, B. S. Afr. p. 263.

$a$ S. Africa (Askew) 1833. $-b$ (Askew) 1839. $-c($ Wood $)$ 1840.

\section{Chalcopelia.}

Chalcopelia, Bonaparte, Consp. Av: ii. p. 67 (1854). 2688. Chalcopelia afra.

Columba afra, Linn. Syst. Nat. i. p. 284. 
Chalcopelia afra, Bp. Consp. Av. ii. p. 67.

Peristera afra, Strickl. \& Scl. Contr. Orn. 1852, p. 157: Layard, B. S. Afr. p. 262.

a (E. Brown) 1850.

A South-African species.

2689. Chalcopelia tympanistria.

Columba tympanistria, Knip et Temm. Pig. i. Columb. p. 80, pl. 36.

Peristera tympanistria, Layard, B. S. Afr. p. 262 : Schl. Mus. des Pays-Bas, Columbæ, p. 143.

a Cape of Good Hope (Askew) 1839.

Engyptila.

Engyptila, Sundevall, Meth. Av. Tent. p. 156 (1872).

2690. Engyptila ochroptera.

Leptoptila ochroptera, Pelz. Orn. Bras. p. 278.

a $\hat{o}$ S. America (Cashmore) 1839. - b (Arthur Strickland) 1850.

2691. Engyptila rufaxilla.

Columba rufaxilla, Richard et Bernard, Act. Soc. Hist. Nat. Paris, i. p. 118.

$a$ (Arthur Strickland) 1850.

A Guiana species.

2692. Engyptila jamaicensis.

Columba jamaicensis, Linn. Syst. Nat. i. p. 283.

Peristera jamaicensis, Gosse, B. Jam. p. 313.

a Jamaica (P. H. Gosse) 1848.

\section{Geotrygon.}

Geotrygon, Gosse, B. Jam. p. 316 (1847).

2693. Geotrygon montana.

Columba montana, Linn. Syst. Nat. i. p. 281.

Geotrygon montana, Gosse, B. Jam. p. 320.

a Brazil ('Thomas). - b, _c Jamaica (P. H. Gosse) 1848. 
2694. Geotrygon violacea.

Columba violacea, Knip et Temm. Pig. i. Columb. p. 67, pl. 29.

$a($ E. Brown $) 1850$.

A Brazilian species.

2695. Geotrygon cristata.

Columba cristata, Temm. Pig. et Gall. i. p. 108: Knip et Temm. Pig. i. Col.-Gall. p. 10, pl. 4.

Geotrygon sylvatica, Gosse, B. Jam. p. 316; Ill. pl.'84.

a, -b Jamaica (P. H. Gosse) 1848.

\section{SUBFAMILY.-PHAPIDIN Æ.}

\section{Leucosarcia.}

Leucosarcia, Gould, B. Austr. v. pl. 63 (1843).

2696. Leucosarcia picata.

Columba picata, Lath. Ind. Orn. Suppl. p. lix.

Leucosarcia picata, Gould, l. s. c.

Phaps picata, Strickl. Ann. \& Mag. N. H. xi. p. 337.

$a$ New South Wales $\left(M^{\circ}\right.$ Donald) 1838 .

\section{Phaps.}

Phaps, Selby, Nat. Libr. Orn. v. p. 194 (1835). 269\%. Phaps chalcoptera.

Columba chalcoptera, Lath. Ind. Orn. ii. p. 604.

Peristera chalcoptera, Gould, B. Austr. v. pl. 64.

Phaps chalcoptera, Schl. Mus. des Pays-Bas, Columbæ, p. 154.

$a$ New South Wales (McDonald) 1839. - b Australia (Arthur Strickland) 1850.

\section{Phaps elegans.}

Columba elegans, Temm. Pig. et Gall. i. pp. 240, 466.

Peristera elegans, Gould, B. Austr. v. pl. 65.

Phaps elegans, Schl. Mus, des Pays-Bas, Columbæ, p. 154.

a Australia (Bt at Stevens's) 1847. 
Geophaps.

Geophaps, Gould, P. Z. S. 1842, p. 19.

\section{Geophaps scripta.}

Columba scripta, Temm. Pl. Col. 187.

Geophaps scripta, Gould, B. Austr. v. pl. 67.

a Australia (McDonald) 1839.

\section{Chalcophiaps.}

Chalcophaps, Gould, B. Austr. v. pl. 62 (1842).

\section{Chalcophaps indica.}

Columba indica, Linn. Syst. Nat. i. p. 284.

Chalcoplaaps indicus, Strickl. Ann. \& Mag. N. H. xix. p. 101: Jerd. B. Ind. ii. p. 484: Wald. Trans. Z. S. ix. p. 221.

a Malacca (Capt. Nash) 1837. - b (Bt at Stevens's) 1843. -c India (Bt at Stevens's) 1844. —d India ("Chalcophaps indicus, Columba javanica Horsf." E. Blyth) 1846. -e (Artlubr Strickland) 1850.

\section{Phlogennas.}

Phlogoenas, Reichenbach, Tauben, p. 40 (1853). 2701. Phlogœnas luzonica.

Columba luzonica, Scop. Del. Fl. et Faun. Ins. ii. p. 94. Phlogonas luzonica, Wald. Trans. Z. S. ix. p. 221. a Luzon, Philippine Islands, 14 June (H. Cuming) 1839.

\section{Phabotreron.}

Phapitreron, Bonaparte, Consp. Av. ii. p. 28 (1854).

Phabotreron, Wald. Trans. Z. S. ix. p. 214.

2702. Phabotreron leucotis.

Columba leucotis, Temm. Pl. Col. 189.

Phabotreron leucotis, Wald. Trans. Z. S. ix. p. 214.

a Luzon, 14 June (H. Cuming) 1839. —b (Gardner) 1847. 


\section{Geopelia.}

Geopelia, Swainson, Classif. B. ii. p. 348 (1837).

2703. Geopelia humeralis.

Geopelia humeralis, Gould, B. Austr. v. pl. 72: Strickl. Ann. \& Mag. N. H. xix. p. 181.

a Australia (Bt at Stevens's) 1843. - b Australia (Gardner) 1845.

\section{Geopelia cuneata.}

Columba cuneata, Lath. Ind. Orn. Suppl. p. Ixi.

Geopelia cuneata, Gould, B. Austr. v. p]. 74.

a (Bt at Edinburgh).

An Australian species.

2705. Geopelia tranquilla.

Geopelia tranquilla, Gould, P. Z. S. 1844, p. 56; B. Austr. v. pl. 73.

a (Bt at Stevens's).

An Australian species.

\section{ENA.}

Enna, Swainson, B. W. Afr. ii. p. 214 (1S37) ex Selby, MS. 2706. CEna capensis.

Columba capensis, Linn. Syst. Nat. i. p. 286.

Ena capensis, Layard, B. S. Afr. p. 258.

$a$ S. Africa (Askew) 1841. —b, —c (Arthur Strickland) 1850.

\section{SUBFAMILY.-CARPOPHAGIN $Æ$ E.}

\section{Carpophaga.}

Carpophaga, Selby, Nat. Libr. Orn. v. p. 112 (1835).

2\%0\%. Carpophaga ænea.

Columba cenea, Linn. Syst. Nat. i. p. 283.

Carpophaga cenea, Wald. Trans. Z. S. ix. p. 215.

$a$ (Askew) 1833. - b Luzon 14 June (H. Cuming) 1839. 


\section{Carpophaga novæ-zealandiæ.}

Columba novce-seelandice, Gm. Syst. Nat. i. p. 773.

Carpophaga novce-zealandice, Buller, Birds N. Zeal. p. 157, pl. 18.

$a$ New South Wales ( $M^{c}$ Donald) 1838. - b Australia (Dewgard) 1847.

A New Zealand species.

\section{Carpophaga insignis.}

Ducula insignis, Hodgs. As. Res. xix. p. 162.

Carpophaga insignis, Jerd. B. Ind. ii. p. 457.

a Darjeeling ("Carpophaga insignis," E. Blyth) 1850.

\section{MEgALOPREPIA.}

Megaloprepia, Reichenbach, Tauben, p. 101 (1853).

2710. Megaloprepia magnifica.

Columba magnifica, Temm. Pl. Col. 163.

Carpophaga magnifica, Gould, B. Austr. v. pl. 58.

$a$ New South Wales (McDonald) 1838.

\section{LEUCOMEL RENA.}

Leucomelcena, Bonaparte, Compt. Rend. xxxix. p. 1104 (1854). 2711. Ieucomelæna norfolciensis.

Columba norfolciensis, Lath. Ind. Orn. Suppl. p. Ix.

Leucomelcena norfolciensis, Gould, Handb. B. Austr. ii. p. 112.

Carpophaga leucomela, Gould, B. Austr. v. pl. 59 (ex Temminck).

$a$ New South Wales ( $M^{c}$ Donald) 1839. - $b$ Australia (Bt at Stevens's) 1843.

\section{LOPHOL AMUS.}

Lopholcemus, Gray, in Gould's B. Austr. v. sub pl. 61 (1841). 2712. Lopholæmus antarcticus.

Columba antarctica, Shaw, Zool. N. Holl. p. 15, pl. 5.

Lopholaimus antarcticus, Gould, B. Austr. v. pl. 61.

a Australia (Bt at Birmingham) 1843. -b Australia (Bt at Sievens's) 1843. —c (Dewgard) 1851. 


\section{SUBFAMILY.-PTILOPINA.}

Ptilopus.

Ptilonopus, Swainson, Zool. Journ. i. p. 473 (1825).

Ptilopus, Strickland, Ann. \& Mag. N. H. vii. p. 36.

2\%13. Ptilopus swainsoni.

Ptilinopus swainsoni, Gould, P. Z. S. 1842, p. 18; B. Austr. v. pl. 55 .

Ptilopus regina, Elliot, P. Z. S. 1878, p. 531 (ex Swainson)。 a Australia (J. Gould) 1848.

\section{Ptilopus dupetithouarsi.}

Columba Dupetithouarsi, Neboux, Rev. Zool. 1840, p. 289.

Ptilopus dupetithouarsi, Elliot, P. Z. S. 1878, p. 539.

a (Bt at Liverpool) 1853.

A species of the Marquesas Islands.

2715. Ptilopus melanocephalus.

Columba melanocephala, Gm. Syst. Nat. i. p. 781.

Ptilopus melanocephalus, Elliot, P. Z. S. 1878, p. 551.

$a$ (Bt at Birmingham) 1849.

A Javan species.

2716. Ptilopus jambu.

Columba jambu, Gm. Syst. Nat. i. p. 784.

Ptilopus jambu, Elliot, P. Z. S. 1878, p. 554.

a Malacca (Askew) 1839. - b Malacca (Thomas) 1840.

\section{SUBFAMILY.-TRERONIN ÆE.}

\section{SPHENOCERCUS.}

Sphenocercus, Gray, List Gen. B. p. 57 (1840).

271\%. Sphenocercus sphenurus.

Vinago sphenura, Vig. P. Z. S. 1830-1, p. 173: Gould, Cent. B. Him. pl. 57.

Sphenocercus sphenurus, Jerd. B. Ind, ii. p. 453.

Vinago cantillans, Blyth, J. A. S. B. xii. p. 166.

a India (Havell) 1839. - b Himalaya (L. Fraser) 1845. -c India ("Treron sphenura," E. Blyth) 1846. 


\section{Treron.}

Treron, Vieillot, Anal. p. 49 (1816).

\section{Treron calva.}

Columba calva, Temm. Pig. et Gall. i. p. 63.

Vinago australis, Jard. \& Selb. Ill. Orn. ii. pl. 81.

a Africa (W. Kirtland) 1838.

\section{Treron delalandi.}

Phalacotreron delalandi, Bp. Consp. Av. ii. p. 6.

Treron delalandi, Layard, B. S. Afr. p. 255.

a Africa (Dewgard) 1847.

\section{Treron abyssinica.}

Columba abyssinica, Lath. Ind. Orn. Suppl. p. xl.

Phalacotreron abyssinica, Bp. Consp. Av. ii. p. 7.

a Africa (W. Kirtland) 1838.

\section{Treron chlorigaster.}

Treron chlorigaster, Blyth, J. A. S. B. xii. p. 167.

Crocopus chlorigaster, Jerd. B. Ind. ii. p. 448.

Treron chlorigaster, Strickl. Ann. \& Mag. N. H. xiii. p. 205; xiv. p. 116.

Treron jerdoni, Strickl. Ann. \& Mag. N. H. xiii. p. 38. $a$ (Askew) 1833. -b (Arthur Strickland) 1850.

\section{Treron capellii.}

Columba capellei, Knip et Prév. Pig. ii. p. 69, pl. 38. Treron capellii, Strickl. P. Z. S. 1846, p. 105.

Treron magnirostris, Strickl. Ann. \& Mag. N. H. xiv. p. 116. a Malacca (Askew) 1839. - b Malacca (W. Kirtland) 1843.

\section{Treron phœnicoptera.}

Columba phoenicoptera, Lath. Ind. Orn. p. 597.

Crocopus phonicopterus, Jerd. B. Ind. ii. p. 447.

Vinago phonicoptera, Strickl. Ann. \& Mag. N. H. xiii. p. 38. Treron phonicoptera, Strickl. Ann. \& Mag. N. H. xiv. p. 116. a Nepal (B. H. Hodgson, 104) 1845.

\section{Treron vernans.}

Columba vernans, Linn. Mantiss. Plant. p. 526.

Usmotreron vernans, Wald. Trans. Z. S. ix. p. 210. 
Treron vernans, Strickl. Ann. \& Mag. N. H. xiii. p. 38.

a Luzon, 30 May (H. Cuming) 1837. -b (Johnson) 1837. —c India (E. Rlyth) 1846. —d (Bt at Stevens's) 1847.

2725. Treron bicincta.

Vinago bicincta, Jerd. Madr. Journ. xii. p. 13; Ill. Ind. Orn. pl. 21; B. Ind. ii. p. 449: Strickl. Ann. \& Mag. N. H. xiii. p. 38.

a Madras (T. C. Jerdon) 1845. - b India ("Treron bicincta, fem." E. Blyth) 1846. — A Arakan (E. Blyth) 1847. —d (Arthur Strickland) 1850.

\section{Treron axillaris.}

Treron axillaris, Bp. Compt. Rend. xxxix. p. 875 (ex Gray).

Osmotreron axillaris, Wald. Trans. Z. S. ix. p. 211.

a (Bt at Birmingham) 1849.

A species of the Philippine Islands.

272\%. Treron pompadora.

Columba pompadora, Gm. Syst. Nat. i. p. 775: Wald. Trans. Z. S. ix. p. 212.

Treron pompadora, Strickl. Ann. \& Mag. N. H. xix. p. 48.

Osmotreron flavogularis, Jerd. B. Ind. ii. p. 452 (ex Blyth). a Madras ("Vinago pompadora," T. C. Jerdon) 1845.

\section{Treron malabarica.}

Vinago malabarica, Jerd. Ill. Ind. Orn. sub pl. 21.

Osmotreron malabarica, Jerd. B. Ind. ii. p. 450.

Vinago affinis, Wald. Trans. Z. S. ix. p. 212 (ex Jerdon).

a f (Askew) 1839. - b S. India (Bt at Stevens's) 1843. -c f Madras ("Vinago pompadora," T. C. Jerdon) 1845. —d India (E. Blyth) 1846.

\section{Treron nipalensis.}

Toria nipalensis, Hodgs. As. Res. xix. p. 164: Blyth, J. A. S. B. xliii. extr. No. p. 143.

Treron nipalensis, Strickl. Ann. \& Mag. N. H. xix. p. 44 : Jerd. B. Ind. ii. p. 445.

a India ("Treron aromatica," $E$. Blyth) 1846. - b India (E. Blyth) 1846. -c Arakan (E. Blyth) 1847. 


\section{Treron pulverulenta.}

Treron pulverulenta, Wall. Ibis, 1863, p. 319: Wald. Trans. Z. S. ix. p. 212.

Treron aromatica, Strickl. Ann. \& Mag. N. H. xix. p. 44 (nec Gmelin).

a, -b Malacca (Capt. Nash) 1837.

2731. Treron olax.

Columba olax, Temm. Pl. Col. 241: Wald. Trans. Z. S. ix. p. 212.

a Malacca (Bt at Stevens's) 1844.

2732. Treron phayrii.

Osmotreron phayrii, Blyth, J. A. S. B. xxxi. p. 344: Jerd. B. Ind. ii. p. 212: Wald. Trans. Z. S. ix. p. 212.

$a$ India ("Treron pompadora," E. Blyth) 1846.

\section{FAMILY.-PTEROCLIDAE.}

\section{Pterocles.}

Pterocles, Temminck, Man. d'Orn. p. 299 (1815).

\section{Pterocles variegatus.}

Tetrao (Pterocles) variegatus, Burch. Trav. S. Afr. ii. p. 345.

Pterocles variegatus, Strickl. \& Scl. Contr. Orn. 1852, p. 157: Elliot, P. Z. S. 1878, p. 244.

$a,-b$ Damara-land (C. J. Andersson) 1852.

2734. Pterocles arenarius.

Tetrao arenaria, Pall. Nov. Comm. Petrop. xix. p. 418.

Pterocles arenarius, Dresser, B. Eur. vii. p. 61, pl. 466: Elliot, P. Z. S. 1878, p. 245.

$a,-b q$ W. Siberia (J. F. Brandt) 1844.

2735. Pterocles exustus.

Pterocles exustus, Temm. Pl. Col. 354, 360: Jerd. B. Ind, ii. p. 502: Elliot, P. Z. S. 1878, p. 248.

a 9 Africa (Askew) 1839. - b, -c, - d Madras ("Pterocles exustus," T. C. Jerdon) 1845. -e (Arthur Strickland) 1850. 


\section{Pterocles alchata.}

Tetrao alchata, Linn. Syst. Nat. i. p. 276.

Pterocles alchata, Dresser, B. Eur. vii. p. 67, pl. 467 : Elliot, P. Z. S. 1878, p. 250.

Pterocles setarius, Temm. Pig. et Gall. iii. pp. 256, 714.

a Spain (Boissoneau) 1839. - b (Arthur Strickland) 1850. -c (E. Brown) 1850.

\section{3\%. Pterocles fasciatus.}

Tringa fasciata, Scop. Del. Fl. et Faun. Ins. ii. p. 92.

Pterocles fasciatus, Elliot, P. Z. S. 1878, p. 254.

$a$ (Bt at Stevens's) 1844. -b Madras (T. C. Jerdon) 184.⿳. -c S. India (T. C. Jerdon) 1850.

2738. Pterocles bicinctus.

Pterocles bicinctus, Temm. Pig. et Gall. iii. pp. 247, 713: Strickl. \& Scl. Contr. Orn. 1852, p. 157 : Elliot, P. Z. S. 1878, p. 255.

$a,-b$ o Damara-land (C. J. Andersson) 1852.

2739. Pterocles quadricinctus.

Pterocles quadricinctus, Temm. Pig. et Gall. iii. pp. 252, 713: Strickl. P. Z. S. 1850, p. 220: Elliot, P. Z. S. 1878, p. 257.

$a(W$. Kirtland) 1843. - 6 \% Kordofan 14 August (J. Petherick) 1848.

\section{SyrRhaptes.}

Syrrhaptes, Illiger, Prodr. p. 243 (1811).

\section{Syrrhaptes paradoxu.s.}

Tetrao paradoxus, Pall. Reise, ii. p. 712, fig. F.

Syrrhaptes paradoxus, Dresser, B. Eur. vii. p. 75, pl. 468: Elliot, P. Z. S. 1878, p. 261.

$a$ W. Siberia (J. F. Brandt) 1844, -6 W. Siberia (J. $F$. Brandt) 1845 . 


\title{
ORDER XII.-GALLIN无。
}

\author{
FAMILY.-CRACIDIE. \\ SUBFAMILY.-PENELOPIN $A$.
}

Penelope.

Penelope, Merrem, Av. Ic. et Descr. ii. p. 39 (1786).

2741. Penelope purpurascens.

Penelope purpurascens, Wagl. Isis, 1830, p. 1110: Scl. \& Salv. P. Z. S. 1870 , p. 522.

$a$ (Dewgard) 1847.

A Central-American species.

2742. Penelope superciliaris.

Penelope superciliaris, Temm. Pig. et Gall. iii. pp. 72, 693: Scl. \& Salv. P. Z. S. 1870, p. 527.

$a$ (E. Brown) 1850. - b (Arthur Strickland) 1850.

A Brazilian species.

\section{Penelopina.}

Penelopina, Reichenbach, Tauben, p. 152 (1853).

2743. Penelopina nigra.

Penelope nigra, Fraser, P. Z. S. 1850, p. 246, pl. 29.

Penelopina nigra, Scl. \& Salv. P. Z. S. 1870, p. 528.

a ruatemala (J. Constancia) 1851. 


\section{ORTALIS.}

Ortalis, Merrem, Av. Ic. et Descr. ii. p. 40 (1786).

2744. Ortalis motmot.

Phasianus motmot, Linn. Syst. Nat. i. p. 271.

Ortalida motmot, Scl. \& Salv. P. Z. S. 1870, p. 532.

a (Bt. at Liverpool) 1853 .

A skin of Cayenne make.

\section{Ortalis albiventris.}

Penelope albiventris, Wagl. Isis, 1830, p. 1111.

Ortalida albiventris, Scl. \& Salv. P. Z. S. 1870, p. 535.

a (Thomas) 1843.

A species of South-Eastern Brazil.

\section{Ortalis guttata.}

Penelope guttata, Spix, Av. Bras. ii. p. 55, pl. 73.

Ortalida guttata, Scl. \& Salv. P. Z. S. 1870, p. 536.

a (Gardner) 1846.

A common species in the western portion of the Amazon Valley.

\section{FAMILY.-PHASIANIDA.}

\section{SUBFAMILY.-PAVONIN AE.}

\section{Argus.}

Argus, Temminck, Pig. et Gall. ii. p. 410 (1813); iii. p. 678.

\section{4\%. Argus giganteus.}

Phasianus argus, Linn. Syst. Nat. i. p. 272.

Argus giganteus, Temm. Pig. et Gall. ii. p. 410 ; iii. p. 678:

Strickl. P. Z. S. 1842, p. 168: Elliot, Mon. Phas. i. pl. 11.

a f Malacca (Bt at Stevens's) 1842.

s. C. 


\section{SUBFAMILY.-PHASIANIN $Æ$.}

LOPHOPHORUS.

Lophophorus, Temminck, Pig. et Gall. ii. p. 355 (1813); iii. p. 673: Strickl. Ann. \& Mag. N. H. vii. p. 36.

\section{Lophophorus impeyanus.}

Phasianus impejanus, Lath. Ind. Orn. p. 632.

Lophophorus impeyanus, Elliot, Mon. Phas. i. pl. 18.

$a$ India (Btat Stevens's) 1844. $-b,-c$ Himalaya Mts. (Bt at Stevens's) 1848.

\section{Certornis.}

Ceriornis, Swainson, Classif. B. ii. p. 341 (1837).

2749. Ceriornis satyrus.

Meleagris satyra, Linn. Syst. Nat. i. p. 269.

Ceriornis satyra, Elliot, Mon. Phas. i. pl. 22.

$a$ (Bt at Stevens's) 1843. - b, $-c$ q Nepal (B. H. Hodgson) 1845 .

\section{PuCrasia.}

Pucrasia, G. R. Gray, List Gen. B. p. 79 (1841).

2750. Pucrasia macrolopha.

Satyra macrolopha, Less. Dict. Sc. Nat. lix. p. 196.

Pucrasia macrolopha, Elliot, Mon. Phas. i. pl. 30.

$a,-b$ India (Bt at Stevens's) 1844.

\section{Phasianus.}

Phasianus, Linnæus, Syst. Nat. i. p. 270 (1766) partim.

\section{Phasianus colchicus.}

Phasianus colchicus, Linn. Syst. Nat. i. p. 271 : Strickl. P. Z. S. 1836, p. 100: Elliot, Mon. Phas. ii. pl. 2 : Dresser, B. Eur. vii. p. 85, pl. 469.

a A variety with half the plumage white.

\section{Phasianus sp.?}

a (Bt at Stevens's) 1847.

A young bird without history which cannot at present be satisfactorily determined. 


\section{EUPLOCAMUS.}

Euplocomus, Temminck, Pl. Col. Livr. 86 (1830).

2753. Euplocamus albocristatus.

Phasianus albocristatus, Vig. P. Z. S. 1830-1, p. 9: Gould, Cent. B. Him. pls. 66, 67.

Euplocamus albocristatus, Elliot, Mon. Phas. ii. pl. 18.

$a$ India (Bt at Stevens's) 1844. - b Himalaya Mts. (Bt at Stevens's) 1848.

\section{Euplocamus erythrophthalmus.}

Phasianus erythrophthalmus, Raffl. Trans. L. S. xiii. p. 321.

Euplocamus erythrophthalmus, Elliot, Mon. Phas. ii. pl. 28.

a (Bt at Stevens's) 1842.

The skin of a female of Malacca make.

\section{ITHAGINIS.}

Ithaginis, Wagler, Isis, 1832, p. 1228.

\section{Ithaginis cruentus.}

Phasianus cruentus, Hardw. Trans. L. S. xiii. p. 237.

Ithaginis cruentus, Elliot, Mon. Phas. ii. pl. 30.

$a,-b \mathrm{Nepal}$ (B. H. Hodgson, 84) 1845.

\section{Gallus.}

Gallus, Brisson, Ornith. i. p. 165 (1760).

\section{Gallus ferrugineus.}

Tetrao ferrugineus, Gm. Syst. Nat. i. p. 761.

Gallus ferrugineus, Elliot, Mon. Phas. ii. pl. 32.

Gallus bankiva, Temm. et Auctt. plurr.

$a \hat{\delta},-b$ o Sumatra (Bt at Stevens's) 1842. -c Nepal (B. H. Hodgson, 74) 1845. —c India (E. Blyth) 1846. -d, -e Himalaya Mts. (Bt at Stevens's) 1848. $-f,-g,-h,-i$.

\section{5\%. Gallus sonnerati.}

Gallus sonnerati, Temm. Pig. et Gall. iii. p. 659: Elliot, Mon. Phas. ii. pl. 34 .

a Madras (T. C. Jerdon) 1845. - b, -c India (E. Blyth) 1846. $-d$. 


\section{SUBFAMILY._MELEAGRIN A.}

Meieagris.

Meleagris, Linnæus, Syst. Nat. i. p. 268 (1766) partim. 2758. Meleagris gallopavo.

Meleagris gallopavo, Linn. l. s. c.: Elliot, Mon. Phas. i. pl. 27.

a Bred at Apperly Court.

\section{SUBFAMILY.-NUMIDIN A.}

\section{NUMIDA.}

Numida, Linnæus, Syst. Nat. i. p. 273 (1766).

2759. Numida meleagris.

Numida meleagris, Linn. Syst. Nat. i. p. 273: Elliot, Mon. Phas. ii. pl. 39.

a Jamaica (J. Gould) 1846.

A West-African species introduced into Jamaica where it is now common.

2760. Numida coronata.

Numida coronata, Gray, List B. Brit. Mus. Part iii. p. 29 (descr. nulla): Elliot, Mon. Phas. ii. pl. 41.

a (Arthur Strickland) 1850.

A South-African species.

\section{Numida ptilorhyncha.}

Numida ptilorhyncha, Rüpp. Syst. Ueb. p. 105, pl. 39 : Strickl. P. Z. S. 1850, p. 219 : Elliot, Mon. Phas. ii. pl. 42.

$a$ (Bt at Stevens's) 1844. -b $\hat{\delta}$ Kordofan, 20th June (J. Petherick) 1848.

\section{SUBFAMILY.-PERDICIN $Æ$.}

\section{Galloperdix.}

Galloperdix, Blyth, J. A. S. B. xiii. p. 936 (1844). 2762. Galloperdix spadaceus.

Tetrao spadaceus, Gm. Syst. Nat. i. p. 759.

Galloperdix spadaceus, Jerd. B. Ind. ii. p. 541. 
a (Mather) 1840. - b o Madras ("Francolinus spadaceus," T. C. Jerdon) 1845. — India ("Galloperdix spadaceus," $E$. Blyth) 1846. - d $f$ ("Francolinus spadaceus," T. C. Jerdon) 1850. - e.

\section{Galloperdix lunulata.}

Perdix lunulata, Valenc. Dict. Sc. Nat. xxxviii. p. 446.

Galloperdix lunulosus, Jerd. B. Ind. ii. p. 543.

Perdix hardwickii, J. E. Gray, Ill. Ind. Zool. i. pl. 52.

$a \hat{\delta},-b$ q Madras ("Francolinus hardwickii," T. C. Jerdon) 1845. - - India ("Galloperdix bicalcaratus (?), Perdix lunulosus, Valenciennes, Perdix hardwickii, Gray," E. Blyth) 1846. $-d$.

\section{Francolinus.}

Francolinus, Stephens in Shaw's Gen. Zool. xi. p. 316 (1819). 2764. Francolinus swainsoni.

Francolinus swainsoni, Steph. p. 329 : Smith, Zool. S. Afr. Birds, pl. 12: Strickl. \& Scl. Contr. Orn. 1852, p. 157.

Pternises swainsoni, Gurney in And. B. Damara-1. p. 244.

a Damara-land (C. T. Andersson) 1852.

\section{Francolinus bicalcaratus.}

Tetrao bicalcaratus, Linn. Syst. Nat. i. p. 277.

Francolinus bicalcaratus, Hartl. Orn. W. Afr. p. 201.

a (A.D. Bartlett) 1845 .

A West-African species.

\section{Francolinus levaillanti.}

Perdix levaillanti, Valenc. Dict. Sc. Nat. xxxviii.p. 441 : Temm. Pl. Col. 477 : Layard, B. S. Afr. p. 270.

a (Arthur Strickland) 1850.

A South-African species.

\section{6\%. Francolinus gariepensis.}

Francolinus gariepensis, Smith, Zool. S. Afr. Birds, pls. 83, 84: Strickl. \& Scl. Contr. Orn. 1852, p. 157.

Scleroptera gariepensis, Gurney in And. B. Damara-1, p. 245. a Damara-land (C.J.Andersson) 1852. 


\section{Francolinus africanus.}

Francolinus africanus, Steph. in Shaw's Zool. xi. p. 323.

Francolinus afer, Layard, B. S. Afr. p. 270 (nec Müller).

$a \hat{\delta},-b$ \& Veyge Kraal, Cape of Good Hope, June ( $A$. Strickland) 1833. -c (Arthur Strickland) 1850.

2769. Francolinus albigularis.

Francolinus albogularis, Gray, List B. Brit. Mus. pt. iii. p. 3ð (1844), (descr. nulla) : Hartl. Orn. W. Afr. p. 201.

$a$ (Osman) 1848.

A West-African species.

\section{7\%0. Francolinus clappertoni.}

Perdix clappertoni, Child. in Denh. and Clapp. Narr. Journ. Centr. Afr. App. p. 198.

Francolinus clappertoni, Strickl. P. Z. S. 1850, p. 219.

a ồ Kordofan, 5 July, 1848 (J. Petherick) 1848.

\section{7\%1. Francolinus capensis.}

Tetrao capensis, Gm. Syst. Nat. i. p. 759.

Francolinus clamator, Layard, B. S. Afr. p. 268 (ex Temm.).

$a$ S. Africa (Arthur Strickland) 1850. - b S. Africa.

27\%2. Francolinus vulgaris.

Tetrao francolinus, Linn. Syst. Nat. i. p. 275.

Francolinus vulgaris, Strickl. P. Z. S. 1836, p. 100: Jerd. B. Ind. ii. p. 558 (ex Stephens): Dresser, B. Eur. vii. p. 123, pl. 473.

a (Bt at Stevens's) 1844. - b India ("Francolinus vulgaris," E. Blyth) 1846. — Afghanistan (T. C. Eyton) 1846.

\section{Francolinus pictus.}

Perdix pictus, Jard. \& Selby, Ill. Orn. i. pl. 50.

Francolinus pictus, Jerd. B. Ind. ii. p. 561.

a Madras ("Perdix pictus," T. C. Jerdion) 1845.

\section{ORTYGORNIS.}

Ortygornis, Reichenbach, Handb. Spec. Orn. p. 28 (1851). 27r4. Ortygornis ponticeriana.

Tetrao ponticerianus, Gm. Syst. Nat. i. p. 760. 
Ortygornis ponticeriana, Jerd. B. Ind. ii. p. 569. a (Bt at Stevens's) 1844.

An Indian species.

\section{RHIZOTHERA.}

Rhizothera, G. R. Gray, List Gen. B. p. 79 (1841).

\section{2\%\%5. Rhizothera longirostris.}

Perdix longirostris, Temm. Pig. et Gall. iii. p. 323, 721.

Francolinus longirostris, J. E. Gray, Ill. Ind. Zool. ii. pl. 45, f. 2. a Malacca (Dewgard) 1851.

\section{Perdix.}

Perdix, Brisson, Orn. i. p. 219 (1760).

\section{2\%16. Perdix cinerea.}

Tetrao perdix, Linn. Syst. Nat. i. p. 276.

Perdix cinerea, Dresser, B. Eur. vii. p. 131, pl. 474, 475 (ex Latham).

$a$ o Worcestershire, September (H. E. Strickland) 1842. -b Britain (W. Kirtland) 1843.

\section{ARBORICOLA.}

Arborophila, Hodgson, Madr. Journ. v. p. 303 (1837): Strickl. Ann. \& Mag. N. H. vii. p. 37.

Arboricola, Hodgson, Zool. Misc. p. 85 (1844).

\section{2\%1\%. Arboricola torqueola.}

Perdix torqueola, Valenc. Dict. Sc. Nat. xxxviii. p. 435.

Arboricola torqueola, Jerd. B. Ind. ii. p. 577.

Perdix olivacea, J. E. Gray, Ill. Ind. Zool. ii. pl. 57.

Perdix megapodia, Strickl. Ann. \& Mag. N. H. viii. p. 374 (ex Temm.).

a Nepal (B. H. Hodgson, 111) 1845. - b Himalaya Mts. (Bt at Stevens's) 1848.

2\%78. Arboricola rufogularis.

Arboricola rufogularis, Blyth, J. A. S. B. xviii. p. 819 : Jerd. B. Ind. ii. p. 578.

$a$ Darjeeling (E. Blyth) 1850.

In a note on the label of this specimen Blyth says "Arboricola 
rufogularis, N.B. not the female of $A$. torqueola though so much resembling it, but a young male of a species inhabiting a greater altitude and which has commonly a broadish black margin to the rufous throat."

\section{7'79. Arboricola atrogularis.}

Arboricola atrogularis, Blyth, J. A. S. B. xviii. p. 819 ; xxiv. p. 276.

a $\hat{\text { S. }},-b q$ India ("Arboricola atrogularis," E. Blyth) 1851. -c.

Common in the mountains of Assam and Sylhet according to Blyth.

\section{Arboricola intermedia.}

Arboricola intermedia, Blyth, J. A. S. B.xxiv. p. 277; xliii. extra No. p. 150.

a Arakan (E. Blyth) 1847.

Blyth's original label to this specimen is lost.

\section{Coturnix.}

Coturnix, Bonnaterre, Tabl. Enc. Méth. p. Ixxxvii. (1790).

\section{Coturnix communis.}

Tetrao coturnix, Linn. Syst. Nat. i. p. 278.

Coturnix communis, Bonn. Tabl. Enc. Méth. p. ccxvii.: Strickl. P. Z. S. 1842 , p. 167 : Dresser, B. Eur. vii. p. 143, pl. 476.

Coturnix dactylisonans, Meyer, Vög. Liv.-Esthl. p. 167 : Strickl. P. Z. S. 1836, p. 100 ; 1850, p. 220; Strickl. \&.Scl. Contr. Orn. 1852, p. 157.

a Cape of Good Hope (A. Strickland) 1833. -b (Askew) 1841. - c Madras (T. C. Jerdon) 1845. -d, -e Nepal (B. H. Hodgson, 130, 131). - f ^, -g q Benares (W. J. E. Boys) 1847. - $h$ $\hat{\delta}$ Kartoom, October (J. Petherick) 1848. - i Damara-land (C. J. Andersson) 1852.

\section{2\%82. Coturnix coromandelica.}

Tetrao coromandelicus, Gm. Syst. Nat. i. p. 764.

Coturnix coromandelica, Strickl. Ann. \& Mag. N. H. xiii. p. 39 : Jerd. B. Ind. ii. p. 588. 
Perdix textilis, Temm. Pl. Col. 35.

a Nepal (B. H. Hodgson, 119) 1845. - b o Madras ("Coturnix textilis," T. C. Jerdon) 1845. -c \& India ("Coturnix coromandelianus," E. Blyth) 1846. —d S. India ("Coturnix textilis," T. C. Jerdon) 1850.

\section{Coturnix pectoralis.}

Coturnix pectoralis, Gould, P. Z. S. 1837, p. 8; B. Austr. v. pl. 88.

$a,-b$ New South Wales (McDonald) 1838. -c (Bt at Stevens's) 1842.

\section{Coturnix australis.}

Perdix australis, Lath. Ind. Orn. Suppl. p. Ixii.

Synoicus australis, Gould, B. Austr. v. pl. 89.

$a$ N. S. Wales ( $M^{\circ}$ Donald) 1838.

\section{Coturnix erythrorhyncha.}

Coturnix erythrorhyncha, Sykes, P.Z. S. 1832, p. 153: Trans. Z. S. ii. p. 16, pl. 1.

Perdicula erythrorhyncha, Jerd. B. Ind. ii. p. 584: Blyth, Ibis 1867, p. 160.

a Madras ("Coturnix erythrorhyncha," T. C. Jerdon) 1845.

\section{ExCALFACTORIA.}

Excalfactoria, Bonaparte, Compt. Rend. xlii. p. 881 (1856). 2786. Excalfactoria chinensis.

Tetrao chinensis, Linn. Syst. Nat. i. p. 277.

Coturnix chinensis, Strickl. P. Z. S. 1842, p. 167.

Excalfactoria chinensis, Wald. Trans. Z. S. ix. p. 224.

$a$ Philippine Islands (H.Cuming) 1839. - b Malacca (Mather) 1840. —c, —d (Arthur Strickland) 1850. —e (Dewgard) 1851.

\section{Perdicula.}

Perdicula, Hodgson, Bengal Sporting Rev. ix. p. 344 (1837). 278\%. Perdicula cambayensis.

Perdix cambaiensis, Lath. Ind. Orn. p. 655.

Perdicula cambayensis, Blyth, Ibis, 1867, p. 160. 
Coturnix argoondah, Sykes, P. Z. S. 1832, p. 153; Trans. Z. S. ii. p. 17, pl. 2.

Perdicula argoondah, Blyth, Cat. B. Mus. As. Soc. p. 254. Perdicula asiatica, Jerd. B. Ind. ii. p. 583.

a Malacca (Mansfield) 1839. - b, - c "Coturnix argoondah," T. C. Jerdon) 1845. — d S. India (T. C. Jerdon) 1850.

Blyth and Jerdon held opposite views as to the application of Latham's names to this and the following species. I have here followed Blyth, but both names might well be rejected in favour of Sykes's well-defined titles.

2788. Perdicula asiatica.

Perdix asiatica, Lath. Ind. Orn. p. 649.

Perdicula asiatica, Blyth, Cat. B. Mus. As. Soc. p. 254.

Coturnix pentah, Sykes, P. Z. S. 1832, p. 153 ; Trans. Z. S. ii. p. 19, pl. 3 .

Perdicula cambayensis, Jerd. B. Ind. ii. p. 581.

$a,-b$ Madras ("Coturnix pentah," T. C. Jerdon) 1845. -c S. India (T. C. Jerdon) 1850.

\section{Rollulus.}

Rollulus, Bonnaterre, Tabl. Enc. Méth. p. xciii. (1790). 2789. Rollulus roulroul.

Phasianus roulroul, Scop. Del. Faun. et Fl. Ins. ii. p. 93.

Cryptonyx coronatus, Temm. Pl. Col. 350, 351.

Rollulus cristatus, Strickl. P. Z. S. 1842, p. 167 (ex Gmel.) 1840 .

$a$, -b Malacca (Capt. Nash) 1837. — Malacca (Mather)

2790. Rollulus niger.

Cryptonyx niger, Vig. Zool. Journ. iv. p. 349.

Rollulus niger, Strickl. P. Z. S. 1846, p. 105.

a Malacca (Capt. Nash) 1837. -b (Arthur Strickland) 1850.

CACCABIS.

Caccabis, Kaup, Naturl. Syst. p. 183 (1829). 2791. Caccabis saxatilis.

Perdix saxatilis, Meyer, Tasch. deutsch. Vög. i. p. 305: Strickl. P. Z. S. 1836, p. 100. 
Caccabis saxatilis, Dresser, B. Eur. vii. p. 93, pl. 470.

a f Smyrna, 18 January (H. E. Strickland) 1836.

2792. Caccabis chukar.

Perdix chukar, J. E. Gray, Ill. Ind. Zool. i. pl. 54.

Caccabis chukor, Jerd. B. Ind. ii. p. 564: Dresser, B. Eur. vii. p. 97.

$a$ (Bt at Stevens's) 1843. - b (Gardner) 1846.

Apparently of this species which is, however, hardly to be separated from $C$. saxatilis.

\section{Caccabis rufa.}

Tetrao rufus, Linn. Syst. Nat. i. p. 276.

Caccabis rufa, Dresser, B. Eur. vii. p. 103, pl. 471, f. 1.

a.

A common European species.

\section{Caccabis petrosa.}

Tetrao petrosus, Gm. Syst. Nat. i. p. 758.

Caccabis petrosa, Dresser, B. Eur. vii. p. 111, pl. 471, f. 2.

a Spain (Boissoneau) 1839.

\section{Lerwa.}

Lerwa, Hodgson, Madras Journ. v. p. 300 (1837).

\section{Lerwa nivicola.}

Perdix lerwa, Hodgs. P. Z. S. 1833, p. 107.

Lerwa nivicola, Hodgs. Madras Journ. v. p. 300 : Jerd. B. Ind. ii. p. 555.

a Nepal (B. H. Hodgson) 1845. - b, -c Himalaya Mts (Bt. at Stevens's) 1848.

\section{Tetraogallus.}

Tetraogallus, J. E. Gray, Ill. Ind. Zool. ii. sub pl. 46 (1833$34)$.

\section{Tetraogallus himalayensis.}

Tetraogallus himalayensis, G. R. Gray, P. Z. S. 1842, p. 105 : Gould, B. Asia: Jerd. B. Ind. ii. p. 549.

Lophophorus nigelli, J. E. Gray, Ill. Ind. Zool. ii. pl. 46 (nec Jard. \& Selb.).

a N. India (Dewgard) 1848. 


\section{Tetraogallus altaicus.}

Perdix altaica, Gebler, Bull. Ac. Petersb. i. p. 31 (1836).

Tetraogallus altaicus, Gould, B. Asia, Part V.

a Altai Mountains ("Perdix (Megaloperdix) altaica, Gebler," J. F. Brandt) Siberia (1841).

Apparently a typical specimen of this species and that figured by Gould in the Fifth part of "The Birds of Asia."

\section{SUBFAMILY。 ODONTOPHORIN AE.}

DeNDRoRTYX.

Dendrortyx, Gould, Mon. Odont. pl. 20 (1845).

2798. Dendrortyx macrurus.

Ortyx macrurus, Jard. \& Selb. Ill. Orn. pl. 49.

Dendrortyx macrurus, Gould, Mon. Odont. pl. 20.

a Mexico (T. Mann) 1844.

\section{ORTYX.}

Ortyx, Stephens in Shaw's Gen. Zool. xi. p. 376 (1819).

\section{Ortyx virginianus.}

Tetrao virginianus, Linn. Syst. Nat. i. p. 277.

Ortyx virginianus, Gould, Mon. Odont. pl. 1. 1851.

$a$ f, $-b$ o N. America (Thomas) 1842. $-c$ Illinois (T. Dunn)

2800. Ortyx pectoralis.

Ortyx pectoralis, Gould, P. Z. S. 1842, p. 182 ; Mon. Odont. pl. $\check{5}$.

a (E. Brown) 1850.

A Mexican species.

2801. Ortyx sp. inc.

a (Carfrae) 1850.

A young bird, perhaps of $O$. parvicristalus, Gould. 
LOPHORTYX.

Lophortyx, Bonaparte, Comp. List. p. 42 (1838). 2802. Lophortyx californica.

Tetrao californicus, Shaw, Nat. Misc. ix. pl. 345. Callipepla californica, Gould, Mon. Odont. pl. 16. a (Bt at Edinburgh) 1852.

A Californian species.

\section{Cyrtonyx.}

Cyrtonyx, Gould, Mon. Odont. sub pl. 7 (1845).

\section{Cyrtonyx ocellatus.}

Ortyx ocellatus, Gould, P. Z. S. 1836, p. 75.

Cyrtonyx ocellatus, Gould, Mon. Odont. pl. 8. a Guatemala (J. Constancia) 1851.

In a note on the label of this specimen Strickland remarks:"Markings of face incorrectly described and figured by Gould." This is quite correct and the inaccuracy has misled Mr Lawrence into describing the same bird as $C$. sumichrasti. The only specimen that came under Gould's observation at the time he wrote his Monograph, which can be now traced, is that in the Paris Museum and this is undoubtedly the same as Mr Lawrence's bird.

\section{ODONTOPHORUS.}

Odontophorus, Vieillot, Anal. p. 51 (1816).

\section{Odontophorus guianensis.}

Tetrao guianensis, Gm. Syst. Nat. i. p. 767.

Odontophorus guianensis, Gould, Mon. Odont. pl. 23.

a S. America (Cashmore) 1839.

\section{Odontophorus dentatus.}

Perdix dentata, Temm. Pig. et Gall. iii. pp. 418, 734.

Odontophorus dentatus, Gould, Mon. Odont. pl. 26.

Ortyx capistrata, Jard. \& Selb. Ill. Orn. i. pl. 38. a (Mather) 1840.

A Brazilian species. 


\section{SUBFAMILY.-TETRAONIN AE.}

\section{Tetrao.}

Tetrao, Linnæus, Syst. Nat. i. p. 273 (1766) partim.

2806. Tetrao urogallus.

Tetrao urogallus, Linn. l. s. c.: Dresser, B. Eur. vii. p. 223, pl. 490.

a f juv., -b \& Russia (J. F. Brandt) 1841. -c.

\section{- Tetrao.}

$a$ Govt. of St Petersburg (J. F. Brandt) 1841.

A hybrid between $T$. urogallus and $T$. tetrix. See Dresser, B. Eur. vii. pl. 489.

\section{0\%. Tetrao tetrix.}

Tetrao tetrix, Linn. Syst. Nat. i. p. 274: Dresser, B. Eur. vii. p. $205, \mathrm{pl} .487$.

$a$ ^, $-b$ ก Russia (J. F. Brandt) 1841. $-c-d$.

\section{Pedicecetes.}

Pediocaetes, Baird, Birds N. Am. p. 625 (1858). 2808. Pedicectes phasianellus.

B. Tetrao phasianellus, Linn. Syst. Nat. i. p. 273.

Pediecates phasianellus, Elliot, Mon. Tetr. pl. 15. a $\hat{o}$ Illinois (J. G. Kinberg) 1845.

\section{BONASA.}

Bonasa, Stephens, in Shaw's Gen. Zool. xi. p. 298 (1819). 2809. Bonasa umbellus.

Tetrao umbellus, Linn. Syst. Nat. i. p. 275.

Bonasa umbellus, Baird, B. N. Am. p. 630: Elliot, Mon. Tetr. pl. 1.

a Massachusetts (J. G. Kinberg) 1845.

\section{Bonasa betulina.}

Tetrao bonasia, Linn. Syst. Nat. i. p. 275.

Tetrao betulinus, Scop. Ann. i. Hist. Nat. p. 119. 
Bonasa betulina, Strickl. Ann. \& Mag. N. H. viii. p. 544: Dresser, B. Eur. vii. p. 193, pl. 486.

a Switzerland (L. Coulon) 1836. - b f Christiania market, 28 September 1831 (Arthur Strickland) 1850.

\section{LAGOPUS.}

Lagopus, Brisson, Orn. i. p. 181 (1760).

\section{Lagopus scoticus.}

Tetrao scoticus, Lath. Ind. Orn. p. 641.

Lagopus scoticus, Dresser, B. Eur. vii. p. 165, pl. 479.

a §ิ Scotland, September (H. E. Strickland) 1840. -b.

\section{Lagopus albus.}

Tetrao lagopus, Linn. Syst. Nat. i. p. 274.

Tetrao albus, Gm. Syst. Nat. i. p. 750.

Lagopus albus, Dresser, B. Eur. vii. p. 183, pls. 483, 484.

a f Britain (Tucker) 1836. - b \& Norway, March (II. E. Strickland) 1839 -c near Jerken, 18 August 1831 (Arthur Strickland) 1850. - d near Kongwold, 6 May 1831 (Arthur Strickland) 1850.

\section{Lagopus mutus.}

Lagopus mutus, Leach, Cat. Mamm. \& B. Brit. Mus. p. 27 : Dresser, B. Eur. vii. p. 157, pls. 477, 478, 484 .

a Ross-shire (Hugh Frazer) 1837. - b Britain (N. C. Strickland) 1838. -c \$ Scotland, September (H. E. Strickland) 1840. —d Ross-shire, 29 September (W. H. Hyett) 1844.

\section{Lagopus rupestris.}

Lagopus rupestris, Dresser, B. Eur. vii. p. 175, pl. 481 (ex Gmelin).

Tetrao islandorum, Faber, Prodr. Isl. Orn. p. 6.

a Iceland (J. G. Kinberg) 1843.

\section{Lagopus canadensis.}

Tetrao canadensis, Linn. Syst. Nat. i. p. 274.

Canace canadensis, Elliot, Mon. Tetr. pl. 9.

$a,-b$ (Bt at Edinburgh) 1852. 


\section{FAMILY.-TURNICID A.}

\section{TURnIX.}

Turnix, Bonnaterre, Tabl. Enc. Méth. p. Ixxxii (1790).

\section{Turnix sylvatica.}

Tetrao sylvaticus, Desf. Hist. Ac. Roy. des Sc. 1787, p. 500, pl. 13.

Turnix sylvatica, Dresser, B. Eur. vii. p. 249, pl. 494.

$a$ (Askew) 1840. -b (Bt at Stevens's) 1847.

A species of South-west Europe and North Africa.

\section{1\%. Turnix lepurana.}

Hemipodius lepurana, Smith, Ill. Zool. S. Afr. pl. 16.

Turnix lepurana, Strickl. \& Scl. Contr. Orn. 1852, p. 158: Gurney, in And. B. Damara-1. p. 249.

a (Arthur Strickland) 1850. - b Damara-land (C.J. Andersson) 1852.

\section{Turnix sykesi.}

Hemipodius sykesi, Smith, Ill. S. Afr. Zool. sub tab. 16.

Turnix sykesi, Jerd. B. Ind. ii. p. 600.

Turnix dussumieri, Blyth, Ibis, 1867, p. 161 (ex Temminck).

$a$ (Bt at Stevens's) 1844. - b, —c Madras ("Ortygis Sykesii, Smith," T. C. Jerdon) 184.5. —d India ("Turnix variabilis, Hodgs. dussumieri, apud Sykes" E. Blyth) 1846.

To avoid the confusion involved in applying Temminck's name to this species as advocated by Blyth I follow Jerdon in adopting Sir A. Smith's title for it.

\section{Turnix joudera.}

Turnix joudera, Hodgs. Beng. Sport. Mag. 1838, pl. 1. f. 1: Gray \& Mitch. Gen. B. iii. pl. 131.

Turnix tanki, Blyth, J. A. S. B. xii. p. 180*: Ibis, 1867, p. 161.

Tumix dussumieri, Jerd. B. Ind. ii. p. 599 (nec Temm.).

$a$ India (Cashmore) 1839. - b S. India ("Ortygis tanki, apud Blyth," T. C. Jerdon) 1844. — q Benares (W. J. E. Boys) 1847.

If Blyth is right in saying that Temminck's name is not appli- 
cable to this bird. Mr Hodgson's comes next in order of date rather than T. tanki of Blyth.

\section{Turnix pugnax.}

Hemipodius pugnax, Temm. Pig..et Gal. iii. pp. 612, 754.

Turnix pugnax, Strickl. P. Z. S. 1842, p. 167; 1846, p. 105: Blyth, Ibis, 1867, p. 161.

Turnix taigoor, Jerd. B. Ind. ii. p. 595 (ex Sykes).

a Malacca (Capt. Nash) 1837. —b Malacca, 1840. —c Madras ("Ortygis pugnax? taigoor?," T. C. Jerdon) 1845. - d o India ("Turnix bengalensis, fem." E. Blyth) 1846. -e, -f, -g (Arthur" Strickland) 1850.

\section{Turnix varia.}

Perdix varia, Lath. Ind. Orn. Suppl. p. Ixii.

Hemipodius varius, Gould, B. Austr. v. pl. 82.

a Australia (Bt at Birmingham) 1843. - b, - - c Australia (Arthur Strickland) 1850.

\section{ORTYXELOS.}

Ortyxelos, Vieillot, Gal. Ois. ii. p. 91, pl. 300 (1825).

\section{Ortyxelos meiffreni.}

Turnix meiffreni, Vieill. N. Dict. d'Hist. N. xxxv. p. 49.

Ortyxelos meiffreni, Strickl. P. Z. S. 1850, p. 220.

a Kordofan, 12 June 1848 (J. Petherick) 1848.

A fragment without the head. 


\title{
ORDER XIII.-GERANOMORPHÆ.
}

\author{
SUBORDER. - FULICARIE. \\ FAMILY.-RALLID瓜. \\ SUBFAMILY.-RAJLIN
}

HYPOT ANIDIA.

Hypotcenidia, Reichenbach, Handb. Spec. Orn. p. 23 (1851). 2823. Hypotænidia philippensis.

Rallus philippensis, Linn. Syst. Nat. i. p. 263.

Rallus philippensis, Buller, B. N. Zeal. p. 176, pl. 21.

Rallus pectoralis, Gould, B. Austr. vi. pl. 76 (ex Cuvier).

$a$ New South Wales ( $M^{c}$ Donald) 1838. -b Australia (Rev. J. Penrose) 1848. -c Australia (Arthur Strickland) 1850.

2824. Hypotænidia striata.

Rallus striatus, Linn. Syst. Nat. i. p. 262 : Strickl. P. Z. S. 1846, p. 105 : Jerd. B. Ind. ii. p. 726.

Hypotcenidia striata, Wald. Trans. Z. S. ix. p. 232.

a Luzon (H. Cuming) 1839. -b Philippine Islands (H. Cuming) 1839. —c Malacca (Askew) 1841. —d Madras (T. C. Jerdon) 1845. -e, -f, -g, India (E. Blyth) 1846.

\section{RALLINA.}

Rallina, Reichenbach, Handb. Spec. Orn. p. 23 (1851). 2825. Rallina fasciata.

Rallus fasciatus, Raffl. Trans. L. S. xiii. p. 328.

Rallina fasciata, Wald. Trans. Z. S. ix. p. 231. 
Gallinula euryzona, Temm. Pl. Col. 417.

a Malacca (Capt. Nash) 1837. -b Luzon (H. Cuming) 1839. -c Malacca? (Thomas) 1840.

\section{RALLUS.}

Rallus, Linnæus, Syst. Nat. i. p. 261 (1766).

2826. Rallus aquaticus.

Rallus aquaticus, Linn. Syst. Nat. i. p. 262: Strickl. P. Z. S. 1836, p. 101 : Dresser, B. Eur. vii. p. 257, pl. 495.

$a$ o Worcestershire, February (H. E. Strickland) 1835. - b o Smyrna, 14 November (H. E. Strickland) 1835.

282\%. Rallus lewini.

Rallus lewini, Sw. An. in Menag. p. 336 : Gould, B. Austr. vi. pl. 77.

Rallus brachypus, Sw. l. s. c.

a (Argent) 1852.

An Australian species.

\section{Rallus cærulescens.}

Rallus ccerulescens, Gm. Syst. Nat. i. p. 716 : Layard, B. S. Afr. p. 337.

$a$ (Askew) 1834. -b (Arthur Strickland) 1850.

A South-African species.

2829. Rallus virginianus.

Rallus virginianus, Linn. Syst. Nat. i. p. 263 : Baird, B. N. Am. p. 748 : Scl. \& Salv. P. Z. S. 1868, p. 445.

a f̂ Carlisle, Pennsylvania, 14 July 1843 (S. F. Baird) 1847. 2830. Rallus crepitans.

Rallus crepitans, Gm. Syst. Nat. i. p. 713 : Baird, B. N. Am. p. 747 .

Rallus longirostris, Scl. \& Salv. P. Z. S. 1868, p. 444 (partim).

$a$ (Askew) 1834. - -b (Mansfield) 1849.

A North-American species. 


\section{Rallus maculatus.}

Râle tachté de Cayenne, D'Aub. Pl. Enl. 775, undè,

Rallus maculatus, Bodd. Tabl. Pl. Enl. p. 48 : Scl. \& Salv. P. Z. S. 1868, p. 444 .

a (Bt. at Stevens's) 1843.

A South-American species.

\section{Rallus nigricans.}

Rallus nigricans, Vieill. N. Dict. d'Hist. N. xxviii. p. 560 : Scl. \& Salv. P. Z. S. 1868, p. 446.

Gallinula ccesia, Spix, Av. Bras. ii. p. 73, pl. 95.

a Brazil (Askew) 1834.

\section{Rallus gularis.}

Rallus gularis, Less. Traité d'Orn. p. 536: Guérin, Icon. Règn. An. p. 32, pl. 58, f. 1 (ex Cuvier): Schl. \& Pollen, Rech. sur la Faune Madag. ii. p. 134 (nec Horsfield).

a (Askew) 1841.

A species of Madagascar.

\section{Amavrormis.}

Amaurormis, Reichenbach, Handb. Spec. Orn. p. 21 (1851). 2834. Amaurormis olivacea.

Gallinula olivacea, Meyen, Nov. Act. Ac. Leop. xvi. Suppl. p. 233, pl. 30 .

Amaurormis olivacea, Wald. Trans. Z. S. ix. p. 231.

$a$ (Gardner) 1846, -b (Gardner) 1847.

A species found in the Philippine Islands.

\section{ARAMIDES.}

Aramides, Pucheron, Rev. Zool. 1845, p. 277.

\section{Aramides cayennensis.}

Poule d'eau, de Cayenne, D'Aub. Pl. Enl. 352, unde,

Fulica cayennensis, Gm. Syst. Nat. i. p. 700.

Aramides cayennensis, Scl. \& Salv. P. Z. S. 1868, p. 447.

a (Arthur Strickland) 1850.

A species of the northern parts of South America. 


\section{Aramides saracura.}

Gallinula saracura, Spix, Av. Bras. ii. p. 75, pl. 98. Aramides saracura, Scl. \& Salv. P. Z. S. 1868, p. 449. a (Thomas) 1842.

A Brazilian species.

\section{Crex.}

Crex, Bechstein, Gem. Naturg. Deutschl. ii. p. 461 (1805).

\section{Crex pratensis.}

Rallus crex, Linn. Syst. Nat. i. p. 261.

Crex pratensis, Bechst. l.s.c.: Strickl. P. Z. S. 1836, p. 101; 1850, p. 221 : Dresser, B. Eur. vii. p. 291, pl. 499.

$a$ Worcestershire, August (T. Robinson) 1839. - b q Kartoum (J. Petherick) 1848.

\section{Porzana.}

Porzana, Vieillot, Anal. p. 61 (1816).

2838. Porzana maruetta.

Rallus porzana, Linn. Syst. Nat. i. p. 262.

Crex porzana, Strickl. P. Z. S. 1836, p. 101.

Porzana maruetta, Leach, Syst. Cat. B. \& M. Brit. Mus. p. 34: Dresser, B. Eur. vii. p. 267, pl. 496.

a f̂, -bo Lincolnshire, 30 August (H. E. Strickland) 1828. -c (Mansfield) 1833. —d Smyrna, 14 November (H. E. Strickland) 1835. -e India ("Porzana maruetta," E. Blyth) 1846. - $f$ S. India ("Rallus porzana," T. C. Jerdon) 1850.

2839. Porzana fluminea.

Porzana fluminea, Gould, P. Z. S. 1842, p. 139 ; B. Austr. vi. pl. 79 .

$a$ (Dewgard) 1851.

An Australian species.

2840. Porzana palustris.

Porzana palustris, Gould, P. Z. S. 1842, p. 139 : B. Austr. vi. pl. 80.

a New South Wales (McDonald) 1838. 
2841. Porzana bailloni.

Rallus bailloni, Vieill. N. Dict. d'Hist. N. xxviii., p. 548 : Dresser, B. Eur. vii. p. 275, pl. 497.

Porzana pygmaea, Jerd. B. Ind. ii. p. 723 (ex Naumann).

a Madras (T. C. Jerdon) 1845. - b, - - India ("Porzana bailloni," E. Blyth) 1846. —d S. India ("Rallus bailloni," T. C. Jerdon) 1850.

2842. Porzana parva.

Rallus parvus, Scop. Ann. i. Hist. Nat. p. 108.

Porzana parva, Dresser, B. Eur. vii. p. 283, pl. 498.

a Lombardy (Bt at Parma) 1836.

2843. Porzana carolina.

Rallus carolinus, Linn. Syst. Nat. i. p. 363.

Porzana carolina, Scl. \& Salv. P. Z. S. 1868, p. 450. 1848.

$a$ N. America $(K o c h)$ 1842. - b Guatemala (J. Constancia)

\section{Porzana albicollis.}

Rallus albicollis, Vieill. N. Dict. d'Hist. N. xxviii. p. 561.

Porzana albicollis, Scl. \& Salv. P. Z. S. 1868, p. 451.

a Brazil (Askew) 1834.

\section{Porzana cayennensis.}

Râle, de Cayenne, D’Aub. Pl. Enl. 368, undè,

Rallus cayennensis, Gm. Syst. Nat. i. p. 718.

Porzana cayennensis, Scl. \& Salv. P. Z. S. 1868, p. 451.

Crex aurita, Gray, Zool. Misc. p. 13.

$a$ South America (Askew) 1839.

2846. Porzana melanophæa.

Rallus melanophaius, Vieill. N. Dict. d'Hist. N. xxviii. p. 549.

Porzana melanophoea, Scl. \& Salv. P. Z. S. 1869, p. 453.

a Brazil (Mansfield) 1833.

\section{4\%. Porzana ruficollis.}

Rallus cinereus, Vieill. N. Dict. d'Hist. N. xxviii. p. 556, (nec Porphyrio cinereus, Vieill.).

Porzana cinerea, Scl. \& Salv. P. Z. S. 1868, p. 456. 
Gallinula ruficollis, Sw. An. in Menag. p. 349.

Rallus exilis, Temm. Pl. Col. 523.

$a$ (E. Brown) 1850 .

A skin of Cayenne make.

2848. Porzana tabuensis.

Rallus tabuensis, Gm. Syst. Nat. i. p. 717.

Porzana? tabuensis, Gould, Handb. B. Austr. ii. p. 341.

Porzana? immaculata, Gould, B. Austr. vi.pl. 82. a Australia (Askew) 1834.

2849. Porzana cinerea.

Porphyrio cinereus, Vieill. N. Dict. d'Hist. N. xxviii. p. 29. Ortygometra cinerea, Wald. Trans. Z. S. ix. p. 230.

Rallus quadristrigatus, Horsf. Trans. L. S. xiii. 196.

a Philippine Islands, 13 May (H. Cuming) 1840.

\section{Porzana fusca.}

Rallus fuscus, Linn. Syst. Nat. i. p. 262.

Porzana fusca, Jerd. B. Ind. ii. p. 724: Wald. Trans. Z. S。 ix. p. 230.

$a$ Philippine Islands, 30 May (H. Cuming) 1839. - b India ("Porzana fusca P. rubiginosa" E. Blyth) 1846.

2851. Porzana noveboracensis.

Fulica noveboracensis, Gm. Syst. Nat. i. p. 701.

Porzana noveboracensis, Scl. \& Salv. P. Z. S. 1868, p. 457.

a North America (Koch) 1842.

\section{LIMNOCORAX.}

Limnocorax, Peters, Ber. Ak. Berl. 1854, p. 187.

2852. Limnocorax niger.

Rallus niger, Gm. Syst. Nat. i. p. 717.

Gallinula niger, Layard, B. S. Afr. p. 342.

$a$ Africa (W. Kirtland) 1838.

Agrees best with South-African specimens. 


\section{SUBFAMILY.-FULICIN AE.}

Porphyrio.

Porphyrio, Brisson, Orn. v. p. 522 (1760).

2853. Porphyrio cærvleus.

Fulica ccerulea, Vandelli, Mem. Ac. real Lisb. 1797, p. 37.

Porphyrio carulea, Scl., Ibis, 1879, p. 195.

Porphyrio veterum, Dresser, B. Eur. vii. p. 299, pl. 500 (ex Gmelin).

Porphyrio antiquorum, Bp. Comp. List. p. 54: Strickl. Ann. \& Mag. N. H. xiv. p. 432.

a. Sicily (Capt. H. Drummond) 1846.

2854. Porphyrio madagascariensis.

Gallinula madagascariensis, Lath. Ind. Orn. Suppl. p. Ixviii. Porphyrio madagascariensis, Schl. Mus. d. Pays-Bas, Ralli, p. 54 .

Porphyrio chloronotus, Elliot, Stray Feath. vii. p. 20 (ex Vieillot).

a (Askew) 1839.

A species of Madagascar and Africa.

2855. Porphyrio poliocephalus.

Gallinula poliocephala, Lath. Ind. Orn. Suppl. p. Ixviii.

Porphyrio poliocephalus, Jerd. B. Ind. ii. p. 713.

a Madras (T. C. Jerdon) 1845. - b f Rohilkund (W. J. E. Boys) 1847. - C S. India (T. C. Jerdon) 1850.

\section{Porphyrio melanonotus.}

Porphyrio melanotus, Temm. Man. d'Orn. ii. p. 701: Gould, B. Austr. vi. pl. 69: Strickl. Ann. \& Mag. N. H. xi. p. 338. a Australia (Arthur Strickland) 1840.

\section{5\%. Porphyrio martinicus.}

Fulica martinica, Linn. Syst. Nat. i. p. 259.

Porphyrio martinicus, Scl. \& Salv. P. Z. S. 1868, p. 459.

a (Askew) 1834 .

A South-American species. 
PORPHYRIOPS.

Porphyriops, Pucheron, Rev. Zool. 1845, p. 278.

2858. Porphyriops melanops.

Rallus melanops, Vieill. N. Dict. d'Hist. N. xxviii. p. 553.

Porphyriops melanops, Scl. \& Salv. P. Z. S. 1868, p. 461.

Fulica crassirostris, J. E. Gray, Griff. ed. Cuv. An. Kingd. Aves, iii. p. 542.

$a$ Chili (Capt. Brown) 18t:.

\section{Tribonyx.}

Tribonyx, Du Bus, Bull. Ac. Brux. vii. p. 212 (1840).

2859. Tribonyz ventralis.

Gallinula ventralis, Gould, P. Z. S. 1836, p. 85.

T'ribonyx ventralis, Gould, B. Austr. vi. pl. 72. 1853.

a Australia (W. Kirtland) 1847. - b Australia (T. C. Eyton)

\section{Gallinula.}

Gallinula, Brisson, Orn. vi. p. 2 (1760).

2860. Gallinula chloropus.

Fulica chloropus, Linn. Syst. Nat. i. p. 258.

Gallinula chloropus, Strickl. P. Z. S. 1836, p. 101; 1842, p. 167: Jerd. B. Ind. ii. p. 718: Dresser, B. Eur. vii. p. 313, pl. 503.

Gallinula parvifrons, Blyth, Ann. \& Mag. N. H. xiv. p. 121.

a o Worcestershire, January (H. E. Strickland) 1842. -b (Bt at Stevens's) 1843. — C Madras (T. C. Jerdon) 1845. -d, -e India ("Gallinula parvifrons, shield never larger," E. Blyth) 1846. $-f,-g,-h$ (Arthur Strickland) 1850.

\section{Gallinula galeata.}

Crex galeata, Licht. Verz. Doubl. p. 80.

Gallinula galeata, Gosse, B. Jam. p. 381: Scl. \& Salv. P. Z. S. 1868, p. 462.

a Jamaica (J. Gould) 1846. -b, - - Canada (Bames) 1848, $-d$. 
2862. Gallinula phœnicurus.

Rallus phonicurus, Forst. vel Pennant, Zool. Ind. p. 19, pl. 9.

Gallinula phonicura, Strickl. P. Z. S. 1842, p. 167: Jerd. B. Ind. ii. p. 720.

Erythra phœnicura, Legge, B. Ceylon, p. 786.

a Ceylon (Askew) 1834. - b Madras (T. C. Jerdon) 1845. -c (Arthur Strickland) 1850.

\section{Gallicrex.}

Gallicrex, Blyth, Cat. B. Mus. As. Soc. p. 283 (1849). 2863. Gallicrex cristatus.

Gallinula cristata, Lath. Ind. Orn. ii. p. 779.

Gallicrex cristatus, Jerd. B. Ind. ii. p. 716.

a Madras (T. C. Jerdon) 1845. - b India ("Gallicrex cristatus," E. Blyth) 1847.

\section{Fulica.}

Fulica, Linnæus, Syst. Nat. i. p. 257 (1766).

2864. Fulica americana.

Fulica americana, Gm. Syst. Nat. i. p. 704: Scl. \& Salv. P. Z. S. 1868, p. 468.

a N. America, 1838.

2865. Fulica cristata.

Fulica cristata, Gm. Syst. Nat. i. p. 704: Strickl. Ann. \& Mag. N. H. xiv. p. 433: Dresser, B. Eur. vii. p. 323, pl. 504, f. 1.

$a$ Tangier (Favier) 1848. - b S. Africa (Arthur Strickland) 1850.

\section{Fulica armillata.}

Fulica armillata, Vieill. N. Dict. d'Hist. N. xii. p. 47: Scl. \& Salv. P. Z. S. 1868, p. 465; Ex. Orn. p. 115, pl. 58.

a Chili (Capt. Brown) 1842.

286\%. Fulica sp. inc.

F. leucopterce affinis sed crisso omnino nigro distinguenda. a (N. C. Strickland) 1838.

2868. Fulica sp. inc.

$F$. armillatce affinis sed pedibus multo debilioribus differt. a (Thomas) 1842. 


\section{FAMILY.-HELIORNITHIDA.}

HeLioRnis.

Heliornis, Bonnaterre, Tabl. Enc. Méth. p. Ixxxiv. (1790).

\section{Heliornis fulica.}

Le Grebifoulque, de Cayenne, D'Aub. Pl. Enl. 893, undè, Colymbus fulica, Bodd. Tabl. Pl. Enl. p. 54.

Heliornis fulica, Scl. \& Salv. P. Z. S. 1868, p. 469.

$a$ South America (Gardner) 1845. - b.

SUBORDER.-ALECTORIDES.

FAMILY.-ARAMID $\mathbb{E}$.

\section{Aramus.}

Aramus, Vieillot, Anal. p. 58 (1816).

\section{8\%0. Aramus scolopaceus.}

Ardea scolopacea, Gm. Syst. Nat. i. p. 647.

Aramus scolopaceus, Strickl. Ann. \& Mag. N. H. viii., p. 545.

$a$ (E. Brown) 1850.

A South-American species. Scolopax guarana, Linn. which Strickland $l$.s. c. says applies to this bird as an older name for it, is now referred to a Plegadis.

\section{FAMILY.-GRUIDE.}

Grus.

Grus, Bechstein, Nat. Vög. Deutschl. ii. p. 60 (1793).

\section{Grus communis.}

Ardea grus, Linn. Syst. Nat. i. p. 234.

Grus communis, Bechst. l.s.c. : Dresser, B. Eur. vii. p. 337, pl. 505.

Grus cinereus, Bechst. et auctt. Strickl. P. Z. S. 1836, p. 101; 1850 , p. 221.

a (Arthur Strickland) 1850.

A species of Europe and North Africa. 


\section{Grus antigone.}

Ardea antigone, Linn. Syst. Nat. i. p. 235.

Grus antigone, Strickl. Ann. \& Mag. N. H. xi. p. 338 : Jerd. B. Ind. ii. p. 662 : Tegetm. \& Blyth, Nat. Hist. Cranes, p. 47. a (Arthur Strickland) 1850.

An Asiatic species common in India.

\section{ANThropoIdes.}

Anthropoides, Vieillot, Anal. p. 59 (1816).

\section{Anthropoides paradiseus.}

Ardea paradisea, Licht. Cat. Hamb. p. 28.

Grus paradisea, Tegetm. \& Blyth, Nat. Hist. Cranes, p. 23. Anthropoides stanleyanus, Layard, B. S. Afr. p. 303 (ex Vigors).

a (Arthur Strickland) 1850.

A South-African species.

\section{FAMILY.-OTID瓜.}

\section{OTIS.}

Oits, Linnæus, Syst. Nat. i. p. 264 (1766).

\section{Otis bengalensis.}

Otis bengalensis, Gm. Syst. Nat. i. p. 724 : Schl. Mus. PaysBas, Cursores, p. 10.

Spheotides bengalensis, Jerd. B. Ind. ii. p. 616.

a India (Warwick Museum) 1841. - b.

\section{Otis senegalensis.}

Otis senegalensis, Vieill. Enc. Méth. p. 333.

Otis rhaad, Rïpp. Mus. Senkenb. ii. p. 230, pl. 15 : Strickl. P. Z. S. 1850 , p. 220.

a ô Kordofan, 26 August (J. Petherick) 1848. 
2876. Otis ruficrista.

Otis ruficrista, Smith, Ill. Zool. S. Afr. pl. 4 : Strickl. \& Scl. Contr. Orn. 1852, p. 158.

Eupodotis ruficrista, Gurney in And. B. Damara-l. p. 259.

a Damara-land (C. J. Andersson) 1852.

\section{7\%. 0tis afra.}

Otis afra, Gm. Syst. Nat. i. p. 724: Schl. Mus. Pays-Bas, Cursores, p. 6.

Eupodotis afra, Layard, B. S. Afr. p. 286.

a South Africa (Arthur Strickland) 1850. -b (Arthur Strickland) 1850.

\section{Otis tetrax.}

Otis tetrax, Linn. Syst. Nat. i. p. 264 : Strickl. P. Z. S. 1836, p. 101 : Dresser, B. Eur. vii. p. 383, pl. 509.

a f Smyrna, 12 December (H. E. Strickland) 1835.

\section{Otis aurita.}

Otis aurita, Lath. Ind. Orn. ii. p. 660.

Spheotides auritus, Jerd. B. Ind. ii. p. 619.

a Madras ("Otis aurita," T. C. Jerdon) 1845. - b S. India (T. C. Jerdon) 1850. 


\section{ORDER XIV.-LIMICOLA.}

\section{FAMILY.-QEDICNEMIDA.}

Edicnemus, Temminck, Man. d'Orn. p. 321 (1815).

\section{Edicnemus scolopax.}

Charadrius oedicnemus, Linn. Syst. Nat. i. p. 255.

Charadrius scolopax, S. G. Gmelin, Reise iii. p. 87, pl. 16.

Edicnemus scolopax, Dresser, B. Eur. vii. p. 401, pl. 512.

EEdicnemus crepitans, Strickl. Ann. \& Mag. N. H. xiii. p. 39;

P. Z. S. 1836, p. 101 ; 1850, p. 220 (et auctt. ex Temminck).

$a$ (Kirtland) 1838. - $b$ Tangier (Favier) 1848. - $c$ q Kartoum, October (J. Petherick) 1848.

2881. Edicnemus affinis.

Edicnemus affinis, Rüpp. Mus. Senkenb. ii. p. 210 ; Syst. Ueb. p. 117, pl. 42: Strickl. P. Z. S. 1850, p. 220 ; Schl. Mus. Pays-Bas, Cursores, p. 21.

a Kordofan, July (J. Petherick) 1848.

\section{Edicnemus capensis.}

Edicnemus capensis, Licht. Verz. Doubl. p. 69 : Finsch \& Hartl. Vög. Ost. Afr. p. 624.

Edicnemus maculosus, Cuv. Règne An. i. p. 500: Layard, B. S. Afr. p. 288.

$a$ (Arthur Strickland) 1850.

A South-African species.

2883. Edicnemus grallarius.

Charadrius grallarius, Lath. Ind. Orn. Suppl. p. Ixvi.

OEdicnemus grallarius, Gould, B. Austr. vi. pl. 5: Strickl. Ann. \& Mag. N. H. xi. p. 337.

a Australia (McDonald) 1838. 
2884. Edicnemus bistriatus.

Charadrius bistriatus, Wagl. Isis, 1829, p. 648.

Edicnemus bistriatus, Scl. \& Salv. Ex. Orn. p. 60.

$a$ Guatemala (J. Constancia) 1851.

\section{Edicnemus superciliaris.}

Edicnemus superciliaris, Tsch. Arch. f. Naturg. 1843. i. p. 387 : Scl. \& Salv. Ex. Orn. p. 59, pl. 30.

$a$ (Thomas) 1842.

A species of Western Peru.

\section{Esacus.}

Esacus, Lesson, Traité d'Orn. p. 547 (1831).

\section{Esacus recurvirostris.}

Edicnemus recurvirostris, Cuv. Règne An. i. p. 500: Jerd. B. Ind. ii. p. 652 .

$a$ (Carfrae) 1840.

A species of India, Ceylon, \&c.

\section{FAMILY.-PARRID $Æ$.}

\section{PARra.}

Parra, Linnæus, Syst. Av. i. p. 259 (1766) (partim). 288\%. Parra jacana.

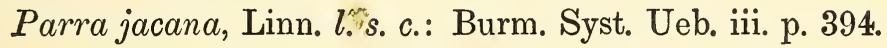

$a$ (Wood) 1840. - b S. America (Bt at Stevens's) 1844. -c, —d (Arthur Strickland) 1850. —e Trinidad (Argent) 1851.

\section{Parra hypomelæna.}

Parra hypomeloena, Gray \& Mitch. Gen. B. iii. p. 589, pl. 159.

Parra nigra, Schl. Mus. Pays-Bas, Ralli, p. 65 (ex Gmelin). $a$ Bogota? (Bt at Stevens's) 1845.

A species of the United States of Colombia. 


\section{Metopidius.}

Metopidius, Wagler, Isis, 1832, p. 279.

2889. Hetopidius africanus.

Parra africana, Gm. Syst. Nat. i. p. 709 : Layard, B. S. Afr. p. 335 : Schl. Mus. Pays-Bas, Ralli, p. 69.

$a$ (Mansfield) 1839. -b (Green) 1849.

A South-African species.

2890. Metopidius indicus.

Parra indica, Lath. Ind. Orn. ii. p. 765 : Schl. Mus. PaysBas, Ralli, p. 67.

Metopidius indicus, Jerd. B. Ind. ii. p. 708.

$a,-b$ India (Havell) 1839. -c.

\section{Hydrophasianus.}

Hydrophasianus, Wagler, Isis, 1832, p. 279.

\section{Hydrophasianus chirurgus.}

Tringa chirurgus, Scop. Del. Fl. et Faun. Ins. ii. p. 92.

Hydrophasianus chirurgus, Jerd. B. Ind. ii. p. 709.

Parra sinensis, Schl. Mus. Pays-Bas, Ralli, p. 71 (ex Gmelin).

$a$ (Havell) 1839. - b India (Bt at Stevens's) 1843. - c Madras (T. C. Jerdon) 1845 .

\section{FAMILY.-GLAREOLID无。}

\section{Glareola.}

Glareola, Brisson, Orn. v. p. 141 (1760).

\section{Glareola pratincola.}

Hirundo pratincola, Linn. Syst. Nat. i. p. 345.

Glareola pratincola, Dresser, B. Eur. vii. p. 411, pl. 513.

Glareola torquata, Strickl. P. Z. S. 1836, p. 101 (ex Meyer). Glareola limbata, Strickl. P. Z. S. 1850, p. 220 (ex Rüppell). $a \hat{\delta},-b$ o Smyrna, April (H. E. Strickland) 1836. -c o Kartoum, November (J. Petherick) 1848. 
2893. Glareola orientalis.

Glareola orientalis, Leach, Trans. L. S. xiii. p. 132, pl. 13 : Jerd. B. Ind. ii. p. 631.

$a,-b$ f India (W. J. E. Boys) 1847. -c India ("Is this torquata or orientalis?" E. Blyth) 1851.

2894. Glareola lactea.

Glareola lactea, Temm. Pl. Col. 399 : Jerd. B. Ind. ii. p. 632. $a$ (N. C. Strickland) 1838. - b India ("Glareola orientalis,"

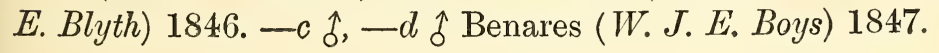

\section{FAMILY.-CHARADRIIDA.}

\section{CURSORIUS.}

Cursorius, Latham, Ind. Orn. ii. p. 751 (1790).

\section{Cursorius gallicus.}

Charadrius gallicus, Gm. Syst. Nat. i. p. 692.

Cursorius gallicus, Strickl. Ann. \& Mag. N. H. viii. p. 544 : Dresser, B. Eur. vii. p. 425, pl. 514.

Cursorius isabellinus, Meyer, Tasch. deutsch. Vög. ii. p. 328.

a ô Susa, Tunis, June (L. Fraser) 1846.

2896. Cursorius coromandelicus.

Charadrius coromandelicus, Gm. Syst. Nat. i. p. 692.

Cursorius coromandelicus, Jerd. B. Ind. ii. p. 626 : Schl. Mus. Pays-Bas, Cursores, p. 13.

a India (Lord A. Hay) 1845. - b Benares (W. J. E. Boys) 1847. —c (Arthur Strickland) 1850.

289\%. Cursorius bicinctus.

Cursorius bicinctus, Temm. Man. d'Orn. ii. p. 515 : Jard. \& Selb. Ill. Orn. i. pl. 48 : Layard, B. S. Afr. p. 290.

a Africa (Arthur Strickland) 1850.

\section{RHINOPTILUS.}

Rhinoptilus, Strickland, P. Z. S. 1850, p. 220. 2898. Rhinoptilus chalcopterus.

Cursorius chalcopterus, Temm. Pl. Col. 298: Schl. Mus. Pays-Bas, Cursores, p. 12.

S. C. 
Rhinoptilus chalcopterus, Strickl. P. Z. S. 1850, p. 220. a $\hat{o}$ Kordofan, August (J. Petherick) 1848.

\section{Rhinoptilus bitorquatus.}

Macrotarsius bitorquatus, Blyth, J. A. S. B. xvii. p. 254 (ex Jerdon MS.).

Rhinoptilus bitorquatus, Strickl. P. Z. S. 1850, p. 220 : Jerd. B. Ind. ii. p. 628.

a Madras ("Charadrius bicinctus," T. C. Jerdon) 1846.

\section{Charadrius.}

Charadrius, Linn. Syst. Nat. i. p. 253 (1766).

2900. Charadrius pluvialis.

Charadrius pluvialis, Linn. l. s. c. p. 254: Dresser, B. Eur. vii. p. 435 , pl. 515 .

a Norway (Dunn) 1838. -b Britain (N. C. Strickland) -c.

\section{Charadrius virginicus.}

Charadrius virginicus, Bechst. Allg. Ueb. der Vög. iii. p. 455 (1812): Coues, Birds of the N. W. p. 448.

$a$ (Askew) 1837. - b Madras (Bt at Stevens's) 1843. — c Canada (Barnes) 1848. - d Lima (Arthur Strickland) 1850. -e (Arthur Strickland) 1850. $-f,-g$.

\section{Squatarola.}

Squatarola, Leach, Syst. Cat. M. \& B. Brit. Mus. p. 29 (1816). 2902. Squatarola helvetica.

Tringa helvetica, Linn. Syst. Nat. i. p. 250.

Squatarola helvetica, Strickl. Ann. \& Mag. N. H. vii. p. 37; P. Z. S. 1850, p. 220: Strickl. \& Scl. Contr. Orn. 1852, p. 158: Dresser, B. Eur. vii. p. 455, pls. 515, 517, 518, 519.

a $\hat{\delta}$ Oxfordshire, February (H. E. Strickland) 1832. -b (Askew) 1837. —c o Kartoum, October (J. Petherick) 1848. -d Damara-land (C. J. Andersson) 1852. 


\section{EUdROMIAS}

Eudromias, C. L. Brehm, Vög. Deutschl. p. ว̌t4 (1831). 2903. Eudromias morinellus.

Charadrius morinellus, Linn. Syst. Nat. i. p. 254.

Eudromias morinellus, Dresser, B. Eur. vii. p. 507, pl. 526. $a,-b$.

A European species.

\section{Eudromias australis.}

Charadrius australis, Gould, P.Z. S. 1840, p. 174.

Eudromias australis, Gould, B. Austr. vi. p. 15.

a Australia (T. C. Eyton) 1849.

\section{EGialitis.}

Agialitis, Boie, Isis, 1826, p. 978.

\section{5, Egialitis asiatica.}

Charadrius asiaticus, Pall. Reise, ii. p. 715.

Eudromias asiaticus, Harting, Ibis, 1870, p. 202.

Agialitis asiatica, Dresser, B. Eur. vii. p. 479, pl. 522.

Charadrius damarensis, Strickl. \& Scl. Contr. Orn. 1852, p. 158.

a $\hat{\delta}$ Kirghis Steppes (J. F. Brandt) 1846, -b Damara-land (C. J. Andersson) 1852.

$b$ is the type of $C$. damarensis, Strickl.

2906. Ifgialitis veredus.

Charadrius veredus, Gould, P. Z. S. 1848, p. 38; B. Austr. vi. pl. 14.

Eudromias veredus, Harting, Ibis, 1870, p. 209.

$a$ (Dewgard) 1851.

A species of Eastern Asia and Australia.

\section{0\%. Ëgialitis geoffroyi.}

Charadrius geoffroyi, Wagl. Syst. Av. fol. 4, p. 13.

Egialitis geoffroyi, Jerd. B. Ind. ii. p. 638: Harting, Ibis, 1870, p. 378: Dresser, B. Eur. vii. p. 475, pl. 521.

$a$ Madras (T. C. Jerdon) 1845. - b India ("Hiaticula geoffroyi, winter dress" E. Blyth) 1846. 


\section{8. सEgialitis mongolica.}

Charadrius mongolus, Pall. Reise, iii. p. 700.

EEgialitis mongolica, Harting, Ibis, 1870, p. 384.

Egialitis pyrrhothorax, Jerd. B. Ind. ii. p. 639 (ex Temm.).

a Madras (T. C. Jerdon) 1845. - b, -c India ("Hiaticula leahenaltii," E. Blyth) 1846. -d Darjeeling (E. Blyth) 1850. -e, -f S. India (T. C. Jerdon) 1850. -g (Arthur Strickland) 1850. $-h,-i$.

\section{Flgialitis hiaticula.}

Charadrius hiaticula, Linn. Syst. Nat. i. p. 253: Strickl. P. Z. S. 1850, p. 221: Strickl. \& Scl. Contr. Orn. 1852, p. 158.

Agialitis hiaticula, Dresser, B. Eur. vii. p. 497, pl. 525.

a o Aberdovey, N. Wales, July (H. E. Strickland) 1833. —b (Askew) 1841. — c Britain (Askew) 1841. —d S. Africa (Bt at Stevens's) 1843. —e Kordofan, February (J. Petherick) 1848. - $f$ Damara-land (C. J. Andersson) 1852.

\section{0. \#gialitis curonica.}

Charadrius curonicus, Gm. Syst. Nat. i. p. 692.

Egialitis curonica, Dresser, B. Eur. vii. p. 491, pl. 524.

Charadrius dubius, Scop. Del. Fl. et Faun. Insubr. ii. p. 93.

Charadrius minor, Wolf \& Meyer, Vög. Deutschl. Heft 15. pl. 5.

Charadrius pusillus, Horsf. Trans. L. S. xiii. p. 187.

$a,-b$ Luzon (H. Cuming) 1839. — $c$ Spain (Boissoneau) 1839. - d Madras (T. C.Jerdon) 1845. - e Nepal (B. H. Hodgson, 628) 1845. - $f$ India ("Hiaticula curonica," E. Blyth) 1846. -g o India (W. J. E. Boys) 1847. - $h$ Malacca (Arthur Strickland) 1850.

\section{1. Ëgialitis cantiana.}

Charadrius cantianus, Lath. Ind. Orn. Suppl. p. Ixvi.

Egialitis cantiana, Dresser, B. Eur. vii. p. 483, pl. 523.

Charadrius alexandrinus, Linn. Syst. Nat. i. p. 253 ? Strickl. P. Z. S. 1850, p. 221.

$a$ Luzon, 14 June (H. Cuming) 1839. - b Philippine Islands, 13 May (H. Cuming) 1839. —c (Askew) 1841. —d ô India (W. J. E. Boys) 1847. - q Kordofan (J. Petherick) 1848. 


\section{Egialitis nivosa.}

Egialitis (Leucopolius) nivosa, Cassin, in Baird's Birds N. Am. p. 696.

Egialitis cantiana var. nivosa, Coues, Birds of the N. W. p. 456 .

$a$ Chili (Capt. Brown).

2913. झ年ialitis collaris.

Charadrius collaris, Vieill. N. Dict. d'Hist. N. xxvii. p. 136 : Schl. Mus. Pays-Bas, Cursores, p. 37.

Charadrius azarce, Licht. Verz. Doubl. p. 71: Cab. in Schomb. Reise n. Guiana, iii. p. 751. 1850.

a ô Guiana (R. Schomburgk) 183- . - b (Arthur Strickland)

\section{4. Ægialitis meloda.}

Charadrius melodus, Ord, ed. Wils. Am. Orn. vii. p. 71: Coues, Birds of the N. W. p. 455 .

$a \mathrm{~N}$. America (Askew) 1837.

2915. झigialitis semipalmata.

Charadrius semipalmatus, Bp. Ann. Lyc. N. Y. ii. p. 296.

Egialitis semipalmata, Coues, Birds of the N. W. p. 453.

$a$ Coast of New Jersey (S. F. Baird) 1847.

\section{6. झEgialitis wilsonia.}

Charadrius wilsonius, Ord, ed. Wils. Arn. Orn. ix. p. 77, pl. 73, f. 5 .

AEgialitis wilsonia, Coues, Birds of the N. W. p. 456.

a America (Askew) 1834. -b (Bt at Stevens's) 1847.

\section{1\%. IEgialitis falklandica.}

Charadrius falklandicus, Lath. Ind. Orn. ii. p. 747 : Schl. Mus. Pays-Bas, Cursores, p. 36.

$a$ (Williams) 1846.

A species of South-America and the Falkland Islands.

\section{8. झ世gialitis vocifera.}

Charadrius vociferus, Linn. Syst. Nat, i. p. 253.

Egialitis vocifera, Coues, Birds of the N. W. p. 4.52. 
a fิ Peru (Mather) 1840. -b N. America (Thomas) 1842. -c Carlisle, Pennsylvania, January 1845 (S. F. Baird) 1847. - $d$ Guatemala (J. Constancia) 1848. —e Chilị (Arthur Strickland) 1850.

\section{Fisialitis marginata.}

Charadrius marginatus, Vieill. N. Dict. d'Hist. N. xxvii. p. 138.

AEgialites marginatus, Gurney in And. B. Damara-l. p. 272. Charadrius leucopolius, Wagl. Syst. Av. fol. 5, p. 1.

Charadrius niveifrons, Less. Traité d'Orn. p. 544: Strickl. \& Scl. Contr. Oru. 1852, p. 159.

a Damara-land (C.J. Andersson) 1852.

\section{Figialitis pallida.}

Charadrius pallidus, Strickl. Contr. Orn. 1852, p. 158.

a Damara-land (C. J. Andersson) 1852.

Mr Gurney thinks that the type of C. pallidus, Strickl, is a very small specimen of $\mathbb{E}$. marginata and unusually pale and grey in its coloration. The differences appear to me to be more than those of a mere variety.

2921. Frialitis tricollaris.

Charadrius tricollaris, Vieill. N. Dict. d'Hist. N. xxvii. p. 147: Layard, B. S. Afr. p. 296.

$a$ S. Africa (Bt at Aberystwith) 1833. - $b$ S. Africa (Bt at Stevens's) 1843._c S. Africa (Gardner) 1846. —d (Arthur Strickland) $1850 .-e$.

\section{Fialitis varia.}

Charadrius varius, Vieill. N. Dict. d'Hist. N. xxvii. p. 143.

AEgialitis varius, Harting, Ibis, 1873, p. 262, pl. 8.

Charadrius pecuarius, Temm. Pl. Col. 183: Strickl. P. Z. S. 1850, p. 221. 1848.

$a$ (Bt at Stevens's) 1847. - b Kordofan, February (J.Petherick)

\section{Wgialitis bicincta.}

Charadrius bicinctus, Jard. et Selb. Ill. Orn. i. pl. 28.

Hiaticula bicincta, Gould, B. Austr. vi. pl. 16.

a New South Wales (McDonald) 1838. 


\section{4. झ出ialitis ruficapillus.}

Charadrius ruficapillus, Temm. Pl. Col. 47, fig. 2.

Hiaticula ruficapilla, Gould, B. Austr. vi. pl. 17.

$a$ (Askew) 1834. - b (Argent) 1851.

An Australian species.

2925. झझgialitis melanops.

Charadrius melanops, Vieill. N. Dict. d'Hist. N. xxvii. p. 139. Hiaticula nigrifrons, Gould, B. Austr. vi. pl. 20 (ex Cuv. et Temm.).

$a$ New South Wales $\left(M^{c}\right.$ Donald) 1838. $-b,-c$ 。

\section{ERYTHROGONYS.}

Erythrogonys, Gould, P. Z. S. 1837, p. 155.

2926. Erythrogonys cinctus.

Erythrogonys cinctus, Gould, l. s. c.; B. Austr. vi. pl. 21.

a Australia (Arthur Strickland) 1850.

\section{Chetrusia.}

Chettusia, Bonaparte, Icon. Faun. Ital. Intr. fol. ******, p. 6 (1841).

\section{Chettusia leucurus.}

Charadrius leucurus, Licht. in Eversm. Reise n. Buch. p. 137.

Chettusia leucura, Dresser, B. Eur. vii. p. 531, pl. 529.

a f Kirghis Steppes (J. F. Brandt) 1846.

\section{Chettusia gregaria.}

Charadrius gregarius, Pall. Reise, i. p. 456.

Chettusia gregaria, Jerd. B. Ind. ii. p. 644: Dresser, B. Eur. vi. p. 527 , pl. 528 .

Chotusia gregaria, Strickl. P. Z. S. 1850, p. 221.

a Altai (J. F. Brandt) 1842. - b o India (W. J. E. Boys) 1847. - c \& Kartoum, October (J. Petherick) 1848.

\section{Chettusia inornata.}

Lobivanellus inornatus, Temm. \& Schl. Faun. Jap. Aves, p. 106, pl. 63.

Chettusia inornata, Jerd. B. Ind. ii. p. 646. 
Lobivanellus cinereus, Blyth, Cat. B. Mus. As. Soc. p. 261. a India ("Lobivanellus cinereus" E. Blyth) 1846.

2930. Chettusia coronata.

Charadrius coronatus, Gm. Syst. Nat. i. p. 691.

Hoplopterus coronatus, Strickl. \& Scl. Contr. Orn. 1852, p. 159.

Chettusia coronata, Gurney in And. B. Damara-1. p. 268.

$a$ (Dewgard) 1849. - b Damara-land (C. J. Andersson) 1852. $-c$.

\section{Pluvianus.}

Pluvianus, Vieillot, N. Dict. d'Hist. N. xxvii. p. 129 (1818). 2931. Pluvianus ægyptius.

Charadrius cegyptius, Linn. Syst. Nat. i. p. 254.

Pluvianus agyptius, Strickl. Ann. \& Mag. N. H. vii. p. 37; xiii. p. 39 ; P. Z. S. 1850 , p. 221 : Dresser, B. Eur. vii. p. 521, pl. 527.

$a$ Africa (Seaman) 1840. - $b$ \& Kartoum, November $(J$. Petherick) 1848.

\section{VANELLUS.}

Vanellus, Brisson, Orn. V. p. 94 (1760).

2932. Vanellus vulgaris.

Tringa vanellus, Linn. Syst. Nat. i. p. 248.

Vanellus vulgaris, Bechst. Orn. Taschenb. ii. p. 313: Dresser, B. Eur. vii. p. 545, pl. 531.

Vanellus cristatus, Wolf \& Meyer, et auctt. plurr. Strickl. P. Z. S. 1836, p. 101: 1841, p. 32.

a.

A common species in Europe and the British Islands.

2933. Vanellus cayennensis.

Parra cayennensis, Gm. Syst. Nat. i. p. 706.

Vanellus cayennensis, Strickl. P. Z. S. 1841, p. 32: Harting, P. Z. S. 1874 , p. 450.

$a$ South America (Havell) 1839. 
2934. Vanellus occidentalis.

Vanellus occidentalis, Harting, P. Z. S. 1874, p. 451.

$a$ Chili (Arthur Strickland) 1850.

\section{HOPLOPTERUS.}

Hoplopterus, Bonaparte, Sagg. Distr. An. Vertebr. p. 56 (1831).

\section{Hoplopterus spinosus.}

Charadrius spinosus, Linn. Syst. Nat. i. p. 256.

Hoplopterus spinosus, Dresser, B. Eur. vii. p. 539, pl. 530.

Charadrius persicus, Bonn. Tabl. Enc. Méth. p. 21.

Hoplopterus persicus, Strickl. P. Z. S. 1850, p. 221.

$a$ (Mansfield) 1839. - b Kordofan (J. Petherick) 1848.

\section{Hoplopterus ventralis.}

Charadrius ventralis, Wagl. Syn. Av. fol. 4, p. 11.

Hoplopterus ventralis, Jerd. B. Ind. ii. p. 650.

a (Arthur Strickland) 1850.

An Indian species.

\section{Hoplopterus cayanus.}

Charadrius cayanus, Lath. Ind. Orn. ii. p. 749.

Hoplopterus cayanus, Scl. \& Salv. P. Z. S. 1867, p. 591.

$a$ (Gardner) 1846. —b Para (S. Stevens) 1849.

$b$ is from Mr A. R. Wallace's collection.

\section{LobIVANELLUS.}

Lobivanellus, Strickland, P. Z. S. 1841, p. 33.

\section{Lobivanellus indicus.}

Vanneau armé, de Goa, D'Aub. PI. Enl. 807, undè, Tringa indica, Bodd. Tabl. Pl. Enl. p. 50.

Parra goensis, Gm. Syst. Nat. i. p. 706.

Lobivanellus goensis, Strickl. l. s. c.: Jerd. B. Ind. ii. p. 648.

a India (Askew) 1834. - b Malacca (Capt. Nash) 1837. -c Nepal (B. H. Hodgson, 228) 1845. 
2939. Lobivanellus lobatus.

Tringa lobata, Lath. Ind. Orn. Suppl. p. lxv.

Lobivanellus lobatus, Gould, B. Austr. vi. pl. 9.

Lobivanellus gallinaceus, Strickl. P. Z. S. 1841, p. 33 (ex Wagler).

a Australia (Thomas) 1842.

2940. Lobivanellus senegalus.

Parra senegala, Linn. Syst. Nat. i. p. 259.

Lobivanellus senegalus, Strickl. P. Z. S. 1841, p. 33.

Lobivanellus albicapillus, Strickl. P. Z. S. 1850, p. 221 (ex Vieillot).

$a$ W. Africa (Thomas) 1842. - b Kordofan, June (J. Petherick) 1848.

\section{SARCIOPHORUS.}

Sarciophorus, Strickland, P. Z. S. 1841, p. 32.

2941. Sarciophorus bilobus.

Charadrius bilobus, Gm. Syst. Nat. i. p. 691.

Sarciophorus bilobus, Strickl. l. s. c.: Jerd. B. Ind. ii. p. 649.

a Cape of Good Hope (Askew) 1834. - $b$ \% Kagudupore ( $W$. J. E. Boys) 1847.

The locality of $a$ is doubtless erroneous.

2942. Sarciophorus tectus.

Pluvier, du Senegal, D'Aub. Pl. Enl. 834, undè,

Charadrius tectus, Bodd. Tabl. Pl. Enl. p. 51.

Sarciophorus pileatus, Strickl. P. Z. S. 1841, p. 33; 1850, p. 221 (ex Gmelin).

$a$ (Mather) 1840.

An African species.

2943. Sarciophorus tricolor.

Charadrius tricolor, Vieill. N. Dict. d'Hist. N. xxvii. p. 147. Sarciophorus tricolor, Strickl. P. Z. S. 1841, p. 33.

Sarciophorus pectoralis, Gould, B. Austr. vi. pl. 11 (ex Cuvier).

$a$ New South Wales ( $M^{c}$ Donald) 1838. 


\section{Strepsilas.}

Strepsilas, Illiger, Prodromus, p. 263 (1811).

2944. Strepsilas interpres.

Tringa interpres, Linn. Syst. Nat. i. p. 248.

Strepsilas interpres, Dresser, B. Eur. vii. p. 555, pl. 532.

a Mersey, 11 September (H. E. Strickland). 1837. -b (Askew) 1837. —c Filey, Yorkshire, 16 August (H. E. Strickland) 1850. -d (Arthur Strickland) 1850.

\section{HAMATOPUS.}

Hcematopus, Linnæus, Syst. Nat. i. p. 257 (1766).

2945. Hæmatopus ostralegus.

Hcematopus ostralegus, Linn. l. s. c.: Dresser, B. Eur. vii. p. 567, pl. 533.

a Isle of Wight (H. E. Strickland) 1831.

\section{Hæmatopus palliatus.}

Hcematopus palliatus, Temm. Man. d'Orn. ii. p. 532: Baird, B. N. Am. p. 699.

a Chili (Capt. Brown) 1842.

\section{Hæmatopus longirostris.}

Hamatopus longirostris, Vieill. N. Dict. d'Hist. N. xv. p. 410: Gould, B. Austr. vi. pl. 7.

Hcematopus australasicus, Gould, P. Z. S. 1837, p. 155.

$a$ New South Wales ( $M^{c}$ Donald) 1838. - $b$ New South Wales (Bt at Birmingham) 1843.

\section{Hæmatopus fuliginosus.}

Hcematopus fuliginosus, Gould, B. Austr. vi. pl. 8. a (Arthur Strickland) 1850.

An Australian species.

\section{Hæmatopus ater.}

Hcematopus ater, Vieill. Gal. Ois. ii. p. 88, pl. 230: Scl. P. Z. S. 1860 , p. 386.

a Chili (Capt. Brown) 1842. 
2950. Hæmatopus niger.

Hoematopus niger, Pall. Zoogr. Rosso-As. ii. p. 131: Baird, B. N. Am. p. 700.

a Kodjak (J. F. Brandt) 1844.

\section{FAMILY.-THINOCORIDA.}

\section{Thinocorus.}

Thinocorus, Eschscholts, Zool. Atlas p. 2, pl. 2 (1829).

2951. Thinocorus rumicivorus.

Thinocorus rumicivorus, Eschsch. l. s. c.: Burm. La Plata Reise, ii. p. 501.

$a$ S. America (N. C. Strickland) 1838.

\section{FAMILY.-SCOLOPACIDE.}

ReCURVIROstra,

Recurvirostra, Linnæus, Syst. Nat. i. p. 256 (1766). 2952. Recurvirostra avocetta.

Recurvirostra avocetta, Linn. l. s. c.: Strickl. P. Z. S. 1836, p. 101 : Harting, Ibis, 1874, p. 245: Dresser, B. Eur. vii. p. 577, pl. 534 .

a f Smyrna, 14 November (H. E. Strickland) 1835.

\section{Recurvirostra novæ-hollandiæ.}

Recurvirostra nova-hollandice, Vieill. N. Dict. d'Hist. N. iii. p. 103: Harting, Ibis, 1874, p. 258.

Recurvirostra rubricollis, Temm. Man. d'Orn. ii. p. 592.

Recurvirostra rubricollis, Gould, B. Austr. vi. pl. 27. a Australia (Cashmore) 1839.

\section{Cladorhynchus.}

Cladorhynchus, Gray, List. Gen. B. p. 69 (1840). 2954. Cladorhynchus leucocephalus.

Recurvirostra leucocephala, Vieill. N. Dict. d'Hist. N. iii. p. 103. 
Cladorhynchus leucocephalus, Salv. Ibis, 1874, p. 252.

Leptorhynchus pectoralis, Du Bus, Bull. Ac. Brux. ii. p. 420, pl. 7.

Cladorhynchus pectoralis, Gould, B. Austr. vi. pl. 26. a Australia (W. Kirtland) 1846.

\section{Himantopus.}

Himantopus, Brisson, Orn. v. p. 33 (1760).

\section{Himantopus candidus.}

Charadrius himantopus, Linn. Syst. Nat. i. p. 255.

Himantopus candidus, Bonn. Tabl. Enc. Méth. p. 24: Dresser, B. Eur. vii. p. 587, pl. 535, 536.

Himantopus melanopterus, Strickl. \& Scl. Contr. Orn. 1852, p. 159 (ex Meyer).

$a$ Tuscany (E. Passerini) 1835. - b, -c India ("Himantopus melanopterus," E. Blyth) 1846. —d Damara-land (C. J. Andersson) 1852.

\section{Himantopus nigricollis.}

Himantopus nigricollis, Vieill. N. Dict. d'Hist. N. x. p. 42: Coues, Birds of the N. W. p. 462.

$a,-b$ Guatemala (J. Constancia) 1851.

295\%. Himantopus brasiliensis.

Himantopus brasiliensis, Brehm. Vög. Deutschl. p. 684: Scl. \& Salv. P. Z. S. 1873 , p. 454.

$a$ Chili (Capt. Brown) 1842.

\section{Phalaropus.}

Phalaropus, Brisson, Orn. vi. p. 12 (1760).

\section{Phalaropus hyperboreus.}

Tringa hyperborea, Linn. Syst. Nat. i. p. 249.

Phalaropus hyperboreus, Dresser, B. Eur. vii. p. 597, pl. 537.

Lobipes hyperboreus, Coues, Birds of the N. W. p. 469.

$a,-b$ Siberia (J. F. Brandt) 1846. —c o Kirghis Steppes, 8 April 1840 (J. F. Brandt) 1846. - d, -e Guatemala (J. Constancia) 1848. - $f$ Guatemala ( $J$. Constancia) 1851. 


\section{Phalaropus fulicarius.}

Tringa fulicaria, Linn. Syst. Nat. i. p. 249.

Phalaropus fulicarius, Coues, Birds of the N. W. p. 471: Dresser, B. Eur. p. 605, pl. 539.

a (J. F. Brandt) 1846. -b Oxfordshire, November (H. E. Strickland) 1846.

\section{SCOLOPAX.}

Scolopax, Linnæus, Syst. Nat. i. p. 242 (1766) partim. 2960. Scolopax rusticula.

Scolopax rusticola, Linn. l.s.c. p. 243: Strickl. P. Z. S. 1836, p. 101: Dresser, B. Eur. vii. p. 615, pl. 540.

a Worcestershire (T. Robinson) 1840. -b Nepal (B. H. Hodgson, 546) 1845. —c $\hat{o}$ Kumaon (W. J. E. Boys) 1847.

\section{Philohela.}

Philohela, Gray, List. Gen. B. p. 90 (1841): Strickl. Ann. \& Mag. N. H. viii. p. 374.

\section{Philohela minor.}

Scolopax minor, Gm. Syst. Nat. i. p. 661.

Philohela minor, Coues, Birds of the N. W. p. 472.

a N. America (Arthur Strickland) 1850. -b.

\section{Gallinago.}

Gallinago, Leach, Syst. Cat. Mamm. \& B. Brit. Mus. p. 30 (1816).

\section{Gallinago major.}

Scolopax major, Gm. Syst. Nat. i. p. 661.

Gallinago major, Dresser, B. Eur. vii. p. 631, pl. 541. 1831.

$a \hat{\delta},-b q$ Jerken, Norway, 8 August (N. C. Strickland)

\section{Gallinago cœlestis.}

Scolopax gallinago, Linn. Syst. Nat. i. p. 244 : Strickl. P. Z. S. 1836, p. 101; Ann. \& Mag. N. H. vii. p. 38.

Scolopax coelestis, Frenzel, Beschr. der Vög. Wittenb. p. 58.

Gallinago coelestis, Dresser, B. Eur. vii. p. 641, pl. 543.

a f Worcestershire, December (H. E. Strickland) 1840. -b 
Dumfriesshire (H. E. Strickland) 1845. —c (Bt at Stevens's) 1845. -d Calcutta ("Scolopax gallinago," E. Blyth) 1846. - e (Arthur Strickland) 1850.

2964. Gallinago wilsoni.

Scolopax wilsoni, Temm. Pl. Col. Livr. 68 (sub Pl. Col. 403). Gallinago wilsoni, Coues, Birds of the N. W. p. 475.

$a$ Carlisle, Pennsylvania 9 December, 1842 (S. F. Baird) 1847. - b Guatemala (J. Constancia) 1848.

\section{Gallinago paraguayæ.}

Scolopax paraguayce, Vieill. N. Dict. d'Hist. N. iii. p. 356.

Gallinago paraguayce, Scl. \& Salv. Ex. Orn. p. 196. a (Arthur Strickland) 1850.

A species of the Southern parts of South America.

2966. Gallinago frenata.

Scolopax frenata, Wied, Beitr. iv. p. 712.

Gallinago frenata, Scl. \& Salv. Ex. Orn. p. 196.

a "Brazil" (Johnson) 1837. -b (Arthur Strickland) 1850.

\section{6\%. Gallinago æquatorialis.}

Scolopax cequatorialis, Rüpp. Syst. Ueb. p. 123: Layard, B. S. Afr. p. 333.

Gallinago nigripennis, Finsch. \& Hartl. Vög. N. O. Afr. p. 769 (ex Bp.).

a S. Africa (Dewgard) 1849.

\section{Gallinago australis.}

Scolopax australis, Lath. Ind. Orn. Suppl. p. lxiv: Gould, B. Austr. vi. pl. 40.

$a$ New South Wales ( $M^{\circ}$ Donald) 1838. - b Australia (Bt at Stevens's) 1843. -c Australia (Bt at Birmingham) 1843.

\section{Gallinago stenura.}

Scolopax stenura, Bp. Osserv. Cuv. Règn. Anim. p. 121: Strickl. Ann. \& Mag. N. H. xiii. p. 40.

Gallinago stenura, Jerd. B. Ind. ii. p. 674.

Gallinago heterura v. biclavata, Hodgs. Zool. Misc. p. 86. a Malacca (Capt. Nash) 1837. -b Nepal (B. H. Hodgson, 544) 1845 


\section{9'70. Gallinago nemoricola.}

Gallinago nemoricola, Hodgs. J. A. S. B. vi. p. 490: Jerd. B. Ind. ii. p. 672.

Scolopax nemoricola, Strickl. Ann. \& Mag. N. H. xv. p. 275. a Nepal (B. H. Hodgson, 540) 1845.

\section{9'1. Gallinago solitaria.}

Gallinago solitaria, Hodgs. J. A. S. B. vi. p. 491 : Jerd. B. Ind. ii. p. 673.

a India ("Scolopax solitaria, Hodgson," E. Blyth) 1846.

\section{Gallinago gallinula.}

Scolopax gallinula, Linn. Syst. Nat. i. p. 244: Strickl. P. Z. S. 1836, p. 101; Ann. \& Mag. N. H. vii. p. 38.

Gallinago gallinula, Dresser, B. Eur. vii. p. 653, pl. 544.

$a$ Worcestershire (T. Robinson) 1840. —b Dumfriesshire, November (H. E. Strickland) 1845. —c India ("Scolopax gallinula," E. Blyth) 1846.

\section{RHYNCHEA.}

Rhynchcea, Cuvier, Règn. An. i. p. 523 (1829).

\section{9'3. Rhynchæa capensis.}

Scolopax capensis, Linn. Syst. Nat. i. p. 246.

Rhynchwa capensis, Strickl. \& Scl. Contr. Orn. 1852, p. 159 : Layard, B. S. Afr. p. 334.

Rhynchcea bengalensis, Jerd. B. Ind. ii. p. 677 (ex Linn.).

a Cape of Good Hope, December (A. Strickland) 1833. - b Cape of Good Hope (Bt at Stevens's) 1843. - c, - d Madras (T. C. Jerdon) 1845. - e, -f (Arthur Strickland) 1850. -g Damara-land (C. J. Andersson) 1852.

29'74. Rhynchæa semicollaris.

Rhynchcea semicollaris, Vieill. N. Dict. d'Hist. N. vi. p. 402. Rhynchoea hilaerea, Burm. Syst. Ueb. iii. p. 378 (ex Valenc.). a Valparaiso (Capt. Brown) 1842. 


\section{Limicola.}

Limicola, Koch, Baier. Zool. i. p. 316 (1816).

\section{Limicola platyrhyncha.}

Tringa platyrincha, Temm. Man. d'Orn. p. 398.

Limicola platyrhyncha, Dresser, B. Eur. viii. p. 3, pl. 545.

a Pomerania (J. G. Kinberg) 1845. -b, -c, -d India ("Tringa platyrhyncha," E. Blyth) 1846. -e S. India (T. C. Jerdon) 1850.

\section{Limicola sibirica.}

Limicola sibirica, Dresser, P. Z. S. 1876, p. 674.

$a$ Siberia (J. F. Brandt) 1846.

The specimen referred to by $\mathrm{Mr}$ Dresser when describing this species.

\section{Tringa.}

Tringa, Linnæus, Syst. Nat. i. p. 247 (1766) partim.

\section{7\%. Tringa minutilla.}

Tringa minutilla, Vieill. N. Dict. d'Hist. N. xxxiv. p. 466: Coues, Birds of the N. W. p. 482: Dresser, B. Eur. viii. p. 51, pl. 552, figs. 2,3 .

$a$ Carlisle, Pennsylvania, 5 August 1845 (S. F. Baird) 1847. -b Guatemala (J. Constancia) 1848. —c Chili (Arthur Strickland) 1850.

\section{9\%8. Tringa temmincki.}

Tringa temminckii, Leisler, Nachtr. z. Bechst. Nat. Deutschl. p. 64: Strickl. P. Z. S. 1836, p. 101 : Dresser, B. Eur. viii. p. 45, pl. $549,550,551$.

a f Smyrna, 27 December (H. E. Strickland) 1835. - b India ("Tringa temmincki," E. Blyth) 1846. —c i (W. J. E. Boys) 1847.

2979. Tringa ruficollis.

Tringa ruficollis, Pall. Reise, iii. p. 700.

Tringa salina, Swinhoe, P. Z. S. 1871, p. 409.

a (W. Kirtland) 1846.

A species of Eastern Asia.

S. C. 


\section{Tringa minuta.}

Tringa minuta, Leisler, Nachtr. z. Bechst. Nat. Deutschl. p. 74: Dresser, B. Eur. viii. p. 29, pls. 549, 550, 551.

Pelidna minuta, Strickl. \& Scl. Contr. Orn. 1852, p. 159.

a Devonshire (Mansfield) 1834. - b (Bt at Stevens's) 1843. -c Nepal (B. H. Hodgson, 629) 1845. —d India (E. Blyth) 1846. - e India ("Tringa minuta," E. Blyth) 1846. - $f$ q Deypalpore (W. J. E. Boys) 1847. -g ô Kartoum, October (J. Petherick) 1848. - h Damara-land (C. J. Andersson) 1852.

2981. Tringa maculata.

Tringa maculata, Vieill. N. Dict. d'Hist. N. xxxiv. p. 465: Coues, Birds of the N. W. p. 486: Dresser, B. Eur. viii. p. 11, pl. 546 .

a ô Carlisle, Pennsylvania, 3 April 1844 (S. F. Baird) 1847. —b (W. Kirtland) 1847. —c (Arthur Strickland) 1850.

\section{Tringa acuminata.}

Totanus acuminatus, Horsf. Trans. L. S. xiii. p. 192.

Schoniclus acuminatus, Gould, B. Austr. vi. pl. 30.

$a,-b$ New South Wales (McDonald) 1838.

2983. Tringa canutus.

Tringa canutus, Linn. Syst. Nat. i. p. 251: Dresser, B. Eur. viii. p. 77 , pls. 555,556 .

a Liverpool, 11 September (H. E. Strickland) 1837. -b Britain (N. C. Strickland) 1838. - c Britain (H. E. Strickland). 2984. Tringa alpina.

Tringa alpina, Linn. Syst. Nat. i. p. 249: Dresser, B. Eur. viii. p. 21 , pl. 548 .

Tringa cinclus, Linn. Syst. Nat. i. p. 251.

Pelidna cinclus, Strickl. Ann. \& Mag. N. H. viii. p. 375.

Tringa variabilis, Strickl. P. Z. S. 1836, p. 101 (ex Meyer).

$a \hat{\delta},-b$ Aberdovey, N. Wales, August (H. E. Strickland) 1833. - c ô Smyrna, 1 December (H. E. Strickland) 1835. -d (W. Kirtland) 1847. -e, $-f$ Britain (H. E. Strickland).

2985. Tringa striata.

Tringa striata, Linn. Syst. Nat. i. Add.: Dresser, B. Eur. viii. p. 69 , pl. 554 . 
Tringa maritima, Brünn. Orn. Bor. p. 54: Coues, Birds of the N. W. p. 488.

a Britain (N. C. Strickland) 1838.

\section{Tringa subarquata.}

Scolopax subarquata, Güld. Nov. Comm. Petr. xix. p. 471, pl. 18.

Tringa subarquata, Dresser, B. Eur. viii. p. 59, pl. 553.

Pelidna subarquata, Strickl. Ann. \& Mag. N. H. viii. p. 375; P. Z. S. 1850, p. 221 : Strickl. \& Scl. Contr. Orn. 1852, p. 159.

a Mersey, 11 September (H. E. Strickland) 1837. - b South Africa (Bt at Stevens's) 1843. — India ("Tringa subarquata," E. Blyth) 1846. - d Kordofan (J. Petherick) 1848. - e Damaraland (C. J. Andersson) 1852.

\section{Ereunetes.}

Ereunetes, Illiger, Prodr. p. 262 (1811). 298\%. Ereunetes pusillus.

Tringa pusilla, Linn. Syst. Nat. i. p. 252.

Ereunetes pusillus, Coues, Birds of the N. W. p. 481.

$a$ Coast of New Jersey (S. F. Baird) 1847.

\section{Calidris.}

Calidris, Cuvier, Leç. d'Anat. Comp. i. tab. ii. (1800). 2988. Calidris arenaria.

Tringa arenaria, Linn. Syst. Nat. i. p. 251.

Calidris arenaria, Dresser, B. Eur. viii. p. 101, pls. 559, 560.

Charadrius calidris, Linn. Syst. Nat. i. p. 255.

$a$ N. America (Havell) 1839. - b United States (S. F. Baird), 1847. —o (Arthur Strickland) 1850.

\section{Machetes.}

Machetes, Cuvier, Règne An. i. p. 490 (1817).

\section{Machetes pugnax.}

Tringa pugnax, Linn. Syst. Nat. i. p. 247.

Machetes pugnax, Strickl. Ann. \& Mag. N. H. xii. p. 421; 
P. Z. S. 1850 , p. 221: Strickl. \& Scl. Contr. Orn. 1852, p. 159 : Dresser, B. Eur. viii. p. 87, pls. 557, 558.

$a$ (Arthur Strickland) 1840. -b i Kartoum, October ( $J$. Petherick) 1848. —c, -d S. India (T. C. Jerdon) 1850. -e (Arthur Strickland) 1850. -f Damara-land (C. J. Andersson) 1852.

\section{Actiturus.}

Actidurus, Bonaparte, Sagg. Distr. Metod. Agg. e Correz. p. 85 (1832).

\section{Actiturus longicauda.}

Tringa longicauda, Bechst. Allg. Ueb. der Vög. iv. p. 453. Actiturus longicaudus, Dresser, B. Eur. viii. p. 119, pl. 562. Tringa bartramia, Wils. Am. Orn. vii. p. 63, pl. 59, fig. 2. Actiturus bartramius, Coues, Birds of the N. W. p. 502. a America (Gardner) 1844. - b o Brazil? (Argent) 1852. $b$ is probably from Bolivia.

\section{Tringoides.}

Tringoides, Bonaparte, Sagg. Distr. An. Vert. p. 58 (1831). 2991. Tringoides hypoleucus.

Tringa hypoleucos, Linn. Syst. Nat. i. p. 250.

Totanus hypoleucos, Strickl. P. Z. S. 1850, p. 221 : Dresser, B. Eur. viii. p. 127, pl. 563.

$a \hat{\delta},-b$ f Aberdovey, August (H. E. Strickland) 1833. - c (Askew) 1837. - d Philippine Islands, 13 May (H. Cuming) 1840. - e q Malwa (W. J. E. Boys) 1847. - $f$ Kordofan (J. Petherick) 1848. -g S. India ("Totanus hypoleucus," T. C. Jerdon) 1850.

\section{Tringoides macularius.}

Tringa macularia, Linn. Syst. Nat. i. p. 249.

Tringoides macularius, Coues, Birds of the N. W. p. 501.

a America (Cashmore) 1839. - b Mexico (T. Mann) 1844. -c ô Mexiana, December 1848 (A. R. Wallace) 1849. -d (Arthur Strickland) 1850. 


\section{Totanus.}

Totanus, Bechstein, Orn. Taschenb. ii. p. 282 (1803).

\section{Totanus calidris.}

Scolopax calidris, Linn. Syst. Nat. i. p. 245.

Totanus calidris, Strickl. P. Z. S. 1836, p. 101 : Dresser, B. Eur. viii. p. 157, pls. 567, 568, 569.

a f Smyrna, 10 December (H. E. Strickland) 1835. -b S. India (T. C. Jerdon) 1850. —c (Arthur Strickland) 1850. -d Britain (H. E. Strickland).

\section{Totanus fuscus.}

Scolopax fusca, Linn. Syst. Nat. i. p. 243.

Totanus fuscus, Dresser, B. Eur. viii. p. 165, pls. 567, 568, 569.

a Britain? (N. C. Strickland) 1838. - b India ("Totanus fuscus," E. Blyth) 1846. —c o Meywar, India (W. J. E. Boys) 1847. -d India ("Totanus fuscus," T. C. Jerdon) 1850.

\section{Totanus flavipes.}

Scolopax flavipes, Gm. Syst. Nat. i. p. 659.

Totanus flavipes, Coues, Birds of the N. W. p. 497.

Gambetta flavipes, Scl. \& Salv. P. Z. S. 1867, p. 592.

$a$ (Askew) 1837. - $b$ o Carlisle, Pennsylvania, 8 May 1844 (S. F. Baird) 1847. —c \& Mexiana, January 1849 (A. R. Wallace) 1849. - d, -e (Arthur Strickland) 1850.

\section{Totanus canescens.}

Scolopax totanus, Linn. Syst. Nat. i. p. 245.

Scolopax canescens, Gm. Syst. Nat. i. p. 668.

Totanus canescens, Strickl. P. Z. S. 1850, p. 221: Dresser, B. Eur. viii. p. 173, pl. 570 .

Glottis canescens, Strickl. \& Scl. Contr. Orn. 1852, p. 159.

Totanus glottis, Strickl. P. Z. S. 1836, p. 101 (ex Bechstein). a Alnwick Fen, Lincolnshire, 20 August (H. E. Stricklaid) 1828. - -b 个 Jerken, Norway, 8 August (N. C. Strickland) 1831. -c $\hat{0}$, -d $q$ Madras (A. Strickland) 1833. - e ô Smyrna, 4 January (H. E. Strickland) 1836. - $f$ India ("Totanus glottis, T. glottoides, Vig." E. Blyth) 1846. -g India (E. Blyth) 1846. 
- $h$ Kordofan (J. Petherick) 1848. - i Damara-land (C. J. Andersson) 1852. - $j$.

\section{9'\%. Totanus melanoleucus.}

Scolopax melanoleuca, Gm. Syst. Nat. i. p. 659.

Totanus melanoleucus, Gosse, B. Jam. p. 352: Coues, Birds of the N. W. p. 496.

a Jamaica (P. H. Gosse) 1856. -b (Arthur Strickland) 1850. $-c$.

\section{Totanus semipalmatus.}

Scolopax semipalmata, Gm. Syst. Nat. i. p. 659.

Totanus semipalmatus, Coues, Birds of the N. W. p. 494.

a $\hat{\delta}$ Jamaica (P. H. Gosse) 1846. $-b$ (Arthur Strickland) 1850. $-c,-d$.

\section{Totanus ochropus.}

Tringa ochrophus, Linn. Syst. Nat. i. p. 250.

Totanus ochropus, Strickl. P. Z. S. 1836, p. 101: Dresser, B. Eur. viii. p. 135, pl. 564.

a Nepal (B. H. Hodgson, 743) 1845. - b o Oxford, 3 January 1833 (Arthur Strickland) 1850. -c.

3000. Totanus solitarius.

Tringa solitaria, Wils. Am. Orn. vii. p. 5อ, pl. 58, f. 3.

Totanus solitarius, Coues, Birds of the N. W. p. 498.

Totanus chloropygius, Vieill. N. Dict. d'Hist. N. vi. p. 401.

a N. America (Johnson) 1837. -b, -c, -d (Arthur Strickland) 1850. -e.

\section{Totanus glarcola.}

Tringa glareola, Linn. Fauna Suec. p, 65.

Tringa ochrophus $\beta$ glareola, Linn. Syst. Nat. i. p. 250.

Totanus glareola, Strickl. Ann. \& Mag. N. H. xiii. p. 40; xiv. p. 120: Strickl. \& Scl. Contr. Orn. 1852, p. 159: Dresser, B. Eur. viii. p. 143, pl. 565 .

a Malacca (Capt. Nash) 1837. -b N. Europe (J. G. Kinberg) 1843. — c f India (W. J. E. Boys) 1847. —d Damara-land (C. J. Andersson, 1847. - e India (Major Stacy). $-f,-g$. 


\section{Totanus stagnatilis.}

Totanus stagnatilis, Bechst. Orn. Taschenb. p. 292: Dresser, B. Eur. viii. p. 151, pl. 566 .

$a$ India ("Totanus stagnatilis, Limosa horsfieldi, Sykes," $E$. Blyth) 1846.

\section{MaCRORHAMPHUS.}

Macrorhamphus, Leach, Syst. Cat. Mamm. \& B. Brit. Mus. p. 31 (1831).

\section{Macrorhamphus griseus.}

Scolopax grisea, Gm. Syst. Nat. i. p. 658.

Macrorhamphus griseus, Coues, Birds of the N. W. p. 476 : Dresser, B. Eur. viii. p. 187, pl, 571.

$a$ (Thomas) 1840. - b (Bt at Stevens's) 1844. -c, $-d$ (Arthur Strickland) 1850.

A North-American species.

\section{TEREKIA.}

Terekia, Bonaparte, Comp. List. p. 52 (1838).

3004. Terekia cinerea.

Scolopax cinerea, Güld. Nov. Comm. Petr. xix. p. 473, pl. 19.

Terekia cinerea, Dresser, B. Eur. viii. p. 195, pl. 572.

Totanus javanicus, Horsf. Trans. L. S. xiii. p. 193.

a Siberia (J. F. Brandt) 1846. - b, -c India ("Terekia javanica," E. Blyth) 1846.

In a note on $b$ and $c$ Blyth says "These two obtained together on October 1st about the extreme amount of variation at any season which I have seen."

\section{LIMOSA.}

Limosa, Brisson, Orn. v. p. 261 (1760). 3005. Limosa lapponica.

Scolopax lapponica, Linn. Syst. Nat. i. p. 246.

Limosa lapponica, Dresser, B. Eur. viii. p. 203, pl. 573, 574. a f Oxfordshire, February (H. E. Strickland) 1832. 
3006. Limosa ægocephala.

Scolopax cegocephala, Linn. Syst. Nat. i. p. 246.

Limosa agocephala, Dresser, B. Eur. viii. p. 211, pls. 573, 574.

$a($ Askew) 1849. $-b,-c$ India (E. Blyth) 1846.

\section{Numenius.}

Numenius, Brisson, Orn. v. p. 311 (1760).

300\%. Numenius arquatus.

Scolopax arquata, Linn. Syst. Nat. i. p. 242.

Numenius arquatus, Strickl. P. Z. S. 1836, p. 101: Dresser, B. Eur. viii. p. 243 , pl. 578.

a f Southampton, February (H. E. Strickland) 1831. -b Madras (T. C. Jerdon) 1845. — C S. Africa (Arthur Strickland) 1850.

\section{Numenius cyanopus.}

Numenius cyanopus, Vieill. N. Dict. d'Hist. N. viii. p. 306: Gould, Handb. B. Austr. ii. p. 277.

Numenius australis, Gould, B. Austr. vi. pl. 42.

a Australia (T. Robinson) 1842.

3009. Numenius longirostris.

Numenius longirostris, Wils. Am. Orn. viii. p. 23, pl. 64, f. 4: Coues, Birds of the N. W. p. 508.

a (Arthur Strickland) 1850.

A North-American species.

3010. Numenius hudsonicus.

Numenius hudsonicus, Lath. Ind. Orn. ii. p. 712: Coues, Birds of the N. W. p. 509.

a Chili (Capt. Brown) 1842.

\section{Numenius phæopus.}

Scolopax phceopus, Linn. Syst. Nat. i. p. 243.

Numenius phceopus, Dresser, B. Eur. viii. p. 227, pl. 576.

a o Mauritius, November (A. Strickland) 1833. - b Britain (N. C. Strickland) 1838. - c (Askew) 1840. - d Madras (T. C. Jerdon) 1845. 
3012. Numenius borealis.

Scolopax borealis, Forst. Phil. Trans. 1xii. p. 411.

Numenius borealis, Coues, Birds of the N. W. p. 510: Dresser, B. Eur. viii. p. 221, pl. 575.

$a$ (Cashmore) 1839.

An American species of wide range.

\section{FAMILY.DROMADIDAE.}

Dromas.

Dromas, Paykull, Handl. Ac. Vet. Stockh. 1805, pl. 3. 3013. Dromas ardeola.

Dromas ardeola, Payk. l. s. c.: Temm. Pl. Col. 362: Strickl. Ann. \& Mag. N. H. vii. p. 38: Jerd. B. Ind. ii. p. 658.

a Madras (T. C. Jerdon) 1845. 


\section{ORDER XV.-GAVIA.}

\section{FAMILY.-LARID庒。 \\ SUBFAMILY.—STERCORARIIN E.}

\section{STERCorarius.}

Stercorarius, Brisson, Orn. vi. p. 149 (1760).

3014. Stercorarius pomatorhinus.

Lestris pomarinus, Temm. Man. d'Orn. p. 514.

Stercorarius pomatorhinus, Saund. P. Z. Ș. 1876, p. 324: Dresser, B. Eur. viii. p. 463, pl. 610.

a Oxfordshire, 24 October (N. C. Strickland) 1834.

\section{Stercorarius crepidatus.}

Larus crepidatus, Banks in Cook's Voy. ii. p. 15 (Hawkesworth's Ed.): Gm. Syst. Nat. i. p. 602.

Stercorarius crepidatus, Saund. P. Z. S. 1876, p. 326: Dresser, B. Eur. viii. p. 471, pls. 611, 612.

Lestris richardsoni, Sw. Faun. Bor.-Am. Birds, p. 433.

Stercorarius richardsoni, Strickl. Ann. \& Mag. N. H. viii. p. 395.

$a \hat{\jmath},-b \hat{\jmath}$ Orkney, August (H. E. Strickland) 1837. -c, -d Britain (N. C. Strickland) 1838.

\section{SUBFAMILY.-LARIN $A$.}

RISSA.

Rissa, Stephens in Shaw's Gen. Zool. xiii. pt. 1, p. 180 (1825).

\section{Rissa tridactyla.}

Larus tridactylus, Linn. Syst. Nat. i. p. 224. 
Rissa tridactyia, Saund. P. Z. S. 1878, p. 163: Dresser, B. Eur. viii. p. 447, pl, 608.

a Britain (H. E. Strickland) 1830. - 6 Dorsetshire (F. O. Morris) 1833. -c ô Orkney, August (H. E. Strickland) 1837. $-d$ found dead near Apperley Court, March 1849.

\section{LARUS.}

Larus, Linnæus, Syst. Nat, i. p. 224 (1766).

\section{1\%. Larus glaucus.}

Larus glaucus, Fabr. Faun. Grœnl. p. 100: Saund. P. Z. S. 1876, p. 165: Dresser, B. Eur. viii. p. 433, pl. 605.

$a$ Yorkshire (Arthur Strickland) 1830.

\section{Larus leucopterus.}

Larus leucopterus, Faber, Prodr. Isl. Orn. p. 91: Saund. P. Z. S. 1878, p. 166: Dresser, B. Eur. viii. p. 439, pl. 606. $a,-b$ (N. C. Strickland) 1838.

A species of the North Atlantic and North Pacific Oceans.

\section{Larus argentatus.}

Larus argentatus, Gm. Syst. Nat. i. p. 600: Saund. P. Z. S. 1878, p. 167: Dresser, B. Eur. viii. p. 399, pl. 602.

$a,-b$ Yorkshire (Arthur Strickland) 1830.

3020. Larus cachinnans.

Larus cachinnans, Pall, Zoogr. Rosso-As. ii. p. 318: Saund. P. Z. S. 1878, p. 169.

Larus lencophceus, Bruch, J. f. Orn. 1853, p. 101 (ex Licht.): Dresser, B. Eur. viii. p. 411, pl. 602.

Larus argentatus, Strickl. P. Z. S. 1836, p. 101.

$a$ Constantinople, March (H. E. Strickland) 1836. - b Madras (T. C. Jerdon) 1845 ?

3021. Larus fuscus.

Larus fuscus, Linn. Syst. Nat. i. p. 225: Saund. P. Z. S. 1878, p. 173: Dresser, B. Eur. viii. p. 421, pl. 603.

$a$ Orkney, August (H. E. Strickland) 1837. $-b,-c$ Britain (N. C. Strickland) 1838. 
3022. Larus delawarensis.

Larus delawarensis, Ord. in Guthrie's Geogr. Ed. 2, ii. p. 319 : Coues, Birds of the N. W. p. 636: Saund. P. Z. S. 1878, p. 176.

a (Arthur Strickland) 1850.

A North-American species.

3023. Larus canus.

Larus canus, Linn. Syst. Nat. i. p. 224: Saund. P. Z. S. 1878, p. 177: Dresser, B. Eur. viii. p. 381, pl. 600.

a, -b Britain (H. E. Strickland) 1830. —c, -d Aberdovey, August (H. E. Strickland) 1833.

3024. Larus marinus.

Larus marinus, Linn. Syst. Nat. i. p. 225 : Saund. P. Z. S. 1878, p. 179: Dresser, B. Eur. viii. p. 427, pl. 604.

$a$ Britain (N. C. Strickland) 1838.

3025. Larus pacificus.

Larus pacificus, Lath. Ind. Orn. Suppl. p. lxviii.: Gould, B. Austr. vii. pl. 19: Saund. P. Z. S. 1878, p. 181.

$a,-b$ (Arthur Strickland) 1850.

An Australian species.

3026. Larus modestus.

Larus modestus, Tsch. Arch. f. Naturg. 1843, i. p. 389; Faun. Per. Av. p. 306, pl. 35: Saund. P. Z. S. 1878, p. 183.

a Chili (Capt. Brown) 1842.

3027. Larus novæ-hollandiæ.

Larus novce-hollandice, Steph. in Shaw's Gen. Zool. xiii. pt. 1, p. 196: Saund. P. Z. S. 1878, p. 185.

Xema jamesoni, Gould, B. Austr. vii. pl. 20 (ex Wils.).

a New Holland (Arthur Strickland) 1850.

3028. Larus atricilla.

Larus atricilla, Linn. Syst. Nat. i. p. 225: Saund. P. Z. S. 1878, p. 194.

$a$ (Arthur Strickland) 1840. -b Jamaica (J. Gould) 1846. $-c,-d,-e$ (Arthur Strickland) 1850. $-f$. 


\section{Larus franklini.}

Larus franklinii, Sw. Faun. Bor.-Am. Birds, p. 424, pl. 71: Saund. P. Z. S. 1878, p. 195.

$a$ Chili (Tucker).

\section{Larus brunneicephalus.}

Larus brunneicephalus, Jerd. Madr. Journ. xii. p. 225: Saund. P. Z. S. 1878, p. 197.

Xema brunneicephala, Jerd. B. Ind, ii. p. 832.

a Malacca (Askew) 1837. $-b,-c,-d$ Madras (T. C. Jerdon) 1845. -e India ("Xema brunneicephala," E. Blyth) 1846.

\section{Larus ridibundus.}

Larus ridibundus, Linn. Syst. Nat. i. p. 225: Strickl. P. Z. S. 1836, p. 101: Saund. P. Z. S. 1878, p. 200: Dresser, B. Eur. viii. p. 357 , pl. 596, 597.

$a,-b$ Britain (Arthur Strickland) 1833. -c ô Smyrna, 10 December (H. E. Strickland) 1835. —d Constantinople, March (H. E. Strickland) 1836. -e India ("Xema ridibundus," $E$. Blyth) 1846. - $f$ Corncockle, Dumfriesshire, July (H. E. Strickland) 1850. $-g$.

\section{Larus phæocephalus.}

Larus poiocephalus, Sw. B. W. Afr. ii. p. 245, pl. 29.

Xema phceocephala, Strickl. \& Scl. Contr. Orn. 1852, p. 160.

Cirrhocephalus poiocephalus, Gurney in And. B. Damara-l. p. 358.

Larus phcocephalus, Saund. P. Z. S. 1878, p. 204.

a Damara-land (C. J. Andersson) 1852.

\section{Larus minutus.}

Larus minutus, Pall. Reise iii. p. 702: Saund. P. Z. S. 1878, p. 206 : Dresser, B. Eur. viii. p. 373, pl. 599.

$a$ N. Europe (J. G. Kinberg) 1843.

\section{Larus philadelphia.}

Sterna philadelphia, Ord. in. Guthrie's Geogr. Ed. 2, ii. p. 319.

Larus philadelphia, Saund. P. Z. S. 1878, p. 206.

a (Arthur Strickland) 1850.

A North-American species. 


\section{SUBFAMILY.-STERNIN A.}

HYDROCHELIDON.

Hydrochelidon, Boie, Isis, 1822, p. 563: Strickl. Ann. \& Mag. N. H. vii. p. 40.

\section{Hydrochelidon hybrida.}

Sterna hybrida, Pall. Zoogr. Rosso-As. ii. p. 338.

Hydrochelidon hybrida, Saund. P. Z. S. 1876, p. 640: Dresser, B. Eur. viii. p. 315, pls. 588, 589.

Sterna leucopareia, Temm. Man. d'Orn. ii. p. 746 (ex Natterer MS.).

a ô Madras, September (A. Strickland) 1833. - - b New Holland? (Askew) 1834. $-c,-d$, - - Madras ("Sterna leucopareia," T. C. Jerdon) 1845. - f India ("Gelochelidon leucopareia," E. Blyth) 1846. -g (Arthur Stricleland) 1850. -h Malacca (Arthur Strickland).

3036. Hydrochelidon leucoptera.

Sterna leucoptera, Schinz in Meisn. \& Schinz, Vög. d. Schweiz. p. 264.

Hydrochelidon leucopterc, Saund. P. Z. S. 1876, p. 641: Dresser, B. Eur. viii. p. 321, pl. 590, 591.

a Italy (Boissoneau) 1839.

\section{3\%. Hydrochelidon nigra.}

Sterna nigra, Linn. Syst Nat. i. p. 227.

Hydrochelidon nigra, Strickl. P. Z. S. 1850, p. 221: Saund. P. Z. S. 1876, p. 642: Dresser, B. Eur. viii. p. 327, pl. 592.

$a$ q, $-b$ q Fernando Po, 8 March (A. Strickland) 1833. -c Neufchatel (L. Coulon) 1836. - d \& Kartoum (J. Petherick) 1848. -e Oxford, 14 October 1834 (Arthur Strickland) 1850.

\section{STERNA.}

Sterna, Linnæus, Syst. Nat. i. p. 227 (1766): Strickl. Ann. \& Mag. N. H. vii. p. 40.

\section{Sterna magnirostris.}

Sterna magnirostris, Licht. Verz. Doubl. p. 81: Saund. P. Z. S. 1876, p. 643.

$a$ S. America (Arthur Strickland). 
3039. Sterna anglica.

Sterna anglica, Mont. Orn. Dict. Suppl. fol. Y. p. 1: Strickl. P. Z. S. 1850, p. 221 : Saund. P. Z. S. 1876, p. 644: Dresser, B. Eur. viii. p. 295, pl. 585 .

$a$ S. Europe (J. G. Kinberg) 1843. - b Madras (T. C. Jerdon) 1845. - c India ("Gelochelidon anglica," E. Blyth) 1846. $-d \hat{o}$ Kartoum, October (J. Petherick) 1848.

3040. Sterna seena.

Sterna seena, Sykes, P. Z. S. 1832, p. 171: Saund. P. Z. S. 1876, p. 645.

Sterna aurantia, Gray, Ill. Ind. Zool. i. pl. 69, fig. 2.

Seena aurantia, Jerd. B. Ind. ii. p. 838.

a India ("Seena aurantia," E. Blyth) 1846. - b S. India (T. C. Jerdon) 1850. -c (Arthur Strickland).

\section{Sterna melanogaster.}

Sterna melanogaster, Temm. Pl. Col. 434: Saund. P. Z. S. 1876, p. 645.

Sterna javanica, Horsf. Trans. L. S. xiii. p. 198 : Jerd. B. Ind. ii. p. 840.

a Malacca (Askew) 1834.

\section{Sterna albigena.}

Sterna albigena, Reichenb. Natatores Suppl. xi. pl. 21, fig. 816: Saund. P. Z. S. 1876, p. 648.

a Madras (T. C. Jerdon) 1845.

\section{Sterna fluviatilis.}

Sterna hirundo, Linn. Syst. Nat. i. p. 227, partim, et Auctt. plurr.

Sterna fuviatilis, Naum. Isis, 1819, p. 1847 : Saund. P. Z. S. 1876, p. 649: Dresser, B. Eur. viii. 263, pl. 580.

$a$ Worcestershire (Mrs Perrott) 1832. - $b$ \& Lago Maggiore, August (H. E. Strickland) 1836. - c Britain (N. C. Strickland) 1838. -d (Arthur Strickland) 1840. -e, -f, -g Britain. - $h$ (Arthur Strickland). - $i$ Newport R. I. (Thomas) 1842. $-j$ S. India ("Sterna hirundo," T. C. Jerdon) 1850. - -,$-l$. 


\section{Sterna macrura.}

Sterna hirundo, Linn. Syst. Nat. i. p. 227, partim: Dresser, B. Eur. viii. p. 255 , pl. 579.

Sterna macrura, Naum. Isis, 1819, p. 1847 : Saund. P. Z. S. 1876 , p. 650.

Sterna arctica, Strickl. Ann. \& Mag. N. H. ix. p. 351.

a §ิ Orkney, August (H. E. Strickland) 1837. - $b$ f Avon, Worcestershire, 8 May (H. E. Strickland) 1842. -c.

\section{Sterna forsteri.}

Sterna forsteri, Nutt. Man. Orn. ii. p. 274: Saund. P. Z. S. 1876, p. 651.

a (Askew) 1834. - b (Arthur Strickland).

A North-American species.

3046. Sterna dougalli.

Sterna dougalli, Mont. Orn. Dict. Suppl. fol. Y. p. 4: Saund. P. Z. S. 1876 , p. 652: Dresser, B. Eur. viii. p. 273, pl. 581.

a Madras, September (A. Strickland) 1833. -b Chili (Capt. Brown) 1842.

304\%. Sterna media.

Sterna media, Horsf. Trans. L. S. xiii. p. 199 : Saund. P. Z. S. 1876, p. 655: Dresser, B. Eur. viii. p. 285, pl. 583.

Thalasseus bengalensis, Jerd. B. Ind. ii. p. 843 (ex Lesson).

$a$ India (Bt at Stevens's) 1843. - b, —c S. India (T. C. Jerdon) 1850.

3048. Sterna maxima.

Hirondelle de Mer, de Cayenne, D'Aub. Pl. Enl. 988, undè

Sterna maxima, Bodd. Tabl. Pl. Enl. p. 58: Scl. \& Salv. P. Z. S. 1871, p. 567: Saund. P. Z. S. 1876 , p. 655.

a (Weaver) 1839.

A species of the tropical and warmer temperate coasts of the Atlantic Ocean.

3049. Sterna caspia.

Sterna caspia, Pall. Nov. Comm. Petrop. xiv. p. 582, pl. 22, f. 2 : Saund. P. Z. S. 1876 , p. 656 : Dresser, B. Eur. viii. p. 289, pl. 584 . 
Sylochelidon caspia, Jerd. B. Ind. ii. p. 83.5.

$a$ New South Wales ( $M^{c}$ Donald) 1838. $-b$ N. Europe (J. G. Kinberg) 1843. - c Madras (T. C. Jerdon) 1845. -d India ("Sylochelidon caspia," E. Blyth) 1846.

3050. Sterna bergii.

Sterna bergii, Licht. Verz. Doubl. p. 80: Saund. P. Z. S. 1876, p. 657.

Thalasseus poliocercus, Gould, B. Austr. vii. pl. 24.

$a$ New South Wales $\left(M^{c}\right.$ Donald) 1838. - b Australia (Arthur Strickland) 1850.

3051. Sterna minuta.

Sterna minuta, Linn. Syst. Nat. i. p. 228: Dresser, B. Eur. viii. p. 279 , pl. 582.

a Southend, Esisex, August (H. E. Strickland) 1822. - b (Askew) 1836. —c (Arthur Strickland).

3052. Sterna antillarum.

Sternula antillarum, Less. Descr. Mamm. et Ois. p. 256.

Sterna antillarum, Saund. P. Z. S. 1876, p. 661.

a Newport, R. I. (Thomas) 1842.

\section{Sterna superciliaris.}

Sterna superciliaris, Vieill. N. Dict. d'Hist. N. xxxii. p. 176 : Saund. P. Z. S. 1876, p. 662.

$a,-b$ (Arthur Strickland).

A South-American species.

3054. Sterna sinensis.

Sterna sinensis, Gm. Syst. Nat. i. p. 608: Saund. P. Z. S. 1876, p. 662.

$a,-b$ Madras ("Sterna minuta," T. C. Jerdon) 1855, - c Australia (Askew) 1839.

3055. Sterna balænarum.

Sternula baloenarum, Strickl. Contr. Orn. 1852, p. 160: Gurney in And. B. Damara-1. p. 362.

Sterna balcenarum, Saund. P. Z. S. 1876 , p. 664.

a Damara-land (C. J. Andersson) 1852.

The type of this species.

S. C. 
3056. Sterna anæstheta.

Sterna anaethetus, Scop. Del. Fl. et Faun. Ins. ii. p. 92.

Sterna ancestheta, Saund. P. Z. S. 1876, p. 664.

Sterna panayensis, Gm. Syst. Nat. i. p. 607.

$a,-b$ Madras, September (A. Strickland) 1833. - $-c$ North America (Arthur Strickland). - d.

305\%. Sterna fuliginosa.

Sterna fuliginosa, Gm. Syst. Nat. i. p. 605 : Saund. P. Z. S. 1876, p. 666.

Onychoprion fuliginosa, Gould, B. Austr. vii. pl. 32.

a Australia (Warwick Museum) 1841. -b (Mansfield) 1834.

\section{NANIA.}

Ncenia, Boie, Isis, 1844, p. 189.

3058. Nænia inca.

Sterna inca, Less. Voy. Coq. Zool. i. p. 731 : Atl. pl. 47.

Ncenia inca, Saund. P. Z. S. 1876, p. 667.

Inca mystacalis, Jard. Contr. Orn. 1850, p. 32.

a (Arthur Strickland).

A species of the West coast of Peru.

\section{GYGIS.}

Gygis, Wagler, Isis, 1832, p. 1223.

3059. Gygis candida.

Sterna candida, Gm. Syst. Nat. i. p. 607.

Gygis candida, Gould, B. Austr. vii. pl. 30 : Saund. P. Z. S. 1876, p. 667.

Sterna alba, Sparrm.? Strickl. Ann. \& Mag. N. H. viii. p. 545. a Australia (Arthur Strickland) 1850.

\section{Anous.}

Anous, Stephens, in Shaw's Gen. Zool. xiii. pt. i. p. 139, (1825).

3060. Anous stolidus.

Sterna stolida, Linn. Syst. Nat. i. p. 227. 
Anous stolidus, Saund. P. Z. S. 1876, p. 669.

$a q,-b q,-c$ off Fernando Po, March (A. Strickland) 1833. $-d$.

3061. Anous melanogenys.

Anous melanogenys, Gray \& Mitch. Gen. B. iii. p. 661, pl. 182; Saund. P. Z. S. 1876 , p. 670.

a f Prince's Island, 12 March (A. Strickland) 1833.

3062. Anous cinereus.

Anous cinereus, Gould, P. Z. S. 1845, p. 104; B. Austr. vii, pl. 37: Sharpe, P. Z. S. 1878, p. 272.

a Australia, 1842.

\section{SUBFAMILY.-RHYNCHOPIN Æ⿸}

RHYNCHOPS.

Rhynchops, Linnæus, Syst. Nat. i. p. 228 (1766).

3063. Rhynchops nigra.

Rhynchops nigra, Linn. l. s. c.: Scl. \& Salv. P. Z. S. 1871, p. 566.

$a$ (Thomas) 1840. $-b$ (Arthur Strickland). -c.

A species of Tropical America.

3064. Rhynchops albicollis.

Rhynchops albicollis, Swains. An. in Menag. p. 360: Gray \& Mitch. Gen. B. iii. pl. 181: Jerd. B. Ind. ii. p. 847.

a Madras (T. C. Jerdon) 1845. 


\section{ORDER XVI.-TUBINARES.}

\section{FAMILY.-PROCELLARIID正.}

Procellaria.

Procellaria, Linnæus, Syst. Nat. i. p. 212 (1766) partim.

\section{Procellaria pelagica.}

Procellaria pelagica, Linn. l.s. c.

Thalassidroma pelagica, Dresser, B. Eur. viii. p. 491, pl. 613. $a$ (Mansfield) 1839. - b, -c.

A species of the North Atlantic Ocean.

\section{Cymochorea.}

Cymochorea, Coues, Proc. Ac. Phil. 1864, p. 75.

3066. Cymochorea leucorrhoa.

Procellaria leucorrhoa, Vieill. N. Dict. d'Hist. N. xxv. p. 422.

Thalassidroma leucorrhoa, Dresser, B. Eur. viii. p. 497, pl. 613.

Procellaria leachii, Temm. Man. d'Orn. ii. p. 812.

$a$ Worcester, December 1839. - b (Arthur Strickland) 1840. -c Flat Dezerta, 7 June (R. T. Lowe) 1850.

\section{OCEANITES.}

Oceanites, Keyseling \& Blasius, Wirb. Eur. p. 238 (1840). 306\%. Oceanites oceanicus.

Procellaria oceanica, Kuhl, Beitr. z. Zool. p. 136. pl. 10. fig. 1.

Oceanites oceanicus, Dresser, B. Eur. viii. p. 505, pl. 614. 
a $q$ March (A. Strickland) 1833.

Algernon Strickland's ship was at Fernando Po on March 10 and at Prince's Island March 12, so that this species was probably caught in the South Atlantic.

\section{OSSIFRAGA.}

Ossifraga, Hombron \& Jaquinot, Compt. Rend. xviii. p. 356 (1844).

3068. Ossifraga gigantea.

Procellaria gigantea, Gm. Syst. Nat. i. p. 563: Gould, B. Austr. vii. pl. 45.

Ossifraga gigantea, Coues, Pr. Ac. Phil. 1866, p. 32.

$a$ S. Seas (T. C. Eyton) 1846. -b (Arthur Strickland) 1850.

A species of the South Seas.

\section{Majaqueus.}

Majaqueus, Reichenbach, Syst. Av. p. iv. (1850).

3069. Majaqueus æquinoctialis.

Procellaria cequinoctialis, Linn. Syst. Nat. i. p. 213.

Majaqueus cequinoctialis, Coues, Pr. Ac. Phil. 1864, p. 118.

$a,-b$ (Arthur Strickland) 1850.

A species of the South Seas.

3070. Majaqueus conspicillatus.

Procellaria conspicillata, Gould, Ann. \& Mag. N. H. xiii. p. 362 ; B. Austr. vii. pl. 46.

Majaqueus conspicillatus, Coues, Pr. Ac. Phil. 1864, p. 118.

$a$ S. Seas (Arthur Strickland) 1850.

Doubtfully distinct from the preceding species.

\section{Estrelata.}

Astrelata, Bonaparte, Consp. Av. ii. p. 188 (1856).

\section{0'71. Estrelata fuliginusa.}

Procellaria fuliginosa, Kuhl, Beitr. z. Zool. p. 142, pl. 10 , fig. 6 . 
Éstrelata fuliginosa, Coues, Pr. Ac. Phil. 1866, p. 157. a (Arthur Strickland) 1850.

A species of the South Seas.

\section{Fulmarus.}

Fulmarus, Stephens, in Shaw's Gen. Zool. xiii. pt. 1, p. 233 (1825).

\section{0'\%. Fulmarus glacialis.}

Procellaria glacialis, Linn. Syst. Nat. i. p. 213.

Fulmarus glacialis, Dresser, B. Eur. viii. p. 535, pl. 617.

$a$ N. Europe (J. G. Kinberg) 1843. - b (Arthur Strickland) 1850.

Thalasseca.

Thalassoica, Reichenbach, Syst. A. p. iv. (1850).

30'73. Thalassœca glacialoides.

Procellaria glacialoides, Smith, Ill. Zool. S. Afr. pl. 51: Gould, B. Austr. vii. pl. 48 : Coues, Pr. Ac. Phil. 1866, p. 30.

$a$ S. Seas (Thomas) 1842.

\section{DAPTION.}

Daption, Stephens in Shaw's Gen. Zool. xiii. pt. i. p. 239 (1825).

\section{Daption capensis.}

Procellaria capensis, Linn. Syst. Nat. i. p. 213.

Daption capensis, Gould, B. Austr. vii. pl. 53 : Coues, Pr. Ac. Phil. 1866, p. 162.

$a$ S. Atlantic (A. Strickland) 1832. - b Lat. $23^{\circ} 3^{\prime}$ S. Long. $58^{\circ} 52^{\prime}$ E. 27 May (A. Strickland) 1832. —c.

\section{Puffinus,}

Puffinus, Brisson, Orn. vi. p. 131 (1760).

3075. Puffinus major.

Puffinus major, Faber, Prod. Isl. Orn. p. 56: Dresser, B. Eur. viii. p. 527, pl. 616 . 
$a$ (Arthur Strickland) 1850. -b.

A species of the North-Atlantic Ocean.

30\%6. Puffinus anglorum.

Procellaria anglorum, Temm. Man. d'Orn. ii. p. 807.

Puffinus anglorum, Dresser, B. Eur. viii. p. 517, pl. 615.

Procellaria yelkouan, Acerbi, Bibl. Ital. cxl. p. 294.

a Constantinople, March (H. E. Strickland) 1836.

\section{7'\%. Puffinus assimilis.}

Puffinus assimilis, Gould, P. Z. S. 1837, p. 156 ; B. Austr. vii. pl. 59.

$a$ New South Wales (McDonald) 1838. -b (Rev. J. Brown) 1845 .

\section{Prion.}

Prion, Lacépède, Mém. l'Inst. p. 514 (1801).

\section{Prion vittatus.}

Procellaria vittata, Gm. Syst. Nat. i. p. 560.

Prion vittatus, Gould, B. Austr. vii. pl. 55.

$a$ \& Fish-hook Bay, Cape of Good Hope, June (A. Strickland) 1833.

\section{0'79. Prion turtur.}

Prion turtur, Gould, Ann. \& Mag。 N. H. xiii. p. 366 ; B. Austr. vii. pl. 54 .

$a \hat{\delta},-b q$ Cape of Good Hope, 10 June (A. Strickland) 1833. $-c$.

\section{Pelecanoides.}

Pelecanoides, Lacépède, Mém. l'Inst. p. 513 (1801).

\section{Pelecanoides garnoti.}

Puffinuria garnoti, Less. Voy. Coq. Zool. i. p. 730, pl. 46.

Pelecanoides garnoti, Coues, Pr. Ac. Phil. 1866, p. 190.

a San Lorenzo, Peru (Arthur Strickland) 1850. 


\section{FAMILY.-DIOMEDIDAE.}

\section{DIOMEDEA.}

Diomedea, Linnæus, Syst. Nat. i. p. 214 (1766) partim.

3081. Diomedea exulans.

Diomedea exulans, Linn. l. s. c. : Gould, B. Austr. vii. pl. 38. a f., May (A. Strickland) 1833.

A species of the South Seas.

3082. Diomedea melanophrys.

Diomedea melanophrys, Temm. Pl. Col. 456: Gould, B. Austr. vii. pl. 43.

$a$.

A head of this species which is widely distributed in the Southern Ocean.

\section{Phoebetria.}

Phoebetria, Reichenbach, Syst. Nat. p. v. (1853).

3083. Phœbetria fuliginosa.

Diomedea fuliginosa, Gm. Syst. Nat. i. p. 568 : Gould, B. Austr. vii. pl. 44.

a (Arthur Strickland) 1850.

Also a widely-ranging southern Species. 


\section{ORDER XVII.-PYGOPODES.}

\section{FAMILY.-COLYMBID庄.}

Colymbus.

Colymbus, Linnæus, Syst. Nat. i. p. 220 (1766) partim. 3084. Colymbus septentrionalis.

Colymbus septentrionalis, Linn. l. s. c.: Dresser, B. Eur. viii. p. 621, pl. 628.

$a,-b$ Orkney (E. Forbes) 1837. — Britain (N. C. Strickland) 1838.

3085. Colymbus arcticus.

Colymbus arcticus, Linn. Syst. Nat. i. p. 221: Dresser, B. Eur. viii. p. 615 , pl. 627 .

$a$ N. Europe (J. G. Kinberg) 1843.

3086. Colymbus glacialis.

Colymbus glacialis, Linn. Syst. Nat. i. p. 221: Dresser, B. Eur. viii. p. 609, pl. 626.

$a,-b$ Orkney (E. Forbes) 1837.

\section{FAMILY.-PODICIPEDIDA.}

Podiceps.

Podiceps, Latham, Gen. Syn. Suppl. i. p. 294 (1787).

3087. Podiceps cristatus.

Colymbus cristatus, Linn. Syst. Nat, i. p. 222.

Podiceps cristatus, Dresser, B. Eur. viii. p. 629, pl. 629.

a Constantinople, March (H. E. Strickland) 1836. -b (N. C. Strickland) 1838. 
3088. Podiceps major.

Grebe de Cayenne, D'Aub. Pl. Enl. 404, f. 1, undè

Colymbus major, Bodd. Tabl. Pl. Enl. p. 24.

Podiceps major, Scl. \& Salv. Ex. Orn. p. 190.

Podiceps bicornis, Licht. Verz. Doubl. p. 88.

$a$ Chili (Capt. Brown) 1843.

\section{Podiceps auritus.}

Colymbus auritus, Linn. Syst. Nat. i. p. 222.

Podiceps auritus, Dresser, B. Eur. viii. p. 645, pl. 631.

Colymbus cornutus, Gm. Syst. Nat. i. p. 591.

$a$ Iceland (Thomas) 1842. — britain (W. Kirtland) 1847. -c (Arthur Strickland) 1850.

\section{Podiceps rollandi.}

Podiceps rollandi, Quoy. \& Gaim. Voy. Uranie. Zool. p. 133, Atl. pl. 36: Scl. \& Salv. Ex. Orn. p. 190.

a Chili (Capt. Brown) 1842.

\section{Podiceps poliocephalus.}

Podiceps poliocephalus, Jard. \& Selb. Ill. Orn. i. pl. 13: Gould, B. Austr. vii. pl. 82.

Podiceps nestor, Gould, P. Z. S. 1836, p. 145; Handb. B. Austr. ii. p. 512.

$a$ Australia (Dewgard) 1847. - $b$ Swan River, Australia ( $P$. L. Sclater) 1847. —c Adelaide, S. Australia (T. C. Eyton) 1850. $-d$ Van Dieman's Land (Arthur Strickland) 1850.

\section{Podiceps fluviatilis.}

Colymbus fluviatilis, Tunst. Orn. Brit. p. 3.

Podiceps fuviatilis, Dresser, B. Eur. viii. p. 659, pl. 633.

Colymbus minor, Gm. Syst. Nat. i. p. 591.

$a$ q Sleaford Fen, Lincolnshire, 8 October (H. E. Strickland) 1826. —b. Orkney (E. Forbes) 1837. —c (N. C. Strickland) 1838. — $d$ Sleaford Fen, Lincolnshire, October (H. E. Strickland) 1828. -e.

\section{Podiceps philippensis.}

Castagneux, des Philippines, D'Aub. Pl. Enl. 945. Colymbus philippensis, Vieill. Enc. Méth. i. p. 58. 
Podiceps philippensis, Jerd. B. Ind. ii. p. 822: Wald. Trans. Z. S. ix. p. 245.

$a$ India (E. Blyth) 1846.

\section{Podiceps dominicus.}

Colymbus dominicus, Linn. Syst. Nat. i. p. 223.

Podiceps dominicus, Gosse, B. Jam. p. 440.

Sylbeocyclus dominicus, Scl. \& Salv. Ex. Orn. p. 190.

$a$ (Mansfield) 1839. - b (Mather) 1840. —c o Yxtlan, Mexico, September (T. Galeotti) 1845. —d Jamaica (P. H. Gosse) 1848. -e (Arthur Strickland) 1850. $f$.

\section{Podilymbus.}

Podilymbus, Lesson, Traité d’Orn. p. 595 (1831) as a subgenus.

\section{Podilymbus podiceps.}

Colymbus podiceps, Linn. Syst. Nat. i. p. 223.

Podilymbus podiceps, Baird, B. N. A.m. p. 898.

Podilymbus carolinensis, Gosse, B. Jam. p. 438 (ex Latham). $a$ (T. Robinson) 1837. - b Jamaica (J. Gould) 1846. -c (Arthur Strickland) 1850. —d Guatemala (J. Constancia) 1851. $-e$.

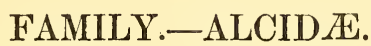

\section{AlCA.}

Alca, Linnæus, Syst. Nat. i. p. 210 (1766) partim. 3096. Alca torda.

Alca torda, Linn. l. s. c.: Dresser, B. Eur. viii. p. 557, pl. 619. a Yorkshire (Arthur Strickland) 1830. - b Isle of Wight, August (H. E. Strickland) 1831. — c Yorkshire (Arthur Strickland) 1833. - d Tenby, August (H. E. Strickland) 1835. -e Britain (N. C. Strickland) 1838. 


\section{URIA.}

Uria, Brisson, Orn. vi. p. 70 (1760).

\section{9\%. Uria troile.}

Colymbus troile, Linn. Syst. Nat. i. p. 220.

Alca troile, Dresser, B. Eur. viii. p. 567, pl. 621.

$a,-b,-c$ Yorkshire (Arthur Strickland) 1830. —d Yorkshire, March (H. E. Strickland) 1833. - e Tenby, August (H. E. Strickland) 1835.

3098. Uria bruennichi.

Uria brünnichi, Sabine, Trans. L. S. xii. p. 538.

Alca bruennichi, Dresser, B. Eur. viii. p. 575, pl. 622. a (Thomas) 1842.

A species of the Arctic Oceans.

\section{Cepphus.}

Cepphus, Pallas, Spic. Zool. v. p. 33 (1769).

\section{Cepphus grylle.}

Colymbus grylle, Linn. Syst. Nat. i. p. 220.

Uria grylle, Dresser, B. Eur. viii. p. 581, pl. 623.

a Yorkshire (Arthur Strickland) 1833. -b o Orkney, August (H. E. Strickland) 1837. —c I. of Skye, 19 August (H. E. Strickland) 1837.

\section{Mergulus.}

Mergulus, Vieillot, Anal. p. 67 (1816).

3100. Mergulus alle.

Alca alle, Linn. Syst. Nat. i. p. 211.

Mergulus alle, Dresser, B. Eur. viii. p. 591, p1. 624.

Mergulus melanoleucos, Leach, Syst. Cat. Mamm. \& Birds Brit. Mus. p. 42.

a (Arthur Stricliland) 1833.

A species of the North-Atlantic Ocean. 
OMBRIA.

Ombria, Eschscholtz, Zool. Atlas, iv. p. 3, pl. 17 (1831). 3101. Ombria psittacula.

Alca psittacula, Pall. Spic. Zool. p. 13, pls. 2, 5.

Simorhynchus psittaculus, Coues, Pr. Ac. Phil. 1868, p. 36.

Ombria psittacula, Brandt, Mel. Biol. vii. p. 237.

$a \hat{\delta} \mathrm{N}$. W. America ("Alca psittacula, Wosnessenski," J. F. Brandt) 1846.

\section{Simorhynchus.}

Simorhynchus, “Merrem (1819)," fide G. R. Gray, Cat. Gen. B. p. 127 (1856).

\section{Simorhynchus pusillus.}

Uria pusilla, Pall. Zoogr. ii. p. 373.

Simorhynchus pusillus, Brandt, Mel. Biol. vii. p. 230.

Phalaris microceros, Brandt, Bull. Ac. Petersb. ii. p. 346 (1837).

Simorhynchus microceros, Coues, Pr. Ac. Phil. 1868, p. 46.

$a \mathrm{~N}$. W. America ("Alca pygmcea, Wosnessenski," J. $F$. Brandt) 1846. -b (J. G. Kinberg) 1845.

3103. Simorhynchus cristatellus.

Alca cristatella, Pall. Spic. Zool. v. p. 18, pl. 3.

Simorhynchus cristatellus, Coues, Pr. Ac. Phil. 1868, p. 37 : Brandt, Mel. Biol. v. p. 223.

$a$ N. Pacific (J. G. Kinberg) 1845.

Fratercula.

Fratercula, Brisson. Orn. vi. p. 81 (1760).

3104. Fratercula arctica.

Alca arctica, Linn. Syst. Nat. i. p. 211.

Fratercula arctica, Dresser, B. Eur. viii. p. 599, pl. 625.

$a$ Yorkshire (Arthur Strickland) 1830. - b Tenby, September (H. E. Strickland) 1835.

3105. Fratercula corniculata.

Mormon corniculata, Naum. Isis, 1821, p. 782.

Fratercula corniculata, Coues, Pr. A.. Phil. 1868, p. 24. 
Lunda (Ceratoblepharum) corniculata, Brandt, Mel. Biol. vii. p. 242.

$a$ N. W. America (J. F. Brandt) 1846.

3106. Fratercula cirrata.

Alca cirrhata, Pall. Spic. Zool. v. p. 7, pls. 1, 5.

Lunda cirrhata, Coues, Pr. Ac. Phil. 1868, p. 26.

Lunda (Gymnoblepharum) cirrata, Brandt, Mel. Biol. vii. p. 244.

$a(J . G$. Kinberg) 1845.

A species of the North-Pacific Ocean. 


\title{
ORDER XVIII.-IMPENNES.
}

\section{FAMILY.-SPHENISCID A.}

\author{
EUDYPTES.
}

Eudyptes, Vieillot, Anal. p. 67 (1816).

310\%. Eudyptes undina.

Aptenodytes undina, Gould, P. Z. S. 1844, p. 57.

Spheniscus undina, Gould, B. Austr. vii. pl. 85.

$a$ S. Seas (Arthur Strickland) 1850.

\section{SpHeniscus.}

Spheniscus, Brisson, Orn. vi. p. 96 (1760).

3108. Spheniscus humboldti.

Spheniscus humboldti, Meyen, Nov. Act. Ac. Leop. xvi. Suppl. p. 234, pl. 31: Scl. Voy. Chall. Zool. ii. Aves, p. 126.

a Chili (Capt. Brown) 1842.

3109. Spheniscus demersus.

Diomedea demersa, Linn. Syst. Nat. i. p. 214.

Spheniscus demersus, Scl. \& Salv. P. Z. S. 1878, p. 653: Scl. Voy. Chall. Zool. ii. Aves, p. 124, pl. 27.

$a$ S. Seas (Thomas) 1842. 


\section{ORDER XIX.-CRYPTURI.}

Tinamus.

Tinamus, Latham, Ind. Orn. ii. p. 633 (1790).

\section{Tinamus subcristatus.}

Trachypelmus subcristatus, Cab.sin Schomb. Reise n. Guiana, iii. p. 749 .

Tinamus major, Schl. Mus. P. B. Tinami, p. 13 (partim). a (Bt. at Liverpool) 1853.

A skin of Cayenne make.

\section{Crypturus.}

Crypturus, Illiger, Prodr. p. 244 (1811).

\section{Crypturus variegatus.}

Tetrao variegatus, Gm. Syst. Nat. i. p. 768.

Crypturus variegatus, Schl. Mus. P. B. Tinami, p. 32.

$a$ S. America (Carfrae) 1840. -b (Bt. at Liverpool) 1853.

$b$ is a skin of Cayenne make.

\section{Crypturus obsoletus.}

Tinamus obsoletus, Temm. Pig. et Gall. iii. pp. 588, 751 ; Pl. Col. 196.

Crypturus obsoletus, Schl. Mus. P. B. Tinami, p. 22.

a (Arthur Strickland) 1850.

A species of South Brazil.

\section{Crypturus tataupa.}

Tinamus tataupa, Temm. Pig. et Gall. iii. pp. 590, 752; Pl. Col. 415.

Crypturus tataupa, Schl. Mus. P. B. Tinami, p. 21. a Brazil (Bt at Aberystwith) 1838. - b Brazil (Askew) 1840. 
RHYNChotus.

Rhynchotus, Spix, Av. Bras. ii. p. 60 (1825).

3114. Rhynchotus rufescens.

Tinamus rufescens, Temm. Pig. et Gall. iii. pp. 552, 747 ; Pl. Cl. 412.

Rhynchotus rufescens, Schl. Mus. P. B. Tinami, p. 35. a (Mansfield) 1849.

A species of South Brazil and the Argentine Republic.

\section{Nothura.}

Nothura, Wagler, Syst. Av. fol. 19, p. 9 (1827).

\section{Nothura maculosa.}

Tinamus maculosus, Temm. Pig. et Gall. iii. pp. 557, 748.

Nothura maculosa, Schl. Mus. P. B. Tinami, p. 42.

Tinamus major, Spix, Av. Bras. ii. p. 64, pl. 80. a (Thomas) 1842.

A species of South Brazil and the Argentine Republic.

\section{Nothura affinis.}

Nothura affinis, G. R. Gray, List of Birds, Pt. v. Gallinæ, p. 105 (1867).

$a($ Osman $) 1848$.

$\mathrm{Mr}$ Sharpe has identified this specimen as belonging to this species, the precise locality of which is at present unknown.

\section{NOTHOPROCTA.}

Nothoprocta, Sclater \& Salvin, Nomencl. Av. Neotr. p. 156 (1873).

\section{Nothoprocta perdicaria.}

Crypturus perdicarius, Kittl. Mém. prés. Ac. Petersb. i. p. 192, pl. 12.

Nothura perdicaria, Schl. Mus. P. B. Tinami, p. 40.

a Chili (Capt. Brown) 1842. 


\title{
ADDENDA.
}

\author{
[p. 15.] \\ Cercotrichas.
}

Cercotrichas, Boie, Isis, 1831, p. 542.

73*. Cercotrichas erythroptera.

Turdus erythropterus, Gm. Syst. Nat. i. p. 835.

Melcenornis? erythropterus, Strickl. P. Z. S. 1850, p. 217.

Cercotrichas erythroptera, Cab. Mus. Hein. i. p. 41.

a \& Kordofan, February (J. Petherick) 1848.

86. Chætornis striata.

$$
\text { [p. 18.] }
$$

Add $c$ India (E. Blyth) 1846.

180. Sialia wilsoni.

$$
\text { [p. 38.] }
$$

Add $f$ Guatemala ( $J$. Constancia) 1845.

$$
\text { [p. 41.] }
$$

195. Calliope camtschatkensis.

Add $c$ India (E. Blyth) 1846.

$$
\begin{aligned}
& \text { [p. 92.] } \\
& \text { ICTERIA. }
\end{aligned}
$$

Icteria, Vieillot, Ois. Am. Sept. i. pp. iii, 85 (1807).

454*. Icteria viridis.

Muscicapa viridis, Gm. Syst. Nat. i. p. 936.

Icteria viridis, Salv. \& Godm. Biol. Centr. Am. Aves i. p. 157.

Icteria velasquezi', Bp. P. Z. S. 1837, p. 117.

$a$ N. America (Askew). - b Guatemala (J. Constancia) 1845. 


$$
\text { [p. 94.] }
$$

$461^{*}$. Basileuterus mesochrysus.

Basileuterus mesochrysus, Scl. P. Z. S. 1860, p. 251 : Salv. \& Godm. Biol. Centr. Am. Aves i. p. 176.

a Bogota (Bt at Stevens's) 184ّ̌.

$$
\text { [p. 105.] }
$$

512. Rubigula flaviventris.

Add $b$ India (Bt at Stevens's) 1844.

$$
\text { [p. 143.] }
$$

Parisoma.

Parisoma, Swainson, Faun. Bor. Am. ii. p. 490 (1831). 689*. Parisoma plumbeum.

Stenostira plumbea, Hartl. J. f. Orn. 1858, p. 41.

Parisoma plumbeum, Sharpe, Cat. B. iv. p. 269.

a (Johnson) 1837.

Agrees with specimens in the British Museum of this tropical African species.

$$
\text { [p. 152.] }
$$

728. Cotyle riparia.

Add $c$ Cape of Good Hope (A. Strickland) 1833.

$$
\text { [p. 216.] }
$$

105\%*. Sycalis citrina.

Sycalis citrina, Pelz. Orn. Bras. pp. 232, 333 : Scl. Ibis, 1872, p. 48.

Fringilla xanthorrhoa, Bp. Journ. Ac. Phil. iv. p. 350 ?

a (N. C. Strickland) 1838.

Apparently a skin of this little-known Brazilian species.

$$
\text { [p. 270.] }
$$

135\%. Quiscalus tenuirostris.

Add c (N. C. Strickland) 1838. 


$$
\text { [p. 430.] }
$$

2100*. Bucco maculatus.

Alcedo maculata, Gm. Syst. Nat. i. p. 451.

Bucco maculatus, Scl. Mon. Galb. \& Bucc. p. 99, pl. 32.

$a,-b$ Brazil (N. C. Strickland) 1838.

$$
\text { [p. 482.] }
$$

2360. Circus æruginosus.

Add $b$ Madras (T. C. Jerdon) 1845.

\section{6*. Buteo jakal.}

$$
\text { [p. 486.] }
$$

Falco jakal, Daud. Traité d’Orn. ii. p. 161.

Buteo jakal, Strickl. Orn. Syn. p. 33 : Sharpe, Cat. B. i. p. 173.

$a$ S. Africa (Arthur Strickland).

$$
\text { [p. 501.] }
$$

2451*. Falco vespertinus.

Falco vespertinus, Linn. Syst. Nat. i. p. 129 : Strickl. Orn. Syn. p. 93.

Cerchneis vespertina, Sharpe, Cat. B. i. p. 443.

$a,-b$ Tuscany (C. Passerini) 1835.

$$
\text { [p. 502.] }
$$

2453. Tinnunculus tinnunculoides.

Add 6 Azani in Mysia, April (H. E. Strickland) 1836. 


\section{INDEX.}

Acanthiza, 49 .

Acanthogenys, 159.

Acanthorhynchus, 155.

Accentor, 60.

Accipiter, 494.

Acredula, 66.

Acridotheres, 272.

Acrocephalus, 50.

Actinodura, 22.

Actiturus, 612.

Aedon, 42.

Egialitis, 595.

Egithalus, 66.

Egithaliscus, $i b$.

Egithina, 107.

Ælurœdus, 280.

Ethopyga, 163.

Agapornis, 461.

Agelæus, 265.

Agriornis, 296.

Agyrtria, 368.

Aithurus, 362.

Aix, 532.

Alæmon, 258.

Alauda, 254.

Alaudula, 256.

Alca, 632 .

Alcedo, 414.

Alcippe, 27.

Alcurus, 98.

Alcyone, 415.

Alectorurus, 297.

Allotrius, 74.

Alophonerpes, 385.

Amadina, 248.

Amaurormis, 580.

Amazilia, 369.

Amblyrhamphus, 267.

Ammodromus, 232.

Ammomanes, 258.

Ampelis, 147.

Ampelion, 328.
Amydrus, 277.

Anabatoides, 338.

Anabazenops, 339.

Anas, 533.

Anastomus, 523.

Anæretes, 305.

Andigena, 451.

Andropadus, 99.

Anous, 626.

Anser, 528.

Anseranus, $i b$.

Anthochæra, 158.

Anthodiæta, 167.

Anthornis, 158.

Anthothreptes, 168.

Anthracoceros, 422.

Anthropoides, 588.

Anthus, 82.

Antrostomus, 372.

Aphantochroa, 360.

Aphobus, 270.

Aplonis, 277.

Aprosmictus, 459 .

Aquila, 490.

Ara, 464.

Arachnothera, 168.

Aramides, 580.

Aramus, 587.

Arboricola, 567.

Archibuteo, 487.

Ardea, 515.

Ardetta, 518.

Argus, 561 .

Arremon, 198.

Artamus, 282.

Arundinax, 50.

Arundinicola, 297.

Asio, 478.

Astur, 493.

Asturina, 484.

Asturinula, 487.

Athene, 476 .
Atticora, 152。

Attila, 326.

Aulacorhamphus, 452 .

Aulia, 325.

Auripasser, 217.

Automolus, 337.

Barbatula, 448.

Baryphthengus, 413.

Basileuterus, 94, 643.

Basilinna, 360.

Batara, 344.

Batrachostomus, 374 .

Baza, 505.

Bernicla, 529.

Biziura, 540.

Blacicus, 316.

Bolborhynchus, 466.

Bonasa, 574.

Botaurus, 519.

Brachypodius, 105.

Brachypternus, 404.

Bradyornis, 133.

Bringa, 117.

Brotogerys, 469.

Buarremon, 197.

Bubo, 172.

Bucco, 429, 644.

Buchanga, 116.

Buphaga, 279.

Busarellus, 487.

Butastur, 493.

Buteola, 485.

Buteo, 485, 644.

Buthraupis, 187.

Butorides, 518.

Cacatua, 453.

Caccabis, 570.

Cacicus, 261.

Cacomantis, 433.

Caica, 468.

Calandrella, 256. 
Calendula, 255.

Calidris, 611.

Callacanthis, 208.

Calliope, 41, 642.

Calliphlox, 365 .

Calliste, 183.

Callocephalus, 454 .

Calocitta, 293.

Calopsittacus, 454 .

Calorhamphus, 449.

Calornis, 278.

Calothorax, 364.

Calyptomena, 357.

Calyptorhynchus, 454 .

Calyptura, 330.

Campephaga, 122.

Campephilus, 383.

Campothera, 393.

Camptolæmus, 539.

Campylopterus, 360.

Campylorhynchus, 75 .

Cancroma, 522 .

Capito, 449.

Caprimulgus, 376.

Capsiempsis, 306.

Carcineutes, 421.

Cardinalis, 218.

Carduelis, 212.

Carphibis, 524.

Carpodacus, 204.

Carpophaga, 553.

Cassidix, 271.

Cathartes, 509.

Catharus, 1.

Catherpes, 76.

Celeus, 403.

Centrites, 300.

Centropus, 443.

Centurus, 399.

Cephalolepis, 365.

Cepphus, 633.

Cercotrichas, 642.

Ceriornis, 562.

Certhia, 71.

Certhilauda, 257.

Certhiola, 178.

Ceryle, 415.

Ceyx, 418.

Chalcopelia, 549.
Chalcophaps, 552.

Chamæpelia, 547.

Chamæza, 352.

Chaptia, 115.

Charadrius, 594.

Chasmorhynchus, 331.

Chætornis, 18, 642.

Chætura, 372.

Chaulelasmus, 534 .

Chelidon, 152.

Chera, 247.

Chettusia, 599.

Chibia, 115.

Chimarrhornis, 40.

Chiromachæris, 321.

Chiroxiphia, 320.

Chlamydodera, 280.

Chlorochrysa, 182.

Chloronerpes, 394.

Chlorophonia, 179.

Chlorophanes, 177.

Chlorospingus, 196.

Chlorostilbon, 370 .

Chondestes, 234.

Chordeiles, 375 .

Chrysococcyx, 435 .

Chrysocolaptes, 403.

Chrysolampis, 365.

Chrysomitris, 213.

Chrysophlegma, 391.

Chrysoptilus, 396.

Chrysotis, 467.

Chthonicola, 59 .

Ciccaba, 475 .

Ciconia, 522.

Cinchlorhamphus, 86 .

Cinclodes, 333.

Cinclosoma, 23.

Cinclus, 29.

Cinnicerthia, 76.

Cinnyris, 163.

Circaetus, 492.

Circus, 482, 644.

Cissa, 287.

Cissopis, 199.

Cisticola, 53.

Cistothorus, 78.

Cittocincla, 15.

Cladorhynchus, 604.
Clangula, 538.

Climacteris, 70 .

Clytolæma, 367.

Cnipolegus, 298.

Coccothraustes, 211.

Coceygus, 440.

Coccystes, 436.

Cochoa, 74 .

Cœreba, 177.

Colaptes, 400.

Colius, 406.

Collocalia, 372.

Collyriocincla, 134.

Columba, 542.

Colymbus, 636.

Compsocoma, 188.

Conirostrum, 175.

Conopias, 310.

Conopophaga, 353 .

Contopus, 314.

Conurus, 465.

Copsychus, 15.

Copurus, 299.

Coracias, 411.

Corcorax, 286.

Coriphilus, 456.

Corvinella, 129.

Corvultur, 285.

Corvus, 283.

Corydon, 358.

Coryphospingus, 226.

Corythaix, 424.

Corythopis, 354.

Corythornis, 415.

Cosmonetta, 538.

Cossypha, 14.

Cotinga, 329.

Coturniculus, 233.

Coturnix, 568.

Cotyle, 152, 643 .

Cracticus, 281.

Crateropus, 16.

Crex, 581.

Criniger, 98.

Crithagra, 216.

Crotophaga, 443.

Cryptolopha, 145.

Crypsirhina, 288.

Cryptorhina, $i b$. 
Crypturus, 640 .

Cuculus, 431.

Culicipeta, 47.

Curæus, 268.

Cursorius, 593.

Cutia, 74.

Cyanecula, 40 .

Cyanocitta, 290.

Cyanocorax, 292.

Cyanomyia, 368.

Cyanospiza, 224.

Cyanotis, 305.

Cyclorhis, 114.

Cymborhynchus, 357.

Cyornis, 140.

Cyphorhinus, 76.

Cypsnagra, 195.

Cypselus, 370.

Cyrtonyx, 573.

Dacèlo, 421.

Dacnis, 176.

Dafila, 533.

Daption, 630 .

Daulias, 41.

Dendrobates, 392.

Dendrocitta, 287.

Dendrocincla, 340.

Dendrocolaptes, $i b$.

Dendrocygna, 531.

Dendrœca, 89.

Dendrophila, 69 .

Dendroplex, 342.

Dendrornis, 341.

Dendrortyx, 572.

Dicæum, 169.

Dicrurus, 115.

Diglossa, 174.

Diglossopis, 175.

Dilophus, 273.

Diomedea, 632.

Diplopterus, 442

Discura, 364

Dissemurus, 117.

Diuca, 228.

Diucopis, 201.

Diva, 182.

Dolichonyx, 264.

Donacobius, 75 .
Doricha, 365.

Drepanis, 173.

Dromas, 617.

Dromococcyx, 442.

Drymocataphus, 26.

Drymœca, 54.

Dryocopus, 384.

Dryodromas, 59.

Dryoscopus, 131.

Dumetia, 25.

Dysithamnus, 346.

Eclectus, 458.

Elainea, 308.

Elanoides, 507.

Elanus, $i b$.

Elminia, 142.

Embernagra, 233.

Emberiza, 235.

Emberizoides, 234.

Empidias, 316.

Empidonax, 313.

Empidonomus, 317.

Engyptila, 550.

Entomophila, 155.

Entomyza, 160.

Eopsaltria, 135.

Ephthianura, 60.

Ereunetes, 611.

Ergaticus, 96.

Erïsmatura, 541.

Erithacus, 41.

Erythrogonys, 599.

Erythrospiza, 206.

Erythrosterna, 137.

Esacus, 591.

Estrilda, 249.

Eucephala, 369.

Eudocimus, 526.

Eudromias, 595.

Eudynamis, 437.

Eudyptes, 639.

Eulampis, 362.

Eumomota, 413.

Eupetomena, 360.

Euphema, 459.

Euphonia, 179.

Euplocamus, 563.

Eurycephalus, 130
Eurylæmus, 357.

Eurystomus, 412.

Euscarthmus, 302.

Euspiza, 237.

Eustephanus, 367.

Excalfactoria, 569.

Falco, 499, 644.

Falcunculus, 67.

Florisuga, 363.

Fluvicola, 297.

Formicarius, 352.

Formicivora, 348

Foudia, 243.

Francolinus, 565.

Fratercula, 634.

Fringilla, 207.

Fringillaria, 238

Fulica, 586.

Fuligula, 537.

Fulmarus, 630 .

Furnarius, 332.

Galbula, 427.

Galeoscoptes, 11.

Galerita, 257.

Gallicrex, 586.

Gallinago, 606 .

Gallinula, 585 .

Galloperdix, 564.

Gallus, 563.

Gampsonyx, 506.

Gampsorhynchus, 22.

Garrulax, 20.

Garrulus, 288.

Gecinus, 389.

Geobates, 332 .

Geocichla, 8.

Geococcyx, 442.

Geocolaptes, 401.

Geopelia, 553.

Geophaps, 552.

Geositta, 332.

Geospiza, 219.

Geothlypis, 92.

Geotrygon, 550.

Geranoaetus, 488.

Geranospizias, 498.

Glareola, 592. 
Glaucidium, 477.

Glaucis, 359.

Glaucopis, 294.

Glossiptila, 178.

Glyciphila, 155.

Glyphorhynchus, 340.

Gracula, 271.

Grallina, 282.

Grallaria, 353.

Grammatoptila, 23.

Grus, 587.

Guira, 442.

Guiraca, 219.

Gygis, 626.

Gymnopelia, 548.

Gymnomystax, 267.

Gymnorhina, 281.

Gypagus, 509.

Gyps, 510.

Habropyga, 248.

Hadrostomus, 323.

Hæmatopus, 603.

Hagedashia, 525.

Halcyon, 419.

Haliaetus, 491.

Haliastur, 505.

Hapalis, 58.

Haploderma, 427.

Haplopelia, 549.

Haplospiza, 225.

Harelda, 539.

Harpactes, 426.

Harpagus, 506.

Harpiprion, 525.

Harporhynchus, 11.

Harpyhaliaetus, 489.

Hedymeles, 218.

Helianthea, 367.

Heilicura, 320.

Heliochera, 328.

Heliomaster, 367.

Heliornis, 587.

Heliothrix, 366.

Helminthophaga, 88.

Helminthotherus, 89.

Helotarsus, 489.

Hemicercus, 401.

Hemichelidon, 139.
Hemipus, 138.

Hemitricus, 303.

Hemixus, 98.

Henicognathus, 464.

Heniconetta, 539.

Henicurus, 30.

Herpetotheres, 508.

Herpornis, 73.

Herpsilochmus, 347.

Heterocnemis, 350 .

Heteropelma, 321.

Heterura, 86.

Hieracidea, 503.

Hierococcyx, 433.

Himantopus, 605.

Hirundinea, 312.

Hirundo, 148.

Homorus, 336.

Hoplopterus, 601.

Hydrochelidon, 622.

Hydrophasianus, 592.

Hydropsalis, 379.

Hyetornis, 441.

Hylacola, 59.

Hylactes, 355.

Hyliota, 138.

Hylocharis, 369 .

Hylomanes, 413.

Hylophilus, 113.

Hypergerus, 19.

Hyphantornis, 240.

Hypochera, 247.

Hypolais, 40.

Hypotænidia, 578.

Hypothymis, 141.

Hypoxanthus, 398.

Hypsipetes, 97.

Ianthia, 41.

Ibis, 524 .

Ibycter, 508 .

Icteria, 642.

Icterus, 262.

Ictinia, 507.

Indicator, 444.

Inocotis, 525 .

Iodopleura, 330.

Iole, 104.

Irena, 108.
Iridornis, 186.

Irrisor, 407.

Ispidina, 418.

Ithaginis, 563.

Ixodia, 105.

Ixulus, 72.

Ixus, 103.

Iynx, 381 .

Jacamaralcyon, 429.

Jacamerops, 428.

Junco, 232.

Ketupa, 472.

Lagenoplastes, 151.

Lagonosticta, 248.

Lagopus, 575.

Lalage, 121.

Lampornis, 361.

Lamprotes, 193.

Lamprocolius, 275.

Lampropsar, 269.

Lamprospiza, 199.

Lamprotornis, 274.

Laniarius, 131.

Lanio, 193.

Laniisoma, 326.

Lanioturdus, 143.

Lanius, 125.

Larus, 619.

Larvivora, 40.

Lathamus, 462.

Lathria, 325.

Legatus, 308.

Leistes, 267.

Leptasthenura, 334.

Leptodon, 505.

Leptopogon, 306.

Leptoptilus, 523.

Leptornis, 159.

Leptotricus, 304.

Lerwa, 571.

Leucochloris, 368.

Leucomelæna, 554 .

Leuconerpes, 398.

Leucopternis, 488.

Leucosarcia, 551.

Leucosticte, 208. 
Lichenops, 299.

Ligurinus, 209.

Limicola, 609.

Limnocorax, 583.

Limosa, 615.

Linota, 206.

Lioptilus, 74.

Liothrix, 71.

Lipaugus, 326.

Lobivanellus, 601.

Lochmias, 333.

Lophoceros, 423.

Lophocitta, 294.

Lopholæmus, 554.

Lophophanes, 64.

Lophophorus, 562.

Lophortyx, 573.

Lophostrix, 474.

Loriculus, 459.

Lorius, 455.

Loxia, 202.

Loxigilla, 219.

Lurocalis, 375.

Machæropterus, 320.

Machetes, 611.

Machetornis, 300.

Macrodipteryx, 378.

Macronus, 25.

Macronyx, 85.

Macropteryx, 373.

Macropygia, 546.

Macrorhamphus, 615.

Majaqueus, 629.

Malacocercus, 16.

Malacopterus, 24.

Malacoptila, 430.

Malacorhynchus, 537.

Malurus, 57.

Manorhina, 160.

Manucodia, 279.

Mareca, 532.

Margarornis, 339.

Marmaronetta, 534.

Megalæma, 445.

Megaloprepia, 554.

Megalurus, 18.

Megarhynchus, 311.

Meiglyptes, 402.

S. C.
Melanerpes, 397.

Melanochlora, 65.

Melanocorypha, 255.

Melænornis, 133.

Melanotis, 11.

Meleagris, 564.

Melierax, 498.

Meliphaga, 157.

Melithreptus, 161.

Melizophilus, 45.

Melopelia, 548.

Melophus, 238.

Melopsittacus, 460.

Melospiza, 230.

Menura, 295.

Mergus, 541.

Mergulus, 633.

Merops, 408.

Merulaxis, 354.

Mesocalius, 435.

Metopia, 321.

Metopidius, 592.

Metriopelia, 549.

Micrastur, 497.

Micrœea, 139.

Microhierax, 506.

Micropternus, 402.

Microtarsus, 104.

Milvago, 508.

Milvulus, 318.

Milvus, 504.

Mimocichla, 11.

Mimus, 12.

Minla, 72.

Mionectes, 306 .

Mirafra, 258.

Mitrephorus, 313.

Mixornis, 25.

Mniotilta, 87.

Mohoua, 67.

Molothrus, 265.

Momotus, 412.

Monarcha, 135.

Monasa, 431.

Montifringilla, 207.

Morphnus, 489.

Motacilla, 79.

Munia, 251.

Muscicapa, 136.
Muscicapula, 138.

Muscipipra, 299.

Muscisaxicola, 300.

Muscivora, 312.

Musophaga, 424.

Mycteria, 523.

Myiagra, 141.

Myiarchus, 315.

Myiadectes, 61.

Myiobius, 312.

Myiochanes, 315.

Myiodioctes, 93.

Myiodynastes, 311.

Myiophoneus, 13.

Myiozetetes, 309.

Myrmeciza, 351.

Myrmecocichla, 34.

Myrmotherula, 347.

Myzantha, 160.

Myzomela, 154.

Nænia, 626.

Nasica, 341.

Nectarinia, 162.

Nemosia, 195.

Neopus, 490.

Neorhynchus, 223.

Nestor, 463.

Nettapus, 529.

Nigrita, 243.

Nilaus, 129.

Niltava, 139.

Ninox, 476.

Nisaetus, 490.

Nonnula, 430.

Nothoprocta, 641.

Nothura, $i b$.

Nucifraga, 285.

Numenius, 616.

Numida, 564.

Nyctala, 481.

Nyctea, 475.

Nyctherodius, 521.

Nyctibius, 374.

Nycticorax, 521.

Nyctidromus, 380.

Nyctiornis, 410.

Oceanites, 628. 
Odontophorus, 573.

Edemia, 540.

Edicnemus, 590.

(Ena, 553.

Estrelata, 629.

Ombria, 634.

Orchesticus, 201.

Orchilus, 303.

Oreocincla, 7.

Oreœca, 67.

Oreotrochilus, 361 .

Origma, 38.

Oriolus, 109.

Ornithion, 307.

Ortalis, 561.

Orthogonys, 193.

Orthonyx, 294.

Orthotomus, 52.

Ortygornis, 566.

Ortygospiza, 253.

Ortyxelos, 577.

Oryzoborus, 220.

Ossifraga, 629.

Ostinops, 260.

Otis, 588.

Otocorys, 260.

Otogyps, 510.

Oxyrhamphus, 296.

Oxyurus, 334.

Pachycephala, 135.

Pachyrhamphus, 323.

Padda, 251.

Palæornis, 457.

Pandion, 482.

Panoplites, 363.

Panurus, 62.

Panyptila, 371.

Paradoxornis, 254.

Pardalotus, 173.

Parisoma, 67, 643.

Paroaria, 225.

Parra, 591.

Parus, 62.

Passer, 209.

Passerculus, 228.

Passerella, 231.

Pastor, 272.

Patagona, 366.
Pediœcetes, 574.

Pelargopsis, 417.

Pelecanoides, 631.

Pelecanus, 511.

Pellorneum, 26.

Penelope, 560.

Penelopides, 422.

Penelopina, 560.

Penthetria, 245.

Pentholæa, 36.

Percnostola, 350.

Perdicula, 569.

Perdix, 567.

Perisoreus, 289.

Pericrocotus, 122.

Peristera, 549.

Pernis, 505.

Petasophera, 366.

Petrochelidon, 151.

Petrocincla, 9.

Petrœca, 37.

Petronia, 211.

Peucæa, 232.

Pezoporus, 460.

Phabotreron, 552.

Phacelodomus, 336.

Phaethon, 511.

Phaethornis, 359.

Phalacrocorax, 512.

Phalaropus, 605.

Phaps, 551.

Pharomacrus, 426.

Phasianus, 562.

Pheucticus, 218.

Phibalura, 328.

Philentoma, 147.

Philohela, 606.

Philydor, 337.

Phleocryptes, 334.

Phlogœnas, 552

Phodilus, 481.

Phœbetria, 632.

Phœnicocercus, 327.

Phœnicophilus, 198.

Phœnicopterus, 527.

Phœnicothraupis, 193.

Pholidauges, 276.

Phonipara, 224.

Phrygilus, 227.
Phyllomyias, 307.

Phyllornis, 105.

Phylloscartes, 304.

Phylloscopus, 45.

Phyllostrephus, 99.

Phytotoma, 332.

Piaya, 441.

Pica, 286.

Picoides, 389.

Picolaptes, 343.

Picumnus, 381.

Picus, 385.

Pinicola, 204.

Pionopsittacus, 468.

Pionus, 467.

Pipilo, 234.

Pipra, 318.

Pipreola, 328.

Pipridea, 182.

Piprisoma, 171.

Piprites, 318.

Pitangus, 310.

Pithys, 351.

Pitta, 355.

Pitylus, 201.

Platalea, 526.

Platycercus, 461.

Platyrhynchus, 301.

Platystira, 142.

Plectrophanes, 239.

Plegadis, 526.

Plocepasser, 243.

Ploceus, 242.

Plotus, 514.

Pluvianus, 600.

Podager, 375.

Podargus, 373.

Podiceps, 636.

Podilymbus, 638.

Pœcilothraupis, 187.

Pœocephalus, 463.

Poephila, 251.

Pogonornis, 159.

Pogonorhynchus, 445.

Polioaetus, 491.

Poliococcyx, 440.

Polioptila, 61.

Poliospiza, 217.

Polyboroides, 498. 
Polyborus, 509.

Polytelis, 458.

Polytmus, 366.

Pomatorhinus, 28.

Poospiza, 226.

Poœcetes, 229.

Porphyrio, 584.

Porphyriops, 585.

Porphyriospiza, 226.

Porzana, 581.

Pratincola, 35.

Prinia, 56.

Prion, 631.

Prionochilus, 171 .

Prionops, 133.

Procellaria, 628.

Procnias, 178.

Progne, 153.

Promerops, 161.

Propasser, 205.

Prosthemadera, 158.

Psalidoprocne, 152.

Psarisomus, 358.

Psephotis, 462.

Pseudocolaptes, 336.

Pseudogyps, 510.

Pseudoleistes, 267.

Psittacula, 462.

Psittinus, 458.

Psittospiza, 199.

Psophodes, 19.

Pterocles, 558.

Pteroglossus, 451.

Pteroptochus, 354.

Pterythrius, 73.

Ptilogonys, 147.

Ptilopus, 555.

Ptilorhis, 279.

Ptilorhynchus, 280.

Ptilotus, 156.

Pucrasia, 562.

Puffinus, 630.

Pycnonotus, 100.

Pyctorhis, 28.

Pyranga, 191.

Pyrocephalus, 313.

Pyrgisoma, 234.

Pyriglena, 349.

Pyroderus, 331.
Pyromelæna, 244.

Pyrrhocorax, 285.

Pyrrhocheira, 277.

Pyrrhula, 203.

Pyrrhulauda, 255.

Pytetia, 248.

Querquedula, 535.

Querula, 330.

Quiscalus, 269, 643.

Rallina, 578.

Rallus, 579 .

Ramphocœlus, 190.

Recurvirostra, 604.

Regulus, 18.

Rhamphastos, 449 .

Rhamphocœnus, 349 .

Rhinococcyx, 439.

Rhinoplax, 421.

Rhinoptilus, 593.

Rhinortha, 438.

Rhipidura, 143.

Rhizothera, 567.

Rhodonessa, 535.

Rollulus, 570.

Rhopodytes, 438.

Rhopoterpe, 352.

Rhynchæa, 608.

Rhynchops, 627.

Rhynchocyclus, 309.

Rhynchotus, 641.

Rhynchopsitta, 464.

Rissa, 618.

Rubigula, 104, 643.

Rupicola, 327.

Ruticilla, 38.

Saltator, 199.

Saraglossa, 278.

Sarcidiornis, 531.

Sarciophorus, 602.

Sarcops, 271.

Sarcorhamphus, 509.

Sasia, 382.

Saurothera, 441.

Saxicola, 31.

Scardafella, 547.

Schizorhis, 424.
Sclerurus, 333.

Scolecophagus, 268.

Scolopax, 606.

Scops, 473.

Scopus, 522.

Scotornis, 378.

Scythrops, 438.

Selasphorus, 364.

Selenidera, 451.

Sericornis, 48.

Sericulus, 279

Serinus, 215

Serphophaga, 304.

Sirystes, 311.

Setophaga, 95 .

Sialia, 38, 642.

Sibia, 23.

Sigelus, 133.

Simorhynchus, 634 。

Siphia, 140.

Sisopygis, 298.

Sisura, 141.

Sitta, 68.

Sittella, 70.

Sittasomus, 339.

Siurus, 87.

Siva, 72.

Somateria, 539 .

Spatula, 536.

Speotyto, 476.

Spermestes, 247.

Spermophaga, 249.

Spermophila, 220.

Spermospiza, 247.

Sphecotheres, 111.

Sphenæacus, 18.

Spheniscus, 639.

Sphenocercus, 555.

Sphenura, 19.

Sphyropicus, 389.

Spilornis, 492.

Spindalis, 190.

Spizaetus, 492.

Spizalauda, 257.

Spizella, 231.

Sporopipes, 246.

Spreo, 276.

Squatarola, 594

Stachyris, 27. 
Stagonopleura, 251.

Steatornis, 380.

Stelgidopteryx, 153.

Stenostira, 143.

Stephanophorus, 186.

Stercorarius, 618.

Sterna, 622.

Stigmatops, 155.

Stipiturus, 58.

Stoparola, 140.

Strepera, 280.

Strepsilas, 603 .

Strix, 470.

Struthidea, 294.

Sturnella, 268.

Sturnia, 273.

Sturnopastor, 274.

Sturnus, $i b$.

Sula, 512.

Surnia, 475.

Surniculus, 434.

Sycalis, 215, 643.

Sycobrotus, 242 .

Sylvia, 43.

Sylvietta, 59.

Symmorphus, 122.

Synallaxis, 334.

Syrnium, 479.

Syrrhaptes, 559.

Tachyphonus, 194.

Tadorna, 531.

Tænioptera, 296.

Tæniopygia, 251.

Tanagra, 188.

Tanagrella, 181.

Tantalus, 523.

Telephonus, 130.

Temenuchus, 273.

Temnurus, 288.

Tephrodornis, 132.

Terekia, 615.

Terenura, 349.

Terpsiphone, 145.
Tetrao, 574.

Tetraogallus, 571.

Textor, 240.

Thalassœca, 630 .

Thalurania, 362.

Thamnobia, 36.

Thamnomanes, 347 .

Thamnophilus, 344 .

Theristicus, 525.

Thinocorus, 604.

Thripophaga, 336.

Thryophilus, 77.

Thryothorus, $i b$.

Tiaris, 226.

Tichodroma, 69.

Tiga, 405.

Tigrisoma, 520.

Tijuca, 327.

Timelia, 24.

Tinamus, 640.

Tinnunculus, 501, 644 .

Tityra, 322.

Toccus, 422.

Todirhamphus, 421.

Todirostrum, 301.

Todus, 414.

Topaza, 361.

Totanus, 613.

Trachycomus, 99.

Trachyphonus, 448.

Treron, 556.

Tribonyz, 585.

Trichoglossus, 455.

Trichothraupis, 194.

Trichostoma, 26.

Triclaria, 467.

Tringa, 609.

Tringoidis, 612 .

Trochalpoterum, 21. Zanclostomus, 439.

Trochilus, 364.

Troglodytes, 78 .

Trogon, 425.

Tropidorhynchus, 159.

Turdus, 1.
Turdinus, 26.

Turnagra, 24.

Turnix, 576.

Turtur, 544.

Tyrannus, 317.

Tyrannulus, 307.

Upucerthia, 332.

Upupa, 407.

Uragus, 205.

Uria, 633

Urobrachys, 245.

Urochroma, 469.

Urocissa, 287.

Urogalba, 428.

Uroloncha, 253.

Urubitinga, 487.

Vanellus, 600.

Venilia, 402.

Vidua, 246.

Vireo, 113.

Vireosylvia, 111.

Volatinia, 223.

Volvocivora, 121.

Xanthocephalus, 266.

Xantholæma, 447.

Xanthomyza, 156

Xanthopygia, 138.

Xanthosomus, 266.

Xenops, 339.

Xiphocolaptes, 341.

Xipholena, 329.

Xiphorhynchus, 343.

Yuhina, 73.

Zebrilus, 520.

Zenaida, 548.

Zenaidura, $i b$.

Zonotrichia, 229.

Zosterops, 171. 
University Press, Cambridge, Fune, 1882 .

CATALOGUE OF W O R K S

PUBLISHED FOR THE SYNDICS

OF THE

\section{Cambrioge danturatity 稀ess.}

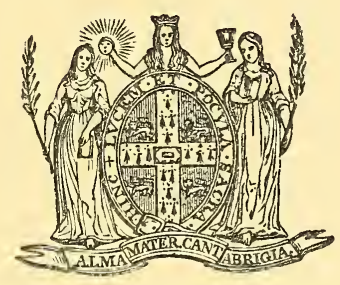

㞷ondon: C. J. CLAY, M.A. AND SON. CAMBRIDGE UNIVERSITY PRESS WAREHOUSE, I 7 PATERNOSTER ROW.

Cambrioge: DEIGHTON, BELL, AND CO. ZLeiptig: F. A. BROCKHAUS. 


\section{PUBLICATIONS OF}

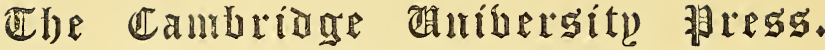

\section{THE HOLY SCRIPTURES, \&C.}

\section{THE CAMBRIDGE PARAGRAPH BIBLE}

of the Authorized English Version, with the Text Revised by a Collation of its Early and other Principal Editions, the Use of the Italic Type made uniform, the Marginal References remodelled, and a Critical Introduction prefixed, by the Rev. F. H. Scrivener, M.A., LL.D., Editor of the Greek Testament, Codex Augiensis, \&c., and one of the Revisers of the Authorized Version. Crown 4to. cloth. gilt. 2 Is.

From the Times.

"Students of the Bible should be particularly grateful to (the Cambridge University Press) for having produced, with the able assistance of Dr Scrivener, a complete critical edition of the Authorized Version of the English Bible, an edition such as, to use the words of the Editor, "would have been executed long ago had this version been nothing more than the greatest and best known of English classics.' Falling at a time when the formal revision of this version has been undertaken by a distinguished company of scholars and divines, the publication of this edition must be considered most opportune."

\section{From the Athenceum.}

"Apart from its religious importance, the English Bible has the glory, which but few sister versions indeed can claim, of being the chief classic of the language, of having, in conjunction with Shakspeare, and in an immeasurable degree more than he, fixed the language beyond any possibility of important change. Thus the recent contributions to the literature of the subject, by such workers as Mr Francis Fry and Canon Westcott, appeal to a wide range of sympathies; and to these may now be added Dr Scrivener, well known for his labours in the cause of the Greek Testament criticisin, who has brought out, for the Syndics of the Cambridge University Press, an edition of the English Bible, according to the text of $\mathrm{r} 6 \mathrm{Ir}$, revised by a comparison with later issues on principles stated by him in his Introduction. Here he enters at length into the history of the chief editions of the version, and of such features as the marginal notes, the use of italic type, and the changes of orthography, as well as into the most interesting question as to the original texts from which our translation is produced."

\section{From the Methodist Recorder.}

" This noble quarto of over I300 pages is in every respect worthy of editor and publishers alike. The name of the Cambridge University Press is guarantee enough for its perfection in outward form, the name of the editor is equal guarantee for the worth and accuracy of its contents. Without question, it is the best Paragraph Bible ever published, and its reduced price of a guinea brings it within reach of a large number of students. . But the volume is much more than a Paragraph Bible. It is an attempt, and a successful attempt, to give a critical edition of the Authorised English Version, not (let it be marked) a revision, but an exact reproduction of the original Authorised Version, as published in r6rI, minus patent mistakes. This is doubly necessary at a time when the version is about to undergo revision. .. To all who at this season seek a suitable volume for presentation to ministers or teachers we earnestly commend this work."

From the London Quarterly Review.

"The work is worthy in every respect of the editor's fame, and of the Cambridge University Press. The noble English Version, to which our country and religion owe so much, was probably never presented before in so perfect a form."

\section{THE CAMBRIDGE PARAGRAPH BIBLE.} STUDFNT'S EDITION, on good writing paper, with one column of print and wide margin to each page for MS. notes. This edition will be found of great use to those who are engaged in the task of Biblical criticism. Two Vols. Crown 4to. cloth. gilt. 3 Is. $6 d$.

THE LECTIONARY BIBLE, WITH APOCRYPHA, divided into Sections adapted to the Calendar and Tables of Lessons of $187 \mathrm{I}$. Crown 8vo. cloth. $35.6 d$.

London: Cambridge Warehouse, I7 Paternoster Row. 


\section{BREVIARIUM}

\section{AD USUM INSIGNIS ECCLESIAE SARUM.}

Fasciculus II. In quo continentur PSALTERIUM, cum ordinario Officii totius hebdomadae juxta Horas Canonicas, et proprio Completorii, Litania, COMMUNe SANCTORUM, ORDINARIUM Missae CUM CANONE ET XIII Missis, \&c. \&c. juxta Editionem maximam pro Claudio Chevallon et Francisco Regnault A.D. Mdxxxi. in Alma Parisiorum Academia impressam : labore ac studio FRANCISCI Procter, A.M., et Christophori Wordsworth, A.M. Demy 8vo. cloth. I $2 s$.

FASCiCUlus I. In quo continentur Kalendarium, et ORdo TemPORALIS sive PROPRIUM DE TEMPORE TOTIUS ANNI, una cum ordinali suo quod usitato vocabulo dicitur PICA SIVE DIRECTORIUM SACERDotUm. Demy 8vo. cloth. £ I.

"Not only experts in liturgiology, but all persons interested in the history of the Anglican Book of Common Prayer, will be grateful to the Syndicate of the Cambridge University Press for forwarding the publication of the volume which bears the above title, and which has recently appeared under their auspices." -Notes and Queries.

"We have here the first instalment of the celebrated Sarum Breviary, of which no entire edition has hitherto been printed since the year $1557 \ldots$ Of the valuable explanatory notes, as well as the learned introduction to this volume, we can only speak in terms of the very highest commendation."-The $E_{x}$ aminer.

\section{GREEK AND ENGLISH TESTAMENT,}

in parallel Columns on the same page. Edited by J. SCHOLEFIELD, M.A. late Regius Professor of Greek in the University. Small Octavo. New Edition, with the Marginal References as arranged and revised by Dr SCRIVENER. Cloth, red edges. 7s. $6 \mathrm{~d}$.

\section{GREEK AND ENGLISH TESTAMENT,}

The StUdent's Edition of the above, on large writing paper. 4 to. cloth. I2s.

\section{GREEK TESTAMENT,}

ex editione Stephani tertia, I550 Small 8vo. 3s. $6 d$.

\section{THE NEW TESTAMENT IN GREEK}

according to the text followed in the Authorised Version, with the Variations adopted in the Revised Version. Edited by F. H. A. Scrivener, M.A., D.C.L., LL.D. Crown 8vo. 6s. Morocco boards or limp. I2s.

\section{THE BOOK OF ECCLESIASTES,}

With Notes and Introduction. By the Very Rev. E. H. Plumptre, D.D., Dean of Wells. Large Paper Edition. Demy 8vo. $7 s .6 d$.

"No one can say that the Old Testament which we can point in English exegesis of is a dull or worn-out subject after reading the Old Testament; indeed, even Delitzsch, this singularly attractive and also instructive whose pride it is to leave no source of illuscommentary. Its wealth of literary and his- tration unexplored, is far inferior on this head torical illustration surpasses anything to to Dr Plumptre."-Academy, Sept. 1o, 188ז. THE GOSPEL ACCORDING TO ST MATTHEW in Anglo-Saxon and Northumbrian Versions, synoptically arranged: with Collations of the best Manuscripts. By J. M. Kemble, M.A. and Archdeacon HaRDwICK. Demy 4to. Ios.

THE GOSPEL ACCORDING TO ST MARK in Anglo-Saxon and Northumbrian Versions synoptically arranged: with Collations exhibiting all the Readings of all the MSS. Edited by the Rev. Professor SkEat, M.A. late Fellow of Christ's College, and author of a McEso-GothiC Dictionary. Demy 4to. Ios.

\section{London: Cambridge Warehouse, I 7 Paternoster Row.}


THE GOSPEL ACCORDING TO ST LUKKE, uniform with the preceding, edited by the Rev. Professor SkEAT. Demy 4to. Ios.

THE GOSPEL ACCORDING TO ST JOHN, uniform with the preceding, by the same Editor. Demy 4to. Ios.

"The Gospel according to St Fohn, in Anglo-Saxon and Northumbrian Versions: Edited for the Syndics of the University Press, by the Rev. Walter W. Skeat, M.A., Elrington and Bosworth Professor of Anglo: Saxon in the University of Cambridge, completes an undertaking designed and ccmnenced by that distinguished scholar J. M. Kemble, some forty years ago. He was not himself permitted to execute his scheme; he died before it was completed for St Matthew. The edition of that Gospel was finished by Mr., subsequently Archdeacon, Hardwick. The remaining Gospels

have had the good fortune to be edited by Professor Skeat, whose competency and zeal have left nothing undone to prove himself equal to his reputation, and to produce a work of the highest value to the student of Anglo-Saxon. The design was indeed worthy of its author. It is difficult to exaggerate the value of such a set of parallel texts. . O Of the particular volume now before us, we can only say it is worthy of its two predecessors. We repeat that the service rendered to the study of Anglo-Saxon by this Synoptic collection cannot easily be overstated."-Contemporary Review.

\section{THE POINTED PRAYER BOOK,}

being the Book of Common Prayer with the Psalter or Psalms of David, pointed as they are to be sung or said in Churches. Royal $24 \mathrm{mo}$. Cloth. Is. 6 .

The same in square 32mo. cloth. $6 d$.

"The 'Pointed Prayer Book' deserves and still more for the terseness and clearmention for the new and ingenious system ness of the directions given for using it." on which the pointing has been marked,

Times.

\section{THE CAMBRIDGE PSALTER,}

for the use of Choirs and Organists. Specially adapted for Congregations in which the "Cambridge Pointed Prayer Book" is used. Demy 8vo. cloth extra, 3s. $6 d$. Cloth limp, cut flush. 2s. $6 d$.

\section{THE PARAGRAPH PSALTER,}

arranged for the use of Choirs by BROOKE Foss WESTCOTT, D.D., Canon of Peterborough, and Regius Professor of Divinity in the University of Cambridge. Fcap. 4to. $5 s$.

\section{The same in royal $32 \mathrm{mo}$. Cloth $1 s$. Leather $1 s .6 \mathrm{~d}$.}

"The Paragraph Psalter exhibits all the care, thought, and learning that those acquainted with the works of the Regius Professor of Divinity at Cambridge would

expect to find, and there is not a clergyman or organist in England who should be without this Psalter as a work of reference."Morning Post.

THE MISSING FRAGMENT OF THE LATIN TRANSLATION OF THE FOURTH BOOK OF EZRA, discovered, and edited with an Introduction and Notes, and a facsimile of the MS., by ROBERT L. Bensly, M.A., Sub-Librarian of the University Library, and Reader in Hebrew, Gonville and Caius College, Cambridge. Demy 4to. Cloth. Ios.

"Edited with true scholarly complete- added a new chapter to the Bible, and, startness."-Westminster Review.

"Wer sich je mit dem 4 Buche Esra eingehender beschäftigt hat, wird durch die obige, in jeder Beziehung musterhafte Publication in freudiges Erstaunen versetzt werden."-Theologische Literaturzeitung.

"It has been said of this book that it has

ling as the staptent thay at first sight startpear, it is no exaggeration of the actual fact, if by the Bible we understand that of the larger size which contains the Apocrypha, and if the Second Book of Esdras can be fairly called a part of the Apocrypha."Saturday Review.

\section{THEOLOGY-(ANCIENT).}

THE PALESTINIAN MISHNA,

By W. H. Lowe, M.A. Lecturer in Hebrew at Christ's College, Cambridge.

In the Press. 


\section{SAYINGS OF THE JEWISH FATHERS,} comprising Pirqe Aboth and Pereq R. Meir in Hebrew and English, with Critical and Illustrative Notes. By CHARLeS TAYLOR, D.D. Master of St John's College, Cambridge, and Honorary Fellow of King:s College, London. Demy 8vo. cloth. Ios.

"The 'Masseketh Aboth' stands at the in an ordinary critical edition. . The Talhead of Hebrew non-canonical writings. It is of ancient date, claiming to contain the dicta of teachers who flourished from B.C. 200 to the same year of our era. The precise time of its conpilation in its present form is, of course, in doubt. Mr Taylor's explanatory and illustrative commentary is very full and satisfactory."-Spectator.

"If we mistake not, this is the first pre. cise translation into the English language accompanied by scholarly notes, of any portion of the Talmud. In other words, it is the first instance of that most valuable and neglected portion of Jewish literature being treated in the same way as a Greek classic neglected, we foresee will be the most important aids of the future for the proper understanding of the Bible. .. The Sayings of the Fewish Fathers may claim to be scholar$1 y$, and, moreover, of a scholarship unusually thorough and finished."-Dublin University Magazine.

"A careful and thorough edition which does credit to English scholarship, of a short treatise from the Mishna, containing a series of sentences or maxims ascribed mostly to Jewish teachers immediately preceding, or immediately following the Christian era. ..." - Contemporary Review.

\section{THEODORE OF MOPSUESTIA'S COMMENTARY ON THE MINOR EPISTLES OF S. PAUL。}

The Latin Version with the Greek Fragments, edited from the MSS. with Notes and an Introduction, by H. B. SWETE, D.D., Rector of Ashdon, Essex, and late Fellow of Gonville and Caius College, Cambridge. In Two Volumes. Vol. I., containing the Introduction, with Facsimiles of the MSS., and the Commentary upon GalatiansColossians. Demy 8vo. I2s.

"In dem oben verzeichneten Buche liegt uns die erste Hälfte einer vollständigen, ebenso sorgfältig gearbeiteten wie schön ausgestatteten Ausgabe des Commentars mit ausführlichen Prolegomena und reichhaltigen kritischen und erläuternden Anmerkungen vor."-Literarisches Centralblutt.

"It is the result of thorough, careful, and patient investigation of all the points bearing on the subject, and the results are presented with admirable good sense and modesty. $\mathrm{Mr}$ Swete has prepared himself for his task by a serious study of the literature and history which are connected with it; and he has produced a volume of high value to the student, not merely of the theology of the fourth and fifth centuries, but of the effect of this theology on the later developments of doctrine and methods of interpretation, in the ages immediately following, and in the middle ages."-Guardian.

Auf Grund dieser Quellen ist der Text bei Swete mit musterhafter Akribie hergestellt. Aber auch sonst hat der Herausgeber

mit unermüdlichem Fleisse und eingehend. ster Sachkenntniss sein Werk mit allen denjenigen Zugaben ausgerüstet, welche bei einer solchen Text-Ausgabe nur irgend erwartet werden können. . . Von den drei Haupthandschriften ... sind vortreffiche photographische Facsimile's beigegeben, wie überhaupt das ganze Werk von der University Press zu Cambridge mit bekannter Eleganz ausgestattet ist." - Theologische Literatur. zeitung.

"It is a hopeful sign, amid forebodings which arise about the theological learning of the Universities, that we have before us the first instalment of a thoroughly scientific and painstaking work, commenced at Cambridge and completed at a country rectory." -Church Quarterly Review (Jan. I88I).

"Hernn Swete's Leistung ist eine so tüchtige dass wir das Werk in keinen besseren Händen wissen möchten, und mit den sichersten Erwartungen auf das Gelingen der Fortsetzung entgegen sehen."-Göttingisch. gelehrte Anzeigen (Sept. I88I).

VOLUME II., containing the Commentary on I ThessaloniansPhilemon, Appendices and Indices. I $2 s$.

\section{SANCTI IRENÆI EPISCOPI LUGDUNENSIS}

libros quinque adversus Hæreses, versione Latina cum Codicibus Claromontano ac Arundeliano denuo collata, præmissa de placitis Gnosticorum prolusione, fragmenta necnon Græce, Syriace, Armeliace, commentatione perpetua et indicibus variis edidit W. WIGAN HARVEY, S.T.B. Collegii Regalis olim Socius. 2 Vols. Demy 8vo. I $8 s$. 


\section{MINUCII FELICIS OCTAVIUS.}

The text newly revised from the original MS., with an English Commentary, Analysis, Introduction, and Copious Indices. Edited by H. A. Holden, LL.D. Head Master of Ipswich School, late Fellow of Trinity College, Cambridge. Crown 8vo. $7 s .6 d$.

\section{THEOPHILI EPISCOPI ANTIOCHENSIS}

\section{LIBRI TRES AD AUTOLYCUM}

edidit, Prolegomenis Versione Notulis Indicibus instruxit GULIELmus Gilson Humphry, S.T.B. Collegii Sanctiss. Trin. apud Cantabrigienses quondam Socius. Post 8vo. 5 s.

\section{THEOPHYLACTI IN EVANGELIUM \\ S. MATTHAEI COMMENTARIUS,}

edited by W. G. Humphry, B.D. Prebendary of St Paul's, late Fellow of Trinity College. Demy Svo. $7 s .6 d$.

TERTULLIANUS DE CORONA MILITIS, DE SPECTACULIS, DE IDOLOLATRIA, with Analysis and English Notes, by George CURREy, D.D. Preacher at the Charter House, late Fellow and Tutor of St John's College Crown 8vo. 5 s.

\section{THEOLOGY-(ENGLISH).}

\section{WORKS OF ISAAC BARROW,}

compared with the Original MSS., enlarged with Materials hitherto unpublished. A new Edition, by A. NAPIER, M.A. of Trinity College, Vicar of Holkham, Norfolk. 9 Vols. Demy 8vo. £3. 3 s.

TREATISE OF THE POPE'S SUPREMACY, and a Discourse concerning the Unity of the Church, by ISAAC BARROW. Demy 8vo. 7s. $6 d$.

PEARSON'S EXPOSITION OF THE CREED, edited by Temple Chevallier, B.D. late Fellow and Tutor of St Catharine's College, Cambridge. New Edition. Revised by R. Sinker, B.D., Librarian of Trinity College. Demy 8vo. I2s.

"A new edition of Bishop Pearson's famous work On the Creed has just been issued by the Cambridge University Press. It is the well-known edition of Temple Chevallier, thoroughly overhauled by the Rev. R. Sinker, of Trinity College. The whole text and notes have been most carefully examined and corrected, and special pains have been taken to verify the almost innumerable references. These have been more clearly and accurately given in very many places, and the citations

themselves have been adapted to the best and newest texts of the several authorstexts which have undergone vast improvements within the last two centuries. The Indices have also been revised and enlarged. ......Altogether this appears to be the most complete and convenient edition as yet published of a work which has long been recognised in all quarters as a standard one."Guardian.

\section{AN ANALYSIS OF THE EXPOSITION OF THE CREED}

written by the Right Rev. John PEARson, D.D. late Lord Bishop of Chester, by W. H. MiLL, D.D. late Regius Professor of Hebrew in the University of Cambridge. Demy 8vo. cloth. $5 s$.

\section{London: Cambridge Warehouse, 17 Paternoster Row.}


WHEATLY ON THE COMMON PRAYER, edited by G. E. Corrie, D.D. Master of Jesus College, Examining Chaplain to the late Lord Bishop of Ely. Demy 8vo. 7 s. $6 d$.

\section{CAEAR MORGAN'S INVESTIGATION OF THE TRINITY OF PLATO,}

and of Philo Judæus, and of the effects which an attachment to their writings had upon the principles and reasonings of the Fathers of the Christian Church. Revised by H. A. Holden, LL.D. Head Master of Ipswich School, late Fellow of Trinity College, Cambridge. Crown 8 vo. $4 s$.

\section{TWO FORMS OF PRAYER OF THE TIME OF QUEEN ELIZABETH. Now First Reprinted. Demy 8vo. $6 d$.}

"From 'Collections and Niotes' 18671876, by W. Carew Hazlitt (p. 340), we learn that-'A very remarkable volume, in the original vellum cover, and containing 25 Forms of Prayer of the reign of Elizabeth, each with the autograph of Humphrey Dyson has lately fallen into the hands of my friend Mr H. Pyne. It is mentioned specially in the Preface to the Parker Society's volume

of Occasional Forms of Prayer, but it had been lost sight of for 200 years.' By the kindness of the present possessor of this valuable volume, containing in all 25 distinct publications, I am enabled to reprint in the following pages the two Forms of Prayer supposed to have been lost." -Extract from the PREFACE.

\section{SELECT DISCOURSES,}

by John Smith, late Fellow of Queens' College, Cambridge. Edited by H. G. Williams, B.D. late Professor of Arabic. Royal 8vo. 7s. 6d.

“The 'Select Discourses' of John Smith, collected and published from his papers after his death, are, in my opinion, much the most considerable work left to us by this Cambridge School [the Cambridge Platonists]. They have a right to a place in English literary history."-Mr MATThew ARNOLD, in the Contemporary Review.

"Of all the products of the Cambridge School, the 'Select Discourses' are perhaps the highest, as they are the most accessible and the most widely appreciated....and indeed no spiritually thoughtful mind can read them unmoved. They carry us so directly into an atmosphere of divine philosophy, luminous

with the richest lights of meditative genius... He was one of those rare thinkers in whom largeness of view, and depth, and wealth of poetic and speculative insight, only served to evoke more fully the religious spirit, and while he drew the mould of his thought from Plotinus, he vivified the substance of it from St Paul."-Principal Tulloch, Rational Theology in England in the I7th Century.

"We may instance Mr Henry Griffin Williams's revised edition of Mr John Smith's 'Select Discourses,' which have won Mr Matthew Arnold's admiration, as an example of worthy work for an University Press to undertake."-Times.

\section{THE HOMILIES,}

with Various Readings, and the Quotations from the Fathers given at length in the Original Languages. Edited by G. E. Corrie, D.D. Master of Jesus College. Demy 8vo. $7 s .6 d$.

\section{DE OBLIGATIONE CONSCIENTIE PRALEC-}

TIONES decem Oxonii in Schola Theologica habitæ a RoBERTo SANDERSON, SS. Theologiæ ibidem Professore Regio. With English Notes, including an abridged Translation, by W. WhewelL, D.D. late Master of Trinity College. Demy 8vo. $7 s .6 d$.

London: Cambridge Warehouse, I7 Paternoster Row. 
ARCHBISHOP USHER'S ANSWER TO A JESUIT, with other Tracts on Popery. Edited by J. ScHOLEFIELD, M.A. late Regius Professor of Greek in the University. Demy 8vo. $7 s .6 d$.

\section{WILSON'S ILLUSTRATION OF THE METHOD} of explaining the New Testament, by the early opinions of Jews and Christians concerning Christ. Edited by T. TURTON, D.D. late Lord Bishop of Ely. Demy 8vo. 5s.

\section{LECTURES ON DIVINITY}

delivered in the University of Cambridge, by JoHN HEY, D.D. Third Edition, revised by T. TURTON, D.D. late Lord Bishop of Ely. 2 vols. Demy 8 vo. I 5 s.

\section{ARABIC, SANSKRIT AND SYRIAC.}

\section{POEMS OF BEHÁ ED DÍN ZOHEIR OF EGYPT.}

With a Metrical Translation, Notes and Introduction, by E. H. PALmer, M.A., Barrister-at-Law of the Middle Temple, Lord Almoner's Professor of Arabic and Fellow of St John's College in the University of Cambridge. 3 vols. Crown 4 to.

Vol. I. The ARABIC TEXT. IOs. 6d.; Cloth extra. I5s.

Vol. II. English Translation. Ios. 6d.; Cloth extra. I $5 s$.

"Professor Palmer's activity in advancing Arabic scholarship has formerly shown itself in the production of his excellent Arabic Grammar, and his Descriptive Catalogue of Arabic MSS. in the Library of Trinity College, Cambridge. He has now produced an admirable text, which illustrates in a remarkable manner the flexibility and graces of the language he loves so well, and of which he seems to be perfect master.... The Syndicate of Cambridge University must not pass without the recognition of their liberality in bringing out, in a worthy form, so important an Arabic text. It is not the first time that Oriental scholarship has thus been wisely subsidised by Cambridge."-Indian Mail.

"It is impossible to quote this edition without an expression of admiration for the perfection to which Arabic typography has been brought in England in this magnificent Oriental work, the production of which redounds to the imperishable credit of the University of Cambridge. It may be pronounced one of the most beautiful Oriental books that have cver been printed in Europe: and the learning of the Editor worthily rivals the technical get-up of the creations of the soul of one of the most tasteful poets of Islâm, the study of which will contribute not a little to save honour of the poetry of the Arabs."Mythology among the HeBRews (Engl. Transl.), p. I94.

"For ease and facility, for variety of metre, for imitation, either designed or unconscious, of the style of several of our own poets, these versions deserve high praise..... We have no hesitation in saying that in both Prof. Palmer has made an addition to Oriental literature for which scholars should be grateful; and that, while his knowledge of Arabic is a sufficient guarantee for his mastery of the original, his English compositions are distinguished by versatility, command of language, rhythmical cadence, and, as we have remarked, by not unskilful imitations of the styles of several of our own favourite poets, living and dead."-Saturday Review.

"This sumptuous edition of the poems of

Behá-ed-dín Zoheir is a very welcome addition to the small series of Eastern poets accessible to readers who are not Orientalists. ... In all there is that exquisite finish of which Arabic poetry is susceptible in so rare a degree. The form is almost always beautiful, be e thought what it may. But this, of course, can only be fully appreciated by Orientalists. And this brings us to the translation. It is excellently well done. $\mathrm{Mr}$ Palmer has tried to imitate the fall of the original in his selection of the English metre for the various pieces, and thus contrives to convey a faint idea of the graceful flow of the Arabic. ..... Altogether the inside of the book is worthy of the beautiful arabesque binding that rejoices the eye of the lover of Arab art."-Academy.

THE CHRONICLE OF JOSHUA THE STYLITE, composed in Syriac A.D. 507 with an English translation and notes, by W. Wright, LL.D., Professor of Arabic. Demy 8vo. cloth. Ios. 6d.

London: Cambridge Warehouse, I7 Paternoster Roze. 
NALOPÁKHYÄNAM, OR, THE TALE OF NALA; containing the Sanskrit Text in Roman Characters, followed by a Vocabulary in which each word is placed under its root, with references to derived words in Cognate Languages, and a sketch of Sanskrit Grammar. By the late Rev. Thomas JARretT, M.A. Trinity College, Regius Professor of Hebrew, late Professor of Arabic, and formerly Fellow of St Catharine's College, Cambridge. Demy 8vo. Ios.

NOTES ON THE TALE OF NALA, for the use of Classical Students, by J. PeILE, M.A. Fellow and Tutor of Christ's College. Demy 8vo. I2s.

\section{GREEK AND LATIN CLASSICS, \&c. (See also pp. 24-27.)}

A SELECTION OF GREEK INSCRIPTIONS,

With Introductions and Annotations by E. S. RoBERTS, M.A. Fellow and Tutor of Caius College.

\section{THE AGAMEMNON OF AESCHYLUS.}

With a Translation in English Rhythm, and Notes Critical and Explanatory. New Edition Revised. By Benjamin Hall Kennedy, D.D., Regius Professor of Greek. "One of the best editions of the masterpiece of Greek tragedy."-Athencem.

"It is needless to multiply proofs of the value of this volume alike to the poetical translator, the critical scholar, and the ethical student. We must be. contented to thank Professor Kennedy for his admirable execution of a great undertaking."-Sat. Rev.

"Let me say that I think it a most admirable piece of the highest criticism. .... I like

\section{THE CEDIPUS TYRANNUS OF SOPHOCLES by}

the same Editor. Crown 8vo. Cloth $6 s$.

"Dr Kennedy's edition of the CEdipus lation, and three indices. The first of these Tyranmus is a worthy companion to his consists of a list of words and phrases either Agamemnon, and we may say at once that uncommon in themselves, or employed in no more valuable contribution to the study unusual ways: in the second we find various of Sophocles has appeared of late years. particles as exhibited in the play; while the Besides the text and notes, the volume con- third gives valuable information on gramtains a most interesting introduction to and matical points as illustrated by the usage of analysis of the play, a rhythmical trans-

Sophocles."-Saturday Revieze.

THE THEAETEUS OF PLATO by the same Editor.

Crown 8vo. Cloth. $7 s .6 d$.

\section{PLATO'S PHADO,}

literally translated, by the late E. M. COPE, Fellow of Trinity College, Cambridge. Demy 8vo. $5 s$.

\section{ARISTOTLE.-ПEPI $\triangle I K A I O \Sigma \Upsilon N$ N $\Sigma$.}

THE FIFTH BOOK OF THE NICOMACHEAN ETHICS OF ARISTOTLE. Edited by Henry JACKSON, M.A., Fellow of Trinity College, Cambridge. Demy 8vo. cloth. $6 s$.

"It is not too much to say that some of Scholars will hope that this is not the only the points he discusses have never had so portion of the Aristotelian writings which he much light thrown upon them before... is likely to edit."-Athenceum.

\section{ARISTOTLE'S PSYCHOLOGY,}

with a Translation, Critical and Explanatory Notes, by EDwiN Wallace, M.A., Fellow and Tutor of Worcester College, Oxford. Demy 8 vo. cloth. I $8 s$.

\section{London: Cambridge Warchouse, I7 Paternoster Row.}


ARISTOTLE.

THE RHETORIC. With a Commentary by the late E. M. Cope, Fellow of Trinity College, Cambridge, revised and edited by J. E. Sandys, M.A., Fellow and Tutor of St John's College, Cambridge, and Public Orator. With a biographical Memoir by H. A. J. MunRo, M.A. Three Volumes, Demy 8vo.

"This work is in many ways creditable to the University of Cambridge. And while it must ever be regretted that a work so laborious should not have received the last touches of its author, the warmest admiration is due to Mr Sandys, for the manly, unselfish, and unflinching spirit in which he has performed his most difficult and delicate task. If an English student wishes to have a full conception of

\section{fi. I I $s .6 d$.}

what is contained in the Rhetoric of Aristotle, to Mr Cope's edition he must go."Academy.

"Mr Sandys has performed his arduous duties with marked ability and admirable tact. . . . . . In every part of his work -revising, supplementing, and completinghe has done exceedingly well."-Exammer.

\section{PRIVATE ORATIONS OF DEMOSTHENES,} with Introductions and English Notes, by F. A. PALEY, M.A. Editor of Aeschylus, etc. and J. E. SANDys, M.A. Fellow and Tutor of St John's College, and Public Orator in the University of Cambridge.

PART I. Contra Phormionem, Lacritum, Pantaenetum, Boeotum de Nomine, Boeotum de Dote, Dionysodorum. Crown 8vo. cloth. $6 s$.

"Mr Paley's scholarship is sound and literature which bears upon his author, and accurate, his experience of editing wide, and if he is content to devote his learning and abilities to the production of such manuals as these, they will be received with gratitude throughout the higher schools of the country. $\mathrm{Mr}$ Sandys is deeply read in the German

the elucidation of matters of daily life, in the delineation of which Demosthenes is so rich, obtains full justice at his hands..... We hope this edition may lead the way to a more general study of these speeches in schools than has hitherto been possible."-Academy.

PART II. Pro Phormione, Contra Stephanum I. II.; Nicostratum, Cononem, Calliclem. $7 s .6 d$.

"To give even a brief sketch of these speeches [Pro Phormione and Contra Stephanum] would be incompatible with our limits, though we can hardly conceive a task more useful to the classical or professional schelar than to make one for himself. .... $Y_{t}$ is a great boon to those who set themselves to unravel the thread of arguments pro and con to have the aid of Mr Sandys's excellent running commentary ..... and no one can say that he is ever deficient

in the needful help which enables us to form a sound estimate of the rights of the case. . . . . . It is long since we have come upon a work evincing more pains, scholarship, and varied research and illustration than Mrr Sandys's contribution to the 'Private Orations of Demosthenes'."-Sat. Rev.

"..... the edition reflects credit on Cambridge scholarship, and ought to be extensively used."-A thenceum.

DEMOSTHENES AGAINST ANDROTION AND AGAINST TIMOCRATES, with Introductions and English Commentary, by WiLliam WAYTE, M.A., late Professor of Greek, University College, London, Formerly Fellow of King's College, Cambridge, and Assistant Master at Eton. [In the Press.

\section{PINDAR.}

OLYMPIAN AND PYTHIAN ODES. With Notes Explanatory and Critical, Introductions and Introductory Essays. Edited by C. A. M. Fennell, M.A., late Fellow of Jesus College. Crown 8vo. cloth. $9 s$.

"Mr Fennell deserves the thanks of all classical studentsfor his careful and scholarly edition of the Olympian and Pythian odes. $\mathrm{He}$ brings to his task the necessary enthusiasm for his author, great industry, a sound judgment, and, in particular, copious and minute learning in comparative philology. To his qualifications in this last respect every page bears witness."-A thenaum.

"Considered simply as a contribution to

the study and criticism of Pindar, Mr Fennell's edition is a work of great merit. But it has a wider interest, as exemplifying the change which has come over the methods and aims of Cambridge scholarship within the last ten or twelve years. ... Altogether, this edition is a welcome and wholesome sign of the vitality and development of Cambridge scholarship, and we are glad to see that it is to be continued."-Saturday Review.

THE NEMEAN AND ISTHMIAN ODES. [In the Press.

\section{London: Cambridge Warehouse, I7 Paternoster Row.}




\section{THE BACCHAE OF EURIPIDES.}

with Introduction, Critical Notes, and Archæological ${ }^{*}$ Illustrations, by J. E. SAnDys, M.A., Fellow and Tutor of St John's College, Cambridge, and Public Orator. Crown 8vo cloth. Ios. $6 d$.

"Of the present edition of the Bacche by $\mathrm{Mr}$ Sandys we may safely say that never before has a Greek play, in England at least, had fuller justice done to its criticism, interpretation, and archæological illustration, whether for the young student or the more advanced scholar. The Cambridge Public Orator may be said to have taken the lead in issuing a complete edition of a Greek play, which is destined perhaps to gain redoubled favour now that the study of ancient monuments has been applied to its illustration."-Saturday Review.-

"Mr Sandys has done well by his poet and by his University. He has given a most welcome gift to scholars both at home and abroad. The illustrations are aptly chosen and delicately executed, and the apparatus criticus, in the way both of notes and indices is very complete."-Notes and Queries.

"The volume is interspersed with wellexecuted woodcuts, and its general attractiveness of form reflects great credit on the University Press. In the notes Mr Sandys has more than sustained his well-earned reputation as a careful and learned editor, and shows considerable advance in freedom and lightness of style. .... Under such circumstances it is superfluous to say that for the purposes of teachers and advanced students this handsome edition far surpasses all its predecessors. The volume will add to the already wide popularity of a unique drama, and must be reckoned among the most important classical publications of the year."-
Athencum.

"This edition of a Greek play deserves more than the passing notice accorded to ordinary school editions of the classics. It has not, like so many such books, been hastily produced to meet the momentary need of some particular examination; but it has employed for some years the labour and thought of a highly finished scholar, whose aim seems to have been that his book should go forth totus teres atque rotundus, armed at all points with all that may throw light upon its subject. The result is a work which will not only assist the schoolboy or undergraduate in his tasks, but will adorn the library of the scholar.". " The description of the woodcuts abounds in interesting and suggestive information upon various points of ancient art, and is a further instance of the very thorough as well as scholarlike manner in which Mr Sandys deals with his subject at every point. 'The commentary (pp. $87-24$ ) bears the same stamp of thoroughness and high finish as the rest of the work. While questions of technical grammar receive due attention, textual criticism, philology, history, antiquities, and art are in turn laid under contribution for the elucidation of the poet's meaning. We must leave our readers to use and appreciate for themselves Mr Sandys' assistance."-The Guardian.

LECTURES ON THE TYPES OF GREEK COINS. By Percy Gardner, M.A., Disney Professor of Archæology. Royal 4 to.

IIn the Press.

\section{TULLI CICERONIS DE FINIBUS BONORUM} ET MALORUM LIBRI QUINQUE. The text revised and explained; With a Translation by JAMES S. REID, M.L., Fellow and Assistant Tutor of Gonville and Caius College.

In the Press.

\section{T. CICERONIS DE OFFICIIS LIBRI TRES,} with Marginal Analysis, an English Commentary, and copious Indices, by H. A. Holden, LL.D. Head Master of Ipswich School, late Fellow of Trinity College, Cambridge, Classical Examiner to the University of London. Fourth Edition. Revised and considerably enlarged. Crown 8vo. 9 s.

"Dr Holden truly states that "Text, Analysis, and Commentary in this third edition have been again subjected to a thorough revision.' It is now certainly the best edition extant. . . . The Introduction (afterHeine) and notes leave nothing to be desired in point of fulness, accuracy, and neatness; the typographical execution will satisfy the most fastidious eye."-Notes and Queries.

"Dr Holden has issued an edition of what is perhaps the easiest and most popular of Cicero's philosophical works, the de Officiis, which, especially in the form which it has now assumed after two most thorough revisions, leaves little or nothing to be desired in the fullness and accuracy of its treatment alike of the matter and the language."-Academy.

London: Cambridge Warehouse, in Paternoster Row. 
M. TULLII CICERONIS DE NATURA DEORUM Libri Tres, with Introduction and Commentary by JOSEPH B. MAYOR, M.A., Professor of Moral Philosophy at King's College, London, formerly Fellow and Tutor of St John's College, Cambridge, together with a new collation of several of the English MSS. by J. H. SWAINSON, M.A., formerly Fellow of Trinity Coll., Cambridge. Vol. I. Demy 8vo. IOS. $6 d$.

"Such editions as that of which Prof. Mayor has given us the first instalment will doubtless do much to remedy this undeserved neglect. It is one on which great pains and much learning have evidently been expended, and is in every way admirably suited to meet the needs of the student. ... . The notes of the editor are all that could be expected from his well-known learning and scholarship. ..... It is needless, therefore, to say

[Vol. II. In the Press. that all points of syntax or of Ciceronian usage which present themselves have been treated with full mastery. .... The thanks of many students will doubtless be given to Prof. Mayor for the amount of historical and biographical information afforded in the commentary, which is, as it should be, supplemented and not replaced by references to the usual authorities."-Academy.

\section{P. VERGILI MARONIS OPERA}

cum Prolegomenis et Commentario Critico pro Syndicis Preli Academici edidit Benjamin Hall Kennedy, S.T.P., Graecae Linguae Professor Regius. Extra Fcap. 8vo. cloth. $5 s$.

\section{IIATHEMATICS, PHYSICAL SCIENCE, \&c.}

\section{MATHEMATICAL AND PHYSICAL PAPERS.}

By Sir W. Thomson, LL.D., D.C.L., F.R.S., Professor of Natural Philosophy, in the University of Glasgow. Collected from different Scientific Periodicals from May I84I, to the present time.

\section{MATHEMATICAL AND PHYSICAL PAPERS,} By George Gabriel Stokes, M.A., D.C.L., LL.D., F.R.S., Fellow oí Pembroke College, and Lucasian Professor of Mathematics in the University of Cambridge. Reprinted from the Original Journals and Transactions, with Additional Notes by the Author. Vol. I. Demy 8 vo. cloth. I 5 s.

"The volume of Professor Stokes's papers contains much more than his hydrodynamical papers. The undulatory theory of light is treated, and the difficulties connected with its application to certain phenomena, such as aberration, are carefully examined and resolved. Such difficulties are commonly passed over with scant notice in the text-books... Those to whom difficulties like these are real stumbling-blocks will still turn for enlightenment to Professor Stokes's old, but still fresh VOL. II.

and still necessary, dissertations. There nothing is slurred over, nothing extenuated. We learn exactly the weaknesses of the theory, and the direction in which the completer theory of the future must be sought for. The same spirit pervades the papers on pure mathematics which are included in the volume. They have a severe accuracy of style which well befits the subtle nature of the subjects, and inspires the completest confidence in their author."-The Times.

THE SCIENTIFIC PAPERS OF THE LATE PROF. J. CLERंK MAXWELL. Edited by W. D. NIVEN, M.A. In 2 vols. Royal 4to.

In the Press.

A TREATISE ON NATURAL PHILOSOPHY.

By Sir W. Thomson, LL.D., D.C.L., F.R.S., Professor of Natural Philosophy in the University of Glasgow, and P. G. TAIT, M.A., Professor of Natural Philosophy in the University of Edinburgh. Vol. I. Part I. Demy 8vo. I6s.

"In this, the second edition, we notice a large amount of new matter, the importance of which is such that any opinion which we

could form within the time at our disposal would be utterly inadequate."-Nature. Part II. In the Press.

London: Cambridge Warehouse, I7 Paternoster Row. 


\section{ELEMENTS OF NATURAL PHILOSOPHY.}

By Professors Sir W. Thomson and P. G. TAIt. Part I. Demy 8vo. cloth. Second Edition. 9s.

"This work is designed especially for the use of schools and junior classes in the Universities, the mathematical methods being limited almost without exception to those of the most elementary geumetry, algebra, and

trigonometry. Tiros in Natural Philosophy cannot be better directed than by being told to give their diligent attention to an intelligent digestion of the contents of this excel. lent vade mecum."-Iron.

\section{A TREATISE ON THE THEORY OF DETER- MINANTS AND THEIR APPLICATIONS IN ANALYSIS AND GEOMETRY, by ROBERT FORSYTH SCOTT, M.A., of St John's College, Cambridge. Demy 8vo. I2s. \\ "This able and comprehensive treatise will be welcomed by the student as bringing within his reach the results of many impor- tant researches on this subject which have hitherto been for the most part inaccessible to him..... It would be presumptuous on \\ ture of the subject than Mr Scott to express an opinion as to the amount of his own re- search contained in this work, but all will appreciate the skill with which the results of his industrious reading have been arranged into this interesting treatise."-A thenaum.} the part of any one less learned in the litera-

\section{HYDRODYNAMICS,}

A Treatise on the Mathematical Theory of the Motion of Fluids, by HoRACE LAMb, M.A., formerly Fellow of Trinity College, Cambridge; Professor of Mathematics in the University of Adelaide. Demy 8vo. I2s.

\section{THE ANALYTICAL THEORY OF HEAT,} By Joseph Fourier. Translated, with Notes, by A. Freeman, M.A. Fellow of St John's College, Cambridge. Demy 8vo. I6s.

" It is time that Fourier's masterpiece, The Analytical Theory of Heat, translated by Mr Alex. Freeman, should be introduced to those English students of Mathematics who do not follow with freedom a treatise in any language but their own. It is a model of mathematical reasoning applied to physical phenomena, and is remarkable for the ingenuity of the analytical process employed by the author." - Contemporary Review, October, 1878 .

"There cannot be two opinions as to the

value and importance of the Theorie de la Chaleur. It has been called 'an exquisite mathematical poem,' not once but many times, independently, by mathematicians of different schools. Many of the very greatest of modern mathematicians regard it, justly, as the key which first opened to them the treasurehouse of mathematical physics. It is still the text-book of Heat Conduction, and there seems little present prospect of its being superseded, thongh it is already more than half a century old."-Nature.

\section{THE ELECTRICAL RESEARCHES OF THE} HONOURABLE HENRY CAVENDISH, F.R.S.

Written between $\mathrm{I} 77 \mathrm{I}$ and $\mathrm{I} 78 \mathrm{I}$, Edited from the original manuscripts in the possession of the Duke of Devonshire, K. G., by J. CLERK MAXWELl, F.R.S. Demy 8vo. cloth. I $8 s$.

"This work, which derives a melancholy interest from the lamented death of the editor following so closely upon its publication, is a valuable addition to the history of electrical research. ... The papers themselves are most carefully reproduced, with fac-similes of the author's sketches of experimental apparatus.

. . Every department of editorial duty appears to have been most conscientiously performed; and it must have been no small satisfaction to Prof. Maxwell to see this goodly volume completed before his life's work was done."-A thenaum.

AN ELEMENTARY TREATISE ON QUATERNIONS, By P. G. TAIT, M.A., Professor of Natural Philosophy in the University of Edinburgh. Second Edition. Demy 8vo. I4s.

\section{London: Cambridge Warehouse, I 7 Paternoster Row.}


A TREATISE ON THE PHYSIOLOGY OF PLANTS, by S. H. VINEs, M.A., Fellow of Christ's College.

[In the Press.

THE MATHEMATICAL WORKS OF ISAAC BARROW, D.D.

Edited by W. WHEwELL, D.D. Demy 8vo. $7 s .6 d$.

\section{COUNTERPOINT.}

A Practical Course of Study, by Professor G. A. MACFARRen, M.A., Mus. Doc. Third Edition, revised. Demy 4to. cloth. 7s. 6d.

\section{ASTRONOMICAL OBSERVATIONS}

made at the Observatory of Cambridge by the Rev. JAmes Challis, M.A., F.R.S., F.R.A.S., Plumian Professor of Astronomy and Experimental Philosophy in the University of Cambridge, and Fellow of Trinity College. For various Years, from I 846 to 1860.

\section{ASTRONOMICAL OBSERVATIONS}

from I86I to I865. Vol. XXI. Royal 4to. cloth. I5s.

A CATALOGUE OF THE COLLECTION OF BIRDS formed by the late HUGH EDWIN STRICKLAND, now in the possession of the University of Cambridge. By Osbert Salvin, M.A., F.R.S., \&c. Strickland Curator in the University of Cambridge. Demy 8vo. ŁI. Is.

\section{A CATALOGUE OF AUSTRALIAN FOSSILS}

(including Tasmania and the Island of Timor), Stratigraphically and Zoologically arranged, by RoBERT ETHERIDGE, Jun., F.G.S., Acting Palæontologist, H.M. Geol. Survey of Scotland, (formerly AssistantGeologist, Geol. Survey of Victoria). Demy 8vo. cloth. Ios. $6 d$.

"The work is arranged with great clear- papers consulted by the author, and an index ness, and contains a full list of the books and to the genera."-Saturday Revierv.

\section{ILLUSTRATIONS OF COMPARATIVE ANA-} TOMY, VERTEBRATE AND INVERTEBRATE, for the Use of Students in the Museum of Zoology and Comparative Anatomy. Second Edition. Demy 8vo. cloth. 2s. $6 d$.

\section{A SYNOPSIS OF THE CLASSIFICATION OF} THE BRITISH PALAEOZOIC ROCKS,

by the Rev. AdAM SEDGwick, M.A., F.R.S., and Frederick M ${ }^{\mathrm{C}} \mathrm{Cov}$, F.G.S. One vol., Royal 4to. Hlates, £I. Is.

A CATALOGUE OF THE COLLECTION OF CAMBRIAN AND SILURIAN FOSSILS contained in the Geological Museum of the University of Cambridge, by J. W. Salter, F.G.S. With a Portrait of Professor SEdGwick. Royal 4to. cloth. $7 s .6 d$.

CATALOGUE OF OSTEOLOGICAL SPECIMENS contained in the Anatomical Museum of the University of Cambridge. Demy 8 vo. $2 s .6 d$.

London: Cambridge Warehouse, I 7 Paternoster Row. 


\section{LAW.}

\section{AN ANALYSIS OF CRIMINAL LIABILITY.}

By E. C. ClaRK, LL.D., Regius Professor of Civil Law in the University of Cambridge, also of Lincoln's Inn, Barrister at Law. Crown 8vo. cloth. $7 s .6 d$.

"Prof Clark's little book is the substance of lectures delivered by him upon those portions of Austin's work on jurisprudence which deal with the "operation of

sanctions" ... Students of jurisprudence will find much to interest and instruct them in the work of Prof. Clark." Athencum.

\section{A SELECTION OF THE STATE TRIALS.}

By J. W. Willis-Bund, M.A., LL.B., Barrister-at-Law, Professor of Constitutional Law and History, University College, London. Vol. I. Trials for Treason (1327-I660). Crown 8vo. cloth, I $8 s$.

"A great and good service has been done to all students of history, and especially to those of them who look to it in a legal aspect, by Prof. J. W. Willis-Bund in the publication of a Selection of Cases from the State Trials. ... Professor Willis-Bund has been very careful to give such selections from the State Trials as will best illustrate those points in what may be called the growth of the Law of Treason which he wishes to bring clearly under the notice of the student, and the result is, that there is not a page in the book which has not its own lesson..... In all respects, so far as we have been able to test it, this book is admirably done."Scotsman.

"Mr Willis-Bund has edited 'A Selection of Cases from the State 'Trials' which is likely to form a very valuable addition to the standard literature. . . There can be no doubt, therefore, of the interest that can be found in the State trials. But they are large and unwieldy, and it is impossible for the general reader to come across them. Mr Willis-Bund has therefore done good service in making a selection that is in the first volume reduced to a commodious form." -The Examiner.

"This work is a very useful contribution to that important branch of the constitutional history of England which is concerned with the growth and development of the law of treason, as it may be gathered from trials before the ordinary courts The author has very wisely distinguished these cases from

those of impeachment for treason before Parliament, which he proposes to treat in a future volume under the general head "Proceedings in Parlianient." - The Academy.

"This is a work of such obvious utility that the only wonder is that no one should have undertaken it before.... In many respects therefore, although the trials are more or less abridged, this is for the ordinary student's purpose not only a more handy, but a more useful work than Howell's." Saturday Reviezw.

"Within the boards of this useful and handy book the student will find everything he can desire in the way of lists of cases given at length or referred to, and the statutes bearing on the text arranged chronologically. The work of selecting from Howell's bulky series of volumes has been done with much judgment, merely curious cases being excluded, and all iucluded so treated as to illustrate some important point of constitutional law."-Glasgow Herald.

"Mr Bund's object is not the romance, but the constitutional and legal bearings of that great series of causes célèbres which is unfortunately not within easy reach of readers not happy enough to possess valuable libraries. ... Of the importance of this subject, or of the want of a book of this kind, referring not vaguely but precisely to the grounds of constitutional doctrines, both of past and present times, no reader of history can feel any doubt."-Daily Newes.

VOL. II. In two parts. Price I4s. each,

Vol. III. In the Press.

\section{THE FRAGMENTS OF THE PERPETUAL EDICT OF SALVIUS JULIANUS,}

collected, arranged, and annotated by BRYAN WALKER, M.A. LL.D., Law Lecturer of St John's College, and late Fellow of Corpus Christi College, Cambridge. Crown 8vo., Cloth, Price $6 s$.

"This is one of the latest, we believe quite the latest, of the contributions made to legal scholarship by that revived study of the Roman Law at Cambridge which is now so marked a feature in the industrial life of the University..... In the present book we have the fruits of the same kind of thorough and well-ordered study which was brought to bear upon the notes to the Com-

mentaries and the Institutes... Hitherto the Edict has been almost inaccessible to the ordinary English student, and such a student will be interested as well as perhaps surprised to find how abundantly the extant fragments illustrate and clear up points which have attracted his attention in the Cummentaries, or the Institutes, or the Digest." Law Times.

\section{London: Cambridge Warehouse, I 7 Paternoster Row,}




\section{THE COMMENTARIES OF GAIUS AND RULES OF ULPIAN. (New Edition, revised and enlarged.)}

With a Translation and Notes, by J. T. ABDy, LL.D., Judge of County Courts, late Regius Professor of Laws in the University of Cambridge, and BRYAN WALKER, M.A., LL.D., Law Lecturer of St John's College, Cambridge, formerly Law Student of Trinity Hall and Chancellor's Medallist for Legal Studies. Crown 8vo. I6s.

"As scholars and as editors Messrs Abdy and Walker have done their work well. ..... For one thing the editors deserve special commendation. They have presented Gaius to the reader with few notes and those merely by way of reference or necessary

explanation. Thus the Roman jurist is allowed to speak for himself, and the reader feels that he is really studying Roman law in the original, and not a fanciful representation of it."-Athenaum.

\section{THE INSTITUTES OF JUSTINIAN,}

translated with Notes by J. T. ABDY, LL.D., Judge of County Courts, late Regius Professor of Laws in the University of Cambridge, and formerly Fellow of Trinity Hall ; and BRYAN WALKER, M.A., LL.D., Law Lecturer of St John's College, Cambridge; late Fellow and Lecturer of Corpus Christi College; and formerly Law Student of Trinity Hall. Crown 8vo. I6s.

"We welcome here a valuable contribution to the study of jurisprudence. The text of the Institutes is occasionally perplexing, even to practised scholars, whose knowledge of classical models does not always avail them in dealing with the technicalities of legal phraseology. Nor can the ordinary dictionaries be expected to furnish all the help that is wanted. This translation will then be of great use. To the ordinary student, whose

attention is distracted from the subject-matter by the difficulty of struggling through the language in which it is contained, it will be almost indispensable."-Spectator.

"The notes are learned and carefully compiled, and this edition will be found useful to students."-Law Times.

"Dr Abdy and Dr Walker have produced a book which is both elegant and useful."Athenaum.

\section{SELECTED TITLES FROM THE DIGEST,}

annotated by B. WALKer, M.A., LL.D. Part I. Mandati vel Contra. Digest XVII. I. Crown 8vo. Cloth. 5 s.

"This small volume is published as an experiment. The author proposes to publish an annotated edition and translation of several books of the Digest if this one is received

- with favour. We are pleased to be able to

say that $\mathrm{Mr}$ Walker deserves credit for the way in which he has performed the task undertaken. The translation, as might be expected, is scholarly." Law Times.

Part II. De Adquirendo rerum dominio and De Adquirenda vel amittenda possessione. Digest XLI. I and II. Crown 8vo. Cloth. 6s.

Part III. De Condictionibus. Digest XII. I and $4-7$ and Digest XIII. I-3. Crown 8vo. Cloth. $6 s$.

\section{GROTIUS DE JURE BELLI ET PACIS,}

with the Notes of Barbeyrac and others; accompanied by an abridged Translation of the Text, by W. WHEwELL, D.D. late Master of Trinity College. 3 Vols. Demy 8vo. I2s. The translation separate, $6 s$.

\section{London: Cambridge Warehouse, I7 Paternoster Row.}




\section{HISTORY.}

\section{THE GROWTH OF ENGLISH INDUSTRY AND COMMERCE,}

by W. Cunningham, M.A., late Deputy to the Knightbridge Professor in the University of Cambridge. With Maps and Charts. Crown 8vo. Cloth. I2s.

"He is, however, undoubtedly sound in search in a field in which the labourers have the main, and his work deserves recognition hitherto been comparatively few." - Scots as the result of immense industry and reLIFE AND TIMES OF STEIN, OR GERMANY AND PRUSSIA IN THE NAPOLEONIC AGE, by J. R. Seeley, M.A., Regius Professor of Modern History in the University of Cambridge, with Portraits and Maps. 3 Vols. Demy 8 vo. $48 s$.

"If we could conceive anything similar to a protective system in the intellectual department, we might perhaps look forward to a time when our historians would raise the cry of protection for native industry. Of the unquestionably greatest German men of modern history-I speak of Frederick the Great, Goethe and Stein-the first two found long since in Carlyle and Lewes biographers who have undoubtedly driven their German competitors out of the field. And now in the year just past Professor Seeley of Cambridge has presented us with a biography of Stein which, though it modestly declines competition with German works and disowns the presumption of teaching us Germans our own history, yet casts into the shade by its brilliant superiority all that we have ourselves hitherto written about Stein.... In five long chapters Seeley expounds the legislative and administrative reforms, the emancipation of the person and the soil, the beginnings of free administration and free trade, in short the foundation of modern Prussia, with more exhaustive thoroughness, with more penetrating insight, than any one had done before."-Deutsche Rundschau.

"Dr Busch's volume has made people think and talk even more than usual of Prince Bismarck, and Professor Seeley's very learned work on Stein will turn attention to an earlier and an almost eqvally eminent German statesman. It is soothing to the national self-respect to find a few Englishmen, such as the late Mr Lewes and Professor Leeley,

doing for German as well as English readers what many German scholars have done for us."-Times.

"In a notice of this kind scant justice can be done to a work like the one before us; no short résumé can give even the most meagre notion of the contents of these volumes, which contain no page that is superfluous, and none that is uninteresting. . . . To understand the Germany of to-day one must study the Germany of many yesterdays, and now that study has been made easy by this work, to which no one can hesitate to assign a very high place among those recent histories which have aimed at original research." - Athenaum.

"The book before us fills an important gap in English-nay, European-historical literature, and bridges over the history of Prussia from the time of Frederick the Great to the days of Kaiser Wilhelm. It thus gives the reader standing ground whence he may regard contemporary events in Germany in their proper historic light. . . . We congratulate Cambridge and her Professor of History on the appearance of such a noteworthy production. And we may add that it is something upon which we may congratulate England that on the especial field of the Ger mans, history, on the history of their own country, by the use of their own literary weapons, an Englishman has produced a history of Germany in the Napoleonic age far superior to any that exists in German."Examiner.

\section{THE UNIVERSITY OF CAMBRIDGE FROM THE EARLIEST TIMES TO THE ROYAL INJUNCTIONS OF I535, \\ by JAmes Bass Mullinger, M.A. Demy 8vo. cloth (734 pp.), I2s.}

"We trust Mr Mullinger will yet continue his history and bring it down to our own day."-Academy.

" $\mathrm{He}$ has brought together a mass of instructive details respecting the rise and progress, not only of his own University, but of all the principal Universities of the Middle Ages...... We hope some day that he may continue his labours, and give us a history of

the University during the troublous times of the Reformation and the Civil War."-Athenaum.

"Mr Mullinger's work is one of great learning and research, which can hardly fail to become a standard book of reference on the subject.... We can most strongly recommend this book to our readers."-Spectator.

VOL. II. In the Press.

London: Cambridge Warehouse, I 7 Paternoster Row. 
CHRONOLOGICAL TABLES OF GREEK HISTORY. Accompanied by a short narrative of events, with references to the sources of information and extracts from the ancient authorities, by CARL PETER. Translated from the German by G. CHAWNER, M.A., Fellow and Lecturer of King's College, Cambridge. Demy 4to. Ios.

\section{HISTORY OF THE COLLEGE OF ST JOHN THE EVANGELIST,}

by Thomas Baker, B.D., Ejected Fellow. Edited by John E. B. Mayor, M.A., Fellow of St John's. Two Vols. Demy 8vo. $24 s$.

"To antiquaries the book will be a source of almost inexhaustible amusement, by historians it will be found a work of considerable service on questions respectıng our social progress in past times; and the care and thoroughness with which Mr Mayor has discharged his editorial functions are creditable to his learning and industry."-A thencenem.

"The work displays very wide reading, and it will be of great use to members of the college and of the university, and, perhaps, of still greater use to students of English history, ecclesiastical, political, social, literary and academical, who have hitherto had to be content with 'Dyer." -Academy.

\section{HISTORY OF NEPĀL,}

translated by Munshi SHEw SHUNKER Singh and PANDit SHRí GUNANAND; edited with an Introductory Sketch of the Country and People by Dr D. WRIGHT, late Residency Surgeon at Kāthmāndū, and with facsimiles of native drawings, and portraits of Sir JUNG BAHĀDUR, the KING OF NEPĀL, \&c. Super-royal 8vo. Price 2 I $s$.

"The Cambridge University Press have done well in publishing this work. Such translations are valuable not only to the historian but also to the ethnologist;......Dr Wright's Introduction is based on personal inquiry and observation, is written intelligently and candidly, and adds much to the value of the volume. The coloured litho-

graphic plates are interesting."-Nature.

"The history has appeared at a very opportune moment... The volume... is beautifully printed, and supplied with portraits of Sir Jung Bahadoor and others, and with excellent coloured sketches illustrating Nepaulese architecture and religion."-Examiner.

\section{SCHOLAE ACADEMICAE :}

Some Account of the Studies at the English Universities in the Eighteenth Century. By CHRISTOPHeR WORDSwORTH, M.A., Fellow of Peterhouse; Author of "Social Life at the English Universities in the Eighteenth Century." Demy 8vo. cloth. I $5 s$.

"The general object of Mr Wordsworth's book is sufficiently apparent from its title. He has collected a great quantity of minute and curious information about the working of Cambridge institutions in the last century, with an occasional comparison of the corresponding state of things at Oxford....To a great extent it is purely a book of reference, and as such it will be of permanent value for the historical knowledge of English education and learning."-Saturday Review.

"Only those who have engaged in like labours will be able fully to appreciate the sustained industry and conscientious accuracy discernible in every page. ... Of the whole volume it may be said that it is a genuine service renclered to the study of University history, and that the habits of thought of any writer educated at either seat of learning in the last century will, in many cases, be far better understood after a consideration of the materials here collected."-Acacienty.

\section{THE ARCHITECTURAL HISTORY OF THE UNIVERSITY AND COLLEGES OF CAMBRIDGE,}

By the late Professor Willis, M.A. With numerous Maps, Plans, and Illustrations. Continued to the present time, and edited by JOHN WILLIS CLARK, M.A., formerly Fellow of Trinity College, Cambridge.

[In the Press.

London: Cambridge Warehouse, i 7 Paternoster Row. 


\section{MISCELLANEOUS.}

\section{LECTURES ON TEACHING,}

\section{Delivered in the University of Cambridge in the Lent Term, 1880 By J. G. Fitch, M.A., Her Majesty's Inspector of Schools. \\ Crown 8vo. cloth. New Edition. $6 s$.}

"The lectures will be found most in. teresting, and deserve to be carefully studied, not only by persons directly concerned with instruction, but by parents who wish to be able to exercise an intelligent judgment in the choice of schools and teachers for their children. For ourselves, we could almost wish to be of school age again, to learn history and geography from some one whe could teach them after the pattern set by Mr Fitch to his audience...... But perhaps Mr Fitch's observations on the general conditions of school-work are even more important than what he says on this or that branch of study." - Saturday Reviezw.

"It comprises fifteen lectures, dealing with such subjects as organisation, discipline, examining, language, fact knowledge, science, and methods of instruction; and though the lectures make no pretention to systematic or exhaustive treatment, they yet leave very little of the ground uncovered; and they combine in an admirable way the exposition of sound principles with practical suggestions and illustrations which are evidently derived from wide and varied experience, both in teaching and in examining. While Mr Fitch addresses himself specially to secondary school-masters, he does not by any means disregard or ignore the needs of the primary school."-Scotsman.

"It would be difficult to find a lecturer better qualified to discourse upon the practical aspects of the teacher's work than $\mathrm{Mr}$ Fitch. He has had very wide and varied experience as a teacher, a training college officer, an Inspector of schools, and as Assistant Commissioner to the late Endowed Schools Commission. While it is difficult for anyone to make many original remarks on this subject $\mathrm{Mr} F$ itch is able to speak with authority upon various controverted points, and to give us the results of many years' study, corrected by the observation of the various schemes and methods rursued in schools of all grades and characters." - The Schoolmaster.

"All who are interested in the management of schools, and all who have made the profession of a teacher the work of their lives, will do well to study with care these results of a large experience and of wide observation. It is not, we are told, a manual of method; rather, we should say, it is that and much more. As a manual of method it is far superior to anything we have seen. Its suggestions of practical means and me. thods are very valuable; but it has an element which a mere text-book of rules for imparting knowledge does not contain. Its tone is lofty; its spirit religious; its ideal of the teacher's aim and life pure and good ... The volume is one of great practical value. It should be in the hands of every teacher, and of every one preparing for the office of a teacher. There are many besides these who will find much in it to interest and instruct them, more especially parents who have children whom they can afford to keep at school till their eighteenth or nineteenth year."The Nonconformist and Independent.

"As principal of a training college and as a Government inspector of schools, Mr Fitch has got at his fingers' ends the working of primary education, while as assistant commissioner to the late Endowed Schools Commission he has seen something of the machinery of our higher schools. . . . Mr Fitch's book covers so wide a field and touches on so many burning questions that we nuust be content to reconimend it as the best existing vade mecum for the teacher. ...He is always sensible, always judicious, never wanting in tact. . . Mr Fitch is a scholar; he pretends to no knowledge that he does not possess; he brings to his work the ripe experience of a well-stored mind, and he possesses in a remarkable degree the art of exposition."-Pall Mall Gazette.

"In his acquaintance with all descrip. tions of schools, their successes and their shortconings, Mr Fitch has great advantages both in knowledge and experience; and if his work receives the attention it deserves, it will tend materially to improve and equalize the methods of teaching in our schools, to whatever class they may belong." - $S t$ Fames's Gazette.

"In no other work in the English language, so far as we know, are the principles and methods which most conduce to successful teaching laid down and illustrated with such precision and fulness of detail as they are here."-Leeds Mercury.

6" The book is replete with practical sagacity, and contains on almost all points of interest to the teaching profession sug. gestive remarks resting evidently on a wide and thoughtful experience of school methods. There are few teachers who will not find aids to reflection in the careful analysis of the qualities required for success in teaching, in the admirable exposition of the value of orderly, methodical arrangement both for instruction and discipline, and in the painstaking discussion of school punishments contained in the earlier section of the volume. ... We recommend it in all confidence to those who are interested in the problems with which the teaching profession has to deal."-Galignani's Messenger.

\section{London: Cambridge Warehouse, I7 Paternoster Roze,}


A CATALOGUE OF ANCIENT MARBLES IN GREAT BRITAIN, by Prof. Adolsh Michaelis. Translated by C. A. M. Fennell, M.A., late Fellow of Jesus College. Royal 8vo. £2. $2 s$.

\section{A GRAMMAR OF THE IRISH LANGUAGE.} By Prof. Windisch. Translated by Dr Norman MOore. Crown 8vo. 7 s. $6 d$.

STATUTA ACADEMIE CANTABRIGIENSIS. Demy 8vo. 2s. sewed.

ORDINATIONES ACADEMIÆ CANTABRIGIENSIS.

Demy 8vo. cloth. 3s. $6 d$.

TRUSTS, STATUTES AND DIRECTIONS affecting (I) The Professorships of the University. (2) The Scholarships and Prizes. (3) Other Gifts and Endowments. Demy 8vo. 5 s.

COMPENDIUM OF UNIVERSITY REGULATIONS, for the use of persons in Statu Pupillari. Demy 8vo. $6 d$.

CATALOGUE OF 'THE HEBREW MANUSCRIPTS preserved in the University Library, Cambridge. By Dr S. M. SCHILlER-SzINESSY. Volume I. containing Section I. The Holy Scriptures; Section II. Commentaries on the Bible. Demy 8vo. 9s.

A CATALOGUE OF THE MANUSCRIPTS preserved in the Library of the University of Cambridge. Demy 8 vo. 5 Vols. Ios. each.

INDEX TO THE CATALOGUE. Demy 8vo. Ios.

A CATALOGUE OF ADVERSARIA and printed books containing MS. notes, preserved in the Library of the University of Cambridge. $3 s .6 d$.

THE ILLUMINATED MANUSCRIPTS IN THE LIBRARY OF THE FITZWILLIAM MUSEUM, Catalogued with Descriptions, and an Introduction, by WiLLIAM GEORGE SEARLE, M.A., late Fellow of Queens' College, and Vicar of Hockington, Cambridgeshire. Demy 8vo. $7 s, 6 d$.

A CHRONOLOGICAL LIST OF THE GRACES, Documents, and other Papers in the University Registry which concern the University Library. Demy 8vo. $2 s .6 d$.

CATALOGUS BIBLIOTHECAE BURCKHARDTIANÆ. Demy 4 to. 5 s.

London: Cambridge Warehouse, I 7 Faternoster Row. 


\title{
Tlye (Cambriage bible for srbonls.
}

\author{
General Editor: J. J. S. Perowne, D.D., Dean of \\ Peterborough.
}

The want of an Annotated Edition of the BIBLE, in handy portions, suitable for School use, has long been felt.

In order to provide Text-books for School and Examination purposes, the CAmbridge University Press has arranged to publish the several books of the BIBLE in separate portions at a moderate price, with introductions and explanatory notes.

The Very Reverend J. J. S. Perowne, D.D., Dean of Peterborough, has undertaken the general editorial supervision of the work, and will be assisted by a staff of eminent coadjutors. Some of the books have already been undertaken by the following gentlemen :

Rev. A. CArr, M.A., Assistant Master at Wellington College. Rev. T. K. Cheyne, M.A., Felloro of Balliol College, Oxford. Rev. S. Cox, Nottingham.

Rev. A. B. Davidson, D.D., Professor of Hebrew, Edinburgh.

Rev. F. W. FARrar, D.D., Canon of Westminster.

Rev. A. E. Humphreys, M.A., Fellow of Trinity College, Cambridge.

Rev. A. F. Kirkpatrick, M.A., Fellow of Trinity College, Regins Professor of Hebrew.

Rev. J. J. Lias, M.A., late Professor at St David's College, Lampeter.

Rev. J. R. Lumby, D.D., Norrisian Professor of Divinity.

Rev. G. F. Maclear, D.D., Warden of St Augustine's Coll., Canterbury.

Rev. H. C. G. Moule, M.A., Fellow of Trinity College, Principal of Ridley Hall, Cambridge.

Rev. W. F. Moulton, D.D., Head Master of the Ley's School, Cambridge. Rev. E. H. Perowne, D.D., Master of Corpus Christi College, Cambridge, Examining Chaplain to the Bishop of St Asaph.

The Ven. T. T. Perowne, M.A., Archdeacon of Norwich.

Rev. A. Plummer, M.A., Master of University College, Durham.

The Very Rev. E. H. Plumptre, D.D., Dean of Wells.

Rev. W. Sanday, M.A., Principal of Bishop Hatfield Hall, Durham.

Rev. W. Simcox, M.A., Rector of Weyhill, Hants.

Rev. W. Robertson Smith, M.A., Edinburgh.

Rev. A. W. Streane, M.A., Fellow of Corpus Christi Coll., Cambridge.

The Ven. H. W. Watkins, M.A., Archdeacon of Northumberland.

Rev. G. H. Whitaker, M.A., Fellow of St Fohn's College, Cambridge. Rev. C. WORDSWorth, M.A., Rector of Glaston, Rutland.

London: Cambridge Warehouse, I 7 Paternoster Row. 
THE CAMBRIDGE BIBLE FOR SCHOOLS.-Continued. Now Ready. Cloth, Extra Fcap. 8vo.

THE BOOK OF JOSHUA. Edited by Rev. G. F. MACLEAR, D.D. With 2 Maps. 2s. $6 d$.

THE BOOK OF JUDGES. By the Rev. J. J. LIAS, M.A. With Map. $3 s .6 d$.

THE FIRST BOOK OF SAMUEL. By the Rev. Professor Kirkpatrick, M.A. With Map. 3s. $6 d$.

THE SECOND BOOK OF SAMUEL. By the Rev. Professor Kirkpatrick, M.A. With 2 Maps. $3 s .6 d$.

THE BOOK OF ECCLESIASTES. By the Very Rev. E. H. Plumptre, D.D., Dean of Wells. $5^{s .}$

THE BOOK OF JEREMIAH. By the Rev. A. W. Streane, M.A. 4 s. $6 d$.

THE BOOK OF JONAH. By Archdn. Perowne. is. $6 d$. THE BOOK OF MICAH. By the Rev. T. K. Cheyne, M.A. Is. 6 .

THE GOSPEL ACCORDING TO ST MATTHEW. Edited by the Rev. A. CARr, M.A. With 2 Maps. 2s. $6 d$.

THE GOSPEL ACCORDING TO ST MARK. Edited by the Rev. G.F. MACLEAR, D.D. With 2 Maps. 2s. $6 d$.

THE GOSPEL ACCORDING TO ST LUKE. By the Rev. F. W. FARRAR, D.D. With 4 Maps. $4 s .6 d$.

THE GOSPEL ACCORDING TO ST JOHN. By the Rev. A. Plummer, M.A. With Four Maps. $4 s .6 d$.

THE ACTS OF THE APOSTLES. By the Rev. Professor Lumby, D.D. Part I. Chaps. I-XIV. With 2 Maps. 2s. $6 d$.

PART II. Chaps. XV. to end. Nearly ready.

THE EPISTLE TO THE ROMANS. By the Rev. H. C. G. Moule, M.A. $3 s .6 d$.

THE FIRST EPISTLE TO THE CORINTHIANS. By the Rev. J. J. Lias, M. A. With a Map and Plan. 2s.

THE SECOND EPISTLE TO THE CORINTHIANS. By the Rev. J. J. Lias, M.A. $2 s$.

THE GENERAL EPISTLE OF ST JAMES. By the Very Rev. E. H. Plumptre, D.D., Dean of Wells. is. $6 d$. THE EPISTLES OF ST PETER AND ST JUDE. By the same Editor. 2s. $6 d$.

London: Cambridge Warehouse, I7 Paternoster Rowe. 
THE CAMBRIDGE BIBLE FOR SCHOOLS.-Continucd.

\section{Preparing.}

THE BOOKS OF HAGGAI AND ZECHARIAH. By Archdeacon PEROwne.

THE EPISTLE TO THE HEBREWS. By the Rev. F. W. FARRAR, D.D.

\section{THE CAMBRIDGE GREEK TESTAMENT,}

\section{FOR SCHOOLS AND COLLEGES,}

with a Revised Text, based on the most recent critical authorities, and English Notes, prepared under the direction of the General Editor,

The Very Reverend J. J. S. PEROWNE, D.D., DEAN OF PETERBOROUGH.

\section{Now Ready.}

THE GOSPEL ACCORDING TO ST MATTHEW. By the

Rev. A. CARr, M.A. With 4 Maps. 4 s. $6 d$.

"With the 'Notes,' in the volume before us, we are much pleased; so far as we have searched, they are scholarly and sound. The quotations from the Classics are apt; and the references to modern Greek form a pleasing feature." - The Churchman.

"Mr Carr, whose 'Notes on St Luke's Gospel' must have thoroughly approved themselves to all who have used them, has followed the same line in this volume of St Matthew. In both works a chief object has been 'to connect more closely the study of the Classics with the reading of the New Testament.' .... Copious illustrations, gathered from a great variety of sources, make his notes a very valuable aid to the student. They are indeed remarkably interesting, while all explanations on meanings, applications, and the like are distinguished by their lucidity and good sense."-Pall Mall Gazette.

THE GOSPEL ACCORDING TO ST LUKE. By the Rev. F. W. FARRAR, D.D. [Preparing.

THE GOSPEL ACCORDING TO ST JOHN. By the Rev. A. Plummer, M.A.

[Nearly ready.

The books will be published separately, as in the "Cambridge Bible for Schools."

London: Cambridge Warehouse, I7 Paternoster Row. 


\section{THE PITT PRESS SERIES.}

\section{GREEK.}

THE ANABASIS OF XENOPHON, BOOK IV. With

a Map and English Notes by Alfred Pretor, M.A., Fellow of St Catharine's College, Cambridge ; Editor of Persius and Cicero ad Atticum Book I. Price 2s.

"In Mr Pretor's edition of the Anabasis the text of Kühner has been followed in the main, while the exhaustive and admirable notes of the great German editor have been largely utilised. These notes deal with the minutest as well as the most important difficulties in construction, and all questions of history, antiquity, and geography are briefly but very effectually elucidated."-The Examiner.

"We welcome this addition to the other books of the Anabasis so ably edited by Mr Pretor. Although originally intended for the use of candidates at the university local examinations, yet this edition will be found adapted not only to meet the wants of the junior student, but even advanced scholars will find much in this work that will repay its perusal." - The Schoolmaster.

" $M r$ Pretor's 'Anabasis of Xenophon, Book IV.' displays a union of accurate Cambridge scholarship, with experience of what is required by learners gained in examining middle-class schools. The text is large and clearly printed, and the notes explain all difficulties. . . . Mr Pretor's notes seem to be all that could be wished as regards grammar, geography, and other matters."-The Academy.

BOOKS I. III. \& V. By the same Editor. 2s. each.

BOOKS II. VI. and VII. By the same Editor. 2s. $6 d$. each.

"Another Greek text, designed it would seem for students preparing for the local examinations, is 'Xenophon's Anabasis,' Book II., with English Notes, by Alfred Pretor, M.A. The editor has exercised his usual discrimination in utilising the text and notes of Kuhner, with the occasional assistance of the best hints of Schneider, Vollbrecht and Macmichael on critical matters, and of Mr R. W. Taylor on points of history and geography. . When Mr Pretor commits himself to Commentator's work, he is eminently helpful. . . Had we to introduce a young Greek scholar to Xenophon, we should esteem ourselves fortunate in having Pretor's text-book as our chart and guide."-Contemporary Reviere.

THE ANABASIS OF XENOPHON, by A. PRETOR, M.A.,

Text and Notes, complete in two Volumes. Price $7 s .6 d$.

AGESILAUS OF XENOPHON. The Text revised with Critical and Explanatory Notes, Introduction, Analysis, and Indices. By H. Hailstone, M.A., late Scholar of Peterhouse, Cambridge, Editor of Xenophon's Hellenics, etc. 2s. 6 d.

ARISTOPHANES-RANAE. With English Notes and Introduction by W. C. Green, M.A., Assistant Master at Rugby School. 3 s. $6 d$.

ARISTOPHANES-AVES. By the same Editor. New Edition. 3s. 6d.

"The notes to both plays are excellent. Much has been done in these two volumes to render the study of Aristophanes a real treat to a boy instead of a drudgery, by helping him to understand the fun and to express it in his mother tongue."-The Examiner.

ARISTOPHANES-PLUTUS. By the same Editor. $3 s .6 d$. EURIPIDES. HERCULES FURENS. With Introductions, Notes and Analysis. By J. T. Hutchinson, M. A., Christ's College; and A. Gray, M.A., Fellow of Jesus College. 2s.

"Messrs Hutchinson and Gray have produced a careful and useful edition."-Saturday Review.

THE HERACLEID E OF EURIPIDES, with Introduction and Critical Notes by E. A. BEck, M.A., Fellow of Trinity Hall. $3^{s .6}$.

London: Cambridge Warehouse, I 7 Paternoster Row. 
LUCIANI SOMNIUM CHARON PISCATOR ET DE LUCTU, with English Notes by W. E. Heitland, M.A., Fellow of St John's College, Cambridge. New Edition, with Appendix. 3s. $6 d$.

\section{LATIN.}

M. T. CICERONIS DE AMICITIA. Edited by J. S. Reid, M.L., Fellow and Assistant Tutor of Gonville and Caius College, Cambridge. Price 3 s.

" Mr Reid has decidedly attained his aim, namely, 'a thorough examination of the Latinity of the dialogue.'..... The revision of the text is most valuable, and comprehends sundry acute corrections. ... This volumie, like Mr Reid's other editions, is a solid gain to the scholarship of the country." -A theriaum.

"A more distinct gain to scholarship is Mr Reid's able and thorough edition of the De Amicitiô of Cicero, a work of which, whether we regard the exhaustive introduction or the instructive and most suggestive commentary, it would be difficult to speak too highly. . . . When we come to the commentary, we are only amazed by its fulness in proportion to its bulk. Nothing is overlooked which can tend to enlarge the learner's general knowledge of Ciceronian Latin or to elucidate the text."-Saturday Review.

M. T. CICERONIS CATO MAJOR DE SENECTUTE. Edited by J. S. ReID, M.L. Price $3 s$. 6.

"The notes are excellent and scholarlike, adapted for the upper forms of public schools, and likely to be useful even to more advanced students."-Guardian.

M. T. CICERONIS ORATIO PRO ARCHIA POETA. Edited by J. S. Rerd, M.L. Price Is. $6 d$.

"It is an admirable specimen of careful editing. An Introduction tells us everything we could wish to know about Archias, about Cicero's connexion with him, about the merits of the trial, and the genuineness of the speech. The text is well and carefully printed. The notes are clear and scholar-like. . . . No boy can master this little volume without feeling that he has advanced a long step in scholarship."-The Academy.

M. T. CICERONIS PRO L. CORNELIO BALBO ORA-

TIO. Edited by J.S. ReId, M.L. Fellow of Caius College, Camb. Price is. $6 d$.

"We are bound to recognize the pains devoted in the annotation of these two orations to the minute and thorough study of their Latinity, both in the ordinary notes and in the textual appendices."-Saturday Revieze.

M. T. CICERONIS PRO P. CORNELIO SULLA ORATIO. Edited by J. S. REID, M.L.

In the Press.

M. T. CICERONIS PRO CN. PLANCIO ORATIO. Edited by H. A. Holden, LL.D., Head Master of Ipswich School. Price 4 s. $6 d$.

"As a book for students this edition can have few rivals. It is enriched by an excellent introduction and a chronological table of the principal events of the life of Cicero; while in its appendix, and in the notes on the text which are added, there is much of the greatest value. The volume is neatly got up, and is in every way commendable."-The Scotsman.

"Dr Holden's own edition is all that could be expected from his elegant and practised scholarship. ... Dr Holden has evidently made up lis mind as to the character of the commentary most likely to be generally useful; and he has carried out his views with admirable thoroughness."-Academy.

"Dr Holden has given us here an excellent edition. The commentary is even unusually full and complete; and after going through it carefully, we find little or nothing to criticize. There is an excellent introduction, lucidly explaining the circumstances under which the speech was delivered, a table of events in the life of Cicero and a useful index." Spectator, Oct. 29, 188r.

M. T. CICERONIS IN Q. CAECILIUM DIVINATIO ET IN C. VERREM ACTIO PRIMA. With Introduction and Notes by W. E. Heitland, M.A., and Herbert Cowie, M.A., Fellows of St John's College, Cambridge. Price 3 s.

London: Cambridge Warchouse, I 7 Paternoster Row. 
M. T. CICERONIS ORATIO PRO L. MURENA, with English Introduction and Notes. By W. E. Heitrand, M.A., Fellow and Classical Lecturer of St John's College, Cambridge. Second Edition, carefully revised. Price $3 s$.

"Those students are to be deemed fortunate who have to read Cicero's lively and brilliant oration for L. Murena with Mr Heitland's handy edition, which may be pronounced 'four-square' in point of equipment, and which has, not without good reason, attained the honours of a second edition."-Saturday Review.

\section{M, T. CICERONIS IN GAIUM VERREM ACTIO} PRIMA. With Introduction and Notes. By H. Cowie, M.A., Fellow of St John's College, Cambridge. Price is. $6 d$.

M. T. CICERONIS ORATIO PRO T. A. MILONE, with a Translation of Asconius' Introduction, Marginal Analysis and English Notes. Edited by the Rev. John SMYTh Purton, B.D., late President and Tutor of St Catharine's College. Price $2 s .6 d$.

"The editorial work is excellently done."-The Academy.

\section{P. OVIDII NASONIS FASTORUM LIBER VI. With} a Plan of Rome and Notes by A. Sidgwick, M.A. Tutor of Corpus Christi College, Oxford. Price Is. $6 d$.

" Mr Sidgwick's editing of the Sixth Book of Ovid's Fasti furnishes a careful and serviceable volume for average students. It eschews 'construes' which supersede the use of the dictionary, but gives full explanation of grammatical usages and historical and mythical allusions, besides illustrating peculiarities of style, true and false derivations, and the more remarkable variations of the text."-Saturday Review.

"It is eminently good and useful. . . . The Introduction is singularly clear on the astronomy of Ovid, which is properly shown to be ignorant and confused; there is an excellent little map of Rome, giving just the places mentioned in the text and no more; the notes are evidently written by a practical schoolmaster."-The Academy.

\section{GAI IULI CAESARIS DE BELLO GALLICO COM-} MENT. I. II. With English Notes and Map by A. G. Pesketr, M.A., Fellow of Magdalene College, Cambridge, Editor of Caesar De Bello Gallico, VII. Price $2 s .6 d$.

\section{GAI IULI CAESARIS DE BELLO GALLICO COM-} MENT. III. With Map and Notes by A. G. Pesketr, M.A., Fellow of Magdalene College, Cambridge. Price is. $6 \%$.

"In an unusually succinct introduction he gives all the preliminary and collateral information that is likely to be useful to a young student; and, wherever we have examined his notes, we have found them eminently practical and satisfying. . . The book may well be recommended for careful study in school or college."-Saturday Review.

"The notes are scholarly, short, and a real help to the most elementary beginners in Latin prose."-The Examiner.

BOOKS IV. AND V. AND Book VII. by the same Editor. Price 2s. each.

BOOK VI. by the same Editor. Price I $s_{0} 6 d$.

\section{London: Cambridge Warehouse, I7 Paternoster Row.}


P. VERGILI MARONIS AENEIDOS LIBER II. Edited with Notes by A. Sidgwick, M.A. Tutor of Corpus Christi College, Oxford. is. $6 d$.

BOOKS IV., V., VI., VII., VIII., X., XI., XII. by the same Editor. Is. $6 d$. each.

" Mr Arthur Sidgwick's 'Vergil, Aeneid, Book XII.' is worthy of his reputation, and is distinguished by the same acuteness and accuracy of knowledge, appreciation of a boy's difficulties and ingenuity and resource in meeting them, which we have on other occasions had reason to praise in these pages."-The Academy.

"As masterly in its clearly divided preface and appendices as in the sound and independent character of its annotations. . . There is a great deal more in the notes than mere compilation and suggestion.... No difficulty is left unnoticed or unhandled."-Saturday Review.

"This edition is admirably adapted for the use of junior students, who will find in it the result of much reading in a condensed form, and clearly expressed."-Cambridge Independent Press.

BOOKS VII. VIII. in one volume. Price 3 s.

BOOKS X., XI., XII. in one volume. Price 3s. $6 d$.

Q UIN T US C URTIUS. A Portion of the History. (Alexander in India.) By W. E. Heitland, M.A., Fellow and Lecturer of St John's College, Cambridge, and T. E. Raven, B.A., Assistant Master in Sherborne School. Price $3 s .6 d$.

"Equally commendable as a genuine addition to the existing stock of school-books is Alexander in India, a compilation from the eighth and ninth books of Q. Curtius, edited for the Pitt Press by Messrs Heitland and Raven.... The work of Curtius has merits of its own, which, in former generations; made it a favourite with English scholars, and which still make it a popular text-book in Continental schools. ..... The reputation of Mr Heitland is a sufficient guarantee for the scholarship of the notes, which are ample without being excessive, and the book is well furnished with all that is needful in the nature of maps, indexes, and appendices." - Academy.

\section{ANNAEI LUCANI PHARSALIAE LIBER} PRIMUS, edited with English Introduction and Notes by W. E. HEITLAND, M.A. and C. E. Haskins, M.A., Fellows and Lecturers of St John's College, Cambridge. Price is. $6 d$.

"A careful and scholarlike production."-Times.

"In nice parallels of Lucan from Latin poets and from Shakspeare, Mr Haskins and Mr Heitland deserve praise."-Saturday Review.

\section{BEDA'S ECCLESIASTICAL HISTORY, BOOKS}

III., IV., the Text from the very ancient MS. in the Cambridge University Library, collated with six other MSS. Edited, with a life from the German of EBERT, and with Notes, \&c. by J. E. B. MAYOR, M.A., Professor of Latin, and J. R. Lumby, D.D., Norrisian Professor of Divinity. Revised edition. Price 7 s. $6 d$.

"To young students of English History the illustrative notes will be of great service, while the study of the texts will be a good introduction to Mediaval Latin."-The Nonconformist.

"In Bede's works Englishmen can go back to origines of their history, unequalled for form and matter by any modern European nation. Prof. Mayor has done good service in rendering a part of Bede's greatest work accessible to those who can read Latin with ease. He has adorned this edition of the third and fourth books of the "Ecclesiastical History" with that amazing erudition for which he is unrivalled among Englishmen and rarely equalled by Germans. And however interesting and valuable the text may be, we can certainly apply to his notes the expression, La sauce vaut meeux que le poisson. They are literally crammed with interesting information about early English life. For though ecclesiastical in name, Bede's history treats of all parts of the national life, since the Church had points of contact with all."-Examiner.

Books I. and II. In the Press.

London: Cambridge Warehouse, I 7 Paternoster Row. 


\section{FRENCH.}

LAZARE HOCHE-PAR ÉMILE DE BONNECHOSE. With Three Maps, Introduction and Commentary, by C. ColbECK, M.A., late Fellow of Trinity College, Cambridge; Assistant Master at Harrow School. Price $2 s$.

\section{HISTOIRE DU SIÉCLE DE LOUIS XIV PAR} VOLTAIRE. Part I. Chaps. I.-XIII. Edited with Notes Philological and Historical, Biographical and Geographical Indices, etc. by Gustave Masson, B. A. Univ. Gallic., Officier d'Académie, Assistant Master of Harrow School, and G. W. Prothero, M.A., Fellow and Tutor of King's College, Cambridge. $2 s .6 d$.

"Messrs Masson and Prothero have, to judge from the first part of their work, performed with much discretion and care the task of editing Voltaire's Siècle de Louis XIV for the 'Pitt Press Series.' Besides the usual kind of notes, the editors have in this case, influenced by Voltaire's 'summary way of treating much of the history,' given a good deal of historical information, in which they have, we think, done well. At the beginning of the book will be found excellent and succinct accounts of the constitution of the French army and Parliament at the period treated of."-Saturday Review.

\section{HISTOIRE DU SIE்CLE DE LOUIS XIV PAR} VOLTAIRE. Part II. Chaps. XIV.--XXIV. With Three Maps of the Period, Notes Philological and Historical, Biographical and Geographical Indices, by G. Masson, B.A. Univ. Gallic., Assistant Master of Harrow School, and G. W. Prothero, M.A., Fellow and Tutor of King's College, Cambridge. Price 2s. 6 d.

Part III. Chap. XXV. to the end. By the same Editors. Price $2 s .6 d$.

LE VERRE D'EAU. A Comedy, by Scribe. With a Biographical Memoir, and Grammatical, Literary and Historical Notes. By C. Colbeck, M.A., late Fellow of Trinity College, Cambridge; Assistant Master at Harrow School. Price 2s.

"It may be national prejudice, but we consider this edition far superior to any of the series which hitherto have been edited exclusively by foreigners. Mr Colbeck seems better to understand the wants and difficulties of an English boy. The etymological notes especially are admirable.... The historical notes and introduction are a piece of thorough honest work."-Fournal of Education.

M. DARU, par M. C. A. Sainte-Beuve, (Causeries du Lundi, Vol. IX.). With Biographical Sketch of the Author, and Notes Philological and Historical. By. Gustave Masson. $2 s$.

LA SUITE DU MENTEUR. A Comedy in Five Acts, by P. CoR NEILle. Edited with Fontenelle's Memoir of the Author, Voltaire's Critical Remarks, and Notes Philological and Historical. By Gustave MASSON. Price $2 s$.

\section{LA JEUNE SIBÉRIENNE. LE LÉPREUX DE LA} CITÉ D'AOSTE. Tales by Count Xavier de Maistre. With Bio. graphical Notice, Critical Appreciations, and Notes. By Gustave Masson. Price 2s.

\section{London: Cambridge Warehouse, I 7 Paternoster Row.}


LE DIRECTOIRE. (Considérations sur la Révolution Française. Troisième et quatrième parties.) Par MADAME LA BARONNE DE StaËL-Holstein. With a Critical Notice of the Author, a Chronological Table, and Notes Historical and Philological, by G. MAsson, B.A., and G. W. Prothero, M.A. Revised and enlarged Edition. Price $2 s$.

"Prussia under Frederick the Great, and France under the Directory, bring tis face to face respectively with periods of history which it is right should be known thoroughly, and which are well treated in the Pitt Press volumes. The latter in particular, an extract from the world-known work of Madame de Staël on the French Revolution, is beyond all praise for the excellence both of its style and of its matter."-Times.

DIX ANNÉES D'ÉXIL. LIVRE II. Chapitres I-S.

Par Madame la Baronne De Stä̈l-Holstein. With a Biographical

Sketch of the Author, a Selection of Poetical Fragments by Madame de

Staël's Contemporaries, and Notes Historical and Philological. By Gustave

Masson. Price $2 s$.

"The choice made by M. Masson of the second book of the Memoirs of Madame de Staell appears specially felicitous. . . This is likely to be one of the most favoured of M. Masson's editions, and deservedly so."-Academy.

FRÉDÉGONDE ET BRUNEHAUT. A Tragedy in Five Acts, by N. Lemercier. Edited with Notes, Genealogical and Chronological Tabies, a Critical Introduction and a Biographical Notice. By Gustave Masson. Price $2 s$.

\section{LE VIEUX CÉLIBATAIRE. A Comedy, by Collin}

D'HARLEville. With a Biographical Memoir, and Grammatical, Literary and Historical Notes. By the same Editor. Price $2 s$.

"M. Masson is doing good work in introducing learners to some of the less-known French play-writers. The arguments are admirably clear, and the notes are not too abundant."Academy.

LA MÉTROMANiE, A Comedy, by Piron, with a Biographical Memoir, and Grammatical, Literary and Historical Notes. $\backslash$ By the same Editor. Price 2s.

LASCARIS, OU LES GRECS DU XVE. SIÉCLE, Nouvelle Historique, par A. F. Villemain, with a Biographical Sketch of the Author, a Selection of Poems on Greece, and Notes Historical and Philological. By the same Editor. Price 2s.

\section{GERMAN.}

ERNST, HERZOG VON SCHWABEN. UHLAND. With Introduction and Notes. By H. J. Wolstenholme, B.A. (Lond.), Lecturer in German at Newnham College, Cambridge, Price $3^{s} .6 d$.

ZOPF UND SCHWERT. Lustspiel in fünf Aufzügen von KARL Gutzkow. With a Biographical and Historical Introduction, English Notes, and an Index. By the same Editor. Price 3. $6 d$.

"We are glad to be able to notice a careful edition of K. Gutzkow's amusing comedy 'Zopf and Schwert' by Mr H. J. Wolstenholme. . . . These notes are abundant and contaix references to standard grammatical works."-Academy.

\section{London: Cambridge Warehouse, I 7 Paternoster Row.}


(5)othe'sz suabenjalye. (I749-I759.) GOETHE'S BOYHOOD: being the First Three Books of his Autobiography. Arranged and Annotated by Wilmelm Wagner, $\mathrm{Ph}$. D., late Professor at the Johanneum, Hamburg. Price 2s.

HAUFF. DAS WIRTHSHAUS IM SPESSART. Edited by A. Schlottmann, Ph.D., Assistant Master at Uppingham School. Price 3 s. $6 d$.

DER OBERHOF. A Tale of Westphalian Life, by KARL Immermann. With a Life of Immermann and English Notes, by Wilhelm WAGner, Ph.D., late Professor at the Johanneum, Hamburg. Price $3^{s .}$

A BOOK OF GERMAN DACTYLIC POETRY. Arranged and Annotated by the same Editor. Price $3^{s}$.

Der erfte sreuzzug (THE FIRST CRUSADE), by FRIEDRICH VON RAUMER. Condensed from the Author's 'History of the Hohenstaufen', with a life of Raumer, two Plans and English Notes. By the same Editor. Price $2 s$.

"Certainly no more interesting book could be made the subject of examinations. The story of the First Crusade has an undying interest. The notes are, on the whole, good."-Educational Times.

\section{A BOOK OF BALLADS ON GERMAN HISTORY.}

Arranged and Annotated by the same Editor. Price $2 s$.

"It carries the reader rapidly through some of the most important incidents connected with the German race and name, from the invasion of Italy by the Visigoths under their King Alaric, down to the Franco-German War and the installation of the present Emperor. The notes supply very well the connecting links between the successive periods, and exhibit in its various phases of growth and progress, or the reverse, the vast unwieldy mass which constitutes modern Germany." -Times.

DER STAAT FRIEDRICHS DES GROSSEN. By G.

Freytag. With Notes. By the same Editor. Price 2 s.

"Prussia under Frederick the Great, and France under the Directory, bring us face to face respectively with periods of history which it is right should be known thoroughly, and which are well treated in the Pitt Press volumes."-Times.

\section{GOETHE'S HERMANN AND DOROTHEA. With}

an Introduction and Notes. By the same Editor. Price $3^{s .}$

"The notes are among the best that we know, with the reservation that they are often too abundant."-Academy.

Daz $\mathfrak{J a h r}$ I8I3 (The YEAR I8I3), by F. KohlRaUsCh. With English Notes. By the same Editor. Price $2 s$.

\section{ENGLISH.}

\section{LOCKE ON EDUCATION. With Introduction and Notes} by the Rev. R. H. QuICk, M.A. Price 3 s. $6 d$.

"The work before us leaves nothing to be desired. It is of convenient form and reasonable price, accurately printed, and accompanied by notes which are admirable. There is no teacher too young to find this book interesting; there is no teacher too old to find it profitable."-The School Bulletin, New York.

THE TWO NOBLE KINSMEN, edited with Introduction and Notes by the Rev. Professor Skeat, M.A., formerly Fellow of Christ's College, Cambridge. Price 3s. $6 d$.

"This edition of a play that is well worth study, for more reasons than one, by so careful a scholar as Mr Skeat, deserves a hearty welcome."-A thenaum.

"Mr Skeat is a conscientious editor, and has left no difficulty unexplained."-Times.

London: Cambridge Warehouse, i 7 Paternoster Roue. 
BACON'S HISTORY OF THE REIGN OF KING HENRY VII. With Notes by the Rev. J. Rawson Lumby, D.D., Norrisian Professor of Divinity; late Fellow of St Catharine's College. Price $3 s$.

\section{SIR THOMAS MORE'S UTOPIA. With Notes by the} Rev. J. Rawson Lumby, D.D., Norrisian Professor of Divinity; late Fellow of St Catharine's College, Cambridge. Price $3 s .6 d$.

"To enthusiasts in history matters, who are not content with mere facts, but like to pursue their investigations behind the scenes, as it were, Professor Rawson Lumby has in the work now before us produced a most acceptable contribution to the now constantly increasing store of illustrative reading."-The Cambridge Review.

"To Dr Lumby we must give praise unqualified and unstinted. He has done his work admirably. ..... Every student of history, every politician, every social reformer, every one interested in literary curiosities, every lover of English should buy and carefully read Dr Lumby's edition of the 'Utopia.' We are afraid to say more lest we should be thought extravagant, and our recommendation accordingly lose part of its force."-The Teacher.

"It was originally written in Latin and does not find a place on ordinary bookshelves. A very great boon has therefore been conferred on the general English reader by the managers of the Pitt Press Series, in the issue of a convenient little volume of More's Utopia not in the original Latin, but in the quaint English Translation thereof made by Raphe Robynson, which adds a linguistic interest to the intrinsic merit of the work. . . All this has been edited in a most complete and scholarly fashion by Dr J. R. Lumby, the Norrisian Professor of Divinity, whose name alone is a sufficient warrant for its accuracy. It is a real addition to the modern stock of classical English literature."-Guardian.

\section{SIR THOMAS MORE'S LIFE OF RICHARD III. With Notes, \&c., by Professor Lumby. \\ [Nearly ready.}

\section{A SKETCH OF ANCIENT PHILOSOPHY FROM} THALES TO CICERO, by Joseph B. MAYor, M.A., Professor of Moral Philosophy at King's College, London. Price 3 . $6 d$.

" It may safely be affirmed that Mr Mayor has successfully accomplished all that he here sets out. His arrangement is admirably methodical, his style is simple but nervous, his knowledge of his subject full and accurate, and his analytical expositions lucid and vivid.... It is therefore a manual which will prove of great utility to University undergraduates, for whom it was particularly prepared, and also for all who study Plato, Aristotle, or other philosophers, in the original. Educated readers, generally, will find it an admirable introduction, or epitome, of ancient speculative thought, and ' a key to our present ways of thinking and judging in regard to matters of the highest importance." "-The British Mail.

"In writing this scholarly and attractive sketch, Professor Mayor has had chiefly in view ' indergraduates at the University or others who are commencing the study of the philosophical works of Cicero or Plato or Aristotle in the original language,' but also hopes that it 'may be found interesting and useful by educated readers generally, not merely as an introduction to the formal history of philosophy, but as supplying a key to our present ways of thinking and judging in regard to matters of the highest importance." "-Mind.

"Professor Mayor contributes to the Pitt Press Series A Sketch of Ancient Philosophy in which he has endeavoured to give a general view of the philosophical systems illustrated by the genius of the masters of metaphysical and ethical science from Thales to Cicero. In the course of his sketch he takes occasion to give concise analyses of Plato's Republic, and of the Ethics and Politics of Aristotle; and these abstracts will be to some readers not the least useful portions of the book. It may be objected against his design in general that ancient philosophy is too vast and too deep a subject to be dismissed in a 'sketch' - that it should be left to those who will make it a serious study. But that objection takes no account of the large class of persons who desire to know, in relation to present discussions and speculations, what famous men in the whole world thought and wrote on these topics. They have not the scholarship which would be necessary for original examination of authorities; but they have an intelligent interest in the relations between ancient and modern philosophy, and need just such information as Professor Mayor's sketch will give them."-The Guardian.

\section{[Other Volumes are in preparation.]}

\section{London: Cambridge Warehouse, I 7 'Paternoster Row.}




\section{aruibrotty of cambrioge.}

\section{IOCAL EXAMINATIONS.}

Examination Papers, for various years, with the Regulations for the

Examination. Demy 8vo. 2s. each, or by Post, $2 s, 2 d$.

Class Lists, for various years, Boys is., Girls $6 d$.

Annual Reports of the Syndicate, with Supplementary Tables showing

the success and failure of the Candidates. $2 s_{0}$ each, by Post $2 s .2 d$.

\section{HIGHER LOCAL EXAMINATIONS.}

Examination Papers for 1881, to which are added the Regulations for I882. Demy 8vo. 2s. each, by Post $2 s .2 d$.

Reports of the Syndicate. Demy 8vo. Is., by Post is. Id.

\section{IOCAL LECTURES SYNDICATE.}

Calendar for the years 1875-9. Fcap. 8vo. cloth. $2 s$.

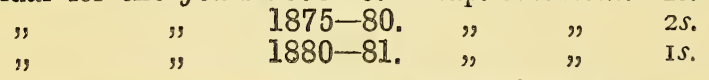

\section{TEACHERS' TRAINING SYNDICATE.}

Examination Papers for 1880 and 1881, to which are added the Regulations for the Examination. Demy 8vo. 6d., by Post $7 d$.

\section{CAMBRIDGE UNIVERSITY REPORTER. Published by Authority.}

Containing all the Official Notices of the University, Reports of Discussions in the Schools, and Proceedings of the Cambridge Philosophical, Antiquarian, and Philological Societies. $3 d$. weekly.

\section{CAMBRIDGE UNIVERSITY EXAMINATION PAPERS.}

These Papers are published in occasional numbers every Term, and in volumes for the Academical year.

Vol. IX. Parts IO5 to II9. PAPERS for the Year I879-80, I2s. cloth. VOL. X. $\quad I 20$ to I38. $", \quad$ I880-8I, I 5s. cloth.

\section{Oxford and Cambridge Schools Examinations.}

Papers set in the Examination for Certificates, July, 1879. Price is. $6 d$. List of Candidates who obtained Certificates at the Examinations held in 1879 and 1880 ; and Supplementary Tables. Price $6 d$. Regulations of the Board for 1882. Price $6 d$.

Report of the Board for the year ending Oct. 31, 1881. Price is.

zlonion: C. J. CLAY, M.A. AND SON. CAMBRIDGE UNIVERSITY PRESS WAREHOUSE, I7 PATERNOSTER ROW. 




